Cochrane Database of Systematic Reviews

\title{
Supportive therapy for schizophrenia (Review)
}

Buckley LA, Maayan N, Soares-Weiser K, Adams CE

Buckley LA, Maayan N, Soares-Weiser K, Adams CE.

Supportive therapy for schizophrenia.

Cochrane Database of Systematic Reviews 2015, Issue 4. Art. No.: CD004716.

DOI: 10.1002/14651858.CD004716.pub4.

www.cochranelibrary.com 
TABLE OF CONTENTS

ABSTRACT

PLAIN LANGUAGE SUMMARY

SUMMARY OF FINDINGS

BACKGROUND

OBJECTIVES

METHODS

RESULTS

Figure 1.

Figure 2.

Figure 3.

DISCUSSION

AUTHORS' CONCLUSIONS

ACKNOWLEDGEMENTS

REFERENCES

CHARACTERISTICS OF STUDIES

DATA AND ANALYSES

Analysis 1.1. Comparison 1 SUPPORTIVE THERAPY versus STANDARD CARE, Outcome 1 Global state: Relapse.

Analysis 1.2. Comparison 1 SUPPORTIVE THERAPY versus STANDARD CARE, Outcome 2 Service outcomes: Hospitalisation. ....

Analysis 1.3. Comparison 1 SUPPORTIVE THERAPY versus STANDARD CARE, Outcome 3 Mental state. 1. No clinically important improvement in general mental state.

Analysis 1.4. Comparison 1 SUPPORTIVE THERAPY versus STANDARD CARE, Outcome 4 Mental state: 2. Average endpoint general mental state score (PANSS, high = poor).

Analysis 1.5. Comparison 1 SUPPORTIVE THERAPY versus STANDARD CARE, Outcome 5 Mental state: 3. Average endpoint negative symptoms score (SANS, skewed data, high = poor).

Analysis 1.6. Comparison 1 SUPPORTIVE THERAPY versus STANDARD CARE, Outcome 6 Mental state: 4. Average endpoint positive symptoms score (PANSS positive subscale, high = poor).

Analysis 1.7. Comparison 1 SUPPORTIVE THERAPY versus STANDARD CARE, Outcome 7 Mental state: 5 . Average endpoint positive symptoms score (BPRS, skewed data, high = poor).

Analysis 1.8. Comparison 1 SUPPORTIVE THERAPY versus STANDARD CARE, Outcome 8 Mental state: 6 . Average endpoint delusions score (PSYRATS delusions score, skewed data, high = poor).

Analysis 1.9. Comparison 1 SUPPORTIVE THERAPY versus STANDARD CARE, Outcome 9 Mental state: 7. Average endpoint hallucinations score (PSYRATS hallucinations score, skewed data, high = poor).

Analysis 1.10. Comparison 1 SUPPORTIVE THERAPY versus STANDARD CARE, Outcome 10 Mental state: 8. Average endpoint depressive symptoms score (CESD, high = poor).

Analysis 1.11. Comparison 1 SUPPORTIVE THERAPY versus STANDARD CARE, Outcome 11 Leaving the study early. ................. Analysis 1.12. Comparison 1 SUPPORTIVE THERAPY versus STANDARD CARE, Outcome 12 General functioning: 2. Average endpoint general functioning score (GAF-M, high $=$ good $)$.

Analysis 1.13. Comparison 1 SUPPORTIVE THERAPY versus STANDARD CARE, Outcome 13 General functioning: 1. Average endpoint general functioning score (GAS, high = good).

Analysis 1.14. Comparison 1 SUPPORTIVE THERAPY versus STANDARD CARE, Outcome 14 General functioning: 3. Average endpoint social functioning score (SFS, high = good).

Analysis 1.15. Comparison 1 SUPPORTIVE THERAPY versus STANDARD CARE, Outcome 15 Satisfaction with treatment: Recipient of care not satisfied with treatment.

Analysis 1.16. Comparison 1 SUPPORTIVE THERAPY versus STANDARD CARE, Outcome 16 Quality of life: 1 . Average endpoint self-esteem score (RSES, high = good).

Analysis 1.17. Comparison 1 SUPPORTIVE THERAPY versus STANDARD CARE, Outcome 17 Quality of life: 2. Average endpoint well-being score (WBS, high = good).

Analysis 1.18. Comparison 1 SUPPORTIVE THERAPY versus STANDARD CARE, Outcome 18 Quality of life: 3 . Average endpoint global health score (GHQ, high = poor).

Analysis 1.19. Comparison 1 SUPPORTIVE THERAPY versus STANDARD CARE, Outcome 19 Death.

Analysis 1.20. Comparison 1 SUPPORTIVE THERAPY versus STANDARD CARE, Outcome 20 Medication: No reduction in dose of antipsychotic medication.

Analysis 2.1. Comparison 2 SUPPORTIVE THERAPY versus ANY OTHER PSYCHOLOGICAL OR PSYCHOSOCIAL TREATMENT, Outcome 1 Global state: 1 . Relapse. 
Analysis 2.2. Comparison 2 SUPPORTIVE THERAPY versus ANY OTHER PSYCHOLOGICAL OR PSYCHOSOCIAL TREATMENT, Outcome 2 Global state: 2 . No remission.

Analysis 2.3. Comparison 2 SUPPORTIVE THERAPY versus ANY OTHER PSYCHOLOGICAL OR PSYCHOSOCIAL TREATMENT, Outcome 3 Service outcomes: Hospitalisation.

Analysis 2.4. Comparison 2 SUPPORTIVE THERAPY versus ANY OTHER PSYCHOLOGICAL OR PSYCHOSOCIAL TREATMENT, Outcome 4 Mental state: 1 . No clinically important improvement in general mental state.

Analysis 2.5. Comparison 2 SUPPORTIVE THERAPY versus ANY OTHER PSYCHOLOGICAL OR PSYCHOSOCIAL TREATMENT, Outcome 5 Mental state: 2. Average endpoint general mental state score (PANSS, high = poor).

Analysis 2.6. Comparison 2 SUPPORTIVE THERAPY versus ANY OTHER PSYCHOLOGICAL OR PSYCHOSOCIAL TREATMENT, Outcome 6 Mental state: 3. Average endpoint general mental state score (BPRS, high = poor).

Analysis 2.7. Comparison 2 SUPPORTIVE THERAPY versus ANY OTHER PSYCHOLOGICAL OR PSYCHOSOCIAL TREATMENT, Outcome 7 Mental state: 4. Average endpoint general mental state score (BPRS short form, high = poor).

Analysis 2.8. Comparison 2 SUPPORTIVE THERAPY versus ANY OTHER PSYCHOLOGICAL OR PSYCHOSOCIAL TREATMENT, Outcome 8 Mental state: 5. Average endpoint general mental state score (CPRS, skewed data, high = poor).

Analysis 2.9. Comparison 2 SUPPORTIVE THERAPY versus ANY OTHER PSYCHOLOGICAL OR PSYCHOSOCIAL TREATMENT, Outcome 9 Mental state: 6 . Average endpoint general mental state score (PANSS general subscale, high $=$ poor).

Analysis 2.10. Comparison 2 SUPPORTIVE THERAPY versus ANY OTHER PSYCHOLOGICAL OR PSYCHOSOCIAL TREATMENT, Outcome 10 Mental state: 7 . No clinically important improvement in negative symptoms.

Analysis 2.11. Comparison 2 SUPPORTIVE THERAPY versus ANY OTHER PSYCHOLOGICAL OR PSYCHOSOCIAL TREATMENT, Outcome 11 Mental state: 8 . No clinically important improvement in depressive symptoms.

Analysis 2.12. Comparison 2 SUPPORTIVE THERAPY versus ANY OTHER PSYCHOLOGICAL OR PSYCHOSOCIAL TREATMENT, Outcome 12 Mental state: 9. Episode of affective symptoms.

Analysis 2.13. Comparison 2 SUPPORTIVE THERAPY versus ANY OTHER PSYCHOLOGICAL OR PSYCHOSOCIAL TREATMENT, Outcome 13 Mental state: 10. Average endpoint negative symptoms score (PANSS negative, high=poor).

Analysis 2.14. Comparison 2 SUPPORTIVE THERAPY versus ANY OTHER PSYCHOLOGICAL OR PSYCHOSOCIAL TREATMENT, Outcome 14 Mental state: 11. Average endpoint negative symptoms score (SANS, high=poor).

Analysis 2.15. Comparison 2 SUPPORTIVE THERAPY versus ANY OTHER PSYCHOLOGICAL OR PSYCHOSOCIAL TREATMENT, Outcome 15 Mental state: 12. Average endpoint negative symptoms score (SANS, skewed data, high = poor).

Analysis 2.16. Comparison 2 SUPPORTIVE THERAPY versus ANY OTHER PSYCHOLOGICAL OR PSYCHOSOCIAL TREATMENT, Outcome 16 Mental state: 13. Average endpoint positive symptoms score (PANSS positive subscale, high $=$ poor).

Analysis 2.17. Comparison 2 SUPPORTIVE THERAPY versus ANY OTHER PSYCHOLOGICAL OR PSYCHOSOCIAL TREATMENT, Outcome 17 Mental state: 14. Average endpoint positive symptoms score (SAPS, skewed data, high = poor).

Analysis 2.18. Comparison 2 SUPPORTIVE THERAPY versus ANY OTHER PSYCHOLOGICAL OR PSYCHOSOCIAL TREATMENT, Outcome 18 Mental state: 15. Average endpoint positive symptoms score (BPRS, skewed data, high = poor).

Analysis 2.19. Comparison 2 SUPPORTIVE THERAPY versus ANY OTHER PSYCHOLOGICAL OR PSYCHOSOCIAL TREATMENT, Outcome 19 Mental state: 16. Average endpoint score (PANSS thought disturbance cluster, high = poor).

Analysis 2.20. Comparison 2 SUPPORTIVE THERAPY versus ANY OTHER PSYCHOLOGICAL OR PSYCHOSOCIAL TREATMENT, Outcome 20 Mental state: 17. Average endpoint delusions score (PSYRATS delusions score, skewed data, high $=$ poor). ..........

Analysis 2.21. Comparison 2 SUPPORTIVE THERAPY versus ANY OTHER PSYCHOLOGICAL OR PSYCHOSOCIAL TREATMENT, Outcome 21 Mental state: 18. Average endpoint hallucinations score (PSYRATS hallucinations score, skewed data, high = poor).

Analysis 2.22. Comparison 2 SUPPORTIVE THERAPY versus ANY OTHER PSYCHOLOGICAL OR PSYCHOSOCIAL TREATMENT, Outcome 22 Mental state: 19. Average endpoint voices score (PSYRATS voices score, high = poor).

Analysis 2.23. Comparison 2 SUPPORTIVE THERAPY versus ANY OTHER PSYCHOLOGICAL OR PSYCHOSOCIAL TREATMENT, Outcome 23 Mental state: 20. Average endpoint beliefs about voices score (BAVQ, high = poor, skewed data).

Analysis 2.24. Comparison 2 SUPPORTIVE THERAPY versus ANY OTHER PSYCHOLOGICAL OR PSYCHOSOCIAL TREATMENT, Outcome 24 Mental state: 21 . Average endpoint depression score (MADRS, skewed data, high = poor).

Analysis 2.25. Comparison 2 SUPPORTIVE THERAPY versus ANY OTHER PSYCHOLOGICAL OR PSYCHOSOCIAL TREATMENT, Outcome 25 Mental state: 22. Average endpoint depression score (BDI-II, skewed data, high = poor).

Analysis 2.26. Comparison 2 SUPPORTIVE THERAPY versus ANY OTHER PSYCHOLOGICAL OR PSYCHOSOCIAL TREATMENT, Outcome 26 Leaving the study early.

Analysis 2.27. Comparison 2 SUPPORTIVE THERAPY versus ANY OTHER PSYCHOLOGICAL OR PSYCHOSOCIAL TREATMENT, Outcome 27 General functioning: 1. Average endpoint general functioning score (GAF \& GAS, high = good).

Analysis 2.28. Comparison 2 SUPPORTIVE THERAPY versus ANY OTHER PSYCHOLOGICAL OR PSYCHOSOCIAL TREATMENT, Outcome 28 General functioning: 2. Average endpoint social functioning score (SFS, high = good).

Analysis 2.29. Comparison 2 SUPPORTIVE THERAPY versus ANY OTHER PSYCHOLOGICAL OR PSYCHOSOCIAL TREATMENT, Outcome 29 General functioning: 3. No paid work. 
Analysis 2.30. Comparison 2 SUPPORTIVE THERAPY versus ANY OTHER PSYCHOLOGICAL OR PSYCHOSOCIAL TREATMENT, Outcome 30 General functioning: 4. Admission to residential placement.

Analysis 2.31. Comparison 2 SUPPORTIVE THERAPY versus ANY OTHER PSYCHOLOGICAL OR PSYCHOSOCIAL TREATMENT, Outcome 31 General functioning: 5. Admission to jail.

Analysis 2.32. Comparison 2 SUPPORTIVE THERAPY versus ANY OTHER PSYCHOLOGICAL OR PSYCHOSOCIAL TREATMENT, Outcome 32 Satisfaction with treatment: Recipient of care not satisfied with treatment.

Analysis 2.33. Comparison 2 SUPPORTIVE THERAPY versus ANY OTHER PSYCHOLOGICAL OR PSYCHOSOCIAL TREATMENT, Outcome 33 Quality of life: Average endpoint quality of life score (QLS, high = good).

Analysis 2.34. Comparison 2 SUPPORTIVE THERAPY versus ANY OTHER PSYCHOLOGICAL OR PSYCHOSOCIAL TREATMENT, Outcome 34 Death.

Analysis 2.35. Comparison 2 SUPPORTIVE THERAPY versus ANY OTHER PSYCHOLOGICAL OR PSYCHOSOCIAL TREATMENT, Outcome 35 Behaviour: 1 . Social impairment on SBAS.

Analysis 2.36. Comparison 2 SUPPORTIVE THERAPY versus ANY OTHER PSYCHOLOGICAL OR PSYCHOSOCIAL TREATMENT, Outcome 36 Behaviour: 2. Poor coping style with relatives.

Analysis 2.37. Comparison 2 SUPPORTIVE THERAPY versus ANY OTHER PSYCHOLOGICAL OR PSYCHOSOCIAL TREATMENT, Outcome 37 Engagement with services: Poor attendance at appointments.

Analysis 2.38. Comparison 2 SUPPORTIVE THERAPY versus ANY OTHER PSYCHOLOGICAL OR PSYCHOSOCIAL TREATMENT, Outcome 38 Insight: Average endpoint insight score (SAI, high = good, skewed data).

Analysis 2.39. Comparison 2 SUPPORTIVE THERAPY versus ANY OTHER PSYCHOLOGICAL OR PSYCHOSOCIAL TREATMENT, Outcome 39 Compliance: 1 . Poor compliance to therapy.

Analysis 2.40. Comparison 2 SUPPORTIVE THERAPY versus ANY OTHER PSYCHOLOGICAL OR PSYCHOSOCIAL TREATMENT, Outcome 40 Compliance: 2 . adherence to medication (self-report).

Analysis 2.41. Comparison 2 SUPPORTIVE THERAPY versus ANY OTHER PSYCHOLOGICAL OR PSYCHOSOCIAL TREATMENT, Outcome 41 Compliance: 3 . average endpoint adherence score (ROMI, high = good).

Analysis 2.42. Comparison 2 SUPPORTIVE THERAPY versus ANY OTHER PSYCHOLOGICAL OR PSYCHOSOCIAL TREATMENT, Outcome 42 Compliance: 4. average endpoint non-adherence score (ROMI, high = poor).

Analysis 2.43. Comparison 2 SUPPORTIVE THERAPY versus ANY OTHER PSYCHOLOGICAL OR PSYCHOSOCIAL TREATMENT, Outcome 43 Medication: Prescribed IM depot medication.

Analysis 2.44. Comparison 2 SUPPORTIVE THERAPY versus ANY OTHER PSYCHOLOGICAL OR PSYCHOSOCIAL TREATMENT, Outcome 44 Attitude to medication: 1. Average endpoint attitude to medication score (AMQ, high = good).

Analysis 2.45. Comparison 2 SUPPORTIVE THERAPY versus ANY OTHER PSYCHOLOGICAL OR PSYCHOSOCIAL TREATMENT, Outcome 45 Attitude to medication: 2. Average endpoint attitude to medication score (DAl, high = good).

Analysis 2.46. Comparison 2 SUPPORTIVE THERAPY versus ANY OTHER PSYCHOLOGICAL OR PSYCHOSOCIAL TREATMENT, Outcome 46 Economic outcomes: Direct costs (skewed data, not ITT).

Analysis 3.1. Comparison 3 SUPPORTIVE THERAPY versus COGNITIVE BEHAVIOURAL THERAPY, Outcome 1 Global state: Relapse.

Analysis 3.2. Comparison 3 SUPPORTIVE THERAPY versus COGNITIVE BEHAVIOURAL THERAPY, Outcome 2 Service outcomes: Hospitalisation.

Analysis 3.3. Comparison 3 SUPPORTIVE THERAPY versus COGNITIVE BEHAVIOURAL THERAPY, Outcome 3 Mental state: 1 . No clinically important improvement in general mental state.

Analysis 3.4. Comparison 3 SUPPORTIVE THERAPY versus COGNITIVE BEHAVIOURAL THERAPY, Outcome 4 Mental state: 2. Average endpoint general mental state score (PANSS total, high = poor).

Analysis 3.5. Comparison 3 SUPPORTIVE THERAPY versus COGNITIVE BEHAVIOURAL THERAPY, Outcome 5 Mental state: 3. Average endpoint general mental state score (BPRS, high = poor).

Analysis 3.6. Comparison 3 SUPPORTIVE THERAPY versus COGNITIVE BEHAVIOURAL THERAPY, Outcome 6 Mental state: 4. Average endpoint general mental state score (BPRS short form, high = poor).

Analysis 3.7. Comparison 3 SUPPORTIVE THERAPY versus COGNITIVE BEHAVIOURAL THERAPY, Outcome 7 Mental state: 5. Average endpoint general mental state score (CPRS, skewed data, high = poor).

Analysis 3.8. Comparison 3 SUPPORTIVE THERAPY versus COGNITIVE BEHAVIOURAL THERAPY, Outcome 8 Mental state: 6. Average endpoint general mental state score (PANSS general subscale, high = poor).

Analysis 3.9. Comparison 3 SUPPORTIVE THERAPY versus COGNITIVE BEHAVIOURAL THERAPY, Outcome 9 Mental state: 7 . No clinically important improvement in negative symptoms.

Analysis 3.10. Comparison 3 SUPPORTIVE THERAPY versus COGNITIVE BEHAVIOURAL THERAPY, Outcome 10 Mental state: 8 . No clinically important improvement in depressive symptoms.

Analysis 3.11. Comparison 3 SUPPORTIVE THERAPY versus COGNITIVE BEHAVIOURAL THERAPY, Outcome 11 Mental state: 9. Episode of affective symptoms. 
Analysis 3.12. Comparison 3 SUPPORTIVE THERAPY versus COGNITIVE BEHAVIOURAL THERAPY, Outcome 12 Mental state: 10. Average endpoint negative symptoms score (PANSS negative subscale, high = poor).

Analysis 3.13. Comparison 3 SUPPORTIVE THERAPY versus COGNITIVE BEHAVIOURAL THERAPY, Outcome 13 Mental state: 11. Average endpoint negative symptoms score (SANS, high = poor).

Analysis 3.14. Comparison 3 SUPPORTIVE THERAPY versus COGNITIVE BEHAVIOURAL THERAPY, Outcome 14 Mental state: 12. Average endpoint negative symptoms score (SANS, skewed data, high = poor).

Analysis 3.15. Comparison 3 SUPPORTIVE THERAPY versus COGNITIVE BEHAVIOURAL THERAPY, Outcome 15 Mental state: 13. Average endpoint positive symptoms score (PANSS positive subscale, high = poor).

Analysis 3.16. Comparison 3 SUPPORTIVE THERAPY versus COGNITIVE BEHAVIOURAL THERAPY, Outcome 16 Mental state: 14. Average endpoint positive symptoms score (SAPS, skewed data, high = poor).

Analysis 3.17. Comparison 3 SUPPORTIVE THERAPY versus COGNITIVE BEHAVIOURAL THERAPY, Outcome 17 Mental state: 15. Average endpoint positive symptoms score (BPRS, skewed data, high = poor).

Analysis 3.18. Comparison 3 SUPPORTIVE THERAPY versus COGNITIVE BEHAVIOURAL THERAPY, Outcome 18 Mental state: 16. Average endpoint score (PANSS thought disturbance cluster, high = poor).

Analysis 3.19. Comparison 3 SUPPORTIVE THERAPY versus COGNITIVE BEHAVIOURAL THERAPY, Outcome 19 Mental state: 17. Average endpoint delusions score (PSYRATS delusions score, skewed data, high = poor).

Analysis 3.20. Comparison 3 SUPPORTIVE THERAPY versus COGNITIVE BEHAVIOURAL THERAPY, Outcome 20 Mental state: 18. Average endpoint hallucinations score (PSYRATS hallucinations score, skewed data, high = poor).

Analysis 3.21. Comparison 3 SUPPORTIVE THERAPY versus COGNITIVE BEHAVIOURAL THERAPY, Outcome 21 Mental state: 19. Average endpoint voices score (PSYRATS voices score, high = poor).

Analysis 3.22. Comparison 3 SUPPORTIVE THERAPY versus COGNITIVE BEHAVIOURAL THERAPY, Outcome 22 Mental state: 20. Average endpoint beliefs about voices score (BAVQ, high = poor, skewed data).

Analysis 3.23. Comparison 3 SUPPORTIVE THERAPY versus COGNITIVE BEHAVIOURAL THERAPY, Outcome 23 Mental state: 21. Average endpoint depression score (MADRS, skewed data, high = poor).

Analysis 3.24. Comparison 3 SUPPORTIVE THERAPY versus COGNITIVE BEHAVIOURAL THERAPY, Outcome 24 Mental state: 22. Average endpoint depression score (BDI-II, skewed data, high = poor).

Analysis 3.25. Comparison 3 SUPPORTIVE THERAPY versus COGNITIVE BEHAVIOURAL THERAPY, Outcome 25 Leaving the study early.

Analysis 3.26. Comparison 3 SUPPORTIVE THERAPY versus COGNITIVE BEHAVIOURAL THERAPY, Outcome 26 General functioning: 1. Average endpoint general functioning score (GAF \& GAS, high = good).

Analysis 3.27. Comparison 3 SUPPORTIVE THERAPY versus COGNITIVE BEHAVIOURAL THERAPY, Outcome 27 General functioning: 2. Average endpoint social functioning score (SFS, high = good).

Analysis 3.28. Comparison 3 SUPPORTIVE THERAPY versus COGNITIVE BEHAVIOURAL THERAPY, Outcome 28 Satisfaction with treatment: Recipient of care not satisfied with treatment.

Analysis 3.29. Comparison 3 SUPPORTIVE THERAPY versus COGNITIVE BEHAVIOURAL THERAPY, Outcome 29 Quality of life: Average endpoint score (RSES, high $=$ good).

Analysis 3.30. Comparison 3 SUPPORTIVE THERAPY versus COGNITIVE BEHAVIOURAL THERAPY, Outcome 30 Death. .............. Analysis 3.31. Comparison 3 SUPPORTIVE THERAPY versus COGNITIVE BEHAVIOURAL THERAPY, Outcome 31 Insight:1. Average endpoint insight score (SAI, skewed data, high = good).

Analysis 3.32. Comparison 3 SUPPORTIVE THERAPY versus COGNITIVE BEHAVIOURAL THERAPY, Outcome 32 Insight: 2 . Average endpoint insight score (BCIS composite, skewed data, high = good).

Analysis 3.33. Comparison 3 SUPPORTIVE THERAPY versus COGNITIVE BEHAVIOURAL THERAPY, Outcome 33 Medication: no reduction in dose of antipsychotic medication.

Analysis 3.34. Comparison 3 SUPPORTIVE THERAPY versus COGNITIVE BEHAVIOURAL THERAPY, Outcome 34 Attitude to medication: 1. Average endpoint attitude to medication score (AMQ, high = good).

Analysis 3.35. Comparison 3 SUPPORTIVE THERAPY versus COGNITIVE BEHAVIOURAL THERAPY, Outcome 35 Attitude to medication: 2. Average endpoint attitude to medication score (DAl, high = good).

Analysis 3.36. Comparison 3 SUPPORTIVE THERAPY versus COGNITIVE BEHAVIOURAL THERAPY, Outcome 36 Economic outcomes: Direct costs (skewed data).

Analysis 4.1. Comparison 4 SUPPORTIVE THERAPY versus FAMILY THERAPY, Outcome 1 Global state: 1 . Relapse. .................... Analysis 4.2. Comparison 4 SUPPORTIVE THERAPY versus FAMILY THERAPY, Outcome 2 Global state: 2 . No remission. ............ Analysis 4.3. Comparison 4 SUPPORTIVE THERAPY versus FAMILY THERAPY, Outcome 3 Service outcomes: Hospitalisation. ..... Analysis 4.4. Comparison 4 SUPPORTIVE THERAPY versus FAMILY THERAPY, Outcome 4 Mental state: Episode of affective symptoms.

Analysis 4.5. Comparison 4 SUPPORTIVE THERAPY versus FAMILY THERAPY, Outcome 5 Leaving the study early (by long term). Analysis 4.6. Comparison 4 SUPPORTIVE THERAPY versus FAMILY THERAPY, Outcome 6 General functioning: 1. No paid work. . . 
Analysis 4.7. Comparison 4 SUPPORTIVE THERAPY versus FAMILY THERAPY, Outcome 7 General functioning: 2. Admission to residential placement.

Analysis 4.8. Comparison 4 SUPPORTIVE THERAPY versus FAMILY THERAPY, Outcome 8 General fuctioning: 3. Admission to jail.

Analysis 4.9. Comparison 4 SUPPORTIVE THERAPY versus FAMILY THERAPY, Outcome 9 Behaviour: 1. Social impairment on SBAS.

Analysis 4.10. Comparison 4 SUPPORTIVE THERAPY versus FAMILY THERAPY, Outcome 10 Behaviour: 2. Poor coping style with relatives.

Analysis 4.11. Comparison 4 SUPPORTIVE THERAPY versus FAMILY THERAPY, Outcome 11 Engagement with services: Poor attendance at appointments.

Analysis 4.12. Comparison 4 SUPPORTIVE THERAPY versus FAMILY THERAPY, Outcome 12 Compliance: Poor compliance to therapy.

Analysis 4.13. Comparison 4 SUPPORTIVE THERAPY versus FAMILY THERAPY, Outcome 13 Medication: Prescribed IM depot medication.

Analysis 5.1. Comparison 5 SUPPORTIVE THERAPY versus PSYCHOEDUCATION, Outcome 1 Service outcomes: Hospitalisation.

Analysis 5.2. Comparison 5 SUPPORTIVE THERAPY versus PSYCHOEDUCATION, Outcome 2 Mental state. 1. No clinically important improvement in general mental state.

Analysis 5.3. Comparison 5 SUPPORTIVE THERAPY versus PSYCHOEDUCATION, Outcome 3 Mental state: 2. Average endpoint general score (PANSS general subscale, high = poor).

Analysis 5.4. Comparison 5 SUPPORTIVE THERAPY versus PSYCHOEDUCATION, Outcome 4 Mental state: 3. Average endpoint negative symptoms score (PANSS negative subscale, high = poor).

Analysis 5.5. Comparison 5 SUPPORTIVE THERAPY versus PSYCHOEDUCATION, Outcome 5 Mental state: 4. Average endpoint positive symptoms score (PANSS positive subscale, high = poor).

Analysis 5.6. Comparison 5 SUPPORTIVE THERAPY versus PSYCHOEDUCATION, Outcome 6 Mental state: 5. Average endpoint depression score (CDRS, high = poor).

Analysis 5.7. Comparison 5 SUPPORTIVE THERAPY versus PSYCHOEDUCATION, Outcome 7 Leaving the study early. ...............

Analysis 5.8. Comparison 5 SUPPORTIVE THERAPY versus PSYCHOEDUCATION, Outcome 8 Quality of life: Average endpoint quality of life score (QLS, high $=$ good).

Analysis 5.9. Comparison 5 SUPPORTIVE THERAPY versus PSYCHOEDUCATION, Outcome 9 Death.

Analysis 5.10. Comparison 5 SUPPORTIVE THERAPY versus PSYCHOEDUCATION, Outcome 10 Behaviour: Average endpoint score (composed of ROMI and ITAQ items, high = good).

Analysis 5.11. Comparison 5 SUPPORTIVE THERAPY versus PSYCHOEDUCATION, Outcome 11 Insight: Average endpoint treatment attitude score (ITAQ, high = good).

Analysis 5.12. Comparison 5 SUPPORTIVE THERAPY versus PSYCHOEDUCATION, Outcome 12 Compliance: 1 . Poor compliance to therapy.

Analysis 5.13. Comparison 5 SUPPORTIVE THERAPY versus PSYCHOEDUCATION, Outcome 13 Compliance: 2 . Adherence to medication (self-report).

Analysis 5.14. Comparison 5 SUPPORTIVE THERAPY versus PSYCHOEDUCATION, Outcome 14 Compliance: 3 . Average endpoint adherence score (ROMI, high = good).

Analysis 5.15. Comparison 5 SUPPORTIVE THERAPY versus PSYCHOEDUCATION, Outcome 15 Compliance: 4. Average endpoint nonadherence score (ROMI, high = poor).

Analysis 6.1. Comparison 6 SUPPORTIVE THERAPY versus REHABILITATION PROGRAMME, Outcome 1 Service outcomes: Hospitalisation.

Analysis 6.2. Comparison 6 SUPPORTIVE THERAPY versus REHABILITATION PROGRAMME, Outcome 2 Leaving the study early. . Analysis 6.3. Comparison 6 SUPPORTIVE THERAPY versus REHABILITATION PROGRAMME, Outcome 3 General functioning: No gainful employment.

Analysis 7.1. Comparison 7 SUPPORTIVE THERAPY versus SKILLS TRAINING, Outcome 1 Service outcomes: Hospitalisation. ... Analysis 7.2. Comparison 7 SUPPORTIVE THERAPY versus SKILLS TRAINING, Outcome 2 Leaving the study early. ................... Analysis 7.3. Comparison 7 SUPPORTIVE THERAPY versus SKILLS TRAINING, Outcome 3 Death.

Analysis 8.1. Comparison 8 SUPPORTIVE THERAPY versus PSYCHODYNAMIC PSYCHOTHERAPY, Outcome 1 Leaving the study early.

Analysis 9.1. Comparison 9 SUPPORTIVE THERAPY versus COMBINATION OF OTHER PSYCHOSOCIAL INTERVENTIONS, OUtCome 1 Global state: Relapse.

Analysis 9.2. Comparison 9 SUPPORTIVE THERAPY versus COMBINATION OF OTHER PSYCHOSOCIAL INTERVENTIONS, OUtCome 2 Mental state: Episode of affective symptoms.

Analysis 9.3. Comparison 9 SUPPORTIVE THERAPY versus COMBINATION OF OTHER PSYCHOSOCIAL INTERVENTIONS, Outcome 3 Leaving the study early: Treatment-related reasons. 
Analysis 10.1. Comparison 10 SUPPORTIVE THERAPY PLUS CLIENT-FOCUSED CASE MANAGEMENT versus CLIENT-FOCUSED CASE MANAGEMENT, Outcome 1 Global state: Relapse.

Analysis 10.2. Comparison 10 SUPPORTIVE THERAPY PLUS CLIENT-FOCUSED CASE MANAGEMENT versus CLIENT-FOCUSED CASE MANAGEMENT, Outcome 2 Leaving the study early.

Analysis 10.3. Comparison 10 SUPPORTIVE THERAPY PLUS CLIENT-FOCUSED CASE MANAGEMENT versus CLIENT-FOCUSED CASE MANAGEMENT, Outcome 3 Death.

Analysis 11.1. Comparison 11 SUPPORTIVE THERAPY PLUS CLIENT-FOCUSED CASE MANAGEMENT versus STANDARD CASE MANGEMENT, Outcome 1 Leaving the study early.

Analysis 11.2. Comparison 11 SUPPORTIVE THERAPY PLUS CLIENT-FOCUSED CASE MANAGEMENT versus STANDARD CASE MANGEMENT, Outcome 2 Death.

Analysis 12.1. Comparison 12 SUPPORTIVE THERAPY PLUS SKILLS TRAINING versus SKILLS TRAINING, Outcome 1 Global state: 1. Relapse.

Analysis 12.2. Comparison 12 SUPPORTIVE THERAPY PLUS SKILLS TRAINING versus SKILLS TRAINING, Outcome 2 Global state: 2. No remission.

Analysis 12.3. Comparison 12 SUPPORTIVE THERAPY PLUS SKILLS TRAINING versus SKILLS TRAINING, Outcome 3 Service outcomes: Not discharged from hospital.

Analysis 12.4. Comparison 12 SUPPORTIVE THERAPY PLUS SKILLS TRAINING versus SKILLS TRAINING, Outcome 4 Mental state: 1. Average endpoint score for 'inability to feel' (CPRS, high = poor).

Analysis 12.5. Comparison 12 SUPPORTIVE THERAPY PLUS SKILLS TRAINING versus SKILLS TRAINING, Outcome 5 Mental state: 2. Average endpoint score for 'derealisation' (CPRS, skewed data, high = poor).

Analysis 12.6. Comparison 12 SUPPORTIVE THERAPY PLUS SKILLS TRAINING versus SKILLS TRAINING, Outcome 6 Leaving the study early.

Analysis 12.7. Comparison 12 SUPPORTIVE THERAPY PLUS SKILLS TRAINING versus SKILLS TRAINING, Outcome 7 General functioning: 1. Average endpoint score for 'free time activities' (KAS, high = good).

Analysis 12.8. Comparison 12 SUPPORTIVE THERAPY PLUS SKILLS TRAINING versus SKILLS TRAINING, Outcome 8 General functioning: 2. Average endpoint score for 'withdrawal' (KAS, skewed data, high = good).

Analysis 12.9. Comparison 12 SUPPORTIVE THERAPY PLUS SKILLS TRAINING versus SKILLS TRAINING, Outcome 9 Death. ...... ADDITIONAL TABLES

APPENDICES

WHAT'S NEW

HISTORY

CONTRIBUTIONS OF AUTHORS 
[Intervention Review]

\section{Supportive therapy for schizophrenia}

Lucy A Buckley ${ }^{1}$, Nicola Maayan², Karla Soares-Weiser ${ }^{3}$, Clive E Adams ${ }^{4}$

1Sunderland Psychotherapy Service, Northumberland, Tyne and Wear NHS Foundation Trust, Sunderland, UK. 2Cochrane Response, Cochrane, London, UK. ${ }^{3}$ Cochrane Editorial Unit, Cochrane, London, UK. ${ }^{4}$ Cochrane Schizophrenia Group, The University of Nottingham, Nottingham, UK

Contact: Lucy A Buckley, Sunderland Psychotherapy Service, Northumberland, Tyne and Wear NHS Foundation Trust, Cherry Knowle Hospital, Upper Poplars, Ryhope, Sunderland, Tyne and Wear, SR2 0NB, UK. Lucy.Buckley@ntw.nhs.uk.

Editorial group: Cochrane Schizophrenia Group.

Publication status and date: Edited (no change to conclusions), published in Issue 11, 2017.

Citation: Buckley LA, Maayan N, Soares-Weiser K, Adams CE. Supportive therapy for schizophrenia. Cochrane Database of Systematic Reviews 2015, Issue 4. Art. No.: CD004716. DOI: 10.1002/14651858.CD004716.pub4.

Copyright @ 2017 The Cochrane Collaboration. Published by John Wiley \& Sons, Ltd.

\section{A B S T R A C T}

\section{Background}

Supportive therapy is often used in everyday clinical care and in evaluative studies of other treatments.

\section{Objectives}

To review the effects of supportive therapy compared with standard care, or other treatments in addition to standard care for people with schizophrenia.

\section{Search methods}

For this update, we searched the Cochrane Schizophrenia Group's register of trials (November 2012).

\section{Selection criteria}

All randomised trials involving people with schizophrenia and comparing supportive therapy with any other treatment or standard care.

\section{Data collection and analysis}

We reliably selected studies, quality rated these and extracted data. For dichotomous data, we estimated the risk ratio (RR) using a fixedeffect model with 95\% confidence intervals (Cls). Where possible, we undertook intention-to-treat analyses. For continuous data, we estimated the mean difference (MD) fixed-effect with $95 \% \mathrm{Cls}$. We estimated heterogeneity ( 12 technique) and publication bias. We used GRADE to rate quality of evidence.

\section{Main results}

Four new trials were added after the 2012 search. The review now includes 24 relevant studies, with 2126 participants. Overall, the evidence was very low quality.

We found no significant differences in the primary outcomes of relapse, hospitalisation and general functioning between supportive therapy and standard care.

There were, however, significant differences favouring other psychological or psychosocial treatments over supportive therapy. These included hospitalisation rates ( $4 \mathrm{RCTs}, \mathrm{n}=306$, RR $1.82 \mathrm{Cl} 1.11$ to 2.99 , very low quality of evidence), clinical improvement in mental state ( 3 RCTs, $n=194$, RR $1.27 \mathrm{Cl} 1.04$ to 1.54 , very low quality of evidence) and satisfaction of treatment for the recipient of care ( $1 \mathrm{RCT}, \mathrm{n}=45, \mathrm{RR}$ $3.19 \mathrm{Cl} 1.01$ to 10.7 , very low quality of evidence). For this comparison, we found no evidence of significant differences for rate of relapse, leaving the study early and quality of life. 
When we compared supportive therapy to cognitive behavioural therapy CBT), we again found no significant differences in primary outcomes. There were very limited data to compare supportive therapy with family therapy and psychoeducation, and no studies provided data regarding clinically important change in general functioning, one of our primary outcomes of interest.

\section{Authors' conclusions}

There are insufficient data to identify a difference in outcome between supportive therapy and standard care. There are several outcomes, including hospitalisation and general mental state, indicating advantages for other psychological therapies over supportive therapy but these findings are based on a few small studies where we graded the evidence as very low quality. Future research would benefit from larger trials that use supportive therapy as the main treatment arm rather than the comparator.

\section{PLAIN LANGUAGE SUMMARY}

\section{Supportive therapy for schizophrenia}

Schizophrenia is a severe mental illness with 'positive symptoms' such as hallucinations (hearing voices and seeing things) and delusions (having strange beliefs). People with schizophrenia also suffer from disorganisation and 'negative symptoms' (such as tiredness, apathy and loss of emotion). People with schizophrenia may find it hard to socialise and find employment. Schizophrenia is considered one of the most burdensome illnesses in the world. For some people it can be a lifelong condition. Most people with schizophrenia will be given antipsychotic medications to help relieve the symptoms. In addition to this they can also receive therapy, of which there are various types.

One therapy often given to people with schizophrenia is supportive therapy, where typically after a person is established in the care of mental health services, they will receive general support rather than specific talking therapies such as cognitive behavioural therapy (CBT). For example, in consultations with health professionals there will often be time given to listening to people's concerns, providing encouragement, or even arranging basic help with daily living. Many people with schizophrenia also receive support from their family and friends. Supportive therapy has been described as the treatment of choice for most people with mental illness and may be one of the most commonly practiced therapies in mental health services.

It is, however, difficult to answer the question of exactly what supportive therapy is. It is difficult to find a widely accepted definition of supportive therapy. For the purposes of this review, supportive therapy includes any intervention from a single person aimed at maintaining a person's existing situation or assisting in people's coping abilities. This includes interventions that require a trained therapist, such as supportive psychotherapy, as well as other interventions that require no training, such as 'befriending'. Supportive therapy does not include interventions that seek to educate, train or change a person's way of coping.

The aim of this review is to assess the effectiveness of supportive therapy compared to other specific therapies or treatment as usual. This update is based on a search run in 2012; the review now includes 24 randomised studies with a total of 2126 people. The studies compared supportive therapy either with standard care alone or a range of other therapies such as CBT, family therapy and psychoeducation. The participants continued to receive their antipsychotic medication and any other treatment they would normally receive during the trials. Overall, the quality of evidence from these studies was very low. There is not enough information or data to identify any real therapeutic difference between supportive therapy and standard care. There are several outcomes, including hospitalisation, satisfaction with treatment and general mental state, indicating advantages for other psychological therapies over supportive therapy. However, these findings are limited because they are based on only a few small studies where the quality of evidence is very low. There was very limited information to compare supportive therapy with family therapy and psychoeducation as most studies in this review focused on other psychological therapies, such as CBT. Apart from one study presenting data on death, there was no information on the adverse effects of supportive therapy. In summary, there does not seem to be much difference between supportive therapy, standard care and other therapies. Future research would benefit from larger studies where supportive therapy is the main treatment.

Ben Gray, Senior Peer Researcher, McPin Foundation: http://mcpin.org/ 


\begin{tabular}{|c|c|c|c|c|c|c|}
\hline \multicolumn{7}{|c|}{$\begin{array}{l}\text { S U M M A R Y O F F I N D I N G S } \\
\text { Summary of findings for the main comparison. SUPPORTIVE THERAPY versus STANDARD CARE for schizophrenia }\end{array}$} \\
\hline \multicolumn{7}{|c|}{ SUPPORTIVE THERAPY versus STANDARD CARE for schizophrenia } \\
\hline \multicolumn{7}{|c|}{$\begin{array}{l}\text { Patient or population: patients with schizophrenia } \\
\text { Settings: inpatients and outpatients } \\
\text { Intervention: SUPPORTIVE THERAPY } \\
\text { Comparison: STANDARD CARE }\end{array}$} \\
\hline \multirow[t]{3}{*}{ Outcomes } & \multicolumn{2}{|c|}{ Illustrative comparative risks* $(95 \% \mathrm{Cl})$} & \multirow{3}{*}{$\begin{array}{l}\text { Relative effect } \\
(95 \% \mathrm{CI})\end{array}$} & \multirow{3}{*}{$\begin{array}{l}\text { No of Partici- } \\
\text { pants } \\
\text { (studies) }\end{array}$} & \multirow{3}{*}{$\begin{array}{l}\text { Quality of the } \\
\text { evidence } \\
\text { (GRADE) }\end{array}$} & \multirow[t]{3}{*}{ Comments } \\
\hline & Assumed risk & Corresponding risk & & & & \\
\hline & Control & $\begin{array}{l}\text { SUPPORTIVE THERAPY versus } \\
\text { STANDARD CARE }\end{array}$ & & & & \\
\hline $\begin{array}{l}\text { Global state: Relapse } \\
\text { Follow-up: } 2 \text { years }\end{array}$ & 321 per 1000 & $\begin{array}{l}309 \text { per } 1000 \\
(141 \text { to } 678)\end{array}$ & $\begin{array}{l}\text { RR } 0.96 \\
(0.44 \text { to } 2.11)\end{array}$ & $\begin{array}{l}54 \\
\text { (1 study) }\end{array}$ & $\begin{array}{l}\oplus \oplus \ominus \ominus \\
\text { very low 1,2, } 3\end{array}$ & \\
\hline $\begin{array}{l}\text { Service outcomes: Hospitalisa- } \\
\text { tion } \\
\text { Follow-up: } 6 \text { months }\end{array}$ & 42 per 1000 & $\begin{array}{l}42 \text { per } 1000 \\
(3 \text { to } 628)\end{array}$ & $\begin{array}{l}\text { RR } 1 \\
(0.07 \text { to } 15.08)\end{array}$ & $\begin{array}{l}48 \\
\text { (1 study) }\end{array}$ & $\begin{array}{l}\oplus \odot \ominus \ominus \\
\text { very low } 1,2,3\end{array}$ & \\
\hline $\begin{array}{l}\text { Mental state: no clinically im- } \\
\text { portant improvement } \\
\text { Follow-up: } 1 \text { to } 2 \text { years }\end{array}$ & 898 per 1000 & $\begin{array}{l}\mathbf{8 5 3} \text { per } 1000 \\
(736 \text { to } 997)\end{array}$ & $\begin{array}{l}\text { RR } 0.95 \\
(0.82 \text { to } 1.11)\end{array}$ & $\begin{array}{l}98 \\
\text { ( } 2 \text { studies) }\end{array}$ & $\begin{array}{l}\oplus \odot \odot \odot \\
\text { very low } 1,2,3\end{array}$ & \\
\hline $\begin{array}{l}\text { Leaving the study early } \\
\text { Follow-up: } 10 \text { weeks to } 2 \text { years }\end{array}$ & 166 per 1000 & $\begin{array}{l}143 \text { per } 1000 \\
\text { (88 to } 232 \text { ) }\end{array}$ & $\begin{array}{l}\text { RR } 0.86 \\
(0.53 \text { to } 1.4)\end{array}$ & $\begin{array}{l}354 \\
\text { (4 studies) }\end{array}$ & $\begin{array}{l}\oplus \ominus \ominus \ominus \\
\text { very low } 1,2,4\end{array}$ & \\
\hline $\begin{array}{l}\text { General functioning } \\
\text { GAS } \\
\text { Follow-up: } 1 \text { years }\end{array}$ & & $\begin{array}{l}\text { The mean general functioning in the } \\
\text { intervention groups was } \\
\mathbf{1 . 4} \text { higher } \\
\text { ( } 5.09 \text { lower to } 7.89 \text { higher) }\end{array}$ & & $\begin{array}{l}29 \\
\text { (1 study) }\end{array}$ & $\begin{array}{l}\oplus \odot \odot \ominus \\
\text { very low } 1,2,5,6\end{array}$ & \\
\hline $\begin{array}{l}\text { Satisfaction with treatment: } \\
\text { Recipient of care not satisfied } \\
\text { with treatment } \\
\text { Follow-up: } 1 \text { years }\end{array}$ & 238 per 1000 & $\begin{array}{l}\mathbf{4 3 6} \text { per } \mathbf{1 0 0 0} \\
(179 \text { to } 1000)\end{array}$ & $\begin{array}{l}\text { RR } 1.83 \\
(0.75 \text { to } 4.47)\end{array}$ & $\begin{array}{l}44 \\
\text { (1 study) }\end{array}$ & $\begin{array}{l}\oplus \odot \odot \ominus \\
\text { very low 1,2,5 }\end{array}$ & \\
\hline
\end{tabular}

Settings: inpatients and outpatients

Comparison: STANDARD CARE 
*The basis for the assumed risk (e.g. the median control group risk across studies) is provided in footnotes. The corresponding risk (and its $95 \%$ confidence interval) is based on the assumed risk in the comparison group and the relative effect of the intervention (and its $95 \% \mathrm{Cl}$ ).

Cl: Confidence interval; RR: Risk ratio;

GRADE Working Group grades of evidence

High quality: Further research is very unlikely to change our confidence in the estimate of effect.

Moderate quality: Further research is likely to have an important impact on our confidence in the estimate of effect and may change the estimate.

Low quality: Further research is very likely to have an important impact on our confidence in the estimate of effect and is likely to change the estimate.

Very low quality: We are very uncertain about the estimate.

1 Imprecision: serious - relatively few participants were included and few events; confidence intervals are wide.

2 Publication bias: strongly suspected - four studies or fewer reported data for this outcome.

3 Risk of bias: serious - one study had an unclear risk of bias for randomisation, allocation concealment and blinding.

4 Risk of bias: serious - one study had an unclear risk of bias for randomisation and allocation concealment; two studies had an unclear risk for blinding.

5 Risk of bias: serious - one study had an unclear risk of bias for blinding.

6 Indirectness: serious - we wanted to collect binary data for this outcome, however, only a proxy scale measure was available.

Summary of findings 2. SUPPORTIVE THERAPY versus ANY OTHER PSYCHOLOGICAL OR PSYCHOSOCIAL TREATMENT fOr SChIZOphrenia

\section{SUPPORTIVE THERAPY verSUS ANY OTHER PSYCHOLOGICAL OR PSYCHOSOCIAL TREATMENT for SChizophrenia}

Patient or population: patients with schizophrenia

Settings: inpatients and outpatients

Intervention: SUPPORTIVE THERAPY

Comparison: ANY OTHER PSYCHOLOGICAL OR PSYCHOSOCIAL TREATMENT

\begin{tabular}{|c|c|c|c|c|c|c|}
\hline \multirow[t]{3}{*}{ Outcomes } & \multicolumn{2}{|c|}{ Illustrative comparative risks ${ }^{\star}(95 \% \mathrm{Cl})$} & \multirow{3}{*}{$\begin{array}{l}\text { Relative effect } \\
(95 \% \mathrm{Cl})\end{array}$} & \multirow{3}{*}{$\begin{array}{l}\text { No of Partici- } \\
\text { pants } \\
\text { (studies) }\end{array}$} & \multirow{3}{*}{$\begin{array}{l}\text { Quality of the } \\
\text { evidence } \\
\text { (GRADE) }\end{array}$} & \multirow[t]{3}{*}{ Comment: } \\
\hline & Assumed risk & Corresponding risk & & & & \\
\hline & & $\begin{array}{l}\text { SUPPORTIVE THERAPY versUS } \\
\text { ANY OTHER PSYCHOLOGICAL } \\
\text { OR PSYCHOSOCIAL TREAT- } \\
\text { MENT }\end{array}$ & & & & \\
\hline
\end{tabular}




\begin{tabular}{|c|c|c|c|c|c|c|}
\hline $\begin{array}{l}\text { Service outcomes: Hospitalisation } \\
\text { Follow-up: } 12 \text { weeks to } 2 \text { years }\end{array}$ & 122 per 1000 & $\begin{array}{l}222 \text { per } 1000 \\
(135 \text { to } 365)\end{array}$ & $\begin{array}{l}\mathbf{R R} 1.82 \\
\text { (1.11 to } 2.99)\end{array}$ & $\begin{array}{l}306 \\
\text { (4 studies) }\end{array}$ & $\begin{array}{l}\oplus \ominus \ominus \ominus \\
\text { very low } 3,4,5\end{array}$ & \\
\hline $\begin{array}{l}\text { Mental state: No clinically important } \\
\text { improvement } \\
\text { Follow-up: } 1 \text { to } 2 \text { years }\end{array}$ & 594 per 1000 & $\begin{array}{l}754 \text { per } 1000 \\
(618 \text { to } 915)\end{array}$ & $\begin{array}{l}\text { RR } 1.27 \\
\text { (1.04 to } 1.54)\end{array}$ & $\begin{array}{l}194 \\
\text { (3 studies) }\end{array}$ & $\begin{array}{l}\oplus \ominus \ominus \ominus \\
\text { very low } 2,3,4,6\end{array}$ & \\
\hline $\begin{array}{l}\text { Leaving the study early } \\
\text { Follow-up: } 10 \text { weeks to } 3 \text { years }\end{array}$ & 249 per 1000 & $\begin{array}{l}\mathbf{2 5 7} \text { per } 1000 \\
\text { (217 to } 302)\end{array}$ & $\begin{array}{l}\text { RR } 1.03 \\
\text { (0.87 to } 1.21)\end{array}$ & $\begin{array}{l}1412 \\
\text { (19 studies) }\end{array}$ & $\begin{array}{l}\oplus \oplus \oplus \ominus \\
\text { moderate } 7\end{array}$ & \\
\hline $\begin{array}{l}\text { General functioning } \\
\text { GAF and GAS } \\
\text { Follow-up: } 12 \text { to } 18 \text { months }\end{array}$ & See comment & See comment & Not estimable & $\begin{array}{l}78 \\
\text { ( } 2 \text { studies) }\end{array}$ & $\begin{array}{l}\oplus \ominus \ominus \ominus \\
\text { very low } 3,8,9,10\end{array}$ & $\begin{array}{l}\text { There was very } \\
\text { high hetero- } \\
\text { geneity for this } \\
\text { outcome so } \\
\text { data were not } \\
\text { pooled. } 11\end{array}$ \\
\hline $\begin{array}{l}\text { Satisfaction with treatment: Recip- } \\
\text { ient of care not satisfied with treat- } \\
\text { ment } \\
\text { Follow-up: } 1 \text { years }\end{array}$ & 136 per 1000 & $\begin{array}{l}435 \text { per } 1000 \\
(138 \text { to } 1000)\end{array}$ & $\begin{array}{l}\text { RR } 3.19 \\
\text { (1.01 to } 10.07)\end{array}$ & $\begin{array}{l}45 \\
\text { (1 study) }\end{array}$ & $\begin{array}{l}\oplus \ominus \ominus \ominus \\
\text { very low } 4,12,13\end{array}$ & \\
\hline $\begin{array}{l}\text { Quality of life } \\
\text { QLS }\end{array}$ & & $\begin{array}{l}\text { The mean quality of life in the } \\
\text { intervention groups was } \\
\mathbf{0 . 0 7} \text { lower } \\
\text { ( } 21.11 \text { lower to } 20.97 \text { higher) }\end{array}$ & & $\begin{array}{l}19 \\
\text { (1 study) }\end{array}$ & $\begin{array}{l}\oplus \ominus \ominus \ominus \\
\text { very low } \\
4,10,13,14\end{array}$ & \\
\hline
\end{tabular}

*The basis for the assumed risk (e.g. the median control group risk across studies) is provided in footnotes. The corresponding risk (and its $95 \%$ confidence interval) is based on the assumed risk in the comparison group and the relative effect of the intervention (and its $95 \% \mathrm{Cl}$ ).

CI: Confidence interval; RR: Risk ratio;

GRADE Working Group grades of evidence

High quality: Further research is very unlikely to change our confidence in the estimate of effect.

Moderate quality: Further research is likely to have an important impact on our confidence in the estimate of effect and may change the estimate.

Low quality: Further research is very likely to have an important impact on our confidence in the estimate of effect and is likely to change the estimate.

Very low quality: We are very uncertain about the estimate.

1 Risk of bias: serious - four studies had an unclear risk of bias for randomisation and allocation concealment. All studies had an unclear risk of bias for blinding of participants and two had a high risk of bias for blinding of outcome assessments.

2 Inconsistency: serious - there was high heterogeneity for this outcome.

3 Imprecision: serious - relatively few participants were included and few events; confidence intervals are wide.

4 Publication bias: strongly suspected - four studies or fewer reported data for this outcome.

5 Risk of bias: serious - three studies had an unclear risk of bias for randomisation and allocation concealment. All studies had an unclear risk of bias for blinding of participants

and two were unclear for blinding of outcome assessments. 
6 Risk of bias: serious - one study had an unclear risk of bias for randomisation. All studies had an unclear risk of bias for blinding of participants and one had an unclear risk of bias for blinding of outcome assessments.

7 Risk of bias: serious - 13 studies had an unclear risk of bias for randomisation and 14 for allocation concealment. Fifteen studies had an unclear risk of bias for blinding of participants and one had a high risk of bias. Three studies two had a high risk of bias for blinding of outcome assessments and eight were unclear. One study had a high risk of bias for incomplete outcome data, and in three it was unclear.

8 Risk of bias: serious - one study had an unclear risk of bias for allocation concealment. Both studies had an unclear risk of bias for blinding of participants and outcome assessments.

9 Inconsistency: very serious - there was high heterogeneity for this outcome and data were not pooled.

10 Indirectness: serious - we wanted to collect binary data for this outcome, however, only a proxy scale measure was available.

11 One study found no difference in general functioning on the GAS, the other study found a difference in favour of supportive therapy on the GAF.

12 Risk of bias: serious - the study had an unclear risk of bias for blinding of participants and outcome assessors.

13 Imprecision: very serious - relatively few participants were included and few events; confidence intervals are very wide.

14 Risk of bias: serious - the study had an unclear risk of bias for blinding of participants and outcome assessments.

Summary of findings 3. SUPPORTIVE THERAPY versus COGNITIVE BEHAVIOURAL THERAPY for schizophrenia

\section{SUPPORTIVE THERAPY versUS COGNITIVE BEHAVIOURAL THERAPY for schizophrenia}

Patient or population: patients with schizophrenia

Settings: inpatients and outpatients

Intervention: SUPPORTIVE THERAPY

Comparison: COGNITIVE BEHAVIOURAL THERAPY

\begin{tabular}{|c|c|c|c|c|c|c|}
\hline \multirow[t]{2}{*}{ Outcomes } & \multicolumn{2}{|c|}{ Illustrative comparative risks ${ }^{\star}(95 \% \mathrm{Cl})$} & \multirow{2}{*}{$\begin{array}{l}\text { Relative effect } \\
(95 \% \mathrm{CI})\end{array}$} & \multirow{2}{*}{$\begin{array}{l}\text { No of Partici- } \\
\text { pants } \\
\text { (studies) }\end{array}$} & \multirow{2}{*}{$\begin{array}{l}\text { Quality of the } \\
\text { evidence } \\
\text { (GRADE) }\end{array}$} & \multirow[t]{2}{*}{ Comments } \\
\hline & Assumed risk & Corresponding risk & & & & \\
\hline $\begin{array}{l}\text { Global state: Relapse } \\
\text { Follow-up: } 2 \text { to } 3 \text { years }\end{array}$ & 429 per 1000 & $\begin{array}{l}\mathbf{4 7 1} \text { per } 1000 \\
\text { (219 to } 1000)\end{array}$ & $\begin{array}{l}\text { RR } 1.1 \\
\text { (0.51 to } 2.41)\end{array}$ & $\begin{array}{l}181 \\
\text { (4 studies) }\end{array}$ & $\begin{array}{l}\oplus \ominus \ominus \ominus \\
\text { very low } 1,2,3,4\end{array}$ & \\
\hline $\begin{array}{l}\text { Service outcomes: Hospitalisation } \\
\text { Follow-up: } 12 \text { weeks to } 18 \text { months }\end{array}$ & 156 per 1000 & $\begin{array}{l}249 \text { per } 1000 \\
\text { (132 to } 468)\end{array}$ & $\begin{array}{l}\text { RR } 1.6 \\
(0.85 \text { to } 3)\end{array}$ & $\begin{array}{l}153 \\
\text { (3 studies) }\end{array}$ & $\begin{array}{l}\oplus \ominus \ominus \ominus \\
\text { very low } 3,4,5\end{array}$ & \\
\hline $\begin{array}{l}\text { Mental state: No clinically important } \\
\text { improvement } \\
\text { Follow-up: } 1 \text { to } 2 \text { years }\end{array}$ & 594 per 1000 & $\begin{array}{l}731 \text { per } 1000 \\
(529 \text { to } 1000)\end{array}$ & $\begin{array}{l}\text { RR } 1.23 \\
(0.89 \text { to } 1.70)\end{array}$ & $\begin{array}{l}194 \\
\text { (3 studies) }\end{array}$ & $\begin{array}{l}\oplus \ominus \ominus \ominus \\
\text { very low } 2,4,6\end{array}$ & \\
\hline $\begin{array}{l}\text { Leaving the study early } \\
\text { Follow-up: } 10 \text { weeks to } 3 \text { years }\end{array}$ & 150 per 1000 & $\begin{array}{l}\mathbf{1 5 8} \text { per } \mathbf{1 0 0 0} \\
\text { (116 to } 217)\end{array}$ & $\begin{array}{l}\text { RR } 1.05 \\
\text { (0.77 to } 1.44)\end{array}$ & $\begin{array}{l}812 \\
\text { (12 studies) }\end{array}$ & $\oplus \oplus \oplus \ominus$ & \\
\hline
\end{tabular}




\begin{tabular}{|c|c|c|c|c|c|c|}
\hline $\begin{array}{l}\text { General functioning } \\
\text { GAF and GAS }\end{array}$ & See comment & See comment & Not estimable & $\begin{array}{l}78 \\
\text { ( } 2 \text { studies) }\end{array}$ & $\begin{array}{l}\oplus \odot \odot \ominus \\
\text { very low } \\
3,4,8,9,10\end{array}$ & $\begin{array}{l}\text { There was very } \\
\text { high hetero- } \\
\text { geneity for this } \\
\text { outcome so } \\
\text { data were not } \\
\text { pooled. } 11\end{array}$ \\
\hline $\begin{array}{l}\text { Satisfaction with treatment: Recip- } \\
\text { ient of care not satisfied with treat- } \\
\text { ment } \\
\text { Follow-up: } 1 \text { years }\end{array}$ & 136 per 1000 & $\begin{array}{l}\mathbf{4 3 5} \text { per } 1000 \\
(138 \text { to } 1000)\end{array}$ & $\begin{array}{l}\text { RR } 3.19 \\
\text { (1.01 to } 10.07)\end{array}$ & $\begin{array}{l}45 \\
\text { (1 study) }\end{array}$ & $\begin{array}{l}\oplus \ominus \odot \odot \\
\text { very low 4,12,13 }\end{array}$ & \\
\hline $\begin{array}{l}\text { Quality of life } \\
\text { RSES } \\
\text { Follow-up: } 12 \text { weeks }\end{array}$ & & $\begin{array}{l}\text { The mean quality of life in the } \\
\text { intervention groups was } \\
\mathbf{1 . 7} \text { lower } \\
\text { ( } 5.19 \text { lower to } 1.79 \text { higher) }\end{array}$ & & $\begin{array}{l}65 \\
\text { (1 study) }\end{array}$ & $\begin{array}{l}\oplus \ominus \ominus \ominus \\
\text { very low } 3,4,10\end{array}$ & \\
\hline
\end{tabular}

*The basis for the assumed risk (e.g. the median control group risk across studies) is provided in footnotes. The corresponding risk (and its $95 \%$ confidence interval) is based on the assumed risk in the comparison group and the relative effect of the intervention (and its $95 \% \mathrm{Cl}$ ).

Cl: Confidence interval; RR: Risk ratio;

GRADE Working Group grades of evidence

High quality: Further research is very unlikely to change our confidence in the estimate of effect.

Moderate quality: Further research is likely to have an important impact on our confidence in the estimate of effect and may change the estimate.

Low quality: Further research is very likely to have an important impact on our confidence in the estimate of effect and is likely to change the estimate.

Very low quality: We are very uncertain about the estimate.

1 Risk of bias: serious - three trials had an unclear risk of bias for randomisation and allocation concealment. All studies had an unclear risk for blinding of participants, while two studies were rated as high risk for blinding of outcome assessors.

2 Inconsistency: serious - there was high heterogeneity for this outcome.

3 Imprecision: serious - relatively few participants were included and few events; confidence intervals are wide.

4 Publication bias: strongly suspected - four studies or fewer reported data for this outcome.

5 Risk of bias: serious - one study had an unclear risk of bias for randomisation and two for allocation concealment. All were unclear risk for blinding of participants and two for blinding of outcome assessors.

6 Risk of bias: serious - one study had an unclear risk of bias for randomisation. All three had an unclear risk of blinding of participants and one for blinding of outcome assessors.

7 Risk of bias: serious - seven studies had an unclear risk of bias for randomisation and allocation concealment. Nine studies had an unclear risk of bias for blinding of participants. Two studies two had a high risk of bias for blinding of outcome assessments and four were unclear.

8 Risk of bias: serious - one study had an unclear risk of bias for allocation concealment. Both studies had an unclear risk of bias for blinding of participants and outcome assessments.

9 Inconsistency: very serious - there was high heterogeneity for this outcome and data were not pooled.

10 Indirectness: serious - we wanted to collect binary data for this outcome, however, only a proxy scale measure was available.

11 One study found no difference in general functioning on the GAS, the other study found a difference in favour of supportive therapy on the GAF. 


\begin{tabular}{|c|c|c|c|c|c|c|}
\hline \multicolumn{7}{|c|}{$\begin{array}{l}12 \text { Risk of bias: serious - the study had an unclear risk of bias for blinding of participants and outcome assess } \\
13 \text { Imprecision: very serious - relatively few participants were included and few events; confidence intervals } \\
\text { Summary of findings 4. SUPPORTIVE THERAPY versus FAMILY THERAPY for schizophrenia }\end{array}$} \\
\hline \multicolumn{7}{|c|}{ SUPPORTIVE THERAPY versus FAMILY THERAPY for schizophrenia } \\
\hline \multicolumn{7}{|c|}{$\begin{array}{l}\text { Patient or population: patients with schizophrenia } \\
\text { Settings: inpatients and outpatients } \\
\text { Intervention: SUPPORTIVE THERAPY } \\
\text { Comparison: FAMILY THERAPY }\end{array}$} \\
\hline \multirow[t]{3}{*}{ Outcomes } & \multicolumn{2}{|c|}{ Illustrative comparative risks* $(95 \% \mathrm{CI})$} & \multirow{3}{*}{$\begin{array}{l}\text { Relative effect } \\
(95 \% \mathrm{Cl})\end{array}$} & \multirow{3}{*}{$\begin{array}{l}\text { No of Partici- } \\
\text { pants } \\
\text { (studies) }\end{array}$} & \multirow{3}{*}{$\begin{array}{l}\text { Quality of the } \\
\text { evidence } \\
\text { (GRADE) }\end{array}$} & \multirow[t]{3}{*}{ Comments } \\
\hline & Assumed risk & Corresponding risk & & & & \\
\hline & Control & $\begin{array}{l}\text { SUPPORTIVE THERAPY } \\
\text { versUS FAMILY THERA- } \\
\text { PY }\end{array}$ & & & & \\
\hline $\begin{array}{l}\text { Global state: Relapse } \\
\text { Follow-up: } 2 \text { to } 3 \text { years }\end{array}$ & See comment & See comment & Not estimable & $\begin{array}{l}87 \\
\text { ( } 2 \text { studies) }\end{array}$ & $\begin{array}{l}\oplus \ominus \ominus \ominus \\
\text { very low } 1,2,3,4\end{array}$ & $\begin{array}{l}\text { There was high het- } \\
\text { erogeneity for this } \\
\text { outcome and data } \\
\text { were not pooled. } 5\end{array}$ \\
\hline $\begin{array}{l}\text { Service outcomes: Hospitalisation } \\
\text { Follow-up: } 2 \text { years }\end{array}$ & 300 per 1000 & $\begin{array}{l}\mathbf{5 7 9} \text { per } \mathbf{1 0 0 0} \\
\text { (267 to } 1000)\end{array}$ & $\begin{array}{l}\text { RR } 1.93 \\
\text { (0.89 to } 4.17)\end{array}$ & $\begin{array}{l}39 \\
\text { (1 study) }\end{array}$ & $\begin{array}{l}\oplus \ominus \ominus \ominus \\
\text { very low 3,4,6 }\end{array}$ & \\
\hline $\begin{array}{l}\text { Mental state: Episode of affective } \\
\text { symptoms }\end{array}$ & 292 per 1000 & $\begin{array}{l}499 \text { per } 1000 \\
(239 \text { to } 1000)\end{array}$ & $\begin{array}{l}\text { RR } 1.71 \\
(0.82 \text { to } 3.6)\end{array}$ & $\begin{array}{l}48 \\
\text { (1 study) }\end{array}$ & $\begin{array}{l}\oplus \ominus \ominus \ominus \\
\text { very low 3,4,7,8 }\end{array}$ & \\
\hline Leaving the study early & 182 per 1000 & $\begin{array}{l}\mathbf{2 3 1} \text { per } \mathbf{1 0 0 0} \\
\text { (102 to } 525)\end{array}$ & $\begin{array}{l}\text { RR } 1.27 \\
\text { (0.56 to } 2.89)\end{array}$ & $\begin{array}{l}87 \\
\text { ( } 2 \text { studies) }\end{array}$ & $\begin{array}{l}\oplus \ominus \ominus \ominus \\
\text { very low } 1,3,4\end{array}$ & \\
\hline General functioning: No paid work & 600 per 1000 & $\begin{array}{l}\mathbf{5 7 6} \text { per } \mathbf{1 0 0 0} \\
\text { (342 to } 978)\end{array}$ & $\begin{array}{l}\mathbf{R R} \mathbf{0 . 9 6} \\
(0.57 \text { to } 1.63)\end{array}$ & $\begin{array}{l}39 \\
\text { (1 study) }\end{array}$ & $\begin{array}{l}\oplus \ominus \ominus \ominus \\
\text { very low } 3,4,6,9\end{array}$ & \\
\hline $\begin{array}{l}\text { Satisfaction with treatment - not re- } \\
\text { ported }\end{array}$ & See comment & See comment & Not estimable & - & See comment & $\begin{array}{l}\text { No studies reported } \\
\text { data for satisfaction } \\
\text { with treatment. }\end{array}$ \\
\hline
\end{tabular}




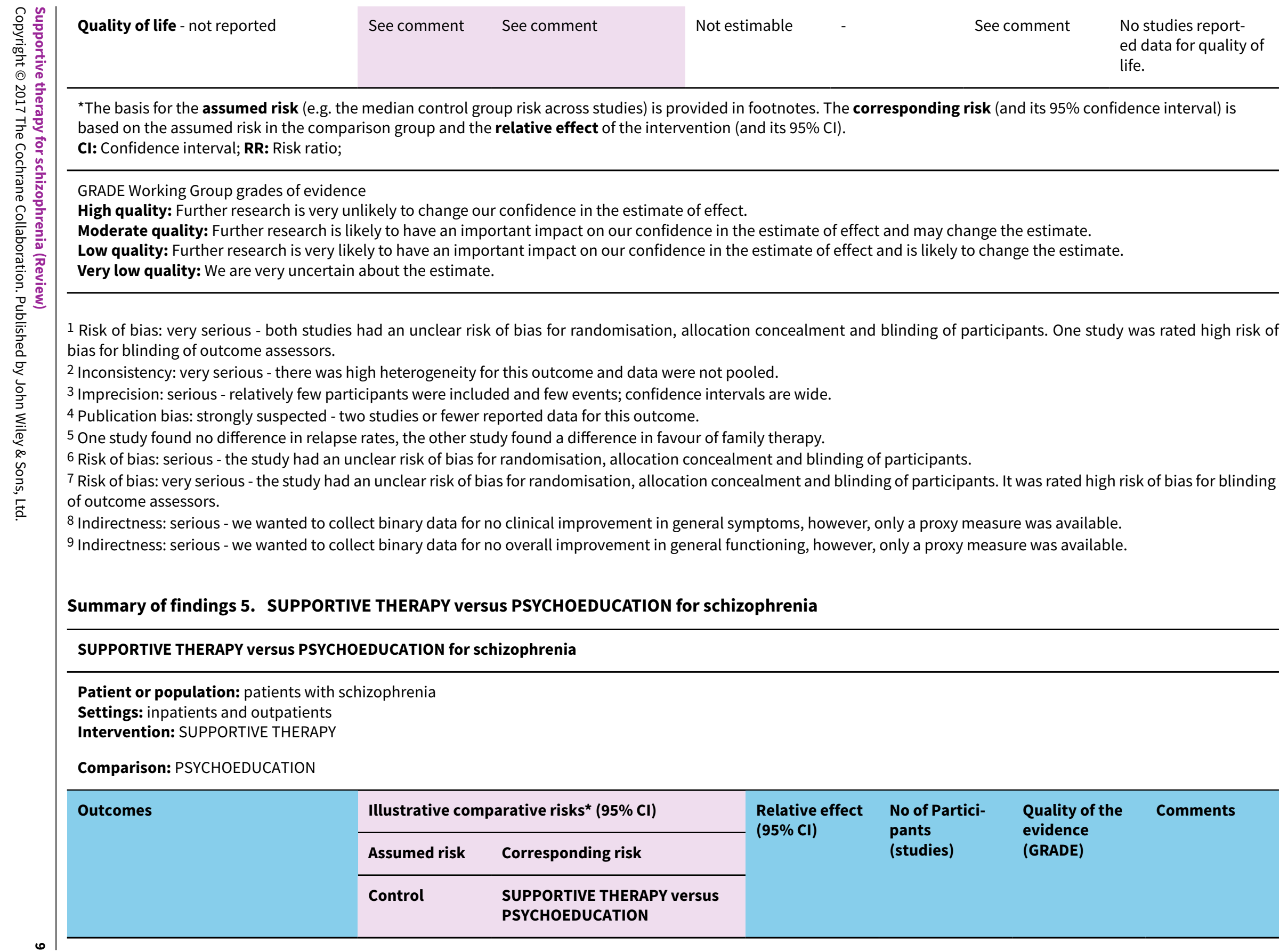




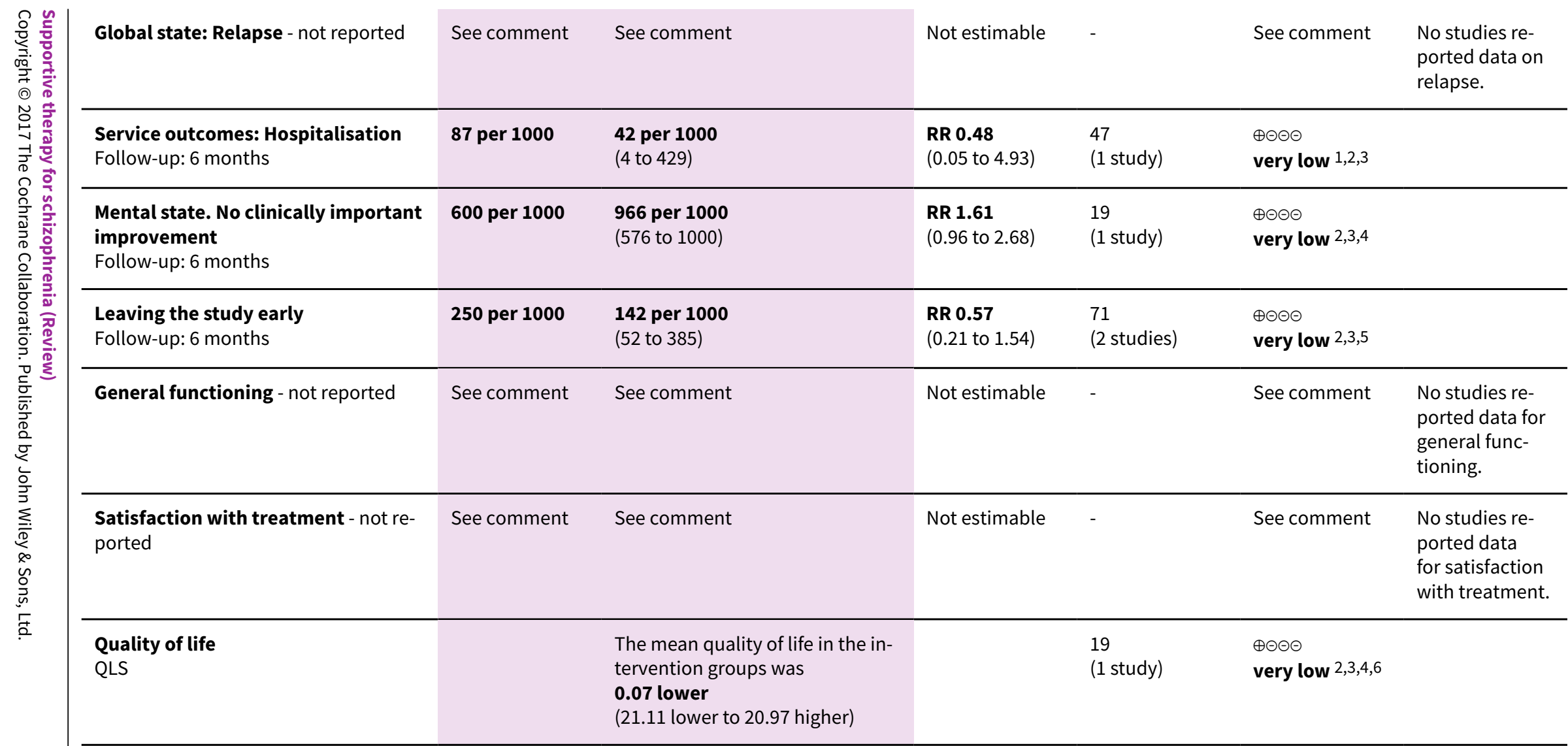

${ }^{\star}$ The basis for the assumed risk (e.g. the median control group risk across studies) is provided in footnotes. The corresponding risk (and its $95 \%$ confidence interval) is based on the assumed risk in the comparison group and the relative effect of the intervention (and its $95 \% \mathrm{Cl}$ ).

Cl: Confidence interval; RR: Risk ratio;

GRADE Working Group grades of evidence

High quality: Further research is very unlikely to change our confidence in the estimate of effect.

Moderate quality: Further research is likely to have an important impact on our confidence in the estimate of effect and may change the estimate.

Low quality: Further research is very likely to have an important impact on our confidence in the estimate of effect and is likely to change the estimate.

Very low quality: We are very uncertain about the estimate.

1 Risk of bias: serious - the study had an unclear risk of bias for randomisation, allocation concealment and blinding of participants and outcome assessors.

2 Imprecision: very serious - relatively few participants were included and few events; confidence intervals are wide.

3 Publication bias: strongly suspected - two studies or fewer reported data for this outcome.

4 Risk of bias: serious - the study had an unclear risk of bias for randomisation, allocation concealment and blinding of participants. 


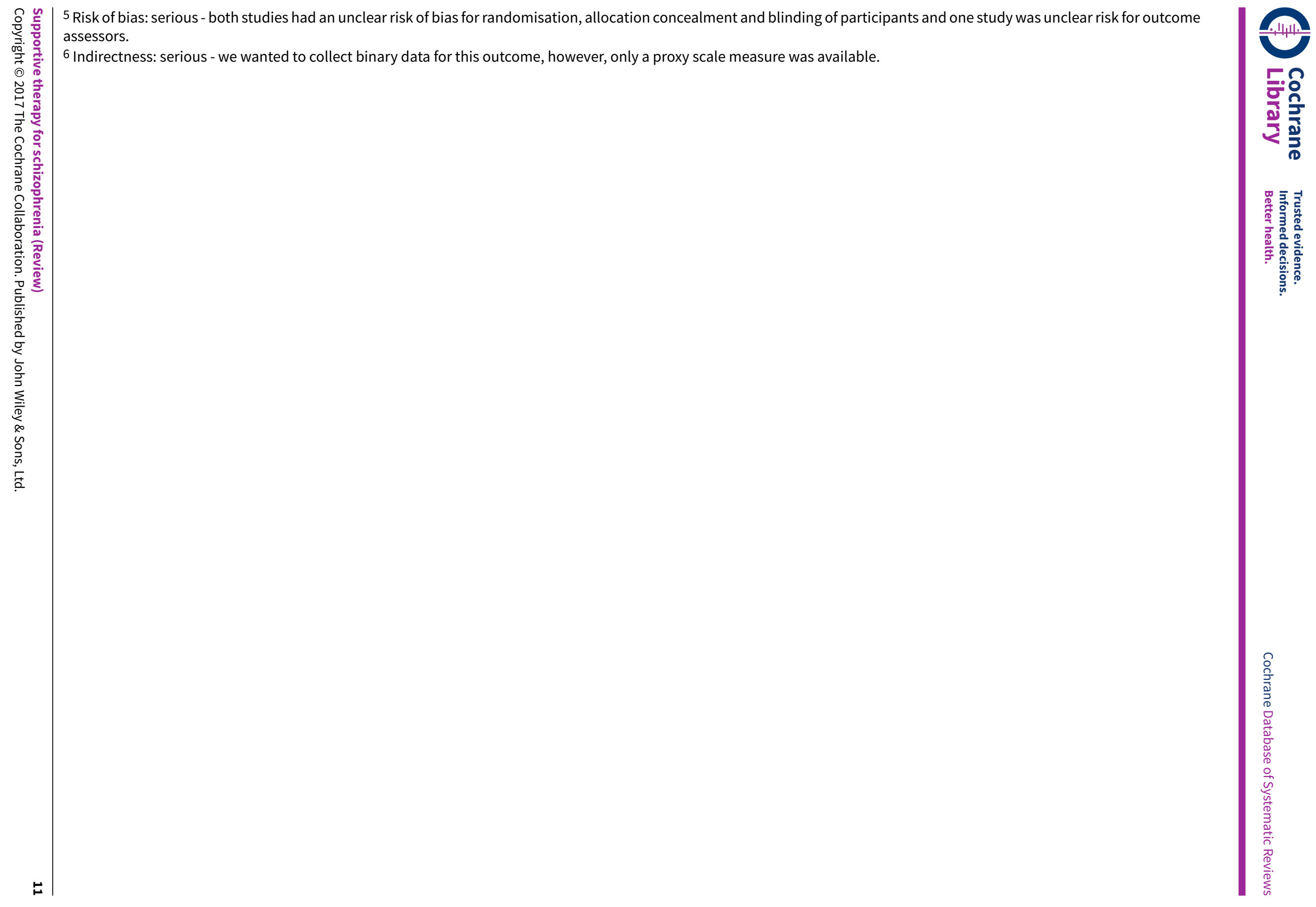




\section{B A C K G R O U N D}

\section{Description of the condition}

One of the common features of psychotherapies and professional interventions for people with schizophrenia is to provide support. For example, in an out-patient consultation there will often be time allocated to listening to patients' concerns, giving encouragement, or even arranging basic help with day to day living, such as access to local resources. The key elements of support are to maintain an existing situation, or assist pre-existing coping abilities. Supportive therapy has been described as the treatment model of choice for most patients (Hellerstein 1994), and may be the most commonly practiced intervention in the mental health service.

\section{Description of the intervention}

It is, however, difficult to answer the question of exactly what is supportive therapy? A starting point is to look at the literature on supportive psychotherapy, which has been defined as "a dyadic treatment characterised by the use of direct measures to ameliorate symptoms and to maintain, restore or improve self esteem, adaptive skills and ego function" (Pinsker 1991). There may also be a difference in the practice of supportive therapy according to country (Holmes 1995). In the UK, supportive therapy implies a frequency of less than once a week, whereas in America, some practitioners would regard any psychodynamic intervention at a frequency of less than four or five times a week as supportive psychotherapy (Werman 1994).

\section{How the intervention might work}

Even though there is no internationally agreed definition, one of the key features is that supportive therapy aims to enhance, rather than challenge, current psychological defence mechanisms. An alternative view is to identify supportive therapy according to the components of the therapy (Misch 2000). For example, one expert proposes seven distinct components; reassurance, explanation, guidance, suggestion, encouragement, affecting change in the environment, and permission for catharsis (Bloch 1996). Bloch also argues that supportive therapy must be a long-term intervention. The difficulty of this proposal is that there is no clear reason why a specific component of therapy should be regarded as supportive. Possible solutions include defining supportive therapy according to outcome, according to the perception of the client or by identifying a feature of therapy that is inherently supportive, regardless of its impact on a client (Barber 2001).

There are many other forms of support that can be given which are distinct from the psychodynamic tradition. For example, there are mental health workers who have the job title of support worker whose role is often to provide practical support such as reminders and transport for other services, or to spend time befriending a client.

\section{Why it is important to do this review}

Ultimately, the lack of a widely accepted definition of supportive therapy means we are not able to avoid an arbitrary element in the definition used for this review. Our definition (see Types of interventions) is influenced by the potential usefulness of this review, which could be:
1. to give information on the effectiveness of a therapy that is commonly used as the control arm of clinical trials for psychotherapies in schizophrenia;

2. to help clinicians with the decision of whether to offer a supportive intervention;

3. to help clinicians understand the value of supportive elements of their individual interactions with people who have schizophrenia.

\section{OBJECTIVES}

To review the effects of supportive therapy compared with standard care, or other treatments in addition to standard care for people with schizophrenia.

\section{METHODS}

\section{Criteria for considering studies for this review}

\section{Types of studies}

We sought all relevant randomised controlled trials. Where a trial was described as 'double-blind', but it was only implied that the study was randomised, we included these trials in a sensitivity analysis. If there was no substantive difference within primary outcomes (see 'Types of outcome measures') when these 'implied randomisation' studies were added, then we included them in the final analyses. If there was a substantive difference, then we only used clearly randomised trials and the results of the sensitivity analysis were described in the text. We excluded quasi-randomised studies, such as those allocating by using alternate days of the week.

\section{Types of participants}

We included people with schizophrenia or schizophrenia-like illnesses using any criteria. We included trials where it was implied that the majority of participants had a severe mental illness that was likely to be schizophrenia. We did not exclude trials due to age, nationality, gender, duration of illness or treatment setting.

\section{Types of interventions}

\section{Supportive therapy and supportive care}

We have used the phrase 'supportive therapy and supportive care' here to indicate that this review covers a wider variety of interventions than supportive psychotherapy alone. However, for the sake of simplicity, we used the term 'supportive therapy' elsewhere in this review. These interventions are provided by a single person with the main purpose of maintaining current functioning or assisting pre-existing coping abilities in people who have a diagnosis of schizophrenia or schizophrenia-like illness. The therapies can be aimed at individuals or groups of people. If the content of the therapy was not sufficiently clear after reading a clinical study, then we included any therapy that had supportive or support in its title. We have included advocacy as a form of supportive therapy. Advocacy is a narrower intervention than other interventions included in the review, but it nevertheless fits our definition as it assists people with their communication and interaction with mental health workers.

A number of common therapies are excluded as they are designed to teach new skills or change pre-existing skills. These include cognitive behavioural therapy(CBT) (Cormac 2004), social skills 
training, psycho-education (Pekkala2002), compliance therapy (McIntosh 2006) and problem-solving therapy.

Some therapies or schemes have been excluded as they involve a team approach rather than an individual worker, or because they are designed to alter a person's environment, rather than to help the person cope with their environment. These include family placement (Pharoah 2006), supported employment and supported accommodation (Chilvers 2006) . An exception was to include an intervention if it was clear that it consisted of a practitioner whose main purpose was to help a client cope with their current situation, rather than alter the situation to make it easier for the client.

We have also excluded counselling (unlike the meta-analysis from the National Institute of Clinical Excellence on supportive therapy (NICE 2003)). The main purpose of counselling is to give an opportunity for a client to explore, discover and clarify ways of living (DoH 2001). Counselling employs a wide variety of techniques, and the purpose may be to facilitate a change in someone's life rather than to maintain the current situation. As counselling has such a broad scope, an exception was to include a clinical trial if there was a clear indication that the main purpose of counselling was to provide support rather than facilitate change or give an opportunity to explore personal issues.

\section{Standard care}

This is defined as the care a person would normally receive had they not been included in the research trial. This would include interventions such as medication, hospitalisation, community psychiatric nursing input and/or day hospital.

\section{Other treatments}

This would include any other treatment (biological, psychological or social) such as medication, problem-solving therapy, psychoeducation, social skills training, CBT, family therapy or psychodynamic psychotherapy.

\section{Types of outcome measures}

We reported outcomes for the short term (up to 12 weeks), medium term (13 to 26 weeks), and long term (more than 26 weeks)

\section{Primary outcomes}

\section{Global state}

1.1 Relapse

\section{Service outcomes}

2.1 Hospitalisation

\section{General functioning}

5.1 No clinically important change in general functioning

\section{Secondary outcomes}

1. Global state

1.2 Time to relapse

1.3 No clinically important change in global state

1.4 Not any change in global state

1.5 Average endpoint global state score

1.6 Average change in global state scores

\section{Service outcomes}

2.2 Time to hospitalisation

\section{Mental state}

3.1 No clinically important improvement 3.2 Not any change in general mental state 3.3 Average endpoint general mental state score 3.4 Average change in general mental state scores 3.5 No clinically important change in specific symptoms 3.6 Not any change in specific symptoms

3.7 Average endpoint specific symptom score 3.8 Average change in specific symptom scores

\section{Leaving the study early}

4.1 For specific reasons 4.2 For general reasons

\section{General functioning}

5.2 Not any change in general functioning

5.3 Average endpoint general functioning score

5.4 Average change in general functioning scores

5.5 No clinically important change in specific aspects of functioning, such as social or life skills

5.6 Not any change in specific aspects of functioning, such as social or life skills

5.7 Average endpoint specific aspects of functioning, such as social or life skills

5.8 Average change in specific aspects of functioning, such as social or life skills

\section{Satisfaction with treatment}

6.1 Recipient of care not satisfied with treatment

6.2 Recipient of care average satisfaction score

6.3 Recipient of care average change in satisfaction scores

6.4 Carer not satisfied with treatment

6.5 Carer average satisfaction score

6.6 Carer average change in satisfaction scores

\section{Quality of life}

7.1 No clinically important change in quality of life

7.2 Not any change in quality of life

7.3 Average endpoint quality of life score

7.4 Average change in quality of life scores

7.5 No clinically important change in specific aspects of quality of life

7.6 Not any change in specific aspects of quality of life

7.7 Average endpoint specific aspects of quality of life

7.8 Average change in specific aspects of quality of life

8. Death - suicide and natural causes

9. Behaviour

9.1 No clinically important change in general behaviour

9.2 Not any change in general behaviour

9.3 Average endpoint general behaviour score

9.4 Average change in general behaviour scores

9.5 No clinically important change in specific aspects of behaviour

9.6 Not any change in specific aspects of behaviour

9.7 Average endpoint specific aspects of behaviour

9.8 Average change in specific aspects of behaviour 


\section{Adverse effects}

10.1 No clinically important general adverse effects

10.2 Not any general adverse effects

10.3 Average endpoint general adverse effect score

10.4 Average change in general adverse effect scores

10.5 No clinically important change in specific adverse effects

10.6 Not any change in specific adverse effects

10.7 Average endpoint specific adverse effects

10.8 Average change in specific adverse effects

\section{Engagement with services}

11.1 No clinically important engagement

11.2 Not any engagement

11.3 Average endpoint engagement score

11.4 Average change in engagement scores

\section{Engagement in structured activities}

12.1 No clinically important change in engagement in structured activities

12.2 Not any change in engagement in structured activities

12.3 Average endpoint engagement in structured activities score

12.4 Average change in engagement in structured activities scores

12.5 No clinically important change in specific activities, such as employment, education or attendance at day centres

12.6 Not any change in specific aspects of functioning, such as employment, education or attendance at day centres

12.7 Average endpoint specific aspects of functioning, such as employment, education or attendance at day centres

12.8 Average change in specific aspects of functioning, such as employment, education or attendance at day centres

\section{Insight}

13.1 Average endpoint insight score

13.2 Average endpoint treatment attitude score

13.3 Average endpoint adherence score

\section{Compliance}

14.1 Adherence

14.2 Poor Compliance

\section{Medication}

15.1 Reduction in dose

15.2 Prescribed intramuscular (IM) depot medication

\section{Attitude to medication}

16.1 Average endpoint attitude to medication score 16.2 Average endpoint need for treatment

\section{Economic outcomes}

17.1 Average change in total cost of medical and mental health care 17.2 Total indirect and direct costs.

\section{8. 'Summary of findings' table}

We used the GRADEapproach to interpret findings (Schünemann 2008) and used the GRADE profiler to import data from Review Manager (RevMan) to create 'Summary of findings' tables. These tables provide outcome-specific information concerning the overall quality of evidence from each included study in the comparison, the magnitude of effect of the interventions examined, and the sum of available data on all outcomes we rated as important to patient-care and decision making. We selected the following main outcomes for inclusion in the 'Summary of findings' tables.

1. Global state: Relapse

2. Service outcomes: Hospitalisation

3. Leaving the study early

4. Mental state: No clinically important change in general mental state

5. General functioning: No clinically important change in general functioning

6. Satisfaction with treatment: Recipient of care not satisfied with treatment

7. Quality of life: No clinically important change in quality of life

\section{Search methods for identification of studies}

\section{Electronic searches}

\section{Cochrane Schizophrenia Group Trials Register}

The Trials Search Co-ordinator searched the Cochrane Schizophrenia Group's Trials Register (28 November 2012)

[((* support $^{\star}$ OR *advoc*) in title of REFERENCE) and ((* support $^{\star}$ or ${ }^{*}$ individual ${ }^{*}$ or * sociotherapy* or *socioenvir*) in intervention of STUDY)]

The Cochrane Schizophrenia Group's Trials Register is compiled by systematic searches of major databases, handsearches of relevant journals and conference proceedings. For details of databases searched see Group Module.

\section{Searching other resources}

\section{Reference searching}

We inspected the reference lists of all identified studies, including existing reviews, for relevant citations.

\section{Personal contact}

If necessary we would have contacted the first author of each relevant study for information on unpublished trials.

\section{Data collection and analysis}

\section{Selection of studies}

For this update review authors NM and KSW independently inspected citations from the new electronic search and identified relevant abstracts. NM and KSW also inspected full articles of the abstracts meeting inclusion criteria. CEA carried out the reliability check of all citations from the new electronic search.

\section{Data extraction and management}

\section{Extraction}

For this update, NM extracted data from included studies. If data were presented only in graphs and figures NM extracted these data whenever possible. If studies were multi-centre, where possible, NM extracted data relevant to each component centre separately. 


\section{Management}

\subsection{Forms}

We extracted data onto standard, simple forms.

\subsection{Scale-derived data}

We included continuous data from rating scales only if: a. the psychometric properties of the measuring instrument have been described in a peer-reviewed journal (Marshall 2000); and b. the measuring instrument has not been written or modified by one of the trialists for that particular trial.

Ideally, the measuring instrument should either be i. a self-report or ii. completed by an independent rater or relative (not the therapist). We realise that this is not often reported clearly; if relevant we noted whether or not this is the case in Description of studies.

\subsection{Endpoint versus change data}

There are advantages of both endpoint and change data. Change data can remove a component of between-person variability from the analysis. On the other hand, calculation of change needs two assessments (baseline and endpoint), which can be difficult in unstable and difficult to measure conditions such as schizophrenia. We decided primarily to use endpoint data, and only use change data if the former were not available. Had we had both change and endpoint data, we would have combined them in the analysis as we used mean differences (MD) rather than standardised mean differences (SMD) throughout (Higgins 2011, Chapter 9.4.5.2).

\subsection{Skewed data}

Continuous data on clinical and social outcomes are often not normally distributed. To avoid the pitfall of applying parametric tests to non-parametric data, we aimed to apply the following standards to all data before inclusion:

- standard deviations (SDs) and means are reported in the paper or obtainable from the authors;

- when a scale starts from the finite number zero, the SD, when multiplied by two, is less than the mean (as otherwise the mean is unlikely to be an appropriate measure of the centre of the distribution (Altman 1996));

- if a scale started from a positive value (such as the Positive and Negative Syndrome Scale (PANSS, (Kay 1986), which can have values from 30 to 210), we modified the calculation described above to take the scale starting point into account. In these cases skew is present if $2 \mathrm{SD}>(\mathrm{S}-\mathrm{S} \mathrm{min})$, where $\mathrm{S}$ is the mean score and $\mathrm{S}$ min is the minimum score.

Endpoint scores on scales often have a finite start and end point and these rules can be applied. We entered skewed endpoint data from studies of fewer than 200 participants as other data within the data and analyses section rather than into a statistical analysis. Skewed data pose less of a problem when looking at mean if the sample size is large; we entered such endpoint data into syntheses.

When continuous data are presented on a scale that includes a possibility of negative values (such as change data), it is difficult to tell whether data are skewed or not, we entered skewed change data into analyses regardless of size of study.

\subsection{Common measure}

To facilitate comparison between trials, we intended to convert variables that can be reported in different metrics, such as days in hospital (mean days per year, per week or per month) to a common metric (e.g. mean days per month).

\subsection{Conversion of continuous to binary}

Where possible, we made efforts to convert outcome measures to dichotomous data. This can be done by identifying cut-off points on rating scales and dividing participants accordingly into 'clinically improved' or 'not clinically improved'. It is generally assumed that if there is a $50 \%$ reduction in a scale-derived score such as the Brief Psychiatric Rating Scale (BPRS, Overall 1962) or the Positive and Negative Syndrome Scale (PANSS, Kay 1986), this could be considered as a clinically significant response (Leucht 2005; Leucht 2005a). If data based on these thresholds were not available, we used the primary cut-off presented by the original authors.

\subsection{Direction of graphs}

Where possible, we entered data in such a way that the area to the left of the line of no effect indicated a favourable outcome for supportive therapy.

\section{Assessment of risk of bias in included studies}

For this update, NM and KSW worked independently by using criteria described in the Cochrane Handbook for Systematic Reviews of Interventions (Higgins 2011) to assess trial quality. This new set of criteria is based on evidence of associations between overestimate of effect and high risk of bias of the article such as sequence generation, allocation concealment, blinding, incomplete outcome data and selective reporting.

Where inadequate details of randomisation and other characteristics of trials were provided, we contacted authors of the studies in order to obtain additional information.

We have noted the level of risk of bias in both the text of the review and in the 'Summary of findings' tables.

\section{Measures of treatment effect}

\section{Binary data}

For binary outcomes, we calculated a standard estimation of the risk ratio (RR) and its 95\% confidence interval $(\mathrm{Cl})$. It has been shown that RR is more intuitive (Boissel 1999) than odds ratios and that odds ratios tend to be interpreted as RR by clinicians (Deeks 2000). The Number Needed to Treat/Harm (NNT/H) statistic with its confidence intervals is intuitively attractive to clinicians but is problematic both in its accurate calculation in meta-analyses and interpretation (Hutton 2009). For binary data presented in the 'Summary of findings' tables, where possible, we calculated illustrative comparative risks.

\section{Continuous data}

For continuous outcomes, we estimated mean difference (MD) between groups. We would prefer not to calculate effect size measures (standardised mean difference (SMD)). However, if scales of very considerable similarity had been used, we would have presumed there was a small difference in measurement, and we would have calculated effect size and transformed the effect back to the units of one or more of the specific instruments. 


\section{Unit of analysis issues}

\section{Cluster trials}

Studies increasingly employ 'cluster randomisation' (such as randomisation by clinician or practice), but analysis and pooling of clustered data poses problems. Authors often fail to account for intra-class correlation in clustered studies, leading to a 'unit of analysis' error (Divine 1992) whereby $P$ values are spuriously low, confidence intervals unduly narrow and statistical significance overestimated. This causes type I errors (Bland 1997; Gulliford 1999).

We did not include any cluster-randomised trials in this version of the review. If we had found trials where clustering was not accounted for in primary studies, we would have presented data in a table, with a $\left(^{\star}\right)$ symbol to indicate the presence of a probable unit of analysis error. In subsequent versions of this review, if we include cluster-randomised trials, we will seek to contact first authors of studies to obtain intra-class correlation coefficients (ICCS) for their clustered data and to adjust for this by using accepted methods (Gulliford 1999).

If we had included trials where clustering had been incorporated into the analysis of primary studies, we would have presented these data as if from a non-cluster randomised study, but adjusted for the clustering effect.

We have sought statistical advice and have been advised that the binary data as presented in a report should be divided by a 'design effect'. This is calculated using the mean number of participants per cluster $(m)$ and the ICC [Design effect $\left.=1+(m-1)^{\star} I C C\right]$ (Donner 2002). If the ICC is not reported it will be assumed to be 0.1 (Ukoumunne 1999).

If cluster studies have been appropriately analysed taking into account ICCs and relevant data documented in the report, synthesis with other studies would be possible using the generic inverse variance technique.

\section{Cross-over trials}

A major concern of cross-over trials is the carry-over effect. It occurs if an effect (e.g. pharmacological, physiological or psychological) of the treatment in the first phase is carried over to the second phase. As a consequence, on entry to the second phase the participants can differ systematically from their initial state despite a wash-out phase. For the same reason, cross-over trials are not appropriate if the condition of interest is unstable (Elbourne 2002). As both effects are very likely in severe mental illness, had we included such trials, we planned to use only the data of the first phase of cross-over studies.

\section{Studies with multiple treatment groups}

Where a study involves more than two treatment arms, if relevant, we presented the additional treatment arms in comparisons. If data were binary, we simply added these and combined within the two-by-two table. If data were continuous, we combined data following the formula in section 7.7.3.8 (Combining groups) of the Cochrane Handbook for Systemic reviews of Interventions (Higgins 2011). Where the additional treatment arms were not relevant, we did not reproduce these data.

\section{Dealing with missing data \\ 1. Overall loss of credibility}

At some degree of loss of follow-up, data must lose credibility (Xia 2009). We chose that, for any particular outcome, should more than $50 \%$ of data be unaccounted for, we would not reproduce these data or use them within analyses. If, however, more than $50 \%$ of those in one arm of a study were lost, but the total loss was less than $50 \%$, we addressed this within the 'Summary of findings' tables by down-rating quality. Finally, we also downgraded quality within the 'Summary of findings' tables where loss was $25 \%$ to $50 \%$ in total.

\section{Binary}

In the case where attrition for a binary outcome was between $0 \%$ and $50 \%$ and where these data were not clearly described, we presented data on a 'once-randomised-always-analyse' basis (an intention-to-treat analysis). Those leaving the study early were all assumed to have the same rates of negative outcome as those who completed, with the exception of the outcome of death and adverse effects. For these outcomes, the rate of those who stayed in the study - in that particular arm of the trial - were used for those who did not. We undertook a sensitivity analysis to test how prone the primary outcomes were to change when 'completer' data only were compared to the intention-to-treat analysis using the above assumptions.

\section{Continuous}

\subsection{Attrition}

In the case where attrition for a continuous outcome is between $0 \%$ and $50 \%$ and completer-only data were reported, we reproduced these.

\subsection{Standard deviations}

If standard deviations (SDs) were not reported, we first tried to obtain the missing values from the authors. If not available, where there are missing measures of variance for continuous data, but an exact standard error (SE) and confidence intervals available for group means, and either a $\mathrm{P}$ value or $\mathrm{T}$ value available for differences in mean, we can calculate them according to the rules described in the Handbook (Higgins 2011): When only the SE is reported, SDs are calculated by the formula $\mathrm{SD}=\mathrm{SE}$ * square root (n). Chapters 7.7.3 and 16.1.3 of the Handbook (Higgins 2011) present detailed formulae for estimating SDs from $P$ values, $T$ or $F$ values, confidence intervals, ranges or other statistics. If these formulae do not apply, we can calculate the SDs according to a validated imputation method, which is based on the SDs of the other included studies (Furukawa 2006). Although some of these imputation strategies can introduce error, the alternative would be to exclude a given study's outcome and thus to lose information. We nevertheless examined the validity of the imputations in a sensitivity analysis excluding imputed values.

\subsection{Last observation carried forward}

We anticipated that in some studies the method of last observation carried forward (LOCF) would be employed within the study report. As with all methods of imputation to deal with missing data, LOCF introduces uncertainty about the reliability of the results (Leucht 2007). Therefore, where LOCF data have been used in the trial, if less than $50 \%$ of the data have been assumed, we reproduced these data and indicated that they are the product of LOCF assumptions. 


\section{Assessment of heterogeneity}

\section{Clinical heterogeneity}

We considered all included studies initially, without seeing comparison data, to judge clinical heterogeneity. We simply inspected all studies for clearly outlying people or situations which we had not predicted would arise. When such situations or participant groups arose, we fully discussed these.

\section{Methodological heterogeneity}

We considered all included studies initially, without seeing comparison data, to judge methodological heterogeneity. We simply inspected all studies for clearly outlying methods which we had not predicted would arise. When such methodological outliers arose, we fully discussed these.

\section{Statistical heterogeneity}

\subsection{Visual inspection}

We visually inspected graphs to investigate the possibility of statistical heterogeneity.

\subsection{Employing the $I^{2}$ statistic}

We investigated heterogeneity between studies by considering the $\mathrm{I}^{2}$ method alongside the $\mathrm{Chi}^{2} \mathrm{P}$ value. The $\mathrm{I}^{2}$ provides an estimate of the percentage of inconsistency thought to be due to chance (Higgins 2003). The importance of the observed value of $1^{2}$ depends on i. magnitude and direction of effects and ii. strength of evidence for heterogeneity (e.g. $\mathrm{P}$ value from $\mathrm{Chi}^{2}$ test, or a confidence interval for $\mathrm{1}^{2}$ ). An $\mathrm{I}^{2}$ estimate greater than or equal to around $50 \%$ accompanied by a statistically significant $\mathrm{Chi}^{2}$ statistic was interpreted as evidence of substantial levels of heterogeneity (Higgins 2011). When substantial levels of heterogeneity were found in the primary outcome, we explored reasons for heterogeneity (Subgroup analysis and investigation of heterogeneity). Where the $\mathrm{I}^{2}$ estimate was equal to or greater than $75 \%$, we interpreted this as indicating the presence of high levels of heterogeneity (Higgins 2003). We did not summate data if inconsistency was high, but presented the data separately and investigated reasons for heterogeneity. If data were heterogeneous we used a random-effects model.

\section{Assessment of reporting biases}

Reporting biases arise when the dissemination of research findings is influenced by the nature and direction of results (Egger 1997). These are described in Section 10 of the Handbook (Higgins 2011). We are aware that funnel plots may be useful in investigating reporting biases but are of limited power to detect small-study effects. We did not use funnel plots for outcomes where there were 10 or fewer studies, or where all studies were of similar sizes. In other cases, where funnel plots are possible, we sought statistical advice in their interpretation.

\section{Data synthesis}

We understand that there is no closed argument for preference for use of fixed-effect or random-effects models. The random-effects method incorporates an assumption that the different studies are estimating different, yet related, intervention effects. This often seems to be true to us and the random-effects model takes into account differences between studies even if there is no statistically significant heterogeneity. There is, however, a disadvantage to the random-effects model: it puts added weight onto small studies which often are the most biased ones. Depending on the direction of effect, these studies can either inflate or deflate the effect size. We chose the fixed-effect model for all analyses. The reader is, however, able to choose to inspect the data using the randomeffects model.

\section{Subgroup analysis and investigation of heterogeneity}

\section{Subgroup analyses - only primary outcomes}

\subsection{Clinical state, stage or problem}

We proposed to undertake this review and provide an overview of the effects of supportive therapy for people with schizophrenia in general. In addition, however, we tried to report data on subgroups of people in the same clinical state, stage and with similar problems. However, there were not enough included studies in the comparisons to undertake subgroup analyses.

\section{Investigation of heterogeneity}

If inconsistency was high, we have reported this. First, we investigated whether data had been entered correctly. Second, if data were correct, we visually inspected the graph and successively removed outlying studies to see if homogeneity was restored. For this review we decided that should this occur with data contributing to the summary finding of no more than around $10 \%$ of the total weighting, we would present these data. If not, then we did not pool data and discussed issues. We know of no supporting research for this $10 \%$ cut-off, but we use prediction intervals as an alternative to this unsatisfactory state.

When unanticipated clinical or methodological heterogeneity was obvious, we simply stated hypotheses regarding these for future reviews or versions of this review. We do not anticipate undertaking analyses relating to these.

\section{Sensitivity analysis}

We applied all sensitivity analyses to the primary outcomes of this review.

\section{Implication of randomisation}

We aimed to include trials in a sensitivity analysis if they were described in some way so as to imply randomisation. For the primary outcomes, we included these studies and if there was no substantive difference when the implied randomised studies were added to those with better description of randomisation, then we entered all data from these studies.

\section{Assumptions for lost binary data}

Where assumptions had to be made regarding people lost to followup (see Dealing with missing data), we compared the findings of the primary outcomes when we used our assumption/s and when we used data only from people who completed the study to that point. If there was a substantial difference, we reported results and discussed them, but continued to employ our assumption.

Where assumptions had to be made regarding missing SDs data (see Dealing with missing data), we compared the findings of the primary outcomes when we used our assumption/s and when we used data only from people who completed the study to that point. A sensitivity analysis was undertaken to test how prone results 
were to change when completer-only data only were compared to the imputed data using the above assumption. If there was a substantial difference, we reported results and discussed them, but continued to employ our assumption

\section{Risk of bias}

We analysed the effects of excluding trials that were judged to be at high risk of bias across one or more of the domains of randomisation (implied as randomised with no further details available): allocation concealment, blinding and outcome reporting for the meta-analysis of the primary outcome. If the exclusion of trials at high risk of bias did not substantially alter the direction of effect or the precision of the effect estimates, then we included data from these trials in the analysis.

\section{Imputed values}

We planned to undertake a sensitivity analysis to assess the effects of including data from trials if we had used imputed values for ICC in calculating the design effect in cluster-randomised trials, however no cluster-randomised trials were included in this version of the review.

If we noted substantial differences in the direction or precision of effect estimates in any of the sensitivity analyses listed above, we did not pool data from the excluded trials with the other trials contributing to the outcome, but presented them separately.

\section{Fixed-effect and random-effects}

We synthesised data using a fixed-effect model, unless data were heterogenous, in which case we used a random-effects model (see Assessment of heterogeneity).

\section{RE S U L T S}

\section{Description of studies}

Please see Characteristics of included studies, Characteristics of excluded studies, Characteristics of studies awaiting classification, and Characteristics of ongoing studies.

\section{Results of the search}

In total, we identified 1396 references from the searches, many of which could be excluded on the basis of their abstracts. We selected 227 references in the previous search and 51 from the update search and obtained full-text papers for assessment. We included an additional four studies (Davidson 2004; Klein 1998; Penn 2009; Uzenoff 2007); the review now includes 24 studies with 56 references (see Figure 1). 
Figure 1. Study flow diagram.

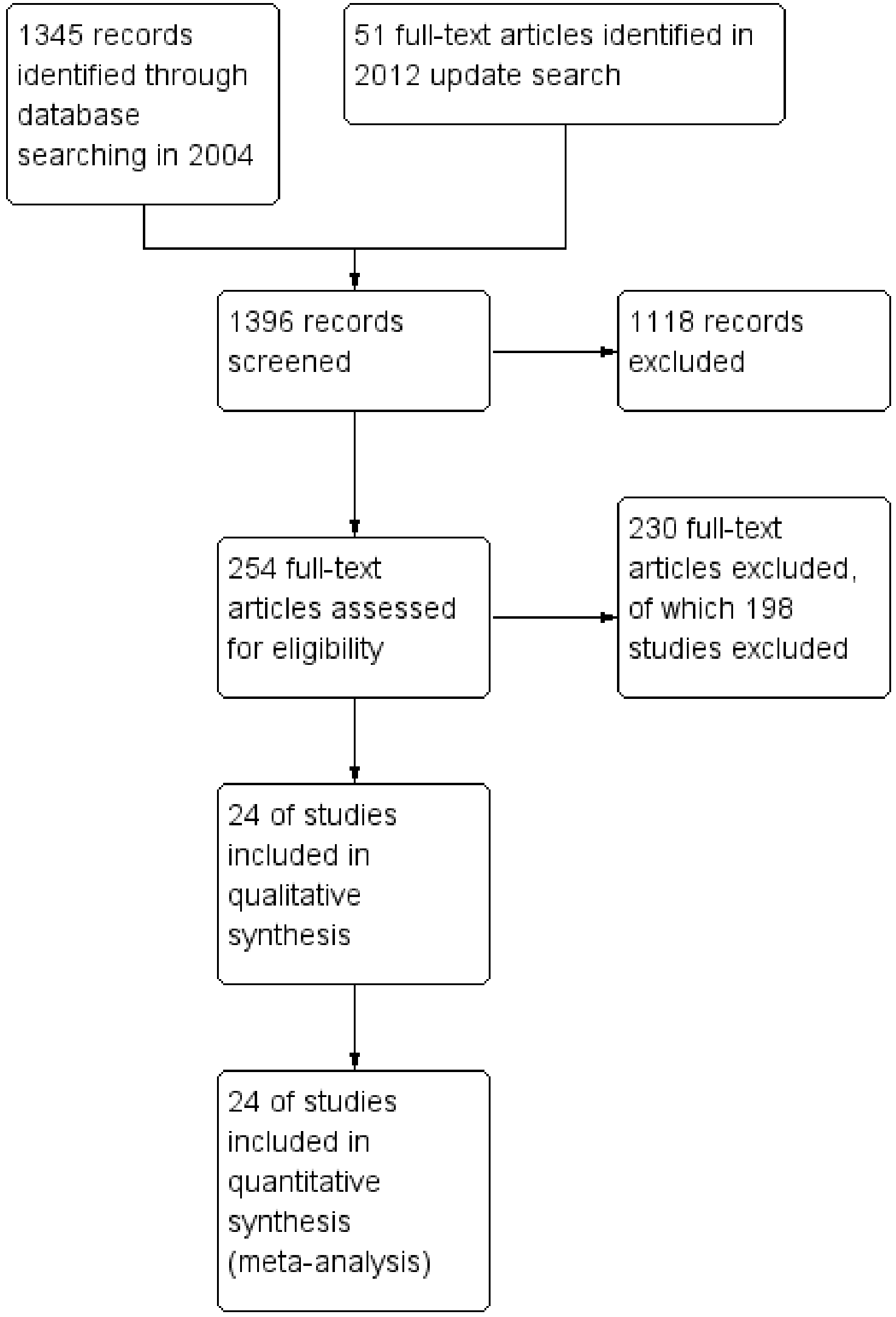




\section{Included studies}

\section{Method}

Due to the nature of the interventions, none of the 24 included studies were able to use a double-blind technique. Some (11/24), however, endeavoured to reduce bias by having some or all outcomes rated by people blind to allocation (Durham 2003; Eckman 1992; Haddock 1999; Kemp 1996; Levine 1998; Lewis 2002b; Sensky 2000b; Spaulding 1999; Stanton 1984; Tarrier 1998; Turkington 2000). Two studies attempted to keep therapists blind to the specific study hypothesis (Coyle 1988; Stanton 1984). Some studies reported outcomes immediately after therapy, whilst others reported outcomes after a follow-up period without therapy.

\section{Duration}

The overall duration of the trials varied between 10 weeks and three years. Three studies (Levine 1998; Lewis 2002b; Penn 2009) were short term (up to 12 weeks). Five studies (Coyle 1988; Klein 1998; Pinto 1999; Turkington 2000; Uzenoff 2007) were medium term (13 to 26 weeks), and the remaining 16 studies were long term (more than 26 weeks).

\section{Participants}

Nineteen included studies had less than 100 participants; the range of the number of participants was 12 to 315 . A total of 2126 people participated in the 24 trials, most of whom had a diagnosis of schizophrenia. Ten studies included only people with schizophrenia, however two of these (Coyle 1988; Malm 1982) did not describe criteria clearly. Sixteen trials included people with other psychotic illnesses (schizoaffective disorder, delusional disorder and other psychoses). Of these, one study also included people with bipolar disorder (O'Donnell 1999) and one included severely disturbed psychiatric patients (Dincin 1982). Twentythree studies employed operational criteria for diagnoses (DSMII, DSM-III, DSM-III-R, DSM-IV, ICD-10, RDC, WHO Discrimination Criteria), however, one of these trials Coyle 1988 did not report how the diagnoses were reached. Malm 1982 described the diagnostic criteria used, but these were not recognised operational criteria. Twenty trials included male and female participants, two studies (Eckman 1992; Wirshing 1991) included men only, and the remaining two studies (Levine 1998; Uzenoff 2007) did not report the sex of participants. Ages of participants ranged from 16 to 72 years.

\section{Setting}

Eleven trials took place in outpatient settings. Three trials were conducted in inpatient settings (Haddock 1999; Spaulding 1999; Stanton 1984), seven were in a mixture of inpatient and outpatient settings (Durham 2003; Lewis 2002b; Malm 1982; O'Donnell 1999; Pinto 1999; Turkington 2000; Wirshing 1991), and the rest did not report on setting.

Thirteen studies were carried out in the USA (Coyle 1988; Davidson 2004; Dincin 1982; Eckman 1992; Falloon 1982; Hogarty 1997study 1; Hogarty 1997-study 2; Klein 1998; Penn 2009; Spaulding 1999; Stanton 1984; Uzenoff 2007; Wirshing 1991); seven in the UK (Durham 2003, Haddock 1999; Kemp 1996; Lewis 2002b; Sensky 2000b; Tarrier 1998; Turkington 2000); one in Australia (O'Donnell 1999); one in Israel (Levine 1998); one in Italy (Pinto 1999); and one in Sweden (Malm 1982).

\section{Interventions}

\subsection{Experimental intervention}

All studies used supportive therapy in addition to standard care (including antipsychotic medication). There were variations between studies with regard to frequency and duration of therapy sessions. Most studies used twice-weekly, weekly and fortnightly sessions, although some studies did not report on the frequency or duration of intervention (Durham 2003; Haddock 1999; O'Donnell 1999; Pinto 1999). The duration of therapy varied between five weeks and three years. Fifteen studies investigated individual treatment, and six studies delivered supportive therapy in a group format (Dincin 1982; Eckman 1992; Levine 1998; Malm 1982; Spaulding 1999; Wirshing 1991). Seven studies reported the use of therapy manuals or protocols (Durham 2003; Hogarty 1997study 1; Hogarty 1997-study 2; Lewis 2002b; Malm 1982; Penn 2009; Spaulding 1999). Eight studies reported that therapists were supervised (Davidson 2004; Haddock 1999; Hogarty 1997-study 1; Hogarty 1997-study 2; Lewis 2002b; Malm 1982; Penn 2009; Sensky $2000 \mathrm{~b})$, and six that audiotapes of sessions were monitored for quality (Haddock 1999; Lewis 2002b; Penn 2009; Sensky 2000b; Turkington 2000; Uzenoff 2007). Six of the studies reported that the same therapists delivered experimental and control interventions, and three studies reported using different therapists for different therapeutic interventions (Durham 2003; O'Donnell 1999; Stanton 1984). Therapists delivering the supportive intervention were trained in a different therapeutic modality, such as cognitive behavioural therapy (CBT) or family therapy, in four studies (Haddock 1999; Lewis 2002b; Penn 2009; Sensky 2000b).

\subsection{Control intervention}

Five studies compared supportive therapy with standard treatment alone (Coyle 1988; Davidson 2004; Durham 2003; Lewis 2002b; Tarrier 1998), the remaining trials used various other psychosocial interventions for comparison. Twelve studies compared supportive therapy with CBT (Durham 2003; Haddock 1999; Hogarty 1997study 1; Hogarty 1997-study 2; Kemp 1996; Levine 1998; Lewis 2002b; Pinto 1999; Sensky 2000b; Spaulding 1999; Tarrier 1998; Turkington 2000). Two studies used family therapy as a comparison (Falloon 1982; Hogarty 1997-study 1). Skills training was investigated in three studies (Coyle 1988; Eckman 1992; Wirshing 1991); other comparisons were personal therapy plus family therapy (Hogarty 1997-study 1), psychoeducation (Coyle 1988; Uzenoff 2007), milieu rehabilitation programme (Dincin 1982) and insight-oriented psychotherapy (Stanton 1984). One study investigated supportive therapy combined with client-focused case management in comparison with client-focused case management (O'Donnell 1999). One trial compared supportive therapy with intensive case management in comparison with intensive case management (Klein 1998). Finally, one trial investigated the effect of adding supportive therapy to a combination of social skills training and medication (Malm 1982). Fourteen of the studies attempted to match experimental and control psychosocial interventions for the amount of therapist contact (Eckman 1992; Falloon 1982; Haddock 1999; Kemp 1996; Levine 1998; Lewis 2002b; Penn 2009; Pinto 1999; Sensky 2000b; Spaulding 1999; Tarrier 1998; Turkington 2000; Uzenoff 2007; Wirshing 1991). In contrast, four studies took the approach that different interventions by their nature involve different amounts of therapist contact (Dincin 1982; Hogarty 1997-study 1; Hogarty 1997-study 2; Stanton 1984). The other studies did not report on this matter. Davidson 2004 gave all participants a $\$ 28$ stipend whether they received supportive care or 
not to control for possible effects of receiving funds to take part in social activities.

\section{Outcomes}

Listed below are the outcomes for which we could obtain usable data, followed by a summary of data that could not be used in the meta-analysis.

\subsection{Outcome scales}

\subsubsection{Mental state}

i. Positive and Negative Syndrome Scale (PANSS) (Kay 1987) This scale assesses the severity of psychotic symptomatology in general. It consists of three subscales, positive symptoms, negative symptoms and general psychopathology, and a total score. This scale was used by Durham 2003; Levine 1998; Lewis 2002b; Penn 2009 and Uzenoff 2007.

\section{ii. Brief Psychiatric Rating Scale (BPRS) (Lukoff 1996)}

This is a semi-structured interview, which assesses the major psychiatric symptoms. It is a 16-item scale, and each item is scored on a seven-point scale, ranging from 'not present' to 'extremely severe'. The range of possible scores is 24 to 168 , and high scores indicate more severe symptoms. Data from the BPRS is reported by Haddock 1999; Kemp 1996; Penn 2009; and Pinto 1999. Kemp 1996 reported data for the full version of the BPRS immediately after treatment. They also used an abridged version of the scale, which contained seven of the 16 items, including psychotic symptoms, negative symptoms and depression. Results for the abridged version were reported immediately after treatment and at six-month, 12-month and 18-month follow-up.

iii. Schedule for Assessment of Negative Symptoms (SANS) (Andreasen 1983)

This scale assesses the negative symptoms of schizophrenia. A six-point scale is used to rate alogia, affective blunting, avolitionapathy, anhedonia-apathy, anhedonia-asociality and attention impairment. Higher scores indicate more severe symptoms. This scale was used by Pinto 1999 and Sensky 2000b.

iv. Schedule for Assessment of Positive Symptoms (SAPS) (Andreasen 1984)

This scale selectively assesses the positive symptoms of psychosis and the higher the score, the more severe the symptoms. Pinto 1999 used this scale.

v. Psychotic Symptoms Rating Scales (PSYRATS) (Haddock 1999b) This consists of two scales, which assess delusional beliefs and auditory hallucinations. There are 11 items in the auditory hallucinations scale, including frequency, duration, level of distress, controllability, loudness, location and beliefs about origin of voices. The delusional beliefs scale has six items, including preoccupation, intensity of distress, conviction and disruption. Each item is rated on a five-point scale. The PSYRATS were used by Lewis 2002b and Penn 2009.

vi. Belief about Voices Questionnaire-Revised (BAVQ-R) (Chadwick 2000)

This scale is a 35-item measure of beliefs about auditory hallucinations and the emotional and behavioural reactions to them. There are five BAVQ-R subscales: malevolence, benevolence, resistance, engagement, and omnipotence. Each item is rated on a four-point scale; a higher score indicates more belief in their voices. This scale was used by Penn 2009.

vii. Comprehensive Psychiatric Rating Scale (CPRS) (Montgomery 1978)

This is a general psychiatric rating scale, which was used by Sensky 2000b and Malm 1982. A high score indicates severe symptoms. Malm 1982 used the schizophrenia subscale, which consists of 45 items and a global rating of degree of illness. The paper reported useable data for only two of the 45 items on this scale.

viii. Beck Depression Inventory II (BDI-II) (Beck 1996)

This scales measures the severity of depression. It has 21 selfreported items measured on a four point scale. A higher score indicates more severe depression. This scale was used by Penn 2009.

ix. Calgary Depression Rating Scale (CDRS) (Addington 1993) This is a nine-item structured interview scale developed specifically for assessing depression in individuals with schizophrenia. The CDRS assesses depression as separate from overlapping negative or extrapyramidal symptoms. Higher scores indicate greater depression. This scale was used by Uzenoff 2007.

x. Center for Epidemiological Studies-Depression Scale (CES-D) (Radloff 1977)

This is a 20-item scale to measure depressive symptomology. The possible range of scores is zero to 60 and higher scores indicate more symptoms. This scale was used by Davidson 2004.

xi. Montgomery-Asberg Depression Rating Scale (MADRS) (Montgomery 1979)

This scale was developed using a 65-item psychopathology scale to identify the 17 most commonly occurring symptoms in primary depressive illness. The maximum score is 30 , and a higher score indicates more severe psychopathology. The scale was used by Sensky 2000b.

\subsubsection{General functioning}

i. Global Assessment of Functioning Scale (GAF) (APA 1987)

The GAF disability scale is taken from the DSM-III-R (APA 1987). The scale has 10 defined anchor points relating to social competence, and scores range from zero to 100 . A higher score indicates better functioning. Durham 2003; Kemp 1996; and Klein 1998 report data from this scale. Davidson 2004 uses the GAF-M, which is a modified version.

ii. Global Assessment Scale (GAS) (Endicott 1976)

This scale provides a rating between zero and 100 , which reflects overall degree of psychological or psychiatric health. A high score indicates better health. This scale was used by Durham 2003.

iii. Katz Adjustment Scales (KAS) (Katz 1963)

This collection of scales measures social adjustment and behaviour. It was used by Malm 1982. It consists of items which can be grouped into subscales including 'performance of socially expected activities' ( 15 items) and 'free-time activities' (23 items), the two subscales chosen by Malm 1982. In this study, the items were also grouped into syndromes, entitled 'offensive behaviour' (two items) and 'withdrawal' (six items).

iv. Social Functioning Scale (SFS) (Birchwood 1990) 
The SFS is a 79-item self-report questionnaire to both the patients and the caregiver, about performance in seven areas: Social Engagement (SE), Interpersonal Communication (IC), Recreational Activities (RA), Social Activities (SA), Independence Competence (INC), Independence Performance (IP) and Occupational Activity $(\mathrm{OA})$. The purpose of the scale is to provide an evaluation of strengths and weaknesses of patient functioning, and it may reveal aims for therapeutic intervention.

\subsubsection{Behaviour}

i. Social Behaviour Adjustment Scale (SBAS) (Platt 1980)

Falloon 1982 was the only study to use this scale. Scoring is based on a structured interview with a key informant from the person's family. Areas covered include household tasks, spare time/leisure activity, work/study, decision making, friendliness/ affection, everyday conversation, relationships outside the family, behavioural disturbance, social and interpersonal role performance, and adverse effects on the family and other people in the community. Each item is scored on a scale from zero to two, and higher scores indicate greater impairment or dissatisfaction.

\subsubsection{Insight}

i. Beck Cognitive Insight Scale - (BCIS) composite (Beck 2004) The $\mathrm{BCIS}$ is a 15-item self-report measure composed of two subscales: self-reflectiveness and self-certainty. Penn 2009 used a composite index of insight, computed from the BCIS. Higher scores reflect greater cognitive insight.

ii. Insight and Treatment Attitudes Questionnaire (ITAQ) (McEvoy 1989)

This scale assesses an individual's recognition of illness and need for treatment and includes 11 questions. Reponses are rated as follows: 2 = good insight, $1=$ partial insight, and $0=$ no insight, which are summed to provide a total insight score. Higher scores correspond to better insight. Uzenoff 2007 reported data on this scale.

iii. Schedule for Assessment of Insight (SAI) (David 1990) The schedule is a semi-structured interview with three components; treatment compliance, awareness of illness and ability to relabel psychotic symptoms correctly. The range of possible scores is zero to 14 , but scores are expressed as a percentage of maximum insight. Low scores therefore indicate poor insight. The expanded version of the SAI was used by Kemp 1996.

\subsubsection{Compliance}

i. Rating of Medication Influences (ROMI) scale (Weiden 1994)

The ROMI is a comprehensive measure of attitudes regarding adherence to antipsychotic medications used by Uzenoff 2007. The measure is divided into two subscales, 'Reasons for Adherence' (nine items) and 'Reasons for Nonadherence' (10 items), each containing attitudinal and behavioural factors thought to influence adherence behaviour. Items are rated on a one through three scale, where $1=$ no influence, $2=$ mild influence, $3=$ strong influence, and $96=$ not applicable. Higher scores on the 'Reasons for Adherence' subscale correspond to an endorsement of factors influencing the individual to take his/her medication, whereas higher scores on the 'Reasons for Nonadherence' subscale correspond to an endorsement of factors influencing the individual to stop taking his/her medication.

\subsubsection{Attitude to medication}

i. Attitudes to Medication Questionnaire (AMQ) (Hayward 1995) The AMQ is a semi-structured interview designed to assess attitudes to drug treatment. It was used by Kemp 1996. A high score indicates a helpful attitude to treatment.

ii. Drug Attitudes Inventory (DAI) (Hogan 1983)

The DAl is a self-report scale used to assess attitude to medication. It has been shown to be highly predictive of compliance. A higher score indicates a better attitude to treatment. This scale was used by Kemp 1996.

\subsubsection{Quality of life}

i. Global Health Questionnaire - GHQ (Goldberg 1972)

This 60 -item scale measures the severity of nonpsychotic psychiatric symptomatology. It was used by Davidson 2004. It assesses the respondent's current state and asks if that differs from his or her usual state and produces an overall score that can be compared with a prescribed cut-off score. All items have a fourpoint Likert scoring (0-1-2-3) system that ranges from a 'better/ healthier than normal' option, through a 'same as usual' and a 'worse/more than usual' to a 'much worse/more than usual' option. The higher the score, the more severe the condition.

ii. Quality of Life Scale - QLS (Heinrichs 1984)

This 21-item scale is based on a semi-structured interview providing information on symptoms and functioning during the preceding four weeks. There are seven severity steps (zero to six; six being adequately functioning and zero being deficient). Four item categories have been identified by factor analysis i) interpersonal relationships (seven items), ii) instrumental role (four items), iii) intrapsychic function (seven items) and iv) common place objects and activities. Higher scores indicate better quality of life. This scale was used by Uzenoff 2007.

\section{iii. Rosenberg Self-Esteem Scale (RSES) (Rosenberg 1965)}

The scale measures state self-esteem by asking the respondents to reflect on their current feelings, with a 10-item Likert-type scale with items answered on a four-point scale - from strongly agree to strongly disagree. Five of the items have positively worded statements and five have negatively worded ones. The scale ranges from zero to 30 . Scores between 15 and 25 are within normal range; scores below 15 suggest low self-esteem. This scale was used by Davidson 2004 and Penn 2009.

\section{iv. Well-Being scale- WBS (Ryff 1989)}

This is a scale to measure positive aspects of psychological functioning along six dimensions in which respondents rate statements on a scale of one to six, with one indicating strong disagreement and six indicating strong agreement: independence and self-determination (autonomy); having satisfying, high quality relationships (positive relations with others); the ability to manage one's life (environmental mastery); being open to new experiences (personal growth); believing that one's life is meaningful (purpose in life); and a positive attitude towards oneself and one's past life (self-acceptance). A high score indicates that the respondent has a mastery of that area in his or her life. Conversely, a low score shows that the respondent struggles to feel comfortable with that particular concept. Davidson 2004 reported data for this scale.

v. Lehman's Quality-of-Life (QOL) Questionnaire (Lehman 1988) 
Lehman's QOL scale uses a response range of one (terrible) to seven (delighted). Klein 1998 used only those domains that had direct applicability - namely, living situation, income, family, friends, social interaction, activities of daily living, and health. They also added a section on drug use, as well as a general list of activities, the response scale was one (daily) to five (never).

\subsection{Missing outcomes}

No studies provided data regarding clinically important change in general functioning, one of our primary outcomes of interest. No studies reported data on time to relapse or hospitalisation, clinically important or any changes in general behaviour scores, adverse events, or in any engagement in structured activities. Economic data, as well as satisfaction with treatment were seldom reported.

\section{Excluded studies}

We excluded 198 reports - most because the reported interventions did not meet our criteria for supportive therapy. In several studies, all participants received supportive therapy, without a comparison group relevant to this review. We excluded some studies because the participants did not have schizophrenia or schizophrenia-like illnesses. Some studies were not randomised controlled trials, and we therefore excluded these. We excluded one study due to inadequate allocation concealment (Slavinsky 1982). Eight studies were excluded as they did not report usable data (Castelein 2008; Castilla-Puentes 2002; Keshavan 2011; Klingberg 2010; Meister 2010; Nelson 2007; Smith 1999; Telles 1995). Details of excluded studies are detailed in the Characteristics of excluded studies.

\section{Awaiting assessment studies}

Three studies are awaiting assessment. Bechdolf 2012, and Blankertz 1997 are awaiting translation from German and Falloon 1983 is awaiting assessment until we obtain a full-text paper.

\section{Ongoing studies}

Sixteen studies seem to be ongoing. We are obtaining more details to ensure that this is indeed the case. These studies are using a variety of interventions, including behavioural treatment, CBT, cognitive enhancement therapy, body orientated therapy, with supportive therapies and counselling as controls.

\section{Risk of bias in included studies}

See also 'Risk of bias' tables in Characteristics of included studies and Figure 2 and Figure 3.

Figure 2.

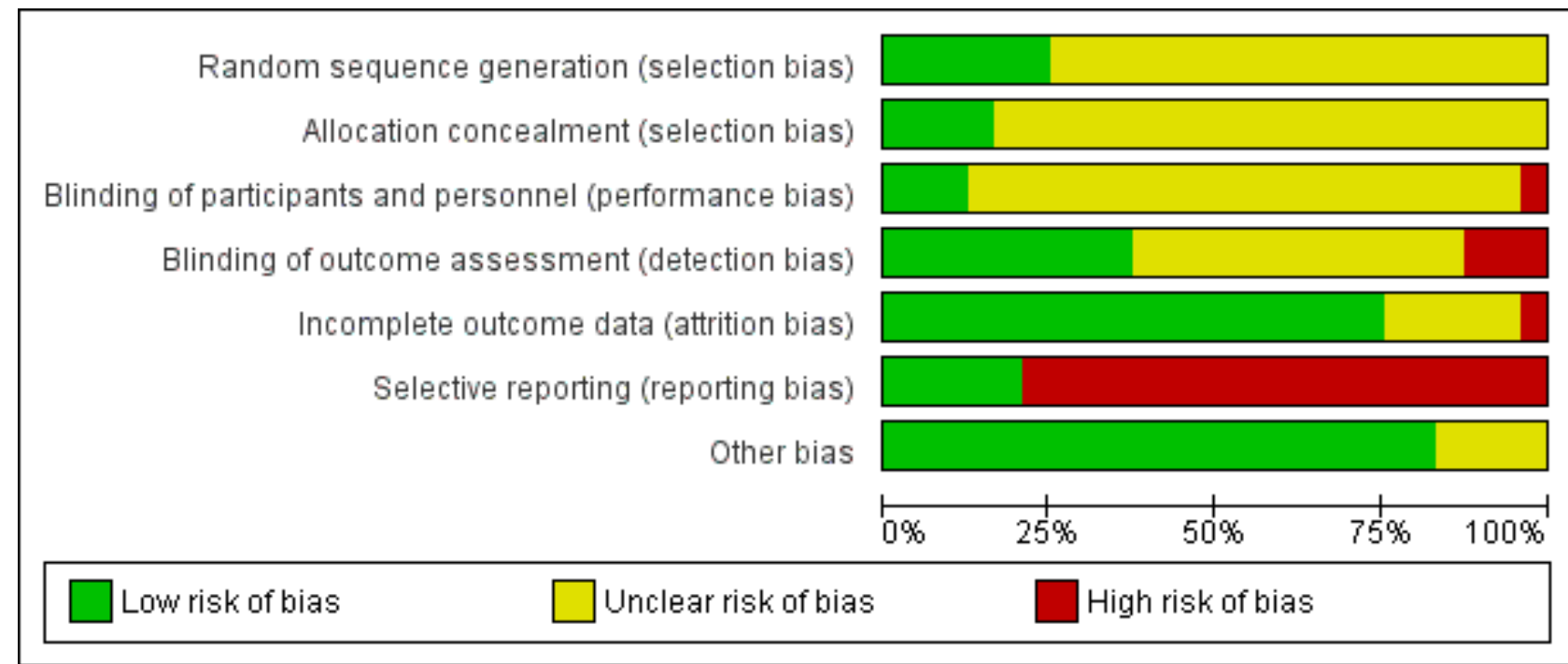


Figure 3.

\begin{tabular}{|c|c|c|c|c|c|c|c|}
\hline & 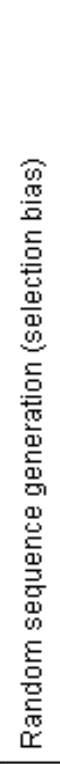 & 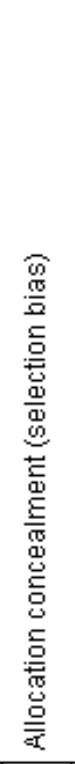 & 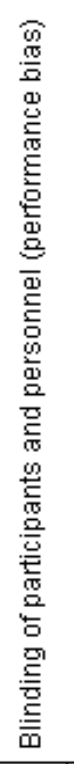 & 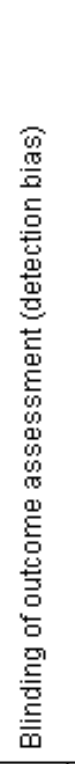 & 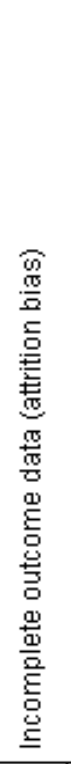 & 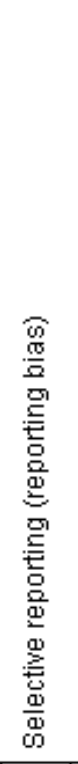 & 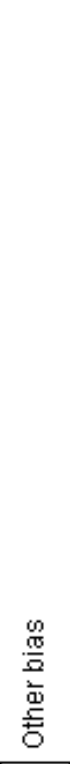 \\
\hline Coyle 1988 & $?$ & $?$ & $?$ & $?$ & + & $\odot$ & $?$ \\
\hline Davidson 2004 & $?$ & $?$ & $?$ & $?$ & $?$ & $\odot$ & + \\
\hline Dincin 1982 & $?$ & $?$ & $?$ & $?$ & $?$ & $\odot$ & $\odot$ \\
\hline Durham 2003 & + & + & $?$ & $?$ & $\odot$ & 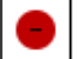 & $\odot$ \\
\hline Eckman 1992 & $?$ & $?$ & $?$ & $?$ & $?$ & $\odot$ & $\odot$ \\
\hline Falloon 1982 & $?$ & $?$ & $?$ & + & + & $\odot$ & $\odot$ \\
\hline Haddock 1999 & $?$ & $?$ & $?$ & + & + & $\Theta$ & $?$ \\
\hline Hogarty 1997 -study 1 & $?$ & $?$ & $?$ & $\Theta$ & + & - & + \\
\hline Hogarty 1997 -study 2 & $?$ & $?$ & $?$ & $\Theta$ & + & 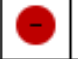 & + \\
\hline Kemp 1996 & + & $?$ & $?$ & $?$ & $\odot$ & $\theta$ & $\odot$ \\
\hline Klein 1998 & $?$ & $?$ & $?$ & $?$ & $\odot$ & $\theta$ & + \\
\hline Levine 1998 & $?$ & $?$ & $?$ & $\odot$ & $?$ & 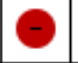 & $?$ \\
\hline Lewis $2002 \mathrm{~b}$ & + & + & $\odot$ & + & $\odot$ & + & + \\
\hline Malm 1982 & $?$ & $?$ & $?$ & $?$ & + & $\theta$ & $\odot$ \\
\hline O'Donnell 1999 & $?$ & $?$ & $?$ & $?$ & + & $\theta$ & + \\
\hline Penn 2009 & + & + & $?$ & + & + & + & + \\
\hline Pinto 1999 & $?$ & $?$ & $?$ & $?$ & + & - & $?$ \\
\hline Sensky $2000 \mathrm{~b}$ & $?$ & + & $?$ & + & + & $\odot$ & $\odot$ \\
\hline Spaulding 1999 & $?$ & $?$ & + & $?$ & + & $\odot$ & + \\
\hline Stanton 1984 & + & $?$ & $\odot$ & $?$ & $\odot$ & $\theta$ & + \\
\hline
\end{tabular}


Figure 3. (Continued)

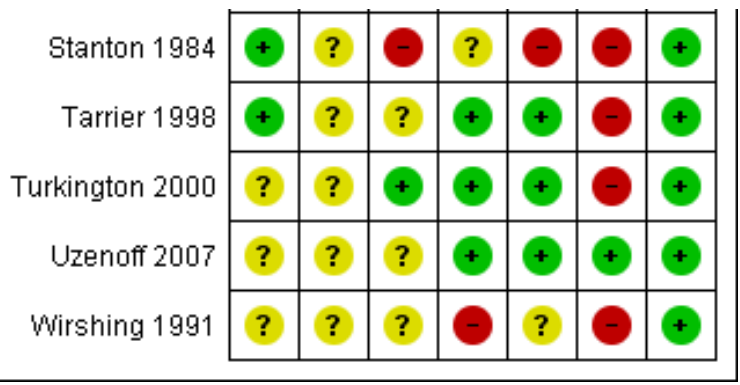

\section{Allocation}

All studies were randomised trials. However, most studies did not provide explicit details of the method of randomisation, and were rated as unclear risk of bias. Six studies were rated low risk of bias. Kemp 1996 reports the use of a table of random numbers. Penn 2009 stratified according to gender, and stratification was also undertaken by Lewis 2002b with the following variables: first or second admission; inpatient or day patient admission; male or female; with the first-episode cases further stratified for duration of symptoms of more or less than six months. Tarrier 1998 used block randomisation, with a block size of nine, stratified by severity of symptoms and sex. Durham 2003 used permuted blocking. Spaulding 1999 matched pairs and assigned each paired participant to one of two groups. The groups were then were randomly assigned to either the treatment or the control intervention. Coyle 1988 describes stratifying participants according to age, sex and race prior to randomisation, but no further details were reported and was rated as unclear.

With regard to allocation concealment, most studies did not report any concealment approach, and were rated as unclear risk of bias. Five studies described the method of allocation concealment, four of which we rated as low risk. Durham 2003 provided a clear description of the randomisation procedure, which was administered centrally by a non-clinical project coordinator, and a sealed envelope technique was used. Sensky $2000 \mathrm{~b}$ reported that randomisation was done by members of the research team not involved with assessment or treatment. Lewis $2002 \mathrm{~b}$ reported that independent, concealed randomisation with minimisation was performed by trial administrators. Penn 2009 reported randomisation by a blinded researcher, using random numbers generated by computer. Tarrier 1998 reported that randomisation was carried out independently by a third party, using sealed envelopes, but not whether the envelopes were opaque and so we rated it as having an unclear risk of bias.

\section{Blinding}

None of the studies were able to use a double-blind technique due to the nature of the interventions, since it would not be possible to have therapists and participants blind to treatment allocation. Turkington 2000, Lewis 2002b, and Spaulding 1999 were the only studies to report appropriate personnel blinding and were rated as low risk of bias. In Stanton 1984, ward and project staff were not blinded, and so was rated as high risk of bias. Blinding of personnel for the remaining trials was not adequately reported and these were rated as unclear risk of bias.
Nine studies stated that outcome raters were blinded to the treatment group (Falloon 1982; Haddock 1999; Levine 1998; Lewis 2002b; Penn 2009; Sensky 2000b; Tarrier 1998; Turkington 2000; Uzenoff 2007) and were rated as low risk of bias for outcome assessment. Two studies (Durham 2003; Eckman 1992) attempted to keep patients from reporting their treatment groups to raters, however both reported that some patients disclosed allocation, and these were rated as unclear risk of bias. Stanton 1984 reported that some outcomes were assessed blindly and others were not. Hogarty 1997-study 1, Hogarty 1997-study 2, and Wirshing 1991 reported raters who were not blinded and were rated as high risk of bias.

Only Coyle 1988 and Stanton 1984 reported that they attempted to keep therapists blinded to the specific study hypothesis.

\section{Incomplete outcome data}

Overall, eighteen studies were rated as low risk of bias for incomplete outcome data. Lewis 2002b, Penn 2009, Sensky 2000b, Tarrier 1998, and Uzenoff 2007 reported outcomes with an intention-to-treat method, although Tarrier 1998 carried out an intention-to-treat analysis post-treatment only, and not at the one- or two-year follow-ups. Levine 1998 reported no participants leaving early. Sixteen studies reported reasons for losses to followup (Coyle 1988; Durham 2003; Falloon 1982; Haddock 1999; Hogarty 1997-study 1; Hogarty 1997-study 2; Kemp 1996; Klein 1998; Lewis 2002b; Malm 1982; O'Donnell 1999; Pinto 1999; Spaulding 1999; Tarrier 1998; Turkington 2000; Uzenoff 2007).

Of the five studies with unclear risk of bias for incomplete outcome data, Davidson 2004, Dincin 1982, Eckman 1992, Levine 1998, and Wirshing 1991 did not report on attrition. Only Stanton 1984 had a high risk of bias due to high rates of attrition reported without reasons.

\section{Selective reporting}

Five studies were of low risk of bias with regard to selective reporting (Davidson 2004, Lewis 2002b, Penn 2009, Sensky 2000b, and Uzenoff 2007). The remaining 19 studies were of high risk of bias, mainly due to poor data reporting - either they did not report data for outcomes or SDs were missing.

\section{Other potential sources of bias}

Four trials were unclear if they were subject to other biases (Coyle 1988; Haddock 1999; Levine 1998; Pinto 1999). All other trials appeared free from other sources of bias. 


\section{Effects of interventions}

See: Summary of findings for the main comparison SUPPORTIVE THERAPY versus STANDARD CARE for schizophrenia; Summary of findings 2 SUPPORTIVE THERAPY versus ANY OTHER PSYCHOLOGICAL OR PSYCHOSOCIAL TREATMENT for schizophrenia; Summary of findings 3 SUPPORTIVE THERAPY versus COGNITIVE BEHAVIOURAL THERAPY for schizophrenia; Summary of findings 4 SUPPORTIVE THERAPY versus FAMILY THERAPY for schizophrenia; Summary of findings $\mathbf{5}$ SUPPORTIVE THERAPY versus PSYCHOEDUCATION for schizophrenia

For dichotomous outcomes, we calulated risk ratios (RR) and for continuous outcomes, we calculated mean differences (MD), both with $95 \%$ confidence intervals $(\mathrm{Cl})$.

\section{Comparison 1: SUPPORTIVE THERAPY versus STANDARD CARE}

Five included studies compared supportive therapy with standard care (Coyle 1988; Davidson 2004; Durham 2003; Lewis 2002b; Tarrier 1998).

\subsection{Global state: relapse}

We found no significant difference in relapse rates between supportive therapy and standard care (Tarrier 1998, medium term, $\mathrm{n}=54$, RR $0.12 \mathrm{Cl} 0.01$ to 2.11 ; long term, $\mathrm{n}=54$, RR $0.96 \mathrm{Cl}$ 0.44 to 2.11 , Analysis 1.1). In this trial, relapses were defined as readmission to hospital for clinical deterioration that lased at least five days and resulted in functional impairment.

\subsection{Service outcomes: hospitalisation}

Hospitalisation rates did not differ significantly between people treated with supportive therapy and standard care ( $1 \mathrm{RCT}, \mathrm{n}=48$, RR $1.00 \mathrm{Cl} 0.07$ to 15.08 , Analysis 1.2).

\subsection{Mental state: general}

\subsubsection{No clinically important improvement in general mental state}

Medium-term data for 'no clinically important improvement in general mental state' were not statistically significant (Tarrier 1998, $\mathrm{n}=54, \mathrm{RR} 0.95 \mathrm{Cl} 0.77$ to 1.17 ), and longer-term data ( $2 \mathrm{RCTs}, \mathrm{n}=98$, $\mathrm{RR} 0.95 \mathrm{Cl} 0.82$ to 1.11 ) were also equivocal (Analysis 1.3).

\subsubsection{Average endpoint general mental state score}

Total scores on the PANSS were not significantly different at both short- (Lewis 2002b, $\mathrm{n}=131, \mathrm{MD}-4.42 \mathrm{Cl}-10.1$ to 1.29 ) and long-term time points (Durham 2003, $\mathrm{n}=36 \mathrm{MD} 4.70 \mathrm{Cl}-6.71$ to 16.11 , Analysis 1.4).

\subsection{Mental state: specific symptoms}

\subsubsection{Average endpoint negative symptoms score}

Skewed data from the SANS scale are presented in tabular form (Analysis 1.5).

\subsubsection{Average endpoint positive symptoms score}

We found that the PANSS positive subscale data from the Lewis 2002b study showed no significant difference between supportive therapy and standard care $(n=131, M D-1.09 \mathrm{Cl}-2.84$ to 0.66 , Analysis 1.6). One study measured severity of positive symptoms using selected items from the BPRS, but data are skewed and are presented as 'Other data' in a table (Analysis 1.7).

\subsubsection{Average endpoint delusions score and hallucinations score}

Skewed data from the PSYRATS scale 'delusions score' and 'hallucinations score' were obtained from one trial and are presented in a tabular form (Analysis 1.8; Analysis 1.9).

\subsubsection{Average endpoint depressive symptoms score}

Davidson 2004 found no statistical significance between the treatment groups for Center for Epidemiological StudiesDepression Scale (CESD) ( $\mathrm{n}=260, \mathrm{MD} 1.61 \mathrm{Cl}-1.61$ to 4.83 , Analysis 1.10).

\subsection{Leaving the study early}

We found no significant difference in rates of attrition between supportive therapy and standard care (4 RCTs, $\mathrm{n}=354, \mathrm{RR} 0.86 \mathrm{Cl}$ 0.53 to 1.40 , Analysis 1.11).

\subsection{General functioning}

\subsubsection{Average endpoint in general functioning score}

This was assessed by Durham 2003 using the GAS. This study found no significant difference between supportive therapy and standard care $(n=29, M D 1.40 \mathrm{Cl}-5.09$ to 7.89, Analysis 1.13). Davidson 2004 used the GAF-M and also found no significant results ( $n=260$, MD $-2.66 \mathrm{Cl}-6.20$ to 0.88 Analysis 1.12 ).

\subsubsection{Average endpoint in social functioning score}

Davidson 2004 found no statistical significance between the treatment groups for social functioning measured on the SFS ( $\mathrm{n}=$ 260, MD $-0.67 \mathrm{Cl}-7.05$ to 5.71 , Analysis 1.14).

\subsection{Satisfaction with treatment: recipient of care not satisfied with treatment}

We found no significant difference in the numbers of recipients of care who were not satisfied with their treatment (Durham 2003, $\mathrm{n}=$ 44, RR $1.83 \mathrm{Cl} 0.75$ to 4.47 , Analysis 1.15 ).

\subsection{Quality of life}

\subsubsection{Average endpoint self-esteem score}

Davidson 2004 found no significant results between standard care and supportive therapy for self-esteem on the Rosenberg SelfEsteem Scale (RSES) ( $n=260$, MD $-1.21 \mathrm{Cl}-2.85$ to 0.43 , Analysis 1.16 ),

\subsubsection{Average endpoint well-being score}

Davidson 2004 also assessed well-being on the WBS and found no significant difference in psychological functioning between treatment groups ( $n=260, \mathrm{MD}-2.73 \mathrm{Cl}-6.04$ to 0.58 , Analysis 1.17 ).

\subsubsection{Average endpoint global health score}

Global health was measured by Davidson 2004 on the GHQ and found no difference in nonpsychotic psychiatric symptomatology ( $\mathrm{n}=260, \mathrm{MD}-2.45 \mathrm{Cl}-2.41$ to 7.31 , Analysis 1.18 )

\subsection{Death}

The number of deaths occurring in the Tarrier 1998 study at medium term, were not significantly different (supportive therapy $1 / 26$, standard care $0 / 28, \mathrm{n}=54, \mathrm{RR} 3.22 \mathrm{Cl} 0.14$ to 75.75 ). Two trials reporting long-term data were also non-significant $(n=92, R R 2.87$ $\mathrm{Cl} 0.31$ to 26.63 , Analysis 1.19). 


\subsection{Medication: no reduction in dose of antipsychotic}

Durham 2003 reported on the number of people who had no reduction in the dose of antipsychotic medication at the endpoint of the trial. We found no statistically significant difference between the groups ( $\mathrm{n}=44, \mathrm{RR} 0.81 \mathrm{Cl} 0.59$ to 1.12 , Analysis 1.20 ).

\subsection{Costs}

Most studies did not measure economic outcomes and no studies reported on costs in a way that could be added to the analysis. Klein 1998 reported that their findings suggest "that if the peer social supporter (the FC) had not been available, the study group may have needed as many inpatient days as the comparison group" and that this would have equated to "250 inpatient days (10 clients x 25 days)" that could have cost $\$ 250,000$ (based on average cost of $\$ 1,000$ per day, per client for inpatient psychiatric care) and compare this to "the cost of $10 \mathrm{FCs}$ for 6 months, including administrative and programmatic costs, was about $\$ 30,000 "$.

\section{Comparison 2: SUPPORTIVE THERAPY versus ANY OTHER PSYCHOLOGICAL OR PSYCHOSOCIAL TREATMENT}

Twenty included studies compared supportive therapy against any other psychological or psychosocial treatment (Coyle 1988; Dincin 1982; Durham 2003; Eckman 1992; Falloon 1982; Haddock 1999; Hogarty 1997-study 1; Hogarty 1997-study 2; Kemp 1996; Levine 1998; Lewis 2002b; Penn 2009; Pinto 1999; Sensky 2000b; Spaulding 1999; Stanton 1984; Tarrier 1998; Turkington 2000; Uzenoff 2007; Wirshing 1991).

\subsection{Global state: relapse}

No significant difference in relapse rates were found in the supportive therapy and other therapies in the medium term $(2$ RCTs, $n=100$, RR $2.86 \mathrm{Cl} 0.32$ to 25.24 , Analysis 2.1 ) or the long term (5 RCTs, $n=270$, RR $1.19 \mathrm{Cl} 0.66$ to 2.16,, Analysis 2.1). Heterogeneity was high $\left(I^{2}=77 \%\right)$, so a random-effects model was used.

\subsection{Global state: no remission}

Falloon 1982 reported the number of people who did not experience remission of symptoms. We found a statistically significant advantage for other psychological therapies (in this case family therapy) over supportive therapy ( $n=39$, RR $1.87 \mathrm{Cl} 1.11$ to 3.15, Analysis 2.2).

\subsection{Service outcomes: hospitalisation}

We found medium-term data for hospitalisation were equivocal (3 RCTs, $n=153$, RR $1.60 \mathrm{Cl} 0.85$ to 3.00 ), but longer-term data were statistically significant, favouring the other psychological or psychosocial therapies over supportive therapy (4 RCTs, $n=306$, RR $1.82 \mathrm{Cl} 1.11$ to 2.99 , Analysis 2.3).

\subsection{Mental state: general}

\subsubsection{No clinically important improvement}

We found a significant difference favouring other therapies over supportive therapy for 'no clinically important improvement' over the long term (3 RCTs, $n=194$, RR 1.27 CI 1.04 to 1.54, Analysis 2.4), but not at medium-term follow-up (Tarrier 1998, $\mathrm{n}=59$, RR $1.27 \mathrm{Cl}$ 0.95 to 1.70$)$.

\subsubsection{Average endpoint score}

\section{i) PANSS total}

A significant difference in favour of other psychological treatment (in this case cognitive behavioural therapy (CBT)) was found for general mental state in the long term ( 2 RCTs, $n=105, \mathrm{MD} 5.82 \mathrm{Cl}$ 1.04 to 10.60 , Analysis 2.5). However, in the short term, the data were highly heterogeneous $\left(1^{2}=98 \%\right)$. We have concerns about the data reported in Levine 1998, (see discussion section Quality of the evidence). We think the data in Levine 1998 may have been incorrectly reported or may have been an anomaly due to the small numbers in each treatment group. Also the standard deviations were very small, and we believe the authors may have erroneously reported standard errors as standard deviations. However, when we remove Levine 1998 from the analysis, heterogeneity is not restored and remains at $83 \%$, therefore data were not pooled for this outcome.

\section{ii) BPRS}

No significant difference in mental state measured on the BPRS was found in the short term (2 RCTs, $n=92, \mathrm{MD}-1.07 \mathrm{Cl}-5.08$ to 2.94); Pinto 1999 found a significant difference in favour of the control group (in this case CBT) in the long term $(n=37, M D 7.60 \mathrm{Cl} 0.90$ to 14.30, Analysis 2.6).

\section{iii) BPRS short form}

Kemp 1996 also used the short form of the BPRS, and found no significant differences at short- $(\mathrm{n}=74, \mathrm{MD}-0.90 \mathrm{Cl}-3.02$ to 1.22$)$, medium- ( $\mathrm{n}=67, \mathrm{MD} 2.20 \mathrm{Cl}-1.18$ to 5.58 ) or long-term follow-up ( $\mathrm{n}$ $=45, \mathrm{MD} 2.30 \mathrm{Cl}-0.54$ to 5.14 , Analysis 2.7 ).

\section{iv) CPRS}

One study presented skewed data from the CPRS (Sensky 2000b). We have reported these in a table (Analysis 2.8).

\section{v) PANSS general subscale}

No significant difference was found for general symptoms measured on the PANSS general subscale in the medium term (1 $\mathrm{RCT}, \mathrm{n}=19, \mathrm{MD} 2.86 \mathrm{Cl}-3.21$ to 8.93 , (Analysis 2.9); in the long term a significant difference was found in favour of the control group (1 $R C T, n=65, M D 3.60 \mathrm{Cl} 0.84$ to 6.36 ). For the short term, we did not pool the data as there was high heterogeneity $\left(1^{2}=97 \%\right)$ and we believe the data reported in Levine 1998 may have been incorrectly reported (see discussion section Quality of the evidence).

\subsection{Mental state: specific symptoms}

2.5.1 No clinically important improvement in negative and depressive symptoms

For the outcome of 'no clinically important improvement in negative symptoms', there was no significant difference between supportive therapy and other therapies (Sensky 2000b, $n=90, R R$ $0.95 \mathrm{Cl} 0.63$ to 1.46 Analysis 2.10). Similarly, for the outcome of 'no important improvement in depressive symptoms', no significant differences were observed (Sensky 2000b, $n=90$, RR $1.53 \mathrm{Cl} 0.92$ to 2.55 Analysis 2.11). However, results show that people who underwent supportive therapy are more likely to have had an episode of affective symptoms than those who underwent other therapies (2 RCTs, $\mathrm{n}=151$, RR $1.84 \mathrm{Cl} 1.15$ to 2.94, Analysis 2.12). 


\subsubsection{Average endpoint in negative symptoms}

\section{i) PANNS negative}

No significant difference was found for general symptoms measured on the PANSS negative subscale in the medium term (1 $\mathrm{RCT}, \mathrm{n}=19, \mathrm{MD} 1.70 \mathrm{Cl}-4.00$ to 7.40 ) or the long term ( $1 \mathrm{RCT}, \mathrm{n}=65$, MD $0.30 \mathrm{Cl}-1.96$ TO 2.56, Analysis 2.13). For the short term, we did not pool the data as there was high heterogeneity $(12=92 \%)$ and we believe the data reported in Levine 1998 may have been incorrectly reported (see discussion section Quality of the evidence).

\section{ii) SANS}

SANS medium-term data (Pinto 1999) were equivocal, Analysis 2.14. Another study also reported SANS data that are skewed, and are therefore reported separately in tabular form, Analysis 2.15.

\subsubsection{Average endpoint in positive symptoms}

\section{i) PANSS positive}

A significant difference in favour of other psychological treatment was found for positive symptoms in the long term ( $1 \mathrm{RCT}, \mathrm{n}=65$, MD $2.30 \mathrm{Cl} 0.60$ to 0.40 , Analysis 2.16). However, in the medium term no significant difference was found ( $1 \mathrm{RCT}, \mathrm{n}=19, \mathrm{MD} 2.71 \mathrm{Cl}-0.71$ to 6.13). For the short term we did not pool data as they were highly heterogeneous $(12=83 \%)$. Furthermore, we have concerns about the data reported Levine 1998, (see discussion section Quality of the evidence). We think the data in Levine 1998 may have been incorrectly reported or may have been an anomaly due to the small numbers in each treatment group. Also the standard deviations were very small, and we believe the authors may have erroneously reported standard errors as standard deviations. However, when we remove Levine 1998 from the analysis, heterogeneity is not restored and remains at $78 \%$.

\section{ii) SAPS}

One study reported data obtained from the SAPS. These data are skewed and are presented as 'Other data' in a table (Analysis 2.17).

\section{iii) BPRS}

One study reported data regarding severity of positive symptoms on the BPRS. However these data are skewed and are reported separately (Analysis 2.18).

\subsubsection{Average endpoint in delusion, hallucination and voices symptoms}

\section{i) PANSS thought disturbance}

Other therapies were found to significantly improve the PANSS thought disturbance cluster score (Levine $1998 \mathrm{n}=12, \mathrm{MD} 4.30 \mathrm{Cl}$ 1.17 to 7.43 , Analysis 2.19 , however, we believe these data may have been incorrectly reported (see above and discussion section Quality of the evidence).

\section{ii) PSYRATS}

Skewed data from the PSYRATS delusions score and hallucinations score are also presented in tabular form (Analysis 2.20; Analysis 2.21). Penn 2009 reported data from the voices score and found no significant difference at short term ( $n=65, M D 0.10 \mathrm{Cl}-3.63$ to 3.83 ) and long term ( $\mathrm{n}=65, \mathrm{MD} 0.00 \mathrm{Cl}-4.76$ to 4.76 , Analysis 2.22$)$.

\section{iii) BAVQ}

Penn 2009 reported skewed data on beliefs about voices using the BAVQ subscales, and are presented as 'Other data' in a table (Analysis 2.23).

\subsubsection{Average endpoint in depressive symptoms}

One study reported results from the MADRS and another from the BDI-II, but data are skewed and are reported in tabular form (Analysis 2.23 and Analysis 2.24, respectively).

\subsection{Leaving the study early}

We found no significant differences between supportive therapy and other treatments for leaving the study early (19 RCTs, $n=1412$, RR $1.03 \mathrm{Cl} 0.87$ to 1.21 , Analysis 2.26). No difference was also found for general reasons for leaving the study early (17 RCTs, $n=1261$, $\mathrm{RR} 0.97 \mathrm{Cl} 0.83$ to 1.15). Treatment-related reasons for leaving the study early were significantly worse in the supportive therapy group (2 RCTs, $\mathrm{n}=151$, RR $2.15 \mathrm{Cl} 1.07$ to 4.31 ).

\subsection{General functioning}

\subsubsection{Average endpoint in general functioning} i) GAF \& GAS

We found GAF scores (Kemp 1996) favoured CBT (short term, $n=70$, $\mathrm{MD}-9.50 \mathrm{Cl}-16.11$ to -2.89 , and medium term, $\mathrm{n}=67, \mathrm{MD}-12.60 \mathrm{Cl}$ -19.43 to -5.77 , Analysis 2.27). When we combined long term data from the GAF and GAS it was heterogeneous $(12=84 \%)$ and so data were not pooled.

\section{ii) SFS}

Penn 2009 measured social functioning on the SFS, and found no significant difference in the short term $(n=65, M D-7.20 \mathrm{Cl}-17.86$ to 3.46) and the long term $(n=65, M D-8.80 \mathrm{Cl}-21.67$ to 4.07 , Analysis 2.28).

\subsubsection{Specific aspects}

No advantage or disadvantages were found for people who had supportive therapy in terms of the number of people not in paid work (2 RCTs, $\mathrm{n}=171$, RR $1.03 \mathrm{Cl} 0.84$ to 1.25 , Analysis 2.29). As regards admission to residential placement $(1 \mathrm{RCT}, \mathrm{n}=39, \mathrm{RR} 1.05$ $\mathrm{Cl} 0.24$ to 4.59 , Analysis 2.30) or admission to jail ( $1 \mathrm{RCT}, \mathrm{n}=39$, RR $1.05 \mathrm{Cl} 0.24$ to 4.59 , Analysis 2.31), similarly, we found no significant differences.

\subsection{Satisfaction with treatment}

One study looked at the proportion of people who were not satisfied with the care they received. We found a significant difference with participants in the other therapies group being less likely to be unsatisfied (Durham 2003, $n=45$, RR 3.19 Cl 1.01 to 10.7,, Analysis 2.32).

\subsection{Quality of life}

There was no significant difference in quality of life scores between the two groups (Uzenoff 2007, $\mathrm{n}=19, \mathrm{MD}-0.07 \mathrm{Cl}-21.11$ to 20.97, Analysis 2.33).

\subsection{Death}

No significant differences were found for deaths during medium and long-term follow-up (Analysis 2.34). 


\subsection{Behaviour}

A significant disadvantage was conferred on people who received supportive therapy in terms of social impairment measured on the SBAS (Falloon 1982, n= 39, RR $1.46 \mathrm{Cl} 1.04$ to 2.04, Analysis 2.35). We found no significant effect on the number of people who showed a poor coping style with relatives (Falloon 1982, $\mathrm{n}=39$, RR $0.90 \mathrm{Cl} 0.37$ to 2.20 , Analysis 2.36 ).

\subsection{Engagement with services}

The rates of poor attendance at appointments were recorded in one study, and no significant differences were found between treatment groups (Falloon 1982, $\mathrm{n}=39$, RR $1.93 \mathrm{Cl} 0.89$ to 4.17, Analysis 2.37.

\subsection{Insight}

Results from the SAl are skewed, and are presented in tabular form (Analysis 2.38).

\subsection{Compliance}

\subsubsection{Poor compliance to therapy}

We found compliance to be significantly worse amongst those who received supportive therapy than those in the control group (in this case, family therapy) ( 2 RCTs, $n=58, \mathrm{RR} 2.63 \mathrm{Cl} 1.30$ to 5.35 , Analysis 2.39). However, by long-term follow-up, data were equivocal (1 RCT, $\mathrm{n}=39$, RR $1.29 \mathrm{Cl} 0.69$ to 2.39 ).

\subsubsection{Medication adherence (self-report)}

No significant difference for self-reported medication adherence was found (Uzenoff 2007, $\mathrm{n}=19, \mathrm{MD} 1.00 \mathrm{Cl} 0.83$ to 1.21 , Analysis 2.40).

\subsubsection{Average endpoint score (ROMI)}

Uzenoff 2007 found no significant difference for medication adherence $(\mathrm{n}=19, \mathrm{MD} 0.45 \mathrm{Cl}-3.58$ to 4.48 , Analysis 2.41) or nonadherence ( $n=19, \mathrm{MD} 1.75 \mathrm{Cl}-0.61$ to 4.11 , Analysis 2.42 ).

\subsection{Medication}

Data from one small trial showed a significant difference, favouring other therapies for prescribed IM depot medication (Falloon 1982, $\mathrm{n}=39$, RR $2.11 \mathrm{Cl} 0.99$ to 4.47 , Analysis 2.43 ).

\subsection{Attitude to medication}

On the AMQ, the other therapies group scored significantly better than those allocated to supportive therapy (Kemp 1996, $n=74$, MD $-4.50 \mathrm{Cl}-6.83$ to -2.17 , Analysis 2.44). The DAl at short-term (Kemp $1996, \mathrm{n}=63, \mathrm{MD}-5.70 \mathrm{Cl}-9.35$ to -2.05 , Analysis 2.45 ) and longterm follow-up (Kemp 1996, $\mathrm{n}=44, \mathrm{MD}-4.90 \mathrm{Cl}-9.38$ to -0.42 ) also favoured the other therapies group.

\subsection{Economic outcomes}

The only study that investigated direct costs of the interventions produced skewed data, which are presented in a table (Analysis 2.46).

\section{Comparison 3: SUPPORTIVE THERAPY versus COGNITIVE BEHAVIOURAL THERAPY (CBT)}

Thirteen included studies compared supportive therapy with CBT (Durham 2003; Haddock 1999; Hogarty 1997-study 1; Hogarty 1997- study 2; Kemp 1996; Levine 1998; Lewis 2002b; Penn 2009; Pinto 1999; Sensky 2000b; Spaulding 1999; Tarrier 1998; Turkington 2000).

\subsection{Global state: relapse}

Relapse rates did not differ significantly between supportive therapy and CBT at medium term ( 2 RCTs, $\mathrm{n}=100$, RR $2.86 \mathrm{Cl} 0.32$ to 25.24 , Analysis 3.1). Longer-term data were heterogeneous $\left(I^{2}=\right.$ $78 \%$ ) and so were not pooled.

\subsection{Service outcomes: hospitalisation}

Hospitalisations did not differ significantly between supportive therapy and CBT groups (medium term, 3 RCTs, $n=153$, RR $1.60 \mathrm{Cl}$ 0.85 to 3.00 , long term, $1 \mathrm{RCT}, \mathrm{n}=65, \mathrm{RR} 0.73 \mathrm{Cl} 0.18$ to 3.00 , Analysis 3.2).

\subsection{Mental state: general}

\subsubsection{No clinically important improvement in general mental state}

We found no significant difference for 'no clinically important improvement in general mental state' over the long term (3 RCTs, $\mathrm{n}=194$, RR $1.23 \mathrm{Cl} 0.89$ to 1.70 ), and at medium-term follow-up (1 $\mathrm{RCT}, \mathrm{n}=59$, RR $1.27 \mathrm{Cl} 0.95$ to 1.70 , Analysis 3.3).

\subsubsection{Average endpoint general mental state score}

\section{i) PANSS total}

A significant difference in general mental state between supportive therapy and CBT was demonstrated in the long term (2 RCTs, n $=105, \mathrm{MD} 5.82 \mathrm{Cl} 1.04$ to 10.60 , Analysis 3.4). However, in the short term, the data were highly heterogeneous $\left(1^{2}=98 \%\right)$. We have concerns about the data reported Levine 1998, (see discussion section Quality of the evidence). We think the data in Levine 1998 may have been incorrectly reported or may have been an anomaly due to the small numbers in each treatment group. Also the standard deviations were very small, and we believe the authors may have erroneously reported standard errors as standard deviations. However, when we remove Levine 1998 from the analysis, heterogeneity is not restored and remains at $83 \%$ so data were not pooled for this outcome.

\section{ii) BPRS}

No significant difference in mental state measured on the BPRS was found in the short term (2 RCTs, $\mathrm{n}=92, \mathrm{MD}-1.07 \mathrm{Cl}-5.08$ to 2.94); Pinto 1999 found a significant difference in favour of CBT in the long term ( $\mathrm{n}=37, \mathrm{MD} 7.60 \mathrm{Cl} 0.90$ to 14.30 , Analysis 3.5).

\section{iii) BPRS short form}

Kemp 1996 also used the short form of the BPRS, and found no significant differences at short- $(n=74, M D-0.90 \mathrm{Cl}-3.02$ to 1.22$)$, medium- ( $\mathrm{n}=67, \mathrm{MD} 2.20 \mathrm{Cl}-1.18$ to 5.58 ) or long-term follow-up ( $\mathrm{n}$ $=45, \mathrm{MD} 2.30 \mathrm{Cl}-0.54$ to 5.14 , Analysis 3.6).

\section{iv) CPRS}

One study presented skewed data from the CPRS (Sensky 2000b). We have reported these in a table, Analysis 3.7.

\section{v) PANSS general subscale}

Levine 1998 reported PANSS general subscale data, which showed an advantage for other therapies over supportive therapy in the short term ( $\mathrm{n}=12$, MD $17.10 \mathrm{Cl} 13.76$ to 20.44), while Penn 2009 
showed also showed a significant difference in favour of CBT in the short term ( $\mathrm{n}=65, \mathrm{MD} 4.40 \mathrm{Cl} 1.36$ to 7.44 ) and long term ( $\mathrm{n}=$ 65 , MD $3.60 \mathrm{Cl} 0.84$ to 6.36 , Analysis 3.8). We did not pool data for this outcome as the short-term results where highly heterogenous $(12=97 \%)$, and we believe the Levine 1998 data may have been incorrectly reported (see above and discussion section Quality of the evidence).

\subsection{Mental state: specific symptoms}

3.4.1 No clinically important improvement in negative and depressive symptoms

We found no significant difference in improvement in negative symptoms between supportive therapy and CBT (Sensky 2000b, $n$ $=90, \mathrm{RR} 0.95 \mathrm{Cl} 0.63$ to 1.46 , Analysis 3.9). Similarly, for depressive symptoms, we found no significant differences (Sensky 2000b, $n=$ 90 , RR $1.53 \mathrm{Cl} 0.92$ to 2.55 , Analysis 3.10). However, results show that people who underwent supportive therapy are more likely to have had an episode of affective symptoms than those who underwent CBT (2 RCTs, n = 101, RR 2.17 Cl 1.16 to 4.06, Analysis 3.11).

\subsubsection{Average endpoint in negative symptoms}

\section{i) PANSS negative}

PANSS negative subscale data reported by Levine 1998 significantly favoured CBT in the short term ( $n=12, M D 7.20 \mathrm{Cl} 3.78$ to 10.62), while Penn 2009 showed no significance in the short term $(n=65$, $\mathrm{MD} 0.30 \mathrm{Cl}-1.55$ to 2.15 ) and long term ( $\mathrm{n}=65, \mathrm{MD} 0.30 \mathrm{Cl}-1.96$ to 2.56, Analysis 3.12). The short-term data were highly heterogenous $(12=92 \%)$ and we have concerns about Levine's data (see above and discussion section Quality of the evidence) and so data were not pooled.

\section{ii) SANS}

Pinto 1999 reported SANS data and found no difference between treatment groups ( $\mathrm{n}=37, \mathrm{MD} 6.60 \mathrm{Cl}-5.81$ to 19.01). Other skewed SANS data for Sensky 2000a and Tarrier 1998 are reported separately in tabular form Analysis 3.14.

\subsubsection{Average endpoint in positive symptoms}

\section{i) PANSS positive}

Three studies evaluated positive symptoms using the PANSS positive subscale, and no significant difference was found Lewis $2002 \mathrm{~b}$ and a significant difference in favour of CBT was found in Levine 1998 and Penn 2009. The pooled data were highly heterogenous $\left(1^{2}=83 \%\right)$; when we removed Levine 1998 , for which we have concerns about the data reported (see above and discussion section Quality of the evidence), the heterogeneity remain high with $\mathrm{L}^{2}=78 \%$ and so data were not pooled (Analysis 3.15). In the long term, Penn 2009 favoured CBT ( $n=65, M D 2.30 \mathrm{Cl}$ 0.60 to 4.00 , Analysis 3.15 ).

\section{ii) SAPS}

Tarrier 1998 reported data obtained from the SAPS, but data are skewed and presented as 'Other data' in a table (Analysis 3.16).

\section{iii) BPRS}

Skewed positive symptoms data from the BPRS were obtained from Tarrier 1998, and are presented as 'Other data' in a table (Analysis 3.17).
3.4.4 Average endpoint in delusion, hallucination and voices symptoms

\section{i) PANSS thought disturbance}

Cognitive behavioural therapy did not improve scores on the PANSS thought disturbance cluster significantly more than supportive therapy (Levine $1998 \mathrm{n}=12$, MD $4.30 \mathrm{Cl} 1.17$ to 7.43 , Analysis 3.18), however, we believe these data may have been incorrectly reported (see above and discussion section Quality of the evidence).

\section{ii) PSYRATS}

Skewed data from the PSYRATS scale, delusions score and hallucinations score are also presented in tabular form (Analysis 3.19; Analysis 3.20). Penn 2009 reported data from the voices score and found no significant difference at short term $(n=65, \mathrm{MD} 0.10 \mathrm{Cl}$ -3.63 to 3.83 ) and long term $(n=65, M D 0.00 \mathrm{Cl}-4.76$ to 4.76 , Analysis $3.21)$.

\section{iii) BAVQ}

Penn 2009 reported skewed data on beliefs about voices using the BAVQ subscales, and are presented as 'Other data' in a table (Analysis 3.22).

\subsubsection{Average endpoint in depressive symptoms}

Sensky $2000 \mathrm{~b}$ reported results from the MADRS and Penn 2009 from the BDI-II, but data are skewed and are reported in tabular form (Analysis 3.23 and Analysis 3.24, respectively).

\subsection{Leaving the study early}

We found no significant differences (general reasons) for leaving the study early, between supportive therapy (10 RCTs, $n=711$, RR $0.93 \mathrm{Cl} 0.66$ to 1.30 , Analysis 3.25) and those allocated to CBT. For those who left due to treatment-related reasons data were again non-significant ( $2 \mathrm{RCTs}, \mathrm{n}=101, \mathrm{RR} 2.34 \mathrm{Cl} 0.90$ to 6.10), but heterogeneous $\left(1^{2}=66 \%\right)$.

\subsection{General functioning}

We found GAF scores (Kemp 1996) favoured CBT (short term, $n=70$, $\mathrm{MD}-9.50 \mathrm{Cl}-16.11$ to -2.89 , and medium term, $\mathrm{n}=67, \mathrm{MD}-12.60 \mathrm{Cl}$ -19.43 to -5.77 , Analysis 3.26). When we combined long-term data from the GAF and GAS they were heterogeneous $\left(1^{2}=84 \%\right)$ and so data were not pooled.

Penn 2009 measured social functioning on the SFS, and found no significant difference in the short term $(n=65, M D-7.20 \mathrm{Cl}-17.86$ to 3.46) and the long term ( $n=65, M D-8.80 \mathrm{Cl}-21.67$ to 4.07 , Analysis 3.27).

\subsection{Satisfaction with treatment}

Durham 2003 looked at the proportion of people who were not satisfied with the care they received. We found a significant difference between treatment groups, with people receiving CBT less likely to be unsatisfied ( $n=45, R R 3.19 \mathrm{Cl} 1.01$ to 10.07 , Analysis 3.28).

\subsection{Quality of life}

One study found no significant results for the RSES (Penn 2009, $\mathrm{n}=$ 65 short term MD $-0.80 \mathrm{Cl}-3.77$ to 2.17 ; long term MD $-1.70 \mathrm{Cl}-5.19$ to 1.79 , Analysis 3.29). 


\subsection{Death}

Two deaths occurred in Tarrier 1998, one from each intervention group, and one death occurred in the Durham 2003 study from the supportive therapy group (Analysis 3.30).

\subsection{Insight}

Results from the Schedule for Assessment of Insight are skewed, and are presented in tabular form Analysis 3.31, as are results from the Beck Cognitive Insight Scale, Analysis 3.32.

\subsection{Medication}

There was no significant difference for no reduction in doses of antipsychotic medication between the CBT and supportive therapy groups (Sensky 2000b, n = 90, MD 0.89 Cl 0.68 to 1.17, Analysis 3.33).

\subsection{Attitude to medication}

On the AMQ, people who were allocated to CBT scored significantly better than those allocated to supportive therapy (Kemp 1996, $\mathrm{n}=$ 74 , MD $-4.50 \mathrm{Cl}-6.83$ to -2.17 , Analysis 3.34). Similarly, the DAl at short-term (Kemp 1996, $\mathrm{n}=63, \mathrm{MD}-5.70 \mathrm{Cl}-9.35$ to -2.05 , Analysis 3.35) and long-term follow-up (Kemp 1996, $n=44, M D-4.90 \mathrm{Cl}-9.38$ to -0.42 ) also significantly favoured CBT compared with supportive therapy.

\subsection{Economic outcomes}

The only study investigating direct costs of the interventions produced skewed data and is reported in tables (Analysis 3.36).

\section{Comparison 4: SUPPORTIVE THERAPY versus FAMILY THERAPY}

Two included studies compared supportive therapy with family therapy (Falloon 1982; Hogarty 1997-study 1).

\subsection{Global state: relapse and remission}

Two trials reported relapse rates, but these showed heterogeneity $\left(I^{2}=87.1 \%\right)$ and so data were not pooled (Analysis 4.1). We found participants in the Falloon 1982 study were more likely to experience remission of their symptoms if they had family therapy rather than supportive therapy $(\mathrm{n}=39, \mathrm{RR} 1.87 \mathrm{Cl} 1.11$ to 3.15 , Analysis 4.2).

\subsection{Service outcome: hospitalisation}

No significant differences were found in hospitalisation rates between the treatment groups (Falloon 1982, $n=39$, RR $1.93 \mathrm{Cl} 0.89$ to 4.17, Analysis 4.3).

\subsection{Mental state: episode of affective symptoms}

Data were only available for one outcome relating to mental state - the likelihood of having an episode of affective symptoms. We found no significant difference between family therapy and supportive therapy (Hogarty 1997-study $1, n=48$, RR $1.71 \mathrm{Cl} 0.82$ to 3.60, Analysis 4.4).

\subsection{Leaving the study early}

We found no significant difference for the number of participants leaving the study early for general reasons (Falloon 1982, $n=39$, RR $0.70 \mathrm{Cl} 0.13$ to 3.75 ) and treatment-related reasons (Hogarty 1997study $1, \mathrm{n}=48 \mathrm{RR} 1.60 \mathrm{Cl} 0.61$ to 4.19 , Analysis 4.5).

\subsection{General functioning}

People who had supportive therapy were no more or less likely to have failed to find paid work than people who had family therapy (Falloon 1982, $\mathrm{n}=39$, RR $0.96 \mathrm{Cl} 0.57$ to 1.63, Analysis 4.6). Rates of admission to residential placement (Falloon 1982, $n=39$, RR 1.05 $\mathrm{Cl} 0.24$ to 4.59, Analysis 4.7) and admission to jail (1 RCT, $n=39$, RR $1.05 \mathrm{Cl} 0.24$ to 4.59 , Analysis 4.8) did not differ significantly between treatment groups.

\subsection{Behaviour}

Falloon 1982 assessed the frequency of social impairment using the Social Behaviour Adjustment Schedule. We found social impairment to be significantly more frequent in the supportive therapy intervention compared with family therapy $(n=39, R R 1.46$ $\mathrm{Cl} 1.04$ to 2.04 , Analysis 4.9). The number of people displaying a poor coping style with relatives did not differ significantly between groups (Falloon 1982, $\mathrm{n}=39$, RR $0.90 \mathrm{Cl} 0.37$ to 2.20, Analysis 4.10).

\subsection{Engagement with services}

The proportion of people who were poor attendees at appointments was not significantly different (Falloon 1982, $\mathrm{n}=39$, RR $1.93 \mathrm{Cl} 0.89$ to 4.17, Analysis 4.11) between groups.

\subsection{Compliance}

We found compliance to be significantly worse amongst those who received supportive therapy than those in the family therapy group (Falloon 1982, $\mathrm{n}=39$, RR $2.63 \mathrm{Cl} 1.30$ to 5.35, Analysis 4.12). However, by long term follow-up data were equivocal $(n=39, R R$ $1.29 \mathrm{Cl} 0.69$ to 2.39$)$.

\subsection{Medication}

The difference in frequency of prescription of antipsychotic depot medication between groups was not statistically significant $(n=39$, RR 2.11 Cl 0.99 to 4.47, Analysis 4.13).

\section{Comparison 5: SUPPORTIVE THERAPY versus PSYCHOEDUCATION}

Two included studies compared supportive therapy with psychoeducation (Coyle 1988; Uzenoff 2007).

\subsection{Service outcomes: hospitalisation}

No significant differences in hospitalisation rates were found (Coyle 1988, n = 47, RR $0.48 \mathrm{Cl} 0.05$ to 4.93, Analysis 5.1).

\subsection{Mental state}

\subsubsection{No clinically important improvement in general mental state}

Uzenoff 2007 found no significant difference between supportive therapy and psychotherapy for 'no clinically important improvement in general mental state' $(\mathrm{n}=19$, MD $1.61 \mathrm{Cl} 0.96$ to 2.68, Analysis 5.2).

\subsubsection{Average endpoint general symptoms score}

There was no significant difference for PANSS general scores between the groups ( $n=19, \mathrm{MD} 2.86 \mathrm{Cl}-3.21$ to 8.93 , Analysis 5.3). 


\subsubsection{Average endpoint negative symptoms score}

PANSS negative subscale data from Uzenoff 2007 showed no significant difference between supportive therapy and psychoeducation ( $\mathrm{n}=19$, MD $1.70 \mathrm{Cl}-4.00$ to 7.40 , Analysis 5.4).

\subsubsection{Average endpoint positive symptoms score}

PANSS positive subscale data from Uzenoff 2007 study showed no significant difference between supportive therapy and psychotherapy ( $n=19, \mathrm{MD} 2.71 \mathrm{Cl}-0.71$ to 6.13 , Analysis 5.5).

\subsubsection{Average endpoint depression score}

No significant difference was found for CDRS between supportive therapy and psychotherapy (Uzenoff 2007, $\mathrm{n}=19$, MD $1.47 \mathrm{Cl}-1.35$ to 4.29 , Analysis 5.6)

\subsection{Leaving the study early}

We found no significant difference in the number of people leaving the study early between treatment groups ( 2 RCTs, $n=71$, RR 0.57 $\mathrm{Cl} 0.21$ to 1.54 , Analysis 5.7).

\subsection{Quality of Life}

There was no significant difference in quality of life scores between the two groups (Uzenoff 2007, $\mathrm{n}=19$, MD $-0.07 \mathrm{Cl}-21.11$ to 20.97, Analysis 5.8).

\subsection{Death}

Death rates were not significantly affected by treatment received (Coyle 1988, n = 47, RR $2.88 \mathrm{Cl} 0.12$ to 67.29, Analysis 5.9).

\subsection{Behaviour}

Uzenoff 2007 found no significant differences in the composite scores from the ROMI and ITAQ for need for treatment $(n=19, M D$ $-0.02 \mathrm{Cl}-0.44$ to 0.40 ) and the benefits of medication ( $n=19, M D$ $-0.19 \mathrm{Cl}-0.56$ to 0.18 , Analysis 5.10$)$.

\subsection{Insight}

No significant difference in insight into treatment scores were found between supportive therapy and psychotherapy (Uzenoff $2007, \mathrm{n}=19, \mathrm{MD}-1.55 \mathrm{Cl}-5.85$ to 2.75 , Analysis 5.11 )

\subsection{Compliance}

\subsubsection{Poor compliance to therapy}

All participants in Uzenoff 2007 attended at least six of the 14 sessions of therapy (Analysis 5.12).

\subsubsection{Medication adherence (self-report)}

No significant difference for self-reported medication adherence was found (Uzenoff 2007, $\mathrm{n}=19, \mathrm{MD} 1.00 \mathrm{Cl}-0.83$ to 1.21 , Analysis 5.13).

\subsubsection{Average endpoint score (ROMI)}

Uzenoff 2007 found no significant difference for medication adherence $(\mathrm{n}=19, \mathrm{MD} .45 \mathrm{Cl}-3.58$ to 4.48 , Analysis 5.14) or nonadherence $(\mathrm{n}=19, \mathrm{MD} 1.75 \mathrm{Cl}-0.61$ to 4.11 , Analysis 5.15) between supportive therapy and psychotherapy.

\section{Comparison 6: SUPPORTIVE THERAPY versus REHABILITATION} PROGRAMME

One study looked at supportive therapy compared to a rehabilitation programme (Dincin 1982).

\subsection{Service outcomes}

Participants in the rehabilitation group were significantly less likely to be hospitalised than those in the supportive therapy group (Dincin 1982, $\mathrm{n}=132$, RR $2.71 \mathrm{Cl} 1.22$ to 6.02 , Analysis 6.1).

\subsection{Leaving the study early}

There were no significant differences in the numbers who left the study early (Dincin 1982, $\mathrm{n}=132, \mathrm{RR} 1.45 \mathrm{Cl} 0.92$ to 2.29 , Analysis 6.2).

\subsection{General functioning: no gainful employment}

We found that the therapeutic intervention did not have a significant impact on the number of people not in gainful employment (Dincin 1982, $\mathrm{n}=132$, RR $1.04 \mathrm{Cl} 0.85$ to 1.29 , Analysis 6.3).

\section{Comparison 7: SUPPORTIVE THERAPY versus SKILLS TRAINING}

Three included studies compared supportive therapy with skills training (Coyle 1988; Eckman 1992; Wirshing 1991).

\subsection{Service outcomes: hospitalisation}

Hospitalisation rates were not found to differ significantly between supportive therapy and skills training (Coyle 1988, $n=47, R R 0.96 \mathrm{Cl}$ 0.06 to 14.43 , Analysis 7.1 ).

\subsection{Leaving the study early}

Attritions rates showed no significant difference between treatment groups ( $3 \mathrm{RCTs}, \mathrm{n}=168$, RR $1.01 \mathrm{Cl} 0.61$ to 1.67 , Analysis 7.2).

\subsection{Death}

Coyle 1988 reports data on death rates, with no significant difference between groups ( $n=47, \operatorname{RR} 2.88 \mathrm{Cl} 0.12$ to 67.29 , Analysis 7.3).

\section{Comparison 8: SUPPORTIVE THERAPY versus PSYCHODYNAMIC PSYCHOTHERAPY}

Stanton 1984 compared supportive therapy with psychodynamic psychotherapy.

\subsection{Leaving the study early}

In the medium term, people in the psychodynamic therapy group were more likely to leave the study early than those in the supportive therapy arm ( $1 \mathrm{RCT}, \mathrm{n}=164$, RR $0.62 \mathrm{Cl} 0.42$ to 0.91 ). However, by long term follow-up, the differences were no longer significant (Stanton 1984, $\mathrm{n}=164$, RR $0.89 \mathrm{Cl} 0.73$ to 1.09 , Analysis 8.1).

\section{Comparison 9: SUPPORTIVE THERAPY versus COMBINATION OF OTHER PSYCHOSOCIAL INTERVENTIONS}

Hogarty 1997-study 1 compared supportive therapy against a combination of other psychosocial interventions. 


\subsection{Global state: relapse}

We found no significant difference for relapse rates between supportive therapy and those receiving a combination of other therapies, in this case CBT and family therapy (Hogarty 1997-study $1, \mathrm{n}=50$, RR $1.48 \mathrm{Cl} 0.86$ to 2.55 , Analysis 9.1).

\subsection{Mental state}

The risk of having an episode of affective symptoms did not differ significantly between groups (Hogarty 1997-study 1, n = 50 RR 1.63 $\mathrm{Cl} 0.81$ to 3.28 , Analysis 9.2 ).

\subsection{Leaving the study early}

The number of people who left the study early for treatment-related reasons were significantly higher in the supportive therapy group than in the combination group (Hogarty 1997-study $1, \mathrm{n}=50$, RR $8.67 \mathrm{Cl} 1.17$ to 64.26 , Analysis 9.3 ).

\section{Comparison 10: SUPPORTIVE THERAPY PLUS CLIENT- FOCUSED CASE MANAGEMENT verSUS CLIENT-FOCUSED CASE MANAGEMENT}

Klein 1998 and O'Donnell 1999 compared supportive therapy and client-focused case management against client-focused case management alone.

\subsection{Global state: relapse}

The number of participants experiencing relapse did not differ between groups (Klein 1998, n =61, RR $0.32 \mathrm{Cl} 0.05$ to 2.14 Analysis 10.1).

\subsection{Leaving the study early}

The frequency of participants leaving the study early was not significantly different ( 2 RCTs, $n=145$, RR $2.38 \mathrm{Cl} 1.15$ to $4 . .3,9$ Analysis 10.2).

\subsection{Death}

Death rates did not differ significantly between the two treatment groups (O'Donnell 1999, $\mathrm{n}=84, \mathrm{RR} 2.61 \mathrm{Cl} 0.11$ to 62.26 , Analysis $10.3)$.

\section{Comparison 11: SUPPORTIVE THERAPY PLUS CLIENT-FOCUSED CASE MANAGEMENT versus STANDARD CASE MANAGEMENT}

O'Donnell 1999 compared supportive therapy and client-focused case management against standard case management.

\subsection{Leaving the study early}

There were no significant differences between the treatment and control group (O'Donnell 1999, $\mathrm{n}=80, \mathrm{RR} 0.88 \mathrm{Cl} 0.52$ to 1.51 , Analysis 11.1).

\subsection{Death}

We found no significant differences in death rates (O'Donnell 1999, $\mathrm{n}=80$, RR $2.35 \mathrm{Cl} 0.10$ to 55.94 Analysis 11.2).

\section{Comparison 12: SUPPORTIVE THERAPY PLUS SKILLS TRAINING versus SKILLS TRAINING}

Malm 1982 compared supportive therapy and skills training with skills training alone.

\subsection{Global state}

Relapse rates were not significantly different between the two treatment regimes (Malm 1982, $\mathrm{n}=80, \mathrm{RR} 1.00 \mathrm{Cl} 0.49$ to 2.04, Analysis 12.1). Similarly, we found no significant difference in the number of people who did not experience remission during the follow-up period (Malm 1982, $\mathrm{n}=80, \mathrm{RR} 0.78 \mathrm{Cl} 0.54$ to 1.12 , Analysis 12.2).

\subsection{Service outcomes}

Data were available for the number of people who were not discharged from hospital during the follow-up period (all participants were inpatients at the start of the study). No significant differences were found between the two treatment groups (Malm 1982, $\mathrm{n}=80$, RR $1.14 \mathrm{Cl} 0.46$ to 2.85, Analysis 12.3).

\subsection{Mental state}

Data were only reported for two out of 45 items on the CPSRS, 'inability to feel' and 'derealisation'. For 'inability to feel', results showed no significant difference between the two treatment groups (Malm 1982, $\mathrm{n}=80, \mathrm{MD} 0.10 \mathrm{Cl}-0.08$ to 0.28 , Analysis 12.4). Data for 'derealisation' are skewed, and are presented as 'Other data' in a table (Analysis 12.5).

\subsection{Leaving the study early}

No significant difference was found for this outcome (Malm 1982, n $=80, \mathrm{RR} 1.00 \mathrm{Cl} 0.35$ to 2.84 , Analysis 12.6 ).

\subsection{General functioning}

Data from the KAS were reported for the subscale entitled 'freetime activities' and for the syndrome entitled 'withdrawal'. For 'free-time activities', we found a statistically significant advantage for supportive therapy plus skills training, compared with skills training alone (Malm 1982, $\mathrm{n}=80, \mathrm{MD} 0.10 \mathrm{Cl} 0.02$ to 0.18 , Analysis 12.8). Data for 'withdrawal' are skewed, and are presented as 'Other data' in a table (Analysis 12.8).

\subsection{Death}

No significant differences were found in death rates between the two treatment groups (Malm 1982, $\mathrm{n}=80, \mathrm{RR} 2.00 \mathrm{Cl} 0.19$ to 21.18, Analysis 12.9). Three deaths occurred, all of which were due to suicide.

\section{DISCUSSION}

\section{Summary of main results}

\section{Supportive therapy versus standard care}

There was a lack of data for this comparison, with only five studies included. Overall, the quality of the evidence was very low for the outcomes in the Summary of findings for the main comparison.

We found no evidence of significant differences between supportive therapy and standard care for rates of relapse and hospitalisation, clinical improvement in mental state, leaving the study early and quality of life and no studies reported data on general functioning and satisfaction with treatment. 


\section{Supportive therapy versus any other psychological or psychosocial treatment}

Twenty studies were included in the comparison, which combines data from the comparisons for other psychological interventions, including cognitive behavioural therapy (CBT), family therapy and the quality of the evidence was very low for all outcomes except leaving the study early, which we rated as moderate in the Summary of findings 2.

For the outcomes rate of hospitalisations, clinical improvement in mental state and satisfaction of treatment for the recipient of care, we found evidence favouring any other psychological or psychosocial treatment. We found no evidence of significant differences for rate of relapse, leaving the study early and quality of life.

\section{Supportive therapy versus cognitive behavioural therapy (CBT)}

Thirteen studies compared supportive therapy with CBT. Again, the quality of the evidence was very low for all outcomes except leaving the study early, which we rated as moderate (see Summary of findings 3 ).

We found no evidence of significant differences between supportive therapy and CBT for rates of relapse and hospitalisation, clinical improvement in mental state, leaving the study early and quality of life. For general functioning, we could not pool data as it showed high heterogeneity; one study showed equivocal data, the other found in favour of supportive therapy.

\section{Supportive therapy versus family therapy}

There was a lack of data for this comparison, with only two studies included. Overall, the quality of the evidence was very low for the outcomes in the Summary of findings 4.

We were not able to pool the data for rate of relapse due to high heterogeneity; one study found no difference in relapse rates, the other study found a difference in favour of family therapy. We found no differences for the rate of hospitalisation and leaving the study early. As there were limited data for this comparison, we had to use proxy data for two outcomes: for mental state we had data for participants experiencing an episode of affective symptoms and for general functioning we had data for participants with no paid work. Neither of the studies reported data for satisfaction with treatment and quality of life.

\section{Supportive therapy versus psychoeducation}

Again, we were only able to include two studies in this comparison and the quality of the evidence was very low (see Summary of findings 5).

We found that there was no evidence of significant differences between supportive therapy and psychoeducation for the outcomes with data - rate of hospitalisation, no clinical improvement in mental state and leaving the study early. No studies reported data for rate of relapse, general functioning, satisfaction with treatment and quality of life.

\section{Overall completeness and applicability of evidence}

\section{Few data}

It is important to point out that the findings in this review are significantly weakened by the limited amount of available data. The search strategy identified five trials, involving 822 people, comparing supportive therapy with standard care. However, few data regarding the predetermined primary outcomes of interest could be extracted. The important clinical question, whether supportive therapy confers any advantage over standard care in the treatment of people with schizophrenia, remains unanswered. It is important to answer this question before results of research comparing supportive therapy with other psychological or psychosocial therapies can be interpreted fully and meaningfully.

More studies compared supportive therapy with other psychological or psychosocial interventions. There were, however, many comparison interventions. The number of studies comparing supportive therapy with each intervention was generally small, the main exception being CBT, for which we identified 13 studies.

\section{Delivery of therapy}

In trials of psychological interventions, there is question about whether the same therapists should provide two different interventions, potentially allowing factors such as level of experience of the therapists and individual differences in personality to be evenly distributed between groups. However, an alternative, and in our opinion more persuasive, argument is that different therapists should provide different therapies. This takes into account the likelihood that therapists have a loyalty to, and training and experience in, one particular type of therapy. This may be particularly important if they have been involved in generating the hypotheses which are being tested. The majority of studies in this review used the same therapists for supportive therapy and other psychological interventions. Most studies did not specify what training the therapists had received or what level of experience they had. Where studies did report details of therapist training, therapists who delivered supportive therapy were sometimes trained in other modalities, such as CBT, but not in supportive therapy. The studies which described standardised supportive therapy, with the use of a manual, were in the minority; as were the studies that evaluated or monitored adherence to the treatment model.

Not all studies attempted to match the amount of therapist contact. For example, Coyle 1988 compared supportive therapy with social skills training and psychoeducation. Supportive therapy sessions, however, were half as long as other therapy sessions, and were delivered individually rather than in a group.

More recent studies, such as Lewis 2002b, Penn 2009 and Sensky $2000 \mathrm{~b}$ are of a much better quality. It seems likely that methodology and reporting have improved considerably over recent years, which will be valuable for future versions of this review. The concerns described above mean that only tentative conclusions can be drawn from this review.

\section{Applicability}

All trials used entry criteria to define who would be included in the study. These, however, varied from study to study. The studies involved people with schizophrenia and schizophrenia-like 
illnesses defined by a range of criteria. Most studies, but not all, used some form of diagnostic criteria. Settings also varied, and both inpatients and outpatients were included. This suggests that the results would be valid for people with a diagnosis of schizophrenia or a related psychotic illness, whether they are being treated as an inpatient or on an outpatient basis. However, the possibility that patients typically studied in randomised controlled trials may differ in important ways from patients typically seen in mental health services should also be considered (Roth 1996). The results of this review should ideally be considered alongside results of effectiveness research. In effectiveness research, generalisability may be higher that for randomised controlled trials, although internal validity is lower (Margison 2000).

One aspect of trial setting which might be particularly important is the country in which trials are conducted. In this review, the majority were set in the USA. Seven were set in the UK, and one each in Israel, Italy, Sweden and Australia. There may be a difference in what is regarded as supportive therapy in the USA and in the UK (Holmes 1995), with, for example, supportive therapy in the USA perhaps being more frequent and more closely related to a psychodynamic approach than in the UK.

One of the difficulties in attempting this systematic review has been finding a clinically useful definition of supportive therapy. Despite the lack of a universally accepted definition, we felt it was important to define the intervention and identify the available evidence. Throughout the process, we have been aware of the risk that interventions included in the review would be too diverse. Readers of this review need to know what is meant by supportive therapy, and to be able to apply the findings in clinical practice. The included interventions do vary, and we have some concerns about whether they have enough in common to be grouped together in a useful way. The wide variation in the duration and frequency of sessions could be taken as evidence of differences between interventions in the included studies. Nevertheless, we note the lack of research in this field, and the lack of specific meta-analyses on supportive therapy. We feel the use of a broad definition of supportive therapy in this review is justified as producing an initial analysis of the available data. It is possible that narrower definitions of supportive therapy, perhaps used in more specific settings, could produce different results. For example, it may be possible that an untrained support worker within a community team could reduce the input required for a patient from other members of the team, or may increase the leisure and community activities of a patient. In this review we are unable to make comments on specific questions such as these.

Treatment manuals, training of therapists and monitoring of treatment fidelity, all help to enhance internal validity of trials. These were used in a minority of trials in this review. However, it can be argued that these measures may increase the apparent effectiveness of interventions over what is possible to reproduce in clinical practice. In many reports, little description of the supportive intervention was provided.

Two studies in particular described interventions which are different from the other supportive interventions. Kemp 1996 reports that therapists delivering supportive therapy were specifically instructed to decline to discuss treatment with people. O'Donnell 1999 also describes a somewhat different intervention. In this study, supportive therapy is more like a client-focused case management with advocacy. Advocacy, which comes within our definition of supportive therapy, was provided by consumer advocates, who could be people who had experienced mental illness, relatives or carers of people with mental illnesses, or interested lay people. No particular frequency or duration of sessions was specified.

\section{Quality of the evidence}

There was little consensus between studies over which outcome variables to use. Definition of outcomes such as relapse also varied between trials.

Sample sizes were small for most studies, perhaps resulting in true beneficial or harmful effects going undetected.

The quality of the available data is also a cause for concern which needs to be borne in mind when interpreting the results of this review. Twenty of the 24 studies did not adequately report the use of allocation concealment, raising concerns about selection bias. We suspect that this would be most likely to act in the direction of biasing results against supportive therapy, as supportive therapy is used as a control treatment in the majority of studies in this review. Although double-blind trials are not possible when testing a psychological intervention, it is possible for therapists to be blinded to the study hypothesis. Only two studies attempted this. Not all studies rated outcomes blindly, resulting in a risk of detection biases. Attrition bias cannot be discounted either, as losses to follow-up were generally poorly reported, and intention-to-treat analyses were the exception rather than the rule.

These potential biases are particularly important in this review. This is because studies are almost all designed with supportive therapy as a comparator for other treatments, rather than being the primary treatment of interest to investigators. The hypothesis and the expectation of investigators is therefore likely to be that supportive therapy will not perform as well as the other treatment under investigation.

Heterogeneity was present, as judged by visual inspection and the $1^{2}$ statistic, for the outcomes: mental state measured on the Positive and Negative Syndrome Scale (PANSS), general functioning on the Global Assessment of Function disability scale (GAF) and Global Assessment Scale (GAS) when supportive therapy was compared with other psychological interventions or CBT alone; relapse when supportive therapy was compared with other psychological interventions CBT alone and family therapy; and leaving the study early when supportive therapy was compared with CBT. This may be due in part to the diversity of the interventions. There is a possibility that our attempt to investigate the effects of supportive therapy may not have been as successful as we might have hoped due to the variety of interventions meeting our criteria for supportive therapy or care. However, as high rates of heterogeneity were not found for other outcomes, this does not seem likely to be a major problem. Another possibility, and probably one that is more likely, is that the heterogeneity was due to diversity of control interventions (family therapy or CBT). Although homogeneity was not restored when data from Levine 1998 were excluded from the pooled analyses (due to the presence of heterogeneity). We think the data in Levine 1998 may have been incorrectly reported or may have been an anomaly due to the small numbers in each treatment group $(\mathrm{n}=$ 7). Also the standard deviations were very small, and we believe the authors may have erroneously reported standard errors as standard deviations. 


\section{Potential biases in the review process}

\section{The limitations of randomised trials of psychotherapy}

In doing this review, we explicitly aimed to include only randomised controlled trials. However, it is important to recognise that evidence from randomised controlled trials is not the only form of evidence which can be used to guide clinical practice. There are particular problems with randomised controlled trials for psychological therapies. Psychotherapy relies on the uniqueness of the clinician-patient relationship, and ways of measuring outcomes which take account of this need to be developed (Holmes 2000). Randomised controlled trials employ techniques to enhance internal validity, such as frequent monitoring, the use of therapy manuals and excluding patients with dual diagnoses. These techniques may increase the apparent effectiveness of the intervention above what would routinely be achieved in clinical practice (Roth 1996). In randomised controlled trials of long-term therapy, there is a risk of contamination by other treatments (Margison 2000).

\section{Definition of supportive therapy}

Distinguishing supportive therapy from standard treatment has also been an issue in this review. We have largely relied on authors' descriptions of the interventions, and have classified some interventions described as treatment as usual as supportive therapy. Similarly, some interventions which were described as supportive in nature were not felt to meet the pre-determined inclusion criteria for this review. Descriptions have not always been of sufficient detail to judge whether a control intervention should be classified as supportive therapy or not, leaving the possibility that some data were in appropriately omitted. The lack of differences in outcomes between supportive therapy and standard care could suggest that this distinction is not a valid one. However, this is not the only interpretation of the results, and a real difference in effectiveness could exist, but may not have been identified due to paucity of data.

\section{Strengths}

Despite the reservations and difficulties outlined above, it is important that this review has been attempted. Supportive therapy is commonly used in clinical practice for people with schizophrenia. It may be cheaper than other therapies, such as CBT but is probably more costly and intensive than what is generally provided as standard care in many places. We also feel it to be a prevalent approach so it is important to systematically examine the effects of supportive therapy as a specific intervention. As far as we are aware, the only published study previously to have attempted this was the meta-analysis undertaken by the Department of Health for the purposes of the NICE Guideline on Schizophrenia (NICE 2003). The scope of this systematic review is not quite as focused as this work and therefore the NICE 2003 review is larger with more studies included. We feel our tighter definitions of supportive therapy are justified, workable and give a clearer picture of the evidence.

\section{Agreements and disagreements with other studies or reviews}

We know of no other reviews on supportive therapy.

\section{AUTHORS' CONCLUSIONS}

\section{Implications for practice}

\section{For people with schizophrenia}

The evidence presented in this review does not allow us to conclude that supportive therapy has any substantial benefit over standard care. Although there is no evidence of a treatment effect when comparing supportive therapy with standard care, the small number and small size of trials means that a treatment effect, either favouring supportive therapy or standard care, cannot be ruled out. This rather unsatisfactory conclusion also applies to other comparisons where supportive therapy is directly compared with several other psychological therapies, including cognitive behavioural therapy (CBT). At present there is not really conclusive evidence for people with schizophrenia to choose one of the other therapies over a more simple supportive approach.

\section{For clinicians}

Guidance encourages use of family and cognitive therapy for people with schizophrenia. From this review, one cannot conclude that supportive therapy offers anything different to other forms of psychological intervention. For some outcomes, including hospitalisation, general mental state and affective symptoms, data suggest an advantage for other therapies over supportive therapy but due to the small amount of data and the lack of consistency between outcomes measuring similar variables, firm conclusions should not be drawn from these results.

\section{For mangers and policy makers}

Unfortunately, the paucity of data means no clear recommendations for clinical practice can be made on the basis of this review. At present, there is no evidence from this review of a beneficial effect for supportive therapy over standard care, although such a beneficial or harmful effect cannot be ruled out. There is some evidence to suggest a disadvantage of supportive therapy in comparison with other therapies but this is scarce and is often derived from studies that may have some inherent bias towards the other therapies. Certainly, these data require replication in large, clinically meaningful randomised controlled trials before they can be used to guide clinical practice. It is important to highlight the lack of data regarding harmful or adverse effects of supportive therapy. This would be deemed unacceptable in a trial relating to a specific medication.

\section{Implications for research}

\section{General}

As with similar studies, public registration of a study before anyone is randomised would ensure that participants could be confident that people would know that the study had at least taken place. Unique study numbers would help researchers to identify single studies from multiple publications and reduce the risk of duplicating the reporting of data. Compliance with CONSORT (Moher 2001), both on the part of authors and editors, would help to clarify methodology and many outcomes. Failure to comply with such standard guidelines results in both loss of data and confusion in the results, neither of which help clinicians, patients or managers. 
Intention-to-treat analysis should be performed on all outcomes and all trial data should be made easily accessible. A minimal requirement should be that all data should, at least, be presented as numbers. In addition, continuous data should be presented with means, standard deviations (or standard errors) and the number of participants. Data from graphs, ' $P$ ' values of differences and statements of significant or non-significant differences are of limited value. Unfortunately, in spite of the large numbers of participants randomised, we were unable to use most of the data in the trials included in this review due to the poor data reporting.

\section{Specific}

\subsection{Reviews}

Many of the excluded studies suggest new comparisons relevant to future reviews (Characteristics of excluded studies), too many for this exhausted group of systematic review authors to currently list.

\subsection{Trials}

Supportive therapy can be usefully investigated using randomised controlled trials. Of course, evidence from randomised controlled trials should be considered alongside evidence from other forms of research, but larger trials do seem warranted and preferably undertaken by those with clear equipoise. A suggestion for the design is outlined in Table 1.

\subsubsection{Methods}

Allocation concealment is vital in the design of future randomised controlled trials to minimise bias. The randomisation process should also be described fully. It is probably not possible to use double-blind methodology in trials of psychological and psychosocial interventions. However, bias can be minimised by ensuring therapists and people are blind to the specific study hypothesis where feasible, and by using blinded or independent outcome raters.

\subsubsection{Participants}

We would suggest that inclusion criteria for participants in such a study would be broad and that the therapy be given in the context of everyday practice.

\subsubsection{Interventions}

\subsubsection{Supportive therapy}

Future trials should clearly explain whether practitioners who deliver supportive therapy have been specifically trained, and if so how. It may make the results more applicable if the therapists are trained but in the context of routine career development, rather than specific highly-trained specialised practitioners. In addition, using a therapy manual or protocol and attempting to ensure adherence to the model may at least increase internal validity of the trial. Ideally, different therapists should be used for different therapies. Finally, it is important to clearly define supportive therapy. We have found no standard definition and it would be beneficial if a standard definition for this commonly practiced intervention were to be developed.

\subsubsection{Comparison group}

Further data comparing supportive therapy both with standard care and with other treatments are needed. These should be clearly defined within the trial, even if it is routine care.

\subsubsection{Outcomes}

Outcomes chosen should be clinically relevant and widely used. Outcomes such as relapse or hospitalisation are less vulnerable to bias than scale data, and may therefore be preferable. Little data are available presently on adverse effects, social functioning, occupational status, quality of life, and economic outcomes, all of which would be of interest. Such outcomes are often not difficult to record over the short, medium or long term.

\section{ACK N O WLEDGEMENTS}

We are grateful to the staff of the Cochrane Schizophrenia Group for valuable support. In particular, for the original version, the search strategy was devised and run by Judith Wright, and statistical advice was provided by John Rathbone. Tessa Grant and Gill Rizzello provided administrative and copy editing support. Tor Pettit helped with protocol development, quality rating and selection of studies, data extraction and was co-author of text of the original version of this review.

For the 2012 update version Samantha Roberts ran the search, Lindsey Air and Claire Irving provided administrative and editorial support.

The Cochrane Schizophrenia Group produce and maintain a template for the methods section of their reviews. For the 2012 update we have used and adapted this.

We would like to thank M. Amr alMukhallalati, Basem Jaber, Bilal AlBaroudi and Diarmid Sinclair for their helpful observations and comments when peer reviewing the 2012 update version, and Heather Maxwell for copy editing. 


\section{R E F E R E N C E S}

\section{References to studies included in this review}

Coyle 1988 \{published data only\}

Coyle P. A comparative analysis of varying treatment approaches on the level of community adjustment among schizophrenic outpatients. PhD dissertation:submitted to the Adelphi University, USA 1988.

\section{Davidson 2004 \{published data only\}}

* Davidson L, Shahar G, Stayner DA, Chinman MJ, Rakfeldt J, Tebes JK. Supported socialization for people with psychiatric disabilities: Lessons from a randomized controlled trial. Journal of Community Psychology 2004;32:453-77.

Shahar G, Kidd S, Styron TH, Davidson L. Consumer support and satisfaction with mental health services in severe mental illness: the moderating role of morale. Journal of Social and Clinical Psychology 2006;25:945-62.

Dincin 1982 \{published data only\}

Dincin J, Witheridge TF. Psychiatric rehabilitation as a deterrent to recidivism. Hospital and Community Psychiatry 1982;33(8):645-50.

\section{Durham 2003 \{published data only\}}

Durham RC, Guthrie M, Morton RV, Reid DA, Treliving LR, Fowler DMR, et al. Tayside-Fife clinical trial of cognitivebehavioural therapy for medication-resistant psychotic symptoms. Results to 3-month follow-up. British Journal of Psychiatry 2003;182:303-11.

\section{Eckman 1992 \{published data only\}}

Eckman TA, Wirshing WC, Marder SR, Liberman RP, JohnstonCronk K, Zimmermann K, et al. Technique for training schizophrenic patients in illness self-management: a controlled trial. American Journal of Psychiatry 1992;149(11):1549-55. [MEDLINE: 93036079; PMID 1384364]

\section{Falloon 1982 \{published data only\}}

Doane JA, Falloon IR, Goldstein MJ, Mintz J. Parental affective style and the treatment of schizophrenia. Predicting course of illness and social functioning. Archives of General Psychiatry 1985;42(1):34-42.

* Falloon IR, Boyd JL, McGill CW, Razani J, Moss HB, Gilderman AM. Family management in the prevention of exacerbations of schizophrenia: a controlled study. New England Journal of Medicine 1982;306(24):1437-40.

Falloon IR, Boyd JL, McGill CW, Williamson M, Razani J, Moss HB, et al. Family management in the prevention of morbidity of schizophrenia. Clinical outcome of a two-year longitudinal study. Archives of General Psychiatry 1985;42(9):887-96.

Falloon IR, McGill CW, Boyd JL, Pederson J. Family management in the prevention of morbidity of schizophrenia: social outcome of a two-year longitudinal study. Psychological Medicine 1987;17(1):59-66.
Falloon IR, Pederson J. Family management in the prevention of morbidity of schizophrenia: the adjustment of the family unit. British Journal of Psychiatry 1985;147:156-63.

Liberman RP, Cardin V, McGill CW, Falloon IR, et al. Behavioral family management of schizophrenia: clinical outcome and costs. University of Maryland School of Medicine Symposium. Psychiatric Annals 1987;17(9):610-9.

McGill CW, Falloon IR, Boyd JL, Siverio CW. Family educational intervention in the treatment of schizophrenia. Hospital and Community Psychiatry 1983;34:934-8.

Rea MM, Strachan AM, Goldstein MJ, Falloon I, Hwang S. Changes in patient coping style following individual and family treatment for schizophrenia. British Journal of Psychiatry 1991;158:642-7.

Strang JS, Falloon IRH, Moss HB, Razani J, Boyd JL. Drug treatment and family intervention during the aftercare treatment of schizophrenics. The effects of family therapy on treatment compliance in schizophrenia. Psychopharmacology Bulletin 1981;17(3):87-8.

Haddock 1999 \{published data only\}

Haddock G, Tarrier N, Morrison AP, Hopkins R, Drake R, Lewis S. A pilot study evaluating the effectiveness of individual inpatient cognitive-behavioural therapy in early psychosis. Social Psychiatry and Psychiatric Epidemiology 1999;34:254-8.

\section{Hogarty 1997-study 1 \{published data only\}}

Hogarty GE, Greenwald D, Ulrich RF, Kornblith SJ, DiBarry AL, Cooley S, et al. Three-year trials of personal therapy among schizophrenic patients living with or independent of family, II: Effects on adjustment of patients. American Journal of Psychiatry 1997;154(11):1514-24.

Hogarty GE, Kornblith SJ, Greenwald D, DiBarry AL, Cooley S, Ulrich RF, et al. Three-year trials of personal therapy among schizophrenic patients living with or independent of family, I: Description of study and effects on relapse rates. American Journal of Psychiatry 1997;154(11):1504-13.

\section{Hogarty 1997-study 2 \{published data only\}}

Hogarty GE, Greenwald D, Ulrich RF, Kornblith SJ, DiBarry AL, Cooley S, et al. Three-year trials of personal therapy among schizophrenic patients living with or independent of family, II: Effects on adjustment of patients. American Journal of Psychiatry 1997;154(11):1514-24.

Hogarty GE, Kornblith SJ, Greenwald D, DiBarry AL, Cooley S, Ulrich RF, et al. Three-year trials of personal therapy among schizophrenic patients living with or independent of family, I: Description of study and effects on relapse rates. American Journal of Psychiatry 1997;154(11):1504-13.

\section{Kemp 1996 \{published data only\}}

Healey A, Knapp M, Astin J, Beecham J, Kemp R, Kirov G, et al. Cost-effectiveness evaluation of compliance therapy for people with psychosis.. British Journal of Psychiatry 1998;172(5):420-4. 
* Kemp R, Hayward P, Applewhaite G, Everitt B, David A. Compliance therapy in psychotic patients: randomised controlled trial. BMJ 1996;312:345-9. [MEDLINE: 96190158; PMID 8611831]

Kemp R, Kirov B, Hayward P, David A. Randonised controlled trial of compliance therapy. 18-month follow-up. British Journal of Psychiatry 1998;172:413-9.

Klein 1998 \{published data only\}

Klein A R, Cnaan R A, Whitecraft J. Significance of peer social support with dually diagnosed clients: findings from a pilot study. Research on Social Work Practice 1998;8:529-51.

\section{Levine 1998 \{published data only\}}

Levine J, Barak Y, Granek I. Cognitive group therapy for paranoid schizophrenics: applying cognitive dissonance. Journal of Cognitive Psychotherapy 1998;12(1):3-12.

\section{Lewis 2002b \{published data only\}}

Kingdon D. SOCRATES - a multicentre, randomized controlled trial of cognitive behavioural therapy in early schizophrenia. National Research Register 2000. [National Research Register N0172004444]

* Lewis S, Tarrier N, Haddock G, Bentall R, Kinderman P, Kingdon D, et al. Randomised controlled trial of cognitivebehavioural therapy in early schizophrenia: acute-phase outcomes. British Journal of Psychiatry - Supplementum 2002;suppl. 43:S91-7. [MEDLINE: 22233037; PMID 12271807]

Lewis SW, Tarrier N, Haddock G, Bentall R, Kinderman P, Kingdon D. Cognitive therapy improves outcomes in first episode psychosis. XIIth World Congress of Psychiatry. Yokohama, Japan, 2002.

Lewis SW, Tarrier N, Haddock G, Bentall R, Kinderman P, Kingdon $\mathrm{D}$, et al. A randomised controlled trial of cognitive behaviour therapy in early schizophrenia. Schizophrenia Research 2001, issue 1,2:263-4. [MEDLINE: 21083242; PMID 11215542; PsycINFO 2001-16630-011]

Lewis SW, Tarrier N, Haddock G, Bentall R, Kinderman P, Kingdon D, et al. Cognitive therapy improves 18-month outcomes but not time to relapse in first episode schizophrenia. Schizophrenia Research 2002;53:14.

Mandelson M. A multi-centre, randomised, controlled trial of cognitive behavioural therapy in early schizophrenia (SOCRATES). National Research Register 2000.

\section{Malm 1982 \{published data only\}}

Malm U. The influence of group therapy on schizophrenia. Acta Psychiatrica Scandinavica Supplementum 1982;Suppl 297:1-65. [MEDLINE: 82280715; PMID 6126069]

\section{O'Donnell 1999 \{published data only\}}

O'Donnell M, Parker G, Proberts M, Matthews R, Fisher D, Johnson $B$, et al. A study of client focused case management and consumer advocacy: The community and consumer service project. Australian and New Zealand Journal of Psychiatry 1999;33(5):684-93. [EMBASE 1999362945]
Penn 2009 \{published data only\}

Penn D L, Meyer PS, Evans E, Wirth RJ, Cai K, Burchinal M. A randomized controlled trial of group cognitive-behavioral therapy vs. enhanced supportive therapy for auditory hallucinations. Schizophrenia Research 2009;109:52-9.

Pinto 1999 \{published data only\}

Pinto A, La-Pia S, Mennella R, Giorgio D, DeSimone L. Cognitive behavioral therapy and clozapine for clients with treatment refractory schizophrenia. Psychiatric Services 1999;50(7):901-4.

\section{Sensky 2000b \{published data only\}}

Sensky T, Turkington D, Kingdon D, Scott JL, Scott J, Siddle R, et al. A randomized controlled trial of cognitive behavioral therapy for persistent symptoms in schizophrenia resistant to medication. Archives of General Psychiatry 2000;57(2):165-72.

Spaulding 1999 \{published data only\}

Spaulding WD, Reed D, Sullivan M, Richardson C, Weiler M. Effects of cognitive treatment in psychiatric rehabilitation. Schizophrenia Bulletin 1999;25(4):657-76.

\section{Stanton 1984 \{published data only\}}

Frank AF, Gunderson JG. The role of the therapeutic alliance in the treatment of schizophrenia. Relationship to course and outcome. Archives of General Psychiatry 1990;47(3):228-36.

Gunderson JG, Frank AF. Effects of psychotherapy in schizophrenia. Yale Journal of Biology and Medicine 1985;58(4):373-81.

Gunderson JG, Frank AF, Katz HM, Vannicelli ML, Frosch JP, Knapp PH. Effects of psychotherapy in schizophrenia: II. Comparative outcome of two forms of treatment. Schizophrenia Bulletin 1984;10(4):564-98.

* Stanton AH, Gunderson JG, Knapp PH, Frank AF, Vannicelli ML, Schnitzer R, et al. Effects of psychotherapy in schizophrenia: I. Design and implementation of a controlled study. Schizophrenia Bulletin 1984;10(4):520-63.

\section{Tarrier 1998 \{published data only\}}

Tarrier N. The use of cognitive behaviour therapy in the treatment of schizophrenia. 8th Congress of the Association of European Psychiatrists; 1996 Jul 7-12; London, UK. 1996. [MEDLINE: 95050364; PMID 7961550]

Tarrier N, Kinney C, McCarthy E, Humphreys L, Wittkowski A, Morris J. Two-year follow-up of cognitive-behavioral therapy and supportive counseling in the treatment of persistent symptoms in chronic schizophrenia. Journal of Consulting and Clinical Psychology 2000;68(5):917-22. [EMBASE 2000386575]

Tarrier N, Kinney C, McCarthy E, Wittkowski A, Yusupoff L, Gledhill A. Are some types of psychotic symptoms more responsive to cognitive-behaviour therapy?. Behavioural and Cognitive Psychotherapy 2001;29:45-55.

Tarrier N, Wittkowski A, Kinney C, McCarthy E, Morris J, Humphreys L. Durability of the effects of cognitive-behavioural therapy in the treatment of chronic schizophrenia: 12-Month 
follow-up. British Journal of Psychiatry 1999;174:500-4. [EMBASE 1999210779]

* Tarrier N, Yusupoff L, Kinney C, McCarthy E, Gledhill A, Haddock G, et al. Randomised controlled trial of intensive cognitive behaviour therapy for patients with chronic schizophrenia. BMJ 1998;317:303-7. [MEDLINE: 98350038; PMID 9685273]

\section{Turkington 2000 \{published data only\}}

Turkington D, Kingdon D. Cognitive-behavioural techniques for general psychiatrists in the management of patients with psychoses. British Journal of Psychiatry 2000;177:101-6.

\section{Uzenoff 2007 \{published data only\}}

* Uzenoff SR. A Preliminary Trial of Adherence-CopingEducation (ACE) Therapy for First-episode Schizophrenia. Chapel Hill: The University of North Carolina, 2007.

Uzenoff SR, Perkins DO, Hamer RM, Wiesen CA, Penn DL. A preliminary trial of Adherence-Coping-Education (ACE) therapy for early psychosis. Journal of Nervous and Mental Disease 2008;196:572-5.

\section{Wirshing 1991 \{published data only\}}

Marder SR, Wirshing DA, Wirshing WC. Psychosocial and pharmacological strategies for improving treatment adherence in schizophrenia. 155th Annual Meeting of the American Psychiatric Association; 2002 May 18-23; Philadelphia, PA, USA. 2002. [No. 3B]

Marder SR, Wirshing DA, Wirshing WC. Psychosocial and pharmacological strategies for improving treatment adherence in schizophrenia. Annual Meeting of the American Psychiatric Association; 2001 May 5-10; LA, USA. Marathon Multimedia, 2001. [MEDLINE: 21185887; PMID 11290639]

Marder SR, Wirshing WC, Mintz J, McKenzie J, Johnston K, Eckman TA, et al. Two-year outcome of social skills training and group psychotherapy for outpatients with schizophrenia. American Journal of Psychiatry 1996;153(12):1585-92.

* Wirshing WC, Eckman T, Liberman RP, Marder SR. Management of risk of relapse through skills training of chronic schizophrenics. In: Tamminga Ca SSC editor(s). Schizophrenia Research: Advances in Neuropsychiatry and Psychopharmacology. Raven Press, 1991:255-67.

Wirshing WC, Marder SR, Eckman T, Liberman RP, Mintz J. Acquisition and retention of skills training methods in chronic schizophrenic outpatients. Psychopharmacology Bulletin 1992;28(3):241-5. [MEDLINE: 93126518; PMID 1480726]

\section{References to studies excluded from this review}

\section{Aberg-Wistedt 1995 \{published data only\}}

Aberg-Wistedt A, Cressell T, Lidberg Y, Liljenberg B, Osby U. Two-year outcome of team-based intensive case management for patients with schizophrenia. Psychiatric Services 1995;12:1263-6. [MEDLINE: 96164410; PMID 8590112]

\section{Abramowitz 1989 \{published data only\}}

Abramowitz IA, Coursey RD. Impact of an educational support group on family participants who take care of their schizophrenic relatives. Journal of Consulting and Clinical Psychology 1989;57:232-6.

\section{Addington 2011 \{published data only\}}

Addington J, Epstein I, Liu L, French P, BoydellK M, Zipursky RB. $A$ randomized controlled trial of cognitive behavioral therapy for individuals at clinical high risk of psychosis. Schizophrenia Research 2011;125:54-61.

\section{Anderson 1982 \{published data only\}}

Anderson CM, Reiss Douglas J. Approaches to psychoeducational family therapy. International Journal of Family Psychiatry 1982;3(4):501-17.

\section{Andres 1998 \{published data only\}}

Andres K, Schindler F, Brenner HD, Garst F, Donzel G, Schaub A. Coping oriented group therapy for patients with schizophrenic or schizoaffective disorders. An exploratory study [Bewaltigungsorientierte Gruppentherapie fur Patienten mit schizophrenen oder schizoaffektiven Storungen. Eine explorative Studie]. Fortschritte der Neurologie-Psychiatrie 1998;5:225-32. [MEDLINE: 98317525; PMID 9653638]

\section{Andres 2000 \{published data only\}}

Andres K, Pfammatter M, Garst F, Teschner C, Brenner HD. Effects of a coping orientated group therapy for schizophrenia and schizoaffective patients: a pilot study. Acta Psychiatrica Scandinavica 2000;101(4):318-22. [MEDLINE: 20242647; PMID 10782553]

\section{Armstrong 1991 \{published data only\}}

Armstrong HE, Cox GB, Short BA, Allmon DJ. A comparative evaluation of two day treatment programs. Psychosocial Rehabilitation Journal 1991;14(4):53-67.

\section{Baker 1994 \{published data only\}}

Baker L. Training mentally ill offenders in problem-solving. PhD dissertation submitted to the University of Pennsylvania 1994.

\section{Beal 1977 \{published data only\}}

Beal D, Duckro P, Elias J, Hecht E. Graded group procedures for long term regressed schizophrenics. Journal of Nervous and Mental Disease 1977;164(2):102-6. [MEDLINE: 77095923; PMID 836481]

\section{Beard 1975 \{published data only\}}

Beard MT, Scott PY. The efficacy of group therapy by nurses for hospitalized patients. Nursing Research 1975;24(2):120-4. [MEDLINE: 75119981; PMID 1090905]

\section{Beard 1978 \{published data only\}}

Beard JH, Malamud TJ, Rossman E. Psychiatric rehabilitation and long-term rehospitalisation rates: the findings of two research studies. Schizophrenia Bulletin 1978;4:622-35.

\section{Becker 1998 \{published data only\}}

Becker DR, Drake RE, Bond GR, Xie H, Dain BJ, Harrison K. Job terminations among persons with severe mental illness 
participating in supported employment. Community Mental Health Journal 1998;34(1):71-82.

\section{Beebe 2001 \{published data only\}}

Beebe LH. Community nursing support for clients with schizophrenia. Archives of Psychiatric Nursing 2001;XV(5):214-22. [MEDLINE: 11584350; CINAHL 2002036665; PMID 21467734]

\section{Bell 1993a \{published data only\}}

Bell MD, Milstein RM, Lysaker PH. Pay and participation in work activity: clinical benefits for clients with schizophrenia. Psychosocial Rehabilitation Journal 1993;17(2):173-7.

Bell 1993b \{published data only\}

Bell MD, Milstein RM, Lysaker PH. Pay as an incentive in work participation by patients with severe mental illness. Hospital and Community Psychiatry 1993;44(7):684-6.

Bell 1998 \{published data only\}

Bell M, Bryson G. Psychological and psychosocial disorders. Rehabilitation effects of pay, activity, and support intensity on schizophrenia. Rehabilitation R\&D Progress Reports 1998;35:228-9.

\section{Bell 2001 \{published data only\}}

Bell MD, Bryson G. Work rehabilitation in schizophrenia: does cognitive impairment limit improvement?. Schizophrenia Bulletin 2001;27:269-79.

\section{Bell 2003 \{published data only\}}

Bell M, Lysaker P, Bryson G. A behavioral intervention to improve work performance in schizophrenia: Work behavior inventory feedback. Journal of Vocational Rehabilitation 2003;1:43-50. [MEDLINE: 2003141918]

\section{Bellack 1986 \{published data only\}}

Bellack AS. Social skills training in the treatment of chronic schizophrenics [Das Training sozialer Fertigkeiten zur Behandlung chronisch Schizophrener]. In: Boeker W BHD editor(s). Bewaltigung der Schizophrenie. Verlag Hans Huber, 1986:121-128. [PSYNDEX 0020596]

\section{Bond 1995 \{published data only\}}

Bond GR, Dietzen LL, McGrew JH, Miller LD. Accelerating entry into supported employment for persons with severe psychiatric disabilities. Rehabilitation Psychology 1995;40(2):75-94.

\section{Brown 1983 \{published data only\}}

Brown MA, Munford AM. Life skills training for chronic schizophrenics. Journal of Nervous and Mental Disease 1983;171(8):466-70. [MEDLINE: 83267491; PMID 6875530]

\section{Buchkremer 1997 \{published data only\}}

Buchkremer G, Klingberg S, Holle R, Monking HS, Hornung WP. Psychoeducational psychotherapy for schizophrenic patients and their key relatives or care-givers: results of a 2-year followup. Acta Psychiatrica Scandinavica 1997;96(6):483-91. [MEDLINE: 98081699; PMID 9421346]
Bush 1990 \{published data only\}

Bush CT, Langford MW, Rosen P, Gott W. Operation outreach: intensive case management for severely psychiatrically disabled adults. Hospital and Community Psychiatry 1990;41(6):647-9. [MEDLINE: 90299261; PMID 2361668]

Canning 1997 \{published data only\}

Canning. The effect of family support on the working alliance in psychotherapy with psychiatrically disturbed parents. PhD dissertation submitted to Yale University, USA 1997:106. [Dissertation Abstracts (order number) AAC 9805882]

Carra 2010 \{published data only\}

Carra G. Multiple group family treatment for schizophrenia in Italy. Schizophrenia Research 2010;117:121.

Castelein 2008 \{published data only\}

Castelein S, Bruggeman R, Van Busschbach JT, Van Der Gaag M, Stant AD, Knegtering $\mathrm{H}$, et al. The effectiveness of minimally guided peer support groups for people suffering from psychosis: a randomized controlled trial. Schizophrenia Research 2008;98:52-3.

Castelein 2008a \{published data only\}

Castelein S, Bruggeman R, Van Busschbach J T, Van Der Gaag M, Stant A D, Knegtering $\mathrm{H}$, et al. The effectiveness of peer support groups in psychosis: a randomized controlled trial. Acta Psychiatrica Scandinavica 2008;118:64-72.

Castilla-Puentes 2002 \{published data only\}

Castilla-Puentes RC, Valero J, Vargas J, Gongora O, Pava C, Perel J. Music therapy and medication compliance in psychotic patients. 155th Annual Meeting of the American Psychiatric Association. Philadelphia, PA, USA, 2002.

Chien 2008 \{published data only\}

Chien WT, Thompson DR, Norman I. Evaluation of a peer-led mutual support group for Chinese families of people with schizophrenia. American Journal of Community Psychology 2008;42:122-34.

\section{Chinman 2010 \{published data only\}}

Chinman M, Shoai R, Cohen A. Using organizational change strategies to guide peer support technician implementation in the veterans administration. Psychiatric Rehabilitation Journal 2010;33:269-77.

\section{Clark 1998 \{published data only\}}

Clark RE, Xie H, Becker DR, Drake RE. Benefits and costs of supported employment from three perspectives. Journal of Behavioral Health Services and Research 1998;25(1):22-34

\section{Cook 2011 \{published data only\}}

Cook JA, Steigman P, Pickett S, Diehl S, Fox A, Shipley P, et al. Randomized controlled trial of peer-led recovery education using building recovery of individual dreams and goals through education and support (bridges). Schizophrenia Research 2012;136(1-3):36-42. 


\section{Cook 2012 \{published data only\}}

Cook J A, Steigman P, Pickett S, Diehl S, Fox A, Shipley P, et al. Randomized controlled trial of peer-led recovery education using building recovery of individual dreams and goals through education and support (bridges). Schizophrenia Research 2012;136:36-42.

\section{Curtis 1992 \{published data only\}}

Curtis JL, Millman EJ, Struening E, D'Ercole A. Effect of case management on rehospitalization and utilization of ambulatory care services. Hospital and Community Psychiatry 1992;43(9):895-9. [MEDLINE: 88251244; PMID 3289523]

\section{Curtis 1996 \{published data only\}}

Curtis JL, Millman EJ, Struening E, D'Ercole A. Deaths among former psychiatric inpatients in an outreach case management program. Psychiatric Services 1996;47(4):398-402. [MEDLINE: 96255404; PMID 8689371]

\section{Czobor 1995 \{published data only\}}

Czobor P, Volavka J, Meibach RC. Effect of risperidone on hostility in schizophrenia. Journal of Clinical Psychopharmacology 1995;15(4):243-9.

D'Ercole 1997 \{published data only\}

D'Ercole A, Struening E, Curtis JL, Millman EJ, Morris A. Effects of diagnosis, demographic characteristics, and case management on rehospitalization. Psychiatric Services 1997;45(5):682-8. [MEDLINE: 97290039; PMID 9144824]

\section{Daumit 2010 \{published data only\}}

Daumit G, Appel L, Leatherman E, Latkin C, Dalcin A, Goggins B, et al. Randomized trial of peer-supported physical activity for persons with severe mental illness in community psychiatry. Journal of General Internal Medicine 2010;25(3 (Suppl.)):378-9.

\section{Dixon 2002 \{published data only\}}

Dixon L, Hoch JS, Clark R, Bebout R, Drake R, McHugo G, et al. Cost-effectiveness of two vocational rehabilitation programs for persons with severe mental illness. Psychiatric Services 2002;53(9):1118-24.

\section{Donlon 1973 \{published data only\}}

Donlon PT, Rada RT, Knight SW. A therapeutic aftercare setting for "refractory" chronic schizophrenic patients. American Journal of Psychiatry 1973;130(6):682-4. [PsycINFO 51-07592]

\section{Drake 1993 \{published data only\}}

Drake RE, Bebout RR, Roach JP. A research evaluation of social network case management for homeless persons with dual disorders. In: Harris H, Bergman HC editor(s). Case Management for Mentally III Patients: Theory and Practice. Chronic mental illness. Pennsylvania: Harwood Academic Publishers, 1993:83-98. [PSYCINFO 1993-98432-005]

\section{Drake 1996 \{published data only\}}

Drake RE, McHugo GJ, Becker DR, Anthony WA, Clark RE. The New Hampshire study of supported employment for people with severe mental illness. Journal of Consulting and Clinical Psychology 1996;64(2):391-9.
Drake 1999 \{published data only\}

Drake RE, McHugo GJ, Bebout RR, Becker DR, Harris M, Bond GR, et al. A randomized clinical trial of supported employment for inner city patients with severe mental disorders. Archives of General Psychiatry 1999;56(7):627-33.

\section{Drury 1996a \{published data only\}}

Drury V, Birchwood M, Cochrane R, MacMillan F. Cognitive therapy and recovery from acute psychosis - A controlled trial. I. Impact on psychotic symptoms. British Journal of Psychiatry 1996;169:593-601.

\section{Drury 1996b \{published data only\}}

Drury V, Birchwood M, Cochrane R, MacMillan F. Cognitive therapy and recovery from acute psychosis - A controlled trial. II. Impact on recovery time. British Journal of Psychiatry 1996;169:602-7.

\section{Drury 2000 \{published data only\}}

Drury V, Birchwood M, Cochrane R. Cognitive therapy and recovery from acute psychosis: a controlled trial. 3. Fiveyear follow-up. British Journal of Psychiatry 2000;177:8-14. [MEDLINE: 20401378; PMID 10945081]

Eack 2007 \{published data only\}

Eack SM, Hogarty GE, Greenwald DP, Hogarty SS, Keshavan MS. Cognitive enhancement therapy improves emotional intelligence in early course schizophrenia: preliminary effects. Schizophrenia Research 2007;89:308-11.

Eack 2010 \{published data only\}

Eack S M, Greenwald D P, Hogarty S S, Keshavan M S. One-year durability of the effects of cognitive enhancement therapy on functional outcome in early schizophrenia. Schizophrenia Research 2010;120:210-6.

Eack 2011 \{published data only\}

Eack S M, Pogue-Geile M F, Greenwald D P, Hogarty S S, Keshavan M S. Mechanisms of functional improvement in a 2-year trial of cognitive enhancement therapy for early schizophrenia. Psychological Medicine 2011;41:1253-61.

Eack 2012 \{published data only\}

Eack S M, Wojtalik J A, Prasad K M, Francis A N, Bhojraj T S, Cho $\mathrm{R} Y$, et al. A broad cortical reserve accelerates response to cognitive enhancement therapy in early course schizophrenia. Clinical and Translational Science 2012;5:152-3.

Essock 1995 \{published data only\}

Essock SM, Kontos N. Implementing assertive community treatment teams. Psychiatric Services 1995;46(7):679-83. [MEDLINE: 96044852; PMID 7552558]

Field 1997 \{published data only\}

Field CD, Galletly C, Anderson D, Walker P. Computer aided cognitive rehabilitation: possible application to the attentional deficit of schizophrenia, a report of negative results. Perceptual and Motor Skills 1997;85:995-1002. 
Ford 1995 \{published data only\}

Ford R, Beadsmoore A, Ryan P, Repper J, Craig T, Muijen M. Providing the safety net: case management for people with a serious mental illness. Journal of Mental Health 1995;1:91-7. [CINAHL 1996004717]

\section{Forsyth 1961 \{published data only\}}

Forsyth RP. MMPI and demographic correlates of post-hospital adjustment in neuropsychiatric patients. Dissertation Abstracts 1961;21:2783-4.

\section{Franklin 1987 \{published data only\}}

Franklin JL, Solovitz B, Mason M, Clemons JR, Miller GE. An evaluation of case management. American Journal of Public Health 1987;77(6):674-8. [MEDLINE: 87210733; PMID 3578614]

\section{Freeman 1998 \{published data only\}}

Freeman D, Garety P, Fowler D, Kuipers E, Dunn G, Bebbington P, et al. The London East Anglia randomized controlled trial of cognitive behaviour therapy for psychosis. IV: Self esteem and persecutory delusions. British Journal of Clinical Psychology 1998;37(4):415-30. [MEDLINE: 99073526; PMID 9856295]

\section{Gaither 2003 \{published data only\}}

Gaither ML, Nazzaro D, Barnett B, Huegel SG, Haas GL. A randomized clinical trial of a brief cognitive-behavioral treatment for the chronic phase of schizophrenia. Schizophrenia Research 2003:322-3. [EMBASE 2003252036]

\section{Garety 1994 \{published data only\}}

Garety PA, Kuipers L, Fowler D, Chamberlain F, Dunn G. Cognitive behavioural therapy for drug-resistant psychosis. British Journal of Medical Psychology 1994;67(Pt 3):259-71. [MEDLINE: 95101546; PMID 7803318]

\section{Glick 1974 \{published data only\}}

Glick ID, Hargreaves WA, Goldfield MD. Short vs long hospitalization: a prospective controlled study: I. The preliminary results of a one-year follow-up of schizophrenics. Archives of General Psychiatry 1974;30(3):363-9.

\section{Glick 1975 \{published data only\}}

Glick ID, Hargreaves WA, Raskin M, Kutner SJ. Short versus long hospitalization: a prospective controlled study. II. Results for schizophrenic inpatients. American Journal of Psychiatry 1975;132(4):385-90.

\section{Glick 1976a \{published data only\}}

Glick ID, Hargreaves WA, Drues J, Showstack JA. Short versus long hospitalization: a prospective controlled study. IV. Oneyear follow-up results for schizophrenia patients. American Journal of Psychiatry 1976;133(5):509-14.

\section{Glick 1976b \{published data only\}}

Glick ID, Hargreaves WA, Drues JMA, Showstack JA. Short versus long hospitalization: a controlled study. III. Inpatient results for non-schizophrenics. Archives of General Psychiatry 1976;33:78-83.

\section{Glick 1976c \{published data only\}}

Glick ID, Hargreaves WA, Drues JMA, Showstack JA. Short versus long hospitalization: a prospective controlled study. V. Oneyear follow up for non-schizophrenics. American Journal of Psychiatry 1976;133:515-7.

Glick 1977 \{published data only\}

Glick ID, Hargreaves WA, Drues JA, Showstack JA, Katzow JJ. Short versus long hospitalization: a prospective controlled study. VII. Two-year follow-up results for non-schizophrenics. Archives of General Psychiatry 1977;34:314-7.

Glick 1979 \{published data only\}

Glick I, Hargreaves W, Drues J, Showstack J. Psychiatric hospital treatment for the 1980 's: a controlled study of short versus long hospitalization. Heath and Company. Lexington Books D.C, 1979.

\section{Glynn 2001 \{published data only\}}

Glynn SM, Marder SR, Liberman RP, Blair K, Wirshing WC, Wirshing DA, et al. Supplementing clinic based skills training for schizophrenia with manualized community support: nine month follow-up effects on social adjustment. Schizophrenia Research 2001;49(12):261.

\section{Grassi 2001 \{published data only\}}

Grassi A, Bruni R, Pileggi F, Chiappelli M, Boldrini M, Franceschi E, et al. Analysis and comparative evaluations of the costs of supports and treatments of schizophrenia, affective psychosis, paranoia and neurosis. Epidemiologia e Psichiatria Sociale 2001;10(2):115-24.

\section{Grawe 1998 \{published data only\}}

Grawe RW, Widen JH. Result of two years optimal out-patient treatment of first episode schizophrenia: a controlled study. 25th Nordic Psychiatric Congress. Nord J Psychiatry. 1998; Vol. Suppl 41.

\section{Haddock 2000a \{published data only\}}

Haddock G. Dual diagnosis project evaluation of family support cognitive behaviour therapy for recent onset schizophrenia sufferers with substance misuse, problems. National Research Register 2000.

\section{Haddock 2000b \{published data only\}}

Haddock G. Evaluation of a family support and cognitivebehavioural treatment service for recent onset schizophrenia sufferers with substance misuse problems. National Research Register 2000.

\section{Hafner 1983 \{published data only\}}

Hafner RJ, Badenoch A, Fisher J, Swift H. Spouse aided versus individual therapy in persisting psychiatric disorders: a systematic comparison. Family Process 1983;22(3):385-99.

\section{Haldun 2002 \{published data only\}}

Haldun S, Mehmet S, Perihan G, Ilkten C, Besti U. Optimal treatment of schizophrenia. XIIth World Congress of Psychiatry; 2002 Aug 24-29; Yokohama, Japan. 2002. [MEDLINE: 92127314; 1773245] 
Hannes 1974 \{published data only\}

Hannes M, Siegel HD. The short term effect of socializing on performance of schizophrenics in recreational therapy. Journal of Community Psychology 1974;1:51-3. [PsycINFO 56-08317]

\section{Hargreaves 1977 \{published data only\}}

Hargreaves WA, Glick ID, Drues J, Showstack JA, Feigenbaum E. Short versus long hospitalization: a prospective controlled study. VI. Two-year follow-up results for schizophrenics. Archives of General Psychiatry 1977;34:305-11.

\section{Harrison-Read 2002 \{published data only\}}

Harrison-Read P, Lucas B, Tyrer P, Ray J, Shipley K, Simmonds S, et al. Heavy users of acute psychiatric beds: randomized controlled trial of enhanced community management in an outer London borough. Psychological Medicine 2002;32(3):403-16. [MEDLINE: 21985356; 11989986]

\section{Harvey 2002 \{published data only\}}

Harvey K, Burns T, Fiander M, Huxley P, Manley C, Fahy T. The effect of intensive case management on the relatives of patients with severe mental illness. Psychiatric Services 2002;53(12):1580-5. [MEDLINE: 22350352; PMID 12461219]

\section{Herz 1974 \{published data only\}}

Herz MI, Spitzer RL, Gibbon M, Greenspan K, Reibel S. Individual versus group aftercare treatment. American Journal of Psychiatry 1974;131:808-12.

\section{Herz 2000 \{published data only\}}

Herz MI, Lamberti JS, Mintz J, Scott R, O'Dell SP, McCartan L, et al. A program for relapse prevention in schizophrenia: a controlled study. Archives of General Psychiatry 2000;57(3):277-83. [MEDLINE: 20175078; PMID 10711914]

\section{Hogarty 1973 \{published data only\}}

Goldberg SC, Schooler NR, Hogarty GE, Roper M. Prediction of relapse in schizophrenic outpatients treated by drug and sociotherapy. Archives of General Psychiatry 1977;34(2):171-84.

Hogarty G, Goldberg SC. Drug and social therapy in schizophrenic outpatients. Unknown.

* Hogarty GE, Goldberg SC. Drug and sociotherapy in the aftercare of schizophrenic patients. One-year relapse rates. Archives of General Psychiatry 1973;28:54-64.

Hogarty GE, Goldberg SC, Schooler NR. Drug and sociotherapy in the aftercare of schizophrenic patients. III. Adjustment of non relapsed patients. Archives of General Psychiatry 1974;31(5):609-18.

Hogarty GE, Goldberg SC, Schooler NR, Ulrich RF. Drug and sociotherapy in the aftercare of schizophrenic patients. II. Two-year relapse rates. Archives of General Psychiatry 1974;31(5):603-8.

\section{Hogarty 1977 \{published data only\}}

Hogarty GE, Ulrich RF. Temporal effects of drug and placebo in delaying relapse in schizophrenic outpatients. Archives of General Psychiatry 1977;34(3):297-301.
Hogarty 1984 \{published data only\}

Hogarty GE, Munetz MR. Pharmacogenic depression among outpatient schizophrenic patients: a failure to substantiate. Journal of Clinical Psychopharmacology 1984;4(1):17-24.

Hogarty 1986a \{published data only\}

Hogarty GE, Anderson C. A controlled trial of family therapy, social skills training and drug therapy in the aftercare of schizophrenics. Effects on relapse and expressed emotion. In: Boeker W BHD editor(s). Bewaeltigung der Schizophrenie. Bern, Switzerland: Verlag Hans Huber, 1986:72-86.

\section{Hogarty 1986b \{published data only\}}

Hogarty GE, Anderson CM. Medication, family psychoeducation, and social skills training: first year relapse results of a controlled study. Psychopharmacology Bulletin 1986;22(3):860-2.

Hogarty GE, Anderson CM, Reiss DJ, Kornblith SJ, Greenwald DP, Javna CD, et al. Environmental/Personal Indicators in the Course of Schizophrenia Research Group. Family psychoeducation, social skills training and maintenance chemotherapy in the aftercare of schizophrenia. I. One-year effects of a controlled study on relapse and expressed emotion.. Archives of General Psychiatry 1986;43:633-42.

\section{Hogarty 1986c \{published data only\}}

Hogarty GE, Anderson CM, Reiss DJ, Kornblith SJ, Greenwald DP, Javna CD, et al. Environmental-personal Indicators in the Course of Schizophrenia Research Group. Family psychoeducation, social skills training, and maintenance chemotherapy in the aftercare treatment of schizophrenia. I. One-year effects of a controlled study on relapse and expressed emotion.. Archives of General Psychiatry 1986;43(7):633-42.

Hogarty GE, Anderson CM, Reiss DJ, Kornblith SJ, Greenwald DP, Ulrich RF, et al. Environmental-Personal Indicators in the Course of Schizophrenia (EPICS) Research Group. Family psychoeducation, social skills training, and maintenance chemotherapy in the aftercare treatment of schizophrenia. II. Two-year effects of a controlled study on relapse and adjustment. Archives of General Psychiatry 1991;48(4):340-7.

\section{Hogarty 1988 \{published data only\}}

Hogarty GE, McEvoy JP, Munetz M, DiBarry AL, Bartone P, Cather R, et al. Dose of fluphenazine, familial expressed emotion, and outcome in schizophrenia. Results of a two-year controlled study. Archives of General Psychiatry 1988;45(9):797-805.

\section{Hornung 1998 \{published data only\}}

Hornung WP, Klingberg S, Feldmann R, Schonauer K, Schulze Monking $\mathrm{H}$. Collaboration with drug treatment by schizophrenic patients with and without psychoeducational training: Results of a 1-year follow-up. Acta Psychiatrica Scandinavica 1998;97(3):213-9. [MEDLINE: 98202311; EMBASE 1998058470; PMID 9543310]

\section{Hornung 1999 \{published data only\}}

Hornung WP, Feldmann R, Klingberg S, Buchkremer G, Reker T. Long term effects of a psychoeducational psychotherapeutic intervention for schizophrenic outpatients and their key 
persons: results of a five year follow-up [Langzeitwirkungen einer psychoedukativen-psychotherapeutischen intervention bei schizophrenen ambulanten patienten und ihren bezugspersonen - ergebnisse einer fuenf-jahres-katamnese]. European Archives of Psychiatry and Clinical Neuroscience 1999;249(3):162-7. [MEDLINE: 99360513; PMID 10433131]

\section{Hurlburt 1996 \{published data only\}}

Hurlburt MS, Hough RL, Wood PA. Effects of substance abuse on housing stability of homeless mentally ill persons in supported housing. Psychiatric Services 1996;47(7):731-6. [EMBASE 1996220189]

\section{Huxley 2001 \{published data only\}}

Huxley P, Evans S, Burns T, Fahy T, Green J. Quality of life outcome in a randomized controlled trial of case management. Social Psychiatry and Psychiatric Epidemiology 2001;36(5):249-55. [MEDLINE: 21406524; PMID 11515703]

\section{Isrctn50487713 2010 \{published data only\}}

ISRCTN50487713. Self help therapy and recovery-trial: Evaluation of a recovery guide for psychosis. http:// www.controlled-trials.com/ISRCTN50487713 (accessed 20 February 2014).

\section{Isrctn69299093 2011 \{published data only\}}

ISRCTN69299093. An evaluation of the feasibility and effectiveness of a supported self management package for relatives of people with recent onset psychosis: Relatives education and coping toolkit (react). http://www.controlledtrials.com/ISRCTN69299093 (accessed 20 February 2014).

\section{Isrctn96754763 2011 \{published data only\}}

ISRCTN96754763. Sos inpatients: A pilot study of enhanced support, openness and supervision for staff working on adult mental health wards. http://www.controlled-trials.com/ ISRCTN96754763 (accessed 20 February 2014).

\section{Issakidis 1999 \{published data only\}}

Issakidis C, Sanderson K, Teesson M, Johnston S, Buhrich N. Intensive case management in Australia: a randomized controlled trial. Acta Psychiatrica Scandinavica 1999;99(5):360-7. [MEDLINE: 99279892; PMID 10353452]

\section{Jones 1994 \{published data only\}}

Jones K, Colson P, Valencia E, Susser E. A preliminary cost effectiveness analysis of an intervention to reduce homelessness among the mentally ill. Psychiatric Quarterly 1994;65(4):243-56. [MEDLINE: 95132734; PMID 7831412]

\section{Kaplan 2011 \{published data only\}}

Kaplan K, Salzer M S, Solomon P, Brusilovskiy E, Cousounis P. Internet peer support for individuals with psychiatric disabilities: A randomized controlled trial. Social Science and Medicine 2011;72:54-62.

\section{Karon 1969 \{published data only\}}

* Karon BP, O'Grady P. Intellectual test changes in schizophrenic patients in the first six months of treatment. Psychotherapy Theory Research and Practice 1969;6(2):88-96.
Karon BP, Vandenbos GR. Experience, medication, and the effectiveness of psychotherapy with schizophrenics. British Journal of Psychiatry 1970;116(533):427-8.

Karon BP, Vandenbos GR. The consequences of psychotherapy for schizophrenic patients. Psychotherapy Theory Research and Practice 1972;9(2):111-9.

\section{Kaufmann 1995 \{published data only\}}

Kaufmann CL. The self help employment center: some outcomes from the first year. Psychosocial Rehabilitation Journal 1995;18(4):145-62. [CINAHL 1995039486]

\section{Keith 1977 \{published data only\}}

Keith RD, Engelkes JR, Winborn BB. Employment-seeking preparation and activity: an experimental job-placement training model for rehabilitation clients. Rehabilitation Counseling Bulletin. 1977;21:159-65.

Kern 2002 \{published data only\}

Kern RS, Liberman RP, Kopelowicz A, Mintz J, Green MF. Applications of errorless learning for improving work performance in persons with schizophrenia. American Journal of Psychiatry 2002;159(11):1921-6.

Keshavan 2011 \{published data only\}

Keshavan M, Eack SM. Brain structure and cognition in schizophrenia and the effects of treatment. Schizophrenia Bulletin 2011;37(Suppl. 1):310.

\section{Kim 1997 \{published data only\}}

Kim C, Kang DH, Jang JH, Cho JS, Youn S, Shim KS. Twoyear effects of psychosocial treatment on relapse of chronic schizophrenia. 150th Annual Meeting of the American Psychiatric Association; 1997 May 17-22; San Diego, USA. 1997. [MEDLINE: 96190158; PMID 8611831]

\section{Klingberg 1999 \{published data only\}}

Klingberg S, Buchkremer G, Holle R, Schulze-Monking H, Hornung WP. Differential therapy effects of psychoeducational psychotherapy for schizophrenic patients: results of a 2 year follow up. European Archives of Psychiatry and Clinical Neuroscience 1999;249(2):66-72. [MEDLINE: 99295826; PMID 10369152]

Klingberg 2010 \{published data only\}

* Klingberg S, Wittorf A, Meisner C, Wolwer W, Wiedemann G, Herrlich J, et al. Cognitive behavioural therapy versus supportive therapy for persistent positive symptoms in psychotic disorders: The positive study, a multicenter, prospective, single-blind, randomised controlled clinical trial. Trials 2010;11:123.

Wagner M. Cognitive biases and cognitive deficits in patients with positive symptoms: Relationship with symptoms and with symptom change during psychotherapy. European Archives of Psychiatry and Clinical Neuroscience 2011;261:S14.

Wittorf A, Klingberg S. The therapeutic alliance in cbt for pychoses: Course and predictive value for outcome. European Archives of Psychiatry and Clinical Neuroscience 2011;261:S13S4. 
Koivunen 2010 \{published data only\}

Koivunen M, Valimaki M, Patel A, Knapp M, Hatonen $\mathrm{H}$, Kuosmanen $\mathrm{L}$, et al. Effects of the implementation of the web-based patient support system on staff's attitudes towards computers and it use: A RANDOMised controlled trial. Scandinavian Journal of Caring Sciences 2010;24:592-9.

Kris 1965 \{published data only\}

Kris EB. Day hospitals. Current Therapeutic Research 1965;7(5):320-3.

\section{Kuipers 1998 \{published data only\}}

Kuipers E, Fowler D, Garety P, Chisholm D, Freeman D, Dunn G, et al. London East Anglia randomised controlled trial of cognitive behavioural therapy for psychosis. III: Follow up and economic evaluation at 18 months. British Journal of Psychiatry 1998;173:61-8. [MEDLINE: 99067191; PMID 9850205]

\section{Lecomte 1999 \{published data only\}}

Lecomte T, Cyr M, Lesage AD, Wilde J, Leclerc C, Ricard N. Efficacy of a self-esteem module in the empowerment of individuals with schizophrenia. Journal of Nervous and Mental Disease 1999;187(7):406-13. [MEDLINE: 99353532; PMID 10426460]

\section{Lehman 1993 \{published data only\}}

Lehman AF, Herron JD, Schwartz RP, Myers CP. Rehabilitation for adults with severe mental illness and substance use disorders. A clinical trial. Journal of Nervous and Mental Disease 1993;181(9):588-9. [MEDLINE: 93147812; PMID 8426176]

\section{Lehman 2001 \{published data only\}}

Lehman AF, Goldberg R, McNary S, Dixon L, Postrado L, Osher F. Improving employment outcomes for persons with schizophrenia and other severe and persistent mental illnesses. Schizophrenia Research 2001;49(12):273-4.

\section{Lehman 2002a \{published data only\}}

Lehman AF. Improving employment outcomes for persons with severe mental illness. 155th Annual Meeting of the American Psychiatric Association; 2002 May 18-23; Philadelphia, PA, USA. 2002. [No. 91D]

\section{Lehman 2002b \{published data only\}}

Lehman AF, Goldberg R, Dixon LB, McNary S, Postrado L, Hackman A, et al. Improving employment outcomes for persons with severe mental illnesses. Archives of General Psychiatry 2002;59:165-72.

\section{Levene 1989 \{published data only\}}

Levene JE, Newman F, Jefferies JJ. Focal family therapy outcome study. I: patient and family functioning. Canadian Journal of Psychiatry (Revue Canadienne de Psychiatrie) 1989;34(7):641-7. [MEDLINE: 90030063; PMID 2804876]

\section{Lewis 2000 \{published data only\}}

Lewis S. Evaluation of family support and cognitive behavioural treatment service for recent onset schizophrenia sufferers with substance misuse problems. National Research Register 2000.
Lewis 2001a \{published data only\}

Lewis SW. SOCRATES: study of cognitive realignment therapy in early schizophrenia. Current Controlled Trials (http:// www.controlled-trials.com/accessed February 2001) 2001.

\section{Lindenmayer 1995 \{published data only\}}

Lindenmayer J, Grochowski S, Hyman RB. Five factor model of schizophrenia - replication across samples. Schizophrenia Research 1995;14(3):229-34.

\section{Linszen 1994 \{published data only\}}

Linszen DH, Dingemans PM, Lenior ME, Nugter MA, Scholte WF, van der Does AJ. Relapse criteria in schizophrenic disorders: different perspectives. Psychiatry Research 1994;54(3):273-81.

\section{Linszen 1996 \{published data only\}}

Linszen D, Dingemans P, van der Does JW, Nugter A, Scholte $P$, Lenior R, et al. Treatment, expressed emotion and relapse in recent onset schizophrenic disorders. Psychological Medicine 1996;26(2):333-42.

\section{Linszen 1997 \{published data only\}}

Linszen DH, Dingemans PM, Nugter MA, van der Does AJ, Scholte WF, Lenior MA. Patient attributes and expressed emotion as risk factors for psychotic relapse. Schizophrenia Bulletin 1997;23:119-30.

\section{Linszen 1998 \{published data only\}}

Linszen D, Dingemans P, Scholte W, Lenior M, Goldstein M. Early recognition, intensive intervention and other protective and risk factors for psychotic relapse in patients with first psychotic episodes in schizophrenia. International Clinical Psychopharmacology 1998;13:S7-S12.

\section{Linszen 2009 \{published data only\}}

Linszen D, Wouters L, Krikke M, Nieman D, Amelsvoort TV, Lenior $\mathrm{M}$, et al. Continuity of treatment and supporting parent groups in early phase schizophrenia: a 5 year randomised trial. Proceedings of the 12th International Congress on Schizophrenia Research; 2009 Mar 28-Apr 1; San Diego, CA. 2009:346.

\section{Macias 1994 \{published data only\}}

Macias C, Kinney R, Farley OW, Jackson R, Vos B. The role of case management within a community support system: partnership with psychosocial rehabilitation. Community Mental Health Journal 1994;30(4):323-39. [MEDLINE: 95044169; EMBASE 1994198866; PMID 7956109]

\section{Marder 1994a \{published data only\}}

Marder SR, Wirshing WC, van Putten T, Mintz J, McKenzie J, Johnston Cronk K, et al. Fluphenazine vs placebo supplementation for prodromal signs of relapse in schizophrenia. Archives of General Psychiatry 1994;51(4):280-7.

\section{Marder 1994b \{published data only\}}

Marder SR, Meibach RC. Risperidone in the treatment of schizophrenia. American Journal of Psychiatry 1994;151(6):825-35. 
Marder 2001b \{published data only\}

Marder SR. Management for risk of relapse in schizophrenia. CRISP database (https:, www-commons.cit.nih.gov/crisp/ index.html accessed 19th February 2001) 2001. [CRISP Grant Number - 2R01MH41573-11A1]

\section{Marshall 1985 \{published data only\}}

Marshall C. Stress reduction through skills training in families of the severely psychiatrically disabled a rehabilitation psychology approach (chronically mentally ill). PhD dissertation submitted to the University of Arizona, USA 1985.

\section{May 1976a \{published data only\}}

May PR, Tuma AH, Dixon WJ. Schizophrenia - a follow-up study of results of treatment. I. Design and other problems. Archives of General Psychiatry 1976;33(4):474-8. [MEDLINE: 76230761; PMID 938184]

May PR, Tuma AH, Dixon WJ, Yale C, Thiele DA, Kraude WH. Schizophrenia. A follow-up study of the results of five forms of treatment. Archives of General Psychiatry 1981;38(7):776-84. [MEDLINE: 81231559; PMID 6113821]

May PR, Tuma AH, Yale C, Potepan P, Dixon WJ. Schizophrenia - a follow-up study of results of treatment. II. Hospital stay over two to five years. Archives of General Psychiatry 1976;33(4):481-6. [MEDLINE: 76230762; PMID 938185]

Tuma AH, May PR, Yale C, Forsythe AB. Therapist characteristics and the outcome of treatment in schizophrenia. Archives of General Psychiatry 1978;35(1):81-5. [MEDLINE: 78079616; PMID 619842]

\section{McEvoy 1994 \{published data only\}}

McEvoy JP. Efficacy of risperidone on positive features of schizophrenia. Journal of Clinical Psychiatry 1994;55(5SUPPL):18-21.

\section{McFarlane 1992 \{published data only\}}

McFarlane WR, Stastny P, Deakins S. Family-aided assertive community treatment: a comprehensive rehabilitation and intensive case management approach for persons with schizophrenic disorders. New Directions for Mental Health Services 1992;53:43-54. [MEDLINE: 92252807; PMID 1579118]

\section{McFarlane 2002 \{published data only\}}

McFarlane WR, Cook W, Balser R, Boyak C, Leavitt R. Effects of fact and an employers consortium on employment in severe mental illness. 155th Annual Meeting of the American Psychiatric Association. Philadelphia, PA, 2002.

\section{McGorry 1999 \{published data only\}}

McGorry P, Adlard S, Yung A, McDonald A, Phillips L, Hearn N. Detection and intervention in pre-psychotic schizophrenia. Current Opinion in Psychiatry 1999;12(Suppl 1):S62. [11th World Congress of Psychiatry [CD-ROM] Conifer, Excerpta Medica Medical Communications BV, 1999 S-17-2]

\section{McGorry 2000 \{published data only\}}

McGorry PD, Phillips LJ, Yung AR, Francey S, Germano D, Bravin J, et al. A randomised controlled trial of interventions in the pre psychotic phase of psychotic disorders. Schizophrenia Research 2000; Vol. Number 1 - Special Issue.

McInnis 1967 \{published data only\}

McInnis TL, Ullmann LP. Positive and negative reinforcement with short and long term hospitalized schizophrenics in a probability learning situation. Journal of Abnormal Psychology 1967;72(2):157-62. [MEDLINE: 70257943; PMID 5623443; PsycINFO 1999-13800-005]

Meisler 2002 \{published data only\}

Meisler N. Rural-based supported employment approaches: results from the South Carolina site of the employment intervention demonstration project. 155th Annual Meeting of the American Psychiatric Association. Philadelphia, PA, USA, 2002.

\section{Meister 2010 \{published data only\}}

Meister K, Rietschel L, Burlon M, Jannsen H, Bock T, Wegscheider $\mathrm{K}$, et al. A cluster-randomized, parallel group, observer blind trial of a group based motivational-behavioural therapy for young people with psychosis and substance use disorder. Early Intervention in Psychiatry 2010;4:163.

Miknyak 2001 \{published data only\}

Miknyak SI, Vlokh IY. To the question of the maintenance therapy of chronic schizophrenia. Schizophrenia Research 2001, issue 1,2:239. [MEDLINE: 21083242; PMID 11215542; PsycINFO 2001-16630-011]

\section{Morse 1997 \{published data only\}}

Morse GA, Calsyn RJ, Klinkenber W, Dean, Trusty ML, Gerber F, et al. An experimental comparison of three types of case management for homeless mentally ill persons. Psychiatric Services 1997;48(4):497-503. [PsycINFO 1997-03557-006]

\section{Mosher 1975 \{published data only\}}

Mosher LR, Menn A, Matthew SM. Soteria: evaluation of a home-based treatment for schizophrenia. American Journal of Orthopsychiatry 1975;45(3):455-67. [MEDLINE: 75202479; PMID 238399]

\section{Mueser 2001a \{published data only\}}

Mueser KT, Becker DR, Wolfe R. Supported employment, job preferences, job tenure and satisfaction. Journal of Mental Health 2001;10(4):411-7.

Mueser 2001b \{published data only\}

Mueser KT, Salyers MP, Mueser PR. A prospective analysis of work in schizophrenia. Schizophrenia Bulletin 2001;27(2):281-96.

NCT 2005 \{published data only\}

NCT00169052. Rehabilitation and health care for elderly with SMI. http://clinicaltrials.gov/show/NCT00169052 (accessed 20 February 2014).

NCT 2005a \{published data only\}

NCT00177983. Support to patients and families. http:// www.clinicaltrials.gov/show/NCT00177983 (accessed 20 February 2014). 
NCT 2009 \{published data only\}

NCT00936351. Mindfulness meditation as a rehabilitation strategy for persons with schizophrenia. http:// www.clinicaltrials.gov/show/ NCT00936351 (accessed 20 February 2014).

\section{NCT 2009a \{published data only\}}

NCT00940394. Effectiveness of family group interventions for people with schizophrenia. http://www.clinicaltrials.gov/show/ NCT00940394 (accessed 20 February 2014).

\section{NCT 2012a \{published data only\}}

NCT01561859. Brain imaging, cognitive enhancement and early schizophrenia. http://ClinicalTrials.gov/show/NCT01561859 (accessed 20 february 2014).

\section{Nelson 2007 \{published data only\}}

Nelson B, Phillips LJ, Yung AR, Francey SM, Leicester S, Baker K, et al. A double blind, placebo-controlled randomized trial of low-dose risperidone, intensive psychological treatment and supportive therapy in young people with subthreshold symptoms at incipient risk of psychotic disorder baseline characteristics of the sample. Schizophrenia Bulletin 2007:33:450

\section{Nugter 1997a \{published data only\}}

Nugter MA, Dingemans PMAJ, Linszen DH, van Der Does AJW, Gersons BPR. Parental communication deviance: its stability and the effect of family treatment in recentonset Schizophrenia. Acta Psychiatrica Scandinavica 1997;95(3):199-204. [MEDLINE: 97266048; PMID 9111852]

\section{Nugter 1997b \{published data only\}}

Nugter A, Dingemans P, van der Does JW, Linszen D, Gersons B. Family treatment, expressed emotion and relapse in recent onset schizophrenia. Psychiatry Research 1997;72(1):23-31.

\section{O'Donnell 2002 \{published data only\}}

O'Donnell C, Sharkey L, O'Donohue G, Owens N, Migone M, Harris R, et al. Influence of compliance therapy and carer education on the outcome of schizophrenia. Schizophrenia Research 2002;3 Suppl.1:253. [MEDLINE: 21372626; PMID 11479066]

\section{Ogrodniczuk 2000 \{published data only\}}

Ogrodniczuk JS, Piper WE, Joyce AS, McCallum M. Different perspectives of the therapeutic alliance and therapist technique in 2 forms of dynamically oriented psychotherapy. Canadian Journal of Psychiatry 2000;45(Revue Canadienne de Psychiatrie):452-8.

\section{Ohlenschlaeger 2002 \{published data only\}}

Ohlenschlaeger J, Nordentoft M, Køster A, Poulsen HD, Bredkjær S. Effect of person continuity on the use of coercion: the Danish opus-project. Schizophrenia Research 2002;53(3SUPPL1):52.

\section{Philipps 2001 \{published data only\}}

Philipps LJ, McGorry P, Yung A, Francey D, Germano F, Bravin J, et al. The development of preventive interventions for early psychosis: early findings and directions for the future. 7 th
World Congress of Biological Psychiatry; 2001 Jul 1-6; Berlin, Germany. 2001, issue Suppl. 1. [7th World Congress of Biological Psychiatry [CD-ROM] Conifer, Excerpta Medica Medical Communications BV, 2001 S066-02]

\section{Phillips 1999 \{published data only\}}

Phillips LJ, McGorry PD, Yung AR, Francey S, Cosgrave L, Germano $D$, et al. The development of preventive interventions for early psychosis: early findings and directions for the future conference abstract. Schizophrenia Research. 1999; Vol. $12 \& 3: 331-2$

\section{Pickett 1997 \{published data only\}}

Pickett SA, Heller T, Cook JA. Professional versus familyled support groups: exploring the differences. 150th Annual Meeting of the American Psychiatric Association. San Diego, USA, 1997.

\section{Pushkaryova 1999 \{published data only\}}

Pushkaryova T. Rehabilitation of the psychotic patients with use of modern methods of efferent therapy and psychotherapy. 11th World Congress of Psychiatry. Hamburg, Germany, 1999.

\section{Razali 1995 \{published data only\}}

Razali MS, Yahya H. Compliance with treatment in schizophrenia - a drug intervention program in a developing country. Acta Psychiatrica Scandinavica 1995;91(5):331-5. [MEDLINE: 95366310; PMID 7639089]

Rector 2002 \{published data only\}

Rector NA, Seeman MV, Segal ZV. Cognitive therapy of schizophrenia: Toronto trial. 155th Annual Meeting of the American Psychiatric Association; 2002 May 18-23; Philadelphia, PA, USA. 2002. [NR219 Tuesday, May 16, 900 a.m.-10 30 a.m]

Reynolds 2002 \{published data only\}

Reynolds W. The Effects of a Transitional Discharge Model for Psychiatric Clients: A pilot study. Unpublished Report. 2002.

\section{Ritch 2001 \{published data only\}}

Ritch JL, Velligan DI, Prihoda TJ, Sui DW, Maples NJ, Miller AL, et al. Effectiveness of compensatory strategies in both recently discharged and stable outpatients with schizophrenia. Schizophrenia Research. 2001, issue 1,2:266. [MEDLINE: 21083242; PMID 11215542; PsycINFO 2001-16630-011]

Ritch 2002 \{published data only\}

Ritch JL, Velligan DI, Dicocco M, Prihoda TJ, Mann TA, Miller AL. The efficacy of cognitive adaptation training versus assertive community treatment for improving functional outcomes. Schizophrenia Research 2002;3 Suppl.1:210. [MEDLINE: 21372626; PMID 11479066]

\section{Rosenheck 2003 \{published data only\}}

Rosenheck R, Kasprow W, Frisman L, Liu-Mare W. Costeffectiveness of supported housing for homeless persons with mental illness. Archives of General Psychiatry 2003;60(9):940-51. [CINAHL 2003153019] 


\section{Rosenthal 2000 \{published data only\}}

Rosenthal RN, Miner CR, Philomena S, Hellerstein DJ, Muran JC. Alliance and engagement in substance-abusing schizophrenia patients. 153rd Annual Meeting of the American Psychiatric Association; 2000 May 13-18; Chicago, USA. 2000. [153rd Annual Meeting of the American Psychiatric Association [CD-ROM] MARATHON Multimedia, 2000 No 30D]

Ro-Trock 1977 \{published data only\}

* Ro-Trock GK, Wellisch DK, Schoolar JC. A family therapy outcome study in an inpatient setting. American Journal of Orthopsychiatry 1977;47(3):514-22.

Wellisch DK, Ro-Trock GK. A three year follow of family therapy. International Journal of Family Therapy 1980;2(3):169-75.

Ruan 2008 \{published data only\}

Ruan S, Zheng WDu L. A control study of clozapine plus fluoxetine in schizophrenia with depressive symptoms. Journal of Clinical Psychosomatic Diseases [\#\#\#\#\#\#\#] 2008;14:211-3.

\section{Serok 1984 \{published data only\}}

Serok S, Rabin C, Spitz Y. Intensive Gestalt group therapy with schizophrenics. International Journal of Group Psychotherapy 1984;43(3):431-50. [MEDLINE: 85029542; EMBASE 1984242594; PMID 6490255]

\section{Shi 2000 \{published data only\}}

Shi Y, Zhao B, Xu D, Sen J. A comparative study of life quality in schizophrenic patients after family intervention. Chinese Mental Health Journal 2000;14(2):135-7.

\section{Shin 2002 \{published data only\}}

Shin SK, Lukens EP. Effects of psychoeducation for Korean Americans with chronic mental illness. Psychiatric Services 2002;53(9):1125-31.

\section{Slavinsky 1982 \{published data only\}}

Slavinsky AT, Krauss JB. Two approaches to the management of long-term psychiatric outpatients in the community. Nursing Research 1982;31:284-9.

\section{Smith 1999 \{published data only\}}

Smith TE, Hull JW, Romanelli S, Fertuck E, Weiss KA. Symptoms and neurocognition as rate limiters in skills training for psychotic patients. American Journal of Psychiatry 1999;156(11):1817-8.

\section{Solomon 1994a \{published data only\}}

Solomon P, Draine J. Family perceptions of consumers as case managers. Community Mental Health Journal 1994;45(2):165-76. [MEDLINE: 94283048; PMID 8013213]

\section{Solomon 1994b \{published data only\}}

Solomon P, Draine J, Meyerson A. Jail recidivism and receipt of community mental health services. Hospital and Community Psychiatry 1994;45(8):793-7. [MEDLINE: 95073747; PMID 7982695]
Solomon 1995a \{published data only\}

Solomon P, Draine J. The efficacy of a consumer case management team: 2-year outcomes of a randomized trial. Journal of Mental Health Administration 1995;22(2):135-46. [MEDLINE: 95271199; SOCIOFILE 95W22429; PMID 10142127]

Solomon 1995b \{published data only\}

Solomon P, Draine J. Consumer case management and attitudes concerning family relations among persons with mental illness. Psychiatric Quarterly 1995;66(3):249-61. [MEDLINE: 96040361; PMID 7568532]

\section{Solomon 1996 \{published data only\}}

Solomon P, Draine J. Perspectives concerning consumers as case managers. Community Mental Health Journal 1996;32(1):41-6. [MEDLINE: 96210695; EMBASE 1996042453; PMID 8635316]

\section{Stant 2011 \{published data only\}}

Stant A, Castelein S, Bruggeman R, Busschbach J, Gaag M, Knegtering $\mathrm{H}$, et al. Economic aspects of peer support groups for psychosis. Community Mental Health Journal 2011:47:99-105.

\section{Stroup 2003 \{published data only\}}

Stroup S, Appelbaum P. The subject advocate: protecting the interests of participants with fluctuating decision making capacity. IRB: a Review of Human Subjects Research 2003;32(3):9-11. [MEDLINE: 22929411; PUBMED 14569988]

Sun ShuMin 2007 \{published data only\}

孙淑敏, 张月卿, 张伟青. Social support schizophrenic patients. Medical Journal of Chinese People's Health [\#\#\#\#\#] 2007;19:1053-4.

\section{Tarrier 2000a \{published data only\}}

Tarrier N. Evaluation of the family support and cognitive service for recent onset schizophrenia sufferers with substance misuse. National Research Register 2000.

\section{Tarrier 2000d \{published data only\}}

Tarrier N. Training family support workers to relieve carer burden. National Research Register 2000.

Telles 1995 \{published data only\}

Telles C, Karno M, Mintz J, Paz G, Arias M, Tucker D, et al. Immigrant families coping with schizophrenia. Behavioral family intervention v. case management with a low-income Spanish-speaking population. British Journal of Psychiatry 1995;167(4):473-9. [MEDLINE: 96113861; PMID 8829715]

Test 1991 \{published data only\}

Test MA, Knoedler WH, Allness DJ, Burke SS, Brown RL, Wallisch LS. Long-term community care through an assertive continuous treatment team. In: Tamminga Ca SSC editor(s). Advances in Neuropsychiatry and Psychopharmacology: Schizophrenia Research. Vol. 1, Raven Press, 1991:239-46. [PsycINFO 91-032024-023] 
Theilemann 1993 \{published data only\}

Theilemann S. Comparing cognitive therapy with sociotherapy in reducing cognitive disturbances of schizoaffective disorders. Nervenarzt 1993;64(9):587-93.

\section{Tong Shouming 2010 \{published data only\}}

童寿明, 张毓茂, 郭玉花, 陈凯, 刘有平, 陈晓燕, et al. social support for the first time the drug compliance of schizophrenia Google Translate. Medical Journal of Chinese People's Health [\#\# \#\#\#\#] 2010;22(5):530-1, 533.

\section{Toprac 2002 \{published data only\}}

Toprac M, Hoppe SK, Daggett P, Wambach K, Onken S, Burek S, et al. The Texas earns supported employment demonstration project. 155th Annual Meeting of the American Psychiatric Association. Philadelphia, PA, USA, 2002.

Torrey 2000 \{published data only\}

Torrey WC, Mueser KT, McHugo GH, Drake RE. Self esteem as an outcome measure in studies of vocational rehabilitation for adults with severe mental illness. Psychiatric Services 2000;51(2):229-33.

\section{Tsang 2001 \{published data only\}}

Tsang HW. Rehab rounds: Social skills training to help mentally ill persons find and keep a job. Psychiatric Services 2001;52(7):891-4.

\section{Tyrer 1995 \{published data only\}}

Tyrer P, Morgan J, van Horn E, Jayakody M, Evans K, Brummell $R$, et al. A randomised controlled study of close monitoring of vulnerable psychiatric patients. Lancet 1995;345(8952):756-9. [MEDLINE: 95198463; PMID 7891486]

\section{Vaughan 1992 \{published data only\}}

Vaughan K, Doyle M, McConaghy N, Blaszczynski A, Fox A, Tarrier N. The Sydney intervention trial: a controlled trial of relatives' counselling to reduce schizophrenic relapse. Social Psychiatry and Psychiatric Epidemiology 1992;27(1):16-21. [MEDLINE: 92213515; PMID 1557677]

\section{Velligan 1999 \{published data only\}}

Velligan DI, Bow-Thomas CC, Eckert SL, Halgunselth LC, Huntzinger CD, Miller AL. Randomized controlled trial of the use of compensatory strategies in schizophrenic outpatients: cognitive adaptation training. Schizophrenia Research. 1999:333.

\section{Walker 1969 \{published data only\}}

Walker R, Winick W, Frost ES, Lieberman JM. Social restoration of hospitalised psychiatric patients through a program of special employment in industry. Rehabilitation Literature 1969;30:297-303.

\section{Wallace 1985 \{published data only\}}

Wallace CJ, Liberman RP. Social skills training for patients with schizophrenia: a controlled clinical trial. Psychiatry Research 1985;15(3):239-47. [MEDLINE: 85299024; PsycINFO 73-20548; PMID 3862158]
Wallace 2001 \{published data only\}

Wallace CJ. Clinical trial of the workplace fundamentals module. CRISP database (https://www-commons.cit.nih.gov/ crisp/index.html accessed 19th February 2001) 2001.

Weinman 1974 \{published data only\}

Weinman B, Kleiner R, Yu JH, Tillson VA. Social treatment of the chronic psychotic patient in the community. Journal of Community Psychology 1974;2(4):358-65.

Wojciechowska 2001 \{published data only\}

Wojciechowska A, Walczewski K, Cechnicki A. Correlations between some features of social networks and treatment outcome in patients with schizophrenia three years after initial hospitalization. Psychiatria Polska 2001;35(1):21-32.

Wolkon 1971 \{published data only\}

Wolkon GH, Karmen M, Tanaka HT. Evaluation of a social rehabilitation program for recently released psychiatric patients. Community Mental Health Journal 1971;7(4):312-22.

Wuerker 2002 \{published data only\}

Wuerker AK, Long JD, Haas GL, Bellack AS. Interpersonal control, expressed emotion, and change in symptoms in families of persons with schizophrenia. Schizophrenia Research 2002;58:281-92.

\section{Yung 2008 \{published data only\}}

Yung A, Nelson B, Phillips L, Francey S, Leicester S, Yuen H, et al. A double blind, placebo-controlled randomized trial of lowdose risperidone, cognitive-behaviour therapy, and supportive therapy in young people at ultra high risk of psychotic disorder: 12 month outcome data. Early Intervention in Psychiatry 2008;2:A12.

\section{Yung 2011 \{published data only\}}

Yung AR, Phillips LJ, Nelson B, Francey SM, PanYuen H, Simmons MB, et al. Randomized controlled trial of interventions for young people at ultra high risk for psychosis: 6-month analysis. Journal of Clinical Psychiatry 2011;72:430-40.

\section{Ziedonis 1997 \{published data only\}}

Ziedonis DM, Harris SA, Wyatt P, Trudeau TP, George K, Johnson D. Motivational enhancement therapy and nicotine replacement improve smoking cessation outcomes. 150th Annual Meeting of the American Psychiatric Association. San Diego, USA, 1997.

\section{References to studies awaiting assessment}

Bechdolf 2012 \{published data only\}

Bechdolf A, Pohlmann B, Guettgemanns J, Geyer C, Lindner K, Ferber $C$, et al. State-dependent motivational interviewing for people with schizophrenia and substance use. Results of a randomised controlled trial. Nervenarzt 2012;83:888-96.

Blankertz 1997 \{published data only\}

Blankertz LE, Keller C. The provision of long-term vocational supports for individuals with severe mental illness. American Sociological Association 1997. 
Falloon 1983 \{published data only\}

Falloon IRH, Razani J, Moss HB, Boyd JL, McGill CW, Pederson J. Community management of schizophrenia. Partnerberatung 1983;20(2/3):73-9.

\section{References to ongoing studies}

\section{ACTRN12610000241033 2010 \{published data only\}}

ACTRN12610000241033. A pilot project investigating the feasibility and effects of a 6 month peer support program commencing 3 months prior to discharge from a specialist firstepisode psychosis treatment center. https://www.anzctr.org.au/ Trial/Registration/TrialReview.aspx?ACTRN=12610000241033 (accessed 20 February 2014).

\section{ACTRN12612000974808 2012 \{published data only\}}

ACTRN12612000974808. Peer delivered support intervention for people who hear voices: Pilot randomised controlled trial. http://www.anzctr.org.au/ACTRN12612000974808.aspx (accessed 20 February 2014).

\section{Bennett 2003 \{published data only\}}

Bennett ME, Bellack S, Gearon JS. Treating substance abuse in schizophrenia: Preliminary analysis of data from a randomized treatment trial. Schizophrenia Research 2003;60(1 Suppl 1):319. [EMBASE 2003252036]

\section{Durham 2000a \{published data only\}}

Durham R. Cognitive-behavioural therapy with medicationresistant psychotic symptoms: a clinical trial with psychiatric nurses as therapists. National Research Register 2000. [National Research Register N0470079877]

\section{Hogarty 2001 \{published data only\}}

Hogarty GE. Environmental-personal treatment of schizophrenia. CRISP database (http://wwwcommons.cit.nih.gov/crisp/index.html accessed 19th February 2001) 2001.

\section{ISRCTN02457313 2006 \{published data only\}}

ISRCTN02457313. The effectiveness of support groups for people suffering from psychosis: A randomized controlled trial. http://www.controlled-trials.com/ISRCTN02457313 (accessed 20 February 2014).

\section{ISRCTN50487713 2011 \{published data only\}}

ISRCTN50487713. An evaluation of different levels of support in using a recovery guide for people with psychosis and the impact of choice on outcomes. http://www.controlled-trials.com/ ISRCTN50487713 (accessed 20 February 2014).

\section{NCT 2007 \{published data only\}}

NCT00495911. A randomized controlled trial of individual therapy for first episode psychosis. http:// www.clinicaltrials.gov/show/NCT00495911 (accessed 20 February 2014).

\section{NCT 2007a \{published data only\}}

NCT00458094. Peer support for increasing physical activity in people with serious mental illnesses. http:// www.clinicaltrials.gov/show/ NCT00458094 (accessed 20 February 2014).

NCT 2007b \{published data only\}

NCT00522613. Recovery group pilot. http:// www.clinicaltrials.gov/show/ NCT00522613 (accessed 20 February 2014).

\section{NCT 2008 \{published data only\}}

NCT00495911. A randomized controlled trial of individual therapy for first episode psychosis. http:// www.clinicaltrials.gov/show/ NCT00495911 (accessed 20 February 2014).

NCT 2012 \{published data only\} NCT01566513. Effectiveness and cost effectiveness of peer mentors in reducing hospital use. http://ClinicalTrials.gov/ show/NCT01566513 (accessed 20 February 2014).

Robinson 2010 \{published data only\}

Robinson J, Bruxner A, Harrigan S, Bendall S, Killackey E, Tonin V, et al. The development, design, and testing of a peer support intervention in youth being discharged from a first episode psychosis clinic. Australian and New Zealand Journal of Psychiatry 2010;4(1 (Suppl.)):43.

\section{Rohricht 2000 \{published data only\}}

Rohricht F. Randomised controlled exploratory trial in the effectiveness of body oriented psychotherapy on anergia/ negative symptoms in patients with chronic schizophrenia. Unpublished Report.

Rohriht F. RCT in effectiveness of body oriented psychotherapy on anergia in patients with chronic schizophrenia. National Research Register 2000.

\section{Sensky 2000a \{published data only\}}

Sensky T. A five year follow-up of patients with schizophrenia with drug-resistant symptoms treated with cognitive behaviour therapy or a befriending intervention. National Research Register 2000.

\section{Tarrier 2000c \{published data only\}}

Tarrier N. A psychological intervention programme to reduce positive symptoms and prevent relapse in psychotic patients. National Research Register 2000.

\section{Additional references}

\section{Addington 1993}

Addington D, Addington J, Maticka-Tyndale E. Assessing depression in schizophrenia: the Calgary Depression Scale. British Journal of Psychiatry 1993;163(Suppl 22):39-44.

\section{Altman 1996}

Altman DG, Bland JM. Detecting skewness from summary information. BMJ 1996;313:1200.

\section{Andreasen 1983}

Andreasen NC. The Scale for the Assessment of Negative Symptoms (SANS). lowa City: University of lowa, 1983. 


\section{Andreasen 1984}

Andreasen NC. The Scale for the Assessment of Positive Symptoms (SAPS). lowa City: University of lowa, 1984.

\section{APA 1987}

American Psychiatric Association. Diagnostic and Statistical Manual of Mental Disorders. 3rd Edition. Washington, DC: APA, 1987.

\section{Barber 2001}

Barber JP, Stratt R, Halperin G, Connolly MB. Supportive techniques: are they found in different therapies?. Journal of Psychotherapy Practice and Research 2001;10(3):165-72.

\section{Beck 1996}

Beck AT, Steer RA, Ball R, Ranieri W. Comparison of Beck Depression Inventories -IA and -II in psychiatric outpatients. Journal of Personality Assessment 1996;67(3):588-97.

\section{Beck 2004}

Beck AT, Baruch E, Balter JM, Steer RA, Warman DM. A new instrument for measuring insight: the Beck Cognitive Insight Scale. Schizophrenia research 2004;68:319-30.

\section{Birchwood 1990}

Birchwood M, Smith J, Cochrane R, Wetton S, Copestake S. The Social Functioning Scale. The development and validation of a new scale of social adjustment for use in family intervention programmes with schizophrenic patients. British Journal of Psychiatry 1990;157:853-9.

\section{Bland 1997}

Bland JM. Statistics notes. Trials randomised in clusters. BMJ 1997;315:600.

\section{Bloch 1996}

Bloch S. Introduction to the Psychotherapies. 3rd Edition. Oxford University Press, 1996.

\section{Boissel 1999}

Boissel JP, Cucherat M, Li W, Chatellier G, Gueyffier F, Buyse $M$, et al. The problem of therapeutic efficacy indices. 3. Comparison of the indices and their use [Apercu sur la problematique des indices d'efficacite therapeutique, 3: comparaison des indices et utilisation. Groupe d'Etude des Indices D'efficacite]. Therapie 1999;54(4):405-11. [PUBMED: 10667106]

\section{Chadwick 2000}

Chadwick P, Lee S, Birchwood M. The revised Beliefs About Voices Questionnaire (BAVQ-R). British Journal of Psychiatry 2000;177:229-32.

\section{Chilvers 2006}

Chilvers R, Macdonald GM, Hayes AA. Supported housing for people with severe mental disorders. Cochrane Database of Systematic Reviews 2006, Issue 4. [DOI: 10.1002/14651858.CD000453.pub2; CD000453]

\section{Cormac 2004}

Cormac I, Jones C, Campbell C. Cognitive behaviour therapy for schizophrenia. Cochrane Database of Systematic Reviews 2004, Issue 4. [DOI: 10.1002/14651858.CD000524.pub2]

\section{Davey 1997}

Davey Smith G, Egger M. Meta-analyses of randomised controlled trials. Lancet 1997;350(9085):1182.

\section{David 1990}

David AS. Insight and psychosis. British Journal of Psychiatry 1990;156:798-808.

\section{Deeks 2000}

Deeks J. Issues in the selection for meta-analyses of binary data. Proceedings of the 8th International Cochrane Colloquium; 2000 Oct 25-28; Cape Town. Cape Town: The Cochrane Collaboration, 2000.

\section{Divine 1992}

Divine GW, Brown JT, Frazier LM. The unit of analysis error in studies about physicians' patient care behaviour. Journal of General Internal Medicine 1992;7(6):623-9.

\section{DoH 2001}

Department of Health. Treatment Choice in Psychological Therapies and Counselling Evidence Based Clinical Practice Guideline. London: Department of Health, 2001.

\section{Donner 2002}

Donner A, Klar N. Issues in the meta-analysis of cluster randomized trials. Statistics in Medicine 2002;21:2971-80.

\section{Egger 1997}

Egger M, Davey Smith G, Schneider M, Minder C. Bias in meta-analysis detected by a simple, graphical test. BMJ 1997;315:629-34.

\section{Elbourne 2002}

Elbourne D, Altman DG, Higgins JPT, Curtina F, Worthingtond HV, Vaile A. Meta-analyses involving crossover trials: methodological issues. International Journal of Epidemiology 2002;31(1):140-9.

\section{Endicott 1976}

Endicott J, Spitzer RL, Fleiss JL, Cohen J. Global Assessment Scale: a procedure for measuring overall severity of psychiatric disturbance. Archives of General Psychiatry 1976;33(6):766-71.

\section{Furukawa 2006}

Furukawa TA, Barbui C, Cipriani A, Brambilla P, Watanabe N. Imputing missing standard deviations in meta-analyses can provide accurate results. Journal of Clinical Epidemiology 2006;59(7):7-10.

\section{Goldberg 1972}

Goldberg D, McDowell I, Newell C. General Health Questionnaire (GHQ), 12 item version, 20 item version, 30 item version, 60 item version [GHQ12, GHQ20, GHQ30, GHQ60]. Measuring health: A guide to rating scales and questionnaire 1972:225-36. 


\section{Gulliford 1999}

Gulliford MC, Ukoumunne OC, Chinn S. Components of variance and intraclass correlations for the design of communitybased surveys and intervention studies: data from the Health Survey for England 1994. American Journal of Epidemiology 1999;149:876-83.

\section{Haddock 1999b}

Haddock G, McCarron J, Tarrier N, Faragher EB. Scales to measure dimensions of hallucinations and delusions: the psychotic symptom rating scales (PSYRATS). Psychological Medicine 1999;29(4):879-89.

\section{Hayward 1995}

Hayward P. Medication self-management: A preliminary report on an intervention to improve medication compliance. Journal of Mental Health 1995;4:511-8.

\section{Heinrichs 1984}

Heinrichs DW, Hanlon TE, Carpenter WT Jr. The quality of life scale. Schizophrenia Bulletin 1984;10:388-98.

\section{Hellerstein 1994}

Hellerstein DJ, Pinsker H, Rosenthal RN, Klee S. Supportive therapy as the treatment model of choice. Journal of Psychotherapy Practice and Research 1994;3(4):300-6.

\section{Higgins 2003}

Higgins JP, Thompson SG, Deeks JJ, Altman DG. Measuring inconsistency in meta-analyses. BMJ 2003;327:557-60.

\section{Higgins 2005}

Higgins JPT, Green S. Cochrane Handbook for Systematic Reviews ofInterventions. Chichester UK: John Wiley and Sons, Ltd., 2005.

\section{Higgins 2011}

Higgins JPT, Green S (editors). Cochrane Handbook for Systematic Reviews of Interventions Version 5.0.2 [updated September 2011]. The Cochrane Collaboration, 2011. Available from www.cochrane-handbook.org..

\section{Hogan 1983}

Hogan TP, Awad AG, Eastwood R. A self report scale predictive of drug compliance in schizophrenics: reliability and discriminative validity. Psychological Medicine 1983;13:177-83.

\section{Holmes 1995}

Holmes J. Supportive psychotherapy. The search for positive meanings. British Journal of Psychiatry 1995;167(4):439-45; 446-7. [MEDLINE: 96113855]

\section{Holmes 2000}

Holmes J. Fitting the biopsychosocial jigsaw together. British Journal of Psychiatry 2000;177:93-4.

\section{Hutton 2009}

Hutton JL. Number needed to treat and number needed to harm are not the best way to report and assess the results of randomised clinical trials. British Journal of Haematology 2009;146(1):27-30.

\section{Katz 1963}

Katz MM, Lyerly SB. Methods for measuring adjustment and social behaviour in the community: I. Rationale, description, discriminative validity and scale development. nt. Psychological Repo 1963;13:503-35.

\section{Kay 1986}

Kay SR, Opler LA, Fiszbein A. Positive and Negative Syndrome Scale (PANSS) Manual. North Tonawanda, NY: Multi-Health Systems, 1986.

\section{Kay 1987}

Kay S, Fitzbein A, Opler L. The Positive and Negative Syndrome Scale (PANSS). Schizophrenia Bulletin 1987;13:261-73.

\section{Lehman 1988}

Lehman AF. A quality of life interview for the chronically mentally ill. Evaluation and Program Planning 1988;11:51-62.

\section{Leucht 2005}

Leucht S, Kane JM, Kissling W, Hamann J, Etschel E, Engel RR. What does the PANSS mean?. Schizophrenia Research 2005;79(2-3):231-8. [PUBMED: 15982856]

\section{Leucht 2005a}

Leucht S, Kane JM, Kissling W, Hamann J, Etschel E, Engel R. Clinical implications of brief psychiatric rating scale scores. British Journal of Psychiatry 2005;187:366-71. [PUBMED: 16199797]

\section{Leucht 2007}

Leucht S, Engel RR, Bauml J, Davis JM. Is the superior efficacy of new generation antipsychotics an artifact of LOCF?. Schizophrenia Bulletin 2007;33(1):183-91. [PUBMED: 16905632]

\section{Lukoff 1996}

Lukoff D, Nuechterlein KH, Ventura J. Manual for the expanded BPRS. Schizophrenia Bulletin 1996;12:594-602.

\section{Margison 2000}

Margison F, Barkham M, Evans C, McGrath G, Mellor CJ, Audin K, et al. Measurement and Psychotherapy. Evidencebased practice and practice-based evidence. British Journal of Psychiatry 2000;177:123-30.

\section{Marshall 2000}

Marshall M, Lockwood A, Bradley C, Adams C, Joy C, Fenton M. Unpublished rating scales: a major source of bias in randomised controlled trials of treatments for schizophrenia. British Journal of Psychiatry 2000;176:249-52.

\section{McEvoy 1989}

McEvoy JP, Apperson LJ, Appelbaum PS, Ortlip P, Brecosky J, Hammill K, et al. Insight in schizophrenia. Its relationship to acute psychopathology. Journal of Nervous and Mental Disease 1989;177(1):43-7.

\section{McIntosh 2006}

McIntosh AM, Conlon L, Lawrie SM, Stanfield AC. Compliance therapy for schizophrenia. Cochrane Database of Systematic Reviews 2006, Issue 3. [DOI: 10.1002/14651858.CD003442.pub2] 


\section{Misch 2000}

Misch DA. Basic strategies of dynamic supportive therapy. Journal of Psychotherapy Practice and Research 2000;9(4):173-89.

\section{Moher 2001}

Moher D, Schulz KF, Altman D. The CONSORT statement: revised recommendations for improving the quality of reports of parallel-group randomized trials. JAMA 2001;285:1987-91.

\section{Montgomery 1978}

Montgomery SA, Taylor P, Montgomery D. Development of a schizophrenia scale sensitive to change. Neuropharmacology 1978;17:1053-71.

\section{Montgomery 1979}

Montgomery SA, Asberg M. A new depression scale designed to be sensitive to change. British Journal of Psychiatry 1979;134:382-9.

\section{NICE 2003}

The National Collaborating Centre for Mental Health. Schizophrenia. Full national clinical guideline on core interventions in primary and secondary care. London: Gaskell, The Royal College of Psychiatrists, 2003.

\section{Overall 1962}

Overall JE, Gorham DR. The brief psychiatric rating scale. Psychological Reports 1962;10:799-812.

\section{Pekkala2002}

Pekkala E, Merinder L. Psychoeducation for schizophrenia. Cochrane Database of Systematic Reviews 2002, Issue 2. [DOI: 10.1002/14651858.CD002831]

\section{Pharoah 2006}

Pharoah FM, Rathbone J, Mari JJ, Streiner D. Family intervention for schizophrenia. Cochrane Database of Systematic Reviews 2006, Issue 4. [DOI: 10.1002/14651858.CD000088.pub2]

\section{Pinsker 1991}

Pinsker H, Rosenthal R, McCullough L. Dynamic supportive therapy. Handbook of Short-term Dynamic Psychotherapy. New York: BasicBooks, 1991:220-47.

\section{Platt 1980}

Platt S, Weyman A, Hirsch S, Hewett S. The social behaviour assessment schedule (SBAS): rationale, contents, scoring and reliability of a new intervention schedule. Social Psychiatry 1980;15:43-55.

\section{Radloff 1977}

Radloff LS. The CES-D Scale: a self-report depression scale for research in the general population. Applied Psychological Measurement 1977;1(3):385-401.

\section{Rosenberg 1965}

Rosenberg M. Rosenberg self-esteem scale (RSE). Acceptance and Commitment Therapy. Measures Package 1965:61.

\section{Roth 1996}

Roth A, Fonagy P, Parry G, Target M, et al. What Works for Whom? A Critical Review of Psychotherapy OResearch. New York: Guilford Press, 1996

\section{Ryff 1989}

Ryff Carol D. Happiness is everything, or is it? Explorations on the meaning of psychological well-being. Journal of Personality and Social Psychology 1989;57:1069-81.

\section{Schulz 1995}

Schulz KF, Chalmers I, Hayes RJ, Altman DG. Empirical evidence of bias: dimensions of methodological quality associated with estimates of treatment effects in controlled trials. JAMA 1995;273:408-12.

\section{Schünemann 2008}

Schünemann HJ, Oxman AD, Vist GE, Higgins JPT, Deeks JJ, Glasziou P, et al. Chapter 12: Interpreting results and drawing conclusions. In: Higgins JPT, Green S editor(s). Cochrane Handbook for Systematic Reviews of Interventions. The Cochrane Collaboration, 2008:359-83.

\section{Ukoumunne 1999}

Ukoumunne OC, Gulliford MC, Chinn S, Sterne JAC, Burney PGJ. Methods for evaluating area-wide and organisation-based intervention in health and health care: a systematic review. Health Technology Assessment 1999;3(5):1-75.

\section{Weiden 1994}

Weiden P, Rapkin B, Mott T, Zygmunt A, Goldman D, HorvitzLennon M, et al. Rating of medication influences (ROMI) scale in schizophrenia. Schizophrenia Bulletin 1994;20:297-310.

\section{Werman 1994}

Werman D. The Practice of Supportive Therapy. New York: Brunner, 1994.

\section{Xia 2009}

Xia J, Adams CE, Bhagat N, Bhagat V, Bhoopathi P, El-Sayeh H, et al. Loss to outcomes stakeholder survey: the LOSS study. Psychiatric Bulletin 2009;33(7):254-7.

\section{References to other published versions of this review Buckley 2007a}

Buckley LA, Pettit T. Supportive therapy for schizophrenia. Cochrane Database of Systematic Reviews 2007, Issue 1. [DOI: 10.1002/14651858.CD004716.pub2]

\section{Buckley 2007b}

Buckley LA, Pettit TACL, Adams CE. Supportive therapy for schizophrenia. Cochrane Database of Systematic Reviews 2007 Issue 3. [DOI: 10.1002/14651858.CD004716.pub3]

* Indicates the major publication for the study 
CHARACTERISTICS OF STUDIES

Characteristics of included studies [ordered by study ID]

Coyle 1988

Methods Allocation: stratified random assignment.

Blindness: therapists not aware of specific study hypothesis.

Duration: approximately 6 months.

Design: parallel.

Setting: outpatient.

Country: USA.

\begin{tabular}{|c|c|}
\hline Participants & $\begin{array}{l}\text { Diagnosis: schizophrenia. } \\
\mathrm{N}=94 \text {. } \\
\text { Age: mean } ~ 47 \text { years, range } 21-72 \text { years. } \\
\text { Sex: } 40 \mathrm{M}, 38 \mathrm{~F} \text { (data not reported for } 16 \text { participants). } \\
\text { History: long duration of illness. } \\
\text { Exclusions: a diagnosis of drug and/or alcohol abuse. }\end{array}$ \\
\hline Interventions & $\begin{array}{l}\text { 1. Individual supportive therapeutic method: dose } 10 \text { fortnightly } 90 \text {-minute sessions. } \mathrm{N}=24 \text {. } \\
\text { 2. Social skills training method. } \mathrm{N}=23 \text {. } \\
\text { 3. Psychoeducational method. } \mathrm{N}=23 \text {. } \\
\text { 4. Psychotropic medication alone. } \mathrm{N}=24 \text {. }\end{array}$ \\
\hline Outcomes & $\begin{array}{l}\text { Death. } \\
\text { Service outcomes: hospitalisation. } \\
\text { Leaving the study early. } \\
\text { Unable to use - } \\
\text { General functioning: KAS (no SD reported). }\end{array}$ \\
\hline
\end{tabular}

Notes

\section{Risk of bias}

\begin{tabular}{lll}
\hline Bias & Authors' judgement & Support for judgement \\
\hline $\begin{array}{l}\text { Random sequence genera- } \\
\text { tion (selection bias) }\end{array}$ & Unclear risk & $\begin{array}{l}\text { Stratified random assignment according to age, sex and race. No further de- } \\
\text { tails reported. }\end{array}$ \\
\hline $\begin{array}{l}\text { Allocation concealment } \\
\text { (selection bias) }\end{array}$ & Unclear risk & No information reported. \\
\hline $\begin{array}{l}\text { Blinding of participants } \\
\begin{array}{l}\text { and personnel (perfor- } \\
\text { mance bias) }\end{array}\end{array}$ & Unclear risk & No information reported. \\
All outcomes & \\
\hline
\end{tabular}

Blinding of outcome as- Unclear risk No information reported.

sessment (detection bias)

All outcomes

Incomplete outcome data Low risk

(attrition bias)

All outcomes

\begin{abstract}
"Out of the 94 subjects entering the study, 14 did not complete the treatment program. In the individual therapy method, one subject died of medical causes, one subject was hospitalised and one subject did not complete the data;
\end{abstract}


in the social skills training method, three subjects dropped out and one was hospitalised; in the psychoeducational method, four subjects dropped out and two were hospitalised; in the medication only group, one subject dropped out, one was hospitalised and one subject did not complete the data. In addition, there were two subjects who completed the program but a full data package could not be collected on them."
Selective reporting (reporting bias)
Reports results incompletely, no SD reported for KAS scale.

Other bias Unclear risk Not enough information provided to make a judgement.

Davidson 2004

$\begin{array}{ll}\text { Methods } & \text { Allocation: randomised. } \\ \text { Blinding: open-label. } & \text { Duration: } 9 \text { months. } \\ \text { Design: parallel. } \\ \text { Setting: outpatients. } \\ \text { Country: United States. }\end{array}$

Participants

Diagnosis: mood, anxiety, or schizophrenia-spectrum disorder; DSM-IIIR.

$N=260$.

Age: Mean 42 years (SD 11).

Sex: $112 \mathrm{M}, 148 \mathrm{~F}$

History: seriously ill, psychiatrically stable for the past 6 months (not hospitalised or institutionalised) and have moderate to severe impairments in social and occupational functioning.

Exclusions:

1. Supportive therapy with consumer partner (volunteer with similar psychiatric history who has recovered and has his/her social network) with shared interests, within the same age range and gender of the participant. $\mathrm{N}=95$.

2. Supportive therapy with non-consumer partner (volunteer without psychiatric history) with shared interests, within the same age range and gender of the participant. $\mathrm{N}=95$.

3. TAU: not matched with a partner. $\mathrm{N}=70$.

Participants in each treatment group were given a \$28 stipend each month.

$\begin{array}{ll}\text { Outcomes } & \text { Mental state: BPRS, CESD, GHQ. } \\ & \text { Global State: GAF. } \\ & \text { Quality of Life: RSES, WBS. } \\ & \text { General Functioning: SFS. } \\ & \text { Unable to use - } \\ & \text { Satisfaction with service: SWS ( scale not validated). }\end{array}$


Davidson 2004 (Continued)

Risk of bias

\begin{tabular}{|c|c|c|}
\hline Bias & Authors' judgement & Support for judgement \\
\hline $\begin{array}{l}\text { Random sequence genera- } \\
\text { tion (selection bias) }\end{array}$ & Unclear risk & "Randomly assigned", no further details reported. \\
\hline $\begin{array}{l}\text { Allocation concealment } \\
\text { (selection bias) }\end{array}$ & Unclear risk & No information reported. \\
\hline $\begin{array}{l}\text { Blinding of participants } \\
\text { and personnel (perfor- } \\
\text { mance bias) } \\
\text { All outcomes }\end{array}$ & Unclear risk & No information reported. \\
\hline $\begin{array}{l}\text { Blinding of outcome as- } \\
\text { sessment (detection bias) } \\
\text { All outcomes }\end{array}$ & Unclear risk & No information reported. \\
\hline $\begin{array}{l}\text { Incomplete outcome data } \\
\text { (attrition bias) } \\
\text { All outcomes }\end{array}$ & Unclear risk & Losses to follow-up not reported. \\
\hline $\begin{array}{l}\text { Selective reporting (re- } \\
\text { porting bias) }\end{array}$ & Low risk & All expected outcomes are reported. \\
\hline Other bias & Low risk & The study appears to be free of other sources of bias. \\
\hline
\end{tabular}

\section{Dincin 1982}

$\begin{array}{ll}\text { Methods } & \text { Allocation: randomised. } \\ \text { Blindness: none. } \\ \text { Duration: } 9 \text { months. } \\ \text { Design: parallel. } \\ \text { Setting: outpatient. } \\ \text { Country: USA. }\end{array}$

Participants Diagnosis: severely disturbed psychiatric patients (75\% schizophrenia) (DSM-II).

$\mathrm{N}=132$.

Age: mean 25 years.

Sex: 49M, 44F.

History: frequent referral diagnosis is schizophrenia; who are not primarily alcoholic, drug-dependent, or mentally retarded; and whose psychiatric histories include an average of three hospitalisations.

Exclusions: under 19 years old or attended fewer than three days during the first month after intake.

Interventions
2. Comprehensive treatment: dose $>6$ hours per week, full-time milieu rehabilitation programme. $\mathrm{N}=$
66 .
66.

\section{Outcomes}

Service outcomes: hospitalisation.

General functioning: gainful employment.

Unable to use - 
Dincin 1982 (Continued)

Service outcomes: time to hospitalisation and time spent in hospital (no SD reported).
Percentage of participants for whom no outcome data is available:

1. Supportive therapy $44 \%$.

2. Rehabilitation programme $30 \%$.

\section{Risk of bias}

\begin{tabular}{|c|c|c|}
\hline Bias & Authors' judgement & Support for judgement \\
\hline $\begin{array}{l}\text { Random sequence genera- } \\
\text { tion (selection bias) }\end{array}$ & Unclear risk & "Randomly assigned" no further details reported. \\
\hline $\begin{array}{l}\text { Allocation concealment } \\
\text { (selection bias) }\end{array}$ & Unclear risk & No information reported. \\
\hline $\begin{array}{l}\text { Blinding of participants } \\
\text { and personnel (perfor- } \\
\text { mance bias) } \\
\text { All outcomes }\end{array}$ & Unclear risk & No information reported. \\
\hline $\begin{array}{l}\text { Blinding of outcome as- } \\
\text { sessment (detection bias) } \\
\text { All outcomes }\end{array}$ & Unclear risk & No information reported. \\
\hline $\begin{array}{l}\text { Incomplete outcome data } \\
\text { (attrition bias) } \\
\text { All outcomes }\end{array}$ & Unclear risk & $\begin{array}{l}\text { "Unable to contact } 5 \text { subjects from the comprehensive treatment and } 14 \text { from } \\
\text { the supportive treatment programs. In the majority of these cases we recon- } \\
\text { structed rehospitalisation data by talking to reliable informants and corrobo- } \\
\text { rating their reports with hospital records. Thus we were able to obtain verified } \\
\text { outcome data for } 50 \text { of the } 51 \text { active subjects in the comprehensive treatment } \\
\text { group and for } 43 \text { of the } 51 \text { active subjects in the supportive treatment group." }\end{array}$ \\
\hline $\begin{array}{l}\text { Selective reporting (re- } \\
\text { porting bias) }\end{array}$ & High risk & $\begin{array}{l}\text { Reports results incompletely, no SDs reported for time to hospitalisation and } \\
\text { time spent in hospital. }\end{array}$ \\
\hline Other bias & Low risk & $\begin{array}{l}\text { "Research was supported by grant } 518 \text { from the Illinois Department of Mental } \\
\text { Health and Developmental Disabilities". }\end{array}$ \\
\hline
\end{tabular}

\section{Durham 2003}

\begin{tabular}{ll}
\hline Methods & Allocation: randomised permuted blocking. \\
Blindness: outcome assessors blind. \\
Duration: 9 months treatment plus 3 months follow-up. \\
Design: parallel. \\
Setting: Inpatient and outpatient. \\
Country: UK. \\
Diagnosis: schizophrenia or schizoaffective disorder or delusional disorder (DSM-IV and ICD-10). \\
N =66. \\
Age: mean 36 years. \\
Sex: $45 \mathrm{M}, 21 \mathrm{~F}$. \\
History: persistent and distressing hallucinations or delusions or both, stable on anti-psychotic med- \\
ication for at least 6 months, duration of illness mean 13 years, range 2-31 years.
\end{tabular}


Durham 2003 (Continued)

Exclusions: primary diagnosis of alcoholism or drug misuse, evidence of organic brain injury, history of violence,

Interventions

1. Supportive psychotherapy plus treatment as usual: psychodynamic in orientation, protocol-based, delivered by staff in a community mental health team, different therapists from cognitive behavioural therapy intervention, tape recordings blindly assessed for treatment adherence. $\mathrm{N}=23$.

2. Cognitive behavioural therapy plus treatment as usual: protocol-based, delivered by clinical nurse specialists, different therapists from supportive psychotherapy intervention, tape recordings blindly assessed for treatment adherence. $\mathrm{N}=22$.

3. Treatment as usual. $\mathrm{N}=21$.

$\begin{array}{ll}\text { Outcomes } & \text { Mental state: PANSS, PSYRATS - delusions score, PSYRATS - hallucinations score. } \\ \text { Global state: GAS. } \\ \text { Satisfaction with treatment: patients' attitude to treatment. } \\ \text { Unable to use - } \\ \text { Global state: self-rating of degree of improvement (results not reported by group). } \\ \text { Mental state: self-report measure of symptom severity (data not reported), self-report measure of self- } \\ \text { esteem (data not reported), self-report measure of attitude to illness (data not reported). }\end{array}$

Notes Percentage of participants for whom no outcome data is available:

1. Supportive therapy $17 \%$.

2. CBT $5 \%$.

3. Standard care $19 \%$.

\section{Risk of bias}

Bias Authors' judgement Support for judgement

Random sequence genera- Low risk tion (selection bias)

"Randomisation procedure (sealed envelope technique) was devised by the project statistician, and administered centrally by the non-clinical project coordinator, It was carried out separately within each treatment centre using randomised permuted blocking".

\begin{tabular}{ll}
\hline $\begin{array}{l}\text { Allocation concealment } \quad \text { Low risk } \\
\text { (selection bias) }\end{array}$ & $\begin{array}{l}\text { "Randomisation procedure (sealed envelope technique) was devised by the } \\
\text { project statistician, and administered centrally by the non-clinical project co- } \\
\text { ordinator". }\end{array}$ \\
\hline
\end{tabular}

\begin{tabular}{|c|c|c|}
\hline $\begin{array}{l}\text { Blinding of participants } \\
\text { and personnel (perfor- } \\
\text { mance bias) }\end{array}$ & Unclear risk & $\begin{array}{l}\text { Patients not blinded: "Patients also were asked not to mention any details of } \\
\text { their treatment during post-treatment assessments, but three patients did." } \\
\text { No details reported for personnel. }\end{array}$ \\
\hline
\end{tabular}

All outcomes

Unclear risk

Blinding of outcome as-

All outcomes
"Work of the independent assessors and therapists were kept strictly separate in order to maintain the blindness of the assessor".
Incomplete outcome data Low risk

(attrition bias)

All outcomes

\begin{abstract}
Losses to followup or missing data balanced across intervention groups, with similar reasons for missing data. Withdrew from treatment: 1 from CBT, 4 from SPT, 2 from TAU; died from natural causes: 1 from SPT; lost to follow-up: 1 from CBT, 4 from SPT, 4 from TAU.

"The analyses were repeated with the missing values replaced either with previous values carried forward or with group means."
\end{abstract}

\begin{tabular}{|c|c|c|}
\hline $\begin{array}{l}\text { Selective reporting (re- } \\
\text { porting bias) }\end{array}$ & High risk & $\begin{array}{l}\text { Reports results incompletely, no data reported for self-reported symptom } \\
\text { severity, self-esteem, and attitude to illness, results not reported by group for } \\
\text { self-rating of degree of improvement. }\end{array}$ \\
\hline
\end{tabular}


Durham 2003 (Continued)

Other bias Low risk The study appears to be free of other sources of bias.

\section{Eckman 1992}

Methods
Blindness: attempts made to keep outcome assessors blind, blindness broken in a few cases.
Duration: 18 months.
Design: parallel.
Setting: not reported.
Country: USA.

Diagnosis: schizophrenia (DSM-III-R).
$\mathrm{N}=41^{\star}$.
Age: mean 40 years.
Sex: all male.
History: veterans receiving constant maintenance neuroleptic drug therapy, and are able to tolerate
5-10 mg fluphenazine decanoate every other week.
Exclusions: evidence of mental retardation or organic brain syndrome, currently abusing alcohol or
drugs.

Interventions 1. Supportive group psychotherapy: dose twice-weekly 90 minute sessions for 6 months, then once weekly 90-minute sessions for 1 year; insight-oriented and supportive, information and education about schizophrenia provided, . $\mathrm{N}=20$.

2. Skills training group: dose twice-weekly 90 -minute sessions for 6 months, then once-weekly 90 minute sessions for a year. $\mathrm{N}=21$.

Leaving the study early.
Unable to use -
Mental state: BPRS (data not reported by group), SANS (data not reported by group).
General functioning: skill assessment by role play (SD not reported, scale not validated).

$\begin{array}{ll}\text { Notes } & \text { *number randomised not reported, data reported only for participants who completed at least } 6 \\ \text { months of psychosocial treatment and all pre- and post-treatment assessments. }\end{array}$
months of psychosocial treatment and all pre- and post-treatment assessments.

Percentage of participants for whom no outcome data is available:

1. Supportive therapy $43 \%$.

2. Skills training $25 \%$.

\section{Risk of bias}

\section{Bias}

Random sequence genera- Unclear risk tion (selection bias)

\section{Authors' judgement Support for judgement}

"Randomly assigned" no further details reported.

No information reported.

$\begin{aligned} & \text { Allocation concealment } \\ & \text { (selection bias) }\end{aligned}$ Unclear risk No information reported.


Eckman 1992 (Continued)
Blinding of outcome as-
Unclear risk
"Although attempts were made to keep the raters blind to the subjects' treat- sessment (detection bias) ment conditions, a few patients revealed this information".

All outcomes

\begin{tabular}{|c|c|c|}
\hline $\begin{array}{l}\text { Incomplete outcome data } \\
\text { (attrition bias) } \\
\text { All outcomes }\end{array}$ & Unclear risk & $\begin{array}{l}\text { "Over the full } 18 \text { month study period, more subjects in the skills training }(15 / 21) \\
\text { than in the supportive therapy }(12 / 21) \text { remained in the study". }\end{array}$ \\
\hline $\begin{array}{l}\text { Selective reporting (re- } \\
\text { porting bias) }\end{array}$ & High risk & $\begin{array}{l}\text { Reports results incompletely, data not reported by group for BPRS and SANS, } \\
\text { SD not reported for skills assessment. }\end{array}$ \\
\hline
\end{tabular}

\begin{tabular}{ll}
\hline Other bias $\quad$ Low risk & "Supported by Medical Research Service, US Department of Veterans Affair- \\
s and by NIMH grants".
\end{tabular}

\section{Falloon 1982}

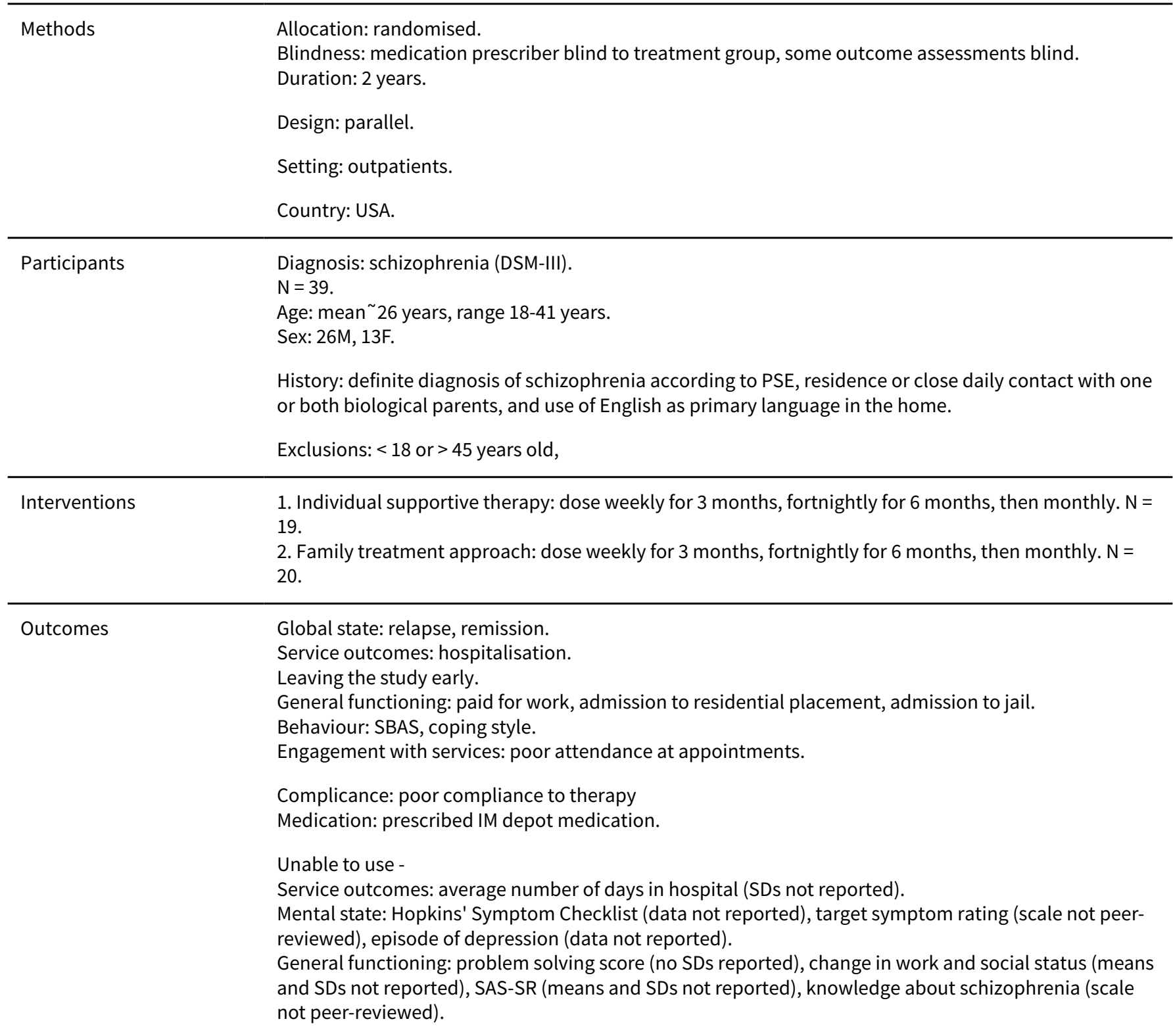


Falloon 1982 (Continued)

Family coping score (no SDs reported).

Antipsychotic drug dose (no SDs reported), antipsychotic drug plasma level (data not reported), co-ef-

ficient of variation in plasma drug level/prescribed dose ratio (data not reported).

Economic outcomes: direct costs (data not reported), indirect costs (data not reported).

Percentage of participants for whom no outcome data is available at 2 years:
1. Supportive psychotherapy $11 \%$.

2. Family therapy $15 \%$.

\section{Risk of bias}

\begin{tabular}{|c|c|c|}
\hline Bias & Authors' judgement & Support for judgement \\
\hline $\begin{array}{l}\text { Random sequence genera- } \\
\text { tion (selection bias) }\end{array}$ & Unclear risk & "Randomized", no further details reported. \\
\hline $\begin{array}{l}\text { Allocation concealment } \\
\text { (selection bias) }\end{array}$ & Unclear risk & No information reported. \\
\hline $\begin{array}{l}\text { Blinding of participants } \\
\text { and personnel (perfor- } \\
\text { mance bias) } \\
\text { All outcomes }\end{array}$ & Unclear risk & $\begin{array}{l}\text { "All patients were seen monthly at the clinic by a research psychiatrist or a } \\
\text { clinical pharmacist, who was blinded to the type of treatment and responsible } \\
\text { for managing the pharmacologic aspects of treatment." No details reported } \\
\text { about other staff. }\end{array}$ \\
\hline $\begin{array}{l}\text { Blinding of outcome as- } \\
\text { sessment (detection bias) } \\
\text { All outcomes }\end{array}$ & Low risk & $\begin{array}{l}\text { Outcomes either self-assessments or by blinded assessors. "The raters were } \\
\text { the prescribing doctors who were blinded to the assignment to treatment." }\end{array}$ \\
\hline $\begin{array}{l}\text { Incomplete outcome data } \\
\text { (attrition bias) } \\
\text { All outcomes }\end{array}$ & Low risk & $\begin{array}{l}\text { "Two patients receiving family therapy and one receiving individual therapy } \\
\text { withdrew from the study in the early stages of treatment". Patients who with- } \\
\text { drew were not analysed for any outcome. Losses to follow-up or missing data } \\
\text { balanced across intervention groups, with similar reasons for missing data. }\end{array}$ \\
\hline $\begin{array}{l}\text { Selective reporting (re- } \\
\text { porting bias) }\end{array}$ & High risk & $\begin{array}{l}\text { Reports results incompletely, data not reported for Hopkins' Symptom Check- } \\
\text { list and episode of depression, SDs not reported for average number of days in } \\
\text { hospital. }\end{array}$ \\
\hline Other bias & Low risk & Funded by an NIH grant. \\
\hline
\end{tabular}

Haddock 1999

$\begin{array}{ll}\text { Methods } & \text { Allocation: randomised. } \\ \text { Blindness: outcome assessor blind to treatment allocation. } \\ \text { Duration: } 5 \text { months treatment plus } 2 \text { years follow-up. } \\ \text { Design: parallel. } \\ \text { Setting: inpatient } \\ \text { Country: UK. } \\ \text { Diagnosis: schizophrenia or schizoaffective disorder (DSM-IV). } \\ \text { N = 21. } \\ \text { Age: mean } 29 \text { years } \\ \text { Sex: } 19 M, 2 F . \\ \text { History: first treatment for psychosis less than five years ago. Admitted to an acute general psychiatric } \\ \text { ward for psychotic symptoms at time of entry into study. }\end{array}$


Haddock 1999 (Continued)

Exclusions: not reported.

Interventions $\quad 1$. Supportive counselling and psychoeducation: manualised, delivered by therapists with expertise in CBT for psychosis who also delivered the CBT, supervised using tape recordings, therapy given during an inpatient stay over 5 weeks or shorter if the patient was discharged, then monthly booster sessions for 4 months. $\mathrm{N}=11$.

2. Cognitive-behavioural treatment: manualised, delivered by therapists with expertise in CBT for psychosis who also delivered the supportive therapy, supervised using tape recordings, therapy given during an inpatient stay over 5 weeks or shorter if the patient was discharged, then monthly booster sessions for 4 months. $\mathrm{N}=10$.

$\begin{array}{ll}\text { Outcomes } & \text { Global state: relapse. } \\ \text { Mental state: BPRS, PANSS. } \\ \text { Leaving the study early. } \\ \text { Unable to use - } \\ \text { Global state: number of relapses per participant (SDs not reported). } \\ \text { Mental state: PSYRATS (data not reported). } \\ \text { Not used in review - } \\ \text { Service outcomes: number of days in hospital before first discharge, tre } \\ \text { during 2-year study duration (not used in review). } \\ \text { Percentage of participants for whom no outcome data is available: } \\ \text { 1. Supportive therapy 0\%. } \\ \text { 2. CBT 10\%. }\end{array}$

\section{Risk of bias}

\begin{tabular}{lll}
\hline Bias & Authors' judgement & Support for judgement \\
\hline $\begin{array}{l}\text { Random sequence genera- } \\
\text { tion (selection bias) }\end{array}$ & Unclear risk & "Randomly allocated" no further details reported. \\
\hline $\begin{array}{l}\text { Allocation concealment } \\
\text { (selection bias) }\end{array}$ & Unclear risk & No information reported. \\
\hline $\begin{array}{l}\text { Blinding of participants } \\
\text { and personnel (perfor- } \\
\text { mance bias) }\end{array}$ & Unclear risk & No information reported. \\
$\begin{array}{l}\text { All outcomes } \\
\text { Blinding of outcome as- } \\
\text { sessment (detection bias) } \\
\text { All outcomes }\end{array}$ & Low risk & \\
\hline & $\begin{array}{l}\text { "Independent assessment using the BPRS and PSYRATS was carried out by the } \\
\text { project psychiatrist, who was blind to treatment allocation, and took place on } \\
\text { entry to the study, at end of treatment and following the final booster session } \\
\text { (4 months following discharge). Days in hospital for the initial episode and any } \\
\text { subsequent episodes, number of relapses, time to relapse of psychotic symp- } \\
\text { toms and time to first readmission were recorded for each patient from entry } \\
\text { to the study and for } 2 \text { years following entry to the study by a blind independent } \\
\text { assessor using patient case notes". }\end{array}$
\end{tabular}

$\begin{array}{ll}\text { Incomplete outcome data } & \text { Low risk } \\ \begin{array}{l}\text { (attrition bias) } \\ \text { All outcomes }\end{array} & \begin{array}{l}\text { "One patient in the CBT treatment withdrew from the study after three ses- } \\ \text { sions because he did not find it was helpful." Unclear if patient who withdrew } \\ \text { imputed into outcomes. }\end{array}\end{array}$

\begin{tabular}{|c|c|c|}
\hline $\begin{array}{l}\text { Selective reporting (re- } \\
\text { porting bias) }\end{array}$ & High risk & $\begin{array}{l}\text { Reports results incompletely, SDs not reported for global state: number of re- } \\
\text { lapses per participant; data not reported for mental state: PSYRATS. }\end{array}$ \\
\hline
\end{tabular}


Haddock 1999 (Continued)

Other bias Unclear risk Not enough information provided to make a judgement.

Hogarty 1997-study 1

\begin{tabular}{|c|c|}
\hline Methods & $\begin{array}{l}\text { Allocation: randomised. } \\
\text { Blindness: none. } \\
\text { Duration: } 3 \text { years. } \\
\text { Design: parallel. } \\
\text { Setting: outpatients. } \\
\text { Country: USA. }\end{array}$ \\
\hline Participants & $\begin{array}{l}\text { Diagnosis: schizophrenia or schizoaffective disorder (RDC). } \\
\mathrm{N}=97 . \\
\text { Age: mean } 29 \text { years, range } 16-55 \text { years. } \\
\text { Sex: } 56 \mathrm{M}, 41 \mathrm{~F} \text {. } \\
\text { History: living with family, IQ above } 75 \text {, absence of organic brain syndrome and serious alcohol or drug } \\
\text { abuse or dependence in the previous } 6 \text { months, recruited whilst in hospital, treated in the community. } \\
\text { Exclusions: Age }<16 \text {, or }>55 \text {; medical contraindications that precluded taking maintenance antipsy- } \\
\text { chotic medication. }\end{array}$ \\
\hline Interventions & $\begin{array}{l}\text { 1. Supportive therapy: biweekly, provided by the same therapists as personal therapy or family thera- } \\
\text { py, manualised, supervised. } N=24 \text {. } \\
\text { 2. Personal therapy: } C B T, 3 \text {-stage approach, sought to enhance personal and social adjustment } \\
\text { through the identification and effective management of affect dysregulation, manualised, weekly but } \\
\text { with less contact in year } 3 \text { for those who completed treatment objectives, provided by therapists who } \\
\text { also provided supportive therapy, but not family therapy. } N=23 \text {. } \\
\text { 3. Family therapy: manualised, biweekly, provided by therapists who also provided supportive therapy } \\
\text { but not personal therapy. } N=24 \text {. } \\
\text { 4. Personal therapy plus family therapy. } N=26 \text {. }\end{array}$ \\
\hline
\end{tabular}

Outcomes

Global state: relapse.

Mental state: episode of affective symptoms.

Leaving the study early: treatment-related reasons.

Unable to use -

Global state: GAS, Subjective Response Questionnaire (data not reported).

Mental state: BPRS, Raskin Depression Scale, Covi Anxiety Scale, Wing Negative Symptom Scale, Every-

day Worries Scale (data not reported).

General functioning: KAS, SAS II, Major Role Adjustment Inventory (data not reported).

Satisfaction with treatment (data not reported by group).

Notes

Percentage of participants for whom no outcome data is available:

1. Supportive psychotherapy $33 \%$.

2. CBT $4 \%$.

3. Family therapy $21 \%$.

4. CBT plus family therapy $4 \%$.

\section{Risk of bias}

Bias Authors' judgement Support for judgement

\footnotetext{
Random sequence genera- Unclear risk

"Randomly assigned" no further details reported. 
Hogarty 1997-study 1 (Continued)

\begin{tabular}{lll}
$\begin{array}{l}\text { Allocation concealment } \\
\text { (selection bias) }\end{array}$ & Unclear risk & No information reported. \\
\hline $\begin{array}{l}\text { Blinding of participants } \\
\text { and personnel (perfor- }\end{array}$ & Unclear risk & No information reported. \\
mance bias) & & \\
All outcomes &
\end{tabular}

\begin{tabular}{lll}
\hline $\begin{array}{l}\text { Blinding of outcome as- } \\
\text { sessment (detection bias) } \\
\text { All outcomes }\end{array}$ & High risk & $\begin{array}{l}\text { "The ratings of treating clinicians who were not blind to the treatment con- } \\
\text { ditions, thereby raising the question of rater bias", "results should be viewed } \\
\text { cautiously in the absence of independent and blind clinical assessors." }\end{array}$ \\
\hline $\begin{array}{l}\text { Incomplete outcome data } \\
\begin{array}{l}\text { (attrition bias) } \\
\text { All outcomes }\end{array}\end{array}$ & Low risk & $\begin{array}{l}8 \text { patients in the supportive therapy group had treatment-related termina- } \\
\text { tions, one in the personal therapy group, five in the family therapy group and } 1 \\
\text { in the combined personal and family therapy group. }\end{array}$
\end{tabular}

Selective reporting (re- High risk Reports results incompletely, data not reported for Global state: GAS, Subjecporting bias) tive Response Questionnaire; Mental state: BPRS, Raskin Depression Scale, Covi Anxiety Scale, Wing Negative Symptom Scale, Everyday Worries Scale; General functioning: KAS, SAS II, Major Role Adjustment Inventory; data not reported by group for Satisfaction with treatment.

Other bias Low risk "Supported by a MERIT extension of NIMH grant."

\section{Hogarty 1997-study 2}

\begin{tabular}{ll}
\hline Methods & Allocation: randomised. \\
Blindness: none. \\
Duration: 3 years. \\
Design: parallel. \\
Setting: outpatient. \\
Country: USA. \\
\hline
\end{tabular}

Diagnosis: schizophrenia or schizoaffective disorder (RDC).
P $=54$.
Age: mean $\sim 33$ years, $S D^{\sim} 8$ years, range $16-55$ years.
Sex: $24 \mathrm{M}, 30 \mathrm{~F}$.
History: living independent of family, IQ above 75 , absence of organic brain syndrome and serious al-
cohol or drug abuse or dependence in the previous 6 months, recruited whilst in hospital, treated in the
community.

Exclusions: Age $<16,>55$; medical contraindications that precluded taking maintenance antipsychotic medication.

Interventions
1. Supportive therapy: biweekly, provided by the same therapists as personal therapy or family thera-
2. Personal therapy: CBT, 3-stage approach, sought to enhance personal and social adjustment
through the identification and effective management of affect dysregulation, manualised, weekly but
with less contact in year 3 for those who completed treatment objectives, provided by therapists who
also provided supportive therapy, but not family therapy. $\mathrm{N}=25$.

Outcomes

Global state: relapse.

Mental state: episode of affective symptoms.

Leaving the study early: treatment-related reasons. 
Hogarty 1997-study 2 (Continued)

Unable to use -

Global state: GAS, Subjective Response Questionnaire (data not reported).

Mental state: BPRS, Raskin Depression Scale, Covi Anxiety Scale, Wing Negative Symptom Scale, Every-

day Worries Scale (data not reported).

General functioning: KAS, SAS II, Major Role Adjustment Inventory (data not reported).

Satisfaction with treatment (data not reported by group).

Notes Percentage of participants for whom no outcome data is available:

1. Supportive psychotherapy $17 \%$.

2. CBT $16 \%$.

\section{Risk of bias}

\begin{tabular}{|c|c|c|}
\hline Bias & Authors' judgement & Support for judgement \\
\hline $\begin{array}{l}\text { Random sequence genera- } \\
\text { tion (selection bias) }\end{array}$ & Unclear risk & "Randomly assigned" no further details reported. \\
\hline $\begin{array}{l}\text { Allocation concealment } \\
\text { (selection bias) }\end{array}$ & Unclear risk & No information reported. \\
\hline $\begin{array}{l}\text { Blinding of participants } \\
\text { and personnel (perfor- } \\
\text { mance bias) } \\
\text { All outcomes }\end{array}$ & Unclear risk & No information reported. \\
\hline $\begin{array}{l}\text { Blinding of outcome as- } \\
\text { sessment (detection bias) } \\
\text { All outcomes }\end{array}$ & High risk & $\begin{array}{l}\text { "The ratings of treating clinicians who were not blind to the treatment con- } \\
\text { ditions, thereby raising the question of rater bias", "results should be viewed } \\
\text { cautiously in the absence of independent and blind clinical assessors." }\end{array}$ \\
\hline $\begin{array}{l}\text { Incomplete outcome data } \\
\text { (attrition bias) } \\
\text { All outcomes }\end{array}$ & Low risk & $\begin{array}{l}\text { Five patients in the supportive therapy group and four in the personal therapy } \\
\text { group had treatment-related terminations. }\end{array}$ \\
\hline $\begin{array}{l}\text { Selective reporting (re- } \\
\text { porting bias) }\end{array}$ & High risk & $\begin{array}{l}\text { Reports results incompletely, data not reported for Global state: GAS, Sub- } \\
\text { jective Response Questionnaire; Mental state: BPRS, Raskin Depression Scale, } \\
\text { Covi Anxiety Scale, Wing Negative Symptom Scale, Everyday Worries Scale; } \\
\text { General functioning: KAS, SAS II, Major Role Adjustment Inventory; data not re- } \\
\text { ported by group for Satisfaction with treatment. }\end{array}$ \\
\hline Other bias & Low risk & "Supported by a MERIT extension of NIMH grant." \\
\hline
\end{tabular}

Kemp 1996

$\begin{array}{ll}\text { Methods } & \text { Allocation: randomised. } \\ \text { Blindness: some outcome assessments blind. } \\ \text { Duration: } 18 \text { months follow-up. } \\ \text { Design: parallel. } \\ \text { Setting: outpatients. } \\ \text { Country: UK. } \\ \text { Diagnosis: schizophrenia or severe affective disorder or schizophreniform disorder or schizoaffective } \\ \text { disorder or delusional disorder or psychotic disorder not otherwise classified (DSM-III-R). } \\ \text { N }=74 .\end{array}$


Kemp 1996 (Continued)

Age: mean 36 years.

Sex: 39M, 35F.

History: no people with low IQ, no deafness, no organic brain disease, no non-English speakers, hospitalised.

Interventions
sions, counselling non-specific, concerns listened to but therapists declined to discuss treatment. $\mathrm{N}=$
35.
2. Compliance therapy: dose $4-6$ sessions plus 3 booster sessions of cognitive behavioural and motiva-
tional interviewing techniques. $\mathrm{N}=39$.

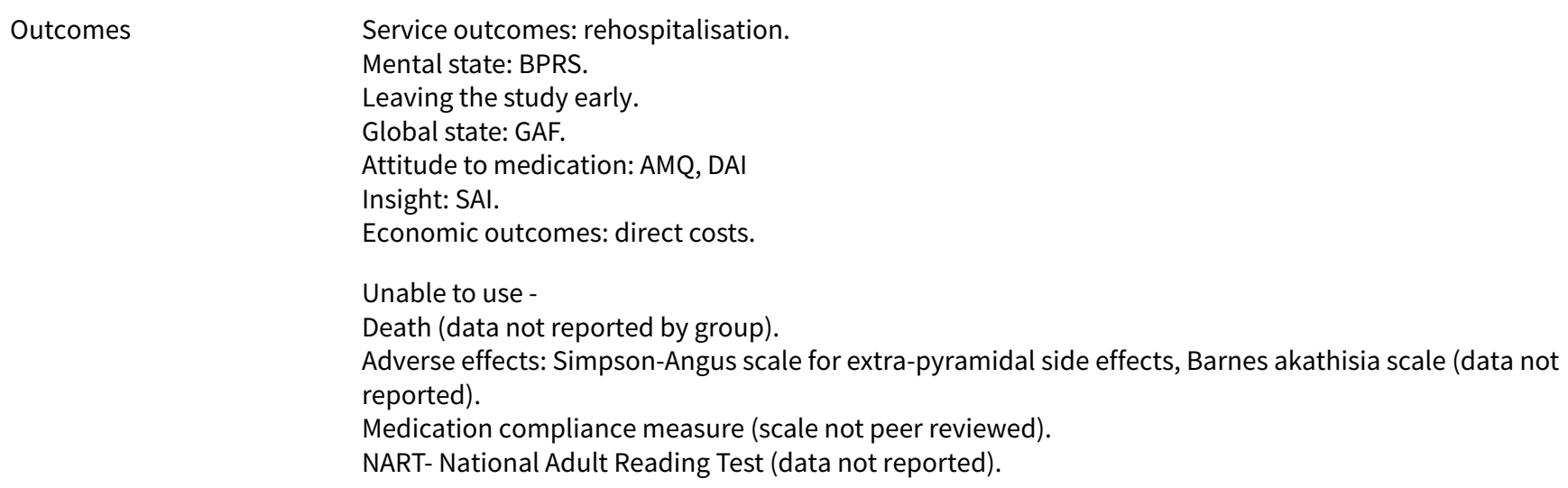

Notes

Percentage of participants for whom no outcome data is available:

1. Supportive therapy $43 \%$.

2. CBT $28 \%$.

\section{Risk of bias}

\begin{tabular}{|c|c|c|}
\hline Bias & Authors' judgement & Support for judgement \\
\hline $\begin{array}{l}\text { Random sequence genera- } \\
\text { tion (selection bias) }\end{array}$ & Low risk & $\begin{array}{l}\text { "Randomly assigned by means of a table of random numbers to compliance } \\
\text { therapy or control treatment" }\end{array}$ \\
\hline $\begin{array}{l}\text { Allocation concealment } \\
\text { (selection bias) }\end{array}$ & Unclear risk & No information reported. \\
\hline $\begin{array}{l}\text { Blinding of participants } \\
\text { and personnel (perfor- } \\
\text { mance bias) } \\
\text { All outcomes }\end{array}$ & Unclear risk & No information reported. \\
\hline $\begin{array}{l}\text { Blinding of outcome as- } \\
\text { sessment (detection bias) } \\
\text { All outcomes }\end{array}$ & Unclear risk & $\begin{array}{l}\text { "Initial compliance was rated blind to intervention by the patients' prima- } \\
\text { ry nurses,"; "The evaluations at six months were carried out by an indepen- } \\
\text { dent assessor (a community psychiatric nurse) blind to intervention."; "Most } \\
\text { of the ratings of functioning and compliance initially and at three months were } \\
\text { made by a research psychiatrist, who was not blind to intervention group. } \\
\text { However, the compliance ratings were based on information from impartial } \\
\text { sources, including community psychiatric nurses and outpatient doctors on } \\
\text { the clinical teams. By contrast, the ratings at six months were carried out by a } \\
\text { researcher trained in the use of all the ratings, who was blind to intervention } \\
\text { group." Some outcome assessors blinded, except third month assessment. }\end{array}$ \\
\hline
\end{tabular}

\begin{tabular}{|c|c|c|}
\hline $\begin{array}{l}\text { Incomplete outcome data } \\
\text { (attrition bias) } \\
\text { All outcomes }\end{array}$ & Low risk & $\begin{array}{l}\text { "Overall, } 35 \% \text { were lost during the follow-up period ( } 11(28 \%) \text { from the com- } \\
\text { pliance therapy group and } 15 \text { from. the control group ( } 43 \%) \text {; Ten participants } \\
\text { were lost during the first six months of follow-up, five from each group; Sub- } \\
\text { sequently, a further } 14 \text { could not be directly re-interviewed at } 12 \text {-month fol- }\end{array}$ \\
\hline
\end{tabular}


low-up, Post-treatment drop-out occurred due to refusal (11), patients either being uncontactable (7) or moving out of the area (2) or recovered patients who were working or at college and no longer having psychiatric out-patient treatment (2). One patient was in prison, There were two deaths, one resulting from stroke, and one from myocardial infarction". Details reported for imputing missing data by method of maximum likelihood.

Selective reporting (re- High risk
porting bias)

Reports results incompletely, data not reported for adverse effects: Simpson-Angus scale for extra-pyramidal side effects, Barnes akathisia scale, NART; data not reported by group for death; scale not peer reviewed for medication compliance measure.

Other bias Low risk Funding from the Medical Research Council.

\section{Klein 1998}

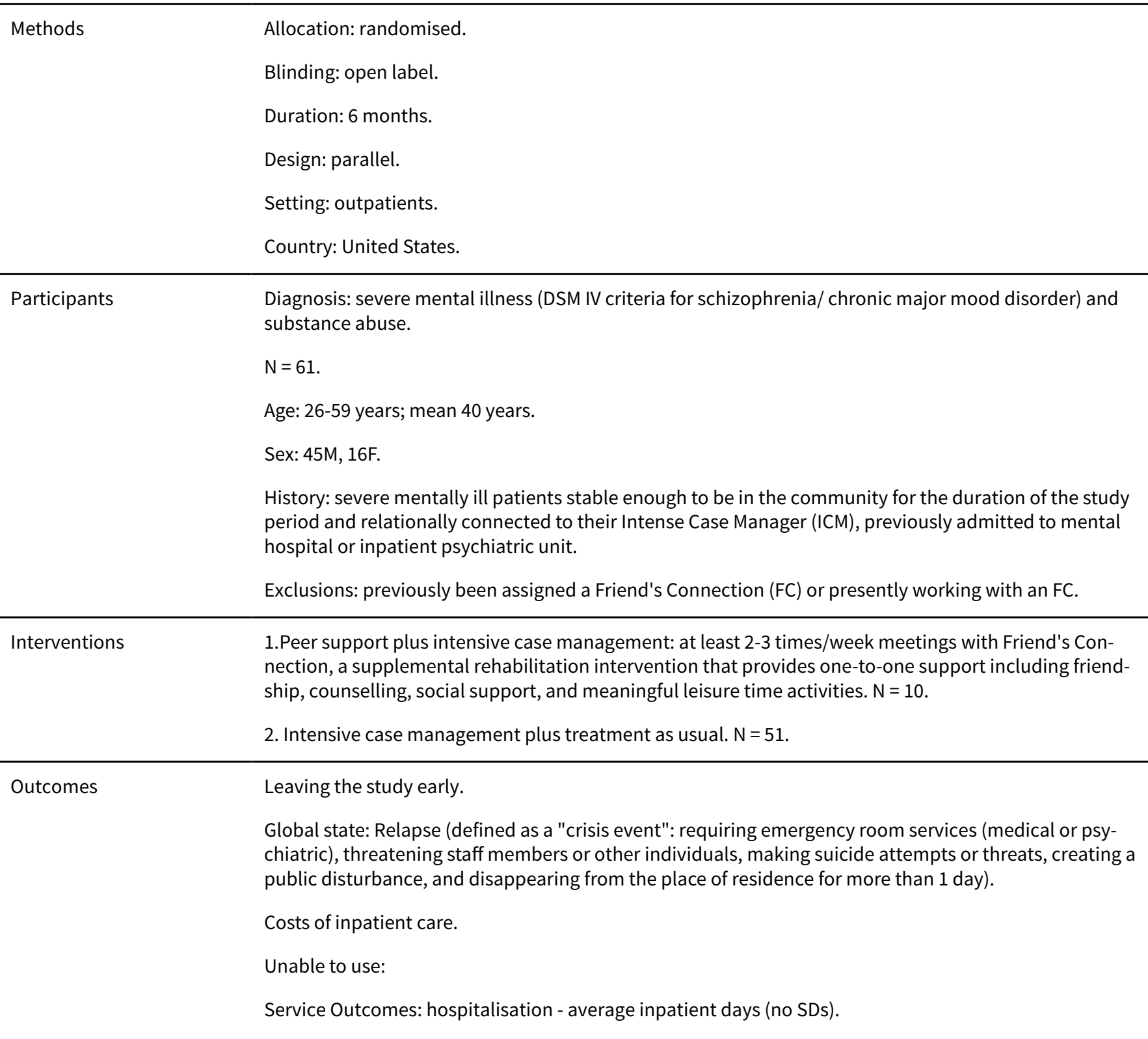


Klein 1998 (Continued)

Quality of life: Lehman's Quality of Life Questionnaire (no SDs).

Global state: GAF, average social interaction per week (no SDs).

Activities of daily living - mean frequency of activities (no SDs).

Costs - use of community resources (no data).

Notes

2 alternates were randomly selected for each of the 10 clients. $9 / 10$ in the study sample were alternates. The 9 clients originally selected for the sample refused to participate and were included in the comparison group.

\section{Risk of bias}

\begin{tabular}{|c|c|c|}
\hline Bias & Authors' judgement & Support for judgement \\
\hline $\begin{array}{l}\text { Random sequence genera- } \\
\text { tion (selection bias) }\end{array}$ & Unclear risk & $\begin{array}{l}\text { "The study sample was randomly selected from ICM caseload lists...The re- } \\
\text { maining } 51 \text { clients in the study population served as a comparison group", no } \\
\text { further details reported. }\end{array}$ \\
\hline $\begin{array}{l}\text { Allocation concealment } \\
\text { (selection bias) }\end{array}$ & Unclear risk & No information reported. \\
\hline $\begin{array}{l}\text { Blinding of participants } \\
\text { and personnel (perfor- } \\
\text { mance bias) } \\
\text { All outcomes }\end{array}$ & Unclear risk & No information reported. \\
\hline $\begin{array}{l}\text { Blinding of outcome as- } \\
\text { sessment (detection bias) } \\
\text { All outcomes }\end{array}$ & Unclear risk & No information reported. \\
\hline $\begin{array}{l}\text { Incomplete outcome data } \\
\text { (attrition bias) } \\
\text { All outcomes }\end{array}$ & Low risk & $\begin{array}{l}\text { "One participant who originally consented to the study and was assigned an } \\
\text { FC withdrew after a few weeks. We did not replace her as she was already part } \\
\text { of the study group, and all analyses include her". }\end{array}$ \\
\hline $\begin{array}{l}\text { Selective reporting (re- } \\
\text { porting bias) }\end{array}$ & High risk & $\begin{array}{l}\text { The study did not report SDs for hospitalisation, quality of life, activities of dai- } \\
\text { ly living and social functioning. No data were reported for use of community } \\
\text { resources. }\end{array}$ \\
\hline Other bias & Low risk & Appears free of other sources of bias. \\
\hline
\end{tabular}

Levine 1998

\begin{tabular}{ll}
\hline Methods & Allocation: randomised. \\
Blindness: outcome assessment blind. \\
Duration: 6 weeks treatment plus 4 weeks follow-up. \\
Design: parallel. \\
Setting: not reported. \\
Country: Israel.
\end{tabular}

Participants Diagnosis: schizophrenia (DSM-III-R).

$\mathrm{N}=12$.

Age: mean 35 years, range $24-42$ years.

Sex: not reported. 
Levine 1998 (Continued)

History: mean duration of illness ${ }^{2} 11$ years, range 6-20 years, 8 years of schooling or more, active delusional system, no change of antipsychotic drugs given in standard doses in the last 3 months

Exclusions: alcohol and/or drug abuse, chronic physical condition, orthodox (Jewish) religious conviction.

Interventions
cussion to delusional experiences. $\mathrm{N}=6$.
2. Cognitive-dissonance group therapy: dose weekly for 6 weeks plus one follow-up session; modified
cognitive therapy. $\mathrm{N}=6$.

Outcomes Mental state: PANSS.

\section{Notes}

\section{Risk of bias}

\begin{tabular}{lll}
\hline Bias & Authors' judgement & Support for judgement \\
\hline $\begin{array}{l}\text { Random sequence genera- } \\
\text { tion (selection bias) }\end{array}$ & Unclear risk & "Randomly assigned" . No further details reported. \\
\hline $\begin{array}{l}\text { Allocation concealment } \\
\text { (selection bias) }\end{array}$ & Unclear risk & No information reported. \\
\hline $\begin{array}{l}\text { Blinding of participants } \\
\text { and personnel (perfor- } \\
\text { mance bias) }\end{array}$ & Unclear risk & No information reported. \\
$\begin{array}{l}\text { All outcomes } \\
\text { Blinding of outcome as- } \\
\text { sessment (detection bias) } \\
\text { All outcomes }\end{array}$ & Low risk & "The PANSS scale was scored by an independent, board certified, senior psy- \\
\hline $\begin{array}{l}\text { Incomplete outcome data } \\
\text { (attrition bias) } \\
\text { All outcomes }\end{array}$ & Unclear risk & the group assignment of the subjects." No further details reported. \\
\hline $\begin{array}{l}\text { Selective reporting (re- } \\
\text { porting bias) }\end{array}$ & High risk & Losses to follow-up not reported. \\
\hline \begin{tabular}{l} 
Other bias \\
\hline
\end{tabular} & Unclear risk & Not enough information provided to make a judgement. \\
\hline
\end{tabular}

Lewis $2002 b$

\begin{tabular}{ll}
\hline Methods & Allocation: randomised with stratification. \\
Blindness: outcome assessment blind. \\
Duration: 5 weeks therapy plus 5 weeks follow-up. \\
Design: parallel. \\
Setting: inpatients \& outpatients. \\
Country: UK. \\
\hline Participants \\
$\mathrm{N}=315^{\star}$.
\end{tabular}


Lewis 2002b (Continued)

Age: median $^{2} 27$ years.

Sex: 216M, 93F.

History: inpatients or day patients, $83 \%$ in first admission and $17 \%$ in second admission, positive psychotic symptoms for 4 weeks or more, moderate or severe score on PANSS for delusions or hallucinations, neither substance misuse nor organic disorder judged to be the major cause of psychotic symptoms.

Exclusions: Substance misuse or organic disorder judged to be the major cause of psychotic symptoms. Patients legally detained in hospital.

Interventions
sions; manualised, supervised, audio taped sessions blindly rated for treatment fidelity and quality,
provided by therapists trained in CBT in psychosis who also provided the CBT intervention. $\mathrm{N}=106$.
2. Cognitive- behavioural therapy plus routine care: dose $15-20$ hours of therapy within 5 weeks plus
3 booster sessions; manualised, supervised, audio taped sessions blindly rated for treatment fidelity
and quality, provided by therapists trained in CBT in psychosis who also provided the supportive coun-
selling intervention. $\mathrm{N}=101$.
3. Routine care: not standardised. $\mathrm{N}=102$.

\begin{tabular}{ll}
\hline Outcomes & Mental state: PANSS, PSYRATS. \\
& Leaving the study early. \\
\hline Notes & *Seven participants excluded after randomisation. \\
& Percentage of participants for whom no outcome data is available: \\
& 1. Supportive psychotherapy $17 \%$. \\
& 2. CBT $5 \%$. \\
3. Treatment as usual $19 \%$.
\end{tabular}

\section{Risk of bias}

\begin{tabular}{lll}
\hline Bias & Authors' judgement & Support for judgement \\
\hline $\begin{array}{l}\text { Random sequence genera- } \\
\text { tion (selection bias) }\end{array}$ & Low risk & $\begin{array}{l}\text { "Independent, concealed, randomisation of individuals with minimisation was } \\
\text { then performed by trial administrator at each centre. Stratification was under- } \\
\text { taken with variables". }\end{array}$ \\
\hline
\end{tabular}

\begin{tabular}{ll}
\hline $\begin{array}{l}\text { Allocation concealment } \\
\text { (selection bias) }\end{array}$ & Low risk \\
& $\begin{array}{l}\text { "independent, concealed, randomisation of individuals with minimisation was } \\
\text { then performed by trial administrator at each centre. Stratification was under- } \\
\text { taken with variables" }\end{array}$
\end{tabular}

\begin{tabular}{ll}
\hline $\begin{array}{l}\text { Blinding of participants } \\
\text { and personnel (perfor- }\end{array}$ & Low risk \\
manterventions carried out independently of clinical staff who were kept un- \\
aware of treatment allocation."
\end{tabular}
mance bias)

All outcomes

\begin{tabular}{|c|c|c|}
\hline $\begin{array}{l}\text { Blinding of outcome as- } \\
\text { sessment (detection bias) } \\
\text { All outcomes }\end{array}$ & Low risk & $\begin{array}{l}\text { "All outcome assessments were made blind to treatment allocation. Extensive } \\
\text { steps were taken to maintain blindness of raters. In all cases randomisation } \\
\text { was carried out by a trial administrator independently of rater or therapist. } \\
\text { Therapist and rater were not to communicate details about individual patients } \\
\text { to each other. Office space and data storage were kept separate and secure. } \\
\text { Clinical staff were instructed not to divulge details of therapist contacts to the } \\
\text { raters." }\end{array}$ \\
\hline
\end{tabular}

\begin{tabular}{ll}
\hline $\begin{array}{l}\text { Incomplete outcome data } \\
\text { (attrition bias) }\end{array}$ & "Fow risk \\
All outcomes & consent to participate during the follow-up period, but are included in the \\
& analysis prior to their withdrawal; 13 of these withdrawals occurred during \\
& the first 2 weeks. One patient died during the follow-up period (in the support- \\
& ive counselling group). "Analyses were conducted on an intention-to-treat
\end{tabular}


Lewis 2002b (Continued)

basis, with patients analysed in the treatment group to which they were randomised"

\begin{tabular}{|c|c|c|}
\hline $\begin{array}{l}\text { Selective reporting (re- } \\
\text { porting bias) }\end{array}$ & Low risk & All expected outcomes are reported \\
\hline Other bias & Low risk & $\begin{array}{l}\text { The trial was funded as follows: UK Medical Research Council (41\%): North- } \\
\text { west England NHSE Office ( } 27 \%) \text {; Trent NHSE Office (7\%); the following health } \\
\text { authorities: Manchester (8\%); Salford and Trafford (2\%); Liverpool (3\%); Sefton } \\
(3 \%) \text {; St Helens and Knowsley (3\%); North Nottinghamshire (6\%). }\end{array}$ \\
\hline
\end{tabular}

\section{Malm 1982}

\begin{tabular}{|c|c|}
\hline Methods & $\begin{array}{l}\text { Allocation: randomised. } \\
\text { Blindness: none. } \\
\text { Duration: } 12 \text { months treatment plus } 12 \text { months follow-up. } \\
\text { Design: parallel. } \\
\text { Setting: inpatients \& outpatients. } \\
\text { Country: Sweden. }\end{array}$ \\
\hline Participants & $\begin{array}{l}\text { Diagnosis: schizophrenic psychosis. } \\
\mathrm{N}=80 \text {. } \\
\text { Age: } 18-50 \text { years. } \\
\text { Sex: } 43 \mathrm{M}, 25 \mathrm{~F} \text {. } \\
\text { History: total period of hospitalisation less than } 3 \text { years, no organic brain damage, no serious somatic } \\
\text { illness, IQ > } 70 \text {. } \\
\text { Exclusions: Gross organic brain damage, serious somatic illness, epilepsy or pathological EEG (which } \\
\text { motivated treatment with antiepileptic drugs), narcotic drug addictions, dependence on alcohol, } \\
\text { known sex chromosome aberration, mental retardation (IQ < } 701 \text {, and previous social skills training or } \\
\text { group therapy, patients who recovered after the first month of treatment with neuroleptics alone. }\end{array}$ \\
\hline Interventions & $\begin{array}{l}\text { 1. Group therapy plus fluphenazine depot plus social skills training: dose weekly sessions of } 60-90 \mathrm{~min}- \\
\text { utes, group therapy lasting } 1 \text { year; therapists not formally trained, manualised and supervised, commu- } \\
\text { nication-oriented. } \mathrm{N}=40 \text {. } \\
\text { 2. Fluphenazine depot plus social skills training: dose } 10 \text { hours per week of individual and group train- } \\
\text { ing. } \mathrm{N}=40 \text {. }\end{array}$ \\
\hline Outcomes & $\begin{array}{l}\text { Death. } \\
\text { Global state: relapse, no remission. } \\
\text { Service outcomes: not discharged from hospital. } \\
\text { Mental state: CPRS - inability to feel and derealisation items. } \\
\text { Leaving the study early. } \\
\text { General functioning: KAS - free time activities subscale and withdrawal syndrome. } \\
\text { Unable to use - } \\
\text { Mental state: CPRS - global rating of illness, all items except derealisation and inability to feel (data not } \\
\text { reported). } \\
\text { General functioning: KAS - socially expected activities subscale, offensive behaviour syndrome and } \\
\text { work and study item (data not reported), life events (data not reported). }\end{array}$ \\
\hline Notes & $\begin{array}{l}\text { Percentage of participants for whom no outcome data is available: } \\
\text { 1. Group therapy plus fluphenazine depot plus social skills training 15\%. } \\
\text { 2. Fluphenazine depot plus social skills training } 15 \%\end{array}$ \\
\hline
\end{tabular}


Malm 1982 (Continued)

Risk of bias

\begin{tabular}{|c|c|c|}
\hline Bias & Authors' judgement & Support for judgement \\
\hline $\begin{array}{l}\text { Random sequence genera- } \\
\text { tion (selection bias) }\end{array}$ & Unclear risk & $\begin{array}{l}\text { "By randomisation they were allocated to the therapy or the control group.", } \\
\text { stratified randomisation based on sex. }\end{array}$ \\
\hline $\begin{array}{l}\text { Allocation concealment } \\
\text { (selection bias) }\end{array}$ & Unclear risk & No information reported. \\
\hline $\begin{array}{l}\text { Blinding of participants } \\
\text { and personnel (perfor- } \\
\text { mance bias) } \\
\text { All outcomes }\end{array}$ & Unclear risk & No information reported. \\
\hline $\begin{array}{l}\text { Blinding of outcome as- } \\
\text { sessment (detection bias) } \\
\text { All outcomes }\end{array}$ & Unclear risk & $\begin{array}{l}\text { "Ratings agreed closely with those of the independent blind raters." Raters } \\
\text { that were blinded not specified, "There were } 7 \text { independent raters, } 5 \text { physi- } \\
\text { cians and } 2 \text { psychologists." }\end{array}$ \\
\hline $\begin{array}{l}\text { Incomplete outcome data } \\
\text { (attrition bias) } \\
\text { All outcomes }\end{array}$ & Low risk & $\begin{array}{l}\text { "Six patients in the therapy group and six in the control group either did not } \\
\text { start or did not complete the study" Details of dropouts further reported in ta- } \\
\text { ble. Dropouts not included in analysis. }\end{array}$ \\
\hline $\begin{array}{l}\text { Selective reporting (re- } \\
\text { porting bias) }\end{array}$ & High risk & $\begin{array}{l}\text { Reports results incompletely, data not reported for CPRS - global rating of ill- } \\
\text { ness, all items except derealisation and inability to feel; KAS - socially expected } \\
\text { activities subscale, offensive behaviour syndrome and work and study item }\end{array}$ \\
\hline Other bias & Low risk & $\begin{array}{l}\text { The study appears to be free of other sources of bias. The study has been sup- } \\
\text { ported by grants from the Einar Hansen Fund for Research, the Leo Research } \\
\text { Fund, the Lundbeck Foundation and the Stiftelsen Psykiatriska Forsknings- } \\
\text { fonden, Goteborg. }\end{array}$ \\
\hline
\end{tabular}

O'Donnell 1999

\begin{tabular}{|c|c|}
\hline \multirow[t]{4}{*}{ Methods } & $\begin{array}{l}\text { Allocation: randomised. } \\
\text { Blindness: none. } \\
\text { Duration: } 12 \text { months. }\end{array}$ \\
\hline & Design: parallel. \\
\hline & Setting: inpatients \& outpatients. \\
\hline & Country: Australia. \\
\hline \multirow[t]{2}{*}{ Participants } & $\begin{array}{l}\text { Diagnosis: schizophrenia (79) or schizoaffective disorder (19) or schizophreniform disorder (7) or bipo- } \\
\text { lar affective disorder (14) (DSM-IV). } \\
\mathrm{N}=119 . \\
\text { Age: mean } 36 \text { years. } \\
\text { Sex: } 68 \mathrm{M}, 51 \mathrm{~F} \text {. } \\
\text { History: English-speaking, mean duration of illness }{ }^{\sim} 10 \text { years, referred for case management by com- } \\
\text { munity health services. }\end{array}$ \\
\hline & $\begin{array}{l}\text { Exclusions: Age }<18 \text { or }>65 \text { years, co-diagnosis substance dependence disorder, no significant history of } \\
\text { violence unless associated with acute psychosis, no extant community treatment order or community } \\
\text { counselling order. }\end{array}$ \\
\hline
\end{tabular}


O'Donnell 1999 (Continued)

Interventions
1. Advocacy plus client-focused case management: advocacy provided by people who had experienced mental illness themselves, siblings or carers of people who had experienced mental illness and nonconsumers interested in working with people with mental illness. $\mathrm{N}=45$.

2. Client-focused case management. $\mathrm{N}=39$.

3. Standard case management. $\mathrm{N}=35$.

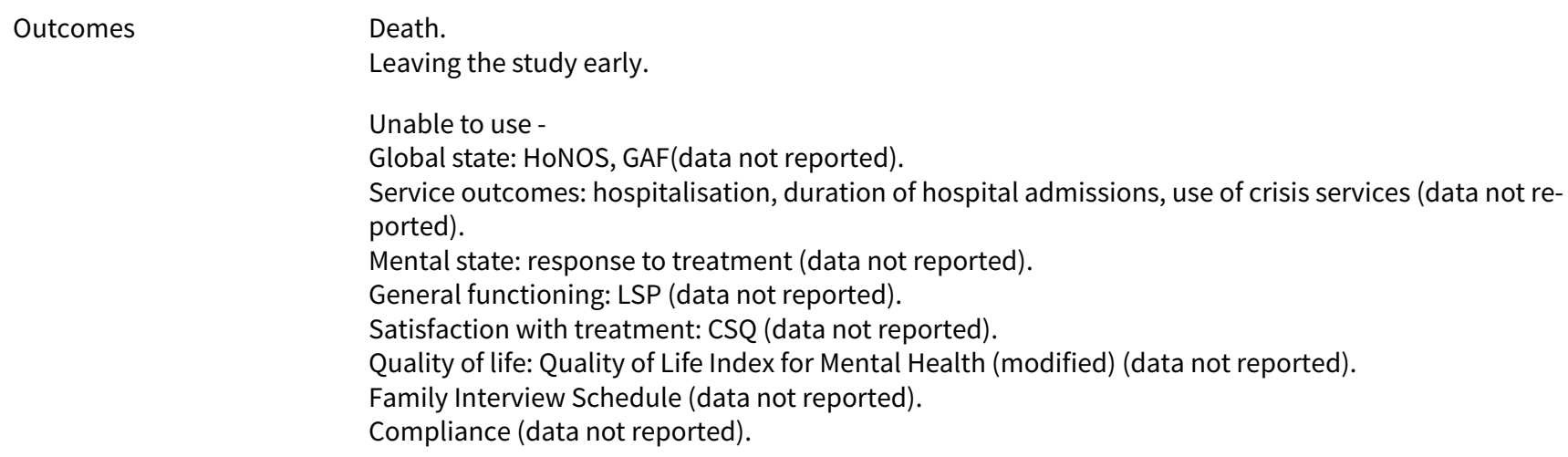

2. Client-focused case management $18 \%$.

3. Standard case management $43 \%$.

\section{Risk of bias}

\begin{tabular}{|c|c|c|}
\hline Bias & Authors' judgement & Support for judgement \\
\hline $\begin{array}{l}\text { Random sequence genera- } \\
\text { tion (selection bias) }\end{array}$ & Unclear risk & "Randomly allocated" . No further details reported. \\
\hline $\begin{array}{l}\text { Allocation concealment } \\
\text { (selection bias) }\end{array}$ & Unclear risk & No information reported. \\
\hline $\begin{array}{l}\text { Blinding of participants } \\
\text { and personnel (perfor- } \\
\text { mance bias) } \\
\text { All outcomes }\end{array}$ & Unclear risk & No information reported. \\
\hline $\begin{array}{l}\text { Blinding of outcome as- } \\
\text { sessment (detection bias) } \\
\text { All outcomes }\end{array}$ & Unclear risk & No information reported. \\
\hline $\begin{array}{l}\text { Incomplete outcome data } \\
\text { (attrition bias) } \\
\text { All outcomes }\end{array}$ & Low risk & $\begin{array}{l}15 \text { participants from the standard case management group left the study early, } \\
7 \text { from the client-focused case management group, } 18 \text { from the client-focused } \\
\text { case management plus advocate group. Reasons similar across groups. }\end{array}$ \\
\hline $\begin{array}{l}\text { Selective reporting (re- } \\
\text { porting bias) }\end{array}$ & High risk & $\begin{array}{l}\text { Reports results incompletely, data not reported for global state: HoNOS, gen- } \\
\text { eral functioning; service outcomes: hospitalisation, duration of hospital ad- } \\
\text { missions, use of crisis services, mental state: response to treatment; satisfac- } \\
\text { tion with treatment; quality of life; Family Interview Schedule and compliance. }\end{array}$ \\
\hline Other bias & Low risk & $\begin{array}{l}\text { "Commonwealth Innovative Grants Program of the National Mental Health } \\
\text { Strategy (Grant No. 22004) for funding this study" }\end{array}$ \\
\hline
\end{tabular}


Penn 2009

Methods $\quad$ Allocation: randomised.
Blinding: single.
Duration: 12 weeks.
Design: parallel.
Setting: outpatients.
Country: United States.

\begin{tabular}{|c|c|c|}
\hline \multirow[t]{7}{*}{ Participants } & \multirow{2}{*}{\multicolumn{2}{|c|}{ Diagnosis: schizophrenia or schizoaffective disorder; DSM IV criteria. }} \\
\hline & & \\
\hline & \multicolumn{2}{|l|}{ Age: $18-65}$. \\
\hline & \multicolumn{2}{|l|}{ Sex: 33M, 32F. } \\
\hline & \multicolumn{2}{|c|}{$\begin{array}{l}\text { History: 1)diagnosis of schizophrenia or schizoaffective disorder; } 2 \text { ) age } 18-65 \text { years; } 3 \text { ) presence of cur- } \\
\text { rent persistent auditory hallucinations of at least moderate severity (i.e. }\end{array}$} \\
\hline & \multicolumn{2}{|c|}{$\begin{array}{l}\text { had a rating of at least } 4 \text { on the Positive and Negative Syndrome Scale) (PANSS); 4) have undergone at } \\
\text { least two pharmacological trials, one of which being an atypical neuroleptic or clozapine for } 8 \text { weeks } \\
\text { prior to randomisation. }\end{array}$} \\
\hline & \multicolumn{2}{|c|}{$\begin{array}{l}\text { Exclusions: 1) mental retardation (based on both IQ and functional impairment criteria) 2) current sub- } \\
\text { stance dependence. }\end{array}$} \\
\hline \multirow[t]{2}{*}{ Interventions } & \multirow{2}{*}{\multicolumn{2}{|c|}{$\begin{array}{l}\text { 1. Cognitive-behavioural therapy (CBT). } \mathrm{N}=32 \text {. } \\
\text { 2. Enhanced supportive therapy (ST). } \mathrm{N}=33 \text {. }\end{array}$}} \\
\hline & & \\
\hline \multirow[t]{8}{*}{ Outcomes } & \multicolumn{2}{|l|}{ Leaving the study early. } \\
\hline & \multicolumn{2}{|c|}{ Service use: hospitalisation. } \\
\hline & \multicolumn{2}{|c|}{ Mental state: BVAQ-R, BDI-II, PSYRATS voices scale. } \\
\hline & \multicolumn{2}{|c|}{ General functioning: SFS. } \\
\hline & \multicolumn{2}{|l|}{ Insight: BCIS. } \\
\hline & \multicolumn{2}{|l|}{ Quality of life: RSES. } \\
\hline & \multicolumn{2}{|l|}{ Not used in review - } \\
\hline & \multicolumn{2}{|c|}{ Average number of days hospitalised. } \\
\hline \multicolumn{3}{|l|}{ Notes } \\
\hline \multicolumn{3}{|l|}{ Risk of bias } \\
\hline Bias & Authors' judgement & Support for judgement \\
\hline $\begin{array}{l}\text { Random sequence genera- } \\
\text { tion (selection bias) }\end{array}$ & Low risk & $\begin{array}{l}\text { "Randomisation was stratified by gender to ensure equal numbers across } \\
\text { groups using a computer randomisation generator". }\end{array}$ \\
\hline $\begin{array}{l}\text { Allocation concealment } \\
\text { (selection bias) }\end{array}$ & Low risk & $\begin{array}{l}\text { "Randomisation to treatment condition (with condition being designated by a } \\
\text { random number), was conducted by two research assistants who were blind to } \\
\text { the correspondence between random number and treatment group." }\end{array}$ \\
\hline
\end{tabular}


Penn 2009 (Continued)

Blinding of participants Unclear risk Participants not blinded. No information reported about personnel. and personnel (performance bias)

All outcomes

\section{Blinding of outcome as- Low risk} sessment (detection bias)

All outcomes

\begin{abstract}
"All...research evaluations were conducted by two research assistants (RAs) who were blind to treatment assignment. Blindness was maintained by asking participants not to talk to the RAs about their treatment. In addition, the RAs had minimal contact with the study therapists. Finally, RAs were kept blind to the coding system used to denote group membership."

"Participants were assessed...by research assistants blind to treatment group."
\end{abstract}

\begin{tabular}{|c|c|c|}
\hline $\begin{array}{l}\text { Incomplete outcome data } \\
\text { (attrition bias) } \\
\text { All outcomes }\end{array}$ & Low risk & $\begin{array}{l}\text { "We analysed data on all participants irrespective of treatment adherence (i.e., } \\
\text { intent-to-treat analyses) and number of post treatment assessments". }\end{array}$ \\
\hline $\begin{array}{l}\text { Selective reporting (re- } \\
\text { porting bias) }\end{array}$ & Low risk & All expected outcomes are reported. \\
\hline Other bias & Low risk & Appears free of other sources of bias. \\
\hline
\end{tabular}

\section{Pinto 1999}

Allocation: randomised.
Blindness: none.
Duration: 6 months.
Design: parallel.
Setting: inpatients \& outpatients.
Country: Italy.

Participants Diagnosis: schizophrenia (DSM-IV).

$\mathrm{N}=41$.

Age: mean 35 years, SD 11 years.

Sex: 28M, 13F.

History: treatment-refractory psychosis, recently started clozapine, illness duration mean 12 years.

Exclusions: evidence of current substance abuse or organic pathology.

Interventions
training. $\mathrm{N}=21$.
2. Cognitive-behavioural therapy plus social skills training. $\mathrm{N}=20$.

\begin{tabular}{ll}
\hline Outcomes & Global state: relapse. \\
& Service outcomes: hospitalisation. \\
& Mental state: BPRS, SAPS, SANS. \\
& Leaving the study early. \\
& Unable to use - \\
& Dose of antipsychotic medication (data not reported). \\
\hline \multirow{2}{*}{ Notes } & Percentage of participants for whom no outcome data is available: \\
& 1. Supportive psychotherapy $14 \%$. \\
2. CBT $5 \%$.
\end{tabular}


Pinto 1999 (Continued)

Risk of bias

\begin{tabular}{|c|c|c|}
\hline Bias & Authors' judgement & Support for judgement \\
\hline $\begin{array}{l}\text { Random sequence genera- } \\
\text { tion (selection bias) }\end{array}$ & Unclear risk & "Randomly assigned " No further details reported. \\
\hline $\begin{array}{l}\text { Allocation concealment } \\
\text { (selection bias) }\end{array}$ & Unclear risk & No information reported.. \\
\hline $\begin{array}{l}\text { Blinding of participants } \\
\text { and personnel (perfor- } \\
\text { mance bias) } \\
\text { All outcomes }\end{array}$ & Unclear risk & No information reported. \\
\hline $\begin{array}{l}\text { Blinding of outcome as- } \\
\text { sessment (detection bias) } \\
\text { All outcomes }\end{array}$ & Unclear risk & No information reported. \\
\hline $\begin{array}{l}\text { Incomplete outcome data } \\
\text { (attrition bias) } \\
\text { All outcomes }\end{array}$ & Low risk & $\begin{array}{l}\text { "One subject in the cognitive-behavioral group did not complete the study; the } \\
\text { client developed leukopenia, and clozapine was discontinued. In the support- } \\
\text { ive therapy group, two subjects withdrew because of refusal to participate fur- } \\
\text { ther, and a third developed seizures and his clozapine was stopped." Losses to } \\
\text { followup not included in analysis. }\end{array}$ \\
\hline $\begin{array}{l}\text { Selective reporting (re- } \\
\text { porting bias) }\end{array}$ & High risk & $\begin{array}{l}\text { Reports results incompletely, data not reported for dose of antipsychotic med- } \\
\text { ication. }\end{array}$ \\
\hline Other bias & Unclear risk & Not enough information provided to make a judgement. \\
\hline
\end{tabular}

Sensky 2000b

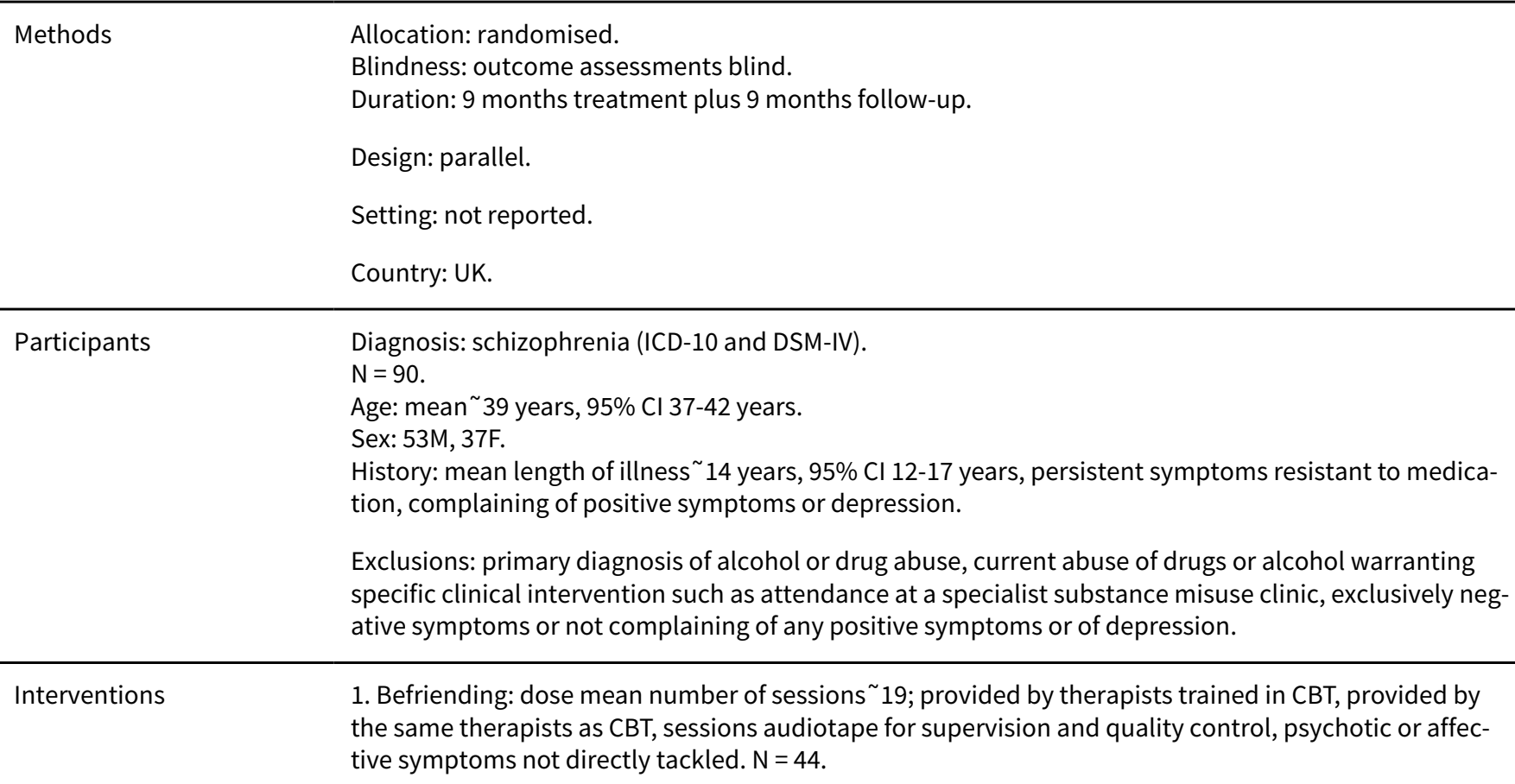


Sensky 2000b (Continued)

2. Cognitive-behavioural therapy: dose mean number of sessions ${ }^{\sim} 19$; manualised, provided by therapists trained in CBT, provided by the same therapists as befriending, sessions audiotape for supervision and quality control. $\mathrm{N}=46$.

\begin{tabular}{|c|c|c|}
\hline Notes & $\begin{array}{l}\text { Percentage of particip } \\
\text { 1. Supportive psychot } \\
\text { 2. CBT } 20 \% \text {. }\end{array}$ & $\begin{array}{l}\text { nts for whom no outcome data is available: } \\
\text { erapy } 14 \% \text {. }\end{array}$ \\
\hline \multicolumn{3}{|l|}{ Risk of bias } \\
\hline Bias & Authors' judgement & Support for judgement \\
\hline $\begin{array}{l}\text { Random sequence genera- } \\
\text { tion (selection bias) }\end{array}$ & Unclear risk & "Simple randomisation applied". No further details reported. \\
\hline $\begin{array}{l}\text { Allocation concealment } \\
\text { (selection bias) }\end{array}$ & Low risk & $\begin{array}{l}\text { "The randomisation was by members of the research team not involved with } \\
\text { either the assessments or the treatments." }\end{array}$ \\
\hline $\begin{array}{l}\text { Blinding of participants } \\
\text { and personnel (perfor- } \\
\text { mance bias) } \\
\text { All outcomes }\end{array}$ & Unclear risk & No information reported. \\
\hline $\begin{array}{l}\text { Blinding of outcome as- } \\
\text { sessment (detection bias) } \\
\text { All outcomes }\end{array}$ & Low risk & $\begin{array}{l}\text { "Assesors were independent of the randomisation process and remained blind } \\
\text { to each patient's assigned group throughout the study". }\end{array}$ \\
\hline $\begin{array}{l}\text { Incomplete outcome data } \\
\text { (attrition bias) } \\
\text { All outcomes }\end{array}$ & Low risk & $\begin{array}{l}\text { Missing data have been imputed using appropriate methods "intention to } \\
\text { treat analysis". }\end{array}$ \\
\hline $\begin{array}{l}\text { Selective reporting (re- } \\
\text { porting bias) }\end{array}$ & Low risk & Patient satisfaction score scale not peer reviewed. \\
\hline Other bias & Low risk & $\begin{array}{l}\text { Funded by grant from Welcome trust, further financial support was provided } \\
\text { by Hounslow and Spelthorne community and mental health service trust. }\end{array}$ \\
\hline
\end{tabular}

Mental state: CPRS, MADRS, SANS.

Leaving the study early.

Medication: no reduction in dose of antipsychotic medication.

Unable to use -

Satisfaction with treatment: patient satisfaction score (scale not peer reviewed).

1. Supportive psychotherapy $14 \%$.

2. CBT $20 \%$

\section{Risk of bias}

Spaulding 1999

Allocation: matched pair randomisation.
Blindness: some outcomes assessments blind.
Duration: 6 months treatment plus 1 year of continued standard rehabilitation.
Design: parallel.
Setting: inpatient.
Country: USA.

Participants Diagnosis: schizophrenia (DSM-III-R).


Spaulding 1999 (Continued)

\author{
$N=91$. \\ Age: mean $~ 36$ years, SD 10 years. \\ Sex: 56M, 35F. \\ History: hospitalised, failure in all other available treatment settings, not responding to short-term in- \\ patient \\ treatment sufficiently enough to allow discharge, having a primary chart diagnosis of an Axis I psychi- \\ atric \\ disorder, $\mathrm{IQ}>70$.
}

Exclusions: primary diagnosis of mental retardation or substance abuse, no dangerous behaviour requiring a higher security setting.

\begin{tabular}{|c|c|}
\hline Interventions & $\begin{array}{l}\text { 1. Supportive group therapy plus standard regimen: dose } 3 \text { sessions per week lasting } 45-60 \text { minutes; } \\
\text { manualised, videotaped sessions assessed using Q-sort instrument, therapists experienced in support- } \\
\text { ive group therapy, standard regimen consists of comprehensive psychiatric rehabilitation. } \mathrm{N}=42 \text {. } \\
\text { 2. Cognitive treatment: dose } 3 \text { sessions per week lasting } 45-60 \text { minutes cognitive sub programmes of } \\
\text { Integrated Psychological Therapy, group, manualised, therapist trained in Integrated Psychological } \\
\text { Therapy, standard regimen consists of comprehensive psychiatric rehabilitation. } \mathrm{N}=49 \text {. }\end{array}$ \\
\hline Outcomes & $\begin{array}{l}\text { Leaving the study early. } \\
\text { Unable to use - } \\
\text { Mental state: BPRS, PANSS, TLC, NOSIE-30 (data not reported). } \\
\text { General functioning: AIPSS, Symptom Management skill assessment, Medication Management skill as- } \\
\text { sessment, Leisure Skills assessment, Conversational Skills assessment, COGLAB cognitive assessment } \\
\text { battery, Trailmaking test, Tactile Performance Test, Categories, Rey Auditory and Visual Learning Tasks, } \\
\text { Denman Neuropsychological Memory Test (data not reported). }\end{array}$ \\
\hline
\end{tabular}

Notes

Percentage of participants for whom no outcome data is available:

1. Supportive psychotherapy $0 \%$.

2. CBT $2 \%$.

\title{
Risk of bias
}

\begin{tabular}{lll}
\hline Bias & Authors' judgement & Support for judgement \\
\hline $\begin{array}{ll}\text { Random sequence genera- } \\
\text { tion (selection bias) }\end{array}$ & Unclear risk & "Patients in the cohort were matched pair-wise, as closely as possible, for gen- \\
& & $\begin{array}{l}\text { der, chronicity, and overall levels of personal and social functioning. Each pa- } \\
\text { tient in the pair was then assigned to one of two groups. When there was an } \\
\text { odd number of patients in the cohort, the unmatched individual was randomly } \\
\text { assigned to one of the groups. One of the groups was then randomly assigned } \\
\text { to the experimental condition and the other to the control condition". No fur- } \\
\text { ther details reported. }\end{array}$
\end{tabular}

\begin{tabular}{|c|c|c|}
\hline $\begin{array}{l}\text { Allocation concealment } \\
\text { (selection bias) }\end{array}$ & Unclear risk & Matched pair randomisation. No further details reported. \\
\hline $\begin{array}{l}\text { Blinding of outcome as- } \\
\text { sessment (detection bias) } \\
\text { All outcomes }\end{array}$ & Unclear risk & $\begin{array}{l}\text { "All NOSIE-30 raters were blind to treatment group assignment" ; "There defi- } \\
\text { nitely were no breaches with any staff directly involved in clinical or laborato- } \\
\text { ry assessments". "All clinical and research personnel were blind to experimen- } \\
\text { tal condition, except for the cognitive and supportive therapists, the observers } \\
\text { who performed the process measures in the project's first year, and the princi- } \\
\text { pal investigator." }\end{array}$ \\
\hline
\end{tabular}


Spaulding 1999 (Continued)

Incomplete outcome data Low risk "One subject who dropped out after the intensive treatment period began." (attrition bias)

All outcomes

\section{Selective reporting (re- High risk} porting bias)
Reports results incompletely, data not reported for BPRS, PANSS, TLC, NOSIE-30, AIPSS, Symptom Management skill assessment, Medication Management skill assessment, Leisure Skills assessment, Conversational Skills assessment, COGLAB cognitive assessment battery, Trailmaking test, Tactile Performance Test, Categories, Rey Auditory and Visual Learning Tasks, Denman Neuropsychological Memory Test

Other bias Low risk Funded by an NIH grant

\section{Stanton 1984}

Allocation: randomised.
Blindness: some outcome assessments blind, attempts made to blind therapists to hypothesis and
outcomes.
Duration: 2 years, had to stay in therapy for 6 months to be included in 2 year follow-up.
Design: parallel.
Setting: inpatient.
Country: USA.

Participants

Diagnosis: schizophrenia or probable schizophrenia (Psychiatric Status Schedule and WHO Discrimination Criteria for Schizophrenia).

$\mathrm{N}=164$.

Age: mean 22 years.

Sex: $112 \mathrm{M}, 52 \mathrm{~F}$.

History: minimal prior treatment, can function outside of a hospital in some major role, without medications for 4 consecutive months preceding 2 years.

Exclusions: history of alcohol dependency or drug use, obvious organic impairments.

Interventions
2. Exploality-adaptive supportive psychotherapy (RAS): dose up to once weekly. $\mathrm{N}=76$.
psychotherapy. $\mathrm{N}=88$.

Outcomes Leaving the study early.

Unable to use -

Service outcomes: rehospitalisation, number of days hospitalised ( $>50 \%$ lost to follow-up). Mental state: Camarillo Dynamic Assessment Scale, IMPS, Psychotherapy Outcome Interview (> 50\% lost to follow-up).

General functioning: number of days dependent, units of productive activity, days employed full-time, occupational level reached, self-support, household responsibilities, significant relationships (> 50\% lost to follow-up). Number of job changes ( $>50 \%$ lost to follow-up).

Days on antipsychotic medication (> $50 \%$ lost to follow-up).

Visual-Verbal Test - total misses (> $>0 \%$ lost to follow-up).

Global state: Menninger Health-Sickness Rating Scale, Psychotherapy Outcome Interview (data not reported).

Mental state: Psychiatric Status Schedule, IMPS, Inpatient Multidimensional Psychiatric Scale, Camarillo Dynamic Assessment Scale (data not reported).

General functioning: WAIS, KAS, Gottschalk Social Alienation/Personal Disorganisation Scale (data not reported). 


Percentage of participants for whom no outcome data is available at 6 months:
1. Supportive psychotherapy $32 \%$.
2. Psychodynamic therapy $51 \%$.
Percentage of participants for whom no outcome data is available at 12 months:
1. Supportive psychotherapy $51 \%$.
2. Psychodynamic therapy $75 \%$.
Percentage of participants for whom no outcome data is available at 24 months:
1. Supportive psychotherapy $67 \%$.
2. Psychodynamic therapy $75 \%$.
> $50 \%$ of participants lost to follow-up at 12 months and 24 months, therefore data excluded.

\section{Risk of bias}

\begin{tabular}{lll}
\hline Bias & Authors' judgement & Support for judgement \\
\hline $\begin{array}{ll}\text { Random sequence genera- } \\
\text { tion (selection bias) }\end{array}$ & Low risk & $\begin{array}{l}\text { "Assignment to EIO or RAS was achieved by a random numbering system with- } \\
\text { in an unrelated office."; "On those occasions where imbalances appeared, a } \\
\text { weighting was introduced into the randomisation procedure to reestablish } \\
\text { comparability of the EIO and RAS groups at baseline." }\end{array}$ \\
& &
\end{tabular}

\begin{tabular}{|c|c|c|}
\hline $\begin{array}{l}\text { Allocation concealment } \\
\text { (selection bias) }\end{array}$ & Unclear risk & $\begin{array}{l}\text { "Assignment to EIO or RAS was achieved by a random numbering system with- } \\
\text { in an unrelated office" Details of method not reported. }\end{array}$ \\
\hline
\end{tabular}

\begin{tabular}{|c|c|c|}
\hline $\begin{array}{l}\text { Blinding of participants } \\
\text { and personnel (perfor- }\end{array}$ & High risk & $\begin{array}{l}\text { "We knew that significant others, ward staff, and project staff also would not } \\
\text { be blind to the patient's experimental condition." }\end{array}$ \\
\hline
\end{tabular}
mance bias)

All outcomes

\begin{tabular}{|c|c|c|}
\hline $\begin{array}{l}\text { Blinding of outcome as- } \\
\text { sessment (detection bias) } \\
\text { All outcomes }\end{array}$ & Unclear risk & $\begin{array}{l}\text { "Four different groups of people administered the measures: (1) project psy- } \\
\text { chiatrists; (2) blind evaluators; (3) psychological testers; and (4) research as- } \\
\text { sistants."; "Psychological testers were clinical psychologists with expertise in } \\
\text { psychological testing. They were blind to the experimental conditions to which } \\
\text { patients were assigned and also unfamiliar with the study design and hypothe- } \\
\text { ses." }\end{array}$ \\
\hline
\end{tabular}

\begin{tabular}{lll}
\hline $\begin{array}{l}\text { Incomplete outcome data } \\
\text { (attrition bias) } \\
\text { All outcomes }\end{array}$ & High risk & $\begin{array}{l}\text { High attrition rates reported throughout both groups during all three fol- } \\
\text { low-up periods (See Notes above). Reasons for attrition not reported. }\end{array}$ \\
\hline $\begin{array}{l}\text { Selective reporting (re- } \\
\text { porting bias) }\end{array}$ & High risk & $\begin{array}{l}\text { Reports results incompletely, data not reported for Menninger Health-Sickness } \\
\text { Rating Scale, Psychotherapy Outcome Interview, Psychiatric Status Schedule, } \\
\text { IMPS, Inpatient Multidimensional Psychiatric Scale, Camarillo Dynamic Assess- } \\
\text { ment Scale, WAIS, KAS, Gottschalk Social Alienation/ Personal Disorganisation } \\
\text { Scale, Rorschach test, Thematic Apperception Test, Visual-Verbal Test, Soskis } \\
\text { Attitude Toward Illness Questionnaire; }\end{array}$ \\
\hline
\end{tabular}

Other bias Low risk "supported in part by NIMH Grant "

Tarrier 1998

$\begin{array}{ll}\text { Methods } & \text { Allocation: stratified block randomisation. } \\ \text { Blindness: outcome assessments blind. } & \text { Duration: } 3 \text { months treatment plus } 2 \text { years follow-up. }\end{array}$


Tarrier 1998 (Continued)

Design: parallel.

Setting: outpatient.

Country: UK.

Diagnosis: schizophrenia or schizoaffective psychosis or delusional disorder (DSM-III-R).
N=87.
Age: mean 39 years.
Sex: $69 \mathrm{M}, 18 \mathrm{~F}$.
History: median length of illness $\sim 11$ years, range $1-42$ years, persistent hallucinations or delusions or
both for minimum of 6 months and at least 1 month of stabilisation if they had experienced an exac-
erbation within this period, stable medication.

Exclusions: organic brain disease, substance abuse as primary problem, threat of violence towards assessors, psychological or family intervention.

Interventions
monthly booster sessions for 4 months. $N=26$.
2. Intensive cognitive behaviour therapy plus routine care: dose 20 sessions of 1 hour twice a week for
3 months plus monthly booster sessions for 4 months; coping strategy enhancement, problem solving
and relapse reduction strategies used. $N=33$.
3. Routine care: standard psychiatric management with medication and monitoring outpatient fol-
low-up and the care programme approach. $\mathrm{N}=28$.

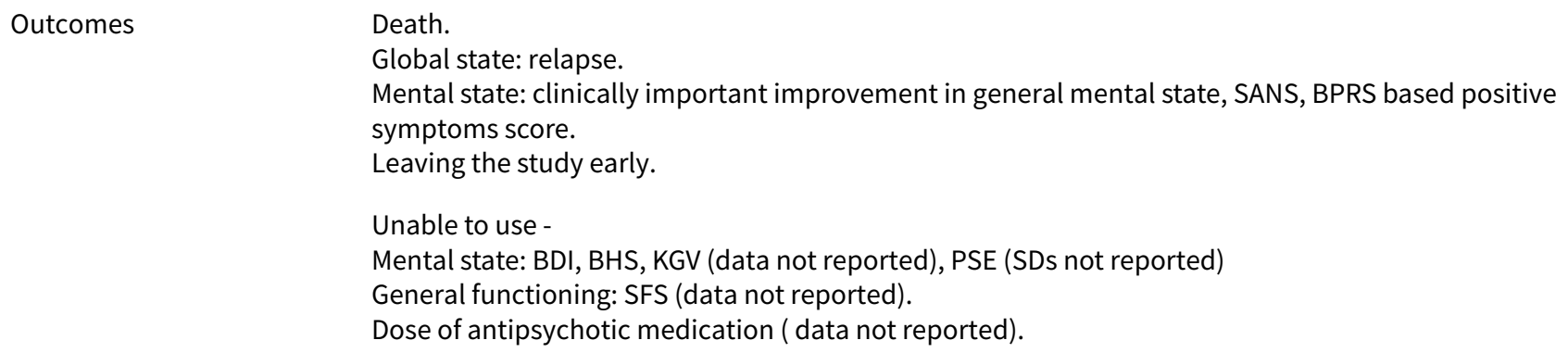

Percentage of participants for whom no outcome data is available:
1. Supportive therapy $4 \%$.
2. CBT $12 \%$.
3. Standard care $4 \%$.

\section{Risk of bias}

\begin{tabular}{|c|c|c|}
\hline Bias & Authors' judgement & Support for judgement \\
\hline $\begin{array}{l}\text { Random sequence genera- } \\
\text { tion (selection bias) }\end{array}$ & Low risk & $\begin{array}{l}\text { "Patients were allocated on a stratified block randomised procedure with } \\
\text { block size equal to nine by using severity of symptoms and sex to one of three } \\
\text { treatment groups" }\end{array}$ \\
\hline $\begin{array}{l}\text { Allocation concealment } \\
\text { (selection bias) }\end{array}$ & Unclear risk & $\begin{array}{l}\text { "Allocation, contained in sealed envelopes, was carried out independently by } \\
\text { a third party." }\end{array}$ \\
\hline $\begin{array}{l}\text { Blinding of participants } \\
\text { and personnel (perfor- } \\
\text { mance bias) } \\
\text { All outcomes }\end{array}$ & Unclear risk & No information reported. \\
\hline $\begin{array}{l}\text { Blinding of outcome as- } \\
\text { sessment (detection bias) } \\
\text { All outcomes }\end{array}$ & Low risk & $\begin{array}{l}\text { "Effort was made to blind the independent assessors to treatment allocation } \\
\text { by using separate offices in a different part of the hospital for the assessors } \\
\text { and therapists, using separate administrative procedures, instructing patients }\end{array}$ \\
\hline
\end{tabular}


Tarrier 1998 (Continued)

to not reveal details of their care, data entry being carried out independently of the assessors, and using a multiple coding system for treatment groups."

\begin{tabular}{ll}
\hline $\begin{array}{l}\text { Incomplete outcome data } \\
\text { (attrition bias) }\end{array}$ & "Analysis by intention to treat was completed on the 87 allocated patients, \\
All outcomes & with last observations (scores before treatment) being carried forward for the \\
& eight patients for whom scores after treatment were missing". Missing data \\
& have been imputed using appropriate methods (intention to treat analysis), \\
& and losses to follow-up or missing data balanced across intervention groups.
\end{tabular}

\begin{tabular}{lll}
\hline $\begin{array}{l}\text { Selective reporting (re- } \\
\text { porting bias) }\end{array}$ & High risk & $\begin{array}{l}\text { Reports results incompletely, data not reported for BDI, BHS, KGV, SFS, and } \\
\text { dose of antipsychotic medication; SDs not reported for PSE. }\end{array}$ \\
\hline Other bias & Low risk & Funded by a Wellcome Trust grant. \\
\hline
\end{tabular}

\section{Turkington 2000}

\begin{tabular}{|c|c|}
\hline \multirow[t]{4}{*}{ Methods } & $\begin{array}{l}\text { Allocation: randomised. } \\
\text { Blindness: patients, assessors and clinical teams blind to allocation. } \\
\text { Duration: } 2 \text { months therapy plus } 4 \text { months follow-up. }\end{array}$ \\
\hline & Design: parallel. \\
\hline & Setting: inpatient \& outpatient. \\
\hline & Country: UK. \\
\hline \multirow[t]{2}{*}{ Participants } & $\begin{array}{l}\text { Diagnosis: schizophrenia (ICD-10). } \\
\mathrm{N}=19 . \\
\text { Age: mean } 41 \text { years. } \\
\text { Sex: } 9 \mathrm{M}, 9 \mathrm{~F} \text {. } \\
\text { History: mean duration of illness for befriending group } 13 \text { years, mean duration of illness for cognitive } \\
\text { therapy group } 9.2 \text { years. }\end{array}$ \\
\hline & Exclusions: not reported. \\
\hline Interventions & $\begin{array}{l}\text { 1. Befriending: dose } 6 \text { sessions over } 2 \text { months; structured and supportive, some sessions audio taped } \\
\text { and analysed for treatment fidelity. } N=6 \text {. } \\
\text { 2. Cognitive techniques: dose } 6 \text { sessions over } 2 \text { months; manualised, some sessions audio taped and } \\
\text { assessed for treatment fidelity. } N=13 \text {. }\end{array}$ \\
\hline \multirow[t]{2}{*}{ Outcomes } & Leaving the study early. \\
\hline & $\begin{array}{l}\text { Unable to use - } \\
\text { Service outcomes: time spent in hospital (SDs not reported). } \\
\text { Mental state: CPRS, MADRS (SDs not reported). }\end{array}$ \\
\hline Notes & $\begin{array}{l}\text { Percentage of participants for whom no outcome data is available: } \\
\text { 1. Supportive psychotherapy } 0 \% \text {. } \\
\text { 2. CBT } 20 \% \text {. }\end{array}$ \\
\hline
\end{tabular}

\section{Risk of bias}

\begin{tabular}{lll}
\hline Bias & Authors' judgement & Support for judgement \\
\hline $\begin{array}{l}\text { Random sequence genera- } \\
\text { tion (selection bias) }\end{array}$ & Unclear risk & $\begin{array}{l}\text { Patients randomised on 2:1 basis after initial assessments made. No further } \\
\text { details reported. }\end{array}$ \\
\hline
\end{tabular}


Turkington 2000 (Continued)

$\begin{aligned} & \text { Allocation concealment } \\ & \text { (selection bias) }\end{aligned}$ Unclear risk Details of method not reported.

\begin{tabular}{ll}
\hline $\begin{array}{l}\text { Blinding of participants } \\
\text { and personnel (perfor- } \\
\text { mance bias) }\end{array}$ & "Patients randomly allocated to two groups...with the patients, assessors, and \\
clinical team remaining blind to the allocation."
\end{tabular}

All outcomes

\begin{tabular}{|c|c|c|}
\hline $\begin{array}{l}\text { Blinding of outcome as- } \\
\text { sessment (detection bias) }\end{array}$ & Low risk & $\begin{array}{l}\text { "Patients randomly allocated to two groups... with the patients, assessors, and } \\
\text { clinical team remaining blind to the allocation. }\end{array}$ \\
\hline
\end{tabular}

All outcomes

\begin{tabular}{lll}
\hline $\begin{array}{l}\text { Incomplete outcome data } \\
\text { (attrition bias) } \\
\text { All outcomes }\end{array}$ & Low risk & $\begin{array}{l}\text { "One patient in the cognitive techniques group withdrew consent after the ini- } \\
\text { tial interview, and so had to be excluded from the study", "Calculations were } \\
\text { also made excluding his results, from the analysis" }\end{array}$ \\
\hline $\begin{array}{l}\text { Selective reporting (re- } \\
\text { porting bias) }\end{array}$ & High risk & $\begin{array}{l}\text { Reports results incompletely, SDs not reported for time spent in hospital, } \\
\text { CPRS, MADRS. }\end{array}$ \\
\hline Other bias & Low risk & The study appears to be free of other sources of bias.
\end{tabular}

\section{Uzenoff 2007}

Methods
Blinding: unclear.
Duration: 6 months.
Design: parallel.
Setting: outpatients.
Country: USA.

Participants

Diagnosis: schizophrenia, schizoaffective disorder or schizophreniform. DSM IV criteria.

$N=24$.

Age: $\geq 16$.

Sex: $14 \mathrm{M}, 10 \mathrm{~F}$

History: clinically stable outpatients recovering from a first psychotic episode (in treatment $<12$ months).

Exclusions: not reported.

\begin{tabular}{ll}
\hline Interventions & 1. Adherence-Coping Education (ACE). $\mathrm{N}=13$. \\
& 2. Supportive Therapy (ST). $\mathrm{N}=11$. \\
\hline Outcomes & Leaving the study early. \\
& Medication: adherence, ROMI (adherence and nonadherence). \\
& Need for treatment and benefits of medication (composed of ROMI and ITAQ items). \\
& Mental state: not clinically important improvement (<50\% improvement on the PANSS), PANSS (posi- \\
tive, negative and general subscales).
\end{tabular}


Uzenoff 2007 (Continued)

Compliance: poor compliance (attended less than 6 sessions of therapy).

Behaviour: treatment attitude (ITAQ).

Quality of life: QLS.

Notes

\section{Risk of bias}

\begin{tabular}{|c|c|c|}
\hline Bias & Authors' judgement & Support for judgement \\
\hline $\begin{array}{l}\text { Random sequence genera- } \\
\text { tion (selection bias) }\end{array}$ & Unclear risk & "Randomised",' no further details reported. \\
\hline $\begin{array}{l}\text { Allocation concealment } \\
\text { (selection bias) }\end{array}$ & Unclear risk & No information reported. \\
\hline $\begin{array}{l}\text { Blinding of participants } \\
\text { and personnel (perfor- } \\
\text { mance bias) } \\
\text { All outcomes }\end{array}$ & Unclear risk & No information reported. \\
\hline $\begin{array}{l}\text { Blinding of outcome as- } \\
\text { sessment (detection bias) } \\
\text { All outcomes }\end{array}$ & Low risk & $\begin{array}{l}\text { "Assessments were conducted at baseline, mid treatment ( } 3 \text { months), and } \\
\text { posttreatment ( } 6 \text { months) by interviewers blind to treatment condition." }\end{array}$ \\
\hline $\begin{array}{l}\text { Incomplete outcome data } \\
\text { (attrition bias) } \\
\text { All outcomes }\end{array}$ & Low risk & $\begin{array}{l}\text { All outcome analyses were completed using a modified intent-to-treat sample } \\
(\mathrm{N}=19) \text { consisting of individuals who completed both a baseline assessment } \\
\text { and at least } 1 \text { follow-up assessment, and who had attended at least } 1 \text { session } \\
\text { of their assigned intervention". } \\
\text { "Of the } 24 \text { participants randomised to receive treatment, } 3 \text { participants did not } \\
\text { attend and follow-up assessments". } \\
\text { "Of the } 24 \text { participants randomized to receive treatment, a total of } 5 \text { partici- } \\
\text { pants were excluded from outcome data analyses" }\end{array}$ \\
\hline $\begin{array}{l}\text { Selective reporting (re- } \\
\text { porting bias) }\end{array}$ & Low risk & All expected outcomes are reported. \\
\hline Other bias & Low risk & The study appears to be free of other sources of bias. \\
\hline
\end{tabular}

Wirshing 1991

\begin{tabular}{ll}
\hline Methods & Allocation: randomised. \\
Blindness: none. \\
Duration: 2 years. \\
Design: $2 \times 2$ factorial. \\
Setting: inpatient \& outpatient. \\
Country: USA. \\
\hline Participants \\
Diagnosis: schizophrenia (DSM-III-R). \\
Age: mean 38 years.
\end{tabular}


Wirshing 1991 (Continued)

Sex: all male.

History: stabilised for 2 months on low dose fluphenazine decanoate, no organic brain disorder, no mental retardation, no severe alcohol or substance abuse, no history of suicidal or homicidal behaviour, mean duration of illness ${ }^{\sim} 13$ years.

Exclusions: not reported.

Interventions

1. Supportive group psychotherapy: dose 90 minutes twice weekly for 6 months, 90 minutes weekly for up to 18 months; goal-setting, information provision. $\mathrm{N}=37$.

2. Behaviourally-oriented social skills training: dose 90 minutes twice weekly for 6 months, 90 minutes weekly for up to 18 months; group setting. $\mathrm{N}=43$.

Leaving the study early.
Unable to use -
Mental state: BPRS, SANS, exacerbation, severity of exacerbation, prodrome, latency from prodrome to
exacerbation (data not reported).
General functioning: SAS-II, degree of life disruption during an exacerbation (data not reported), symp-
tom management skills test, medication management skills test (scale unpublished).
Amount of medication needed to control an exacerbation (data not reported).

\section{Notes}

2x2 factorial design: first two conditions were behaviourally-oriented skills training program versus supportive psychotherapy control group; the other two conditions were oral neuroleptic supplementation versus placebo given in double blind fashion for the duration of each prodromal period.

\section{Risk of bias}

\begin{tabular}{|c|c|c|}
\hline Bias & Authors' judgement & Support for judgement \\
\hline $\begin{array}{l}\text { Random sequence genera- } \\
\text { tion (selection bias) }\end{array}$ & Unclear risk & "Randomly assigned" no further details reported. \\
\hline $\begin{array}{l}\text { Allocation concealment } \\
\text { (selection bias) }\end{array}$ & Unclear risk & Details of method not reported. \\
\hline $\begin{array}{l}\text { Blinding of participants } \\
\text { and personnel (perfor- } \\
\text { mance bias) } \\
\text { All outcomes }\end{array}$ & Unclear risk & No information reported. \\
\hline $\begin{array}{l}\text { Blinding of outcome as- } \\
\text { sessment (detection bias) } \\
\text { All outcomes }\end{array}$ & High risk & $\begin{array}{l}\text { "The rater was a clinician who was not blind to the psychosocial treatment } \\
\text { condition." }\end{array}$ \\
\hline $\begin{array}{l}\text { Incomplete outcome data } \\
\text { (attrition bias) } \\
\text { All outcomes }\end{array}$ & Unclear risk & Losses to follow-up not reported. \\
\hline $\begin{array}{l}\text { Selective reporting (re- } \\
\text { porting bias) }\end{array}$ & High risk & $\begin{array}{l}\text { Reports results incompletely, data not reported for BPRS, SANS, exacerbation, } \\
\text { severity of exacerbation, prodrome, latency from prodrome to exacerbation, } \\
\text { SAS-II, Amount of medication needed to control an exacerbation, and degree } \\
\text { of life disruption during an exacerbation. }\end{array}$ \\
\hline Other bias & Low risk & $\begin{array}{l}\text { "This project was supported in part by research grants from the Veterans Ad- } \\
\text { ministration Medical Research Service and the National Institute of Mental } \\
\text { Health (MH141573 and MH30911)". }\end{array}$ \\
\hline
\end{tabular}

\section{Diagnosis}

RDC - Research Diagnostic Criteria 
DSM - Diagnostic and Statistical Manual ICD - International Classification of Diseases

\section{Global state}

GAS - Global Assessment Scale

CGI - Clinical Global Improvement

HoNOS - Health of the Nation Outcome Scales

\section{Mental state}

BDI - Beck Depression Inventory

BHS - Beck Hopelessness Scale

BPRS - Brief Psychiatric Rating Scale

BVAQ-R - Revised Beliefs About Voices Questionnaire

CESD - Center for Epidemiological Studies-Depression Scale

CPRS - Comprehensive Psychiatric Rating Scale

IMPS - Inpatient Multidimensional Psychiatric Scale

KGV - Psychiatric Assessment Scale

MADRS - Montgomery-Asberg Depression Rating Scale

NOSIE-30 - Nurses Observation Scale for Inpatient Evaluation

PANSS - Positive and Negative Syndrome Scale

PSE - Present State Examination

PSYRATS - Psychotic Symptom Rating Scale

SANS - Schedule for Assessment of Negative Symptoms

SAPS - Schedule for Assessment of Positive Symptoms

TLC - Thought, Language and Communication

\section{Medication compliance}

MCS - Medication Compliance Survey

ROMI - Rating of Medication Influences

\section{Attitude to medication}

AMQ - Attitudes to Medication Questionnaire

Insight

BCIS - Beck Cognitive Insight

ITAQ - Insight and Treatment Attitudes

SAI - Schedule for Assessment of Insight (SAI)

\section{General functioning}

AIPSS - Assessment of Interpersonal Problem-Solving Skills

GAF - Global Assessment of Function disability scale

IMPS - Inpatient Multidimensional Psychiatric Scale

KAS - Katz Adjustment Scales

LSP - Life Skills Profile

SAS II - Social Adjustment Scale II

SAS-SR - Social Adjustment Scale - Self Report

SFS - Social Functioning Scale

WAIS - Wechsler Adult Intelligence Scale

\section{Behaviour}

SBAS - Social Behaviour Adjustment Schedule

\section{Satisfaction with treatment}

CSQ - Client Service Satisfaction Questionnaire

NART - National Adult Reading Test

\section{Quality of Life}

GHQ - Global Health Questionnaire

RSES - Rosenberg Self-Esteem Scale

QLS - Quality of Life Scale

\section{General}

CBT - cognitive behavioural therapy

ST - supportive therapy

TAU - treatment as usual

Characteristics of excluded studies [ordered by study ID] 


\section{Study}

Aberg-Wistedt 1995

\section{Reason for exclusion}

Allocation: randomised.

Participants: people with schizophrenia or other long-term psychotic disorders.

Interventions: case management group vs standard services, not supportive therapy or supportive care.

\begin{tabular}{ll}
\hline Abramowitz 1989 & Allocation: not randomised. \\
\hline Addington 2011 & $\begin{array}{l}\text { Allocation: randomised. } \\
\text { Participants: people with a high risk of developing psychosis. }\end{array}$ \\
\hline Anderson 1982 & $\begin{array}{l}\text { Allocation: randomised. } \\
\text { Participants: people with schizophrenia. } \\
\text { Interventions: family therapy vs social skills training, not supportive therapy or supportive care. }\end{array}$ \\
\hline Andres 1998 & Allocation: not randomised. \\
\hline Andres 2000 & Allocation: not randomised. \\
\hline Armstrong 1991 & $\begin{array}{l}\text { Allocation: randomised. } \\
\text { Participants: people with major affective disorder (56\%) or schizophrenia (29\%) or other psychi- } \\
\text { atric disorders (16\%). } \\
\text { Interventions: life skills program vs supportive psychotherapeutic milieu. } \\
\text { Outcomes: no data were reported separately for people with schizophrenia. }\end{array}$
\end{tabular}

Allocation: randomised.

Participants: people with schizophrenia.

Interventions: problem solving training sessions vs no problem solving training, not supportive therapy or supportive care.

\begin{tabular}{ll}
\hline Beal 1977 & Allocation: randomised. \\
& Participants: people with schizophrenia. \\
& Interventions: activities group vs remotivation group vs social living group vs waiting list control \\
& group, not supportive therapy or supportive care.
\end{tabular}

\section{Beard 1975}

Allocation: randomised.

Participants: people with chronic psychiatric disorders.

Interventions: group therapy sessions vs standard care, not supportive therapy or supportive care.

\begin{tabular}{ll}
\hline Beard 1978 & Allocation: randomised. \\
& Participants: people with schizophrenia (approximately 90\%). \\
Interventions: reaching out service vs community placement service vs other community facilities, \\
not supportive therapy or supportive care.
\end{tabular}

\begin{tabular}{ll}
\hline Becker 1998 & Allocation: randomised. \\
Participants: people with severe mental illness (44.4\% schizophrenia and related psychotic disor- \\
ders, $49.2 \%$ bipolar disorder and other severe mood disorders, 6.3\% other diagnoses). \\
Interventions: employment program with individual placement and support vs group skills train- \\
ing, not supportive therapy or supportive care.
\end{tabular}

Beebe 2001

Allocation: randomised.

Participants: people with schizophrenia.

Interventions: telephone nursing intervention vs informational calls, not supportive therapy or supportive care.

\section{Bell 1993a}

\section{Allocation: randomised.}

Participants: people with schizophrenia, schizoaffective disorder. 


\begin{tabular}{ll}
\hline Study & Reason for exclusion \\
\hline & $\begin{array}{l}\text { Interventions: paid work participation vs unpaid work participation, not supportive therapy or } \\
\text { supportive care. }\end{array}$ \\
\hline
\end{tabular}

\begin{tabular}{ll}
\hline Bell 1993b & Allocation: randomised. \\
& Participants: people with schizophrenia or schizoaffective disorder. \\
& Interventions: paid work participation vs unpaid work participation, not supportive therapy or \\
& supportive care.
\end{tabular}

Bell 1998
$\begin{aligned} & \text { Allocation: randomised. } \\ & \text { Participants: people with schizophrenia or schizoaffective disorder. } \\ & \text { Interventions:paid work participation vs unpaid work participation, not supportive therapy or sup- } \\ & \text { portive care. }\end{aligned}$

\begin{tabular}{ll}
\hline Bell 2001 & Allocation: randomised. \\
& Participants: people with schizophrenia or schizoaffective disorder. \\
& Interventions: paid work participation vs unpaid work participation, not supportive therapy or \\
& supportive care.
\end{tabular}

Bell $2003 \quad \begin{aligned} & \text { Allocation: randomised. } \\ & \text { Participants: people with schizophrenia or schizoaffective disorder. } \\ & \text { Interventions: paid work participation with behavioural intervention vs paid work participation } \\ & \text { with usual support conditions, not supportive therapy or supportive care. }\end{aligned}$

\begin{tabular}{ll}
\hline Bellack 1986 & Allocation: randomised. \\
& Participants: people with schizophrenia. \\
& Interventions: day hospital treatment vs day hospital treatment and social skills training, all par- \\
& ticipants received supportive care.
\end{tabular}

\begin{tabular}{ll}
\hline Bond 1995 & Allocation: randomised. \\
& Participants: people with serious mental illness (66\% schizophrenia or schizoaffective disorder). \\
& Interventions: accelerated approach to supported employment vs gradual approach, not support- \\
& ive therapy or supportive care.
\end{tabular}

\begin{tabular}{ll}
\hline Brown 1983 & Allocation: randomised. \\
& Participants: people with schizophrenia. \\
& Interventions: life skills vs rehabilitation programme, not supportive therapy or supportive care.
\end{tabular}

\begin{tabular}{ll}
\hline Buchkremer 1997 & Allocation: randomised. \\
& Participants: people with schizophrenia. \\
& Interventions: psychoeducational medication management training vs cognitive psychotherapy vs \\
& key-person counselling, not supportive therapy or supportive care.
\end{tabular}

\begin{tabular}{ll}
\hline Bush 1990 & Allocation: randomised. \\
& Participants: people with schizophrenia (86\%) or bipolar disorder (7\%) or personality disorder \\
& $(7 \%)$. \\
& Interventions: intensive support from case managers vs less intensive support, not supportive \\
& therapy or supportive care.
\end{tabular}

\begin{tabular}{ll}
\hline Canning 1997 & $\begin{array}{l}\text { Allocation: randomised. } \\
\text { Participants: people with a serious psychiatric illness. } \\
\text { Interventions: psychotherapy + family support versus psychotherapy alone. }\end{array}$ \\
\hline Carra 2010 & $\begin{array}{l}\text { Allocation: randomised. } \\
\text { Participants: family members of people with schizophrenia. }\end{array}$ \\
\hline Castelein 2008 & $\begin{array}{l}\text { Allocation: randomised. } \\
\text { Participants: people with psychosis. } \\
\text { Interventions: peer support vs waiting list. }\end{array}$ \\
\hline
\end{tabular}




\begin{tabular}{ll}
\hline Study & Reason for exclusion \\
\hline & Outcomes: no usable data. \\
\hline Castelein 2008a & Allocation: randomised. \\
& Participants: people with schizophrenia. \\
& Interventions: peer to peer support groups vs waiting list. \\
\hline
\end{tabular}

\begin{tabular}{ll}
\hline Chien 2008 & $\begin{array}{l}\text { Allocation: randomised. } \\
\text { Participants: family caregivers of people with schizophrenia. } \\
\text { Interventions: mutual support group vs standard treatment. }\end{array}$ \\
\hline Chinman 2010 & Allocation: not randomised. \\
\hline Clark 1998 & $\begin{array}{l}\text { Allocation: randomised. } \\
\text { Participants: people with severe mental illness (46.9\% schizophrenia or related psychotic disor- } \\
\text { ders, } 42.7 \% \text { bipolar disorder or other severe mood disorders, } 10.5 \% \text { other disorders). } \\
\text { Interventions: individual placement and support for employment vs group skills training, not sup- } \\
\text { portive therapy or supportive care. }\end{array}$
\end{tabular}

\begin{tabular}{ll}
\hline Cook 2011 & Allocation: randomised. \\
& Participants: persons with serious mental illness, $<50 \%$ schizophrenia. \\
Interventions: Building Recovery of Individual Dreams and Goals through Education and Support \\
(BRIDGES) vs waiting list.
\end{tabular}

Cook $2012 \quad$ Allocation: randomised.

Participants: persons with serious mental illness, $<50 \%$ schizophrenia.

Interventions: Building Recovery of Individual Dreams and Goals through Education and Support (BRIDGES) vs waiting list.

Curtis $1992 \quad$ Allocation: randomised.

Participants: people who were being discharged from psychiatric hospital (44.7\% schizophrenia). Interventions: multidisciplinary intensive outreach case management vs less intensive community support system case management services vs routine aftercare with no case management, not supportive therapy or supportive care.

$\begin{array}{ll}\text { Curtis } 1996 & \text { Allocation: randomised. } \\ & \text { Participants: people who had received inpatient psychiatric care (38.4\% schizophrenia). } \\ \text { Interventions: intensive outreach case management vs standard aftercare services, not supportive } \\ \text { therapy or supportive care. }\end{array}$

\begin{tabular}{ll} 
Czobor 1995 & $\begin{array}{l}\text { Allocation: randomised. } \\
\text { Participants: people with schizophrenia. } \\
\text { Interventions: risperidone vs placebo, not supportive therapy or supportive care. }\end{array}$ \\
\hline D'Ercole 1997 & $\begin{array}{l}\text { Allocation: randomised. } \\
\text { Participants: people with major psychiatric disorder (38\% schizophrenia). } \\
\text { Interventions: intensive outreach case management vs standard aftercare, not supportive therapy } \\
\text { or supportive care. }\end{array}$
\end{tabular}

Daumit 2010 Allocation: randomised. Participants: persons with severe mental illness, 25\% schizophrenia. Interventions: group exercise vs group exercise plus peer support. 


\begin{tabular}{ll}
\hline Study & Reason for exclusion \\
\hline Dixon 2002 & Allocation: randomised. \\
& Participants: people with severe mental illness. \\
& Interventions: individual placement and support vs enhanced vocational rehabilitation, not sup- \\
& portive therapy or supportive care.
\end{tabular}

\begin{tabular}{|c|c|}
\hline Donlon 1973 & $\begin{array}{l}\text { Allocation: randomised. } \\
\text { Participants: people with schizophrenia. } \\
\text { Interventions: group socialisation, refreshments and support versus individual supportive psy- } \\
\text { chotherapy; both interventions are supportive therapy or supportive care. }\end{array}$ \\
\hline
\end{tabular}

\begin{tabular}{ll}
\hline Drake 1993 & $\begin{array}{l}\text { Allocation: randomised. } \\
\text { Participants: people with severe mental illness and substance use disorder. } \\
\text { Interventions: social network treatment vs CBT, not supportive therapy or supportive care. }\end{array}$ \\
\hline Drake 1996 & $\begin{array}{l}\text { Allocation: randomised. } \\
\text { Participants: people with severe mental illness (46.9\% schizophrenia and related psychotic disor- } \\
\text { ders, } 42.7 \% \text { bipolar disorder and other severe mood disorders, } 10.5 \% \text { other disorders). } \\
\text { Interventions: group skills training vs individual placement and support, not supportive therapy or } \\
\text { supportive care. }\end{array}$
\end{tabular}

\begin{tabular}{|c|c|}
\hline Drake 1999 & $\begin{array}{l}\text { Allocation: randomised. } \\
\text { Participants: people with severe mental disorders ( } 67 \% \text { schizophrenia). } \\
\text { Interventions: individual placement and support vs enhanced vocational rehabilitation, not sup- } \\
\text { portive therapy or supportive care. }\end{array}$ \\
\hline
\end{tabular}

\begin{tabular}{ll}
\hline Drury 1996a & Allocation: randomised. \\
& Participants: people with non-affective psychosis. \\
& Interventions: individual and group cognitive therapy vs matched hours of therapy, not supportive \\
& therapy or supportive care. \\
\hline
\end{tabular}

Drury 1996b
$\begin{array}{ll}\text { Allocation: randomised. } \\ \text { Participants: people with non-affective psychosis. } \\ \text { Interventions: individual and group cognitive therapy vs matched hours of therapy, not supportive } \\ \text { therapy or supportive care. }\end{array}$

\begin{tabular}{ll}
\hline Drury 2000 & Allocation: randomised. \\
& Participants: people with non-affective psychosis. \\
& Interventions: cognitive therapy programme vs recreational activities and support, not supportive \\
therapy or supportive care.
\end{tabular}

\begin{tabular}{|c|c|}
\hline Eack 2007 & $\begin{array}{l}\text { Allocation:randomised. } \\
\text { Participants: people with schizophrenia. } \\
\text { Interventions: Enriched Supportive therapy (psycho-education and training) vs CBT. }\end{array}$ \\
\hline Eack 2010 & $\begin{array}{l}\text { Allocation:randomised. } \\
\text { Participants: people with schizophrenia. } \\
\text { Interventions: Enriched Supportive therapy (psycho-education and training) vs CBT. }\end{array}$ \\
\hline Eack 2011 & $\begin{array}{l}\text { Allocation:randomised. } \\
\text { Participants: people with schizophrenia. } \\
\text { Interventions: Enriched Supportive therapy (psycho-education and training) vs CBT. }\end{array}$ \\
\hline Eack 2012 & $\begin{array}{l}\text { Allocation: randomised. } \\
\text { Participants: people with schizophrenia. } \\
\text { Interventions: Enriched Supportive therapy (psycho-education and training) vs CBT. }\end{array}$ \\
\hline Essock 1995 & Allocation: randomised. \\
\hline
\end{tabular}




\begin{tabular}{ll}
\hline Study & Reason for exclusion \\
\hline & $\begin{array}{l}\text { Participants: people with serious mental disorder (67\% schizophrenia or schizoaffective disorder). } \\
\text { Interventions: assertive community treatment vs case management, not supportive therapy or } \\
\text { supportive care. }\end{array}$ \\
\hline Field 1997 & Allocation: not randomised. \\
\hline Ford 1995 & $\begin{array}{l}\text { Allocation: randomised. } \\
\text { Participants: people with mental illness (82\% schizophrenia). } \\
\text { Interventions: case management vs standard psychiatric services, not supportive therapy or sup- } \\
\text { portive care. }\end{array}$
\end{tabular}

\begin{tabular}{ll}
\hline Forsyth 1961 & Allocation: not randomised. \\
\hline Franklin 1987 & Allocation: randomised. \\
& $\begin{array}{l}\text { Participants: people with a minimum of two discharges from a mental hospital (56\% schizophre- } \\
\text { nia). } \\
\text { Interventions: case management services vs any services except case management, not supportive } \\
\text { therapy or supportive care. }\end{array}$
\end{tabular}

Freeman 1998

Allocation: randomised.

Participants: people with schizophrenia or schizoaffective disorder or delusional disorder. Interventions: standard care vs standard care and CBT, not supportive therapy or supportive care.

\begin{tabular}{ll}
\hline Gaither 2003 & $\begin{array}{l}\text { Allocation: randomised. } \\
\text { Participants: people with schizophrenia or schizoaffective disorder. } \\
\text { Interventions: cognitive behavioural intervention vs interpersonal attention control, not support- } \\
\text { ive therapy or supportive care. }\end{array}$ \\
\hline Garety 1994 & Allocation: not randomised. \\
\hline Glick 1974 & $\begin{array}{l}\text { Allocation: randomised. } \\
\text { Participants: people with schizophrenia. } \\
\text { Interventions: short term hospitalisation vs long-term hospitalisation, not supportive therapy or } \\
\text { supportive care. }\end{array}$
\end{tabular}

\begin{tabular}{ll}
\hline Glick 1975 & Allocation: randomised. \\
& Participants: people with schizophrenia. \\
Interventions: short term hospitalisation vs long-term hospitalisation, not supportive therapy or \\
supportive care.
\end{tabular}

\begin{tabular}{ll}
\hline Glick 1976a & Allocation: randomised. \\
& Participants: people with schizophrenia. \\
Interventions: short term hospitalisation vs long-term hospitalisation, not supportive therapy or \\
supportive care.
\end{tabular}

\begin{tabular}{ll}
\hline Glick 1976b & Allocation: randomised. \\
& Participants: people with schizophrenia. \\
& Interventions: short term hospitalisation vs long \\
& -term hospitalisation, not supportive therapy or supportive care. \\
\hline Glick 1976c & $\begin{array}{l}\text { Allocation: randomised. } \\
\text { Participants: people with schizophrenia. } \\
\text { Interventions: short term hospitalisation vs long-term hospitalisation, not supportive therapy or } \\
\text { supportive care. }\end{array}$ \\
\hline Glick 1977 & $\begin{array}{l}\text { Allocation: randomised. } \\
\text { Participants: people with schizophrenia. }\end{array}$ \\
\hline
\end{tabular}




\begin{tabular}{ll}
\hline Study & Reason for exclusion \\
\hline & $\begin{array}{l}\text { Interventions: short term hospitalisation vs long-term hospitalisation, not supportive therapy or } \\
\text { supportive care. }\end{array}$
\end{tabular}

\section{Glick 1979}

Allocation: randomised.

Participants: people admitted to a psychiatric inpatient ward (60\% schizophrenia). Interventions: short term hospitalisation vs long-term hospitalisation, not supportive therapy or supportive care.

\begin{tabular}{ll}
\hline Glynn 2001 & $\begin{array}{l}\text { Allocation: randomised. } \\
\text { Participants: people with schizophrenia. } \\
\text { Interventions: clinic skills training vs clinic skills training with manualised weekly community in- } \\
\text { struction to generalise skills to the natural environment, not supportive therapy or supportive care. }\end{array}$ \\
\hline Grassi 2001 & Allocation: not randomised. \\
\hline Grawe 1998 & $\begin{array}{l}\text { Allocation: randomised. } \\
\text { Participants: people with schizophrenia. } \\
\text { Interventions: optimal multimodal treatment vs treatment as usual, not supportive therapy or } \\
\text { supportive care. }\end{array}$ \\
\hline Haddock 2000a & $\begin{array}{l}\text { Allocation: randomised. } \\
\text { Participants: people with schizophrenia and substance misuse. } \\
\text { Interventions: individual CBT vs family support, not supportive therapy or supportive care. }\end{array}$ \\
\hline Haddock 2000b & $\begin{array}{l}\text { Allocation: randomised. } \\
\text { Participants: people with schizophrenia or schizoaffective disorder or delusional disorder and al- } \\
\text { cohol or drug abuse. } \\
\text { Interventions: individual CBT vs family support, not supportive therapy or supportive care. }\end{array}$
\end{tabular}

\begin{tabular}{ll}
\hline Hafner 1983 & Allocation: randomised. \\
& Participants: people with severe and persisting psychiatric disorders, the majority did not have \\
& schizophrenia. \\
& Interventions: couples therapy vs individual therapy. \\
\hline Haldun 2002 & $\begin{array}{l}\text { Allocation: randomised. } \\
\text { Participants: people with schizophrenia. } \\
\text { Interventions: optimal clinical management vs routine case management, not supportive therapy } \\
\text { or supportive care. }\end{array}$
\end{tabular}

Hannes 1974 Allocation: randomised. Participants: people with schizophrenia. Interventions: recreational therapy vs relaxation period, not supportive therapy or supportive care.

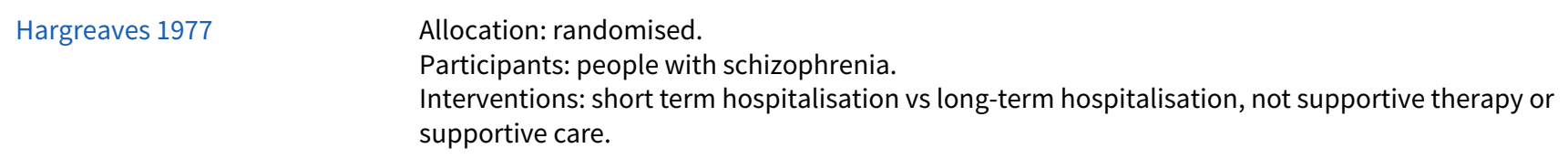

Allocation: randomised.

Participants: people who are heavy users of inpatient psychiatric services (majority are people with schizophrenia or schizotypal disorders or delusional disorders).

Interventions: enhanced community management vs standard care, not supportive therapy or supportive care.

Participants: relatives of people with psychosis. 


\begin{tabular}{|c|c|}
\hline Study & Reason for exclusion \\
\hline & Interventions: standard case management vs intensive case management. \\
\hline Herz 1974 & $\begin{array}{l}\text { Allocation: randomised. } \\
\text { Participants: people with psychiatric illness (66\% schizophrenia). } \\
\text { Interventions: individual supportive psychotherapy versus group supportive psychotherapy. }\end{array}$ \\
\hline Herz 2000 & $\begin{array}{l}\text { Allocation: randomised. } \\
\text { Participants: people with schizophrenia or schizoaffective disorder. } \\
\text { Interventions: program for relapse prevention vs treatment as usual, supportive therapy received } \\
\text { by all participants. }\end{array}$ \\
\hline Hogarty 1973 & $\begin{array}{l}\text { Allocation: randomised. } \\
\text { Participants: people with schizophrenia. } \\
\text { Interventions: major role therapy and chlorpromazine vs major role therapy and placebo vs chlor- } \\
\text { promazine vs placebo, not supportive therapy or supportive care. }\end{array}$ \\
\hline
\end{tabular}

\begin{tabular}{ll}
\hline Hogarty 1977 & Allocation: randomised. \\
& Participants: people with schizophrenia. \\
& Interventions: major role therapy and chlorpromazine vs major role therapy and placebo vs chlor- \\
& promazine vs placebo, not supportive therapy or supportive care.
\end{tabular}

Hogarty $1984 \quad \begin{aligned} & \text { Allocation: randomised. } \\ & \text { Participants: people with schizophrenia. } \\ & \text { Interventions: major role therapy and chlorpromazine vs major role therapy and placebo vs chlor- } \\ & \text { promazine vs placebo, not supportive therapy or supportive care. }\end{aligned}$

\begin{tabular}{ll}
\hline Hogarty 1986a & Allocation: randomised. \\
& Participants: people with schizophrenia. \\
& Interventions: family treatment and medication vs social skills training and medication vs family \\
& treatment + social skills training + medication vs medication.
\end{tabular}

\begin{tabular}{ll}
\hline Hogarty 1986b & Allocation: randomised. \\
& Participants: people with schizophrenia. \\
& Interventions: family treatment and medication vs social skills training and medication vs family \\
& treatment + social skills training + medication vs medication.
\end{tabular}

\begin{tabular}{ll}
\hline Hogarty 1986c & Allocation: randomised. \\
& Participants: people with schizophrenia or schizoaffective disorder. \\
& Interventions: family treatment and medication vs social skills training and medication vs family \\
& treatment + social skills training + medication vs medication, not supportive therapy or supportive \\
& care.
\end{tabular}

\begin{tabular}{ll}
\hline Hogarty 1988 & Allocation: randomised. \\
& Participants: people with schizophrenia. \\
& Interventions: standard dose of fluphenazine decanoate vs minimal dose, not supportive therapy \\
& or supportive care.
\end{tabular}

\begin{tabular}{ll}
\hline Hornung 1998 & Allocation: randomised. \\
& Participants: people with schizophrenia. \\
& Interventions: psychoeducational medication management vs cognitive psychotherapy vs key per- \\
& son counselling, not supportive therapy or supportive care.
\end{tabular}

\begin{tabular}{ll}
\hline Hornung 1999 & Allocation: randomised. \\
& Participants: people with schizophrenia. \\
& Interventions: psychoeducational medication management vs cognitive psychotherapy vs key per- \\
& son counselling, not supportive therapy or supportive care.
\end{tabular}




\begin{tabular}{ll}
\hline Study & Reason for exclusion \\
\hline & $\begin{array}{l}\text { Participants: people with severe mental illness (55.4\% schizophrenia). } \\
\text { Interventions: access to rent subsidy certificate vs no access to rent subsidy certificate vs tradition- } \\
\text { al services vs comprehensive services, not supportive therapy or supportive care. }\end{array}$ \\
\hline Huxley 2001 & $\begin{array}{l}\text { Allocation: randomised. } \\
\text { Participants: people with psychosis. } \\
\text { Interventions: intensive case management vs standard case management, not supportive therapy } \\
\text { or supportive care. }\end{array}$ \\
\hline $\begin{array}{l}\text { Allocation: partially randomised (participants able to choose their preferred treatment option or } \\
\text { elect to be randomised to a treatment option. } \\
\text { Participants: people with schizophrenia. } \\
\text { Interventions: peer support and CBT vs peer support, CBT and group sessions. }\end{array}$
\end{tabular}

$\begin{array}{ll}\text { Isrctn69299093 } 2011 & \text { Allocation: randomised. } \\ & \text { Participants: family members of people with psychotic symptoms. } \\ & \text { Interventions: Relatives' Education and Coping Toolkit (REACT) vs CBT oriented self management } \\ & \text { intervention. }\end{array}$

Isrctn967547632011
Allocation:randomised.
Participants: staff working on acute mental health wards.
Interventions: modified cognitive analytic therapy approach to clinical supervision vs inactive con-
trol arm.

\begin{tabular}{|c|c|}
\hline Issakidis 1999 & $\begin{array}{l}\text { Allocation: randomised. } \\
\text { Participants: people with schizophrenia or bipolar disorder. } \\
\text { Interventions: intensive case management vs standard case management, not supportive therapy } \\
\text { or supportive care. }\end{array}$ \\
\hline
\end{tabular}

$\begin{array}{ll}\text { Jones } 1994 & \text { Allocation: randomised. } \\ & \text { Participants: people with a DSM-III-R Axis I diagnosis (63\% schizophrenia). } \\ & \text { Interventions: critical time intervention vs usual treatment services for homeless people with se- } \\ & \text { vere mental illness, not supportive therapy or supportive care. }\end{array}$

$\begin{array}{ll}\text { Kaplan } 2011 & \text { Allocation:randomised. } \\ & \text { Participants: people with schizophrenia. } \\ & \text { Interventions: experimental peer support listserv (group distribution email list) vs experimental } \\ & \text { peer support online bulletin vs waiting list control group. }\end{array}$

\begin{tabular}{ll}
\hline Karon 1969 & Allocation: randomised. \\
& Participants: people with schizophrenia. \\
Interventions: supportive therapy + medication versus 'active' psychoanalytic therapy + no med- \\
ication versus 'ego-analytic' psychoanalytic therapy + medication as an adjunct; intervention \\
groups received different medication regimes therefore results are confounded.
\end{tabular}

$\begin{array}{ll}\text { Kaufmann } 1995 & \text { Allocation: randomised. } \\ & \text { Participants: people with schizophrenia or schizoaffective disorder or major affective disorder. } \\ & \text { Interventions: self help employment centre services vs customary community service, not sup- } \\ \text { portive therapy or supportive care. }\end{array}$

\begin{tabular}{ll}
\hline Keith 1977 & Allocation: randomised. \\
& Participants: rehabilitation service clients. \\
& Interventions: innovative training program vs usual service + outcome measures vs usual services \\
& only, not supportive therapy or supportive care.
\end{tabular}

Kern $2002 \quad$ Allocation: randomised.

Participants: people with schizophrenia or schizoaffective disorder. 


\begin{tabular}{ll}
\hline Study & Reason for exclusion \\
\hline & $\begin{array}{l}\text { Interventions: work training by errorless learning vs conventional instruction, not supportive ther- } \\
\text { apy or supportive care. }\end{array}$
\end{tabular}

\section{Keshavan 2011}

Allocation: randomised.

Participants: people with schizophrenia or schizoaffective disorder; criteria not reported.

Interventions: cognitive enhancement therapy vs. supportive therapy.

Outcomes: no usable data, abstract only.

\begin{tabular}{ll}
\hline Kim 1997 & Allocation: not randomised. \\
\hline Klingberg 1999 & Allocation: randomised. \\
& Participants: people with schizophrenia. \\
& Interventions: psychoeducational medication management training vs cognitive psychotherapy vs \\
key-person counselling, not supportive therapy or supportive care.
\end{tabular}

Klingberg 2010

Allocation: randomised.

Participants: schizophrenia, schizophreniform disorder, schizoaffective disorder, delusional disorder.

Interventions: CBT vs non-specific supportive therapy.

Outcomes: no usable data.

Koivunen $2010 \quad \begin{aligned} & \text { Allocation:randomised. } \\ & \text { Participants: nurses on acute psychiatric wards. } \\ & \text { Interventions: web-based patient support system vs standard patient education sessions with } \\ & \text { written material. }\end{aligned}$

Kris 1965

Allocation: randomised.

Participants: people who have previously been in a psychiatric hospital, with severe psychotic relapse.

Interventions: day hospital vs state mental hospital, not supportive therapy or supportive care.

\begin{tabular}{ll}
\hline Kuipers 1998 & Allocation: randomised. \\
& Participants: people with schizophrenia or delusional disorder or schizoaffective disorder. \\
Interventions: CBT vs standard care, not supportive therapy or supportive care.
\end{tabular}

Lecomte 1999

Allocation: randomised.

Participants: people with schizophrenia or schizoaffective disorder.

Interventions: self-esteem module vs standard care, not supportive therapy or supportive care.

Lehman 1993

Allocation: randomised.

Participants: people with schizophrenia or schizoaffective disorder or major affective disorder and substance use disorder.

Interventions: innovative group and intensive case management + usual community mental health centre and rehabilitation services vs usual community mental health centre and rehabilitation services, not supportive therapy or supportive care.

Lehman $2001 \quad$ Allocation: randomised.
Participants: people with severe mental illness (70\% schizophrenia).
Interventions: Individual Placement and Support (IPS) employment program vs comparison psy-
chosocial program, not supportive therapy or supportive care.

Lehman 2002a

Allocation: randomised.

Participants: people with severe mental illness ( $75 \%$ chronic psychoses). 


\begin{tabular}{ll}
\hline Study & Reason for exclusion \\
\hline & $\begin{array}{l}\text { Interventions: Individual Placement and Support (IPS) employment program vs psychosocial reha- } \\
\text { bilitation program, not supportive therapy or supportive care. }\end{array}$
\end{tabular}

\begin{tabular}{ll}
\hline Lehman 2002b & Allocation: randomised. \\
& Participants: people with severe mental illness (75\% chronic psychoses). \\
& Interventions: Individual Placement and Support (IPS) employment program vs psychosocial reha- \\
bilitation program, not supportive therapy or supportive care.
\end{tabular}

Levene 1989
$\begin{aligned} & \text { Allocation: randomised. } \\ & \text { Participants: people with schizophrenia. } \\ & \text { Interventions: focal family therapy vs supportive management counselling, not supportive therapy } \\ & \text { or supportive care. }\end{aligned}$

\begin{tabular}{ll}
\hline Lewis 2000 & $\begin{array}{l}\text { Allocation: randomised. } \\
\text { Participants: people with schizophrenia and substance use. } \\
\text { Interventions: family support vs CBT, not supportive therapy or supportive care. }\end{array}$ \\
\hline Lewis 2001a & $\begin{array}{l}\text { Allocation: randomised. } \\
\text { Participants: people with schizophrenia. } \\
\text { Interventions: CBT + drug treatment vs drug treatment, not supportive therapy or supportive care. }\end{array}$ \\
\hline
\end{tabular}

Lindenmayer $1995 \quad$ Allocation: randomised.

Participants: people with schizophrenia.

Interventions: placebo vs haloperidol vs risperidone, not supportive therapy or supportive care.

Linszen 1994

Allocation: randomised.

Participants: people with schizophrenia or schizophreniform disorder or schizoaffective disorder or other schizophrenia-like psychotic disorder.

Interventions: individual treatment versus family treatment + individual treatment.

\begin{tabular}{|c|c|}
\hline Linszen 1996 & $\begin{array}{l}\text { Allocation: randomised. } \\
\text { Participants: people with schizophrenia. } \\
\text { Interventions: individual orientated intervention vs individual and family orientated intervention, } \\
\text { not supportive therapy or supportive care. }\end{array}$ \\
\hline
\end{tabular}

\begin{tabular}{ll}
\hline Linszen 1997 & Allocation: randomised. \\
& Participants: people with schizophrenia or schizophreniform disorder or schizoaffective disorder \\
& or other psychotic disorder. \\
& Interventions: individual treatment versus family treatment + individual treatment, not supportive \\
therapy or supportive care.
\end{tabular}

Linszen $1998 \quad$ Allocation:randomised.

Participants: people with schizophrenia.

Interventions: individual treatment versus family treatment + individual treatment, not supportive therapy or supportive care.

Linszen 2009
$\begin{aligned} & \text { Allocation:randomised. } \\ & \text { Participants: people with schizophrenia and parents of schizophrenic patients. } \\ & \text { Interventions: Continuity of Treatment by professionals Specialized in the treatment of early schiz- } \\ & \text { ophrenia (CST) vs Continuity of Specialized Treatment plus Parent groups (CSTpP) vs Continuity of } \\ & \text { Treatment as Usual (CTU). }\end{aligned}$
$\begin{aligned} & \text { Allocation: randomised. } \\ & \text { Participants: people with serious mental illness (46\% schizophrenia, } 22 \% \text { affective disorder). } \\ & \text { Interventions: team case management vs rehabilitation program, not supportive therapy or sup- } \\ & \text { portive care. }\end{aligned}$




\begin{tabular}{ll}
\hline Study & Reason for exclusion \\
\hline & Participants: people with schizophrenia. \\
Interventions: risperidon vs placebo, not supportive therapy or supportive care.
\end{tabular}

\begin{tabular}{ll}
\hline Marder 1994b & $\begin{array}{l}\text { Allocation: randomised. } \\
\text { Participants: people with schizophrenia. } \\
\text { Interventions: oral fluphenazine vs placebo, not supportive therapy or supportive care. }\end{array}$ \\
\hline Marder 2001b & $\begin{array}{l}\text { Allocation: randomised. } \\
\text { Participants: people with schizophrenia or schizoaffective disorder. } \\
\text { Interventions: Individual Placement and Support + Psychoeducational Skills Training vs Individual } \\
\text { Placement and Support, not supportive therapy or supportive care. }\end{array}$ \\
\hline
\end{tabular}

Allocation: randomised.

Participants: family members of people with schizophrenic disorder.

Interventions: skills training and education vs education only.

May 1976a
Pllocation: randomised.
Participants: people with schizophrenia.
Interventions: individual psychotherapy vs ataraxic drugs vs individual psychotherapy + ataraxic
drugs vs ECT vs milieu treatment alone, not supportive therapy or supportive care.

\begin{tabular}{ll}
\hline McEvoy 1994 & Allocation: randomised. \\
& Participants: people with
\end{tabular}

Participants: people with schizophrenia.

Interventions: risperidone vs haloperidol vs placebo, not supportive therapy or supportive care.

\begin{tabular}{ll} 
McFarlane 1992 & Allocation: not randomised. \\
\hline McFarlane 2002 & Allocation: randomised. \\
& Participants: people with disabling psychiatric disorder. \\
Interventions: Mental Health Employers Consortium vs conventional methods of supported em- \\
ployment, not supportive therapy or supportive care.
\end{tabular}

McGorry $1999 \quad$ Allocation: randomised.

Participants: people with an at risk mental state.

Interventions: supportive psychotherapy alone (control group) or specific cognitive psychotherapy plus low dose risperidone.

Outcome: no usable data, abstract only.

Allocation: randomised.

Participants: people with schizophrenia.

Interventions: risperidone and cognitive therapy vs supportive case management. Outcome: no usable data, abstract only. port-IPS (ACT-IPS), not supportive therapy or supportive care. 


\begin{tabular}{|c|c|}
\hline Study & Reason for exclusion \\
\hline & $\begin{array}{l}\text { Interventions: group-based motivational behavioural therapy for drug abuse vs supportive treat- } \\
\text { ment for addiction recovery. } \\
\text { Outcomes: no usable data. }\end{array}$ \\
\hline Miknyak 2001 & $\begin{array}{l}\text { Allocation: not stated. } \\
\text { Participants: people with schizophrenia. } \\
\text { Interventions: risperidone vs fluanxol-depot vs risperidone + fluanxol-depot, not supportive thera- } \\
\text { py or supportive care. }\end{array}$ \\
\hline Morse 1997 & $\begin{array}{l}\text { Allocation: randomised. } \\
\text { Participants: people with a serious DSM-III-R axis I diagnosis ( } 66 \% \text { schizophrenia). } \\
\text { Interventions: broker care management vs assertive community treatment vs assertive communi- } \\
\text { ty treatment + support from community workers, not supportive therapy or supportive care. }\end{array}$ \\
\hline Mosher 1975 & Allocation: not randomised. \\
\hline Mueser 2001a & $\begin{array}{l}\text { Allocation: randomised. } \\
\text { Participants: people with schizophrenia or other severe mental illness. } \\
\text { Interventions: Individual Placement and Support (IPS) vs psychiatric rehabilitation program vs } \\
\text { standard services, not supportive therapy or supportive care. }\end{array}$ \\
\hline
\end{tabular}

Allocation: randomised.

Participants: people with schizophrenia or schizoaffective disorder or schizophreniform disorder. Interventions: supportive family treatment vs applied family therapy, not supportive therapy or supportive care.

\begin{tabular}{|c|c|}
\hline NCT 2005 & $\begin{array}{l}\text { Allocation: randomised. } \\
\text { Participants: people with schizophrenia. } \\
\text { Interventions: Health Care Management and Supported Rehabilitation (includes skills training) vs } \\
\text { standard treatment. }\end{array}$ \\
\hline NCT 2005a & $\begin{array}{l}\text { Allocation: randomised. } \\
\text { Participants: people with schizophrenia and their families. } \\
\text { Interventions: online advice from healthcare professionals, social support, and information vs } \\
\text { standard treatment. }\end{array}$ \\
\hline NCT 2009 & $\begin{array}{l}\text { Allocation: randomised. } \\
\text { Participants: people with schizophrenia. } \\
\text { Interventions: support group vs mindfulness meditation. }\end{array}$ \\
\hline NCT 2009a & $\begin{array}{l}\text { Allocation: randomised. } \\
\text { Participants: family of people with schizophrenia. } \\
\text { Interventions: family led mutual support group vs psycho-education and standard treatment. }\end{array}$ \\
\hline NCT 2012a & $\begin{array}{l}\text { Allocation: randomised. } \\
\text { Participants: people with schizophrenia. } \\
\text { Interventions: Enriched Supportive therapy (psycho-education and training) vs Cognitive Enhance- } \\
\text { ment therapy. }\end{array}$ \\
\hline Nelson 2007 & $\begin{array}{l}\text { Allocation: randomised. } \\
\text { Participants: ultra high risk criteria for psychotic disorder. } \\
\text { Interventions: low- dose risperidone + intensive CBT-based psychological treatment vs placebo + } \\
\text { intensive CBT-based psychological treatment vs placebo + control psychological treatment (sup- } \\
\text { portive therapy). } \\
\text { Outcomes: no usable data, abstract only. }\end{array}$ \\
\hline Nugter $1997 a$ & Allocation: randomised. \\
\hline
\end{tabular}




\begin{tabular}{|c|c|}
\hline Study & Reason for exclusion \\
\hline & $\begin{array}{l}\text { Participants: people with schizophrenia or schizophreniform disorder or schizoaffective disorder } \\
\text { or other psychotic disorders. } \\
\text { Interventions: individual treatment versus individual treatment + behavioural family treatment. }\end{array}$ \\
\hline Nugter 1997b & $\begin{array}{l}\text { Allocation: randomised. } \\
\text { Participants: people with schizophrenia or schizophreniform disorder or schizoaffective disorder } \\
\text { or other psychotic disorders. } \\
\text { Interventions: individual treatment vs individual treatment + family treatment, not supportive } \\
\text { therapy or supportive care. }\end{array}$ \\
\hline O'Donnell 2002 & $\begin{array}{l}\text { Allocation: randomised. } \\
\text { Participants: people with schizophrenia. } \\
\text { Interventions: compliance therapy vs non-specific counselling therapy, not supportive therapy or } \\
\text { supportive care. }\end{array}$ \\
\hline Ogrodniczuk 2000 & $\begin{array}{l}\text { Allocation: randomised. } \\
\text { Participants: people with major depression or dysthymia or personality disorder. }\end{array}$ \\
\hline Ohlenschlaeger 2002 & $\begin{array}{l}\text { Allocation: randomised. } \\
\text { Participants: people with schizophrenia or schizotypal disorders or delusional disorders. } \\
\text { Interventions: standard treatment vs hospital based rehabilitation, not supportive therapy or sup- } \\
\text { portive care. }\end{array}$ \\
\hline Philipps 2001 & $\begin{array}{l}\text { Allocation: randomised. } \\
\text { Participants: young people identified as being at high risk of psychosis. } \\
\text { Interventions: combined medical and psychological (specific) approach vs supportive (non-specif- } \\
\text { ic) case management. }\end{array}$ \\
\hline
\end{tabular}

Phillips 1999

Allocation: randomised.

Participants: young people identified as being at high risk of psychosis.

Interventions: combined medical and psychological approach vs supportive case management.

\begin{tabular}{ll}
\hline Pickett 1997 & Allocation: not randomised. \\
\hline Pushkaryova 1999 & Allocation: randomised. \\
& Participants: people with psychosis. \\
& Interventions: enterosorption of silicorganic enterosorbent vs placebo, not supportive therapy or \\
& supportive care. \\
\hline Razali 1995 & Allocation: randomised. \\
& Participants: people with schizophrenia. \\
Interventions: counselled by trained hospital pharmacist vs no counselling, not supportive therapy & or supportive care.
\end{tabular}

Rector 2002

Allocation: randomised.

Participants: people with schizophrenia.

Interventions: cognitive therapy vs standard care, not supportive therapy or supportive care.

\section{Reynolds 2002}

\section{Allocation: randomised.}

Participants: people discharged from a psychiatric inpatient unit.

Interventions: transitional discharge model vs standard discharge care, not supportive therapy or supportive care.
Ritch 2001
Allocation: randomised.

Participants: people with schizophrenia or schizoaffective disorder. 


\begin{tabular}{ll}
\hline Study & Reason for exclusion \\
\hline & Interventions: Cognitive Adaptation Training vs a condition designed to control for therapist time \\
and provide environmental changes not associated with cognitive deficits vs assessment only., not \\
supportive therapy or supportive care.
\end{tabular}

Ritch $2002 \quad \begin{aligned} & \text { Allocation: randomised. } \\ & \text { Participants: people with high service utilisation. } \\ & \text { Interventions: Assertive Community Treatment vs Cognitive Adaptation Training, not supportive } \\ & \text { therapy or supportive care. }\end{aligned}$

\begin{tabular}{ll}
\hline Ro-Trock 1977 & Allocation: randomised. \\
& Participants: adolescents with schizophrenic reaction (57\%), adolescent adjustment reaction or \\
& drug problem \\
& Interventions: family therapy vs individual therapy, not supportive therapy or supportive care.
\end{tabular}

\begin{tabular}{ll}
\hline Rosenheck 2003 & Allocation: randomised. \\
& Participants: people with major psychiatric disorder or an alcohol or drug abuse disorder or both \\
& $\sim 10 \%$ serious psychiatric disorders, $\sim 50 \%$ alcohol or drug disorders, $\sim 35 \%$ dual diagnoses, $\sim 5 \%$ \\
& other psychiatric diagnoses). \\
& Interventions:supported housing program + intensive case management vs case management only \\
& vs standard care.
\end{tabular}

Rosenthal $2000 \quad \begin{aligned} & \text { Allocation: randomised. } \\ & \text { Participants: people with schizophrenia and substance abuse. } \\ & \text { Interventions: outpatient group therapy vs group therapy plus Targeted Assertive Outreach visits, } \\ & \text { all participants received group therapy. }\end{aligned}$

\begin{tabular}{|c|c|}
\hline Ruan 2008 & $\begin{array}{l}\text { Allocation:randomised. } \\
\text { Participants: people with schizophrenia. } \\
\text { Interventions: clozapine vs clozapine plus fluoxetine, both groups received supportive therapy. }\end{array}$ \\
\hline Serok 1984 & $\begin{array}{l}\text { Allocation: randomised. } \\
\text { Participants: people with schizophrenia. } \\
\text { Interventions: Gestalt therapy vs care as usual, not supportive therapy or supportive care. }\end{array}$ \\
\hline Shi 2000 & $\begin{array}{l}\text { Allocation: not clear. } \\
\text { Participants: people with schizophrenia. } \\
\text { Interventions: family therapy vs control, not supportive therapy or supportive care. }\end{array}$ \\
\hline Shin 2002 & $\begin{array}{l}\text { Allocation: randomised. } \\
\text { Participants: people with schizophrenia. } \\
\text { Interventions: psycho-educational group programme + individual supportive therapy vs individual } \\
\text { supportive therapy alone. }\end{array}$ \\
\hline
\end{tabular}

\begin{tabular}{|c|c|}
\hline Slavinsky 1982 & Allocation: randomised (but allocation concealment inadequate). \\
\hline Smith 1999 & $\begin{array}{l}\text { Allocation: randomised. } \\
\text { Participants: people with schizophrenia. } \\
\text { Interventions: supportive group psychotherapy vs skills training. } \\
\text { Outcomes: no usable data. }\end{array}$ \\
\hline Solomon 1994a & $\begin{array}{l}\text { Allocation: randomised. } \\
\text { Participants: people with serious mental illness ( } 85 \% \text { schizophrenia). } \\
\text { Interventions: intensive case management by an assertive community team vs intensive case man- } \\
\text { agement by individual case managers vs community mental health centre, not supportive therapy } \\
\text { or supportive care. }\end{array}$ \\
\hline
\end{tabular}

Solomon 1994b Allocation: randomised.




\begin{tabular}{|c|c|}
\hline Study & Reason for exclusion \\
\hline & $\begin{array}{l}\text { Participants: people with serious mental illness ( } 85.9 \% \text { schizophrenia). } \\
\text { Interventions: consumer case managers vs non-consumer case management, not supportive ther- } \\
\text { apy or supportive care. }\end{array}$ \\
\hline Solomon 1995a & $\begin{array}{l}\text { Allocation: randomised. } \\
\text { Participants: people with serious mental illness ( } 86 \% \text { schizophrenia). } \\
\text { Interventions: consumer case managers vs non-consumer case management, not supportive ther- } \\
\text { apy or supportive care, not supportive therapy or supportive care. }\end{array}$ \\
\hline Solomon 1995b & $\begin{array}{l}\text { Allocation: randomised. } \\
\text { Participants: people with schizophrenia (85\%) or major affective disorder (14\%) or unspecified } \\
\text { psychotic disorder (1\%). } \\
\text { Interventions: consumer case managers vs non-consumer case management, not supportive ther- } \\
\text { apy or supportive care. }\end{array}$ \\
\hline
\end{tabular}

Solomon 1996

Allocation: randomised.

Participants: people with serious mental illness.

Interventions: consumer case managers vs non-consumer case management, not supportive therapy or supportive care.

$\begin{array}{ll}\text { Stant } 2011 & \text { Allocation:randomised. } \\ \text { Participants: people with schizophrenia. } \\ \text { Interventions: peer support group vs standard treatment. }\end{array}$

Stroup $2003 \quad$ Allocation: randomised.

Participants: people with schizophrenia.

Interventions: typical antipsychotics vs atypical antipsychotics, not supportive therapy or supportive care.

Allocation: randomised.

Participants: people with schizophrenia.

Interventions: social support therapy (includes psycho-education, delivered by a group of people) vs standard treatment.

$\begin{array}{ll}\text { Tarrier 2000a } & \text { Allocation: randomised. } \\ & \text { Participants: people with schizophrenia and substance misuse. } \\ \text { Interventions: family support vs cognitive service, not supportive therapy or supportive care. }\end{array}$

$\begin{array}{ll}\text { Tarrier 2000d } & \text { Allocation: randomised. } \\ & \text { Participants: support workers with care load of people with schizophrenia. } \\ \text { Interventions: training in family support vs not reported (trial register). }\end{array}$

Telles $1995 \quad$ Allocation: randomised.

Participants: people with schizophrenia.

Interventions: standard case management vs behavioural family management.

Outcomes: no usable data.

Test 1991

Allocation: randomised.

Participants: people with schizophrenia or schizoaffective disorder or schizotypal personality. Interventions: training in community living vs case management, not supportive therapy or supportive care.

Theilemann 1993

Allocation: randomised.

Participants: people with schizophrenia or schizoaffective disorder.

Interventions: "place-train" supported employment strategies vs employment strategies along with intensive interventions, not supportive therapy or supportive care. 


\begin{tabular}{ll}
\hline Study & Reason for exclusion \\
\hline Tong Shouming 2010 & Allocation: not randomised. \\
\hline Toprac 2002 & $\begin{array}{l}\text { Allocation: randomised. } \\
\text { Participants: people with serious mental illness. } \\
\text { Interventions: integrated supported employment vs group skills training, not supportive therapy } \\
\text { or supportive care. }\end{array}$ \\
\hline Allocation: randomised. \\
Participants: people with severe mental disorders (46.9\% chronic psychotic illnesses, $42.7 \%$ severe \\
affective disorders, $10.4 \%$ other disorders). \\
Interventions: $X X$ vs $Y$ YY, not supportive therapy or supportive care.
\end{tabular}
support vs assessment only, not supportive therapy or supportive care.

Tyrer 1995 Allocation: randomised.
Participants: people on a register for vulnerable psychiatric patients (54\% schizophrenia).
Interventions: supervision by nominated key workers vs standard care, not supportive therapy or
supportive care.

\begin{tabular}{ll}
\hline Vaughan 1992 & $\begin{array}{l}\text { Allocation: randomised. } \\
\text { Participants: people with schizophrenia. } \\
\text { Interventions: relatives' counselling + standard after-care versus standard after-care alone; not } \\
\text { supportive therapy or supportive care. }\end{array}$ \\
\hline Velligan 1999 & $\begin{array}{l}\text { Allocation: randomised. } \\
\text { Participants: people with schizophrenia or schizoaffective disorder. } \\
\text { Interventions: cognitive adaptation training vs attention control vs follow-up only, not supportive } \\
\text { therapy or supportive care. }\end{array}$
\end{tabular}

$\begin{array}{ll}\text { Walker } 1969 & \text { Allocation: randomised. } \\ & \text { Participants: psychiatric inpatients (50\% schizophrenic reaction). } \\ & \text { Interventions: community hospital industrial rehabilitation placement vs waiting list, not support- } \\ & \text { ive therapy or supportive care. }\end{array}$

$\begin{array}{ll}\text { Wallace } 1985 & \text { Allocation: randomised. } \\ & \text { Participants: people with schizophrenia. } \\ & \text { Interventions: intensive social skills training vs holistic health therapy, not supportive therapy or } \\ & \text { supportive care. }\end{array}$

\begin{tabular}{ll}
\hline Wallace 2001 & Allocation: randomised. \\
& Participants: people with a serious and persistent mental illness. \\
& Interventions: Individual Placement and Support vs Individual Placement and Support + work- \\
place fundamentals module, not supportive therapy or supportive care.
\end{tabular}

\begin{tabular}{ll}
\hline Weinman 1974 & Allocation: randomised. \\
& Participants: people with schizophrenia or functional psychosis. \\
Interventions: community placement vs hospital based socio-environmental condition, not sup- \\
portive therapy or supportive care.
\end{tabular}

\begin{tabular}{ll}
\hline Wojciechowska 2001 & Allocation: not randomised. \\
\hline Wolkon 1971 & Allocation: randomised. \\
& Participants: people recently discharged from psychiatric hospital with schizophrenia (78\%) or \\
& psychotic disorder or non-psychotic disorder.
\end{tabular}




\begin{tabular}{ll}
\hline Study & Reason for exclusion \\
\hline Interventions: social rehabilitation program vs control group, not supportive therapy or supportive \\
care.
\end{tabular}

CBT - cognitive behavioural therapy

DSM-III - Diagnostic and Statistical Manual third edition

ECT - electroconvulsive therapy

Characteristics of studies awaiting assessment [ordered by study ID]

Bechdolf 2012

\begin{tabular}{ll}
\hline Methods & Allocation: randomised. \\
Blindness: unclear. \\
Duration: 6 months. \\
Design: parallel. \\
Setting: inpatient. \\
Country: Germany. \\
\hline Participants & N = 60. \\
& Age: unclear. \\
& Sex: unclear. \\
& History: unclear. \\
& Exclusions: unclear. \\
\hline Interventions & 1. Motivational interviewing: four sessions. N= unclear. \\
& 2. Supportive therapy: four sessions. $\mathrm{N}=$ unclear. \\
\hline Outcomes & Unclear. \\
\hline Notes & Paper in German, awaiting translation \\
\hline
\end{tabular}


Blankertz 1997

\begin{tabular}{ll}
\hline Methods & Unclear. \\
\hline Participants & Unclear. \\
\hline Interventions & Unclear. \\
\hline Outcomes & Unclear. \\
\hline Notes & Waiting for a translation of the paper \\
\hline
\end{tabular}

Falloon 1983

\begin{tabular}{ll}
\hline Methods & Unclear. \\
\hline Participants & Unclear. \\
\hline Interventions & Unclear. \\
\hline Outcomes & Unclear. \\
\hline Notes & No full text available. \\
\hline
\end{tabular}

Characteristics of ongoing studies [ordered by study ID]

\section{ACTRN126100002410332010}

Trial name or title A pilot project investigating the feasibility and effects of a 6-month peer support program commencing 3 months prior to discharge from a specialist first-episode psychosis treatment centre.

\begin{tabular}{ll}
\hline Methods & $\begin{array}{l}\text { Randomised controlled trial (computer-generated random numbers). Parallel assignment. Out- } \\
\text { come assessors: blinded. }\end{array}$ \\
\hline Participants & First-episode psychosis. \\
\hline Interventions & Intervention: Individual peer support. \\
& Control: Standard care. \\
\hline Outcomes & Primary outcome: Increased service satisfaction, as measured by the Verona Service Satisfaction \\
& Scale. \\
& Secondary outcome: Reduced risk of suicide, as measured by The Reynolds Suicidal Ideation \\
& Questionnaire. \\
\hline Starting date & $1 / 6 / 2010$. \\
\hline Contact information & Jo Robinson \\
& Orygen Youth Health Research Centre \\
& 35 Poplar Road \\
& Parkville, \\
Vic, 3052 & Australia \\
+61393422866 \\
+61393422941 \\
jo.robinson@mh.org.au
\end{tabular}


Trial name or title Peer-delivered support intervention for people who hear voices: Pilot randomised controlled trial.

\begin{tabular}{ll}
\hline Methods & Randomised controlled trial using computerised sequence generation. \\
& Blinded by masking. \\
& Crossover assignment. \\
\hline Participants & $\begin{array}{l}18-65 \text { years old, Auditory verbal hallucinations at least weekly over the past six months associated } \\
\text { with significant distress or disability. }\end{array}$ \\
\hline Interventions & $\begin{array}{l}\text { Intervention: } 12 \text { weekly one hour one-to-one sessions of a support intervention for hearing voices } \\
\text { (auditory verbal hallucinations) from a peer mental health worker who has had personal lived ex- } \\
\text { perience of hearing voices themselves. The intervention group will receive the intervention along- } \\
\text { side treatment as usual (TAU) in the 3-month period immediately following randomisation. } \\
\text { Control: Treatment as usual (wait list). The control group will receive the intervention after a 3- } \\
\text { month treatment as usual wait list period. Treatment as usual will involve the person's usual men- } \\
\text { tal health care which will ordinarily include prescription of antipsychotic medication, plus meet- } \\
\text { ings with mental health workers and possible attendance at rehabilitative and recovery-oriented } \\
\text { mental health service programmes. }\end{array}$ \\
\hline
\end{tabular}

Outcomes $\quad$ Primary outcome: Subjective Experiences of Psychosis Scale.

Secondary outcome: Recovery Assessment Scale.

\begin{tabular}{ll}
\hline Starting date & $1 / 11 / 2012$ \\
\hline Contact information & Ms Indigo Daya, Voices Vic 15 Cromwell Road South Yarra VIC 314, Australia, \\
& +61396929562, \\
& indigo@prahranmission.org.au \\
\hline
\end{tabular}

Notes

Bennett 2003

Trial name or title Treating substance abuse in schizophrenia: Preliminary analysis of data from a randomised
treatment trial.

\section{Methods}

\begin{tabular}{ll}
\hline Participants & People with schizophrenia. \\
\hline Interventions & $\begin{array}{l}\text { 1 Behavioural treatment for substance abuse. } \\
\text { 2. supportive therapy. }\end{array}$ \\
\hline Outcomes & Treatment retention, treatment utilisation, urine data. \\
\hline
\end{tabular}


Bennett 2003 (Continued)

Starting date

\section{Contact information}

Notes

Durham 2000a

Trial name or title Cognitive behavioural therapy with medication resistant psychotic symptoms: a clinical trial with psychiatric nurses as therapists.

\begin{tabular}{ll}
\hline Methods & \\
\hline Participants & People with schizophrenia. \\
\hline Interventions & $\begin{array}{l}\text { 1. CBT. } \\
\text { 2. Supportive counselling. }\end{array}$ \\
\hline Outcomes & To assess the efficacy of delivery of care by clinical nurse specialist. \\
\hline Starting date & \\
\hline Contact information & \\
\hline Notes
\end{tabular}

Hogarty 2001

Trial name or title

Environmental-personal treatment of schizophrenia.

\section{Methods}

Participants People with schizophrenia.

Interventions $\quad$ 1. Cognitive Enhancement Therapy.

2. Enriched Supportive Therapy.

Outcomes Interpersonal and vocational effectiveness, self-esteem, neuropsychological competence and residual symptoms.

\section{Starting date}

\section{Contact information}

Notes

\section{ISRCTN024573132006}

Trial name or title
The effectiveness of support groups for people suffering from psychosis: a randomised controlled trial. 
ISRCTN02457313 2006 (Continued)

\begin{tabular}{|c|c|}
\hline Methods & Randomised controlled trial. Parallel group. \\
\hline Participants & Patients diagnosed with schizophrenia, psychosis. \\
\hline Interventions & $\begin{array}{l}\text { Intervention: Peer support group. } \\
\text { Control: Waiting list control group. }\end{array}$ \\
\hline Outcomes & $\begin{array}{l}\text { Primary outcome: Quality of Life: WHO Qol Bref. } \\
\text { Secondary outcomes: } \\
\text { 1. Social support } \\
\text { 2. Social network } \\
\text { 3. Self-efficacy } \\
\text { 4. Self esteem } \\
\text { 5. Psychopathology. }\end{array}$ \\
\hline Starting date & Feb 2003. \\
\hline Contact information & Not reported. \\
\hline Notes & Trial ended. \\
\hline
\end{tabular}

\section{ISRCTN504877132011}

Trial name or title

An evaluation of different levels of support in using a recovery guide for people with psychosis and the impact of choice on outcomes

\begin{tabular}{|c|c|}
\hline Methods & $\begin{array}{l}\text { Partially randomised patient preference trial. Participants may choose their preferred treatment } \\
\text { option: Treatment As Usual, low support/high support or elect to be randomised to a treatment op- } \\
\text { tion. }\end{array}$ \\
\hline Participants & $\begin{array}{l}\text { Meeting ICD } 10 \text { criteria for non affective psychosis (schizophrenia,schizophreniform disorder, } \\
\text { schizoaffective disorder, delusional disorder). }\end{array}$ \\
\hline Interventions & $\begin{array}{l}\text { Interventions: } \\
\text { 1. Low support } \\
\text { 2. High support } \\
\text { Control: Treatment as usual. }\end{array}$ \\
\hline Outcomes & Psychotic symptoms, affect, well-being and functioning. \\
\hline Starting date & Not reported. \\
\hline Contact information & $\begin{array}{l}\text { Prof Gillian Haddock } \\
\text { University of Manchester } \\
\text { School of Psychological Sciences } \\
\text { Zochonis Building } \\
\text { Oxford Road } \\
\text { Manchester } \\
\text { Greater Manchester } \\
\text { M13 9PL } \\
\text { UNITED KINGDOM } \\
\text { Tel: } 01612758756 \\
\text { gillian.haddock@manchester.ac.uk }\end{array}$ \\
\hline Notes & The trial is reported as "closed - in follow-up". \\
\hline
\end{tabular}


NCT 2007

\begin{tabular}{|c|c|}
\hline Trial name or title & A randomised controlled trial of individual therapy for first episode psychosis (PSTEP). \\
\hline Methods & Randomised, single-blind (outcomes assessor), placebo-control, single-group assignment. \\
\hline Participants & $\begin{array}{l}\text { Schizophrenia, schizophreniform disorder, brief psychotic disorder, delusional disorder, schizoaf- } \\
\text { fective disorder, substance induced psychotic disorder, or psychotic disorder NOS. }\end{array}$ \\
\hline Interventions & CBT; befriending. \\
\hline Outcomes & $\begin{array}{l}\text { Social functioning; positive \& negative symptoms; individual dimensions of psychotic symptoms; } \\
\text { depression; substance use; alcohol and drug use; medication adherence adaptation to illness; self } \\
\text { esteem; coping skills. }\end{array}$ \\
\hline Starting date & September 2007 \\
\hline Contact information & $\begin{array}{l}\text { Diane Kirsopp, BA } \\
\text { 416-535-8501 ext } 6288 \\
\text { diane_kirsopp@camh.net }\end{array}$ \\
\hline Notes & \\
\hline
\end{tabular}

NCT 2007a

\begin{tabular}{ll}
\hline Trial name or title & Peer support for increasing physical activity in people with serious mental illnesses. \\
\hline Methods & Randomised, single-blind (outcomes assessor), active control, parallel assignment \\
\hline Participants & Patient at the Johns Hopkins Community Psychiatry program: serious mental illnesses. \\
\hline Interventions & $\begin{array}{l}\text { 1. Physical activity intervention with peer support (PA + PS) } \\
\text { 2. Physical activity intervention without peer support (PA) }\end{array}$ \\
\hline Outcomes & $\begin{array}{l}\text { Primary: Cardiorespiratory fitness } \\
\text { Secondary: Weight; waist circumference;physical activity; health status; Center for Epidemiology } \\
\text { Depression Scale; exercise-related self-efficacy; general perceived efficacy; participation. }\end{array}$ \\
\hline Starting date & March 2007. \\
\hline Contact information & $\begin{array}{l}\text { Gail L. Daumit, MD, MHS } \\
\text { gda-614-6460 }\end{array}$ \\
\hline Notes &
\end{tabular}

\section{NCT 2007b}

\begin{tabular}{ll}
\hline Trial name or title & Recovery Group Pilot. \\
\hline Methods & $\begin{array}{l}\text { Randomised,open label, uncontrolled, parallel assignment, delayed control group and 6- and 12- } \\
\text { month post-intervention follow-up. }\end{array}$ \\
\hline
\end{tabular}


NCT 2007b (Continued)

Participants Bipolar disorder, schizophrenia, schizoaffective disorder.

\begin{tabular}{ll}
\hline Interventions & Recovery group workbook \& group sessions \\
\hline Outcomes & $\begin{array}{l}\text { Improvements in short-term recovery and functioning, participant attendance and satisfaction } \\
\text { with the group sessions. }\end{array}$ \\
\hline Starting date & July 2005. \\
\hline Contact information & Not reported. \\
\hline
\end{tabular}

Notes

NCT 2008

\begin{tabular}{|c|c|}
\hline Trial name or title & A randomised controlled trial of individual therapy for first episode psychosis \\
\hline Methods & Randomised, single-blind, active control, parallel assignment. \\
\hline Participants & $\begin{array}{l}\text { DSM-IV criteria for: schizophrenia, schizophreniform disorder, brief psychotic disorder, delusion- } \\
\text { al disorder, schizoaffective disorder, substance induced psychotic disorder, or psychotic disorder } \\
\text { NOS. }\end{array}$ \\
\hline Interventions & $\begin{array}{l}\text { 1. CBT } \\
\text { 2. Befriending } \\
\text { 3. Routine care }\end{array}$ \\
\hline Outcomes & $\begin{array}{l}\text { Social Functioning Scale (SFS), } \\
\text { Positive and Negative Syndrome Scale (PANSS), } \\
\text { Psychotic Symptom Rating Scales (PSYRATS), Calgary Depression Scale for Schizophrenia (CDSS), } \\
\text { The Time-Line Follow Back (TLFB), } \\
\text { Alcohol and Drug Use Scale (AUS; DUS), } \\
\text { Medication Event Monitoring System (MEMS), } \\
\text { Rosenberg Self-Esteem Scale, } \\
\text { Maastrich Assessment of Coping Skills (MACS). }\end{array}$ \\
\hline Starting date & June 2007. \\
\hline Contact information & $\begin{array}{l}\text { Jean Addington, PhD } \\
\text { 416-535-8501 Ext. } 4360 \\
\text { Jean_Addington@camh.net }\end{array}$ \\
\hline
\end{tabular}

Notes

NCT 2012

\begin{tabular}{ll}
\hline Trial name or title & Effectiveness and cost effectiveness of peer mentors in reducing hospital use. \\
\hline Methods & Randomised controlled trial. Parallel assignment. Open label. \\
\hline Participants & Diagnosed with serious mental illness. \\
\hline Interventions & 1.Community Connector. \\
\hline
\end{tabular}


NCT 2012 (Continued)

2. Peer recovery mentor.

3. Peer case manager.

\section{Outcomes}

Primary Outcome: Service use via self-report from the participant. Two time points: 3 months and 9 months.

Secondary Outcome: (measured at 3 months and 9 months)

Psychiatric symptoms measured using the Symptom Distress scale, and the Paranoia and Psychoticism subscales from the Symptom Check List-90 (SCL-90), Quality of life measured using Lehman's Brief Quality of Life scale,

Community inclusion measured using Mancini's Community Connections Inventory.

\begin{tabular}{ll}
\hline Starting date & August 2011. \\
\hline Contact information & Larry Davidson, PhD \\
& $\begin{array}{l}\text { 203-764-7583 } \\
\text { larry.davidson@yale.edu }\end{array}$ \\
\hline Notes & \\
\hline
\end{tabular}

Robinson 2010

$\begin{array}{ll}\text { Trial name or title } & \begin{array}{l}\text { The development, design, and testing of a peer support intervention in youth being discharged } \\ \text { from a first episode psychosis clinic. }\end{array}\end{array}$

\begin{tabular}{ll}
\hline Methods & Randomised controlled trial, open-label, parallel group. \\
\hline Participants & Outpatients diagnosed with psychosis. \\
\hline Interventions & $\begin{array}{l}\text { 1. Peer-support for } 6 \text { months: } 2 \text { hours of contact per fortnight to engage and support the partic- } \\
\text { ipant in transition to the new service (accompanying, helping with attendance, understanding } \\
\text { health services, and motivating to develop contacts) }\end{array}$ \\
\hline 2. Treatment as usual \\
\hline Outcomes & Not reported. \\
\hline Contact information & Not reported. \\
\hline Notes & Jo Robinson, Orygen Youth Health Research Centre, University ofMelbourne, Melbourne, Australia \\
\hline
\end{tabular}

\section{Rohricht 2000}

$\begin{array}{ll}\text { Trial name or title } & \begin{array}{l}\text { RCT in effectiveness of body oriented psychotherapy on anergia in patients with chronic } \\ \text { schizophrenia. }\end{array}\end{array}$

\begin{tabular}{ll}
\hline Methods & \\
\hline Participants & People with schizophrenia. \\
\hline Interventions & $\begin{array}{l}\text { 1. Body oriented psychotherapy. } \\
\text { 2. No information available for comparison group. }\end{array}$ \\
\hline
\end{tabular}


Rohricht 2000 (Continued)

Outcomes

Starting date

Contact information

\section{Notes}

\section{Sensky 2000a}

Trial name or title

A five-year follow-up of patients with schizophrenia with drug-resistant symptoms treated with cognitive behaviour therapy or a befriending intervention.

\begin{tabular}{ll}
\hline Methods & \\
\hline Participants & People with schizophrenia. \\
\hline Interventions & $\begin{array}{l}\text { 1. CBT. } \\
\text { 2. Befriending intervention. }\end{array}$ \\
\hline Outcomes & $\begin{array}{l}\text { Global state: Comprehensive Psychopathological Rating Scale. } \\
\text { Mental state: Montgomery-Asberg Depression Rating Score; } \\
\text { the Schedule for Negative Symptoms. }\end{array}$
\end{tabular}

\section{Starting date}

Contact information

\section{Notes}

\section{Tarrier 2000c}

\begin{tabular}{ll}
\hline Trial name or title & $\begin{array}{l}\text { A psychological intervention programme to reduce positive symptoms and prevent relapse in } \\
\text { psychotic patients. }\end{array}$ \\
\hline Methods & People with schizophrenia. \\
\hline Participants & $\begin{array}{l}\text { 1. Psychological intervention. } \\
\text { 2. Supportive counselling and routine care. }\end{array}$ \\
\hline Interventions & Relapse and social functioning. \\
\hline Outcomes & \\
\hline Starting date & \\
\hline Contact information & \\
\hline Notes
\end{tabular}

CBT - cognitive behavioural therapy

DSM - Diagnostic and Statistical Manual 
ICD - International Classification of Diseases

$\mathrm{RCT}$ - randomised controlled trial

\section{DATA AND ANALYSES}

Comparison 1. SUPPORTIVE THERAPY versus STANDARD CARE

\begin{tabular}{|c|c|c|c|c|}
\hline Outcome or subgroup title & No. of studies & $\begin{array}{l}\text { No. of partici- } \\
\text { pants }\end{array}$ & Statistical method & Effect size \\
\hline 1 Global state: Relapse & 1 & & $\begin{array}{l}\text { Risk Ratio (M-H, Fixed, 95\% } \\
\mathrm{Cl} \text { ) }\end{array}$ & Subtotals only \\
\hline 1.1 medium term & 1 & 54 & $\begin{array}{l}\text { Risk Ratio (M-H, Fixed, 95\% } \\
\mathrm{Cl})\end{array}$ & $0.12[0.01,2.11]$ \\
\hline 1.2 long term & 1 & 54 & $\begin{array}{l}\text { Risk Ratio (M-H, Fixed, 95\% } \\
\mathrm{Cl})\end{array}$ & $0.96[0.44,2.11]$ \\
\hline 2 Service outcomes: Hospitalisation & 1 & & $\begin{array}{l}\text { Risk Ratio (M-H, Fixed, 95\% } \\
\mathrm{Cl})\end{array}$ & Subtotals only \\
\hline 2.1 long term & 1 & 48 & $\begin{array}{l}\text { Risk Ratio (M-H, Fixed, 95\% } \\
\mathrm{Cl} \text { ) }\end{array}$ & $1.0[0.07,15.08]$ \\
\hline $\begin{array}{l}3 \text { Mental state. } 1 \text {. No clinically impor- } \\
\text { tant improvement in general mental } \\
\text { state }\end{array}$ & 2 & & $\begin{array}{l}\text { Risk Ratio (M-H, Fixed, 95\% } \\
\mathrm{Cl})\end{array}$ & Subtotals only \\
\hline 3.1 medium term & 1 & 54 & $\begin{array}{l}\text { Risk Ratio (M-H, Fixed, 95\% } \\
\mathrm{Cl} \text { ) }\end{array}$ & $0.95[0.77,1.17]$ \\
\hline 3.2 long term & 2 & 98 & $\begin{array}{l}\text { Risk Ratio (M-H, Fixed, 95\% } \\
\text { Cl) }\end{array}$ & $0.95[0.82,1.11]$ \\
\hline $\begin{array}{l}4 \text { Mental state: } 2 \text {. Average endpoint } \\
\text { general mental state score (PANSS, } \\
\text { high = poor) }\end{array}$ & 2 & & $\begin{array}{l}\text { Mean Difference (IV, Fixed, } \\
95 \% \mathrm{CI})\end{array}$ & Subtotals only \\
\hline 4.1 short term & 1 & 131 & $\begin{array}{l}\text { Mean Difference (IV, Fixed, } \\
95 \% \mathrm{Cl})\end{array}$ & $-4.42[-10.13,1.29]$ \\
\hline 4.2 long term & 1 & 36 & $\begin{array}{l}\text { Mean Difference (IV, Fixed, } \\
95 \% \mathrm{Cl} \text { ) }\end{array}$ & $4.70[-6.71,16.11]$ \\
\hline $\begin{array}{l}5 \text { Mental state: } 3 \text {. Average endpoint } \\
\text { negative symptoms score (SANS, } \\
\text { skewed data, high = poor) }\end{array}$ & & & Other data & No numeric data \\
\hline 5.1 short term & & & Other data & No numeric data \\
\hline 5.2 long term & & & Other data & No numeric data \\
\hline $\begin{array}{l}6 \text { Mental state: } 4 \text {. Average endpoint } \\
\text { positive symptoms score (PANSS posi- } \\
\text { tive subscale, high = poor) }\end{array}$ & 1 & & $\begin{array}{l}\text { Mean Difference (IV, Fixed, } \\
95 \% \mathrm{CI})\end{array}$ & Subtotals only \\
\hline
\end{tabular}




\begin{tabular}{|c|c|c|c|c|}
\hline Outcome or subgroup title & No. of studies & $\begin{array}{l}\text { No. of partici- } \\
\text { pants }\end{array}$ & Statistical method & Effect size \\
\hline 6.1 short term & 1 & 131 & $\begin{array}{l}\text { Mean Difference (IV, Fixed, } \\
95 \% \mathrm{CI})\end{array}$ & $-1.09[-2.84,0.66]$ \\
\hline $\begin{array}{l}7 \text { Mental state: } 5 . \text { Average endpoint } \\
\text { positive symptoms score (BPRS, } \\
\text { skewed data, high = poor) }\end{array}$ & & & Other data & No numeric data \\
\hline 7.1 short term & & & Other data & No numeric data \\
\hline 7.2 long term & & & Other data & No numeric data \\
\hline $\begin{array}{l}8 \text { Mental state: } 6 \text {. Average endpoint } \\
\text { delusions score (PSYRATS delusions } \\
\text { score, skewed data, high = poor) }\end{array}$ & & & Other data & No numeric data \\
\hline 8.1 short term & & & Other data & No numeric data \\
\hline 8.2 long term & & & Other data & No numeric data \\
\hline $\begin{array}{l}9 \text { Mental state: } 7 \text {. Average endpoint } \\
\text { hallucinations score (PSYRATS hallu- } \\
\text { cinations score, skewed data, high = } \\
\text { poor) }\end{array}$ & & & Other data & No numeric data \\
\hline 9.1 long term & & & Other data & No numeric data \\
\hline $\begin{array}{l}10 \text { Mental state: } 8 \text {. Average endpoint } \\
\text { depressive symptoms score (CESD, } \\
\text { high = poor) }\end{array}$ & 1 & & $\begin{array}{l}\text { Mean Difference (IV, Fixed, } \\
95 \% \mathrm{Cl})\end{array}$ & Subtotals only \\
\hline 10.1 long term & 1 & 260 & $\begin{array}{l}\text { Mean Difference (IV, Fixed, } \\
95 \% \mathrm{Cl})\end{array}$ & $1.61[-1.61,4.83]$ \\
\hline 11 Leaving the study early & 4 & 354 & $\begin{array}{l}\text { Risk Ratio (M-H, Fixed, 95\% } \\
\mathrm{Cl} \text { ) }\end{array}$ & $0.86[0.53,1.40]$ \\
\hline $\begin{array}{l}12 \text { General functioning: } 2 \text {. Average } \\
\text { endpoint general functioning score } \\
\text { (GAF-M, high = good) }\end{array}$ & 1 & & $\begin{array}{l}\text { Mean Difference (IV, Fixed, } \\
95 \% \mathrm{CI})\end{array}$ & Subtotals only \\
\hline 12.1 long term & 1 & 260 & $\begin{array}{l}\text { Mean Difference (IV, Fixed, } \\
95 \% \mathrm{CI})\end{array}$ & $-2.66[-6.20,0.88]$ \\
\hline $\begin{array}{l}13 \text { General functioning: } 1 \text {. Average } \\
\text { endpoint general functioning score } \\
\text { (GAS, high = good) }\end{array}$ & 1 & & $\begin{array}{l}\text { Mean Difference (IV, Fixed, } \\
95 \% \mathrm{CI})\end{array}$ & Subtotals only \\
\hline 13.1 long term & 1 & 29 & $\begin{array}{l}\text { Mean Difference (IV, Fixed, } \\
95 \% \mathrm{CI})\end{array}$ & $1.40[-5.09,7.89]$ \\
\hline $\begin{array}{l}14 \text { General functioning: } 3 \text {. Average } \\
\text { endpoint social functioning score (SFS, } \\
\text { high = good) }\end{array}$ & 1 & & $\begin{array}{l}\text { Mean Difference (IV, Fixed, } \\
95 \% \mathrm{CI})\end{array}$ & Subtotals only \\
\hline
\end{tabular}




\begin{tabular}{|c|c|c|c|c|}
\hline Outcome or subgroup title & No. of studies & $\begin{array}{l}\text { No. of partici- } \\
\text { pants }\end{array}$ & Statistical method & Effect size \\
\hline 14.1 long term & 1 & 260 & $\begin{array}{l}\text { Mean Difference (IV, Fixed, } \\
95 \% \mathrm{CI} \text { ) }\end{array}$ & $-0.67[-7.05,5.71]$ \\
\hline $\begin{array}{l}15 \text { Satisfaction with treatment: Recipi- } \\
\text { ent of care not satisfied with treatment }\end{array}$ & 1 & & $\begin{array}{l}\text { Risk Ratio (M-H, Fixed, 95\% } \\
\text { Cl) }\end{array}$ & Subtotals only \\
\hline 15.1 long term & 1 & 44 & $\begin{array}{l}\text { Risk Ratio (M-H, Fixed, 95\% } \\
\mathrm{Cl} \text { ) }\end{array}$ & $1.83[0.75,4.47]$ \\
\hline $\begin{array}{l}16 \text { Quality of life: } 1 . \text { Average endpoint } \\
\text { self-esteem score (RSES, high = good) }\end{array}$ & 1 & & $\begin{array}{l}\text { Mean Difference (IV, Fixed, } \\
95 \% \mathrm{CI} \text { ) }\end{array}$ & Subtotals only \\
\hline 16.1 long term & 1 & 260 & $\begin{array}{l}\text { Mean Difference (IV, Fixed, } \\
95 \% \mathrm{CI})\end{array}$ & $-1.21[-2.85,0.43]$ \\
\hline $\begin{array}{l}17 \text { Quality of life: } 2 . \text { Average endpoint } \\
\text { well-being score (WBS, high = good) }\end{array}$ & 1 & & $\begin{array}{l}\text { Mean Difference (IV, Fixed, } \\
95 \% \mathrm{CI})\end{array}$ & Subtotals only \\
\hline 17.1 long term & 1 & 260 & $\begin{array}{l}\text { Mean Difference (IV, Fixed, } \\
95 \% \mathrm{CI} \text { ) }\end{array}$ & $-2.73[-6.04,0.58]$ \\
\hline $\begin{array}{l}18 \text { Quality of life: } 3 \text {. Average endpoint } \\
\text { global health score (GHQ, high = poor) }\end{array}$ & 1 & & $\begin{array}{l}\text { Mean Difference (IV, Fixed, } \\
95 \% \mathrm{Cl} \text { ) }\end{array}$ & Subtotals only \\
\hline 18.1 long term & 1 & 260 & $\begin{array}{l}\text { Mean Difference (IV, Fixed, } \\
95 \% \mathrm{CI})\end{array}$ & $2.45[-2.41,7.31]$ \\
\hline 19 Death & 3 & & $\begin{array}{l}\text { Risk Ratio (M-H, Fixed, 95\% } \\
\mathrm{Cl})\end{array}$ & Subtotals only \\
\hline 19.1 medium term & 1 & 54 & $\begin{array}{l}\text { Risk Ratio (M-H, Fixed, 95\% } \\
\mathrm{Cl})\end{array}$ & $3.22[0.14,75.75]$ \\
\hline 19.2 long term & 2 & 92 & $\begin{array}{l}\text { Risk Ratio (M-H, Fixed, 95\% } \\
\mathrm{Cl} \text { ) }\end{array}$ & $2.87[0.31,26.63]$ \\
\hline $\begin{array}{l}20 \text { Medication: No reduction in dose of } \\
\text { antipsychotic medication }\end{array}$ & 1 & & $\begin{array}{l}\text { Risk Ratio (M-H, Fixed, 95\% } \\
\text { Cl) }\end{array}$ & Subtotals only \\
\hline 20.1 long term & 1 & 44 & $\begin{array}{l}\text { Risk Ratio (M-H, Fixed, 95\% } \\
\mathrm{Cl} \text { ) }\end{array}$ & $0.81[0.59,1.12]$ \\
\hline
\end{tabular}

Analysis 1.1. Comparison 1 SUPPORTIVE THERAPY versus STANDARD CARE, Outcome 1 Global state: Relapse.

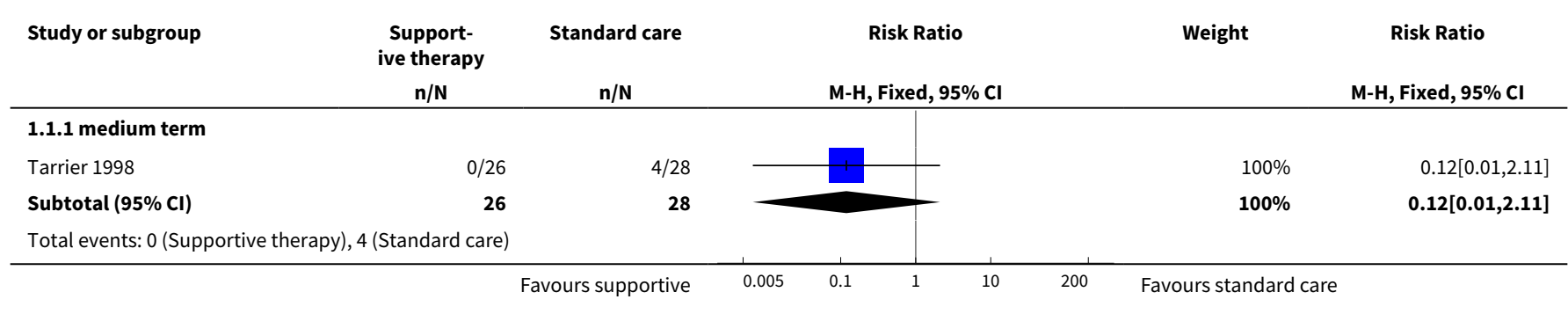




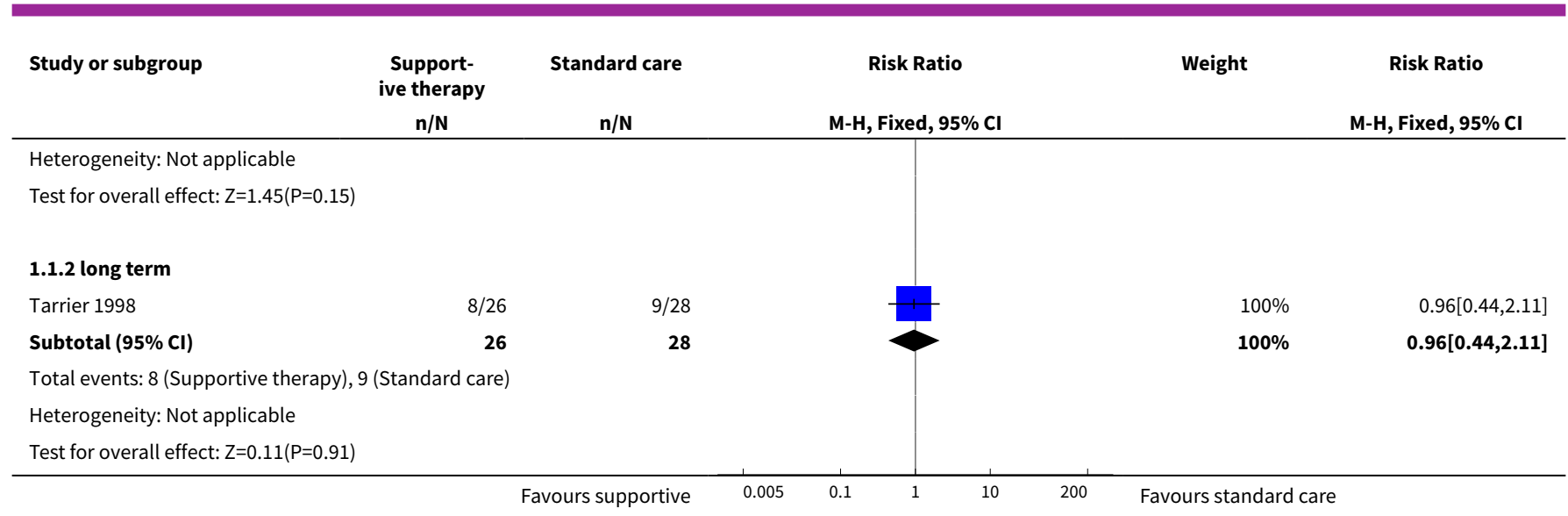

Analysis 1.2. Comparison 1 SUPPORTIVE THERAPY versus STANDARD CARE, Outcome 2 Service outcomes: Hospitalisation.

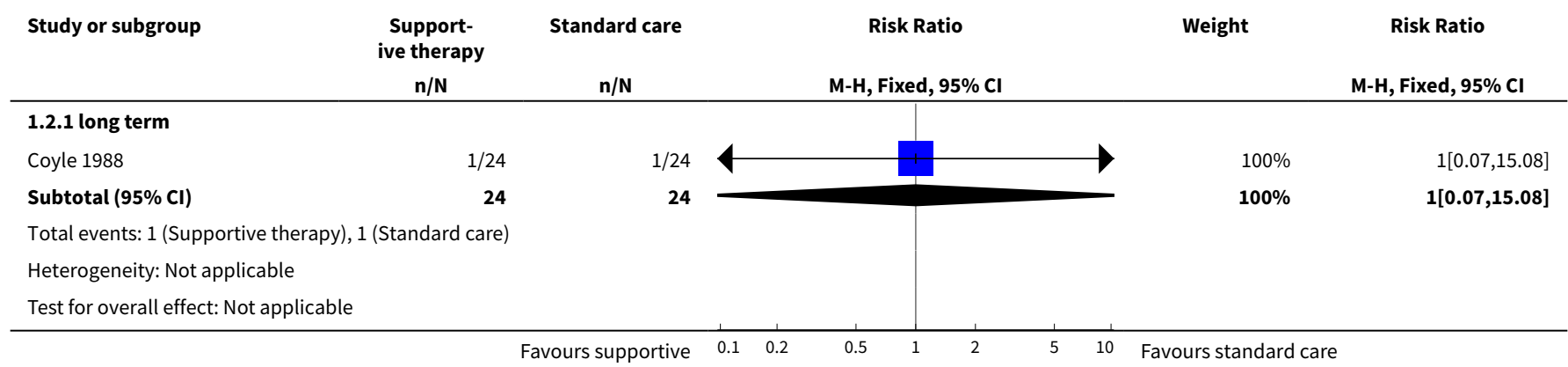

\section{Analysis 1.3. Comparison 1 SUPPORTIVE THERAPY versus STANDARD CARE, Outcome} 3 Mental state. 1. No clinically important improvement in general mental state.

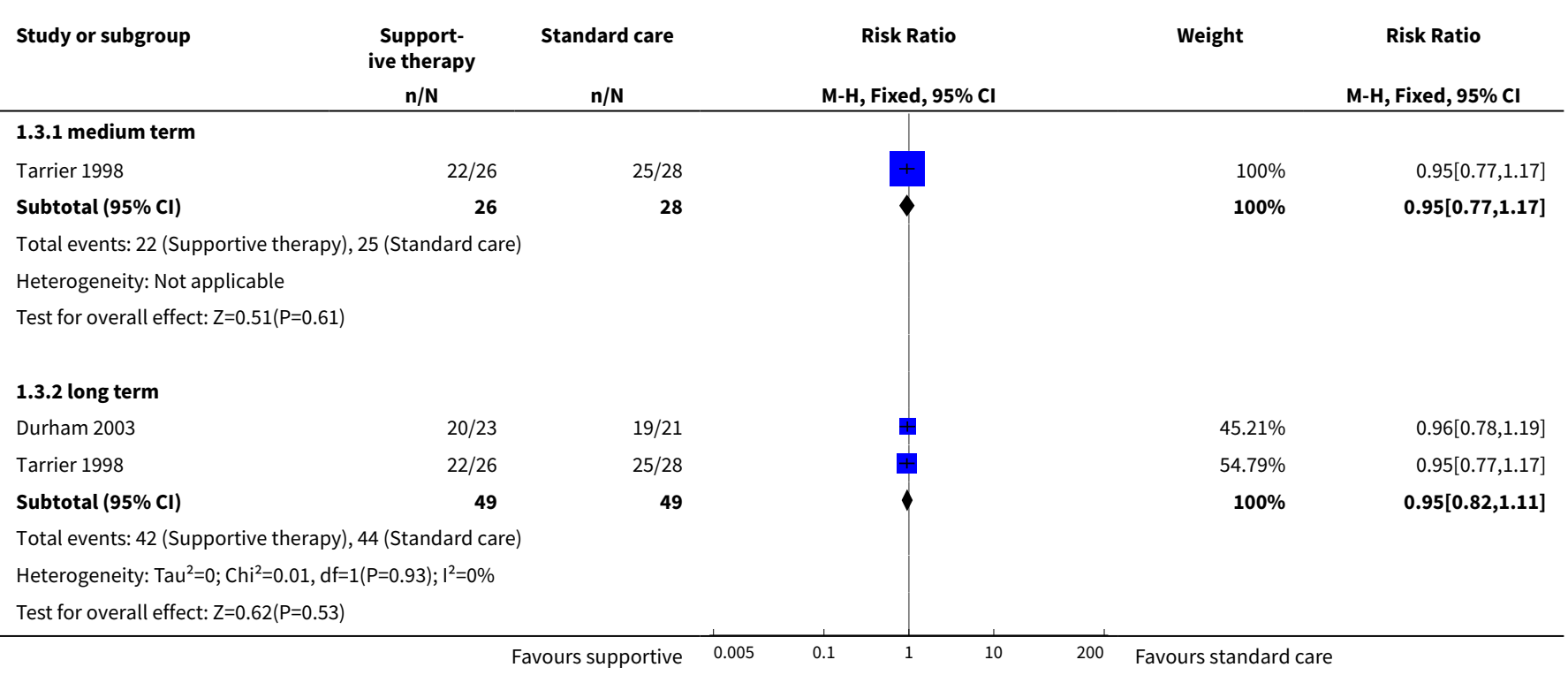


Analysis 1.4. Comparison 1 SUPPORTIVE THERAPY versus STANDARD CARE, Outcome

4 Mental state: 2 . Average endpoint general mental state score (PANSS, high = poor).

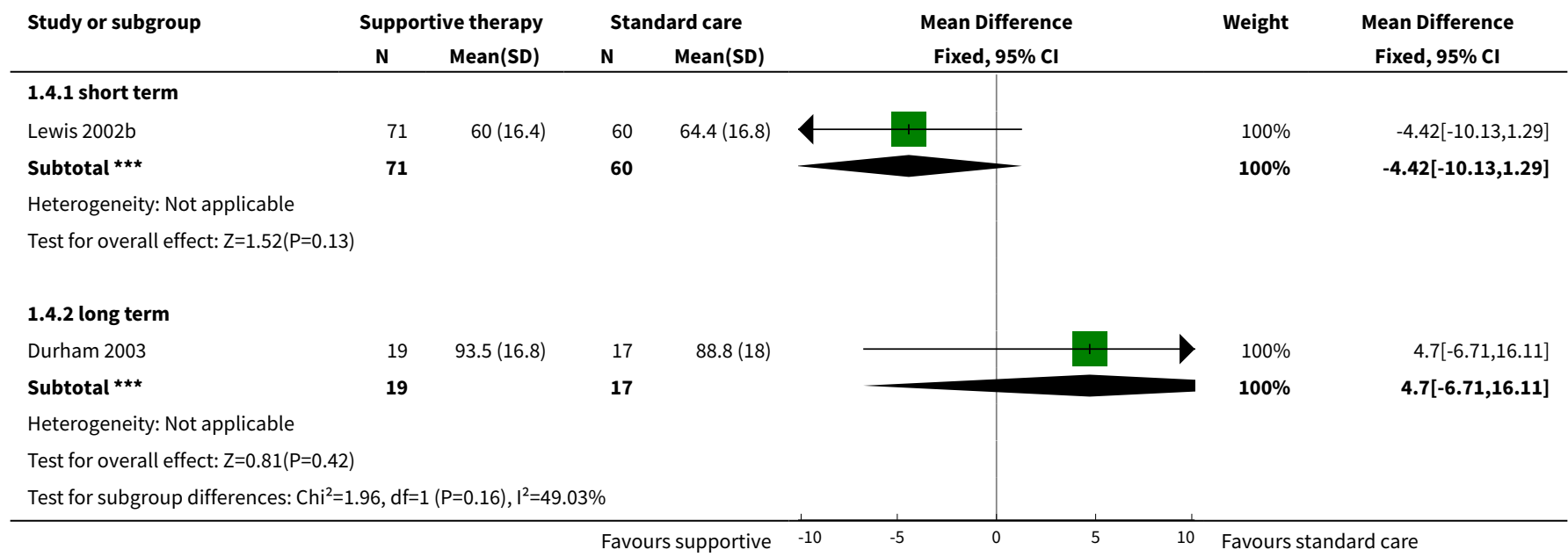

Analysis 1.5. Comparison 1 SUPPORTIVE THERAPY versus STANDARD CARE, Outcome 5 Mental state: 3. Average endpoint negative symptoms score (SANS, skewed data, high = poor).

Mental state: 3. Average endpoint negative symptoms score (SANS, skewed data, high = poor)

\begin{tabular}{|c|c|c|c|c|}
\hline Study & Intervention & $\mathbf{N}$ & Mean & SD \\
\hline \multicolumn{5}{|c|}{ short term } \\
\hline Tarrier 1998 & Supportive therapy & 21 & 10.19 & 5.83 \\
\hline Tarrier 1998 & Standard care & 26 & 10.73 & 4.19 \\
\hline \multicolumn{5}{|c|}{ long term } \\
\hline Tarrier 1998 & Supportive therapy & 21 & 9.90 & 5.44 \\
\hline Tarrier 1998 & Standard care & 26 & 11.46 & 4.34 \\
\hline
\end{tabular}

Analysis 1.6. Comparison 1 SUPPORTIVE THERAPY versus STANDARD CARE, Outcome 6 Mental state: 4. Average endpoint positive symptoms score (PANSS positive subscale, high = poor).

\begin{tabular}{|c|c|c|c|c|c|c|c|}
\hline \multirow[t]{2}{*}{ Study or subgroup } & \multicolumn{2}{|c|}{ Supportive therapy } & \multicolumn{2}{|c|}{ Standard care } & \multirow{2}{*}{$\begin{array}{c}\text { Mean Difference } \\
\text { Fixed, } 95 \% \mathrm{Cl} \\
\end{array}$} & \multirow[t]{2}{*}{ Weight } & \multirow{2}{*}{$\begin{array}{c}\text { Mean Difference } \\
\text { Fixed, } 95 \% \mathrm{Cl}\end{array}$} \\
\hline & $\mathbf{N}$ & Mean(SD) & $\mathbf{N}$ & Mean(SD) & & & \\
\hline \multicolumn{8}{|l|}{ 1.6.1 short term } \\
\hline Lewis 2002b & 71 & $12.6(4.8)$ & 60 & $13.7(5.3)$ & - & $100 \%$ & $-1.09[-2.84,0.66]$ \\
\hline Subtotal $\star \star \star$ & 71 & & 60 & & & $100 \%$ & $-1.09[-2.84,0.66]$ \\
\hline \multicolumn{8}{|c|}{ Heterogeneity: Not applicable } \\
\hline \multicolumn{8}{|c|}{ Test for overall effect: $Z=1.22(P=0.22)$} \\
\hline & & & Favo & supportive & -5 & Favours & ard care \\
\hline
\end{tabular}


Analysis 1.7. Comparison 1 SUPPORTIVE THERAPY versus STANDARD CARE, Outcome 7

Mental state: 5. Average endpoint positive symptoms score (BPRS, skewed data, high = poor).

Mental state: 5 . Average endpoint positive symptoms score (BPRS, skewed data, high = poor)

\begin{tabular}{|c|c|c|c|c|}
\hline Study & Intervention & $\mathbf{N}$ & Mean & SD \\
\hline \multicolumn{5}{|c|}{ short term } \\
\hline Tarrier 1998 & Supportive therapy & 21 & 15.81 & 16.10 \\
\hline Tarrier 1998 & Standard care & 26 & 15.65 & 14.62 \\
\hline \multicolumn{5}{|c|}{ long term } \\
\hline Davidson 2004 & Supportive therapy & 190 & 10.71 & 7.92 \\
\hline Davidson 2004 & Standard care & 70 & 9.14 & 6.92 \\
\hline Tarrier 1998 & Standard care & 26 & 17.63 & 13.51 \\
\hline
\end{tabular}

Analysis 1.8. Comparison 1 SUPPORTIVE THERAPY versus STANDARD CARE, Outcome 8 Mental state: 6. Average endpoint delusions score (PSYRATS delusions score, skewed data, high = poor).

\begin{tabular}{|c|c|c|c|c|}
\hline \multicolumn{5}{|c|}{ Mental state: 6 . Average endpoint delusions score (PSYRATS delusions score, skewed data, high = poor) } \\
\hline Study & Intervention & Mean & SD & $\mathrm{N}$ \\
\hline \multicolumn{5}{|c|}{ short term } \\
\hline Lewis 2002b & Supportive therapy & 6.13 & 6.98 & 67 \\
\hline Lewis $2002 \mathrm{~b}$ & Standard care & 7.52 & 7.15 & 56 \\
\hline \multicolumn{5}{|c|}{ long term } \\
\hline Durham 2003 & Supportive therapy & 9.7 & 6.1 & 19 \\
\hline
\end{tabular}

Analysis 1.9. Comparison 1 SUPPORTIVE THERAPY versus STANDARD CARE, Outcome 9 Mental state: 7. Average endpoint hallucinations score (PSYRATS hallucinations score, skewed data, high = poor).

\begin{tabular}{|c|c|c|c|c|c|c|}
\hline \multicolumn{7}{|c|}{ Mental state: 7. Average endpoint hallucinations score (PSYRATS hallucinations score, skewed data, high = poor) } \\
\hline Study & Intervention & Mean & & SD & & $\mathbf{N}$ \\
\hline \multicolumn{7}{|c|}{ long term } \\
\hline Durham 2003 & Supportive therapy & 18.0 & 12.2 & & 19 & \\
\hline Durham 2003 & Standard care & 17.2 & 11.7 & & 17 & \\
\hline
\end{tabular}

Analysis 1.10. Comparison 1 SUPPORTIVE THERAPY versus STANDARD CARE, Outcome 10 Mental state: 8. Average endpoint depressive symptoms score (CESD, high = poor).

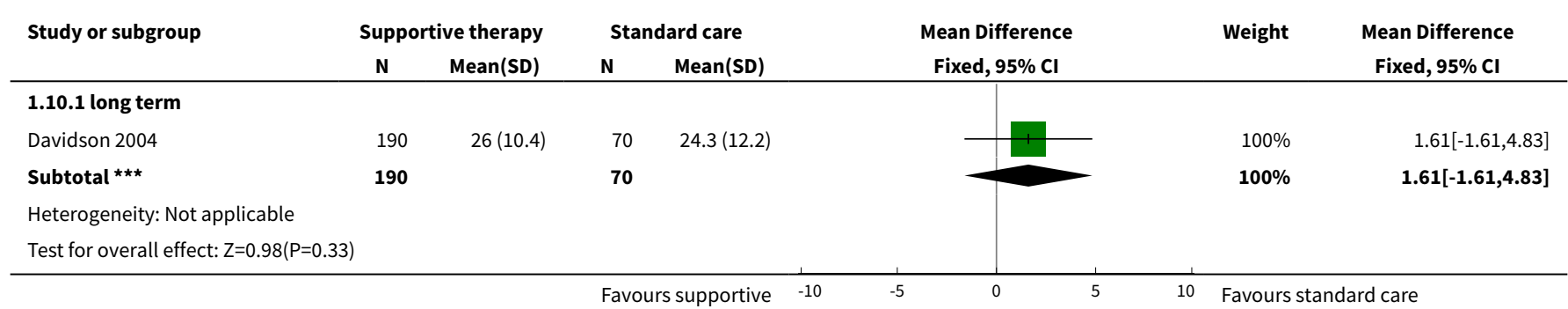


Analysis 1.11. Comparison 1 SUPPORTIVE THERAPY versus STANDARD CARE, Outcome 11 Leaving the study early.

\begin{tabular}{|c|c|c|c|c|c|}
\hline Study or subgroup & $\begin{array}{c}\text { Support- } \\
\text { ive therapy } \\
n / N\end{array}$ & Standard care & M-H, Fixed, 95\% Cl & Weight & $\begin{array}{c}\text { Risk Ratio } \\
\text { M-H, Fixed, } 95 \% \mathrm{Cl}\end{array}$ \\
\hline Coyle 1988 & $3 / 24$ & $3 / 24$ & - & $10.15 \%$ & $1[0.22,4.47]$ \\
\hline Durham 2003 & $4 / 23$ & $4 / 21$ & $\rightarrow$ & $14.15 \%$ & $0.91[0.26,3.2]$ \\
\hline Lewis 2002b & $18 / 106$ & $21 / 102$ & & $72.44 \%$ & $0.82[0.47,1.46]$ \\
\hline Tarrier 1998 & $1 / 26$ & $1 / 28$ & & $3.26 \%$ & $1.08[0.07,16.35]$ \\
\hline Total $(95 \% \mathrm{Cl})$ & 179 & 175 & & $100 \%$ & $0.86[0.53,1.4]$ \\
\hline \multicolumn{6}{|c|}{ Total events: 26 (Supportive therapy), 29 (Standard care) } \\
\hline \multicolumn{6}{|c|}{ Heterogeneity: $\mathrm{Tau}^{2}=0 ; \mathrm{Chi}^{2}=0.09, \mathrm{df}=3(\mathrm{P}=0.99) ; \mathrm{I}^{2}=0 \%$} \\
\hline Test for overall effect & & & & & \\
\hline
\end{tabular}

Analysis 1.12. Comparison 1 SUPPORTIVE THERAPY versus STANDARD CARE, Outcome 12 General functioning: 2. Average endpoint general functioning score (GAF-M, high = good).

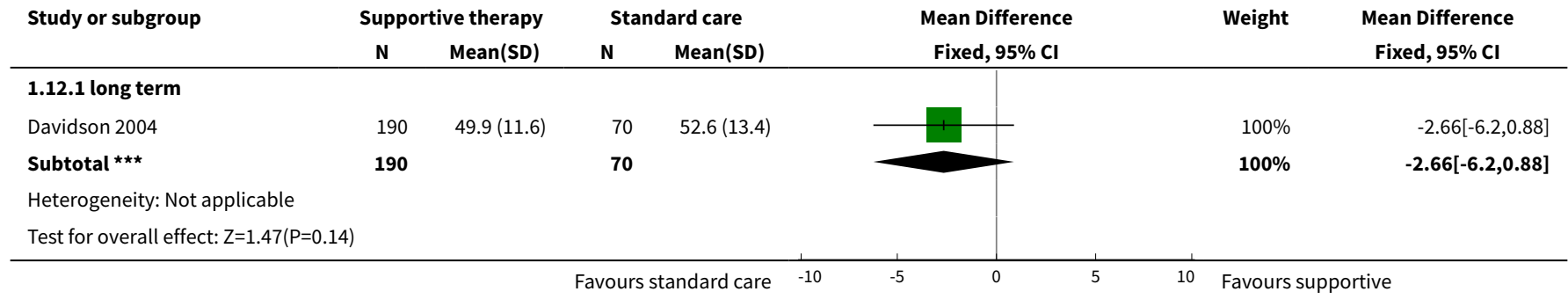

Analysis 1.13. Comparison 1 SUPPORTIVE THERAPY versus STANDARD CARE, Outcome 13 General functioning: 1. Average endpoint general functioning score (GAS, high = good).

\begin{tabular}{|c|c|c|c|c|c|c|c|}
\hline \multirow{3}{*}{$\begin{array}{l}\text { Study or subgroup } \\
1.13 .1 \text { long term }\end{array}$} & \multicolumn{2}{|c|}{ Supportive therapy } & \multicolumn{2}{|c|}{ Standard care } & \multirow{2}{*}{$\begin{array}{c}\text { Mean Difference } \\
\text { Fixed, } 95 \% \mathrm{Cl}\end{array}$} & \multirow[t]{2}{*}{ Weight } & \multirow{2}{*}{$\begin{array}{c}\text { Mean Difference } \\
\text { Fixed, } 95 \% \mathrm{Cl}\end{array}$} \\
\hline & \multirow[t]{2}{*}{$\mathbf{N}$} & \multirow[t]{2}{*}{ Mean(SD) } & \multirow[t]{2}{*}{$\mathbf{N}$} & \multirow[t]{2}{*}{ Mean(SD) } & & & \\
\hline & & & & & & & \\
\hline Durham 2003 & 12 & $36.3(9.8)$ & 17 & $34.9(7.1)$ & & $100 \%$ & $1.4[-5.09,7.89]$ \\
\hline Subtotal $* \star \star$ & 12 & & 17 & & & $100 \%$ & $1.4[-5.09,7.89]$ \\
\hline \multicolumn{8}{|c|}{ Heterogeneity: Not applicable } \\
\hline \multicolumn{3}{|c|}{ Test for overall effect: $Z=0.42(P=0.67)$} & & & & & \\
\hline
\end{tabular}

Analysis 1.14. Comparison 1 SUPPORTIVE THERAPY versus STANDARD CARE, Outcome 14 General functioning: 3. Average endpoint social functioning score (SFS, high = good).

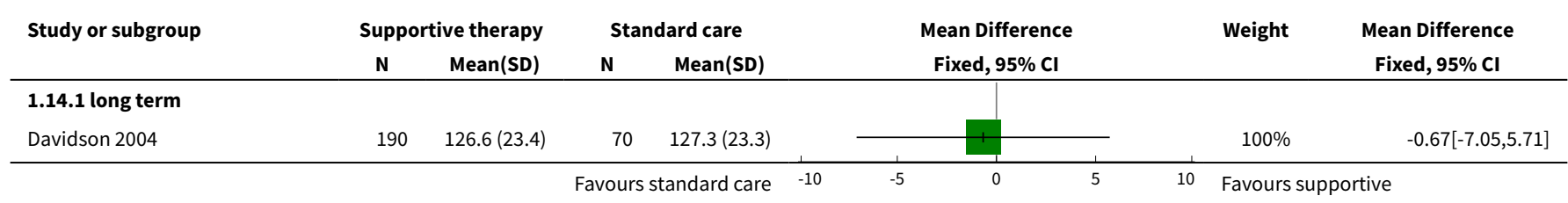




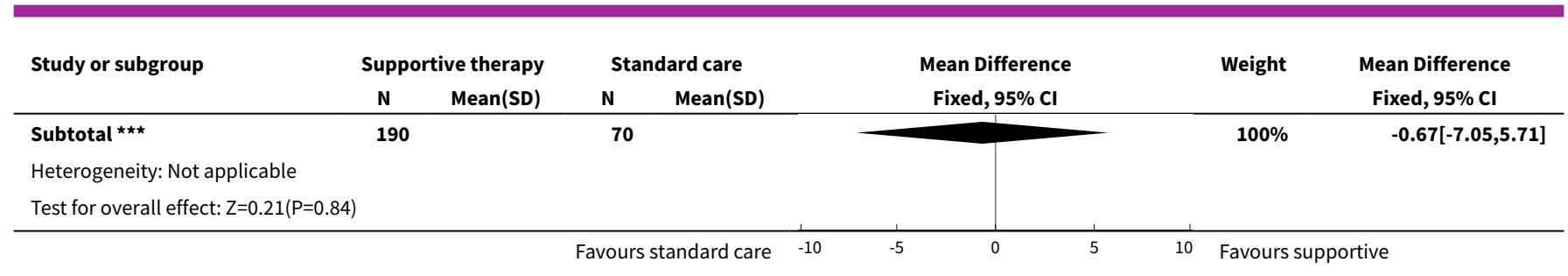

Analysis 1.15. Comparison 1 SUPPORTIVE THERAPY versus STANDARD CARE, Outcome 15 Satisfaction with treatment: Recipient of care not satisfied with treatment.

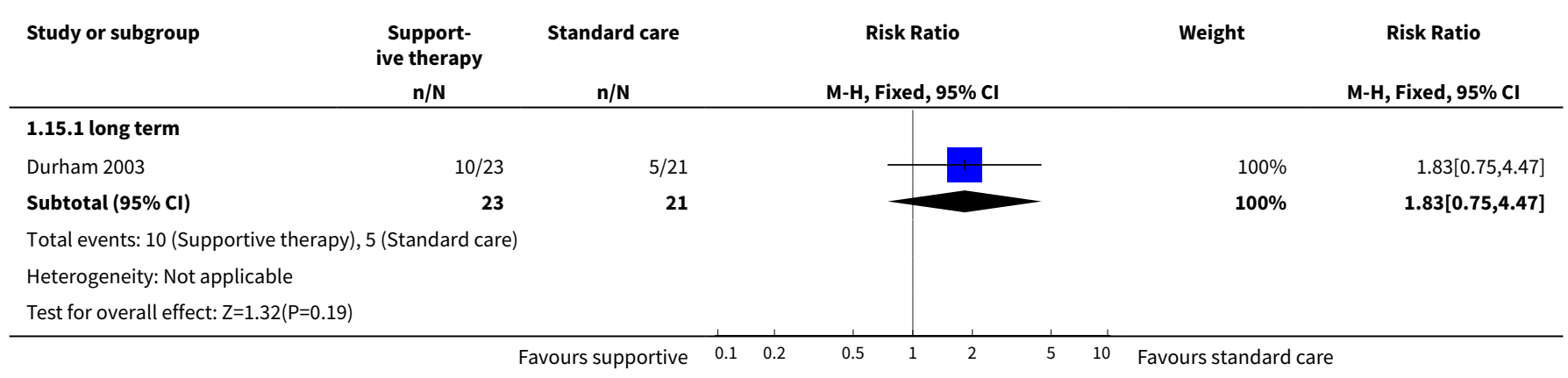

Analysis 1.16. Comparison 1 SUPPORTIVE THERAPY versus STANDARD CARE, Outcome 16 Quality of life: 1. Average endpoint self-esteem score (RSES, high = good).

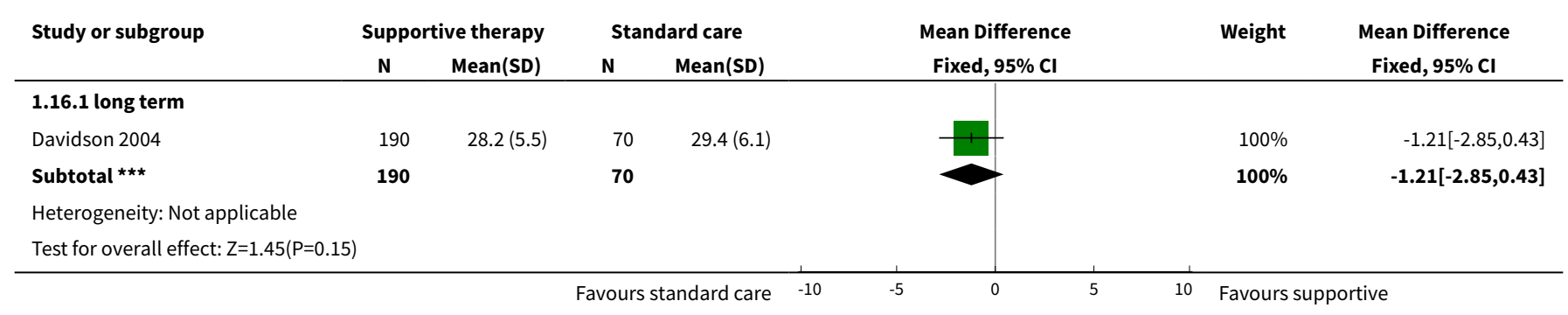

Analysis 1.17. Comparison 1 SUPPORTIVE THERAPY versus STANDARD CARE, Outcome 17 Quality of life: 2. Average endpoint well-being score (WBS, high = good).

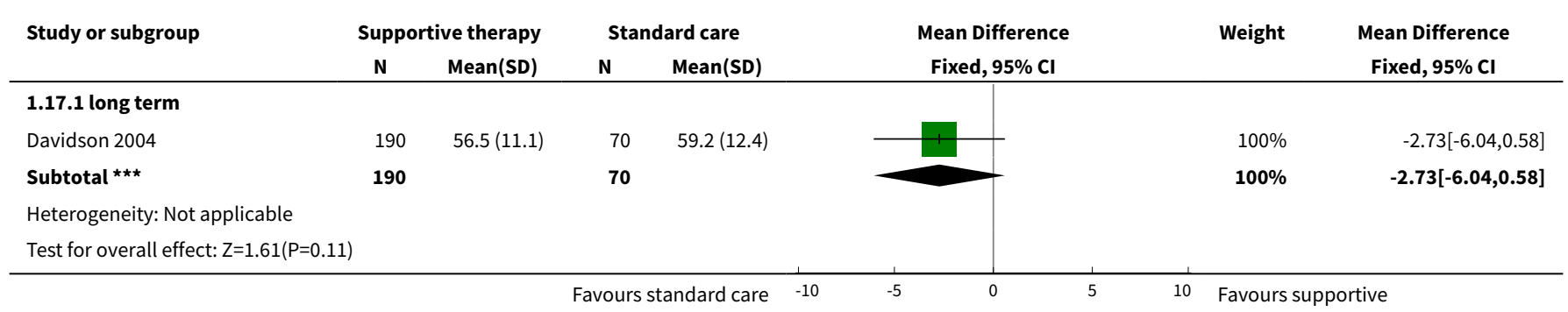


Analysis 1.18. Comparison 1 SUPPORTIVE THERAPY versus STANDARD CARE, Outcome 18 Quality of life: 3. Average endpoint global health score (GHQ, high = poor).

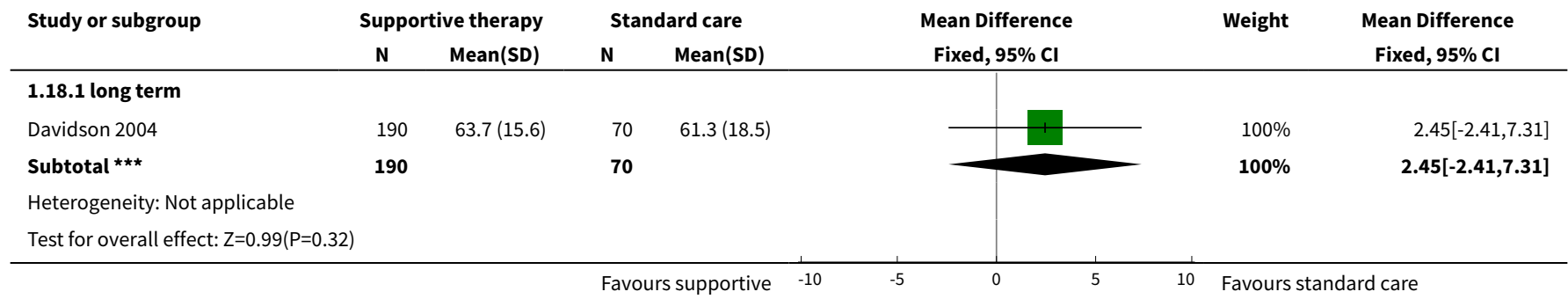

Analysis 1.19. Comparison 1 SUPPORTIVE THERAPY versus STANDARD CARE, Outcome 19 Death.

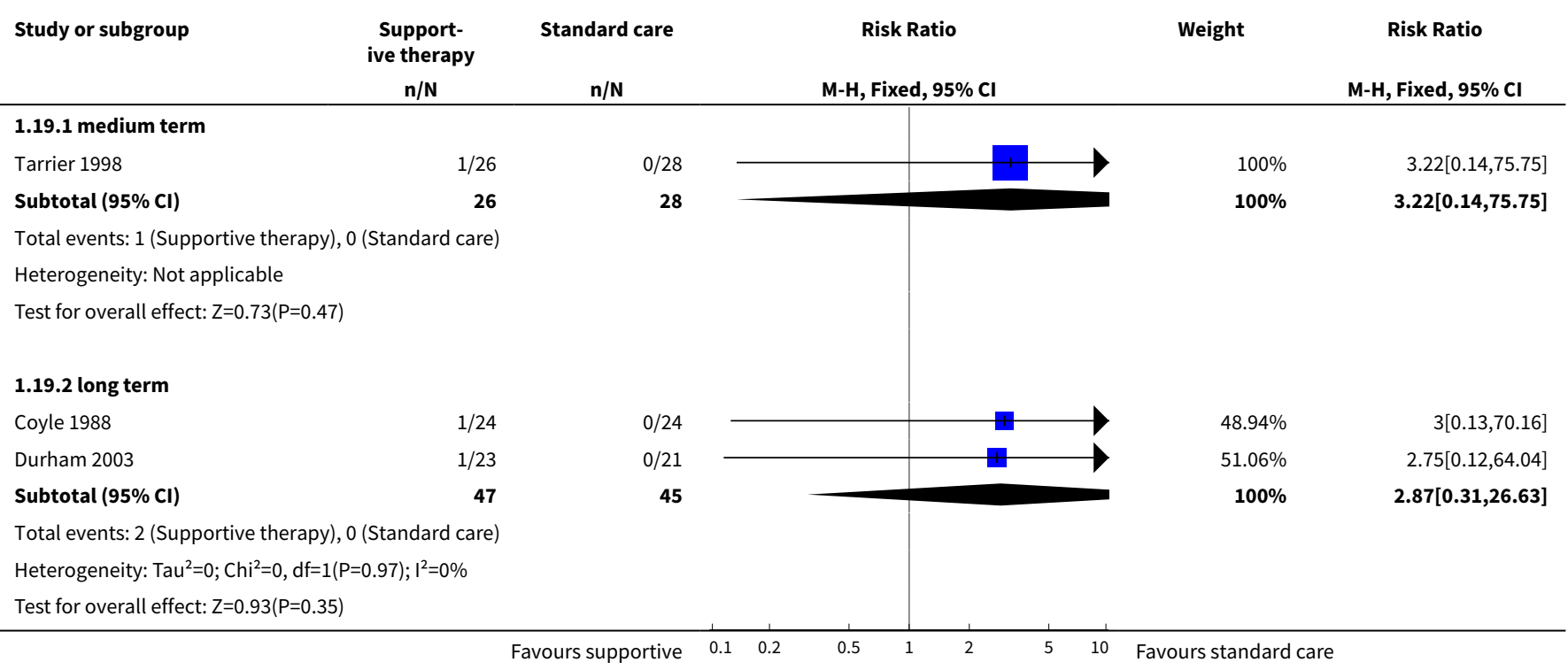

Analysis 1.20. Comparison 1 SUPPORTIVE THERAPY versus STANDARD CARE, Outcome 20 Medication: No reduction in dose of antipsychotic medication.

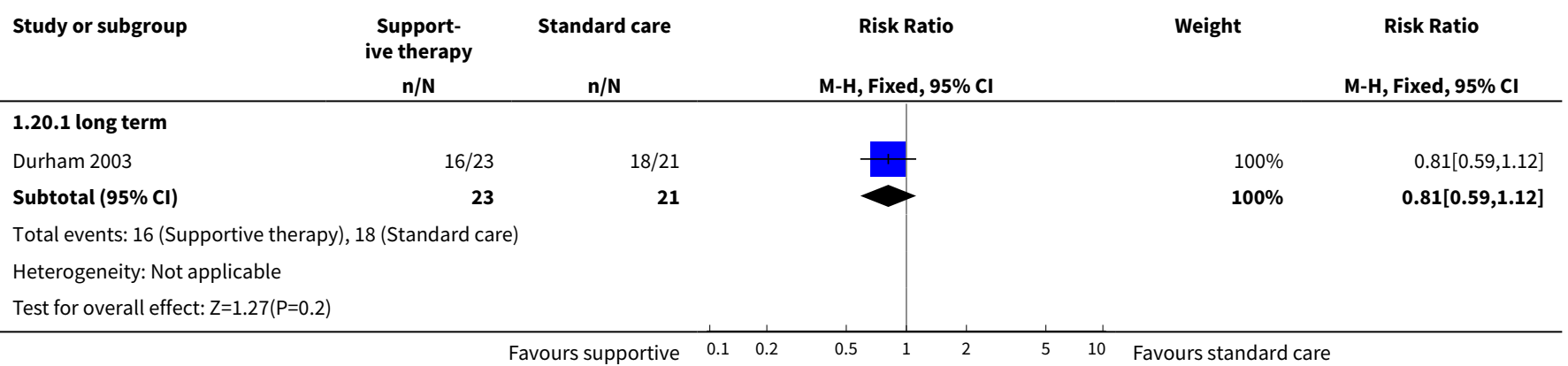


Comparison 2. SUPPORTIVE THERAPY versUS ANY OTHER PSYCHOLOGICAL OR PSYCHOSOCIAL TREATMENT

\begin{tabular}{|c|c|c|c|c|}
\hline Outcome or subgroup title & No. of studies & $\begin{array}{l}\text { No. of partici- } \\
\text { pants }\end{array}$ & Statistical method & Effect size \\
\hline 1 Global state: 1 . Relapse & 6 & & $\begin{array}{l}\text { Risk Ratio (M-H, Random, } \\
95 \% \mathrm{Cl})\end{array}$ & Subtotals only \\
\hline 1.1 medium term & 2 & 100 & $\begin{array}{l}\text { Risk Ratio (M-H, Random, } \\
95 \% \mathrm{Cl})\end{array}$ & $2.86[0.32,25.24]$ \\
\hline 1.2 long term & 5 & 270 & $\begin{array}{l}\text { Risk Ratio (M-H, Random, } \\
95 \% \mathrm{Cl})\end{array}$ & $1.19[0.66,2.16]$ \\
\hline 2 Global state: 2 . No remission & 1 & & $\begin{array}{l}\text { Risk Ratio (M-H, Fixed, 95\% } \\
\mathrm{Cl} \text { ) }\end{array}$ & Subtotals only \\
\hline 2.1 long term & 1 & 39 & $\begin{array}{l}\text { Risk Ratio (M-H, Fixed, 95\% } \\
\mathrm{Cl} \text { ) }\end{array}$ & $1.87[1.11,3.15]$ \\
\hline 3 Service outcomes: Hospitalisation & 6 & & $\begin{array}{l}\text { Risk Ratio (M-H, Fixed, 95\% } \\
\text { Cl) }\end{array}$ & Subtotals only \\
\hline 3.1 medium term & 3 & 153 & $\begin{array}{l}\text { Risk Ratio (M-H, Fixed, 95\% } \\
\mathrm{Cl} \text { ) }\end{array}$ & $1.60[0.85,3.00]$ \\
\hline 3.2 long term & 4 & 306 & $\begin{array}{l}\text { Risk Ratio (M-H, Fixed, 95\% } \\
\mathrm{Cl} \text { ) }\end{array}$ & $1.82[1.11,2.99]$ \\
\hline $\begin{array}{l}4 \text { Mental state: } 1 \text {. No clinically impor- } \\
\text { tant improvement in general mental } \\
\text { state }\end{array}$ & 3 & & $\begin{array}{l}\text { Risk Ratio (M-H, Fixed, 95\% } \\
\mathrm{Cl})\end{array}$ & Subtotals only \\
\hline 4.1 medium term & 1 & 59 & $\begin{array}{l}\text { Risk Ratio (M-H, Fixed, 95\% } \\
\mathrm{Cl} \text { ) }\end{array}$ & $1.27[0.95,1.70]$ \\
\hline 4.2 long term & 3 & 194 & $\begin{array}{l}\text { Risk Ratio (M-H, Fixed, 95\% } \\
\mathrm{Cl} \text { ) }\end{array}$ & $1.27[1.04,1.54]$ \\
\hline $\begin{array}{l}5 \text { Mental state: } 2 \text {. Average endpoint } \\
\text { general mental state score (PANSS, } \\
\text { high = poor) }\end{array}$ & 4 & & $\begin{array}{l}\text { Mean Difference (IV, Ran- } \\
\text { dom, } 95 \% \mathrm{CI} \text { ) }\end{array}$ & Totals not selected \\
\hline 5.1 short term & 3 & & $\begin{array}{l}\text { Mean Difference (IV, Ran- } \\
\text { dom, } 95 \% \mathrm{CI} \text { ) }\end{array}$ & $0.0[0.0,0.0]$ \\
\hline 5.2 long term & 2 & & $\begin{array}{l}\text { Mean Difference (IV, Ran- } \\
\text { dom, } 95 \% \mathrm{CI} \text { ) }\end{array}$ & $0.0[0.0,0.0]$ \\
\hline $\begin{array}{l}6 \text { Mental state: } 3 \text {. Average endpoint } \\
\text { general mental state score (BPRS, } \\
\text { high = poor) }\end{array}$ & 3 & & $\begin{array}{l}\text { Mean Difference (IV, Fixed, } \\
95 \% \mathrm{Cl} \text { ) }\end{array}$ & Subtotals only \\
\hline 6.1 short term & 2 & 92 & $\begin{array}{l}\text { Mean Difference (IV, Fixed, } \\
95 \% \mathrm{CI} \text { ) }\end{array}$ & $-1.07[-5.08,2.94]$ \\
\hline 6.2 medium term & 1 & 37 & $\begin{array}{l}\text { Mean Difference (IV, Fixed, } \\
95 \% \mathrm{CI})\end{array}$ & $7.60[0.90,14.30]$ \\
\hline
\end{tabular}




\begin{tabular}{|c|c|c|c|c|}
\hline Outcome or subgroup title & No. of studies & $\begin{array}{l}\text { No. of partici- } \\
\text { pants }\end{array}$ & Statistical method & Effect size \\
\hline $\begin{array}{l}7 \text { Mental state: } 4 \text {. Average endpoint } \\
\text { general mental state score (BPRS } \\
\text { short form, high = poor) }\end{array}$ & 1 & & $\begin{array}{l}\text { Mean Difference (IV, Fixed, } \\
95 \% \mathrm{Cl} \text { ) }\end{array}$ & Subtotals only \\
\hline 7.1 short term & 1 & 74 & $\begin{array}{l}\text { Mean Difference (IV, Fixed, } \\
95 \% \mathrm{Cl} \text { ) }\end{array}$ & $-0.90[-3.02,1.22]$ \\
\hline 7.2 medium term & 1 & 67 & $\begin{array}{l}\text { Mean Difference (IV, Fixed, } \\
95 \% \mathrm{Cl} \text { ) }\end{array}$ & $2.20[-1.18,5.58]$ \\
\hline 7.3 long term & 1 & 45 & $\begin{array}{l}\text { Mean Difference (IV, Fixed, } \\
95 \% \mathrm{Cl} \text { ) }\end{array}$ & $2.30[-0.54,5.14]$ \\
\hline $\begin{array}{l}8 \text { Mental state: } 5 . \text { Average endpoint } \\
\text { general mental state score (CPRS, } \\
\text { skewed data, high = poor) }\end{array}$ & & & Other data & No numeric data \\
\hline 8.1 medium term & & & Other data & No numeric data \\
\hline 8.2 long term & & & Other data & No numeric data \\
\hline $\begin{array}{l}9 \text { Mental state: } 6 \text {. Average endpoint } \\
\text { general mental state score (PANSS } \\
\text { general subscale, high = poor) }\end{array}$ & 3 & & $\begin{array}{l}\text { Mean Difference (IV, Ran- } \\
\text { dom, } 95 \% \mathrm{CI} \text { ) }\end{array}$ & Totals not selected \\
\hline 9.1 short term & 2 & & $\begin{array}{l}\text { Mean Difference (IV, Ran- } \\
\text { dom, } 95 \% \mathrm{CI} \text { ) }\end{array}$ & $0.0[0.0,0.0]$ \\
\hline 9.2 medium term & 1 & & $\begin{array}{l}\text { Mean Difference (IV, Ran- } \\
\text { dom, } 95 \% \mathrm{CI} \text { ) }\end{array}$ & $0.0[0.0,0.0]$ \\
\hline 9.3 long term & 1 & & $\begin{array}{l}\text { Mean Difference (IV, Ran- } \\
\text { dom, } 95 \% \mathrm{CI} \text { ) }\end{array}$ & $0.0[0.0,0.0]$ \\
\hline $\begin{array}{l}10 \text { Mental state: } 7 \text {. No clinically im- } \\
\text { portant improvement in negative } \\
\text { symptoms }\end{array}$ & 1 & 90 & $\begin{array}{l}\text { Risk Ratio (M-H, Fixed, 95\% } \\
\mathrm{Cl})\end{array}$ & $0.95[0.63,1.46]$ \\
\hline $\begin{array}{l}11 \text { Mental state: } 8 \text {. No clinically im- } \\
\text { portant improvement in depressive } \\
\text { symptoms }\end{array}$ & 1 & 90 & $\begin{array}{l}\text { Risk Ratio (M-H, Fixed, 95\% } \\
\mathrm{Cl} \text { ) }\end{array}$ & $1.53[0.92,2.55]$ \\
\hline $\begin{array}{l}12 \text { Mental state: } 9 \text {. Episode of affec- } \\
\text { tive symptoms }\end{array}$ & 2 & & $\begin{array}{l}\text { Risk Ratio (M-H, Fixed, 95\% } \\
\mathrm{Cl})\end{array}$ & Subtotals only \\
\hline 12.1 long term & 2 & 151 & $\begin{array}{l}\text { Risk Ratio (M-H, Fixed, 95\% } \\
\mathrm{Cl} \text { ) }\end{array}$ & $1.84[1.15,2.94]$ \\
\hline $\begin{array}{l}13 \text { Mental state: } 10 . \text { Average endpoint } \\
\text { negative symptoms score (PANSS } \\
\text { negative, high=poor) }\end{array}$ & 3 & & $\begin{array}{l}\text { Mean Difference (IV, Fixed, } \\
95 \% \mathrm{Cl} \text { ) }\end{array}$ & Totals not selected \\
\hline 13.1 short term & 2 & & $\begin{array}{l}\text { Mean Difference (IV, Fixed, } \\
95 \% \mathrm{CI} \text { ) }\end{array}$ & $0.0[0.0,0.0]$ \\
\hline
\end{tabular}




\begin{tabular}{|c|c|c|c|c|}
\hline Outcome or subgroup title & No. of studies & $\begin{array}{l}\text { No. of partici- } \\
\text { pants }\end{array}$ & Statistical method & Effect size \\
\hline 13.2 medium term & 1 & & $\begin{array}{l}\text { Mean Difference (IV, Fixed, } \\
95 \% \mathrm{CI})\end{array}$ & $0.0[0.0,0.0]$ \\
\hline 13.3 long term & 1 & & $\begin{array}{l}\text { Mean Difference (IV, Fixed, } \\
95 \% \mathrm{CI})\end{array}$ & $0.0[0.0,0.0]$ \\
\hline $\begin{array}{l}14 \text { Mental state: } 11 \text {. Average endpoint } \\
\text { negative symptoms score (SANS, } \\
\text { high=poor) }\end{array}$ & 1 & & $\begin{array}{l}\text { Mean Difference (IV, Fixed, } \\
95 \% \mathrm{CI})\end{array}$ & Subtotals only \\
\hline 14.1 medium term & 1 & 37 & $\begin{array}{l}\text { Mean Difference (IV, Fixed, } \\
95 \% \mathrm{CI})\end{array}$ & $6.60[-5.81,19.01]$ \\
\hline $\begin{array}{l}15 \text { Mental state: } 12 \text {. Average endpoint } \\
\text { negative symptoms score (SANS, } \\
\text { skewed data, high = poor) }\end{array}$ & & & Other data & No numeric data \\
\hline 15.1 short term & & & Other data & No numeric data \\
\hline 15.2 medium term & & & Other data & No numeric data \\
\hline 15.3 long term & & & Other data & No numeric data \\
\hline $\begin{array}{l}16 \text { Mental state: } 13 \text {. Average endpoint } \\
\text { positive symptoms score (PANSS pos- } \\
\text { itive subscale, high = poor) }\end{array}$ & 4 & & $\begin{array}{l}\text { Mean Difference (IV, Fixed, } \\
95 \% \mathrm{CI})\end{array}$ & Totals not selected \\
\hline 16.1 short term & 3 & & $\begin{array}{l}\text { Mean Difference (IV, Fixed, } \\
95 \% \mathrm{Cl})\end{array}$ & $0.0[0.0,0.0]$ \\
\hline 16.2 medium term & 1 & & $\begin{array}{l}\text { Mean Difference (IV, Fixed, } \\
95 \% \mathrm{CI})\end{array}$ & $0.0[0.0,0.0]$ \\
\hline 16.3 long term & 1 & & $\begin{array}{l}\text { Mean Difference (IV, Fixed, } \\
95 \% \mathrm{CI})\end{array}$ & $0.0[0.0,0.0]$ \\
\hline $\begin{array}{l}17 \text { Mental state: } 14 \text {. Average end- } \\
\text { point positive symptoms score (SAPS, } \\
\text { skewed data, high = poor) }\end{array}$ & & & Other data & No numeric data \\
\hline 17.1 medium term & & & Other data & No numeric data \\
\hline $\begin{array}{l}18 \text { Mental state: } 15 . \text { Average endpoint } \\
\text { positive symptoms score (BPRS, } \\
\text { skewed data, high = poor) }\end{array}$ & & & Other data & No numeric data \\
\hline 18.1 short term & & & Other data & No numeric data \\
\hline 18.2 long term & & & Other data & No numeric data \\
\hline $\begin{array}{l}19 \text { Mental state: } 16 . \text { Average endpoint } \\
\text { score (PANSS thought disturbance } \\
\text { cluster, high = poor) }\end{array}$ & 1 & & $\begin{array}{l}\text { Mean Difference (IV, Fixed, } \\
95 \% \mathrm{CI})\end{array}$ & Subtotals only \\
\hline
\end{tabular}




\begin{tabular}{|c|c|c|c|c|}
\hline Outcome or subgroup title & No. of studies & $\begin{array}{l}\text { No. of partici- } \\
\text { pants }\end{array}$ & Statistical method & Effect size \\
\hline 19.1 short term & 1 & 12 & $\begin{array}{l}\text { Mean Difference (IV, Fixed, } \\
95 \% \mathrm{CI})\end{array}$ & $4.3[1.17,7.43]$ \\
\hline $\begin{array}{l}20 \text { Mental state: } 17 \text {. Average endpoint } \\
\text { delusions score (PSYRATS delusions } \\
\text { score, skewed data, high = poor) }\end{array}$ & & & Other data & No numeric data \\
\hline 20.1 short term & & & Other data & No numeric data \\
\hline 20.2 long term & & & Other data & No numeric data \\
\hline $\begin{array}{l}21 \text { Mental state: } 18 \text {. Average endpoint } \\
\text { hallucinations score (PSYRATS hallu- } \\
\text { cinations score, skewed data, high = } \\
\text { poor) }\end{array}$ & & & Other data & No numeric data \\
\hline 21.1 long term & & & Other data & No numeric data \\
\hline $\begin{array}{l}22 \text { Mental state: } 19 . \text { Average endpoint } \\
\text { voices score (PSYRATS voices score, } \\
\text { high = poor) }\end{array}$ & 1 & & $\begin{array}{l}\text { Mean Difference (IV, Fixed, } \\
95 \% \mathrm{Cl} \text { ) }\end{array}$ & Subtotals only \\
\hline 22.1 short term & 1 & 65 & $\begin{array}{l}\text { Mean Difference (IV, Fixed, } \\
95 \% \mathrm{Cl} \text { ) }\end{array}$ & $0.10[-3.63,3.83]$ \\
\hline 22.2 long term & 1 & 65 & $\begin{array}{l}\text { Mean Difference (IV, Fixed, } \\
95 \% \mathrm{Cl} \text { ) }\end{array}$ & $0.0[-4.76,4.76]$ \\
\hline $\begin{array}{l}23 \text { Mental state: } 20 \text {. Average endpoint } \\
\text { beliefs about voices score (BAVQ, } \\
\text { high = poor, skewed data) }\end{array}$ & & & Other data & No numeric data \\
\hline 23.1 Malevolence & & & Other data & No numeric data \\
\hline 23.2 Benevolence & & & Other data & No numeric data \\
\hline 23.3 Resistance & & & Other data & No numeric data \\
\hline 23.4 Engagement & & & Other data & No numeric data \\
\hline 23.5 Omnipotence & & & Other data & No numeric data \\
\hline $\begin{array}{l}24 \text { Mental state: } 21 \text {. Average endpoint } \\
\text { depression score (MADRS, skewed da- } \\
\text { ta, high = poor) }\end{array}$ & & & Other data & No numeric data \\
\hline 24.1 medium term & & & Other data & No numeric data \\
\hline 24.2 long term & & & Other data & No numeric data \\
\hline $\begin{array}{l}25 \text { Mental state: } 22 \text {. Average endpoint } \\
\text { depression score (BDI-II, skewed da- } \\
\text { ta, high = poor) }\end{array}$ & & & Other data & No numeric data \\
\hline
\end{tabular}




\begin{tabular}{|c|c|c|c|c|}
\hline Outcome or subgroup title & No. of studies & $\begin{array}{l}\text { No. of partici- } \\
\text { pants }\end{array}$ & Statistical method & Effect size \\
\hline 26 Leaving the study early & 19 & 1412 & $\begin{array}{l}\text { Risk Ratio (M-H, Fixed, 95\% } \\
\mathrm{Cl})\end{array}$ & $1.03[0.87,1.21]$ \\
\hline 26.1 general reasons & 17 & 1261 & $\begin{array}{l}\text { Risk Ratio (M-H, Fixed, 95\% } \\
\mathrm{Cl})\end{array}$ & $0.97[0.83,1.15]$ \\
\hline 26.2 treatment-related reasons & 2 & 151 & $\begin{array}{l}\text { Risk Ratio (M-H, Fixed, 95\% } \\
\mathrm{CI})\end{array}$ & $2.15[1.07,4.31]$ \\
\hline $\begin{array}{l}27 \text { General functioning: } 1 \text {. Average } \\
\text { endpoint general functioning score } \\
\text { (GAF \& GAS, high = good) }\end{array}$ & 2 & & $\begin{array}{l}\text { Mean Difference (IV, Fixed, } \\
95 \% \mathrm{CI} \text { ) }\end{array}$ & Totals not selected \\
\hline 27.1 short term & 1 & & $\begin{array}{l}\text { Mean Difference (IV, Fixed, } \\
95 \% \mathrm{Cl})\end{array}$ & $0.0[0.0,0.0]$ \\
\hline 27.2 medium term & 1 & & $\begin{array}{l}\text { Mean Difference (IV, Fixed, } \\
95 \% \mathrm{CI} \text { ) }\end{array}$ & $0.0[0.0,0.0]$ \\
\hline 27.3 long term & 2 & & $\begin{array}{l}\text { Mean Difference (IV, Fixed, } \\
95 \% \mathrm{CI})\end{array}$ & $0.0[0.0,0.0]$ \\
\hline $\begin{array}{l}28 \text { General functioning: } 2 \text {. Average } \\
\text { endpoint social functioning score } \\
\text { (SFS, high = good) }\end{array}$ & 1 & & $\begin{array}{l}\text { Mean Difference (IV, Fixed, } \\
95 \% \mathrm{CI} \text { ) }\end{array}$ & Subtotals only \\
\hline 28.1 short term & 1 & 65 & $\begin{array}{l}\text { Mean Difference (IV, Fixed, } \\
95 \% \mathrm{Cl} \text { ) }\end{array}$ & $-7.20[-17.86,3.46]$ \\
\hline 28.2 long term & 1 & 65 & $\begin{array}{l}\text { Mean Difference (IV, Fixed, } \\
95 \% \mathrm{CI} \text { ) }\end{array}$ & $-8.80[-21.67,4.07]$ \\
\hline $\begin{array}{l}29 \text { General functioning: } 3 \text {. No paid } \\
\text { work }\end{array}$ & 2 & & $\begin{array}{l}\text { Risk Ratio (M-H, Fixed, 95\% } \\
\mathrm{Cl})\end{array}$ & Subtotals only \\
\hline 29.1 long term & 2 & 171 & $\begin{array}{l}\text { Risk Ratio (M-H, Fixed, 95\% } \\
\mathrm{Cl})\end{array}$ & $1.03[0.84,1.25]$ \\
\hline $\begin{array}{l}30 \text { General functioning: } 4 \text {. Admission } \\
\text { to residential placement }\end{array}$ & 1 & & $\begin{array}{l}\text { Risk Ratio (M-H, Fixed, 95\% } \\
\mathrm{Cl})\end{array}$ & Subtotals only \\
\hline 30.1 long term & 1 & 39 & $\begin{array}{l}\text { Risk Ratio (M-H, Fixed, 95\% } \\
\mathrm{Cl})\end{array}$ & $1.05[0.24,4.59]$ \\
\hline $\begin{array}{l}31 \text { General functioning: } 5 \text {. Admission } \\
\text { to jail }\end{array}$ & 1 & & $\begin{array}{l}\text { Risk Ratio (M-H, Fixed, 95\% } \\
\mathrm{Cl})\end{array}$ & Subtotals only \\
\hline 31.1 long term & 1 & 39 & $\begin{array}{l}\text { Risk Ratio (M-H, Fixed, 95\% } \\
\mathrm{Cl})\end{array}$ & $1.05[0.24,4.59]$ \\
\hline $\begin{array}{l}32 \text { Satisfaction with treatment: Recip- } \\
\text { ient of care not satisfied with treat- } \\
\text { ment }\end{array}$ & 1 & & $\begin{array}{l}\text { Risk Ratio (M-H, Fixed, 95\% } \\
\mathrm{Cl})\end{array}$ & Subtotals only \\
\hline
\end{tabular}




\begin{tabular}{|c|c|c|c|c|}
\hline Outcome or subgroup title & No. of studies & $\begin{array}{l}\text { No. of partici- } \\
\text { pants }\end{array}$ & Statistical method & Effect size \\
\hline 32.1 long term & 1 & 45 & $\begin{array}{l}\text { Risk Ratio (M-H, Fixed, 95\% } \\
\mathrm{Cl})\end{array}$ & $3.19[1.01,10.07]$ \\
\hline $\begin{array}{l}33 \text { Quality of life: Average endpoint } \\
\text { quality of life score (QLS, high = good) }\end{array}$ & 1 & 19 & $\begin{array}{l}\text { Mean Difference (IV, Fixed, } \\
95 \% \mathrm{CI} \text { ) }\end{array}$ & $-0.07[-21.11,20.97$ \\
\hline 34 Death & 3 & & $\begin{array}{l}\text { Risk Ratio (M-H, Fixed, 95\% } \\
\mathrm{Cl})\end{array}$ & Subtotals only \\
\hline 34.1 medium term & 1 & 59 & $\begin{array}{l}\text { Risk Ratio (M-H, Fixed, 95\% } \\
\mathrm{Cl} \text { ) }\end{array}$ & $1.27[0.08,19.34]$ \\
\hline 34.2 long term & 2 & 115 & $\begin{array}{l}\text { Risk Ratio (M-H, Fixed, 95\% } \\
\mathrm{Cl} \text { ) }\end{array}$ & $3.99[0.44,36.08]$ \\
\hline $\begin{array}{l}35 \text { Behaviour: 1. Social impairment } \\
\text { on SBAS }\end{array}$ & 1 & & $\begin{array}{l}\text { Risk Ratio (M-H, Fixed, 95\% } \\
\mathrm{Cl} \text { ) }\end{array}$ & Subtotals only \\
\hline 35.1 long term & 1 & 39 & $\begin{array}{l}\text { Risk Ratio (M-H, Fixed, 95\% } \\
\mathrm{Cl} \text { ) }\end{array}$ & $1.46[1.04,2.04]$ \\
\hline $\begin{array}{l}36 \text { Behaviour: } 2 \text {. Poor coping style } \\
\text { with relatives }\end{array}$ & 1 & & $\begin{array}{l}\text { Risk Ratio (M-H, Fixed, 95\% } \\
\mathrm{Cl})\end{array}$ & Subtotals only \\
\hline 36.1 long term & 1 & 39 & $\begin{array}{l}\text { Risk Ratio (M-H, Fixed, 95\% } \\
\mathrm{Cl} \text { ) }\end{array}$ & $0.90[0.37,2.20]$ \\
\hline $\begin{array}{l}37 \text { Engagement with services: Poor } \\
\text { attendance at appointments }\end{array}$ & 1 & 39 & $\begin{array}{l}\text { Risk Ratio (M-H, Fixed, 95\% } \\
\mathrm{Cl})\end{array}$ & $1.93[0.89,4.17]$ \\
\hline $\begin{array}{l}38 \text { Insight: Average endpoint insight } \\
\text { score (SAl, high = good, skewed data) }\end{array}$ & & & Other data & No numeric data \\
\hline 38.1 short term & & & Other data & No numeric data \\
\hline 38.2 medium term & & & Other data & No numeric data \\
\hline 38.3 long term & & & Other data & No numeric data \\
\hline $\begin{array}{l}39 \text { Compliance: } 1 \text {. Poor compliance to } \\
\text { therapy }\end{array}$ & 2 & & $\begin{array}{l}\text { Risk Ratio (M-H, Fixed, 95\% } \\
\mathrm{Cl})\end{array}$ & Subtotals only \\
\hline 39.1 medium term & 2 & 58 & $\begin{array}{l}\text { Risk Ratio (M-H, Fixed, 95\% } \\
\mathrm{Cl} \text { ) }\end{array}$ & $2.63[1.30,5.35]$ \\
\hline 39.2 long term & 1 & 39 & $\begin{array}{l}\text { Risk Ratio (M-H, Fixed, 95\% } \\
\mathrm{Cl})\end{array}$ & $1.29[0.69,2.39]$ \\
\hline $\begin{array}{l}40 \text { Compliance: } 2 \text {. adherence to med- } \\
\text { ication (self-report) }\end{array}$ & 1 & 19 & $\begin{array}{l}\text { Risk Ratio (M-H, Fixed, 95\% } \\
\mathrm{Cl} \text { ) }\end{array}$ & $1.0[0.83,1.21]$ \\
\hline $\begin{array}{l}41 \text { Compliance: } 3 \text {. average endpoint } \\
\text { adherence score (ROMI, high = good) }\end{array}$ & 1 & 19 & $\begin{array}{l}\text { Mean Difference (IV, Fixed, } \\
95 \% \mathrm{Cl})\end{array}$ & $0.45[-3.58,4.48]$ \\
\hline
\end{tabular}




\begin{tabular}{|c|c|c|c|c|}
\hline Outcome or subgroup title & No. of studies & $\begin{array}{l}\text { No. of partici- } \\
\text { pants }\end{array}$ & Statistical method & Effect size \\
\hline $\begin{array}{l}42 \text { Compliance: } 4 \text {. average endpoint } \\
\text { non-adherence score (ROMI, high = } \\
\text { poor) }\end{array}$ & 1 & 19 & $\begin{array}{l}\text { Mean Difference (IV, Fixed, } \\
95 \% \mathrm{Cl} \text { ) }\end{array}$ & $1.75[-0.61,4.11]$ \\
\hline $\begin{array}{l}43 \text { Medication: Prescribed IM depot } \\
\text { medication }\end{array}$ & 1 & & $\begin{array}{l}\text { Risk Ratio (M-H, Fixed, 95\% } \\
\text { Cl) }\end{array}$ & Subtotals only \\
\hline 43.1 medium term & 1 & 39 & $\begin{array}{l}\text { Risk Ratio (M-H, Fixed, 95\% } \\
\mathrm{Cl})\end{array}$ & $2.11[0.99,4.47]$ \\
\hline $\begin{array}{l}44 \text { Attitude to medication: } 1 \text {. Aver- } \\
\text { age endpoint attitude to medication } \\
\text { score (AMQ, high = good) }\end{array}$ & 1 & & $\begin{array}{l}\text { Mean Difference (IV, Fixed, } \\
95 \% \mathrm{Cl} \text { ) }\end{array}$ & Subtotals only \\
\hline 44.1 short term & 1 & 74 & $\begin{array}{l}\text { Mean Difference (IV, Fixed, } \\
95 \% \mathrm{CI} \text { ) }\end{array}$ & $-4.50[-6.83,-2.17]$ \\
\hline $\begin{array}{l}45 \text { Attitude to medication: } 2 \text {. Aver- } \\
\text { age endpoint attitude to medication } \\
\text { score (DAl, high = good) }\end{array}$ & 1 & & $\begin{array}{l}\text { Mean Difference (IV, Fixed, } \\
95 \% \mathrm{Cl} \text { ) }\end{array}$ & Subtotals only \\
\hline 45.1 short term & 1 & 63 & $\begin{array}{l}\text { Mean Difference (IV, Fixed, } \\
95 \% \mathrm{CI} \text { ) }\end{array}$ & $-5.70[-9.35,-2.05]$ \\
\hline 45.2 long term & 1 & 44 & $\begin{array}{l}\text { Mean Difference (IV, Fixed, } \\
95 \% \mathrm{Cl} \text { ) }\end{array}$ & $-4.90[-9.38,-0.42]$ \\
\hline $\begin{array}{l}46 \text { Economic outcomes: Direct costs } \\
\text { (skewed data, not ITT) }\end{array}$ & & & Other data & No numeric data \\
\hline 46.1 medium term & & & Other data & No numeric data \\
\hline 46.2 long term & & & Other data & No numeric data \\
\hline
\end{tabular}

Analysis 2.1. Comparison 2 SUPPORTIVE THERAPY versus ANY OTHER PSYCHOLOGICAL OR PSYCHOSOCIAL TREATMENT, Outcome 1 Global state: 1. Relapse.

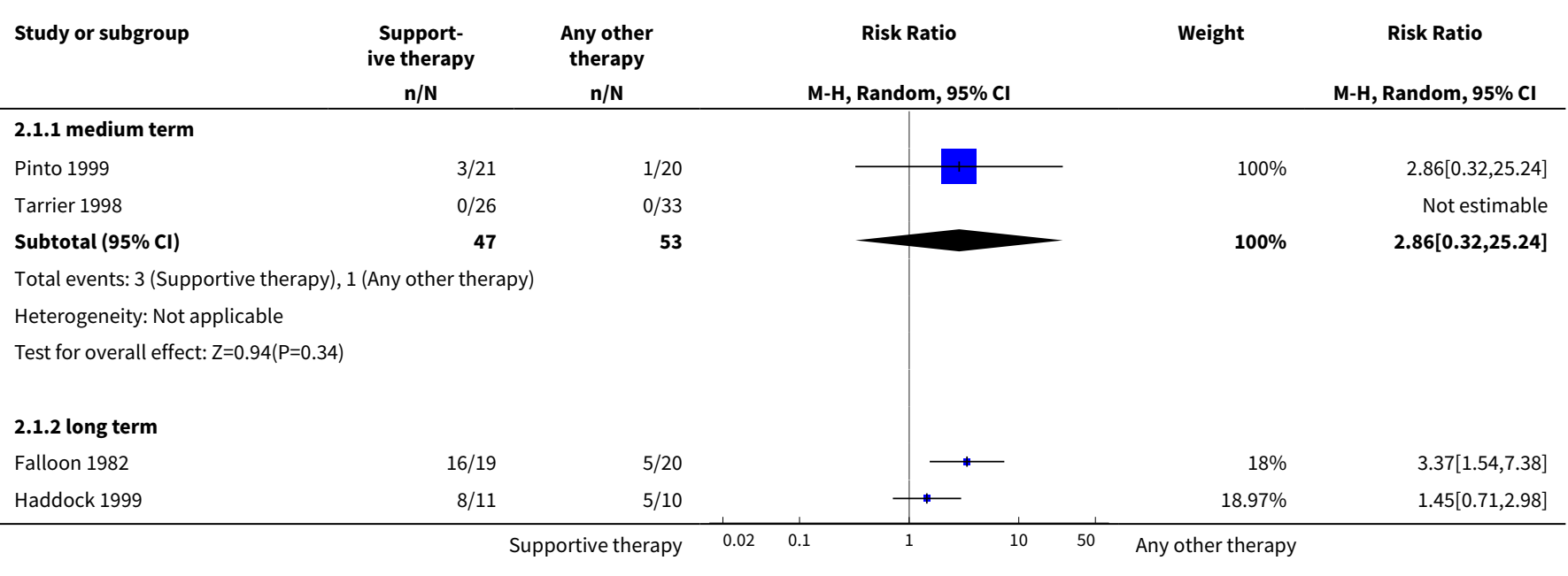




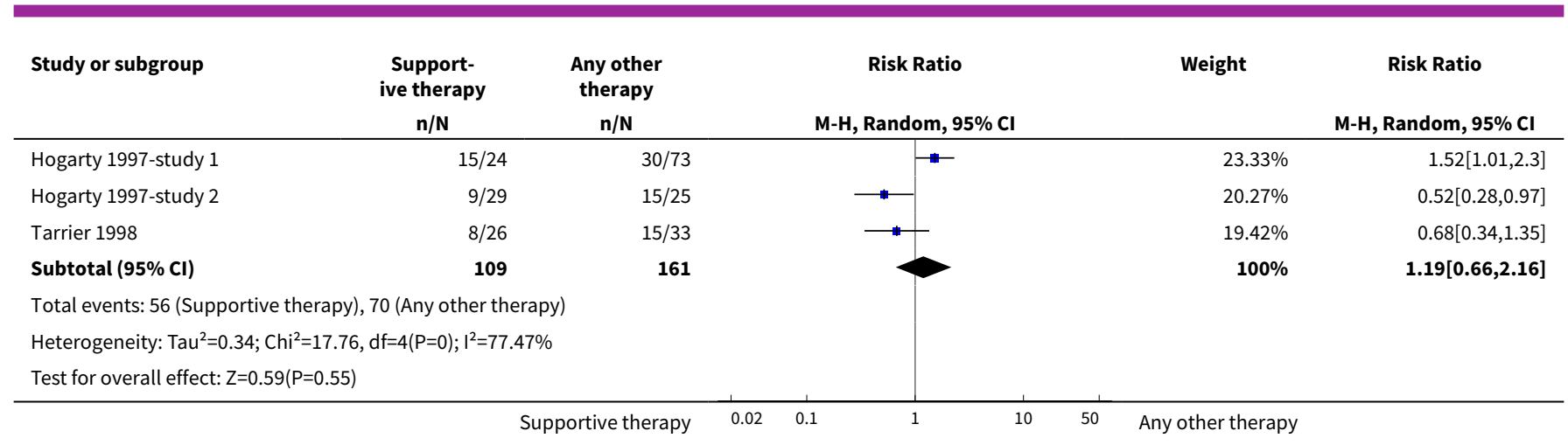

\section{Analysis 2.2. Comparison 2 SUPPORTIVE THERAPY versus ANY OTHER PSYCHOLOGICAL OR PSYCHOSOCIAL TREATMENT, Outcome 2 Global state: 2 . No remission.}

\begin{tabular}{|c|c|c|c|c|c|}
\hline Study or subgroup & $\begin{array}{c}\text { Support- } \\
\text { ive therapy } \\
n / N\end{array}$ & $\begin{array}{c}\text { Any other } \\
\text { therapy } \\
n / N\end{array}$ & $\begin{array}{c}\text { Risk Ratio } \\
\text { M-H, Fixed, 95\% Cl }\end{array}$ & Weight & $\begin{array}{c}\text { Risk Ratio } \\
\text { M-H, Fixed, 95\% Cl }\end{array}$ \\
\hline \multicolumn{6}{|l|}{ 2.2.1 long term } \\
\hline Falloon 1982 & $16 / 19$ & $9 / 20$ & L & $100 \%$ & $1.87[1.11,3.15]$ \\
\hline Subtotal $(95 \% \mathrm{Cl})$ & 19 & 20 & & $100 \%$ & $1.87[1.11,3.15]$ \\
\hline \multicolumn{6}{|c|}{ Total events: 16 (Supportive therapy), 9 (Any other therapy) } \\
\hline \multicolumn{6}{|c|}{ Test for overall effect: $Z=2.35(P=0.02)$} \\
\hline
\end{tabular}

\section{Analysis 2.3. Comparison 2 SUPPORTIVE THERAPY versus ANY OTHER PSYCHOLOGICAL OR PSYCHOSOCIAL TREATMENT, Outcome 3 Service outcomes: Hospitalisation.}

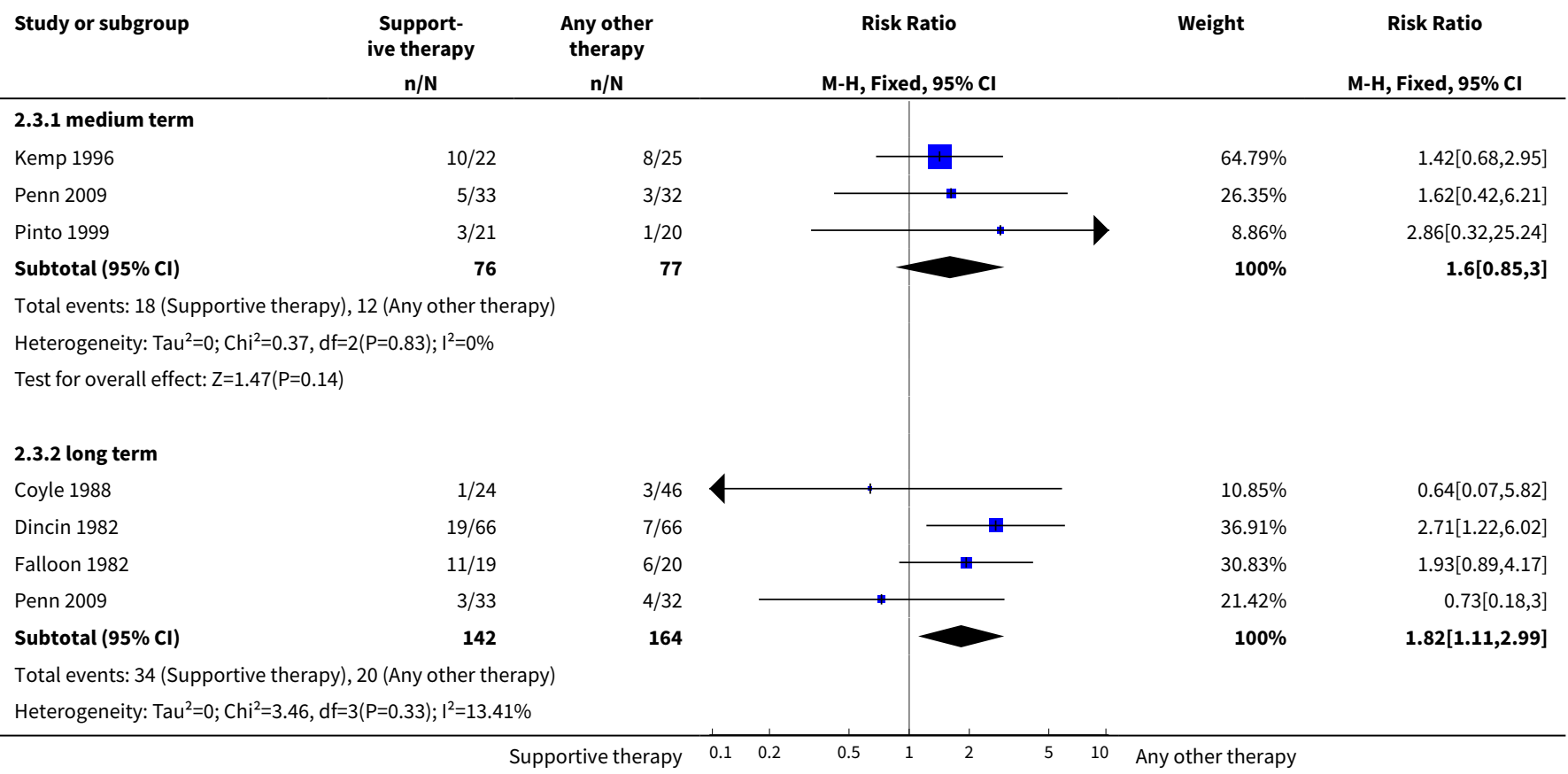




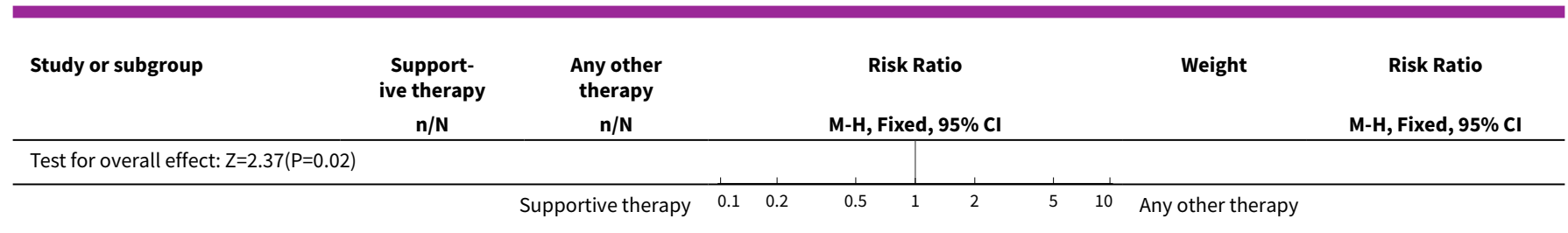

Analysis 2.4. Comparison 2 SUPPORTIVE THERAPY versus ANY OTHER PSYCHOLOGICAL OR PSYCHOSOCIAL TREATMENT, Outcome 4 Mental state: 1 . No clinically important improvement in general mental state.

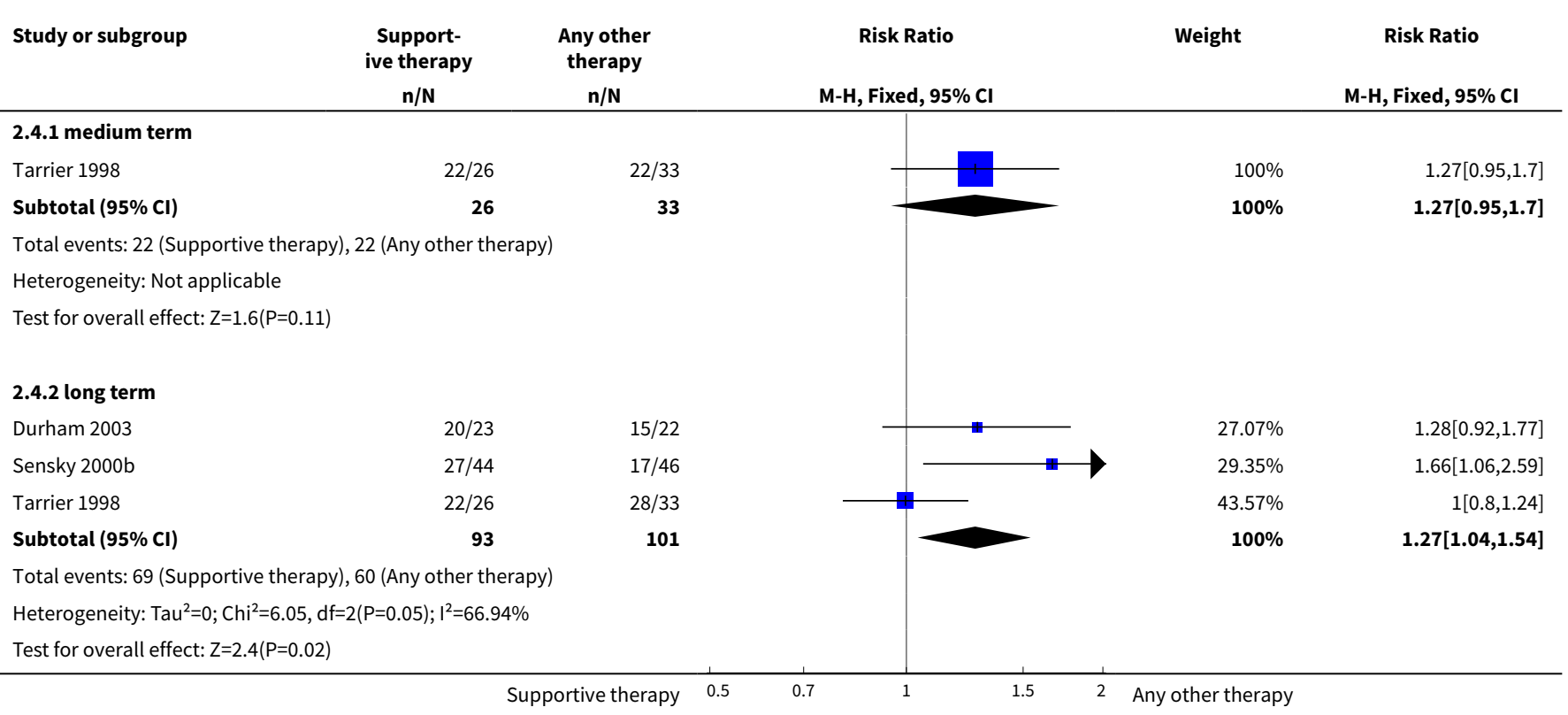

Analysis 2.5. Comparison 2 SUPPORTIVE THERAPY versus ANY OTHER PSYCHOLOGICAL OR PSYCHOSOCIAL TREATMENT, Outcome 5 Mental state: 2. Average endpoint general mental state score (PANSS, high = poor).

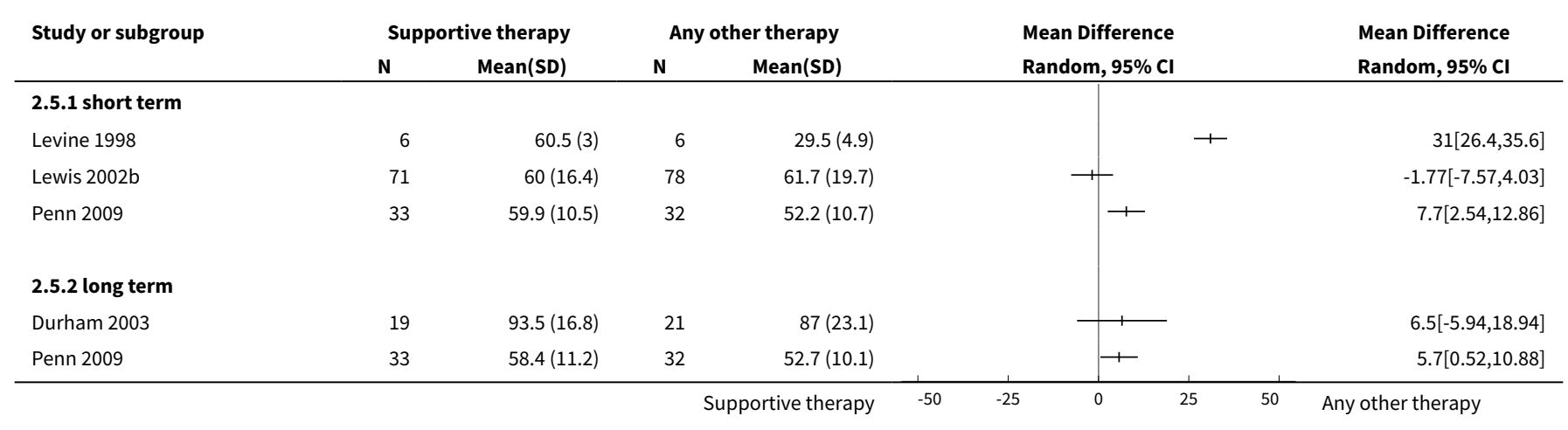


Analysis 2.6. Comparison 2 SUPPORTIVE THERAPY versus ANY OTHER PSYCHOLOGICAL OR PSYCHOSOCIAL TREATMENT, Outcome 6 Mental state: 3. Average endpoint general mental state score (BPRS, high = poor).

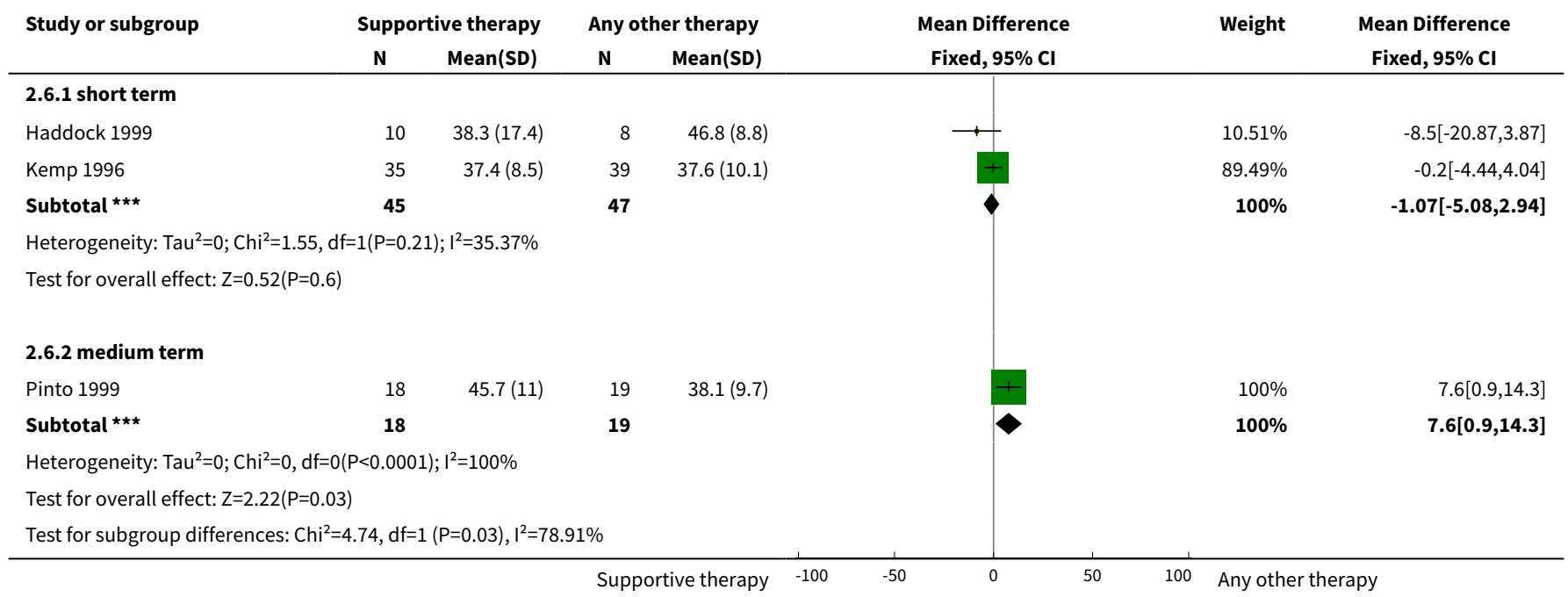

Analysis 2.7. Comparison 2 SUPPORTIVE THERAPY versus ANY OTHER PSYCHOLOGICAL OR PSYCHOSOCIAL TREATMENT, Outcome 7 Mental state: 4. Average endpoint general mental state score (BPRS short form, high = poor).

\begin{tabular}{|c|c|c|c|c|c|c|c|}
\hline \multirow[t]{2}{*}{ Study or subgroup } & \multicolumn{2}{|c|}{ Supportive therapy } & \multicolumn{2}{|c|}{ Any other therapy } & \multirow{2}{*}{$\begin{array}{c}\text { Mean Difference } \\
\text { Fixed, } 95 \% \mathrm{Cl}\end{array}$} & \multirow[t]{2}{*}{ Weight } & \multirow{2}{*}{$\begin{array}{c}\text { Mean Difference } \\
\text { Fixed, } 95 \% \mathrm{Cl}\end{array}$} \\
\hline & $\mathbf{N}$ & Mean(SD) & $\mathbf{N}$ & Mean(SD) & & & \\
\hline \multicolumn{8}{|l|}{ 2.7.1 short term } \\
\hline Kemp 1996 & 35 & $11.7(3.3)$ & 39 & $12.6(5.8)$ & & $100 \%$ & $-0.9[-3.02,1.22]$ \\
\hline Subtotal $\star \star \star$ & 35 & & 39 & & & $100 \%$ & $-0.9[-3.02,1.22]$ \\
\hline \multicolumn{8}{|c|}{ Heterogeneity: Not applicable } \\
\hline \multicolumn{8}{|c|}{ Test for overall effect: $Z=0.83(P=0.41)$} \\
\hline \multicolumn{8}{|l|}{ 2.7.2 medium term } \\
\hline Kemp 1996 & 31 & $16.7(6.9)$ & 36 & $14.5(7.2)$ & & $100 \%$ & $2.2[-1.18,5.58]$ \\
\hline 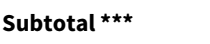 & 31 & & 36 & & & $100 \%$ & $2.2[-1.18,5.58]$ \\
\hline \multicolumn{8}{|c|}{ Heterogeneity: Not applicable } \\
\hline \multicolumn{8}{|c|}{ Test for overall effect: $Z=1.28(P=0.2)$} \\
\hline Kemp 1996 & 20 & $14.8(4.1)$ & 25 & $12.5(5.6)$ & & $100 \%$ & $2.3[-0.54,5.14]$ \\
\hline Subtotal $\star \star \star ~$ & 20 & & 25 & & & $100 \%$ & $2.3[-0.54,5.14]$ \\
\hline \multicolumn{8}{|c|}{ Heterogeneity: $\mathrm{Tau}^{2}=0 ; \mathrm{Chi}^{2}=0, \mathrm{df}=0(\mathrm{P}<0.0001) ; \mathrm{I}^{2}=100 \%$} \\
\hline \multicolumn{8}{|c|}{ Test for overall effect: $Z=1.59(P=0.11)$} \\
\hline \multicolumn{8}{|c|}{ Test for subgroup differences: $\mathrm{Chi}^{2}=4.15, \mathrm{df}=1(\mathrm{P}=0.13), \mathrm{I}^{2}=51.85 \%$} \\
\hline
\end{tabular}


Analysis 2.8. Comparison 2 SUPPORTIVE THERAPY versus ANY OTHER

PSYCHOLOGICAL OR PSYCHOSOCIAL TREATMENT, Outcome 8 Mental state: 5.

Average endpoint general mental state score (CPRS, skewed data, high = poor).

\begin{tabular}{|c|c|c|c|c|}
\hline \multicolumn{5}{|c|}{ Mental state: 5. Average endpoint general mental state score (CPRS, skewed data, high = poor) } \\
\hline Study & Intervention & $\mathbf{N}$ & Mean & SD \\
\hline \multicolumn{5}{|c|}{ medium term } \\
\hline Sensky $2000 \mathrm{~b}$ & Supportive therapy & 44 & 22.9 & 17.3 \\
\hline Sensky $2000 \mathrm{~b}$ & CBT & 46 & 20.5 & 13.1 \\
\hline \multicolumn{5}{|c|}{ long term } \\
\hline Sensky 2000b & Supportive therapy & 44 & 26.6 & 25.3 \\
\hline Sensky $2000 \mathrm{~b}$ & CBT & 46 & 15.1 & 12.0 \\
\hline
\end{tabular}

Analysis 2.9. Comparison 2 SUPPORTIVE THERAPY versus ANY OTHER

PSYCHOLOGICAL OR PSYCHOSOCIAL TREATMENT, Outcome 9 Mental state: 6.

Average endpoint general mental state score (PANSS general subscale, high $=$ poor).

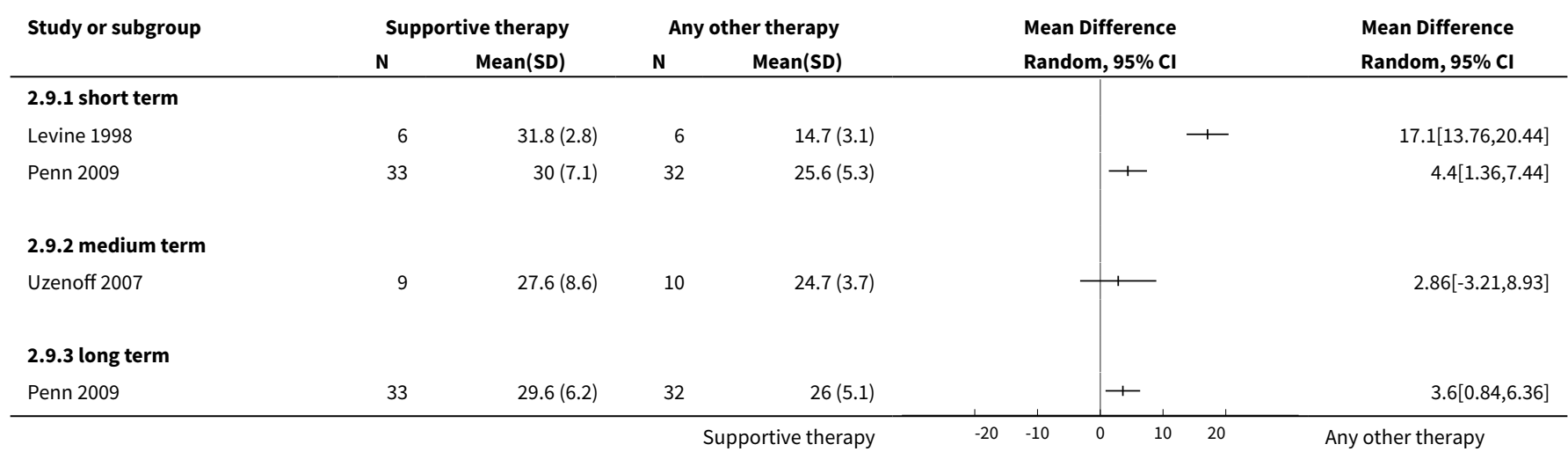

Analysis 2.10. Comparison 2 SUPPORTIVE THERAPY versus ANY OTHER PSYCHOLOGICAL OR PSYCHOSOCIAL TREATMENT, Outcome 10 Mental state: 7. No clinically important improvement in negative symptoms.

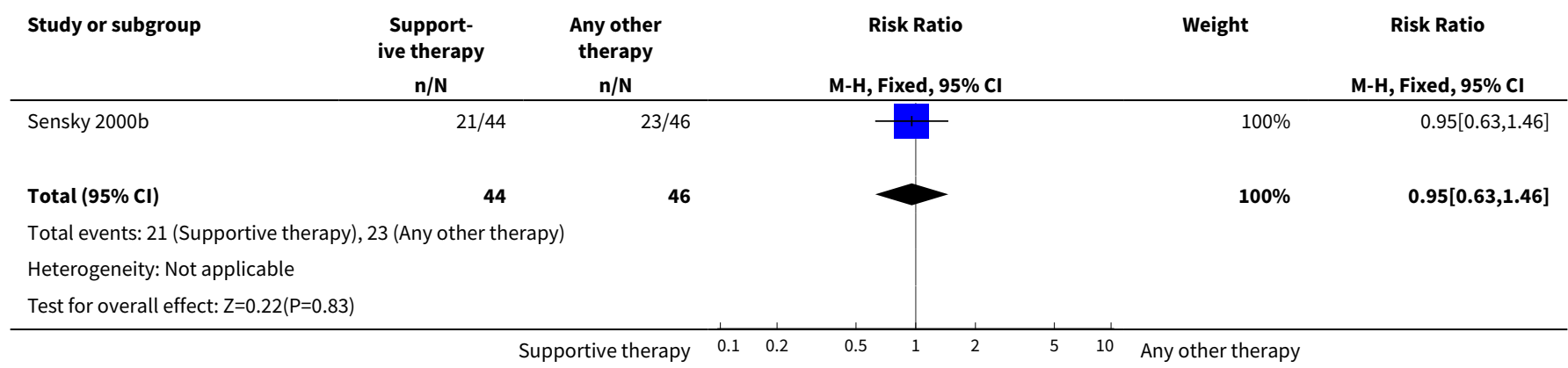


Analysis 2.11. Comparison 2 SUPPORTIVE THERAPY versus ANY OTHER PSYCHOLOGICAL OR PSYCHOSOCIAL TREATMENT, Outcome 11 Mental state: 8. No clinically important improvement in depressive symptoms.

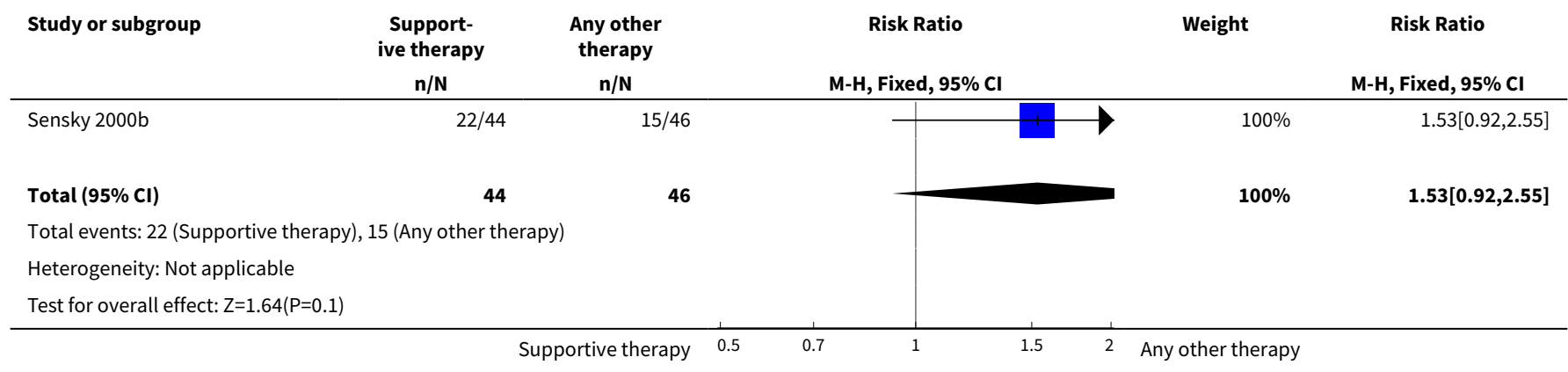

Analysis 2.12. Comparison 2 SUPPORTIVE THERAPY versus ANY OTHER PSYCHOLOGICAL OR PSYCHOSOCIAL TREATMENT, Outcome 12 Mental state: 9. Episode of affective symptoms.

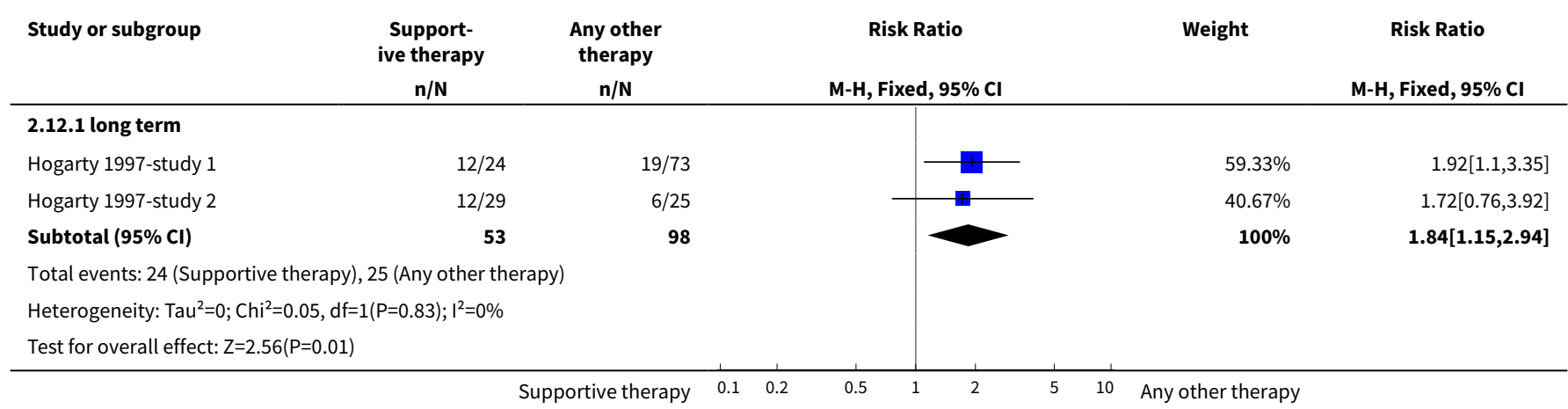

Analysis 2.13. Comparison 2 SUPPORTIVE THERAPY versus ANY OTHER PSYCHOLOGICAL OR PSYCHOSOCIAL TREATMENT, Outcome 13 Mental state: 10. Average endpoint negative symptoms score (PANSS negative, high=poor).

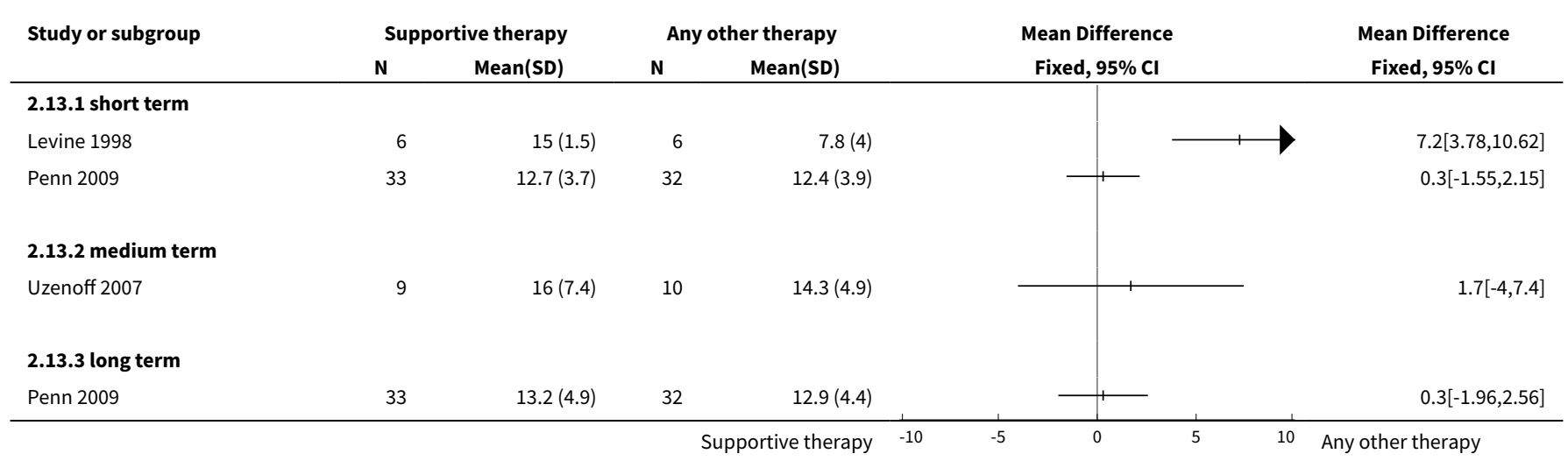


Analysis 2.14. Comparison 2 SUPPORTIVE THERAPY versus ANY OTHER PSYCHOLOGICAL OR PSYCHOSOCIAL TREATMENT, Outcome 14 Mental state: 11. Average endpoint negative symptoms score (SANS, high=poor).

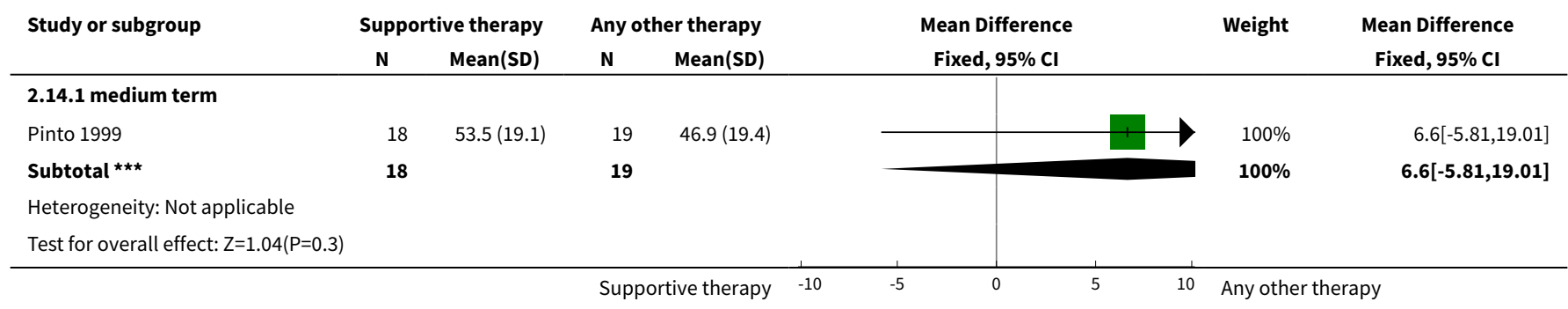

Analysis 2.15. Comparison 2 SUPPORTIVE THERAPY versus ANY OTHER PSYCHOLOGICAL OR PSYCHOSOCIAL TREATMENT, Outcome 15 Mental state: 12. Average endpoint negative symptoms score (SANS, skewed data, high = poor).

\begin{tabular}{|c|c|c|c|c|}
\hline \multicolumn{5}{|c|}{ Mental state: 12. Average endpoint negative symptoms score (SANS, skewed data, high = poor) } \\
\hline Study & Intervention & $\mathbf{N}$ & Mean & SD \\
\hline \multicolumn{5}{|c|}{ short term } \\
\hline Tarrier 1998 & Supportive therapy & 21 & 10.19 & 5.48 \\
\hline Tarrier 1998 & CBT & 24 & 9.83 & 4.43 \\
\hline \multicolumn{5}{|c|}{ medium term } \\
\hline Sensky $2000 b$ & Supportive therapy & 44 & 20.7 & 20.9 \\
\hline \multicolumn{5}{|c|}{ long term } \\
\hline Sensky $2000 b$ & Supportive therapy & 44 & 25.1 & 27.5 \\
\hline Sensky $2000 b$ & CBT & 46 & 18.2 & 17.7 \\
\hline Tarrier 1998 & Supportive therapy & 21 & 9.90 & 5.11 \\
\hline Tarrier 1998 & CBT & 23 & 10.39 & 3.79 \\
\hline
\end{tabular}

Analysis 2.16. Comparison 2 SUPPORTIVE THERAPY versus ANY OTHER PSYCHOLOGICAL OR PSYCHOSOCIAL TREATMENT, Outcome 16 Mental state: 13. Average endpoint positive symptoms score (PANSS positive subscale, high $=$ poor).

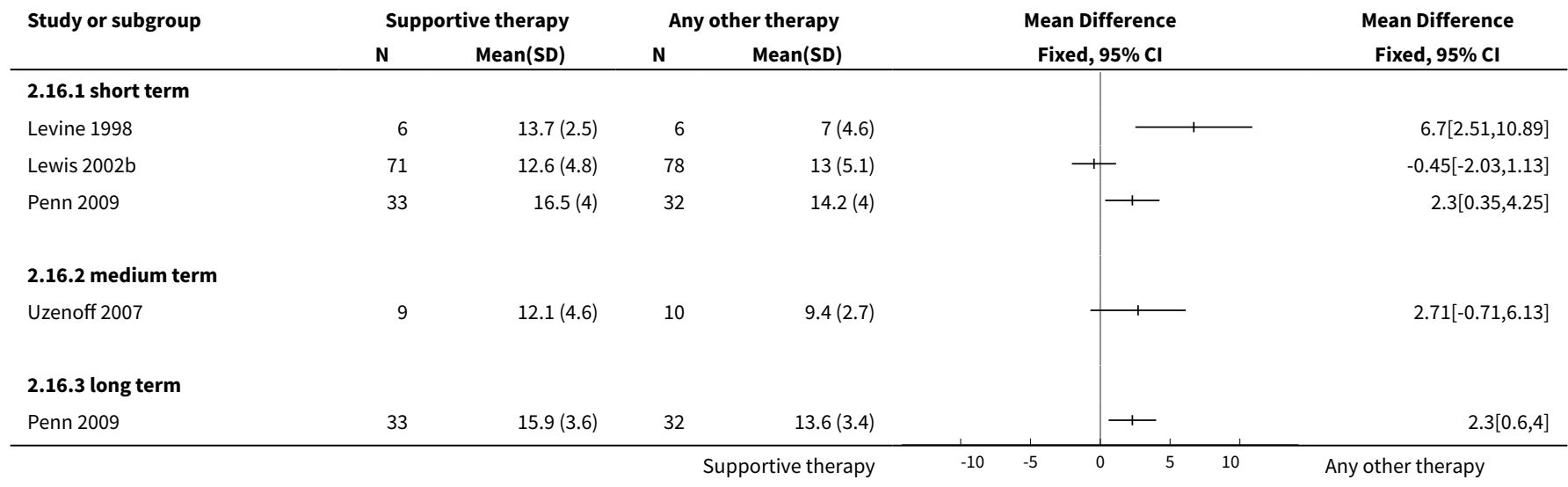


Analysis 2.17. Comparison 2 SUPPORTIVE THERAPY versus ANY OTHER

PSYCHOLOGICAL OR PSYCHOSOCIAL TREATMENT, Outcome 17 Mental state: 14.

Average endpoint positive symptoms score (SAPS, skewed data, high = poor).

\begin{tabular}{|c|c|c|c|c|}
\hline \multicolumn{5}{|c|}{ Mental state: 14. Average endpoint positive symptoms score (SAPS, skewed data, high = poor) } \\
\hline Study & Intervention & Mean & SD & $\mathbf{N}$ \\
\hline \multicolumn{5}{|c|}{ medium term } \\
\hline Pinto 1999 & Supportive therapy & 29.9 & 12.1 & 18 \\
\hline Pinto 1999 & CBT & 17.9 & 17.0 & 19 \\
\hline
\end{tabular}

Analysis 2.18. Comparison 2 SUPPORTIVE THERAPY versus ANY OTHER PSYCHOLOGICAL OR PSYCHOSOCIAL TREATMENT, Outcome 18 Mental state: 15. Average endpoint positive symptoms score (BPRS, skewed data, high = poor).

\begin{tabular}{|c|c|c|c|c|}
\hline \multicolumn{5}{|c|}{ Mental state: 15. Average endpoint positive symptoms score (BPRS, skewed data, high = poor) } \\
\hline Study & Intervention & $\mathbf{N}$ & Mean & SD \\
\hline \multicolumn{5}{|c|}{ short term } \\
\hline Tarrier 1998 & Supportive therapy & 21 & 15.81 & 16.10 \\
\hline Tarrier 1998 & CBT & 23 & 10.67 & 9.42 \\
\hline \multicolumn{5}{|c|}{ long term } \\
\hline Tarrier 1998 & Supportive therapy & 21 & 16.30 & 16.71 \\
\hline
\end{tabular}

Analysis 2.19. Comparison 2 SUPPORTIVE THERAPY versus ANY OTHER PSYCHOLOGICAL OR PSYCHOSOCIAL TREATMENT, Outcome 19 Mental state: 16. Average endpoint score (PANSS thought disturbance cluster, high = poor).

\begin{tabular}{|c|c|c|c|c|c|c|c|}
\hline \multirow[t]{2}{*}{ Study or subgroup } & \multicolumn{2}{|c|}{ Supportive therapy } & \multicolumn{2}{|c|}{ Any other therapy } & \multirow{2}{*}{$\begin{array}{c}\text { Mean Difference } \\
\text { Fixed, } 95 \% \mathrm{Cl}\end{array}$} & \multirow[t]{2}{*}{ Weight } & \multirow{2}{*}{$\begin{array}{c}\text { Mean Difference } \\
\text { Fixed, } 95 \% \mathrm{Cl}\end{array}$} \\
\hline & $\mathbf{N}$ & Mean(SD) & $\mathbf{N}$ & Mean(SD) & & & \\
\hline \multicolumn{8}{|l|}{ 2.19.1 short term } \\
\hline Levine 1998 & 6 & $7.8(2.1)$ & 6 & $3.5(3.3)$ & & $100 \%$ & $4.3[1.17,7.43]$ \\
\hline Subtotal $\star \star \star$ & 6 & & 6 & & & $100 \%$ & $4.3[1.17,7.43]$ \\
\hline \multicolumn{8}{|c|}{ Heterogeneity: Not applicable } \\
\hline \multicolumn{8}{|c|}{ Test for overall effect: $Z=2.69(P=0.01)$} \\
\hline
\end{tabular}

Analysis 2.20. Comparison 2 SUPPORTIVE THERAPY versus ANY OTHER PSYCHOLOGICAL OR PSYCHOSOCIAL TREATMENT, Outcome 20 Mental state: 17. Average endpoint delusions score (PSYRATS delusions score, skewed data, high = poor).

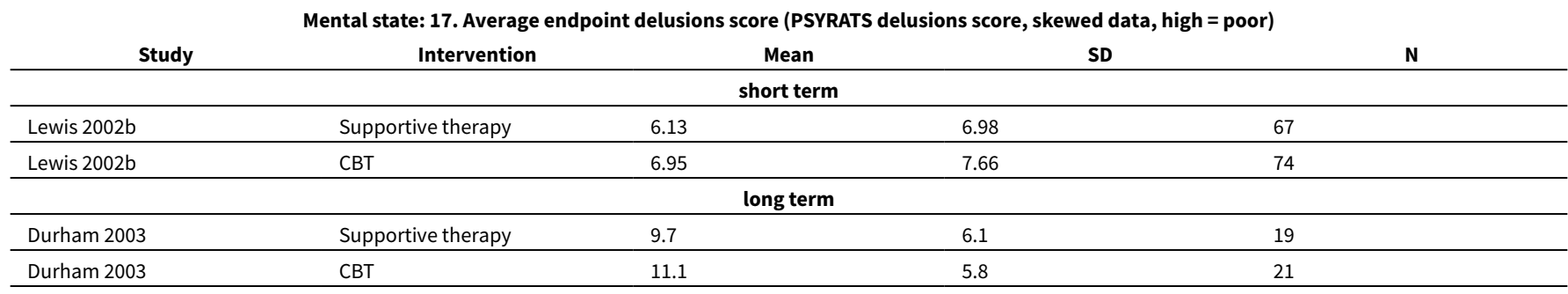


Analysis 2.21. Comparison 2 SUPPORTIVE THERAPY versus ANY OTHER PSYCHOLOGICAL

OR PSYCHOSOCIAL TREATMENT, Outcome 21 Mental state: 18. Average endpoint

hallucinations score (PSYRATS hallucinations score, skewed data, high = poor).

\begin{tabular}{|c|c|c|c|c|}
\hline \multicolumn{5}{|c|}{ Mental state: 18. Average endpoint hallucinations score (PSYRATS hallucinations score, skewed data, high = poor) } \\
\hline Study & Intervention & Mean & SD & $\mathbf{N}$ \\
\hline \multicolumn{5}{|c|}{ long term } \\
\hline Durham 2003 & Supportive therapy & 18.0 & 12.2 & 19 \\
\hline Durham 2003 & CBT & 18.5 & 12.8 & 20 \\
\hline
\end{tabular}

Analysis 2.22. Comparison 2 SUPPORTIVE THERAPY versus ANY OTHER PSYCHOLOGICAL OR PSYCHOSOCIAL TREATMENT, Outcome 22 Mental state: 19. Average endpoint voices score (PSYRATS voices score, high = poor).

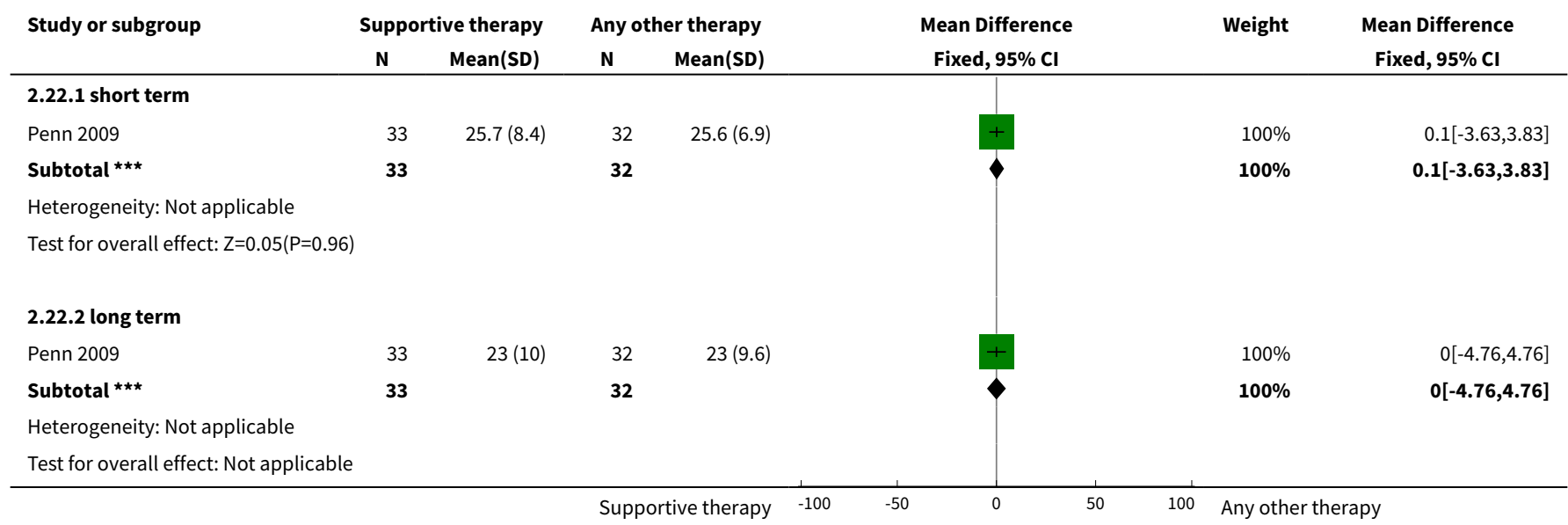

Analysis 2.23. Comparison 2 SUPPORTIVE THERAPY versus ANY OTHER

PSYCHOLOGICAL OR PSYCHOSOCIAL TREATMENT, Outcome 23 Mental state: 20. Average endpoint beliefs about voices score (BAVQ, high = poor, skewed data).

\begin{tabular}{|c|c|c|c|c|c|c|c|}
\hline \multicolumn{7}{|c|}{ Mental state: 20. Average endpoint beliefs about voices score (BAVQ, high = poor, skewed data) } & \\
\hline Study & Follow-up length & Intervention & Mean & & SD & $\mathbf{N}$ & \\
\hline \multicolumn{7}{|c|}{ Malevolence } & \\
\hline Penn 2009 & Short term & Supportive therapy & 8.1 & 5.4 & & 33 & \\
\hline Penn 2009 & Short term & CBT & 6.3 & 5.4 & & 32 & \\
\hline Penn 2009 & Long term & Supportive therapy & 6.7 & 4.9 & & 33 & \\
\hline Penn 2009 & Long term & CBT & 6.3 & 5.4 & & 32 & \\
\hline Penn 2009 & Short term & Supportive therapy & 5.7 & 5.5 & & 33 & \\
\hline Penn 2009 & Short term & CBT & 4.4 & 5.3 & & 32 & \\
\hline Penn 2009 & Long term & Supportive therapy & 6.2 & 5.4 & & 33 & \\
\hline Penn 2009 & Long term & CBT & 5.3 & 5.2 & & 32 & \\
\hline \multicolumn{8}{|c|}{ Resistance } \\
\hline Penn 2009 & Short term & Supportive therapy & 16.3 & 6.6 & & 33 & \\
\hline Penn 2009 & Long term & Supportive therapy & 15.3 & 7.3 & & 33 & \\
\hline Penn 2009 & Long term & CBT & 13.9 & 7.8 & & 32 & \\
\hline \multicolumn{8}{|c|}{ Engagement } \\
\hline Penn 2009 & Short term & Supportive therapy & 7.8 & 7.4 & & 33 & \\
\hline Penn 2009 & Short term & CBT & 5.4 & 5.7 & & 32 & \\
\hline Penn 2009 & Long term & Supportive therapy & 7.3 & 6.8 & & 33 & \\
\hline
\end{tabular}

Supportive therapy for schizophrenia (Review) 
Mental state: 20. Average endpoint beliefs about voices score (BAVQ, high = poor, skewed data)

\begin{tabular}{|c|c|c|c|c|c|}
\hline Study & Follow-up length & Intervention & Mean & SD & $\mathbf{N}$ \\
\hline Penn 2009 & Long term & CBT & 6.4 & 7.2 & 32 \\
\hline \multicolumn{6}{|c|}{ Omnipotence } \\
\hline Penn 2009 & Short term & Supportive therapy & 8.6 & 5 & 33 \\
\hline Penn 2009 & Short term & CBT & 8 & 4.2 & 32 \\
\hline Penn 2009 & Long term & Supportive therapy & 7.7 & 4.3 & 33 \\
\hline Penn 2009 & Long term & CBT & 7.3 & 3.3 & 32 \\
\hline
\end{tabular}

Analysis 2.24. Comparison 2 SUPPORTIVE THERAPY versus ANY OTHER PSYCHOLOGICAL OR PSYCHOSOCIAL TREATMENT, Outcome 24 Mental state: 21. Average endpoint depression score (MADRS, skewed data, high = poor).

\begin{tabular}{|c|c|c|c|c|c|}
\hline \multicolumn{6}{|c|}{ Mental state: 21 . Average endpoint depression score (MADRS, skewed data, high = poor) } \\
\hline Study & Intervention & & $\mathbf{N}$ & Mean & SD \\
\hline \multicolumn{6}{|c|}{ medium term } \\
\hline Sensky $2000 b$ & Supportive therapy & 44 & & 6.0 & 5.4 \\
\hline Sensky $2000 b$ & CBT & 46 & & 4.8 & 3.5 \\
\hline \multicolumn{6}{|c|}{ long term } \\
\hline Sensky $2000 \mathrm{~b}$ & Supportive therapy & 44 & & 6.7 & 7.1 \\
\hline
\end{tabular}

Analysis 2.25. Comparison 2 SUPPORTIVE THERAPY versus ANY OTHER PSYCHOLOGICAL OR PSYCHOSOCIAL TREATMENT, Outcome 25 Mental state: 22. Average endpoint depression score (BDI-II, skewed data, high = poor).

Mental state: 22. Average endpoint depression score (BDI-II, skewed data, high = poor)

\begin{tabular}{|c|c|c|c|c|c|}
\hline Study & Follow-up length & Intervention & Mean & SD & $\mathbf{N}$ \\
\hline Penn 2009 & Short term & Supportive therapy & 13.9 & 10.7 & 33 \\
\hline Penn 2009 & Short term & CBT & 10.5 & 8.5 & 32 \\
\hline Penn 2009 & Long term & Supportive therapy & 17.9 & 13.6 & 33 \\
\hline Penn 2009 & Long term & CBT & 11.5 & 9.4 & 32 \\
\hline
\end{tabular}

Analysis 2.26. Comparison 2 SUPPORTIVE THERAPY versus ANY OTHER PSYCHOLOGICAL OR PSYCHOSOCIAL TREATMENT, Outcome 26 Leaving the study early.

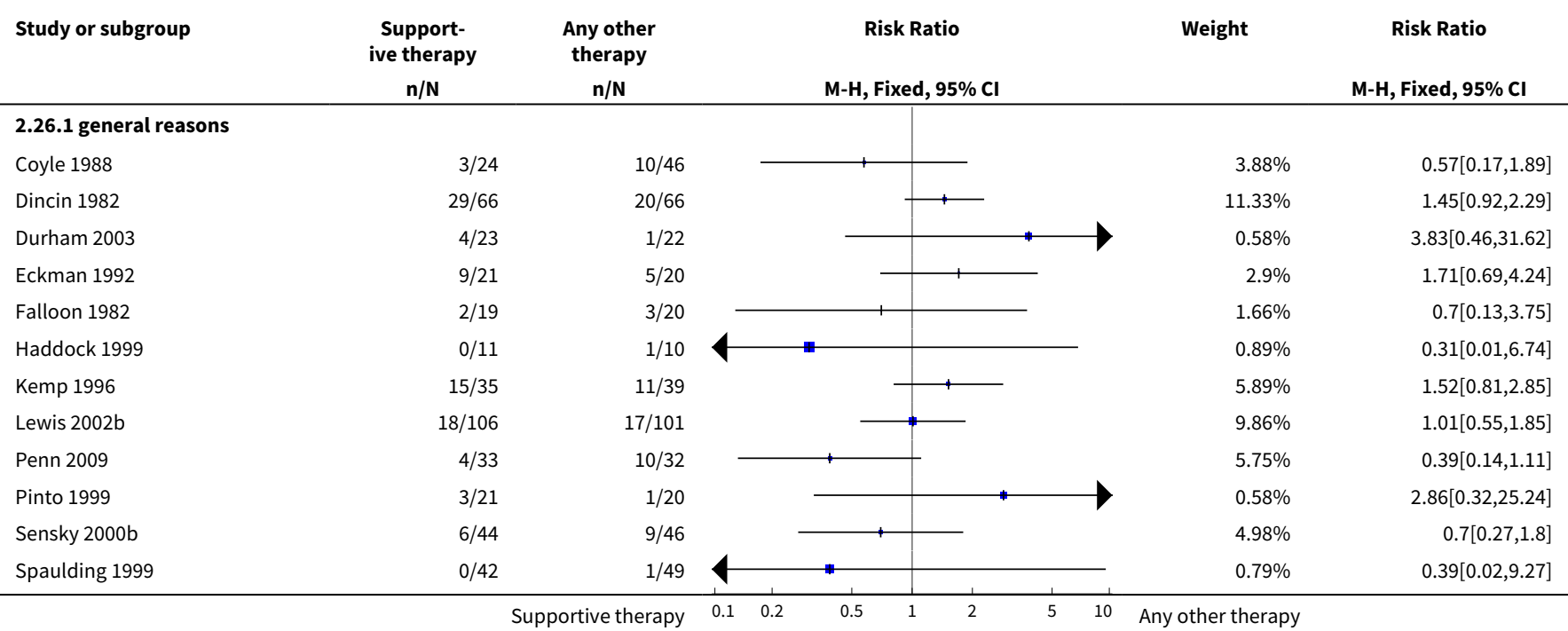




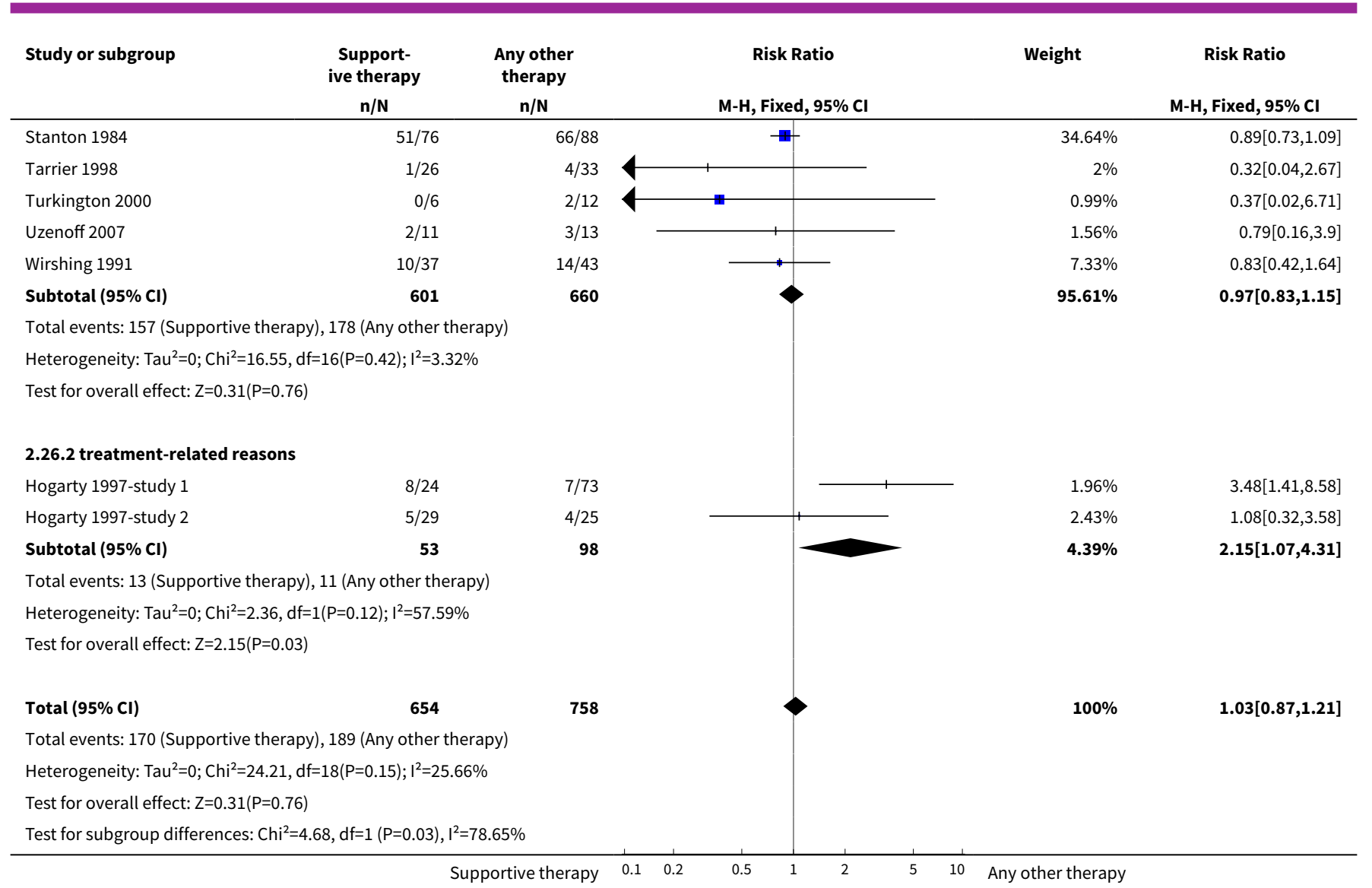

Analysis 2.27. Comparison 2 SUPPORTIVE THERAPY versus ANY OTHER PSYCHOLOGICAL OR PSYCHOSOCIAL TREATMENT, Outcome 27 General functioning: 1. Average endpoint general functioning score (GAF \& GAS, high = good).

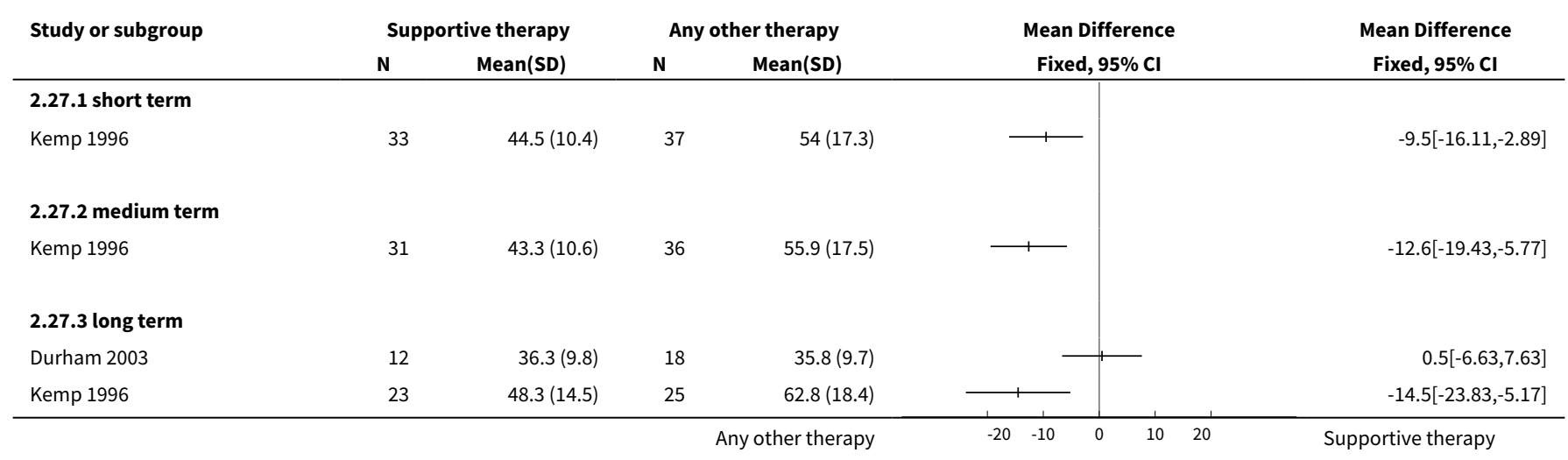


Analysis 2.28. Comparison 2 SUPPORTIVE THERAPY versus ANY OTHER PSYCHOLOGICAL OR PSYCHOSOCIAL TREATMENT, Outcome 28 General functioning: 2 . Average endpoint social functioning score (SFS, high = good).

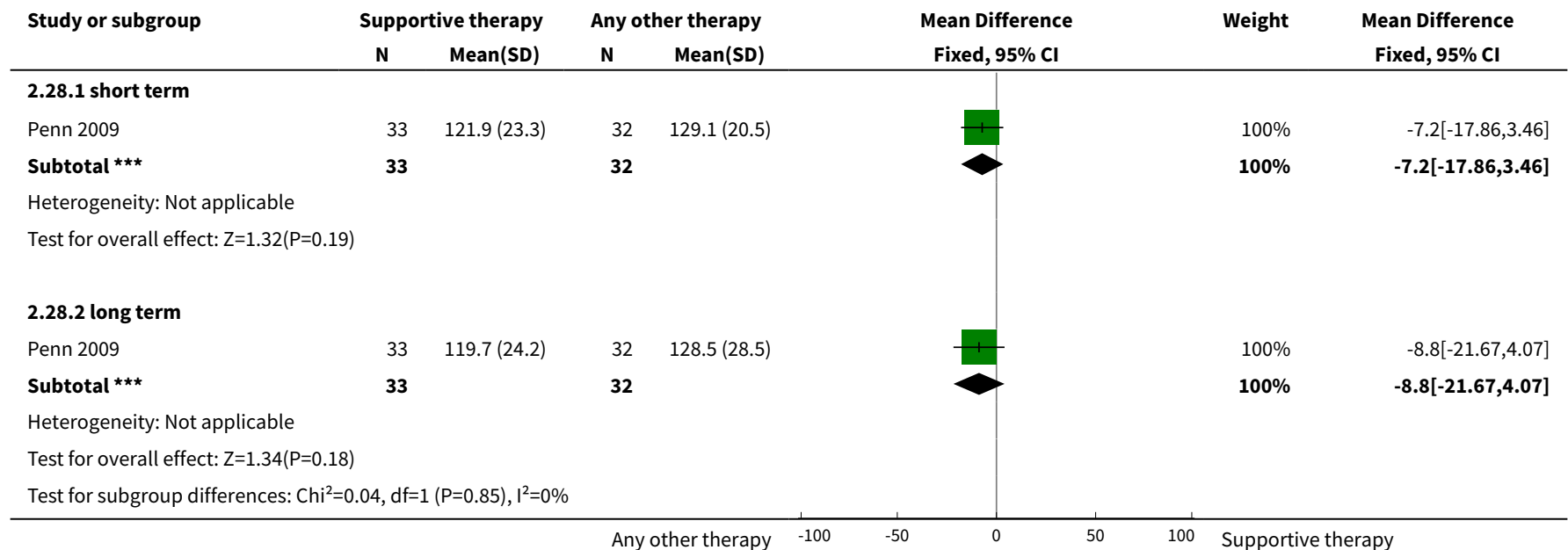

Analysis 2.29. Comparison 2 SUPPORTIVE THERAPY versus ANY OTHER PSYCHOLOGICAL OR PSYCHOSOCIAL TREATMENT, Outcome 29 General functioning: 3. No paid work.

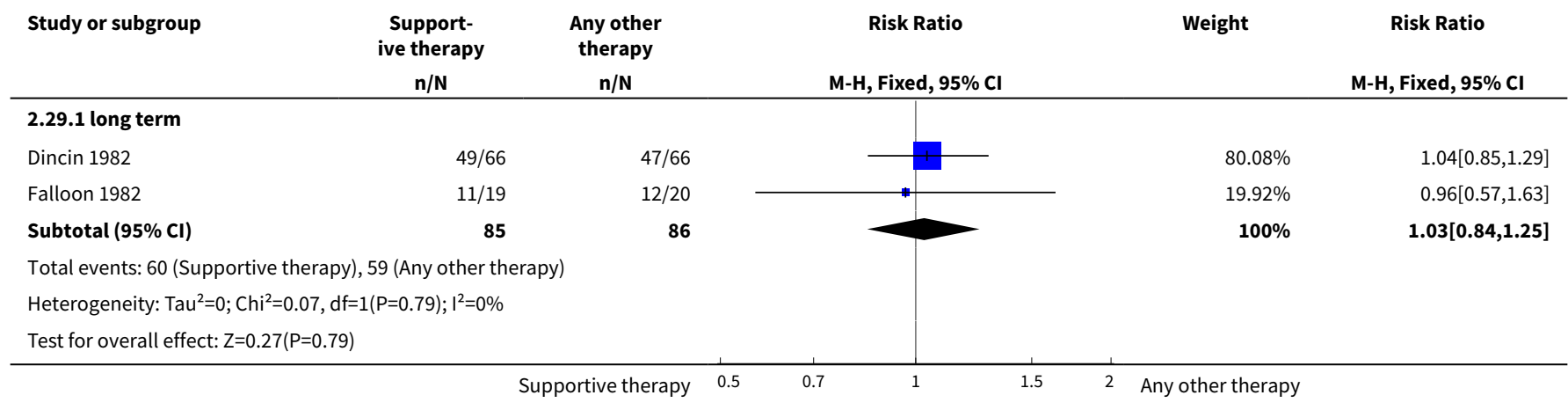

Analysis 2.30. Comparison 2 SUPPORTIVE THERAPY versus ANY OTHER PSYCHOLOGICAL OR PSYCHOSOCIAL TREATMENT, Outcome 30 General functioning: 4. Admission to residential placement.

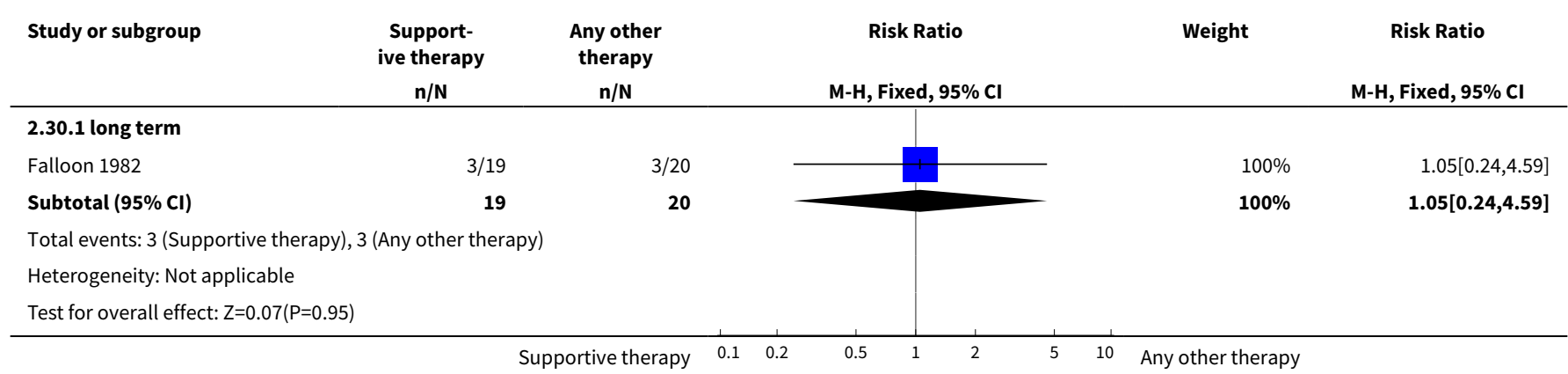


Analysis 2.31. Comparison 2 SUPPORTIVE THERAPY versus ANY OTHER PSYCHOLOGICAL OR PSYCHOSOCIAL TREATMENT, Outcome 31 General functioning: 5. Admission to jail.

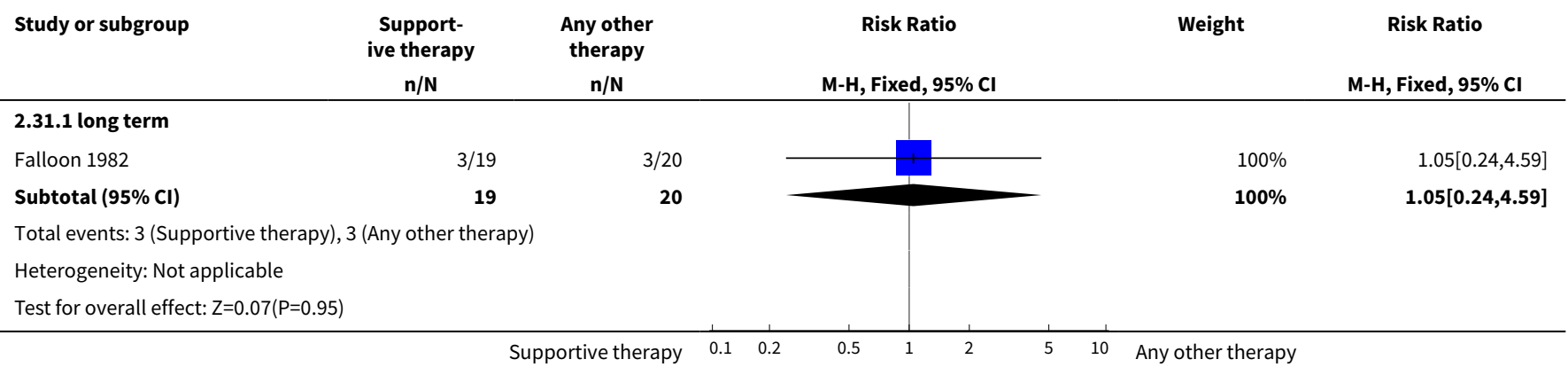

Analysis 2.32. Comparison 2 SUPPORTIVE THERAPY versus ANY OTHER PSYCHOLOGICAL OR PSYCHOSOCIAL TREATMENT, Outcome 32 Satisfaction with treatment: Recipient of care not satisfied with treatment.

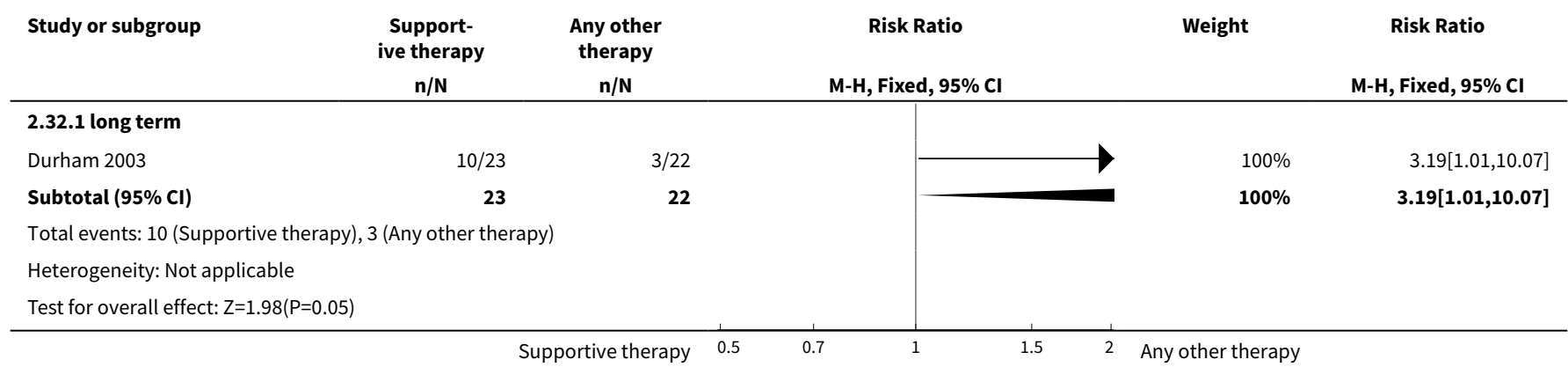

Analysis 2.33. Comparison 2 SUPPORTIVE THERAPY versus ANY OTHER PSYCHOLOGICAL OR PSYCHOSOCIAL TREATMENT, Outcome 33 Quality of life: Average endpoint quality of life score (QLS, high = good).

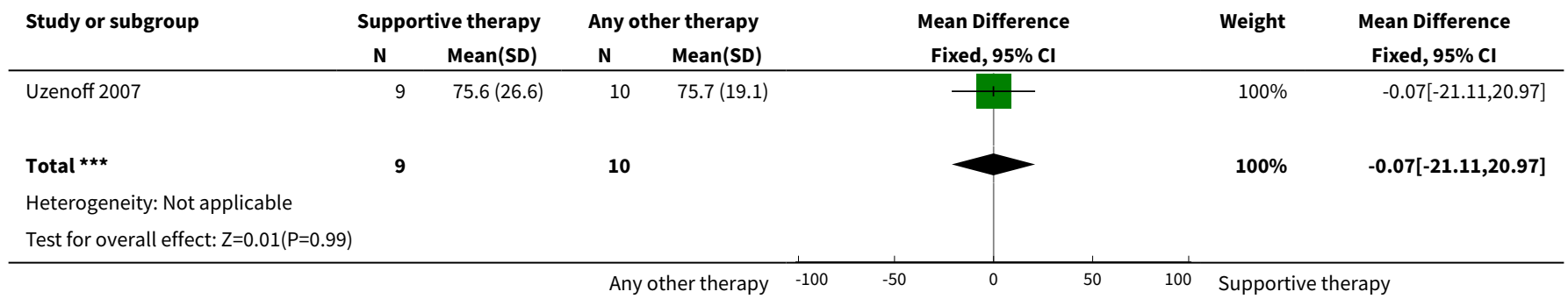

Analysis 2.34. Comparison 2 SUPPORTIVE THERAPY versus ANY OTHER PSYCHOLOGICAL OR PSYCHOSOCIAL TREATMENT, Outcome 34 Death.

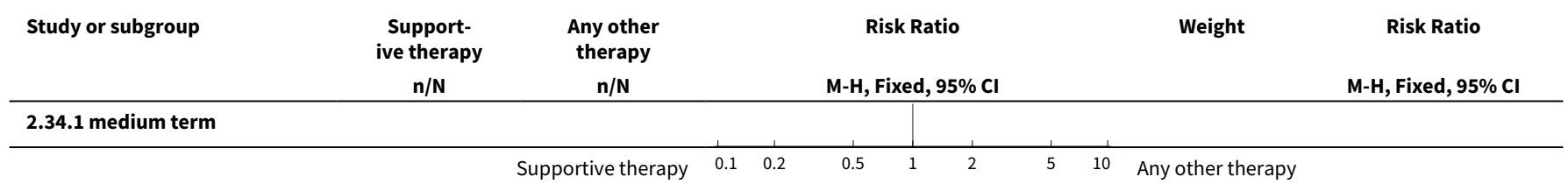




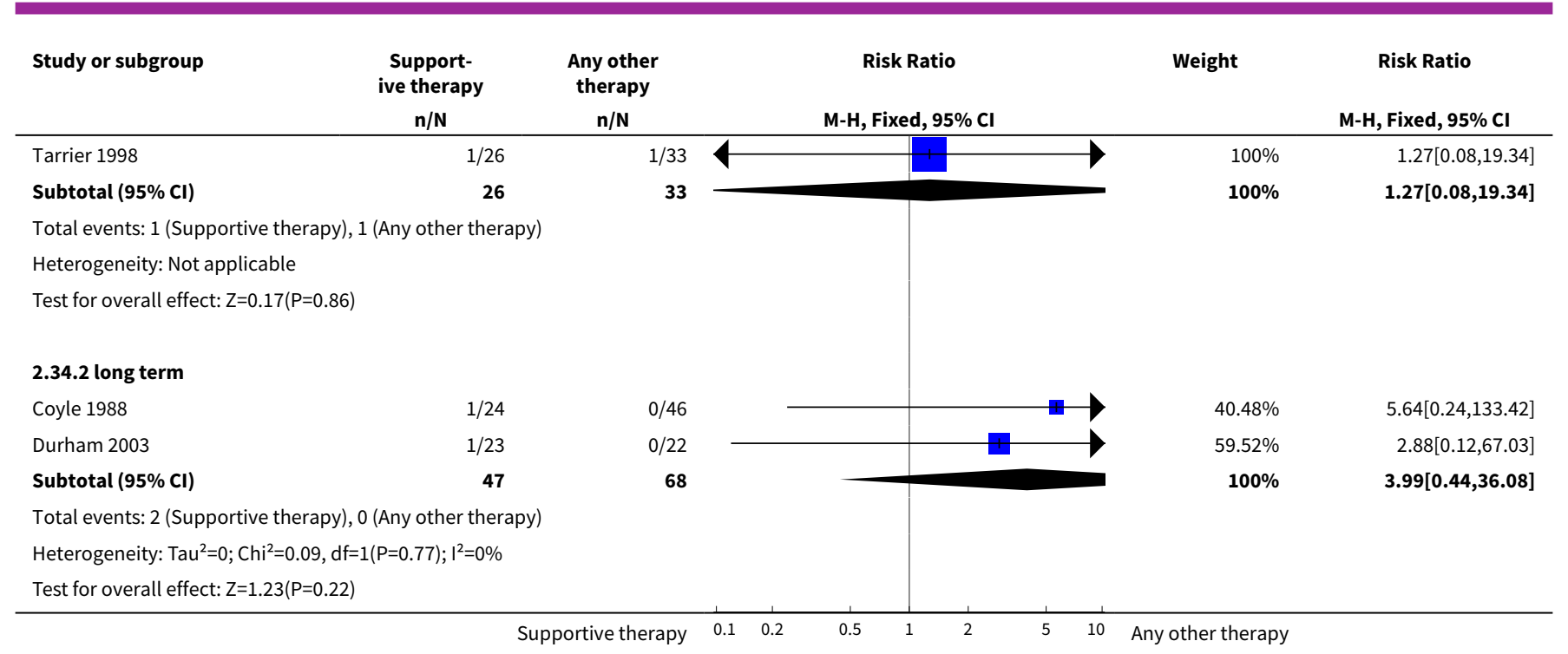

Analysis 2.35. Comparison 2 SUPPORTIVE THERAPY versus ANY OTHER PSYCHOLOGICAL OR PSYCHOSOCIAL TREATMENT, Outcome 35 Behaviour: 1. Social impairment on SBAS.

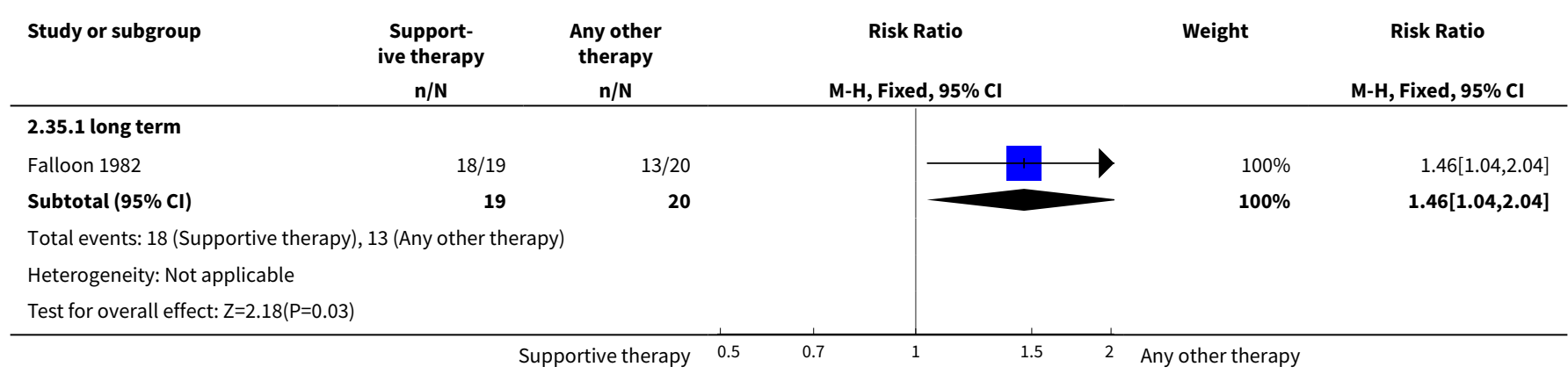

Analysis 2.36. Comparison 2 SUPPORTIVE THERAPY versus ANY OTHER PSYCHOLOGICAL OR PSYCHOSOCIAL TREATMENT, Outcome 36 Behaviour: 2. Poor coping style with relatives.

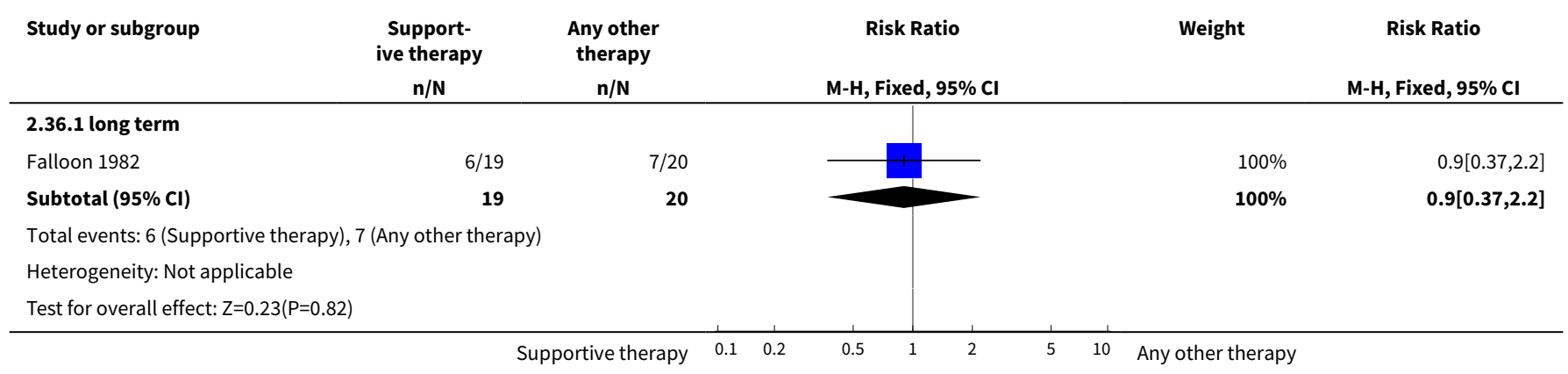


Analysis 2.37. Comparison 2 SUPPORTIVE THERAPY versus ANY OTHER PSYCHOLOGICAL OR

PSYCHOSOCIAL TREATMENT, Outcome 37 Engagement with services: Poor attendance at appointments.

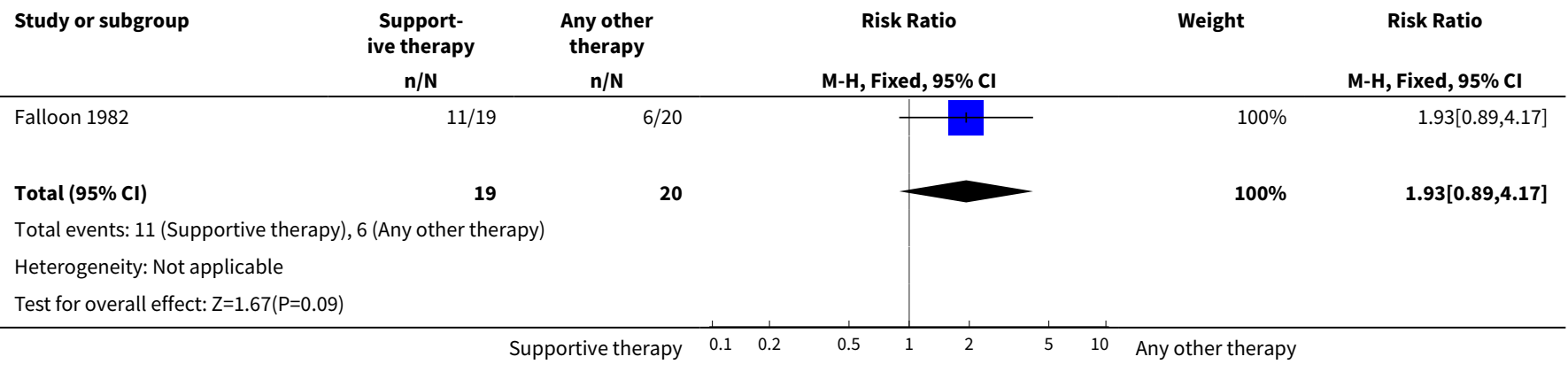

Analysis 2.38. Comparison 2 SUPPORTIVE THERAPY versus ANY OTHER PSYCHOLOGICAL OR PSYCHOSOCIAL TREATMENT, Outcome 38 Insight: Average endpoint insight score (SAI, high = good, skewed data).

Insight: Average endpoint insight score (SAI, high = good, skewed data)

\begin{tabular}{|c|c|c|c|c|}
\hline Study & Intervention & Mean & SD & $\mathbf{N}$ \\
\hline \multicolumn{5}{|c|}{ short term } \\
\hline Kemp 1996 & Supportive therapy & 40.60 & 31.20 & 35 \\
\hline Kemp 1996 & CBT & 63.00 & 23.60 & 39 \\
\hline \multicolumn{5}{|c|}{ medium term } \\
\hline Kemp 1996 & Supportive therapy & 41.90 & 30.80 & 29 \\
\hline Kemp 1996 & CBT & 62.60 & 23.50 & 34 \\
\hline Kemp 1996 & Supportive therapy & 42.60 & 36.50 & 20 \\
\hline Kemp 1996 & CBT & 63.40 & 25.50 & 30 \\
\hline
\end{tabular}

Analysis 2.39. Comparison 2 SUPPORTIVE THERAPY versus ANY OTHER PSYCHOLOGICAL OR PSYCHOSOCIAL TREATMENT, Outcome 39 Compliance: 1. Poor compliance to therapy.

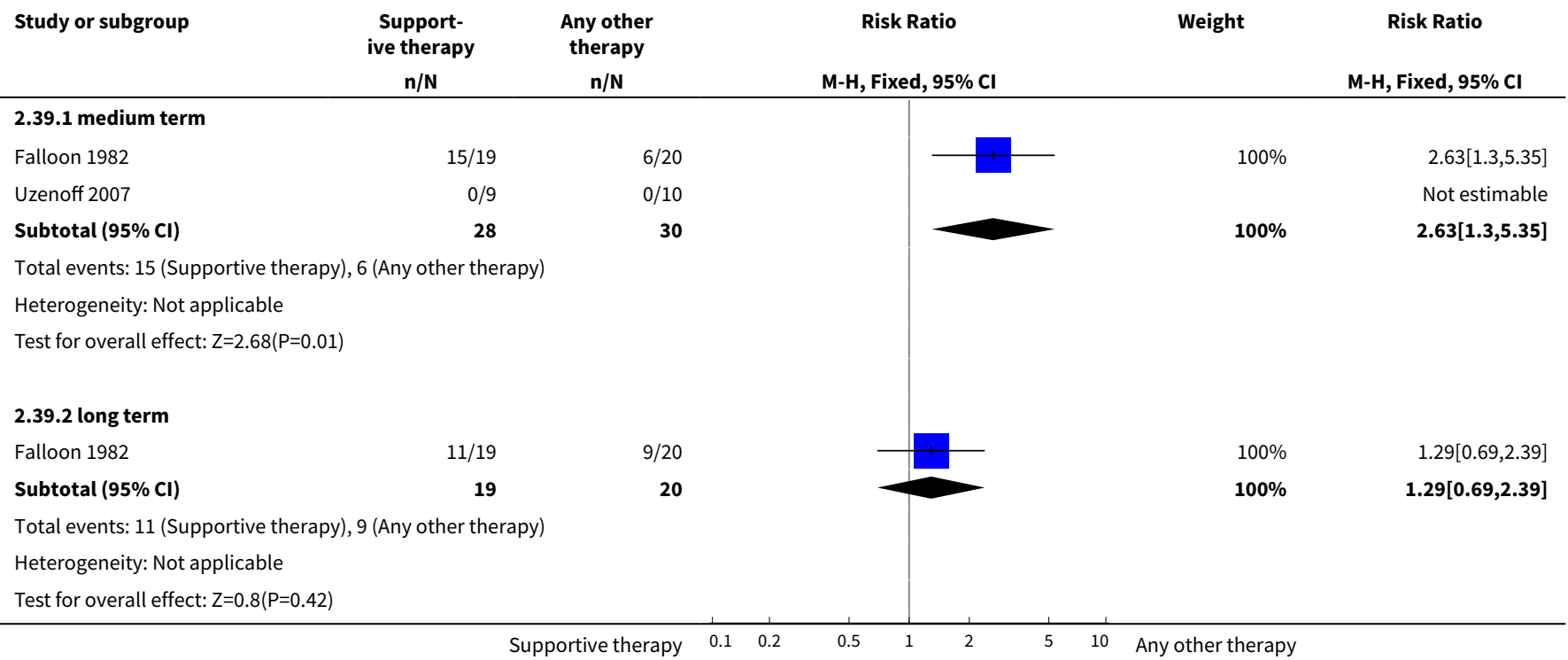


Analysis 2.40. Comparison 2 SUPPORTIVE THERAPY versus ANY OTHER PSYCHOLOGICAL OR PSYCHOSOCIAL TREATMENT, Outcome 40 Compliance: 2 . adherence to medication (self-report).

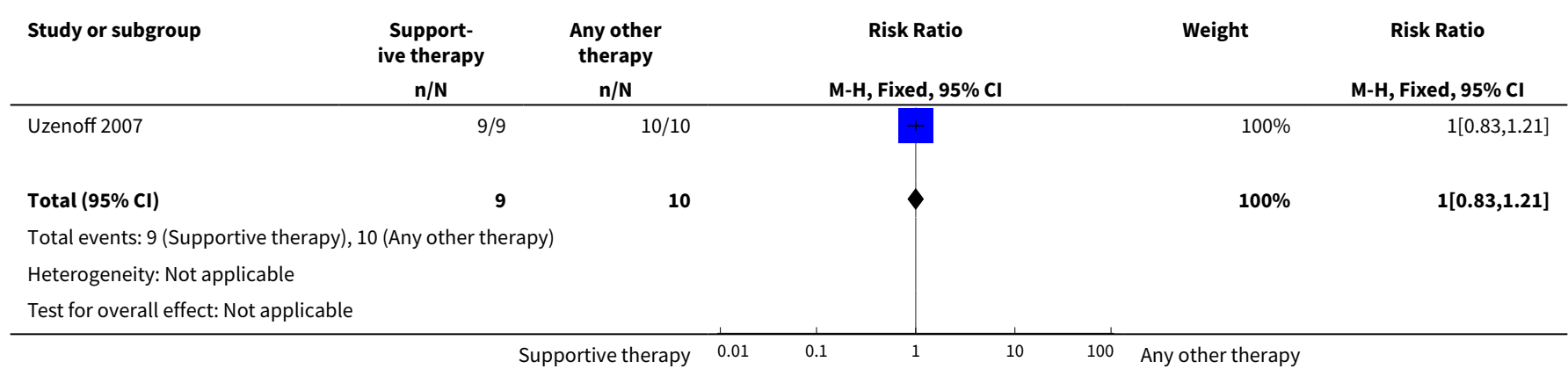

Analysis 2.41. Comparison 2 SUPPORTIVE THERAPY versus ANY OTHER PSYCHOLOGICAL OR PSYCHOSOCIAL TREATMENT, Outcome 41 Compliance: 3. average endpoint adherence score (ROMI, high = good).

\begin{tabular}{|c|c|c|c|c|c|c|c|c|}
\hline \multirow[t]{2}{*}{ Study or subgroup } & \multicolumn{2}{|c|}{ Supportive therapy } & \multicolumn{2}{|c|}{ Any other therapy } & \multirow{2}{*}{\multicolumn{2}{|c|}{$\begin{array}{c}\text { Mean Difference } \\
\text { Fixed, } 95 \% \mathrm{Cl} \\
\end{array}$}} & \multirow[t]{2}{*}{ Weight } & \multirow{2}{*}{$\begin{array}{c}\text { Mean Difference } \\
\text { Fixed, } 95 \% \mathrm{Cl}\end{array}$} \\
\hline & $\mathbf{N}$ & Mean(SD) & $\mathbf{N}$ & Mean(SD) & & & & \\
\hline Uzenoff 2007 & 9 & $16.8(3.7)$ & 10 & $16.3(5.2)$ & & + & $100 \%$ & $0.45[-3.58,4.48]$ \\
\hline Total $\star \star \star ~$ & 9 & & 10 & & & $\gamma$ & $100 \%$ & $0.45[-3.58,4.48]$ \\
\hline \multicolumn{9}{|c|}{ Heterogeneity: Not applicable } \\
\hline \multicolumn{9}{|c|}{ Test for overall effect: $Z=0.22(P=0.83)$} \\
\hline
\end{tabular}
Analysis 2.42. Comparison 2 SUPPORTIVE THERAPY versus ANY OTHER PSYCHOLOGICAL OR PSYCHOSOCIAL
TREATMENT, Outcome 42 Compliance: 4 . average endpoint non-adherence score (ROMI, high = poor).

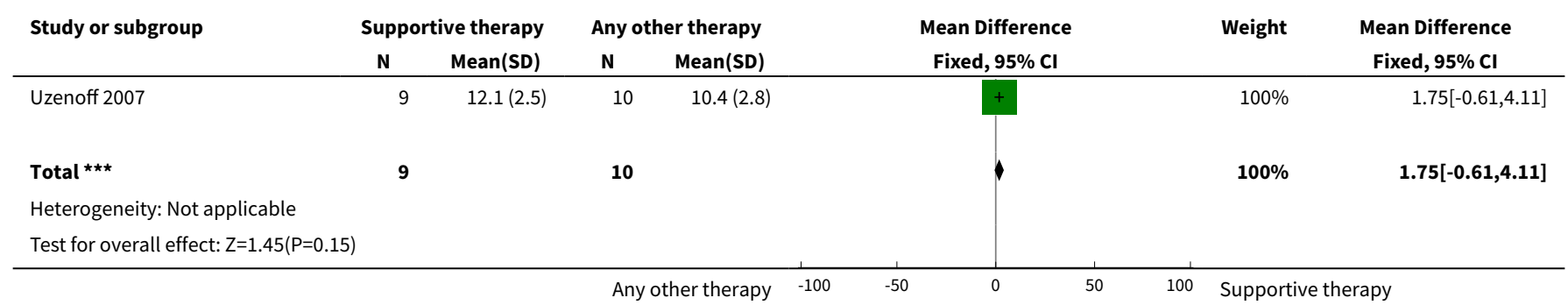

Analysis 2.43. Comparison 2 SUPPORTIVE THERAPY versus ANY OTHER PSYCHOLOGICAL OR PSYCHOSOCIAL TREATMENT, Outcome 43 Medication: Prescribed IM depot medication.

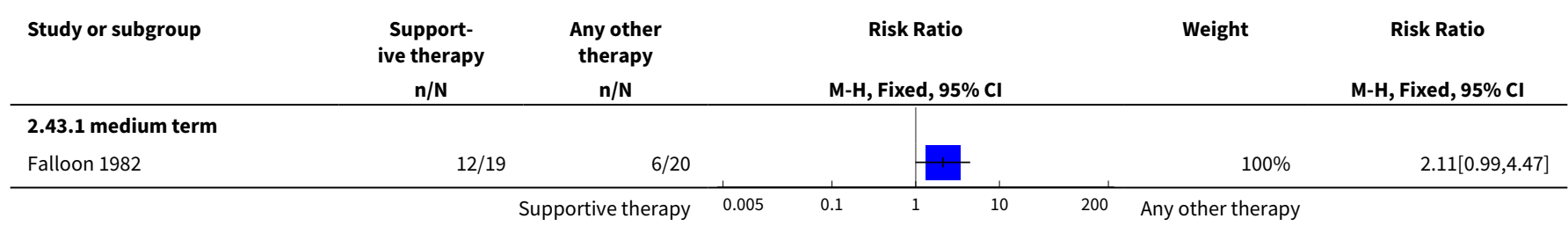




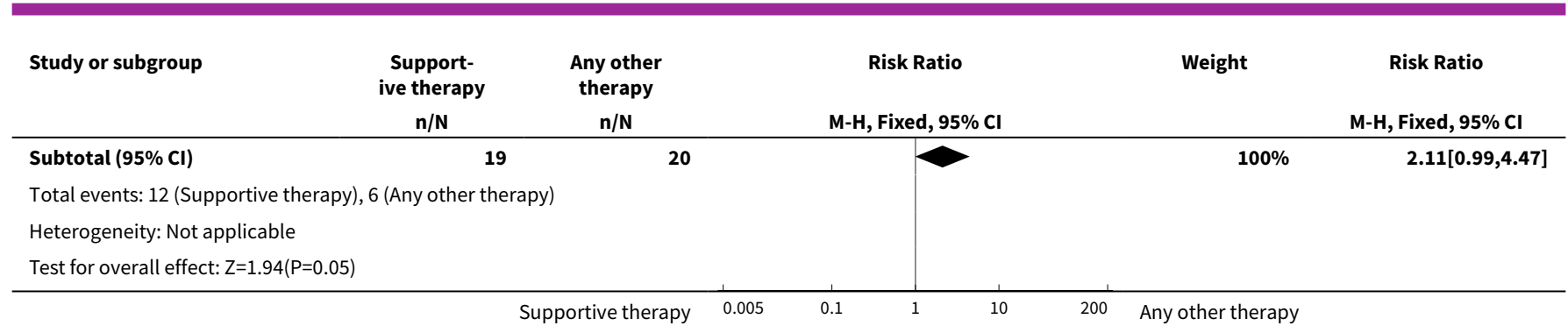

Analysis 2.44. Comparison 2 SUPPORTIVE THERAPY versus ANY OTHER PSYCHOLOGICAL OR PSYCHOSOCIAL TREATMENT, Outcome 44 Attitude to medication: 1. Average endpoint attitude to medication score (AMQ, high = good).

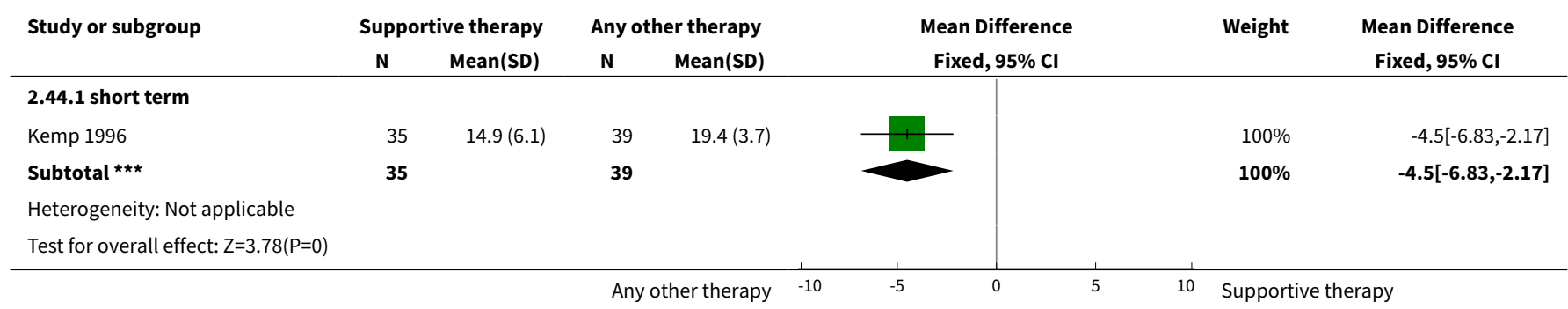

Analysis 2.45. Comparison 2 SUPPORTIVE THERAPY versus ANY OTHER PSYCHOLOGICAL OR PSYCHOSOCIAL TREATMENT, Outcome 45 Attitude to medication: 2. Average endpoint attitude to medication score (DAI, high = good).

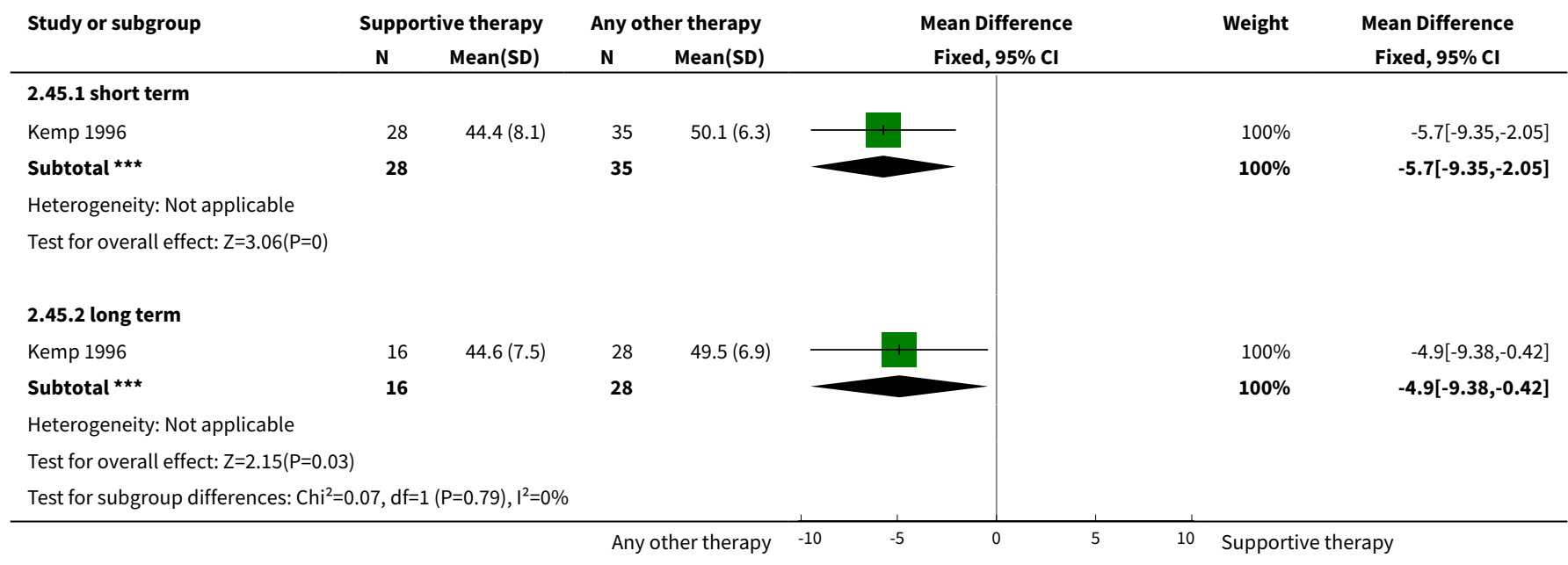

Analysis 2.46. Comparison 2 SUPPORTIVE THERAPY versus ANY OTHER PSYCHOLOGICAL OR PSYCHOSOCIAL TREATMENT, Outcome 46 Economic outcomes: Direct costs (skewed data, not ITT).

Economic outcomes: Direct costs (skewed data, not ITT)

\begin{tabular}{lcccc} 
& Intervention & Mean & SD & \\
& & medium term & & \\
\hline Kemp 1996 & Supportive therapy & 252 & & 234 \\
\hline \hline Supportive therapy for schizophrenia (Review) & & & & \\
\hline
\end{tabular}




\begin{tabular}{|c|c|c|c|c|}
\hline \multicolumn{5}{|c|}{ Economic outcomes: Direct costs (skewed data, not ITT) } \\
\hline Study & Intervention & Mean & SD & $\mathbf{N}$ \\
\hline Kemp 1996 & CBT & 187 & 292 & 36 \\
\hline \multicolumn{5}{|c|}{ long term } \\
\hline Kemp 1996 & Supportive therapy & 326 & 404 & 21 \\
\hline Kemp 1996 & CBT & 239 & 281 & 24 \\
\hline
\end{tabular}

\section{Comparison 3. SUPPORTIVE THERAPY versus COGNITIVE BEHAVIOURAL THERAPY}

\begin{tabular}{|c|c|c|c|c|}
\hline Outcome or subgroup title & No. of studies & $\begin{array}{l}\text { No. of partici- } \\
\text { pants }\end{array}$ & Statistical method & Effect size \\
\hline 1 Global state: Relapse & 5 & & $\begin{array}{l}\text { Risk Ratio (M-H, Fixed, } \\
95 \% \mathrm{Cl})\end{array}$ & Totals not selected \\
\hline 1.1 medium term & 2 & & $\begin{array}{l}\text { Risk Ratio (M-H, Fixed, } \\
95 \% \mathrm{Cl})\end{array}$ & $0.0[0.0,0.0]$ \\
\hline 1.2 long term & 4 & & $\begin{array}{l}\text { Risk Ratio (M-H, Fixed, } \\
95 \% \mathrm{Cl})\end{array}$ & $0.0[0.0,0.0]$ \\
\hline 2 Service outcomes: Hospitalisation & 3 & & $\begin{array}{l}\text { Risk Ratio (M-H, Fixed, } \\
95 \% \mathrm{Cl})\end{array}$ & Subtotals only \\
\hline 2.1 medium term & 3 & 153 & $\begin{array}{l}\text { Risk Ratio (M-H, Fixed, } \\
95 \% \mathrm{Cl})\end{array}$ & $1.60[0.85,3.00]$ \\
\hline 2.2 long term & 1 & 65 & $\begin{array}{l}\text { Risk Ratio (M-H, Fixed, } \\
95 \% \mathrm{Cl})\end{array}$ & $0.73[0.18,3.00]$ \\
\hline $\begin{array}{l}3 \text { Mental state: } 1 . \text { No clinically impor- } \\
\text { tant improvement in general mental } \\
\text { state }\end{array}$ & 3 & & $\begin{array}{l}\text { Risk Ratio (M-H, Random, } \\
95 \% \mathrm{Cl})\end{array}$ & Subtotals only \\
\hline 3.1 medium term & 1 & 59 & $\begin{array}{l}\text { Risk Ratio (M-H, Random, } \\
95 \% \mathrm{Cl} \text { ) }\end{array}$ & $1.27[0.95,1.70]$ \\
\hline 3.2 long term & 3 & 194 & $\begin{array}{l}\text { Risk Ratio (M-H, Random, } \\
95 \% \mathrm{Cl} \text { ) }\end{array}$ & $1.23[0.89,1.70]$ \\
\hline $\begin{array}{l}4 \text { Mental state: } 2 \text {. Average endpoint } \\
\text { general mental state score (PANSS to- } \\
\text { tal, high = poor) }\end{array}$ & 4 & & $\begin{array}{l}\text { Mean Difference (IV, Ran- } \\
\text { dom, } 95 \% \mathrm{Cl} \text { ) }\end{array}$ & Totals not selected \\
\hline 4.1 short term & 3 & & $\begin{array}{l}\text { Mean Difference (IV, Ran- } \\
\text { dom, } 95 \% \mathrm{CI} \text { ) }\end{array}$ & $0.0[0.0,0.0]$ \\
\hline 4.2 long term & 2 & & $\begin{array}{l}\text { Mean Difference (IV, Ran- } \\
\text { dom, } 95 \% \mathrm{CI} \text { ) }\end{array}$ & $0.0[0.0,0.0]$ \\
\hline $\begin{array}{l}5 \text { Mental state: } 3 \text {. Average endpoint } \\
\text { general mental state score (BPRS, high } \\
=\text { poor) }\end{array}$ & 3 & & $\begin{array}{l}\text { Mean Difference (IV, Fixed, } \\
95 \% \mathrm{CI})\end{array}$ & Subtotals only \\
\hline 5.1 short term & 2 & 92 & $\begin{array}{l}\text { Mean Difference (IV, Fixed, } \\
95 \% \mathrm{CI} \text { ) }\end{array}$ & $-1.07[-5.08,2.94]$ \\
\hline
\end{tabular}




\begin{tabular}{|c|c|c|c|c|}
\hline Outcome or subgroup title & No. of studies & $\begin{array}{l}\text { No. of partici- } \\
\text { pants }\end{array}$ & Statistical method & Effect size \\
\hline 5.2 medium term & 1 & 37 & $\begin{array}{l}\text { Mean Difference (IV, Fixed, } \\
95 \% \mathrm{Cl} \text { ) }\end{array}$ & $7.60[0.90,14.30]$ \\
\hline $\begin{array}{l}6 \text { Mental state: } 4 \text {. Average endpoint } \\
\text { general mental state score (BPRS short } \\
\text { form, high = poor) }\end{array}$ & 1 & & $\begin{array}{l}\text { Mean Difference (IV, Fixed, } \\
95 \% \mathrm{CI} \text { ) }\end{array}$ & Subtotals only \\
\hline 6.1 short term & 1 & 74 & $\begin{array}{l}\text { Mean Difference (IV, Fixed, } \\
95 \% \mathrm{Cl} \text { ) }\end{array}$ & $-0.90[-3.02,1.22]$ \\
\hline 6.2 medium term & 1 & 67 & $\begin{array}{l}\text { Mean Difference (IV, Fixed, } \\
95 \% \mathrm{Cl} \text { ) }\end{array}$ & $2.20[-1.18,5.58]$ \\
\hline 6.3 long term & 1 & 45 & $\begin{array}{l}\text { Mean Difference (IV, Fixed, } \\
95 \% \mathrm{Cl} \text { ) }\end{array}$ & $2.30[-0.54,5.14]$ \\
\hline $\begin{array}{l}7 \text { Mental state: } 5 . \text { Average endpoint } \\
\text { general mental state score (CPRS, } \\
\text { skewed data, high = poor) }\end{array}$ & & & Other data & No numeric data \\
\hline 7.1 medium term & & & Other data & No numeric data \\
\hline 7.2 long term & & & Other data & No numeric data \\
\hline $\begin{array}{l}8 \text { Mental state: } 6 \text {. Average endpoint } \\
\text { general mental state score (PANSS gen- } \\
\text { eral subscale, high = poor) }\end{array}$ & 2 & & $\begin{array}{l}\text { Mean Difference (IV, Fixed, } \\
95 \% \mathrm{Cl} \text { ) }\end{array}$ & Totals not selected \\
\hline 8.1 short term & 2 & & $\begin{array}{l}\text { Mean Difference (IV, Fixed, } \\
95 \% \mathrm{Cl} \text { ) }\end{array}$ & $0.0[0.0,0.0]$ \\
\hline 8.2 long term & 1 & & $\begin{array}{l}\text { Mean Difference (IV, Fixed, } \\
95 \% \mathrm{CI})\end{array}$ & $0.0[0.0,0.0]$ \\
\hline $\begin{array}{l}9 \text { Mental state: } 7 . \text { No clinically impor- } \\
\text { tant improvement in negative symp- } \\
\text { toms }\end{array}$ & 1 & 90 & $\begin{array}{l}\text { Risk Ratio (M-H, Fixed, } \\
95 \% \mathrm{Cl})\end{array}$ & $0.95[0.63,1.46]$ \\
\hline $\begin{array}{l}10 \text { Mental state: } 8 . \text { No clinically impor- } \\
\text { tant improvement in depressive symp- } \\
\text { toms }\end{array}$ & 1 & 90 & $\begin{array}{l}\text { Risk Ratio (M-H, Fixed, } \\
95 \% \mathrm{Cl} \text { ) }\end{array}$ & $1.53[0.92,2.55]$ \\
\hline $\begin{array}{l}11 \text { Mental state: } 9 \text {. Episode of affective } \\
\text { symptoms }\end{array}$ & 2 & & $\begin{array}{l}\text { Risk Ratio (M-H, Fixed, } \\
95 \% \mathrm{Cl})\end{array}$ & Subtotals only \\
\hline 11.1 long term & 2 & 101 & $\begin{array}{l}\text { Risk Ratio (M-H, Fixed, } \\
95 \% \mathrm{Cl})\end{array}$ & $2.17[1.16,4.06]$ \\
\hline $\begin{array}{l}12 \text { Mental state: } 10 \text {. Average endpoint } \\
\text { negative symptoms score (PANSS neg- } \\
\text { ative subscale, high = poor) }\end{array}$ & 2 & & $\begin{array}{l}\text { Mean Difference (IV, Fixed, } \\
95 \% \mathrm{CI} \text { ) }\end{array}$ & Totals not selected \\
\hline 12.1 short term & 2 & & $\begin{array}{l}\text { Mean Difference (IV, Fixed, } \\
95 \% \mathrm{CI})\end{array}$ & $0.0[0.0,0.0]$ \\
\hline
\end{tabular}




\begin{tabular}{|c|c|c|c|c|}
\hline Outcome or subgroup title & No. of studies & $\begin{array}{l}\text { No. of partici- } \\
\text { pants }\end{array}$ & Statistical method & Effect size \\
\hline 12.2 long term & 1 & & $\begin{array}{l}\text { Mean Difference (IV, Fixed, } \\
95 \% \mathrm{CI})\end{array}$ & $0.0[0.0,0.0]$ \\
\hline $\begin{array}{l}13 \text { Mental state: } 11 \text {. Average endpoint } \\
\text { negative symptoms score (SANS, high } \\
=\text { poor) }\end{array}$ & 1 & & $\begin{array}{l}\text { Mean Difference (IV, Fixed, } \\
95 \% \mathrm{CI})\end{array}$ & Subtotals only \\
\hline 13.1 medium term & 1 & 37 & $\begin{array}{l}\text { Mean Difference (IV, Fixed, } \\
95 \% \mathrm{CI})\end{array}$ & $6.60[-5.81,19.01]$ \\
\hline $\begin{array}{l}14 \text { Mental state: } 12 \text {. Average endpoint } \\
\text { negative symptoms score (SANS, } \\
\text { skewed data, high = poor) }\end{array}$ & & & Other data & No numeric data \\
\hline 14.1 short term & & & Other data & No numeric data \\
\hline 14.2 medium term & & & Other data & No numeric data \\
\hline 14.3 long term & & & Other data & No numeric data \\
\hline $\begin{array}{l}15 \text { Mental state: } 13 \text {. Average endpoint } \\
\text { positive symptoms score (PANSS posi- } \\
\text { tive subscale, high = poor) }\end{array}$ & 3 & & $\begin{array}{l}\text { Mean Difference (IV, Fixed, } \\
95 \% \mathrm{CI})\end{array}$ & Totals not selected \\
\hline 15.1 short term & 3 & & $\begin{array}{l}\text { Mean Difference (IV, Fixed, } \\
95 \% \mathrm{CI})\end{array}$ & $0.0[0.0,0.0]$ \\
\hline 15.2 long term & 1 & & $\begin{array}{l}\text { Mean Difference (IV, Fixed, } \\
95 \% \mathrm{Cl} \text { ) }\end{array}$ & $0.0[0.0,0.0]$ \\
\hline $\begin{array}{l}16 \text { Mental state: } 14 \text {. Average end- } \\
\text { point positive symptoms score (SAPS, } \\
\text { skewed data, high = poor) }\end{array}$ & & & Other data & No numeric data \\
\hline
\end{tabular}

\begin{tabular}{|c|c|c|c|}
\hline 16.1 medium term & & Other data & No numeric data \\
\hline $\begin{array}{l}17 \text { Mental state: } 15 \text {. Average end- } \\
\text { point positive symptoms score (BPRS, } \\
\text { skewed data, high = poor) }\end{array}$ & & Other data & No numeric data \\
\hline 17.1 short term & & Other data & No numeric data \\
\hline 17.2 long term & & Other data & No numeric data \\
\hline $\begin{array}{l}18 \text { Mental state: } 16 . \text { Average endpoint } \\
\text { score (PANSS thought disturbance } \\
\text { cluster, high = poor) }\end{array}$ & 1 & $\begin{array}{l}\text { Mean Difference (IV, Fixed, } \\
95 \% \mathrm{CI})\end{array}$ & Subtotals only \\
\hline 18.1 short term & 12 & $\begin{array}{l}\text { Mean Difference (IV, Fixed, } \\
95 \% \mathrm{CI})\end{array}$ & $4.3[1.17,7.43]$ \\
\hline $\begin{array}{l}19 \text { Mental state: } 17 . \text { Average endpoint } \\
\text { delusions score (PSYRATS delusions } \\
\text { score, skewed data, high = poor) }\end{array}$ & & Other data & No numeric data \\
\hline
\end{tabular}




\begin{tabular}{|c|c|c|c|c|}
\hline Outcome or subgroup title & No. of studies & $\begin{array}{l}\text { No. of partici- } \\
\text { pants }\end{array}$ & Statistical method & Effect size \\
\hline 19.1 short term & & & Other data & No numeric data \\
\hline 19.2 long term & & & Other data & No numeric data \\
\hline $\begin{array}{l}20 \text { Mental state: } 18 \text {. Average endpoint } \\
\text { hallucinations score (PSYRATS hallu- } \\
\text { cinations score, skewed data, high = } \\
\text { poor) }\end{array}$ & & & Other data & No numeric data \\
\hline 20.1 long term & & & Other data & No numeric data \\
\hline $\begin{array}{l}21 \text { Mental state: } 19 . \text { Average endpoint } \\
\text { voices score (PSYRATS voices score, } \\
\text { high = poor) }\end{array}$ & 1 & & $\begin{array}{l}\text { Mean Difference (IV, Fixed, } \\
95 \% \mathrm{CI})\end{array}$ & Subtotals only \\
\hline 21.1 short term & 1 & 65 & $\begin{array}{l}\text { Mean Difference (IV, Fixed, } \\
95 \% \mathrm{Cl} \text { ) }\end{array}$ & $0.10[-3.63,3.83]$ \\
\hline 21.2 long term & 1 & 65 & $\begin{array}{l}\text { Mean Difference (IV, Fixed, } \\
95 \% \mathrm{Cl} \text { ) }\end{array}$ & $0.0[-4.76,4.76]$ \\
\hline $\begin{array}{l}22 \text { Mental state: } 20 \text {. Average endpoint } \\
\text { beliefs about voices score (BAVQ, high } \\
=\text { poor, skewed data) }\end{array}$ & & & Other data & No numeric data \\
\hline 22.1 Malevolence & & & Other data & No numeric data \\
\hline 22.2 Benevolence & & & Other data & No numeric data \\
\hline 22.3 Resistance & & & Other data & No numeric data \\
\hline 22.4 Engagement & & & Other data & No numeric data \\
\hline 22.5 Omnipotence & & & Other data & No numeric data \\
\hline $\begin{array}{l}23 \text { Mental state: } 21 \text {. Average endpoint } \\
\text { depression score (MADRS, skewed da- } \\
\text { ta, high = poor) }\end{array}$ & & & Other data & No numeric data \\
\hline 23.1 medium term & & & Other data & No numeric data \\
\hline 23.2 long term & & & Other data & No numeric data \\
\hline $\begin{array}{l}24 \text { Mental state: } 22 \text {. Average endpoint } \\
\text { depression score (BDI-II, skewed data, } \\
\text { high = poor) }\end{array}$ & & & Other data & No numeric data \\
\hline 25 Leaving the study early & 12 & & $\begin{array}{l}\text { Risk Ratio (M-H, Fixed, } \\
95 \% \mathrm{Cl})\end{array}$ & Subtotals only \\
\hline 25.1 general reasons & 10 & 711 & $\begin{array}{l}\text { Risk Ratio (M-H, Fixed, } \\
95 \% \mathrm{Cl})\end{array}$ & $0.93[0.66,1.30]$ \\
\hline 25.2 treatment-related reasons & 2 & 101 & $\begin{array}{l}\text { Risk Ratio (M-H, Fixed, } \\
95 \% \mathrm{Cl})\end{array}$ & $2.34[0.90,6.10]$ \\
\hline
\end{tabular}




\begin{tabular}{|c|c|c|c|c|}
\hline Outcome or subgroup title & No. of studies & $\begin{array}{l}\text { No. of partici- } \\
\text { pants }\end{array}$ & Statistical method & Effect size \\
\hline $\begin{array}{l}26 \text { General functioning: } 1 \text {. Average } \\
\text { endpoint general functioning score } \\
\text { (GAF \& GAS, high = good) }\end{array}$ & 2 & & $\begin{array}{l}\text { Mean Difference (IV, Fixed, } \\
95 \% \mathrm{CI})\end{array}$ & Totals not selected \\
\hline 26.1 short term & 1 & & $\begin{array}{l}\text { Mean Difference (IV, Fixed, } \\
95 \% \mathrm{Cl} \text { ) }\end{array}$ & $0.0[0.0,0.0]$ \\
\hline 26.2 medium term & 1 & & $\begin{array}{l}\text { Mean Difference (IV, Fixed, } \\
95 \% \mathrm{Cl} \text { ) }\end{array}$ & $0.0[0.0,0.0]$ \\
\hline 26.3 long term & 2 & & $\begin{array}{l}\text { Mean Difference (IV, Fixed, } \\
95 \% \mathrm{CI})\end{array}$ & $0.0[0.0,0.0]$ \\
\hline $\begin{array}{l}27 \text { General functioning: } 2 \text {. Average } \\
\text { endpoint social functioning score (SFS, } \\
\text { high = good) }\end{array}$ & 1 & & $\begin{array}{l}\text { Mean Difference (IV, Fixed, } \\
95 \% \mathrm{CI} \text { ) }\end{array}$ & Subtotals only \\
\hline 27.1 short term & 1 & 65 & $\begin{array}{l}\text { Mean Difference (IV, Fixed, } \\
95 \% \mathrm{Cl} \text { ) }\end{array}$ & $-7.20[-17.86,3.46]$ \\
\hline 27.2 long term & 1 & 65 & $\begin{array}{l}\text { Mean Difference (IV, Fixed, } \\
95 \% \mathrm{CI} \text { ) }\end{array}$ & $-8.80[-21.67,4.07]$ \\
\hline $\begin{array}{l}28 \text { Satisfaction with treatment: Recipi- } \\
\text { ent of care not satisfied with treatment }\end{array}$ & 1 & & $\begin{array}{l}\text { Risk Ratio (M-H, Fixed, } \\
95 \% \mathrm{Cl})\end{array}$ & Subtotals only \\
\hline 28.1 long term & 1 & 45 & $\begin{array}{l}\text { Risk Ratio (M-H, Fixed, } \\
95 \% \mathrm{Cl})\end{array}$ & $3.19[1.01,10.07]$ \\
\hline $\begin{array}{l}29 \text { Quality of life: Average endpoint } \\
\text { score (RSES, high = good) }\end{array}$ & 1 & & $\begin{array}{l}\text { Mean Difference (IV, Fixed, } \\
95 \% \mathrm{CI})\end{array}$ & Subtotals only \\
\hline 29.1 short term & 1 & 65 & $\begin{array}{l}\text { Mean Difference (IV, Fixed, } \\
95 \% \mathrm{CI})\end{array}$ & $-0.80[-3.77,2.17]$ \\
\hline 29.2 long term & 1 & 65 & $\begin{array}{l}\text { Mean Difference (IV, Fixed, } \\
95 \% \mathrm{Cl} \text { ) }\end{array}$ & $-1.70[-5.19,1.79]$ \\
\hline 30 Death & 2 & & $\begin{array}{l}\text { Risk Ratio (M-H, Fixed, } \\
95 \% \mathrm{Cl})\end{array}$ & Subtotals only \\
\hline 30.1 medium term & 1 & 59 & $\begin{array}{l}\text { Risk Ratio (M-H, Fixed, } \\
95 \% \mathrm{Cl})\end{array}$ & $1.27[0.08,19.34]$ \\
\hline 30.2 long term & 1 & 45 & $\begin{array}{l}\text { Risk Ratio (M-H, Fixed, } \\
95 \% \mathrm{Cl})\end{array}$ & $2.88[0.12,67.03]$ \\
\hline $\begin{array}{l}31 \text { Insight: } 1 \text {. Average endpoint insight } \\
\text { score (SAl, skewed data, high = good) }\end{array}$ & & & Other data & No numeric data \\
\hline 31.1 short term & & & Other data & No numeric data \\
\hline 31.2 medium term & & & Other data & No numeric data \\
\hline 31.3 long term & & & Other data & No numeric data \\
\hline
\end{tabular}




\begin{tabular}{|c|c|c|c|c|}
\hline Outcome or subgroup title & No. of studies & $\begin{array}{l}\text { No. of partici- } \\
\text { pants }\end{array}$ & Statistical method & Effect size \\
\hline $\begin{array}{l}32 \text { Insight: } 2 \text {. Average endpoint insight } \\
\text { score (BCIS composite, skewed data, } \\
\text { high = good) }\end{array}$ & & & Other data & No numeric data \\
\hline $\begin{array}{l}33 \text { Medication: no reduction in dose of } \\
\text { antipsychotic medication }\end{array}$ & 1 & 90 & $\begin{array}{l}\text { Risk Ratio (M-H, Fixed, } \\
95 \% \mathrm{Cl})\end{array}$ & $0.89[0.68,1.17]$ \\
\hline $\begin{array}{l}34 \text { Attitude to medication: } 1 \text {. Average } \\
\text { endpoint attitude to medication score } \\
\text { (AMQ, high = good) }\end{array}$ & 1 & & $\begin{array}{l}\text { Mean Difference (IV, Fixed, } \\
95 \% \mathrm{CI})\end{array}$ & Subtotals only \\
\hline 34.1 short term & 1 & 74 & $\begin{array}{l}\text { Mean Difference (IV, Fixed, } \\
95 \% \mathrm{CI})\end{array}$ & $-4.50[-6.83,-2.17]$ \\
\hline $\begin{array}{l}35 \text { Attitude to medication: } 2 \text {. Average } \\
\text { endpoint attitude to medication score } \\
\text { (DAI, high = good) }\end{array}$ & 1 & & $\begin{array}{l}\text { Mean Difference (IV, Fixed, } \\
95 \% \mathrm{CI})\end{array}$ & Subtotals only \\
\hline 35.1 short term & 1 & 63 & $\begin{array}{l}\text { Mean Difference (IV, Fixed, } \\
95 \% \mathrm{CI})\end{array}$ & $-5.70[-9.35,-2.05]$ \\
\hline 35.2 long term & 1 & 44 & $\begin{array}{l}\text { Mean Difference (IV, Fixed, } \\
95 \% \mathrm{CI})\end{array}$ & $-4.90[-9.38,-0.42]$ \\
\hline $\begin{array}{l}36 \text { Economic outcomes: Direct costs } \\
\text { (skewed data) }\end{array}$ & & & Other data & No numeric data \\
\hline 36.1 medium term & & & Other data & No numeric data \\
\hline 36.2 long term & & & Other data & No numeric data \\
\hline
\end{tabular}

\section{Analysis 3.1. Comparison 3 SUPPORTIVE THERAPY versus COGNITIVE BEHAVIOURAL THERAPY, Outcome $1 \mathrm{Global}$ state: Relapse.}

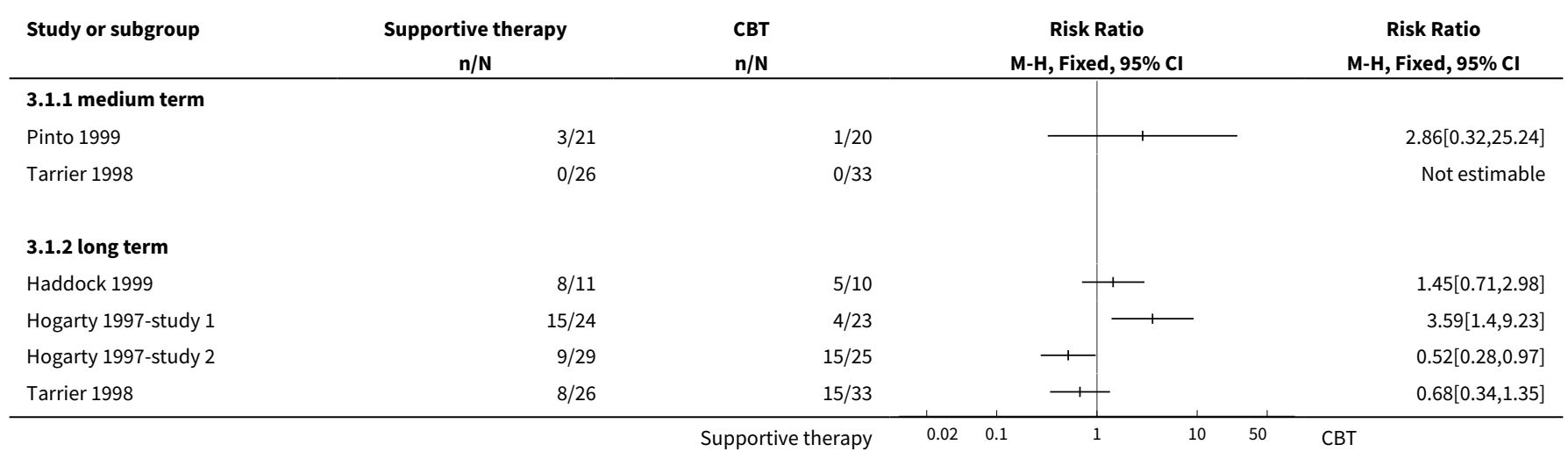


Analysis 3.2. Comparison 3 SUPPORTIVE THERAPY versus COGNITIVE BEHAVIOURAL THERAPY, Outcome 2 Service outcomes: Hospitalisation.

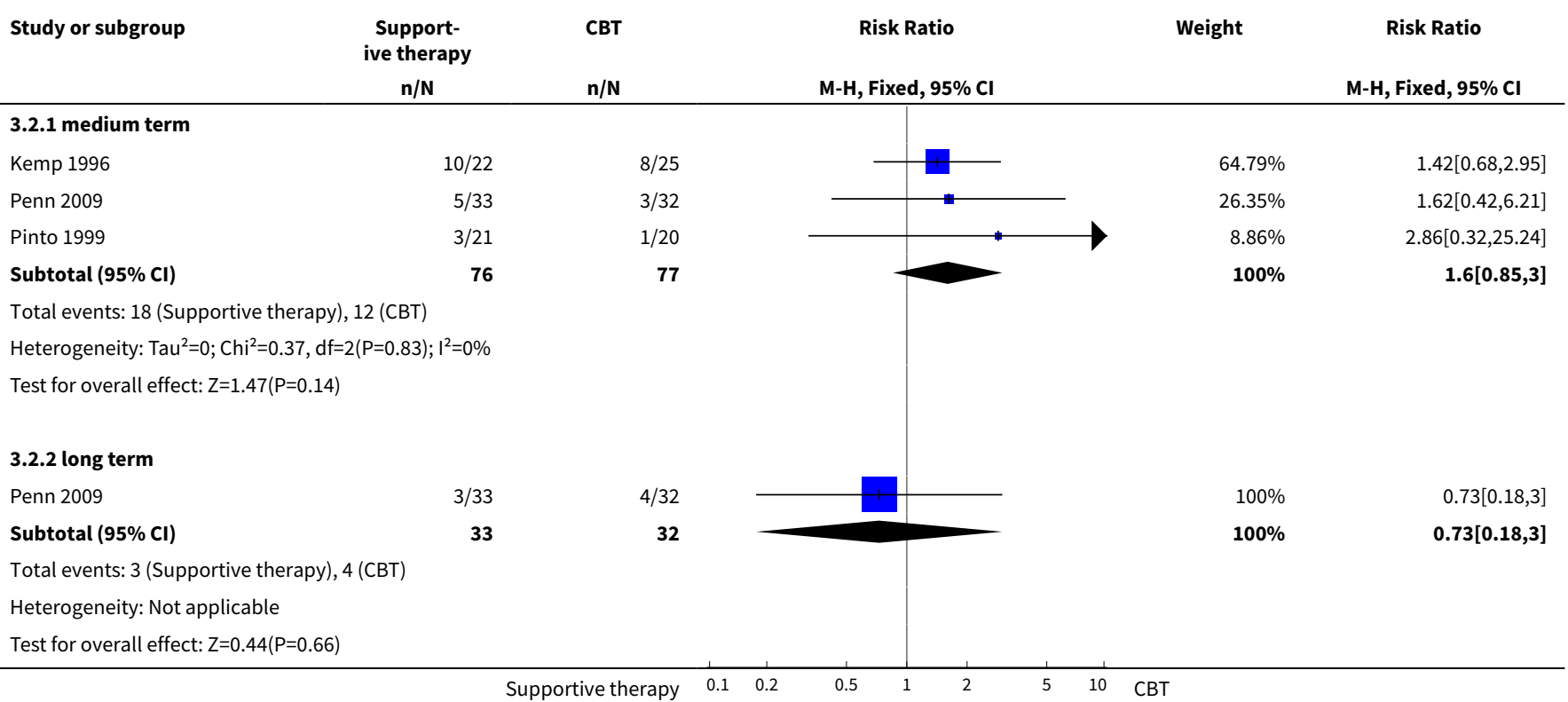

\section{Analysis 3.3. Comparison 3 SUPPORTIVE THERAPY versus COGNITIVE BEHAVIOURAL THERAPY,} Outcome 3 Mental state: 1 . No clinically important improvement in general mental state.

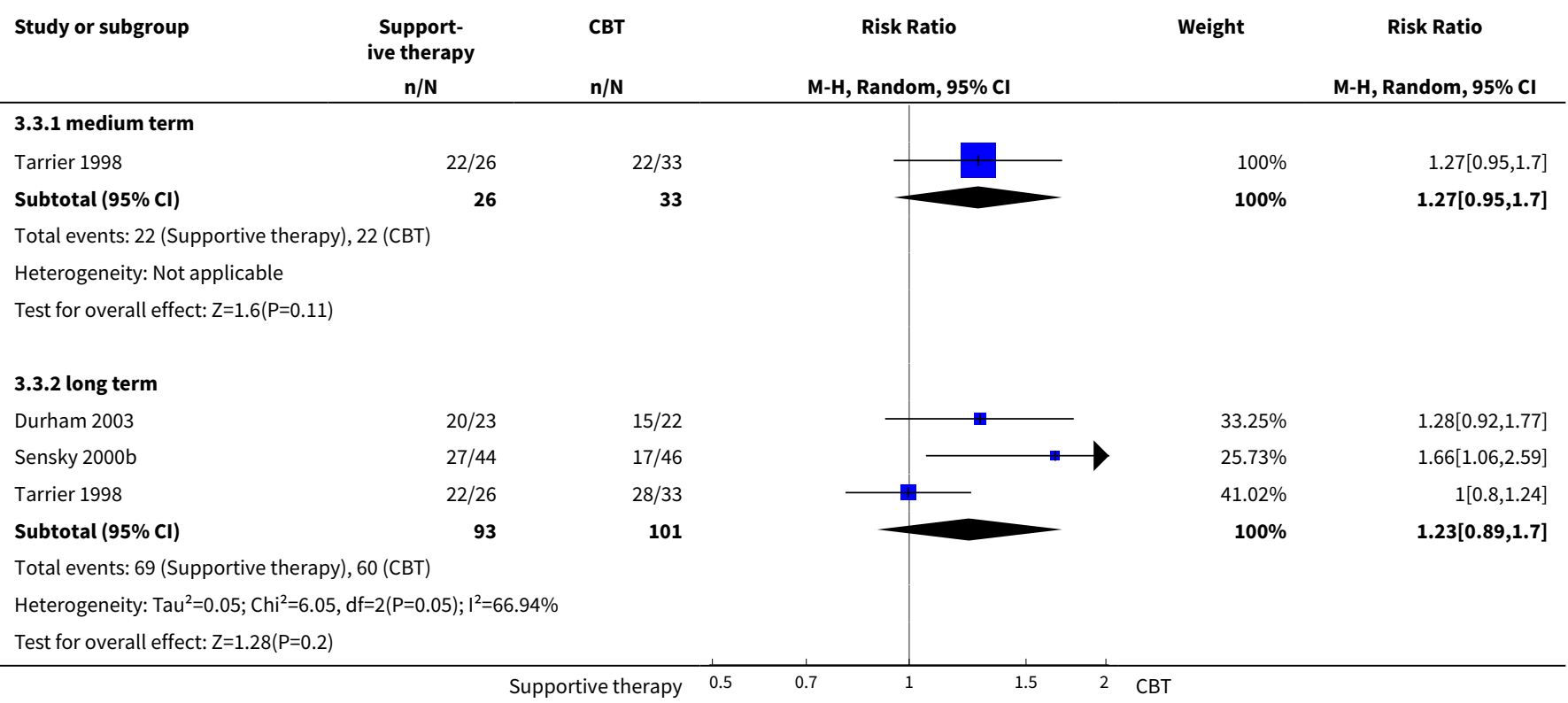


Analysis 3.4. Comparison 3 SUPPORTIVE THERAPY versus COGNITIVE BEHAVIOURAL THERAPY, Outcome 4 Mental state: 2. Average endpoint general mental state score (PANSS total, high = poor).

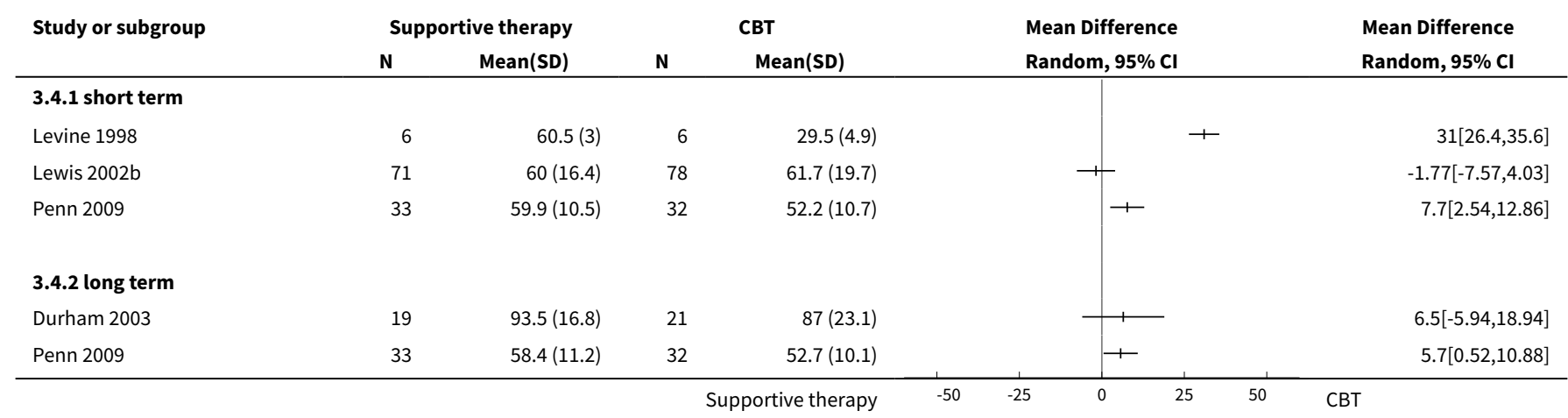

Analysis 3.5. Comparison 3 SUPPORTIVE THERAPY versus COGNITIVE BEHAVIOURAL THERAPY,

Outcome 5 Mental state: 3. Average endpoint general mental state score (BPRS, high = poor).

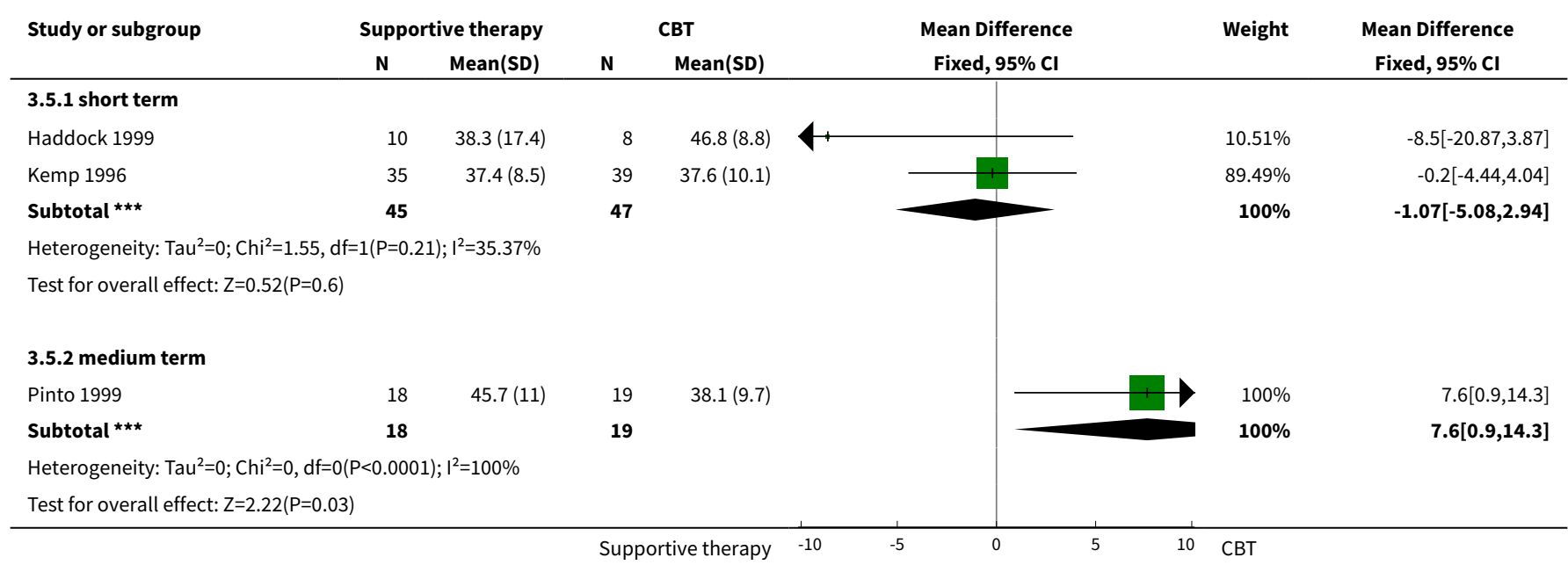

Analysis 3.6. Comparison 3 SUPPORTIVE THERAPY versus COGNITIVE BEHAVIOURAL THERAPY, Outcome 6 Mental state: 4. Average end point general mental state score (BPRS short form, high = poor).

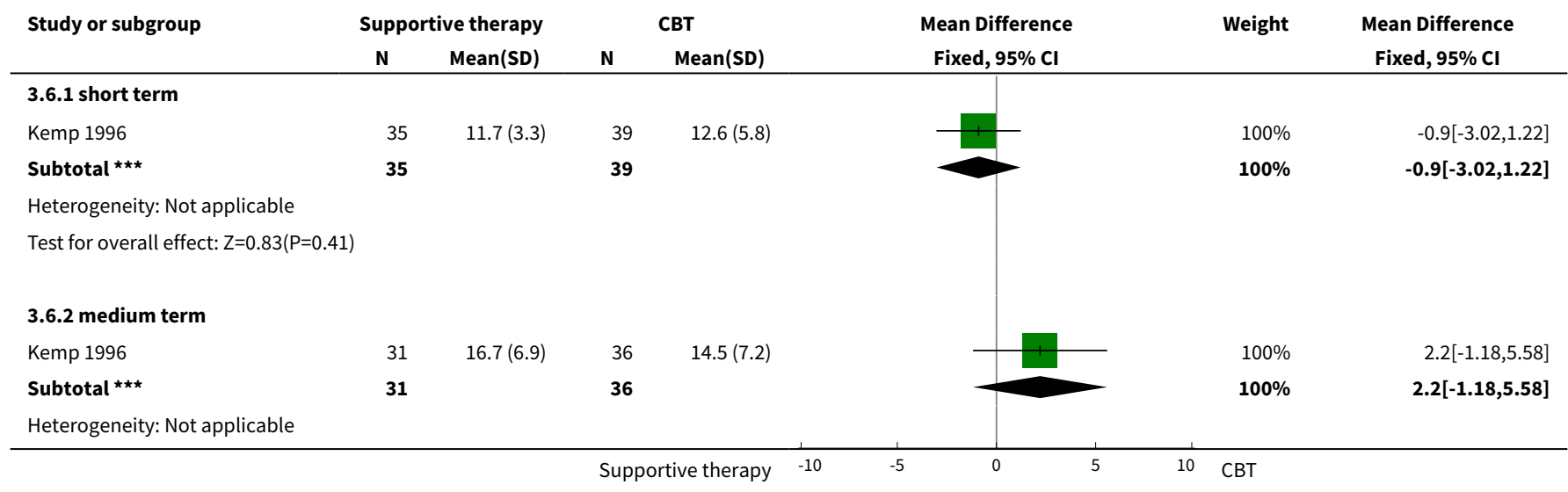




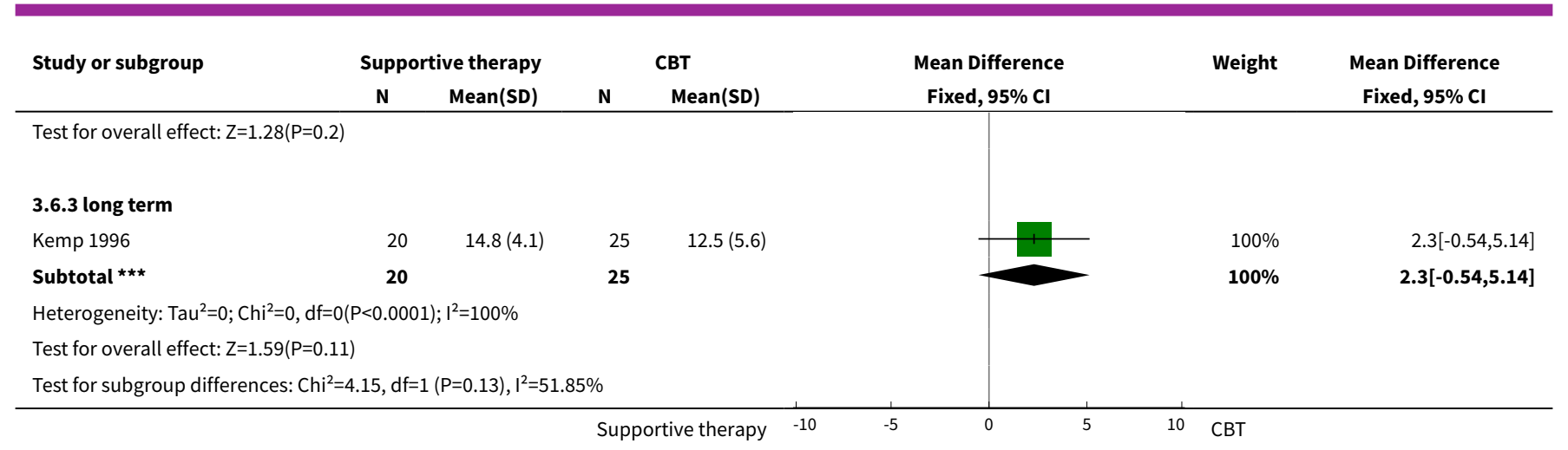

Analysis 3.7. Comparison 3 SUPPORTIVE THERAPY versus COGNITIVE BEHAVIOURAL THERAPY, Outcome 7 Mental state: 5. Average endpoint general mental state score (CPRS, skewed data, high = poor).

\begin{tabular}{|c|c|c|c|c|}
\hline \multicolumn{5}{|c|}{ Mental state: 5. Average endpoint general mental state score (CPRS, skewed data, high = poor) } \\
\hline Study & Intervention & $\mathbf{N}$ & Mean & SD \\
\hline \multicolumn{5}{|c|}{ medium term } \\
\hline Sensky 2000b & Supportive therapy & 44 & 22.9 & 17.3 \\
\hline Sensky 2000b & CBT & 46 & 20.5 & 13.1 \\
\hline \multicolumn{5}{|c|}{ long term } \\
\hline Sensky 2000b & Supportive therapy & 44 & 26.6 & 25.3 \\
\hline Sensky 2000b & CBT & 46 & 15.1 & 12.0 \\
\hline
\end{tabular}

Analysis 3.8. Comparison 3 SUPPORTIVE THERAPY versus COGNITIVE BEHAVIOURAL THERAPY, Outcome 8 Mental state: 6 . Average endpoint general mental state score (PANSS general subscale, high = poor).

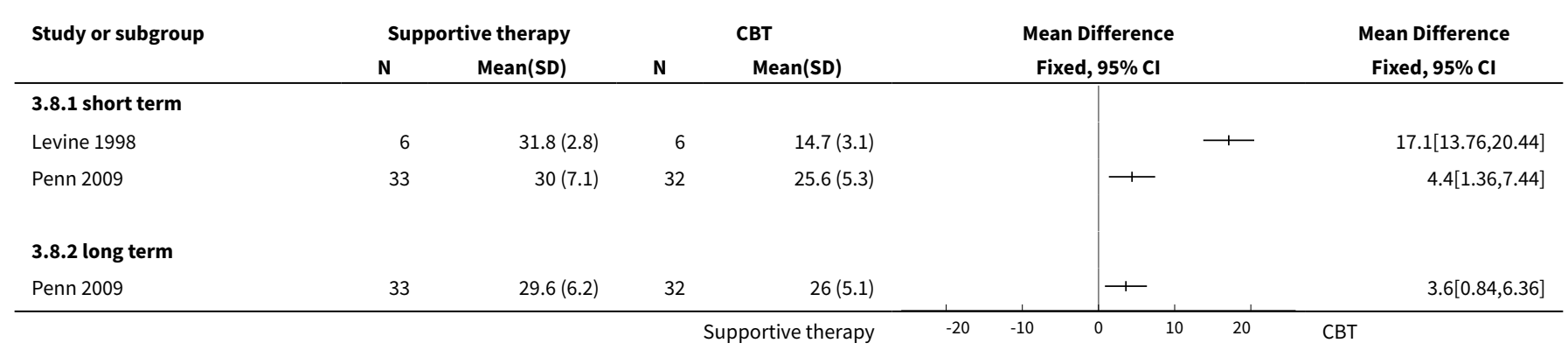

Analysis 3.9. Comparison 3 SUPPORTIVE THERAPY versus COGNITIVE BEHAVIOURAL THERAPY, Outcome 9 Mental state: 7 . No clinically important improvement in negative symptoms.

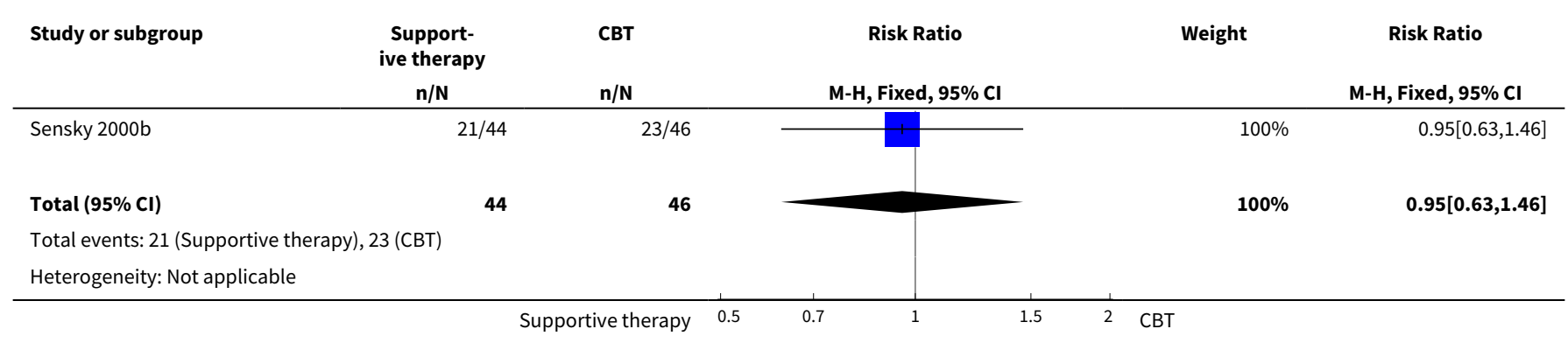




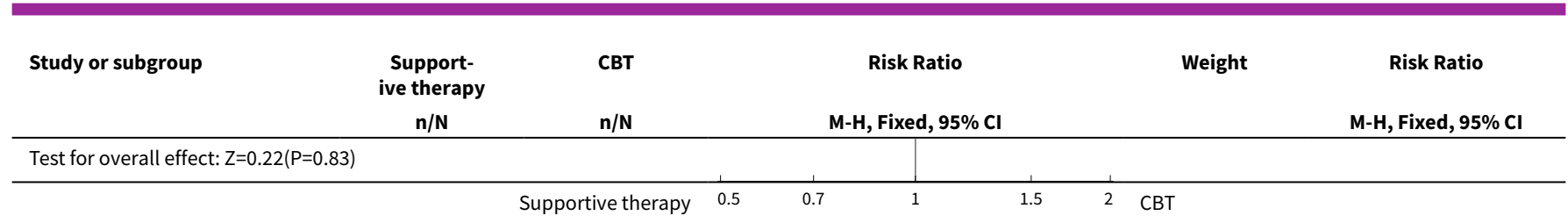

\section{Analysis 3.10. Comparison 3 SUPPORTIVE THERAPY versus COGNITIVE BEHAVIOURAL THERAPY,} Outcome 10 Mental state: 8 . No clinically important improvement in depressive symptoms.

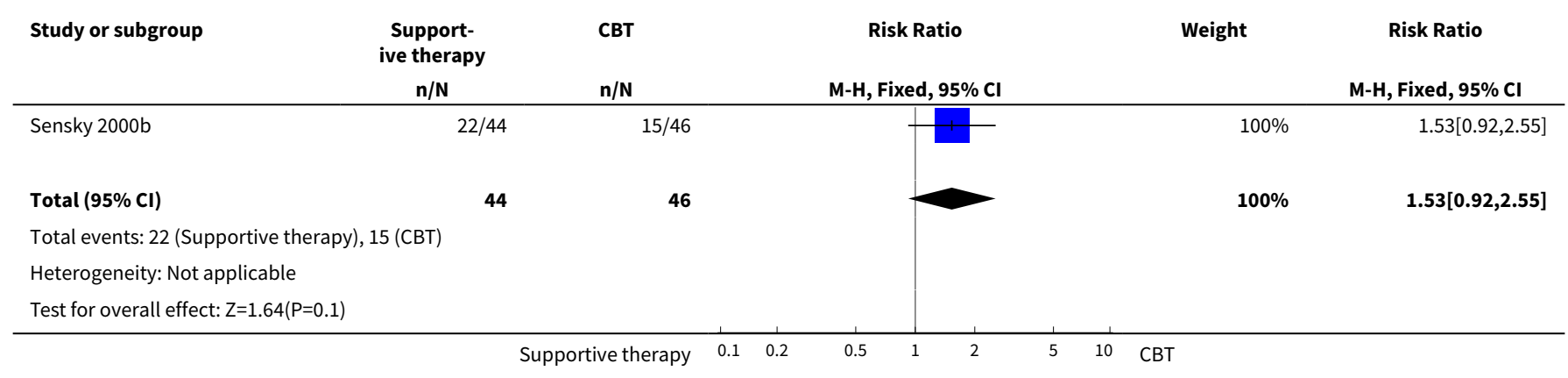

Analysis 3.11. Comparison 3 SUPPORTIVE THERAPY versus COGNITIVE BEHAVIOURAL THERAPY, Outcome 11 Mental state: 9. Episode of affective symptoms.

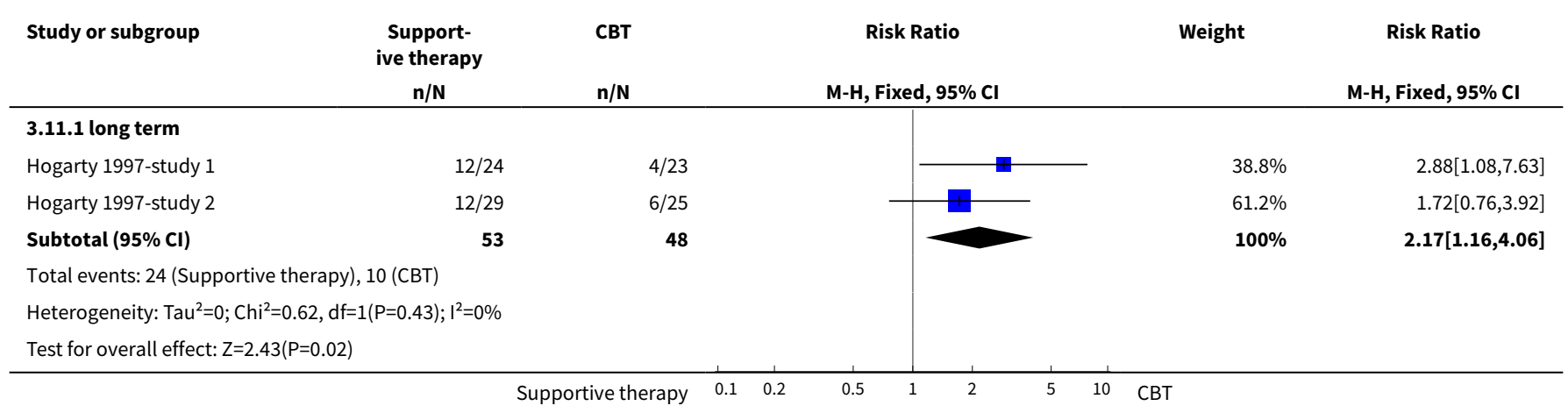

Analysis 3.12. Comparison 3 SUPPORTIVE THERAPY versus COGNITIVE BEHAVIOURAL THERAPY, Outcome 12 Mental state: 10. Average endpoint negative symptoms score (PANSS negative subscale, high = poor).

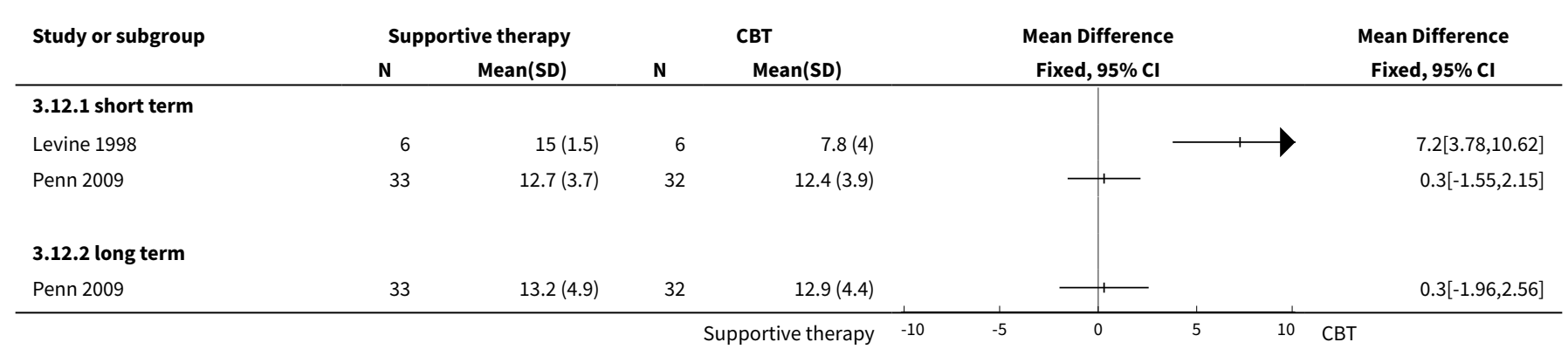


Analysis 3.13. Comparison 3 SUPPORTIVE THERAPY versus COGNITIVE BEHAVIOURAL THERAPY, Outcome 13 Mental state: 11. Average endpoint negative symptoms score (SANS, high = poor).

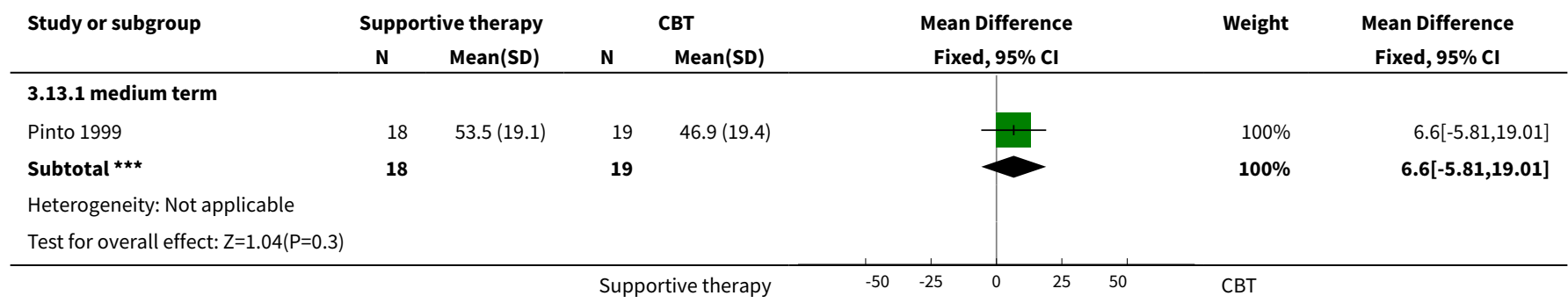

Analysis 3.14. Comparison 3 SUPPORTIVE THERAPY versus COGNITIVE BEHAVIOURAL THERAPY, Outcome 14 Mental state: 12. Average endpoint negative symptoms score (SANS, skewed data, high = poor).

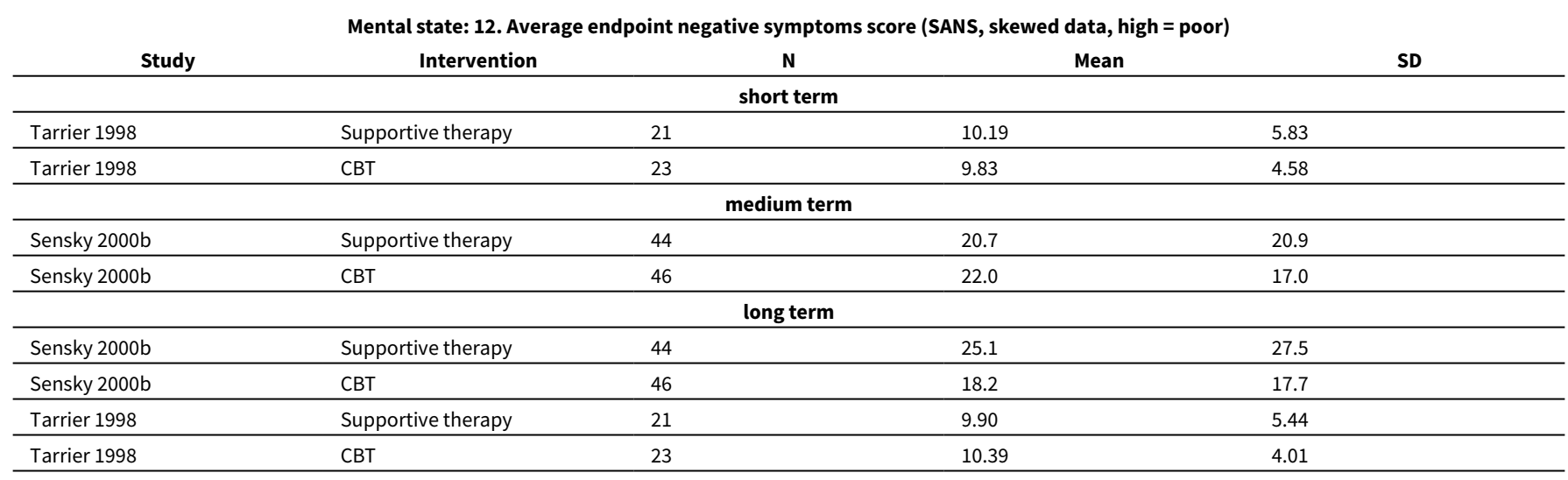

Analysis 3.15. Comparison 3 SUPPORTIVE THERAPY versus COGNITIVE BEHAVIOURAL THERAPY, Outcome 15 Mental state: 13. Average endpoint positive symptoms score (PANSS positive subscale, high = poor).

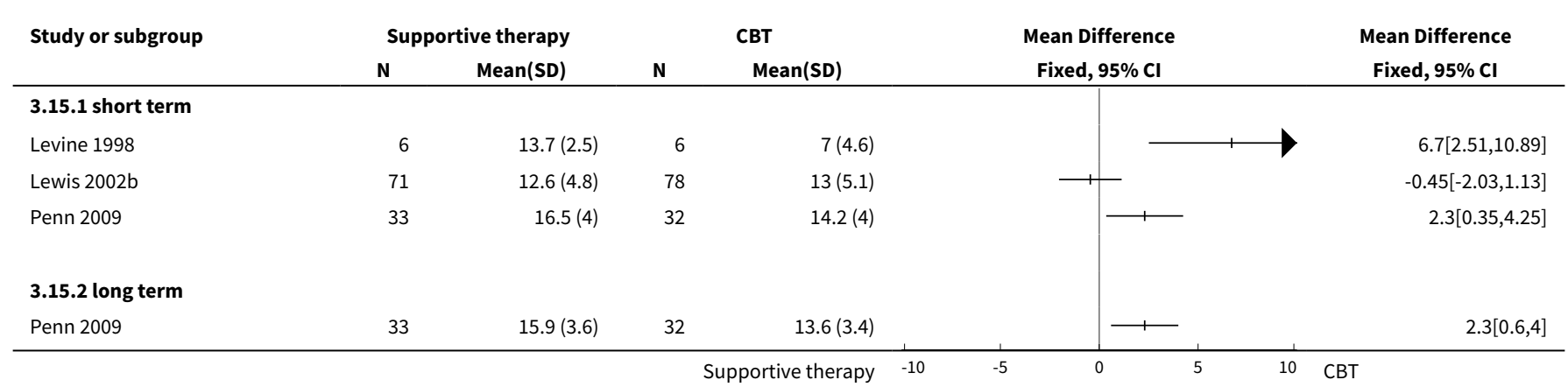


Analysis 3.16. Comparison 3 SUPPORTIVE THERAPY versus COGNITIVE BEHAVIOURAL THERAPY, Outcome 16 Mental state: 14. Average endpoint positive symptoms score (SAPS, skewed data, high = poor).

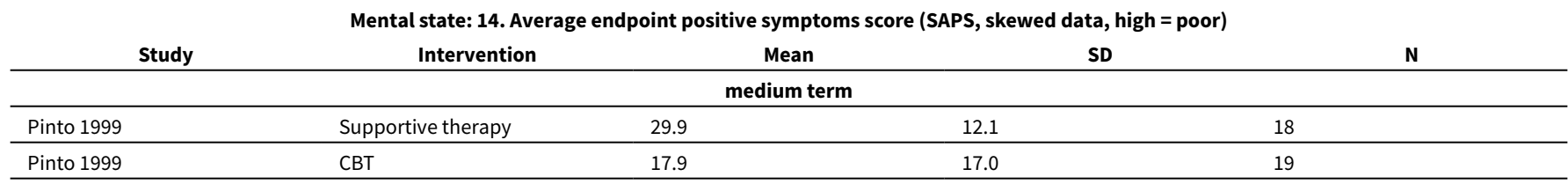

Analysis 3.17. Comparison 3 SUPPORTIVE THERAPY versus COGNITIVE BEHAVIOURAL THERAPY, OUtcome 17 Mental state: 15. Average endpoint positive symptoms score (BPRS, skewed data, high = poor).

\begin{tabular}{|c|c|c|c|c|}
\hline \multicolumn{5}{|c|}{ Mental state: 15. Average endpoint positive symptoms score (BPRS, skewed data, high = poor) } \\
\hline Study & Intervention & $\mathbf{N}$ & Mean & SD \\
\hline \multicolumn{5}{|c|}{ short term } \\
\hline Tarrier 1998 & Supportive therapy & 21 & 15.81 & 16.10 \\
\hline Tarrier 1998 & CBT & 23 & 10.67 & 9.42 \\
\hline \multicolumn{5}{|c|}{ long term } \\
\hline Tarrier 1998 & Supportive therapy & 21 & 16.30 & 16.71 \\
\hline
\end{tabular}

Analysis 3.18. Comparison 3 SUPPORTIVE THERAPY versus COGNITIVE BEHAVIOURAL THERAPY, Outcome 18 Mental state: 16. Average endpoint score (PANSS thought disturbance cluster, high = poor).

\begin{tabular}{|c|c|c|c|c|c|c|c|}
\hline \multirow[t]{2}{*}{ Study or subgroup } & \multicolumn{2}{|c|}{ Supportive therapy } & \multicolumn{2}{|c|}{ CBT } & \multirow{2}{*}{$\begin{array}{c}\text { Mean Difference } \\
\text { Fixed, } 95 \% \mathrm{Cl}\end{array}$} & \multirow[t]{2}{*}{ Weight } & \multirow{2}{*}{$\begin{array}{c}\text { Mean Difference } \\
\text { Fixed, } 95 \% \mathrm{Cl}\end{array}$} \\
\hline & $\mathbf{N}$ & $\operatorname{Mean}(S D)$ & $\mathbf{N}$ & Mean(SD) & & & \\
\hline \multicolumn{8}{|l|}{ 3.18.1 short term } \\
\hline Levine 1998 & 6 & $7.8(2.1)$ & 6 & $3.5(3.3)$ & & $100 \%$ & $4.3[1.17,7.43]$ \\
\hline Subtotal $\star \star \star ~$ & 6 & & 6 & & & $100 \%$ & $4.3[1.17,7.43]$ \\
\hline \multicolumn{8}{|c|}{ Heterogeneity: Not applicable } \\
\hline \multicolumn{8}{|c|}{ Test for overall effect: $Z=2.69(P=0.01)$} \\
\hline
\end{tabular}

Analysis 3.19. Comparison 3 SUPPORTIVE THERAPY versus COGNITIVE BEHAVIOURAL THERAPY, Outcome 19 Mental state: 17. Average endpoint delusions score (PSYRATS delusions score, skewed data, high = poor).

\begin{tabular}{|c|c|c|c|c|}
\hline \multicolumn{5}{|c|}{ Mental state: 17. Average endpoint delusions score (PSYRATS delusions score, skewed data, high = poor) } \\
\hline Study & Intervention & Mean & SD & $\mathbf{N}$ \\
\hline \multicolumn{5}{|c|}{ short term } \\
\hline Lewis $2002 b$ & Supportive therapy & 6.13 & 6.98 & 67 \\
\hline Lewis $2002 b$ & CBT & 6.95 & 7.66 & 74 \\
\hline Penn 2009 & Supportive therapy & 10.4 & 5.9 & 33 \\
\hline Penn 2009 & CBT & 8 & 7.7 & 32 \\
\hline Durham 2003 & Supportive therapy & 9.7 & 6.1 & 19 \\
\hline Durham 2003 & CBT & 11.1 & 5.8 & 21 \\
\hline Penn 2009 & Supportive therapy & 9.0 & 6.8 & 33 \\
\hline Penn 2009 & CBT & s6.9 & 7.0 & 32 \\
\hline
\end{tabular}


Analysis 3.20. Comparison 3 SUPPORTIVE THERAPY versus COGNITIVE BEHAVIOURAL THERAPY, Outcome 20 Mental state: 18. Average endpoint hallucinations score (PSYRATS hallucinations score, skewed data, high = poor).

\begin{tabular}{|c|c|c|c|c|c|c|}
\hline \multicolumn{7}{|c|}{ Mental state: 18. Average endpoint hallucinations score (PSYRATS hallucinations score, skewed data, high = poor) } \\
\hline Study & Intervention & Mean & & SD & & $\mathbf{N}$ \\
\hline \multicolumn{7}{|c|}{ long term } \\
\hline Durham 2003 & Supportive therapy & 18.0 & 12.2 & & 19 & \\
\hline Durham 2003 & CBT & 18.5 & 12.8 & & 20 & \\
\hline
\end{tabular}

Analysis 3.21. Comparison 3 SUPPORTIVE THERAPY versus COGNITIVE BEHAVIOURAL THERAPY, Outcome 21 Mental state: 19. Average endpoint voices score (PSYRATS voices score, high = poor).

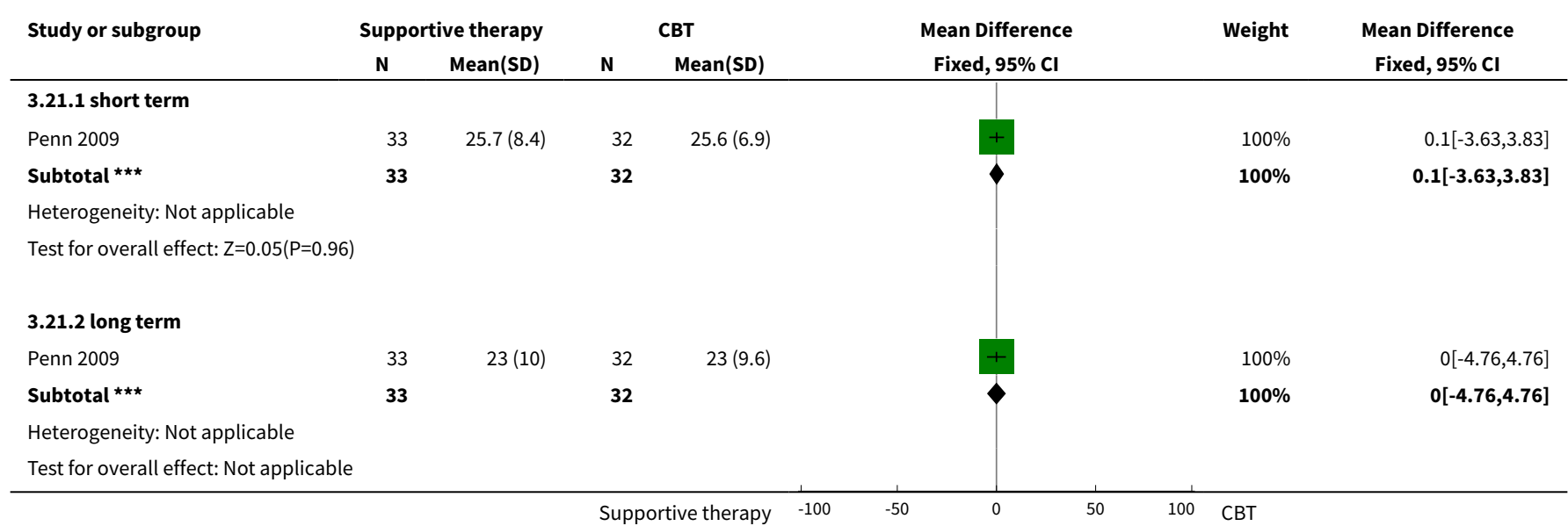

Analysis 3.22. Comparison 3 SUPPORTIVE THERAPY versus COGNITIVE BEHAVIOURAL THERAPY, Outcome 22 Mental state: 20. Average endpoint beliefs about voices score (BAVQ, high = poor, skewed data).

\begin{tabular}{|c|c|c|c|c|c|c|c|}
\hline \multicolumn{7}{|c|}{ Mental state: 20. Average endpoint beliefs about voices score (BAVQ, high = poor, skewed data) } & \\
\hline Study & Follow-up length & Intervention & Mean & & SD & $\mathbf{N}$ & \\
\hline \multicolumn{7}{|c|}{ Malevolence } & \\
\hline Penn 2009 & Short term & Supportive therapy & 8.1 & 5.4 & & 33 & \\
\hline Penn 2009 & Short term & CBT & 6.3 & 5.4 & & 32 & \\
\hline Penn 2009 & Long term & Supportive therapy & 6.7 & 4.9 & & 33 & \\
\hline Penn 2009 & Long term & CBT & 6.3 & 5.4 & & 32 & \\
\hline \multicolumn{8}{|c|}{ Benevolence } \\
\hline Penn 2009 & Short term & Supportive therapy & 5.7 & 5.5 & & 33 & \\
\hline Penn 2009 & Short term & CBT & 4.4 & 5.3 & & 32 & \\
\hline Penn 2009 & Long term & Supportive therapy & 6.2 & 5.4 & & 33 & \\
\hline Penn 2009 & Long term & CBT & 5.3 & 5.2 & & 32 & \\
\hline \multicolumn{8}{|c|}{ Resistance } \\
\hline Penn 2009 & Short term & Supportive therapy & 16.3 & 6.6 & & 33 & \\
\hline Penn 2009 & Short term & CBT & 15 & 7.3 & & 32 & \\
\hline Penn 2009 & Long term & Supportive therapy & 15.3 & 7.3 & & 33 & \\
\hline Penn 2009 & Long term & $\mathrm{CBT}$ & 13.9 & 7.8 & & 32 & \\
\hline \multicolumn{8}{|c|}{ Engagement } \\
\hline Penn 2009 & Short term & Supportive therapy & 7.8 & 7.4 & & 33 & \\
\hline Penn 2009 & Short term & CBT & 5.4 & 5.7 & & 32 & \\
\hline Penn 2009 & Long term & Supportive therapy & 7.3 & 6.8 & & 33 & \\
\hline Penn 2009 & Long term & $\mathrm{CBT}$ & 6.4 & 7.2 & & 32 & \\
\hline \multicolumn{7}{|c|}{ Omnipotence } & \\
\hline
\end{tabular}


Mental state: 20. Average endpoint beliefs about voices score (BAVQ, high = poor, skewed data)

\begin{tabular}{|c|c|c|c|c|c|}
\hline Study & Follow-up length & Intervention & Mean & SD & $\mathbf{N}$ \\
\hline Penn 2009 & Short term & Supportive therapy & 8.6 & 5 & 33 \\
\hline Penn 2009 & Short term & CBT & 8 & 4.2 & 32 \\
\hline Penn 2009 & Long term & Supportive therapy & 7.7 & 4.3 & 33 \\
\hline Penn 2009 & Long term & CBT & 7.3 & 3.3 & 32 \\
\hline
\end{tabular}

Analysis 3.23. Comparison 3 SUPPORTIVE THERAPY versus COGNITIVE BEHAVIOURAL THERAPY, Outcome 23 Mental state: 21 . Average endpoint depression score (MADRS, skewed data, high = poor).

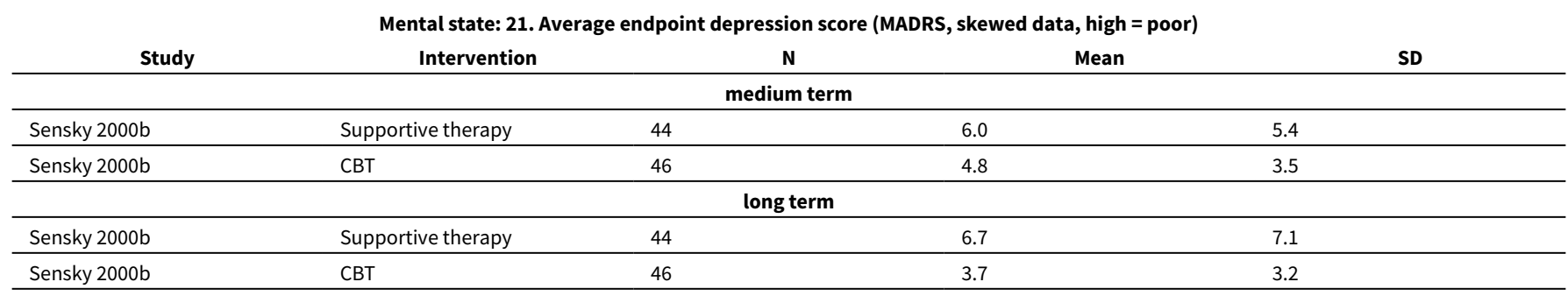

Analysis 3.24. Comparison 3 SUPPORTIVE THERAPY versus COGNITIVE BEHAVIOURAL THERAPY, Outcome 24 Mental state: 22. Average endpoint depression score (BDI-II, skewed data, high = poor).

\begin{tabular}{llllll}
\multicolumn{2}{c}{ Study } & \multicolumn{2}{c}{ Mental state: 22. Average endpoint depression score (BDI-II, skewed data, high = poor) } \\
Follow-up length & \multicolumn{2}{c}{ Intervention } & Mean & SD & N \\
\hline Penn 2009 & Short term & Supportive therapy & 13.9 & 10.7 & 33 \\
\hline Penn 2009 & Short term & CBT & 10.5 & 8.5 & 32 \\
\hline Penn 2009 & Long term & Supportive therapy & 17.9 & 13.6 & 33 \\
\hline Penn 2009 & Long term & CBT & 11.5 & 9.4 & 32 \\
\hline
\end{tabular}

Analysis 3.25. Comparison 3 SUPPORTIVE THERAPY versus COGNITIVE BEHAVIOURAL THERAPY, Outcome 25 Leaving the study early.

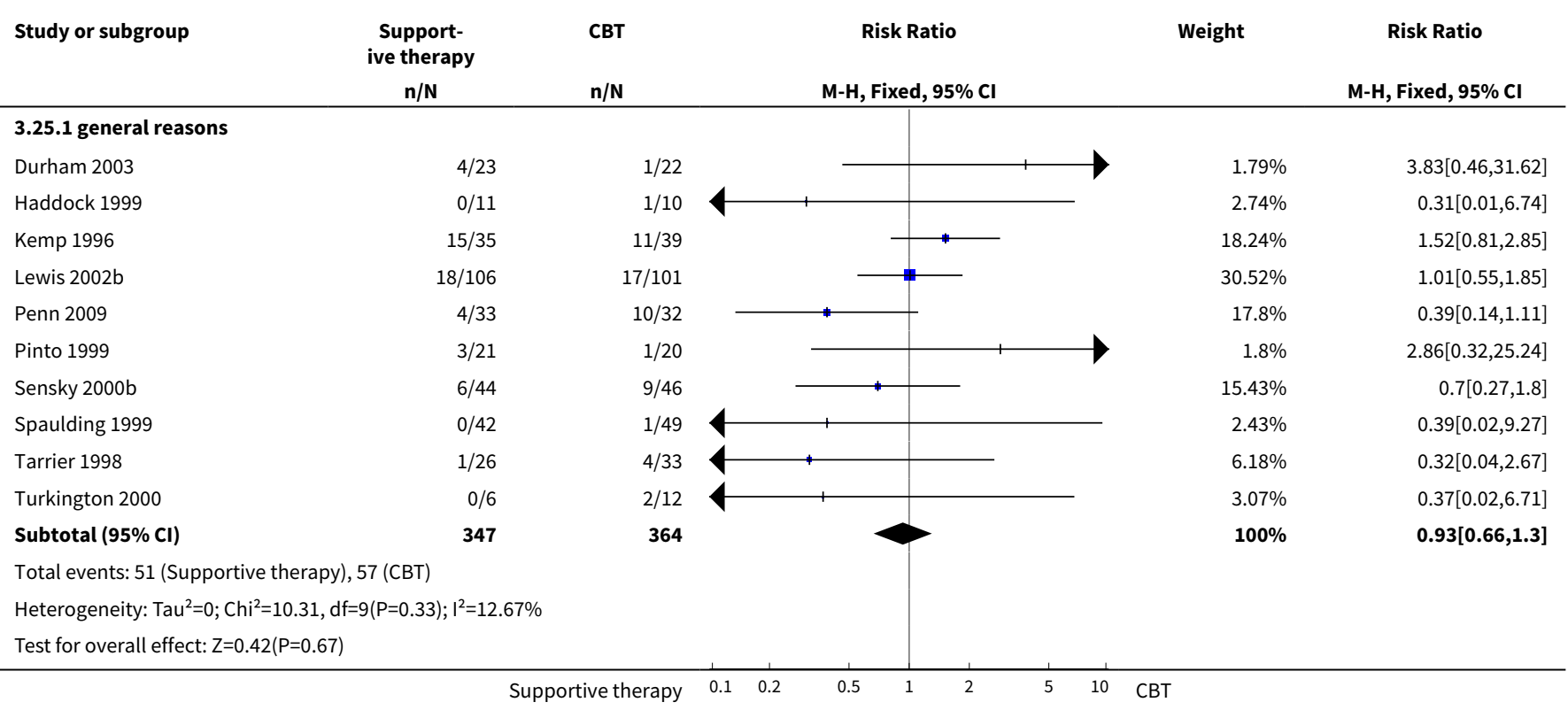




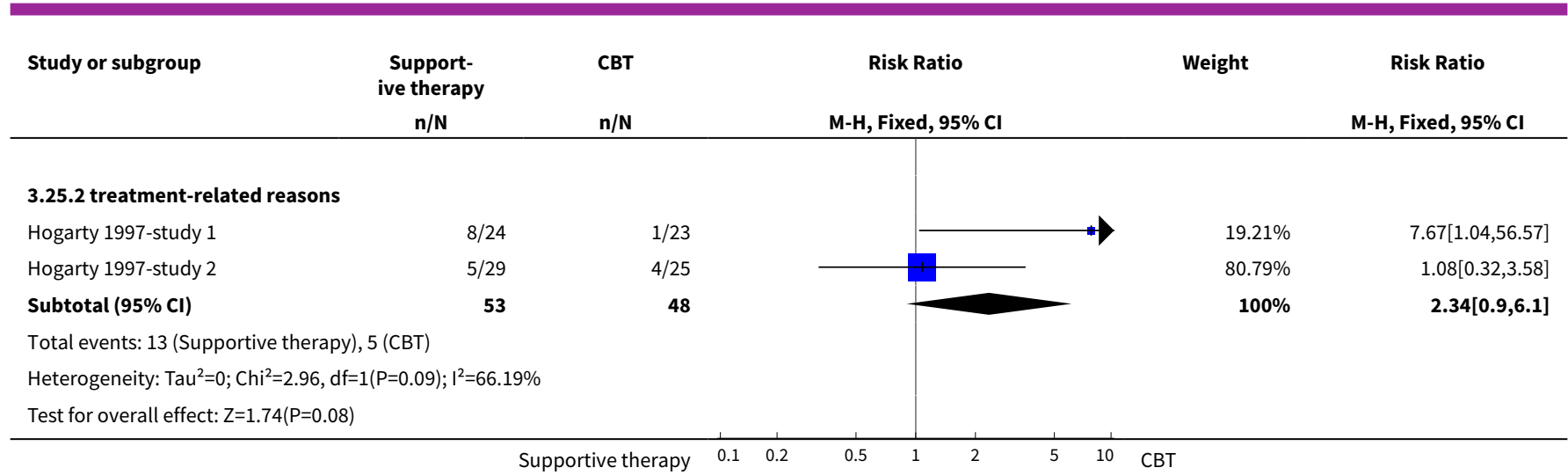

Analysis 3.26. Comparison 3 SUPPORTIVE THERAPY versus COGNITIVE BEHAVIOURAL THERAPY, Outcome 26 General functioning: 1. Average endpoint general functioning score (GAF \& GAS, high = good).

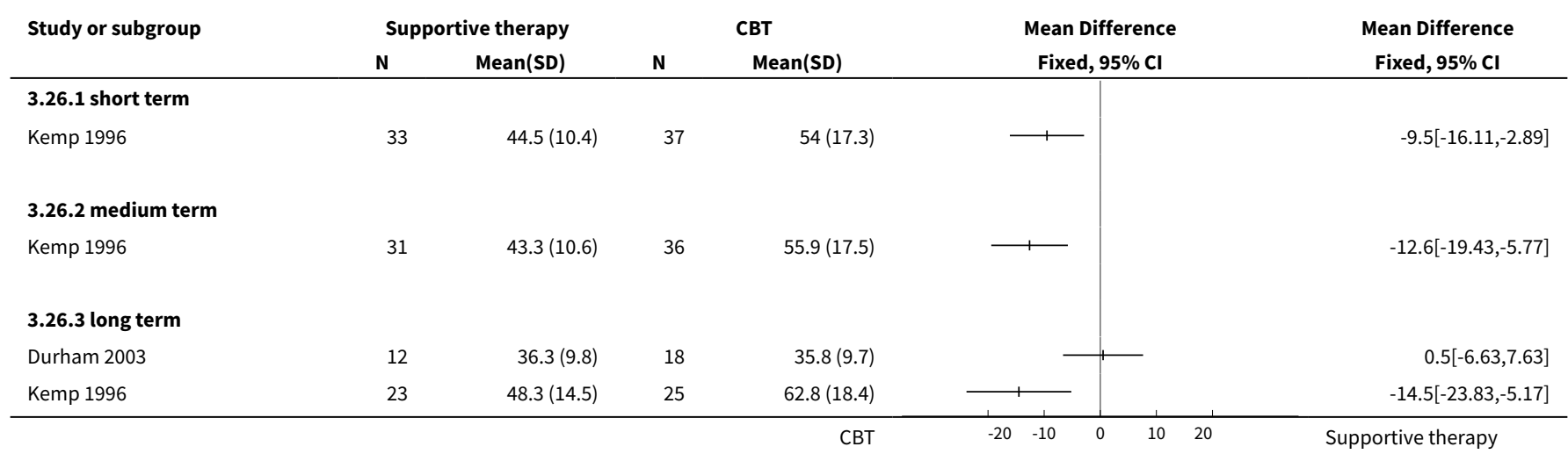

Analysis 3.27. Comparison 3 SUPPORTIVE THERAPY versus COGNITIVE BEHAVIOURAL THERAPY, Outcome 27 General functioning: 2 . Average endpoint social functioning score (SFS, high = good).

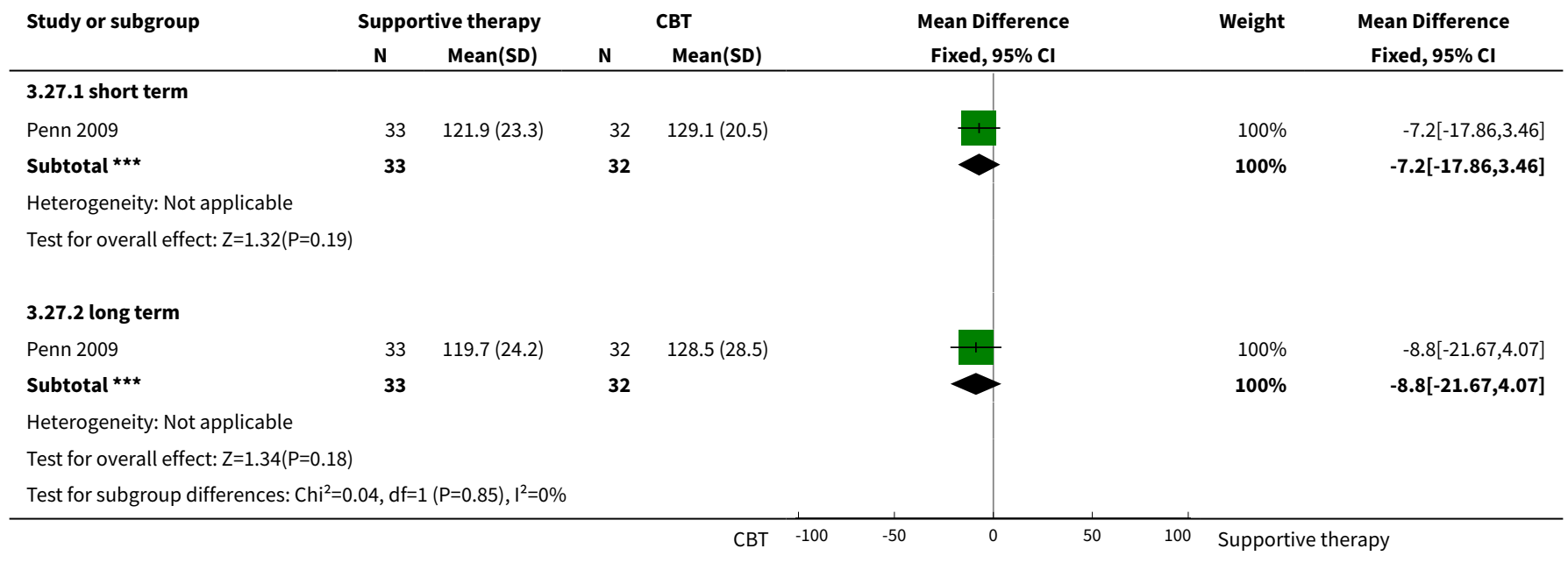


Analysis 3.28. Comparison 3 SUPPORTIVE THERAPY versus COGNITIVE BEHAVIOURAL THERAPY, Outcome 28 Satisfaction with treatment: Recipient of care not satisfied with treatment.

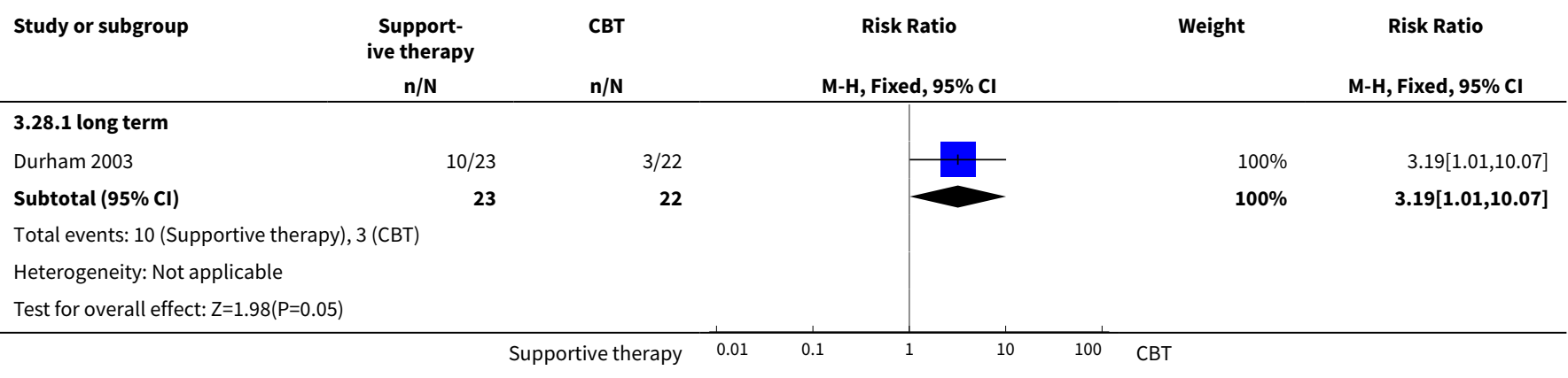

Analysis 3.29. Comparison 3 SUPPORTIVE THERAPY versus COGNITIVE BEHAVIOURAL THERAPY, Outcome 29 Quality of life: Average endpoint score (RSES, high = good).

\begin{tabular}{|c|c|c|c|c|c|c|c|}
\hline \multirow{3}{*}{$\begin{array}{l}\text { Study or subgroup } \\
\text { 3.29.1 short term }\end{array}$} & \multicolumn{2}{|c|}{ Supportive therapy } & \multicolumn{2}{|r|}{ CBT } & \multirow{2}{*}{$\begin{array}{c}\text { Mean Difference } \\
\text { Fixed, } 95 \% \mathrm{Cl} \\
\end{array}$} & \multirow[t]{2}{*}{ Weight } & \multirow{2}{*}{$\begin{array}{c}\text { Mean Difference } \\
\text { Fixed, } 95 \% \mathrm{Cl}\end{array}$} \\
\hline & \multirow[t]{2}{*}{$\mathbf{N}$} & \multirow[t]{2}{*}{ Mean(SD) } & \multirow[t]{2}{*}{$\mathbf{N}$} & \multirow[t]{2}{*}{$\operatorname{Mean}(S D)$} & & & \\
\hline & & & & & & & \\
\hline Penn 2009 & 33 & $28.6(6.2)$ & 32 & $29.4(6)$ & + & $100 \%$ & $-0.8[-3.77,2.17]$ \\
\hline Subtotal $\star \star \star ~$ & 33 & & 32 & & $\checkmark$ & $100 \%$ & $-0.8[-3.77,2.17]$ \\
\hline \multicolumn{8}{|c|}{ Heterogeneity: Not applicable } \\
\hline \multicolumn{8}{|c|}{ Test for overall effect: $Z=0.53(P=0.6)$} \\
\hline \multicolumn{8}{|l|}{ 3.29.2 long term } \\
\hline Penn 2009 & 33 & $27.6(6.7)$ & 32 & $29.3(7.6)$ & + & $100 \%$ & $-1.7[-5.19,1.79]$ \\
\hline 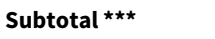 & 33 & & 32 & & $\diamond$ & $100 \%$ & $-1.7[-5.19,1.79]$ \\
\hline \multicolumn{8}{|c|}{ Heterogeneity: $\mathrm{Tau}^{2}=0 ; \mathrm{Chi}^{2}=0, \mathrm{df}=0(\mathrm{P}<0.0001) ; \mathrm{I}^{2}=100 \%$} \\
\hline \multicolumn{8}{|c|}{ Test for overall effect: $Z=0.96(P=0.34)$} \\
\hline \multicolumn{8}{|c|}{ Test for subgroup differences: $\mathrm{Chi}^{2}=0.15, \mathrm{df}=1(\mathrm{P}=0.7), \mathrm{I}^{2}=0 \%$} \\
\hline
\end{tabular}

Analysis 3.30. Comparison 3 SUPPORTIVE THERAPY versus COGNITIVE BEHAVIOURAL THERAPY, Outcome 30 Death.

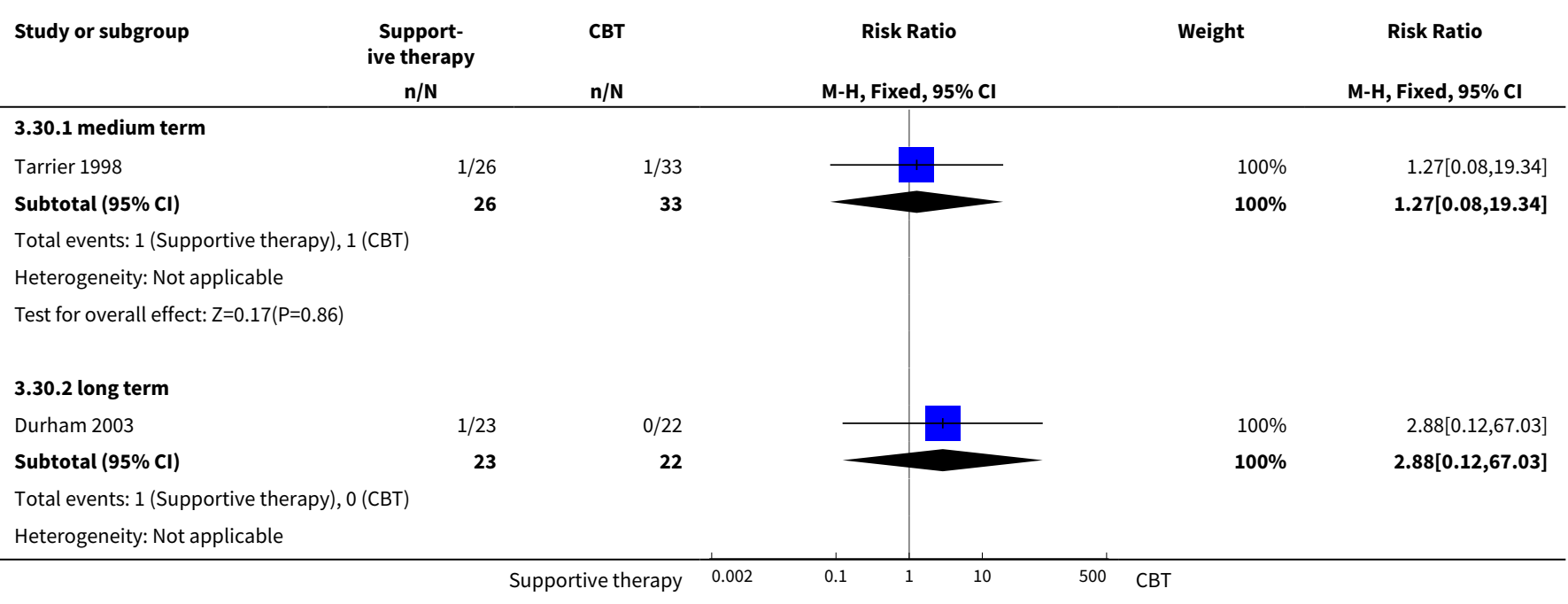




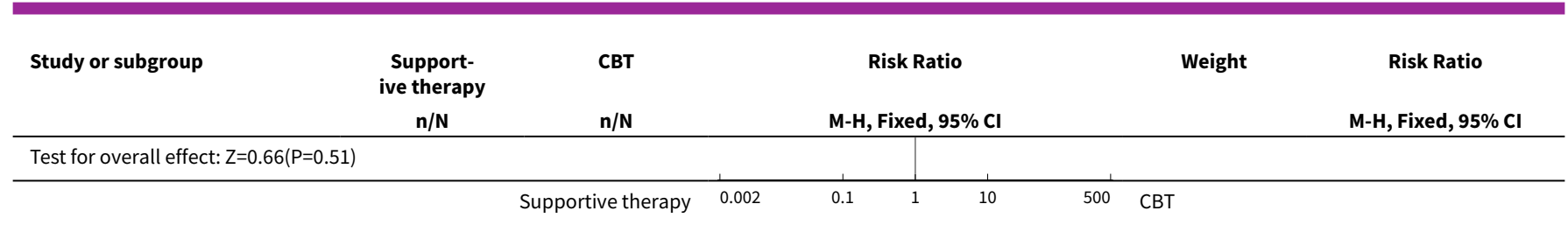

Analysis 3.31. Comparison 3 SUPPORTIVE THERAPY versus COGNITIVE BEHAVIOURAL THERAPY, Outcome 31 Insight:1. Average endpoint insight score (SAI, skewed data, high = good).

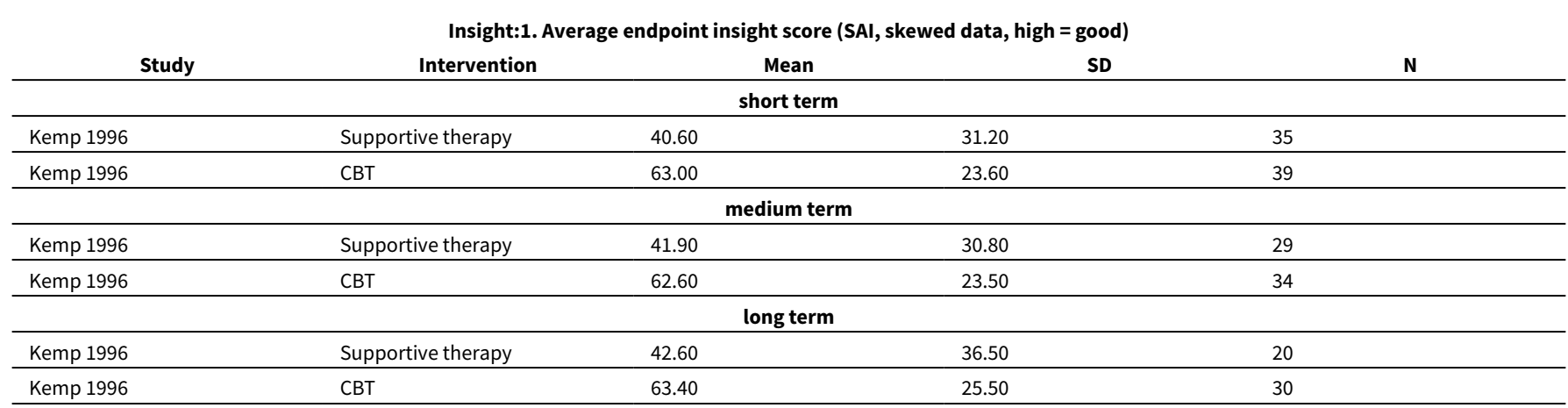

Analysis 3.32. Comparison 3 SUPPORTIVE THERAPY versus COGNITIVE BEHAVIOURAL THERAPY, Outcome 32 Insight: 2. Average endpoint insight score (BCIS composite, skewed data, high = good).

\begin{tabular}{|c|c|c|c|c|c|}
\hline Study & Follow-up length & Intervention & Mean & SD & $\mathbf{N}$ \\
\hline Penn 2009 & Short term & Supportive therapy & 4.7 & 5.4 & 33 \\
\hline Penn 2009 & Short term & CBT & 4.2 & 6.6 & 32 \\
\hline Penn 2009 & Long term & Supportive therapy & 3.7 & 4.4 & 33 \\
\hline Penn 2009 & Long term & CBT & 4.4 & 5.6 & 32 \\
\hline
\end{tabular}

Analysis 3.33. Comparison 3 SUPPORTIVE THERAPY versus COGNITIVE BEHAVIOURAL THERAPY, Outcome 33 Medication: no reduction in dose of antipsychotic medication.

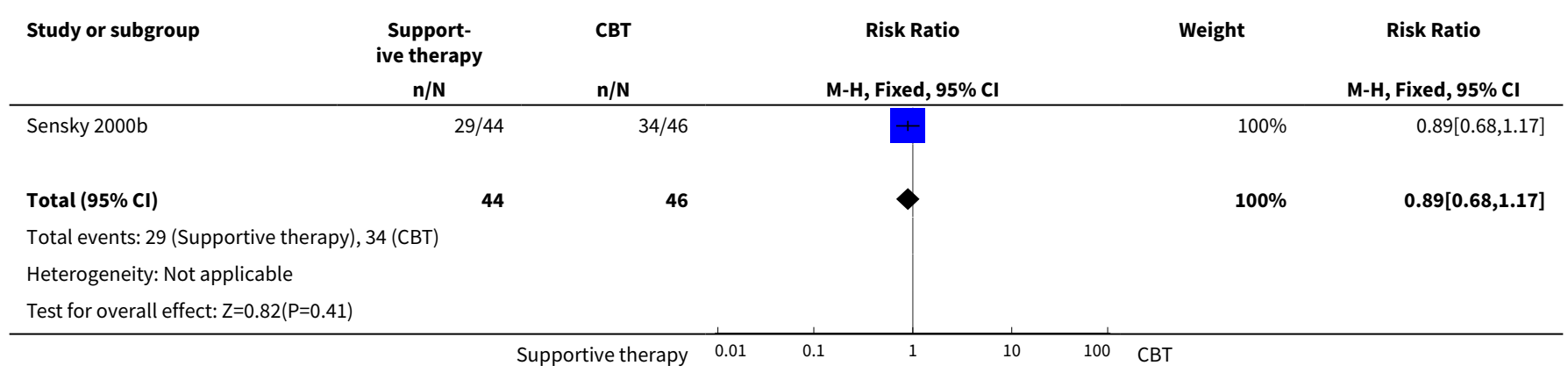


Analysis 3.34. Comparison 3 SUPPORTIVE THERAPY versus COGNITIVE BEHAVIOURAL THERAPY, OUtCome 34 Attitude to medication: 1. Average endpoint attitude to medication score (AMQ, high = good).

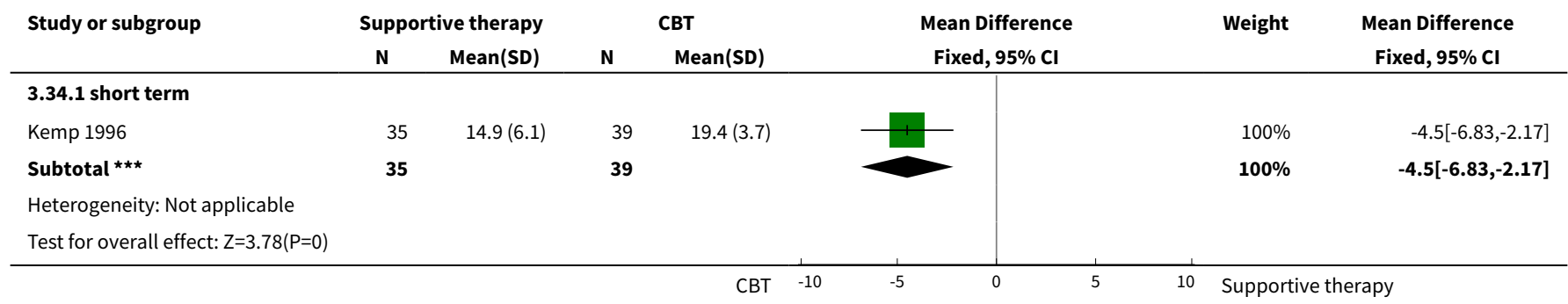

Analysis 3.35. Comparison 3 SUPPORTIVE THERAPY versus COGNITIVE BEHAVIOURAL THERAPY, Outcome 35 Attitude to medication: 2. Average endpoint attitude to medication score (DAl, high = good).

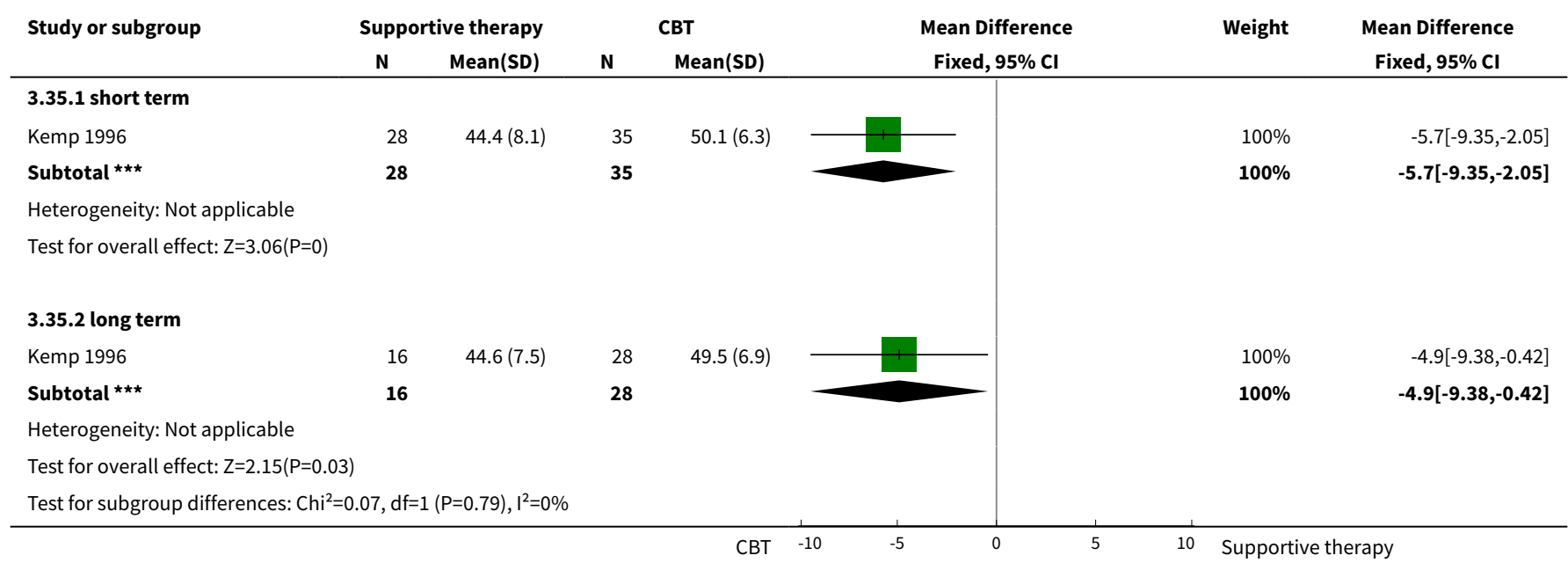

Analysis 3.36. Comparison 3 SUPPORTIVE THERAPY versus COGNITIVE BEHAVIOURAL THERAPY, Outcome 36 Economic outcomes: Direct costs (skewed data).

\begin{tabular}{|c|c|c|c|c|}
\hline \multicolumn{5}{|c|}{ Economic outcomes: Direct costs (skewed data) } \\
\hline Study & Intervention & Mean & SD & $\mathbf{N}$ \\
\hline \multicolumn{5}{|c|}{ medium term } \\
\hline Kemp 1996 & Supportive therapy & 252 & 234 & 34 \\
\hline Kemp 1996 & CBT & 187 & 292 & 36 \\
\hline \multicolumn{5}{|c|}{ long term } \\
\hline Kemp 1996 & Supportive therapy & 326 & 404 & 21 \\
\hline
\end{tabular}

Comparison 4. SUPPORTIVE THERAPY versus FAMILY THERAPY

\begin{tabular}{lllll}
\hline Outcome or subgroup title & No. of studies & $\begin{array}{l}\text { No. of partici- } \\
\text { pants }\end{array}$ & Statistical method & Effect size \\
\hline 1 Global state: 1. Relapse & 2 & Risk Ratio (M-H, Fixed, 95\% Cl) & Totals not selected \\
\hline
\end{tabular}




\begin{tabular}{|c|c|c|c|c|}
\hline Outcome or subgroup title & No. of studies & $\begin{array}{l}\text { No. of partici- } \\
\text { pants }\end{array}$ & Statistical method & Effect size \\
\hline 1.1 long term & 2 & & Risk Ratio (M-H, Fixed, 95\% Cl) & $0.0[0.0,0.0]$ \\
\hline 2 Global state: 2 . No remission & 1 & & Risk Ratio (M-H, Fixed, 95\% Cl) & Subtotals only \\
\hline 2.1 long term & 1 & 39 & Risk Ratio (M-H, Fixed, 95\% Cl) & $1.87[1.11,3.15]$ \\
\hline $\begin{array}{l}3 \text { Service outcomes: Hospitali- } \\
\text { sation }\end{array}$ & 1 & & Risk Ratio (M-H, Fixed, 95\% Cl) & Subtotals only \\
\hline 3.1 long term & 1 & 39 & Risk Ratio (M-H, Fixed, 95\% Cl) & $1.93[0.89,4.17]$ \\
\hline $\begin{array}{l}4 \text { Mental state: Episode of af- } \\
\text { fective symptoms }\end{array}$ & 1 & & Risk Ratio (M-H, Fixed, 95\% Cl) & Subtotals only \\
\hline 4.1 long term & 1 & 48 & Risk Ratio (M-H, Fixed, 95\% Cl) & $1.71[0.82,3.60]$ \\
\hline $\begin{array}{l}5 \text { Leaving the study early (by } \\
\text { long term) }\end{array}$ & 2 & & Risk Ratio (M-H, Fixed, 95\% Cl) & Subtotals only \\
\hline 5.1 general reasons & 1 & 39 & Risk Ratio (M-H, Fixed, 95\% Cl) & $0.70[0.13,3.75]$ \\
\hline 5.2 treatment-related reasons & 1 & 48 & Risk Ratio (M-H, Fixed, 95\% Cl) & $1.6[0.61,4.19]$ \\
\hline $\begin{array}{l}6 \text { General functioning: } 1 \text {. No } \\
\text { paid work }\end{array}$ & 1 & & Risk Ratio (M-H, Fixed, 95\% Cl) & Subtotals only \\
\hline 6.1 long term & 1 & 39 & Risk Ratio (M-H, Fixed, 95\% Cl) & $0.96[0.57,1.63]$ \\
\hline $\begin{array}{l}7 \text { General functioning: } 2 \text {. Ad- } \\
\text { mission to residential place- } \\
\text { ment }\end{array}$ & 1 & & Risk Ratio (M-H, Fixed, 95\% Cl) & Subtotals only \\
\hline 7.1 long term & 1 & 39 & Risk Ratio (M-H, Fixed, 95\% Cl) & $1.05[0.24,4.59]$ \\
\hline $\begin{array}{l}8 \text { General fuctioning: } 3 \text {. Admis- } \\
\text { sion to jail }\end{array}$ & 1 & & Risk Ratio (M-H, Fixed, 95\% Cl) & Subtotals only \\
\hline 8.1 long term & 1 & 39 & Risk Ratio (M-H, Fixed, 95\% Cl) & $1.05[0.24,4.59]$ \\
\hline $\begin{array}{l}9 \text { Behaviour: } 1 \text {. Social impair- } \\
\text { ment on SBAS }\end{array}$ & 1 & & Risk Ratio (M-H, Fixed, 95\% Cl) & Subtotals only \\
\hline 9.1 long term & 1 & 39 & Risk Ratio (M-H, Fixed, 95\% Cl) & $1.46[1.04,2.04]$ \\
\hline $\begin{array}{l}10 \text { Behaviour: } 2 \text {. Poor coping } \\
\text { style with relatives }\end{array}$ & 1 & & Risk Ratio (M-H, Fixed, 95\% Cl) & Subtotals only \\
\hline 10.1 long term & 1 & 39 & Risk Ratio (M-H, Fixed, 95\% Cl) & $0.90[0.37,2.20]$ \\
\hline $\begin{array}{l}11 \text { Engagement with services: } \\
\text { Poor attendance at appoint- } \\
\text { ments }\end{array}$ & 1 & 39 & Risk Ratio (M-H, Fixed, 95\% Cl) & $1.93[0.89,4.17]$ \\
\hline $\begin{array}{l}12 \text { Compliance: Poor compli- } \\
\text { ance to therapy }\end{array}$ & 1 & & Risk Ratio (M-H, Fixed, 95\% Cl) & Subtotals only \\
\hline
\end{tabular}




\begin{tabular}{|c|c|c|c|c|}
\hline Outcome or subgroup title & No. of studies & $\begin{array}{l}\text { No. of partici- } \\
\text { pants }\end{array}$ & Statistical method & Effect size \\
\hline 12.1 medium term & 1 & 39 & Risk Ratio (M-H, Fixed, 95\% Cl) & $2.63[1.30,5.35]$ \\
\hline 12.2 long term & 1 & 39 & Risk Ratio (M-H, Fixed, 95\% Cl) & $1.29[0.69,2.39]$ \\
\hline $\begin{array}{l}13 \text { Medication: Prescribed IM } \\
\text { depot medication }\end{array}$ & 1 & & Risk Ratio (M-H, Fixed, 95\% Cl) & Subtotals only \\
\hline 13.1 medium term & 1 & 39 & Risk Ratio (M-H, Fixed, 95\% Cl) & $2.11[0.99,4.47]$ \\
\hline
\end{tabular}

\section{Analysis 4.1. Comparison 4 SUPPORTIVE THERAPY versus FAMILY THERAPY, Outcome 1 Global state: 1. Relapse.}

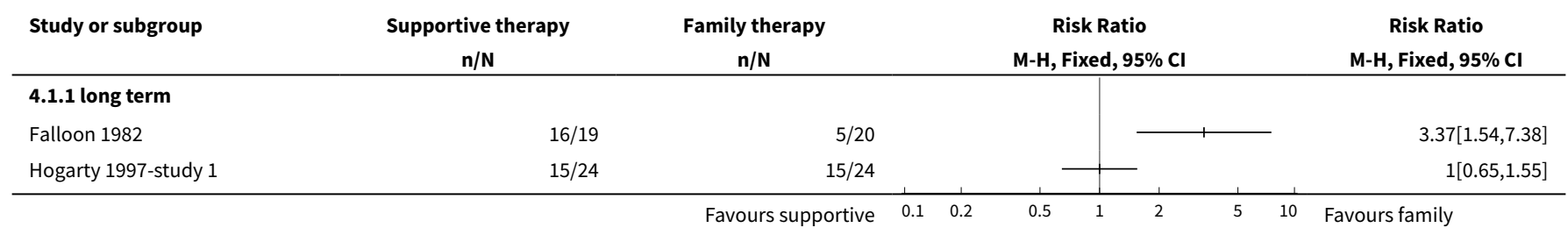

Analysis 4.2. Comparison 4 SUPPORTIVE THERAPY versus FAMILY THERAPY, Outcome 2 Global state: 2 . No remission.

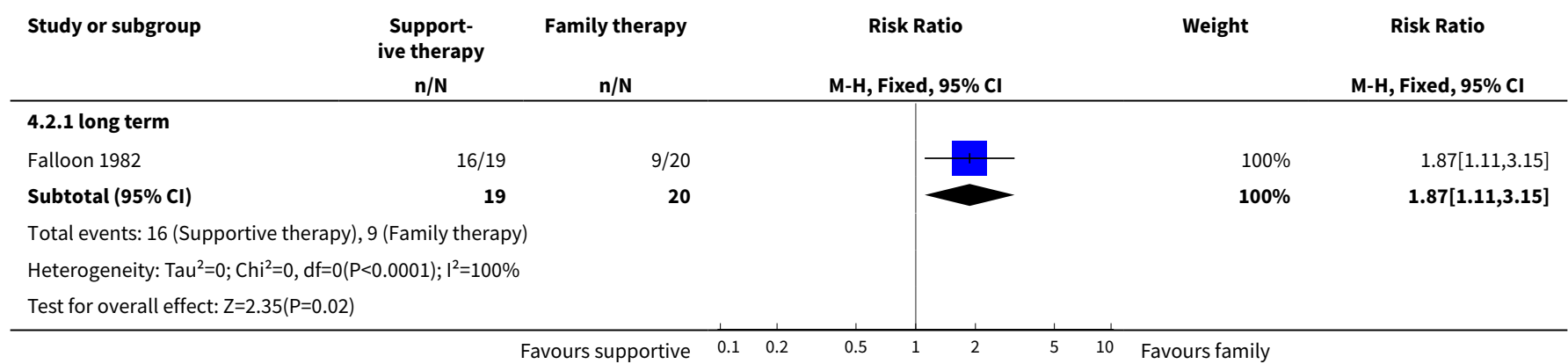

Analysis 4.3. Comparison 4 SUPPORTIVE THERAPY versus FAMILY THERAPY, Outcome 3 Service outcomes: Hospitalisation.

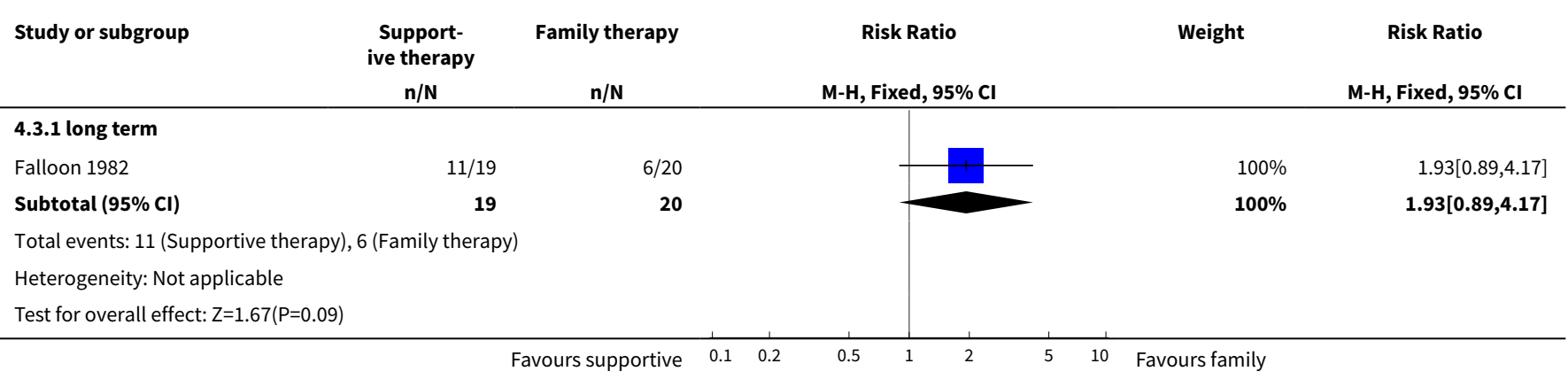


Analysis 4.4. Comparison 4 SUPPORTIVE THERAPY versus FAMILY

THERAPY, Outcome 4 Mental state: Episode of affective symptoms.

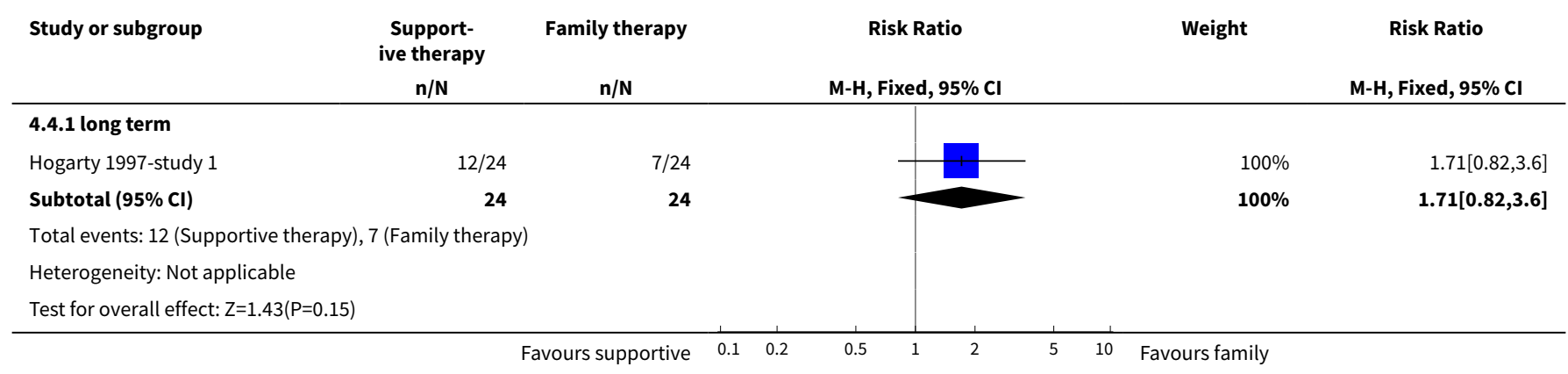

Analysis 4.5. Comparison 4 SUPPORTIVE THERAPY versus FAMILY THERAPY, Outcome 5 Leaving the study early (by long term).

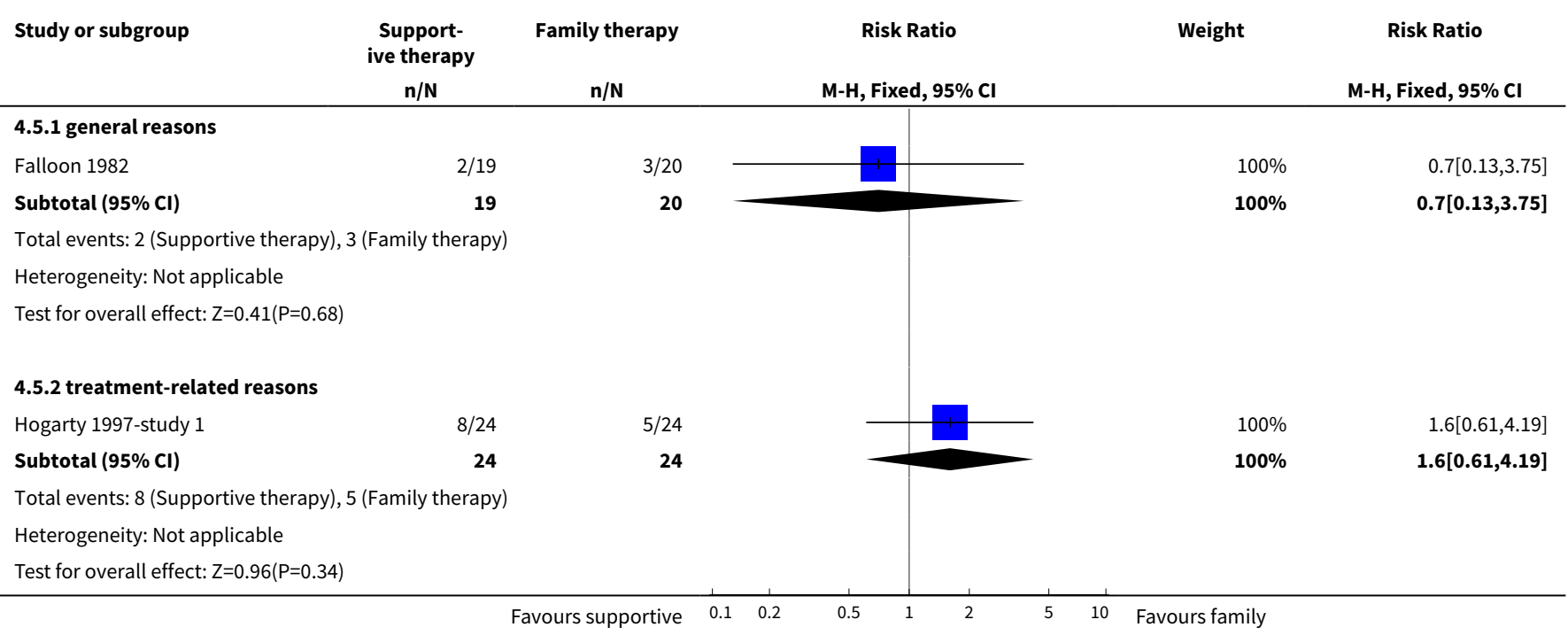

Analysis 4.6. Comparison 4 SUPPORTIVE THERAPY versus FAMILY THERAPY, Outcome 6 General functioning: 1. No paid work.

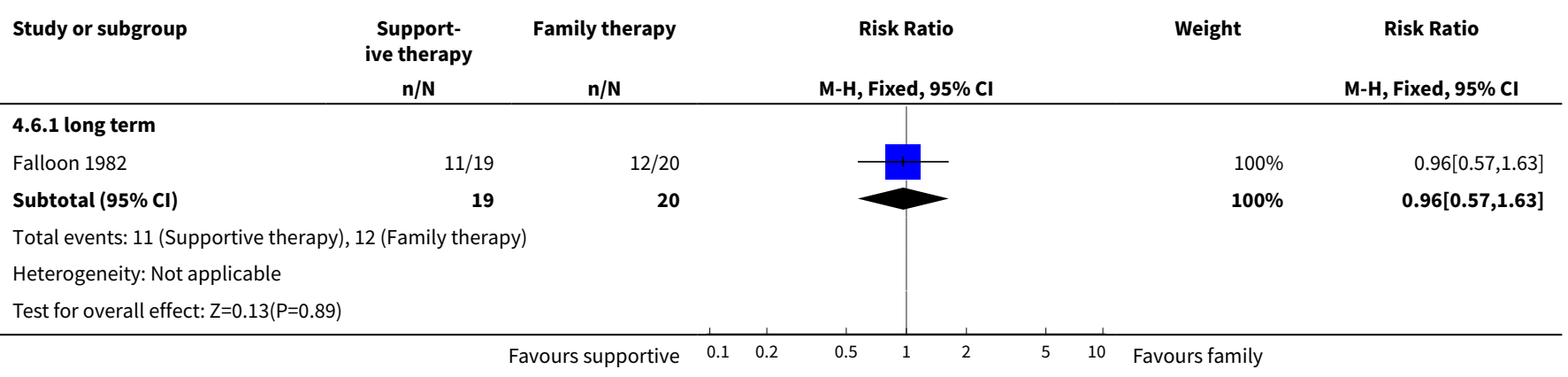


Analysis 4.7. Comparison 4 SUPPORTIVE THERAPY versus FAMILY THERAPY, Outcome 7 General functioning: 2 . Admission to residential placement.

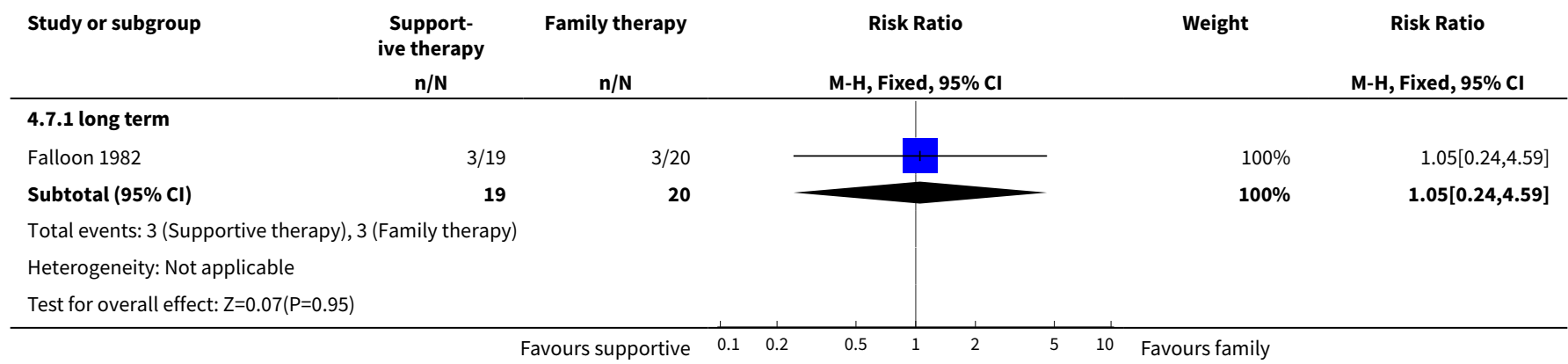

Analysis 4.8. Comparison 4 SUPPORTIVE THERAPY versus FAMILY THERAPY, Outcome 8 General fuctioning: 3 . Admission to jail.

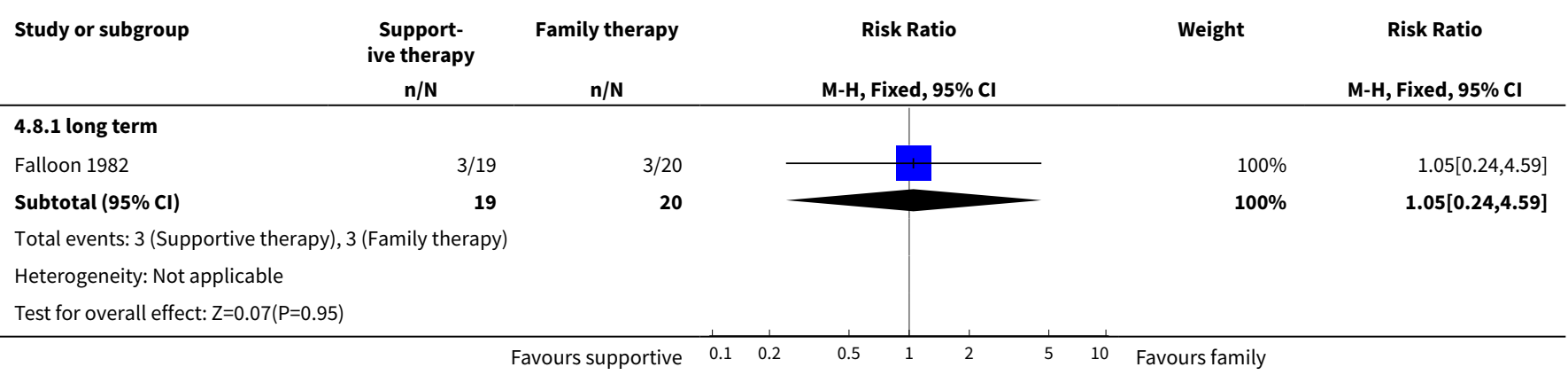

Analysis 4.9. Comparison 4 SUPPORTIVE THERAPY versus FAMILY

THERAPY, Outcome 9 Behaviour: 1. Social impairment on SBAS.

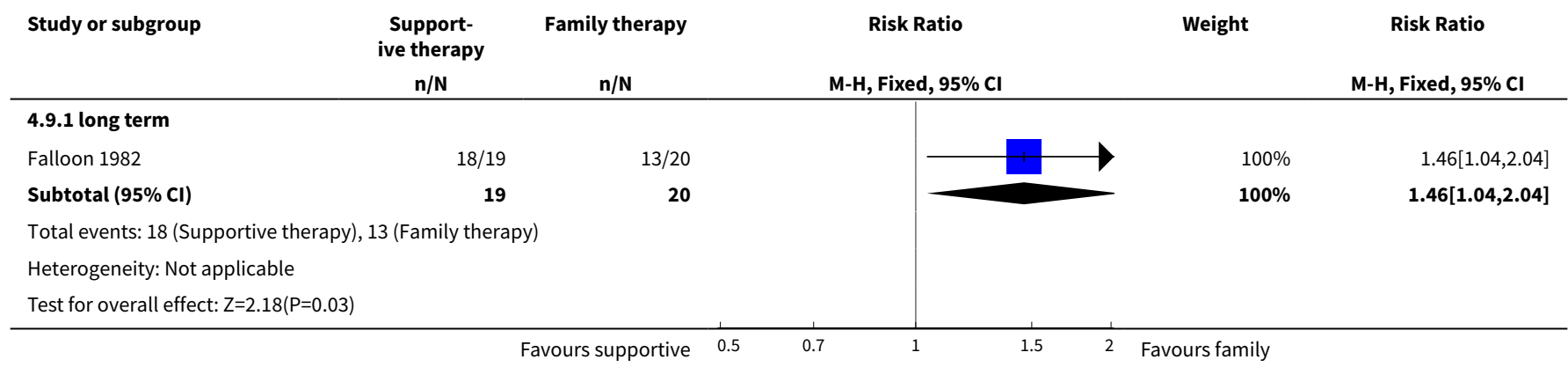


Analysis 4.10. Comparison 4 SUPPORTIVE THERAPY versus FAMILY

THERAPY, Outcome 10 Behaviour: 2. Poor coping style with relatives.

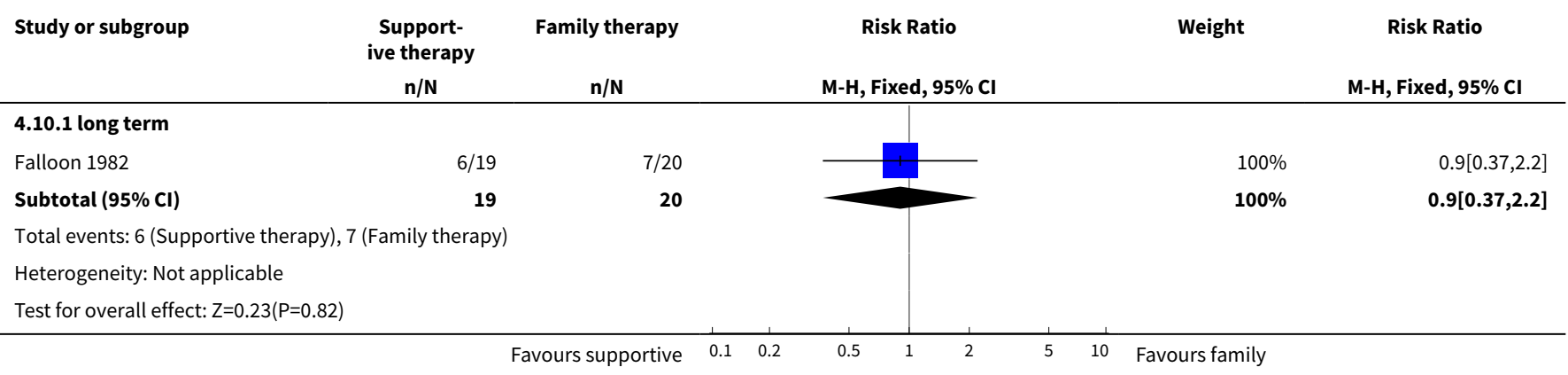

Analysis 4.11. Comparison 4 SUPPORTIVE THERAPY versus FAMILY THERAPY, Outcome 11 Engagement with services: Poor attendance at appointments.

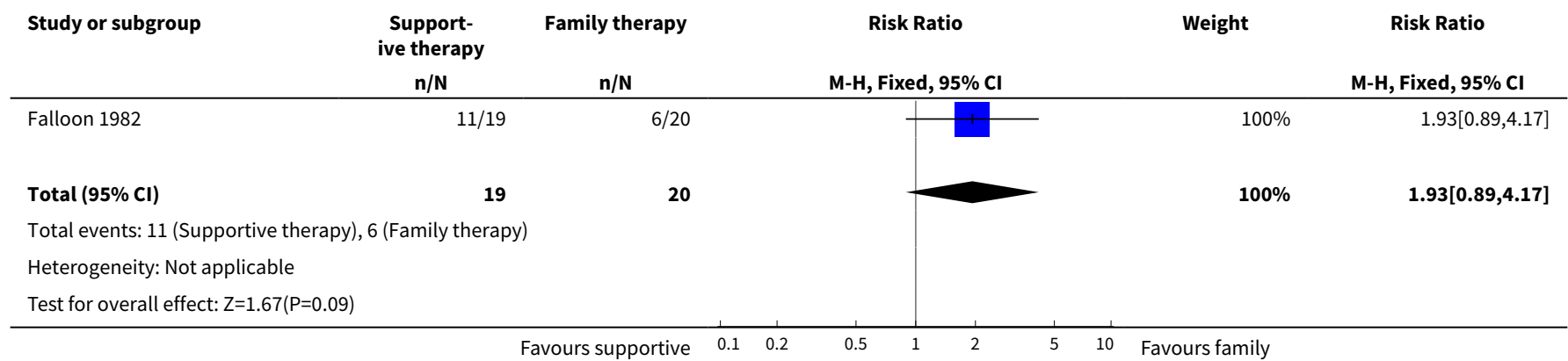

Analysis 4.12. Comparison 4 SUPPORTIVE THERAPY versus FAMILY THERAPY, Outcome 12 Compliance: Poor compliance to therapy.

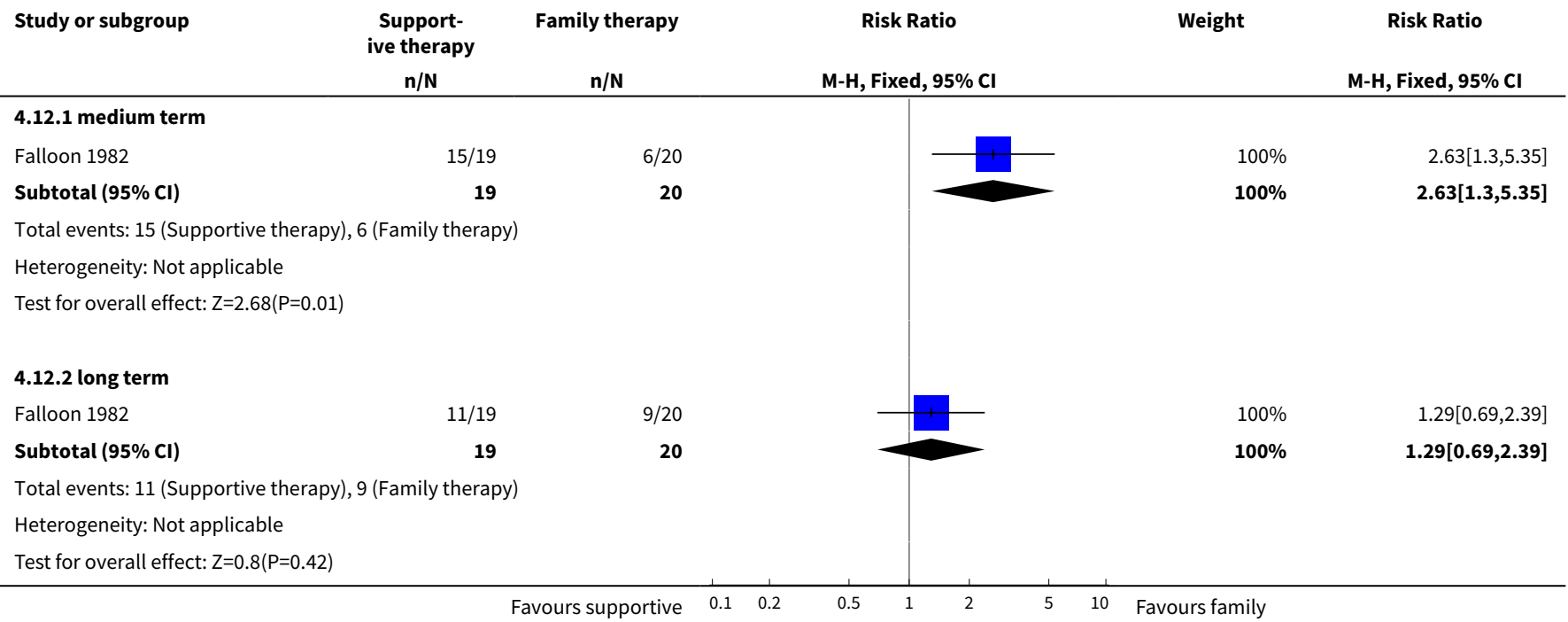


Analysis 4.13. Comparison 4 SUPPORTIVE THERAPY versus FAMILY THERAPY, Outcome 13 Medication: Prescribed IM depot medication.

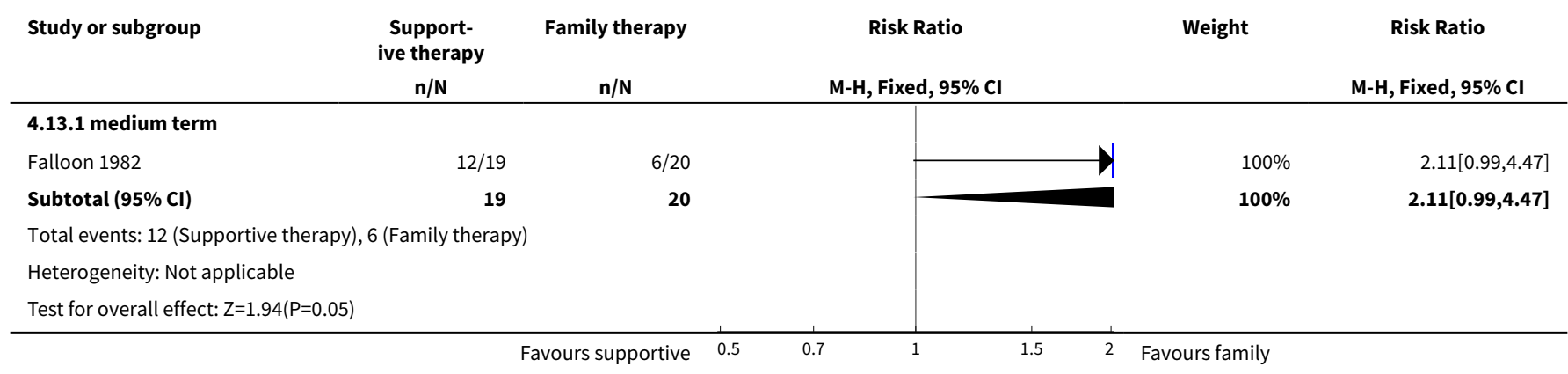

\section{Comparison 5. SUPPORTIVE THERAPY versus PSYCHOEDUCATION}

\begin{tabular}{|c|c|c|c|c|}
\hline Outcome or subgroup title & No. of studies & $\begin{array}{l}\text { No. of partici- } \\
\text { pants }\end{array}$ & Statistical method & Effect size \\
\hline 1 Service outcomes: Hospitalisation & 1 & 47 & $\begin{array}{l}\text { Risk Ratio (M-H, Fixed, } \\
95 \% \mathrm{Cl})\end{array}$ & $0.48[0.05,4.93]$ \\
\hline $\begin{array}{l}2 \text { Mental state. } 1 \text {. No clinically important } \\
\text { improvement in general mental state }\end{array}$ & 1 & 19 & $\begin{array}{l}\text { Risk Ratio (M-H, Fixed, } \\
95 \% \mathrm{Cl})\end{array}$ & $1.61[0.96,2.68]$ \\
\hline $\begin{array}{l}3 \text { Mental state: } 2 \text {. Average endpoint gen- } \\
\text { eral score (PANSS general subscale, high } \\
=\text { poor) }\end{array}$ & 1 & 19 & $\begin{array}{l}\text { Mean Difference (IV, Fixed, } \\
95 \% \mathrm{CI} \text { ) }\end{array}$ & $2.86[-3.21,8.93]$ \\
\hline $\begin{array}{l}4 \text { Mental state: } 3 \text {. Average endpoint neg- } \\
\text { ative symptoms score (PANSS negative } \\
\text { subscale, high = poor) }\end{array}$ & 1 & 19 & $\begin{array}{l}\text { Mean Difference (IV, Fixed, } \\
95 \% \mathrm{CI} \text { ) }\end{array}$ & $1.70[-4.00,7.40]$ \\
\hline $\begin{array}{l}5 \text { Mental state: } 4 \text {. Average endpoint posi- } \\
\text { tive symptoms score (PANSS positive sub- } \\
\text { scale, high = poor) }\end{array}$ & 1 & 19 & $\begin{array}{l}\text { Mean Difference (IV, Fixed, } \\
95 \% \mathrm{CI})\end{array}$ & $2.71[-0.71,6.13]$ \\
\hline $\begin{array}{l}6 \text { Mental state: } 5 \text {. Average endpoint de- } \\
\text { pression score (CDRS, high = poor) }\end{array}$ & 1 & 19 & $\begin{array}{l}\text { Mean Difference (IV, Fixed, } \\
95 \% \mathrm{CI})\end{array}$ & $1.47[-1.35,4.29]$ \\
\hline 7 Leaving the study early & 2 & 71 & $\begin{array}{l}\text { Risk Ratio (M-H, Fixed, } \\
95 \% \mathrm{Cl})\end{array}$ & $0.57[0.21,1.54]$ \\
\hline $\begin{array}{l}8 \text { Quality of life: Average endpoint quality } \\
\text { of life score (QLS, high = good) }\end{array}$ & 1 & 19 & $\begin{array}{l}\text { Mean Difference (IV, Fixed, } \\
95 \% \mathrm{Cl} \text { ) }\end{array}$ & $\begin{array}{l}-0.07[-21.11 \\
20.97]\end{array}$ \\
\hline 9 Death & 1 & 47 & $\begin{array}{l}\text { Risk Ratio (M-H, Fixed, } \\
95 \% \mathrm{Cl})\end{array}$ & $2.88[0.12,67.29]$ \\
\hline $\begin{array}{l}10 \text { Behaviour: Average endpoint score } \\
\text { (composed of ROMI and ITAQ items, high } \\
=\text { good) }\end{array}$ & 1 & & $\begin{array}{l}\text { Mean Difference (IV, Fixed, } \\
95 \% \mathrm{CI})\end{array}$ & Subtotals only \\
\hline 10.1 Need for treatment & 1 & 19 & $\begin{array}{l}\text { Mean Difference (IV, Fixed, } \\
95 \% \mathrm{CI} \text { ) }\end{array}$ & $-0.02[-0.44,0.40]$ \\
\hline
\end{tabular}




\begin{tabular}{|c|c|c|c|c|}
\hline Outcome or subgroup title & No. of studies & $\begin{array}{l}\text { No. of partici- } \\
\text { pants }\end{array}$ & Statistical method & Effect size \\
\hline 10.2 Benefits of medication & 1 & 19 & $\begin{array}{l}\text { Mean Difference (IV, Fixed, } \\
95 \% \mathrm{CI})\end{array}$ & $-0.19[-0.56,0.18]$ \\
\hline $\begin{array}{l}11 \text { Insight: Average endpoint treatment } \\
\text { attitude score (ITAQ, high = good) }\end{array}$ & 1 & 19 & $\begin{array}{l}\text { Mean Difference (IV, Fixed, } \\
95 \% \mathrm{CI})\end{array}$ & $-1.55[-5.85,2.75]$ \\
\hline $\begin{array}{l}12 \text { Compliance: } 1 . \text { Poor compliance to } \\
\text { therapy }\end{array}$ & 1 & 19 & $\begin{array}{l}\text { Odds Ratio (M-H, Fixed, } \\
95 \% \mathrm{Cl})\end{array}$ & $0.0[0.0,0.0]$ \\
\hline $\begin{array}{l}13 \text { Compliance: } 2 \text {. Adherence to medica- } \\
\text { tion (self-report) }\end{array}$ & 1 & 19 & $\begin{array}{l}\text { Risk Ratio (M-H, Fixed, } \\
95 \% \mathrm{Cl})\end{array}$ & $1.0[0.83,1.21]$ \\
\hline $\begin{array}{l}14 \text { Compliance: } 3 \text {. Average endpoint ad- } \\
\text { herence score (ROMI, high = good) }\end{array}$ & 1 & 19 & $\begin{array}{l}\text { Mean Difference (IV, Fixed, } \\
95 \% \mathrm{Cl} \text { ) }\end{array}$ & $0.45[-3.58,4.48]$ \\
\hline $\begin{array}{l}15 \text { Compliance: } 4 \text {. Average endpoint non- } \\
\text { adherence score (ROMI, high = poor) }\end{array}$ & 1 & 19 & $\begin{array}{l}\text { Mean Difference (IV, Fixed, } \\
95 \% \mathrm{CI})\end{array}$ & $1.75[-0.61,4.11]$ \\
\hline
\end{tabular}

Analysis 5.1. Comparison 5 SUPPORTIVE THERAPY versus PSYCHOEDUCATION, Outcome 1 Service outcomes: Hospitalisation.

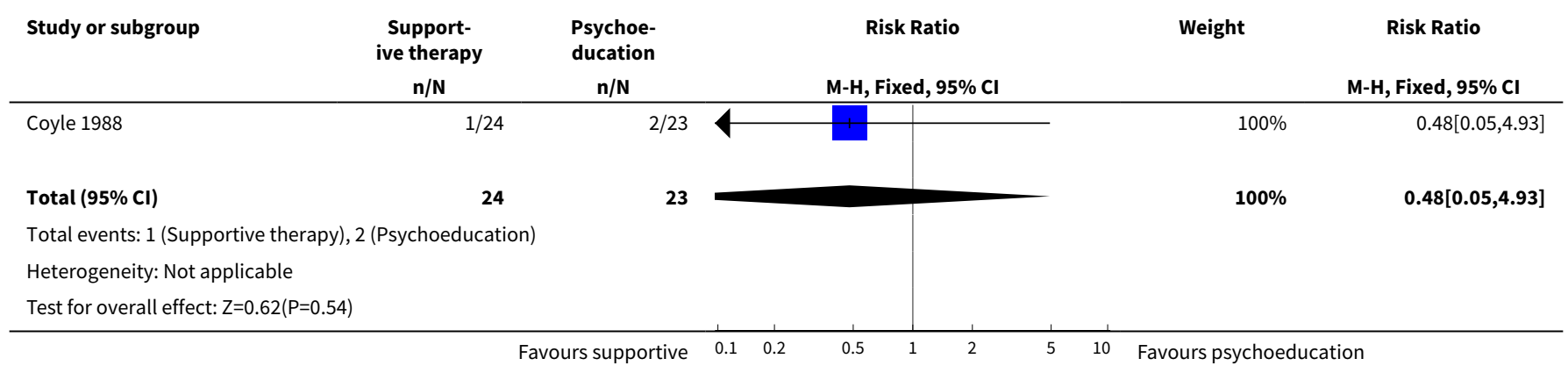

Analysis 5.2. Comparison 5 SUPPORTIVE THERAPY versus PSYCHOEDUCATION, Outcome 2 Mental state. 1. No clinically important improvement in general mental state.

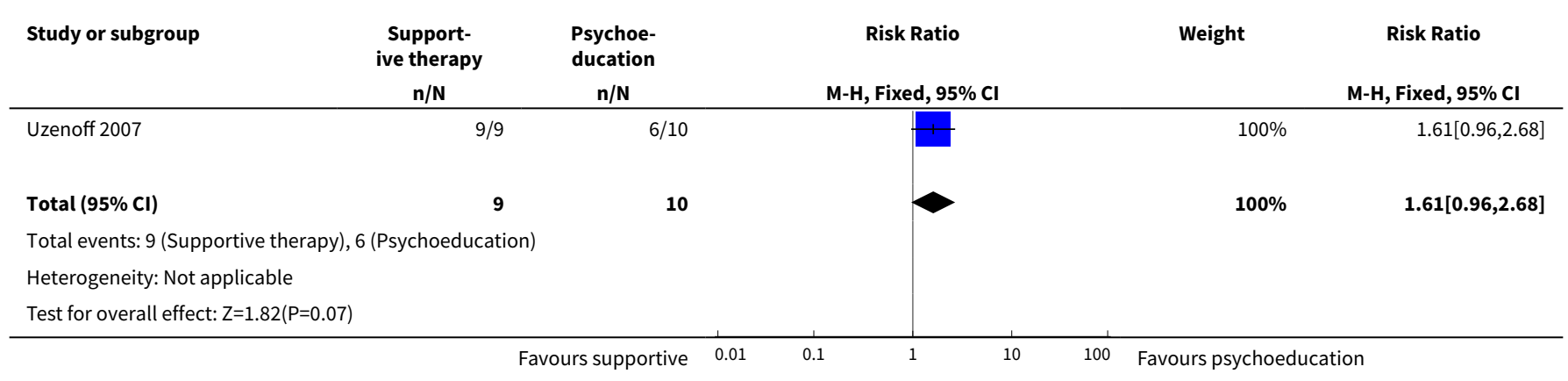


Analysis 5.3. Comparison 5 SUPPORTIVE THERAPY versus PSYCHOEDUCATION, Outcome 3 Mental state: 2. Average endpoint general score (PANSS general subscale, high = poor).

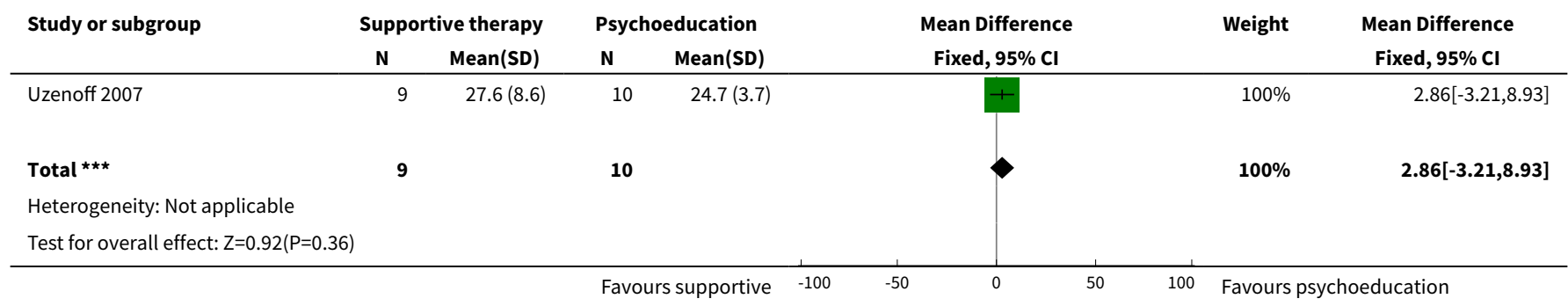

Analysis 5.4. Comparison 5 SUPPORTIVE THERAPY versus PSYCHOEDUCATION, Outcome 4 Mental state: 3. Average endpoint negative symptoms score (PANSS negative subscale, high = poor).

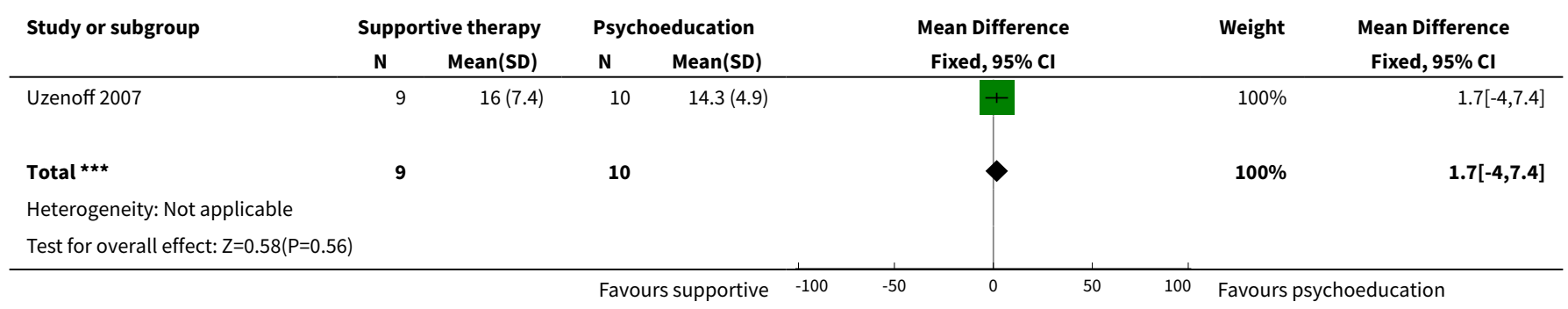

Analysis 5.5. Comparison 5 SUPPORTIVE THERAPY versus PSYCHOEDUCATION, Outcome 5 Mental state: 4. Average endpoint positive symptoms score (PANSS positive subscale, high = poor).

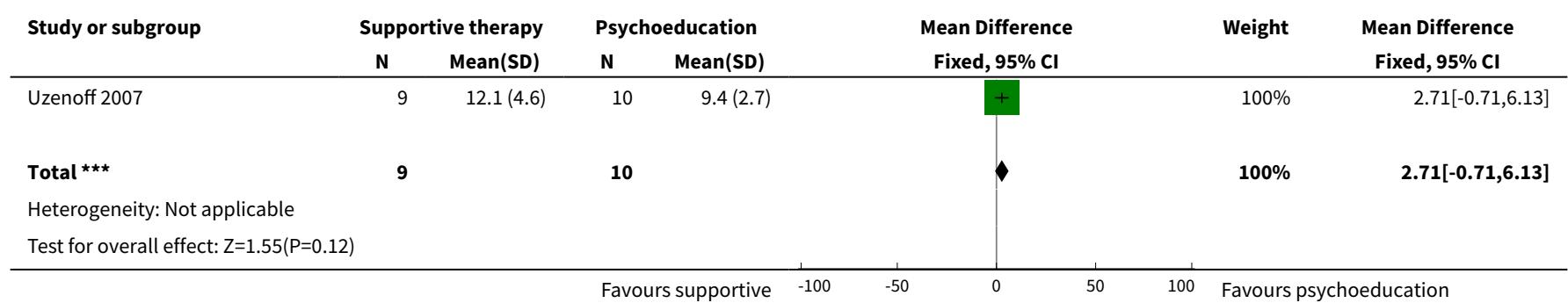

Analysis 5.6. Comparison 5 SUPPORTIVE THERAPY versus PSYCHOEDUCATION, Outcome 6 Mental state: 5. Average endpoint depression score (CDRS, high = poor).

\begin{tabular}{|c|c|c|c|c|c|c|c|c|}
\hline \multirow[t]{2}{*}{ Study or subgroup } & \multicolumn{2}{|c|}{ Supportive therapy } & \multicolumn{2}{|c|}{ Psychoeducation } & \multirow{2}{*}{\multicolumn{2}{|c|}{$\begin{array}{c}\text { Mean Difference } \\
\text { Fixed, } 95 \% \mathrm{Cl}\end{array}$}} & \multirow[t]{2}{*}{ Weight } & \multirow{2}{*}{$\begin{array}{c}\text { Mean Difference } \\
\text { Fixed, } 95 \% \mathrm{Cl}\end{array}$} \\
\hline & $\mathbf{N}$ & $\operatorname{Mean}(S D)$ & $\mathbf{N}$ & Mean(SD) & & & & \\
\hline Uzenoff 2007 & 9 & $11.7(3.9)$ & 10 & $10.2(1.9)$ & & + & $100 \%$ & $1.47[-1.35,4.29]$ \\
\hline Total $\star \star \star ~$ & 9 & & 10 & & & 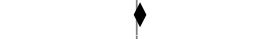 & $100 \%$ & $1.47[-1.35,4.29]$ \\
\hline \multicolumn{9}{|c|}{ Heterogeneity: Not applicable } \\
\hline \multicolumn{9}{|c|}{ Test for overall effect: $Z=1.02(P=0.31)$} \\
\hline
\end{tabular}


Analysis 5.7. Comparison 5 SUPPORTIVE THERAPY versus PSYCHOEDUCATION, Outcome 7 Leaving the study early.

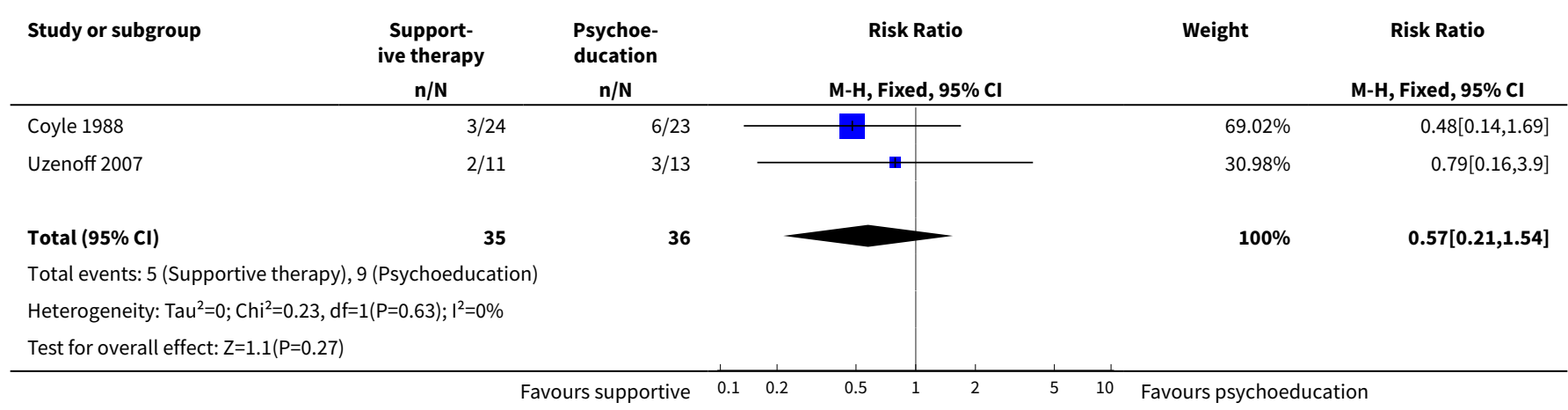

Analysis 5.8. Comparison 5 SUPPORTIVE THERAPY versus PSYCHOEDUCATION, Outcome 8 Quality of life: Average endpoint quality of life score (QLS, high = good).

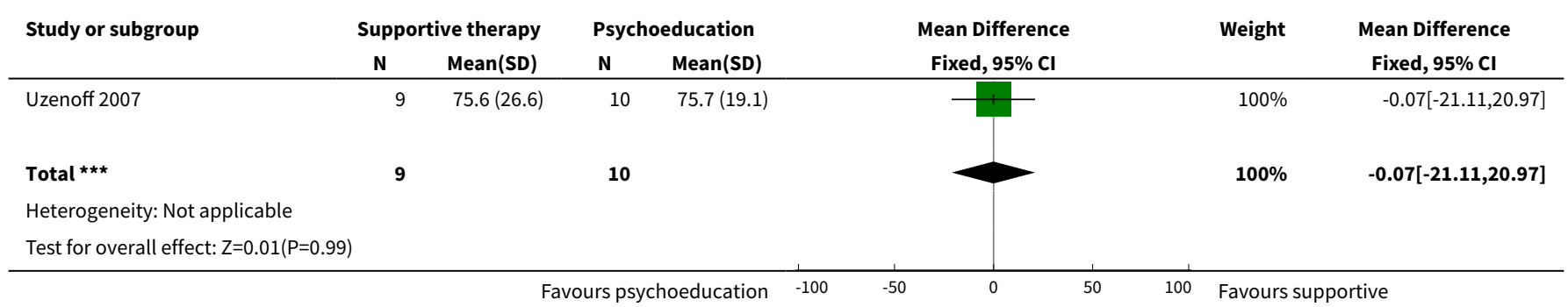

Analysis 5.9. Comparison 5 SUPPORTIVE THERAPY versus PSYCHOEDUCATION, Outcome 9 Death.

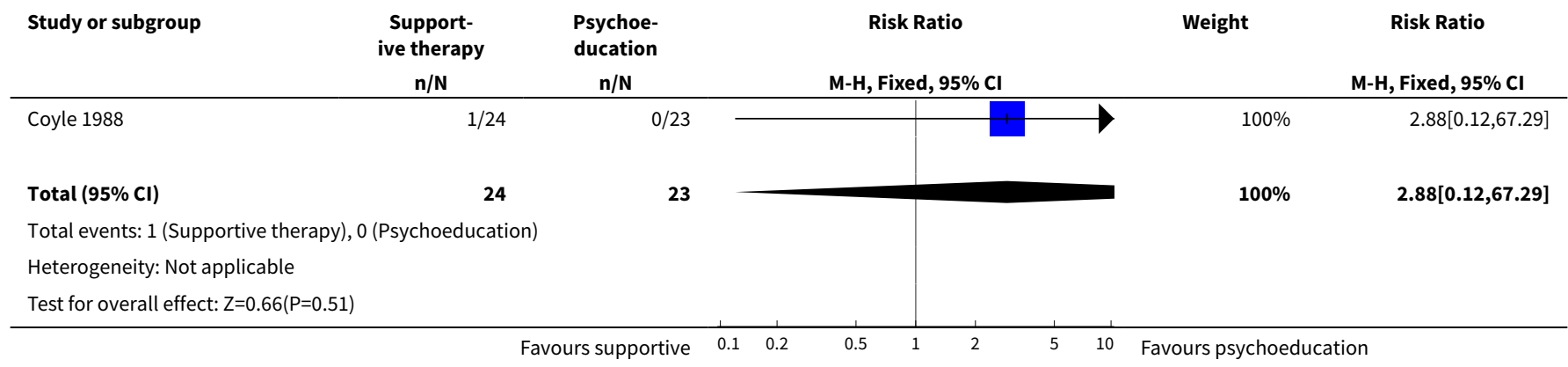

Analysis 5.10. Comparison 5 SUPPORTIVE THERAPY versus PSYCHOEDUCATION, Outcome 10 Behaviour: Average endpoint score (composed of ROMI and ITAQ items, high = good).

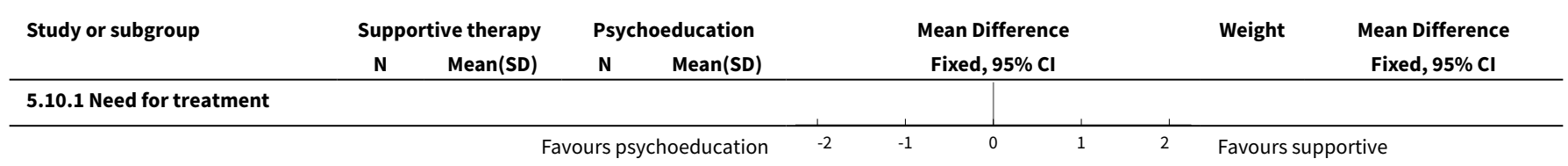




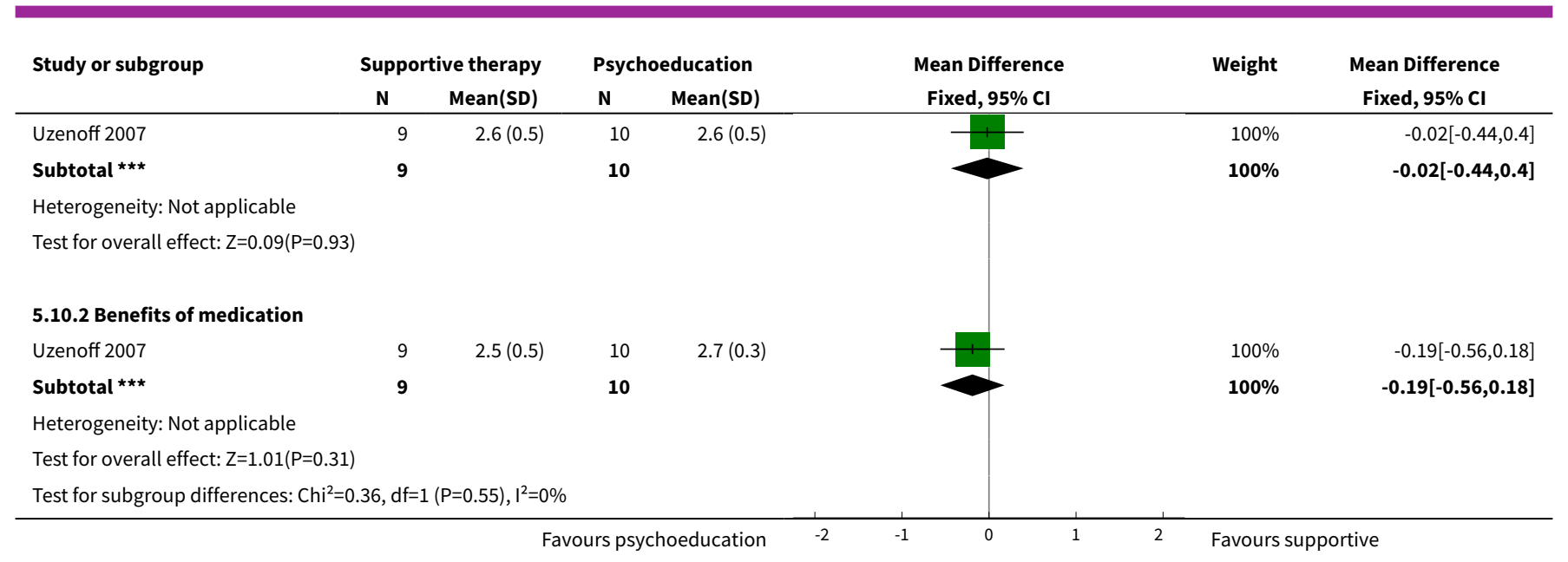

Analysis 5.11. Comparison 5 SUPPORTIVE THERAPY versus PSYCHOEDUCATION, Outcome 11 Insight: Average endpoint treatment attitude score (ITAQ, high = good).

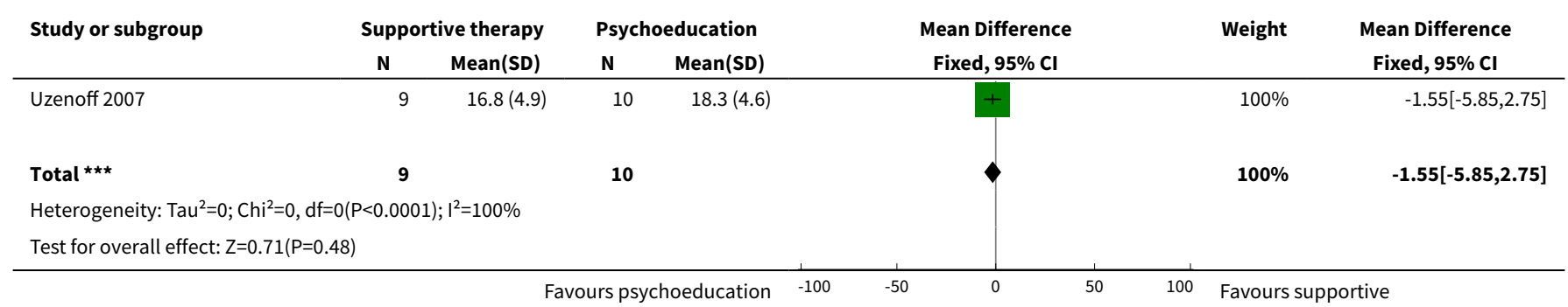

Analysis 5.12. Comparison 5 SUPPORTIVE THERAPY versus PSYCHOEDUCATION, Outcome 12 Compliance: 1 . Poor compliance to therapy.

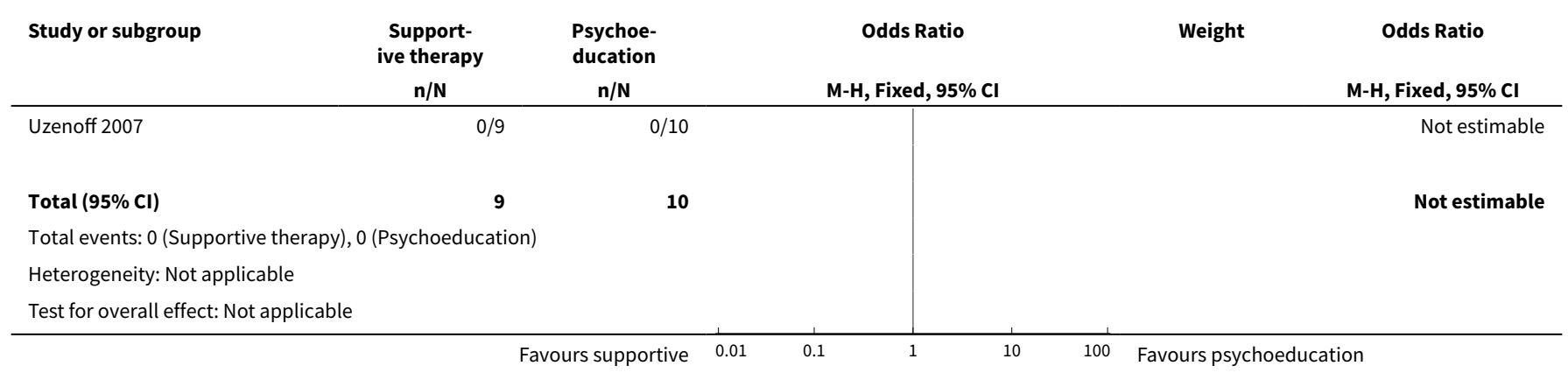


Analysis 5.13. Comparison 5 SUPPORTIVE THERAPY versus PSYCHOEDUCATION, Outcome 13 Compliance: 2 . Adherence to medication (self-report).

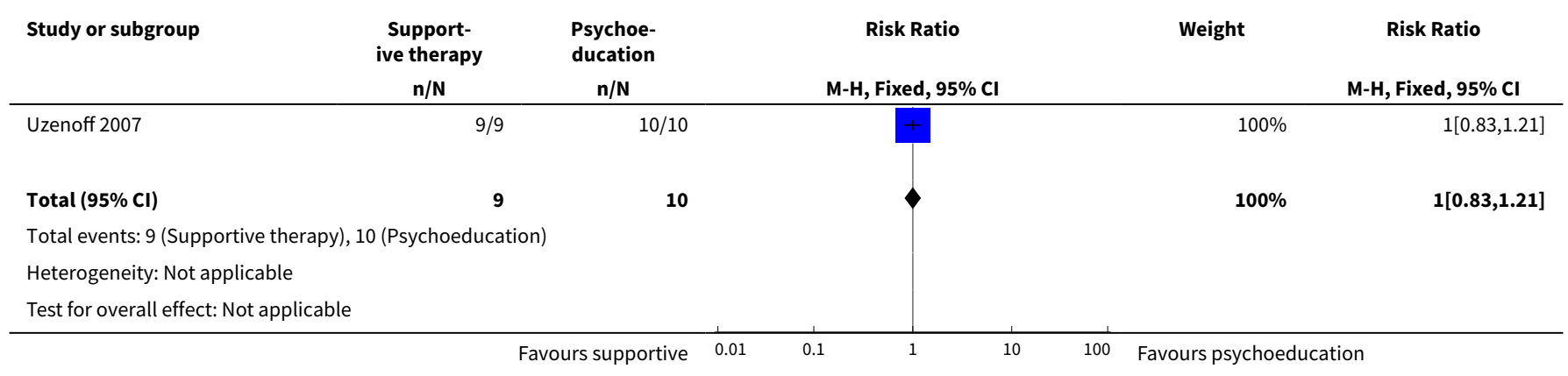

Analysis 5.14. Comparison 5 SUPPORTIVE THERAPY versus PSYCHOEDUCATION, Outcome 14 Compliance: 3. Average endpoint adherence score (ROMI, high = good).

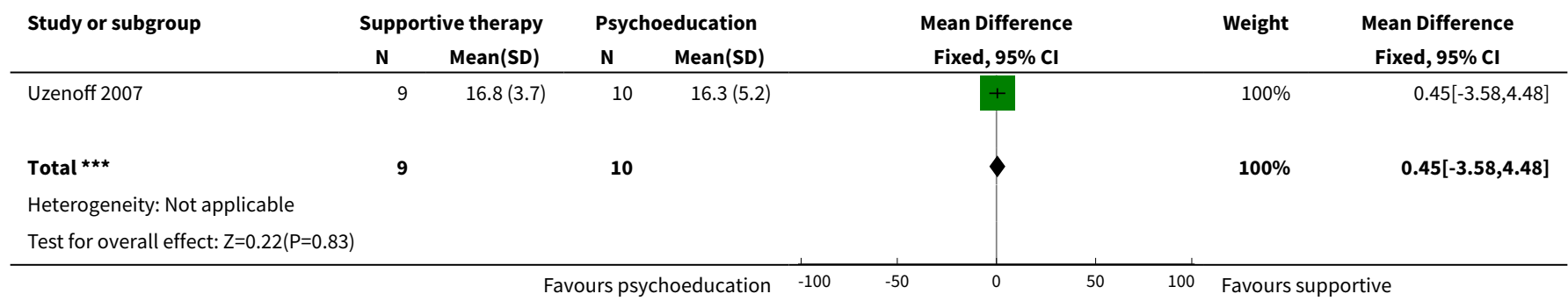

Analysis 5.15. Comparison 5 SUPPORTIVE THERAPY versus PSYCHOEDUCATION, Outcome 15 Compliance: 4. Average endpoint nonadherence score (ROMI, high = poor).

\begin{tabular}{|c|c|c|c|c|c|c|c|}
\hline \multirow[t]{2}{*}{ Study or subgroup } & \multicolumn{2}{|c|}{ Supportive therapy } & \multicolumn{2}{|c|}{ Psychoeducation } & \multirow{2}{*}{$\begin{array}{c}\text { Mean Difference } \\
\text { Fixed, } 95 \% \mathrm{Cl}\end{array}$} & \multirow[t]{2}{*}{ Weight } & \multirow{2}{*}{$\begin{array}{c}\text { Mean Difference } \\
\text { Fixed, } 95 \% \mathrm{Cl}\end{array}$} \\
\hline & $\mathbf{N}$ & Mean(SD) & $\mathbf{N}$ & Mean(SD) & & & \\
\hline Uzenoff 2007 & 9 & $12.1(2.5)$ & 10 & $10.4(2.8)$ & + & $100 \%$ & $1.75[-0.61,4.11]$ \\
\hline Total $* \star \star$ & 9 & & 10 & & 1 & $100 \%$ & $1.75[-0.61,4.11]$ \\
\hline \multicolumn{8}{|c|}{ Heterogeneity: Not applicable } \\
\hline Test for overall effect & & & & & & & \\
\hline
\end{tabular}

\section{Comparison 6. SUPPORTIVE THERAPY versUS REHABILITATION PROGRAMME}

\begin{tabular}{lllll}
\hline Outcome or subgroup title & No. of studies & $\begin{array}{l}\text { No. of partici- } \\
\text { pants }\end{array}$ & Statistical method & Effect size \\
\hline $\begin{array}{l}1 \text { Service outcomes: Hospi- } \\
\text { talisation }\end{array}$ & 1 & Risk Ratio (M-H, Fixed, 95\% Cl) & Subtotals only \\
\hline 1.1 long term & 1 & 132 & Risk Ratio (M-H, Fixed, 95\% Cl) & $2.71[1.22,6.02]$ \\
\hline
\end{tabular}




\begin{tabular}{lllll}
\hline Outcome or subgroup title & No. of studies & $\begin{array}{l}\text { No. of partici- } \\
\text { pants }\end{array}$ & Statistical method & Effect size \\
\hline 2 Leaving the study early & 1 & 132 & Risk Ratio (M-H, Fixed, 95\% Cl) & $1.45[0.92,2.29]$ \\
\hline $\begin{array}{l}\text { 3 General functioning: No } \\
\text { gainful employment }\end{array}$ & 1 & Risk Ratio (M-H, Fixed, 95\% Cl) & Subtotals only \\
\hline 3.1 long term & 1 & 132 & Risk Ratio (M-H, Fixed, 95\% Cl) & $1.04[0.85,1.29]$ \\
\hline
\end{tabular}

Analysis 6.1. Comparison 6 SUPPORTIVE THERAPY versus REHABILITATION PROGRAMME, Outcome 1 Service outcomes: Hospitalisation.

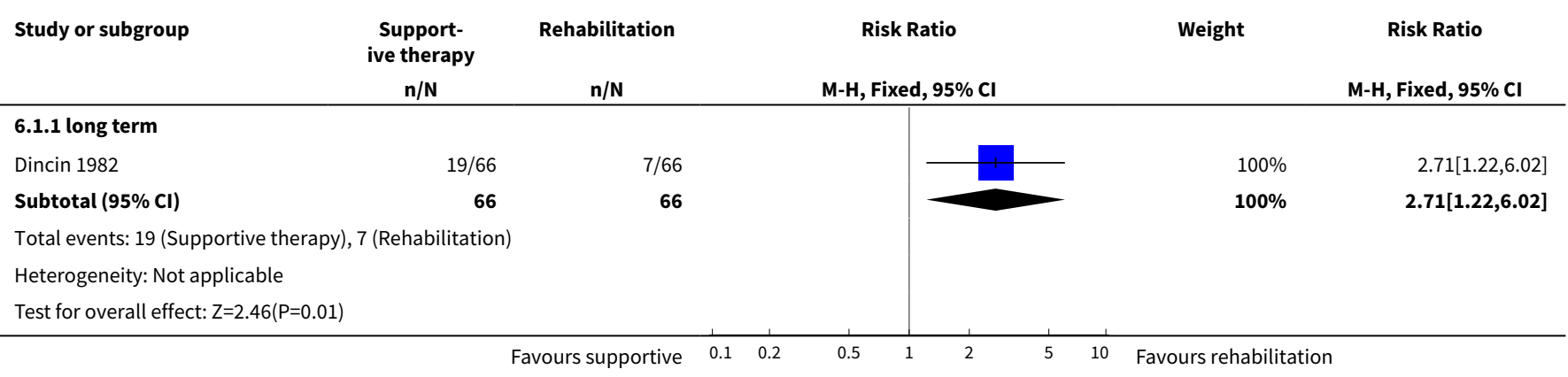

Analysis 6.2. Comparison 6 SUPPORTIVE THERAPY versus REHABILITATION PROGRAMME, Outcome 2 Leaving the study early.

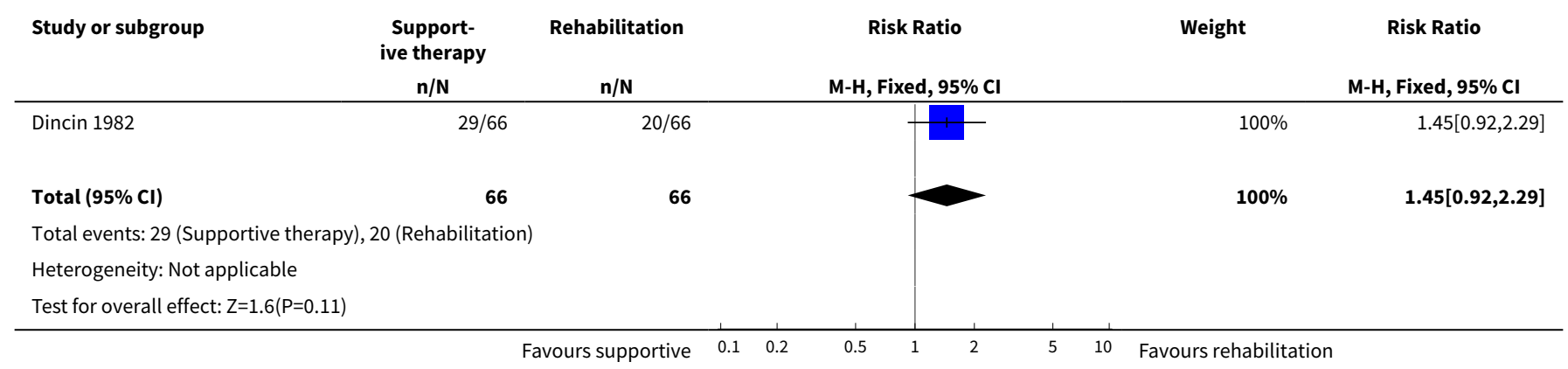

Analysis 6.3. Comparison 6 SUPPORTIVE THERAPY versus REHABILITATION PROGRAMME, Outcome 3 General functioning: No gainful employment.

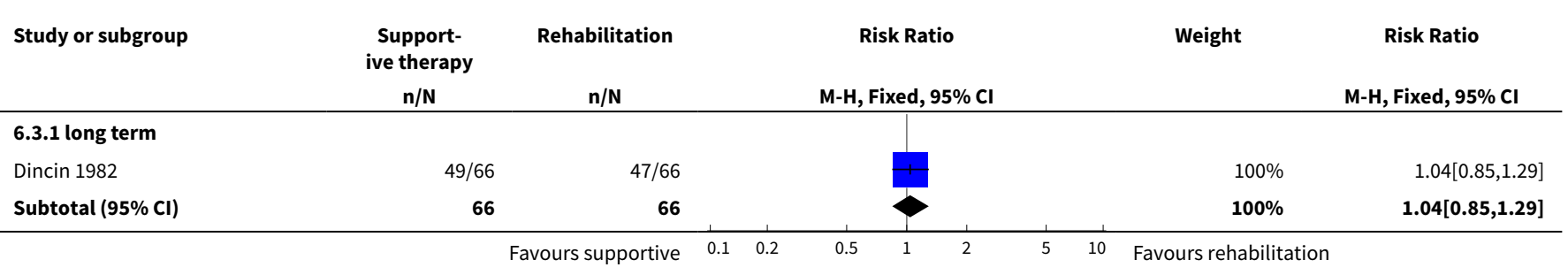




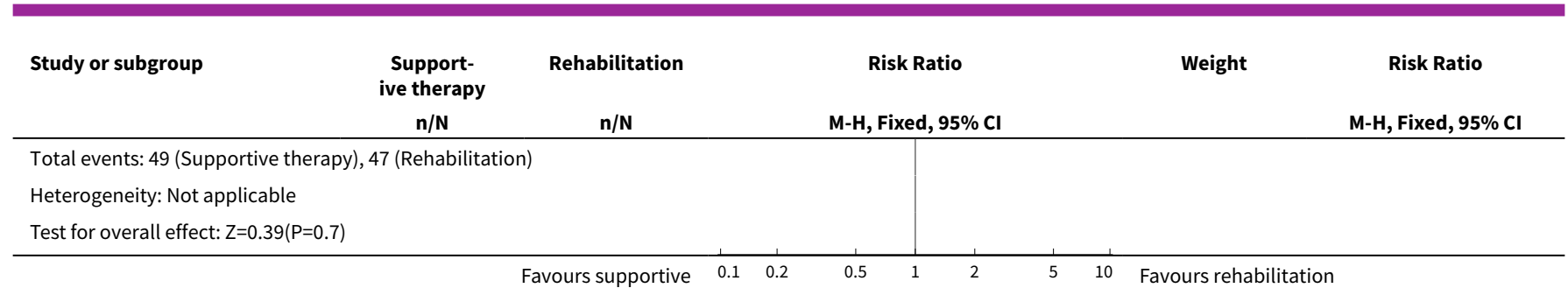

\section{Comparison 7. SUPPORTIVE THERAPY versus SKILLS TRAINING}

\begin{tabular}{lllll}
\hline Outcome or subgroup title & No. of studies & $\begin{array}{l}\text { No. of partici- } \\
\text { pants }\end{array}$ & Statistical method & Effect size \\
\hline $\begin{array}{l}1 \text { Service outcomes: Hospi- } \\
\text { talisation }\end{array}$ & 1 & 47 & Risk Ratio (M-H, Fixed, 95\% Cl) & $0.96[0.06,14.43]$ \\
\hline 2 Leaving the study early & 3 & 168 & Risk Ratio (M-H, Fixed, 95\% Cl) & $1.01[0.61,1.67]$ \\
\hline 3 Death & 1 & 47 & Risk Ratio (M-H, Fixed, 95\% Cl) & $2.88[0.12,67.29]$ \\
\hline
\end{tabular}

Analysis 7.1. Comparison 7 SUPPORTIVE THERAPY versus SKILLS TRAINING, Outcome 1 Service outcomes: Hospitalisation.

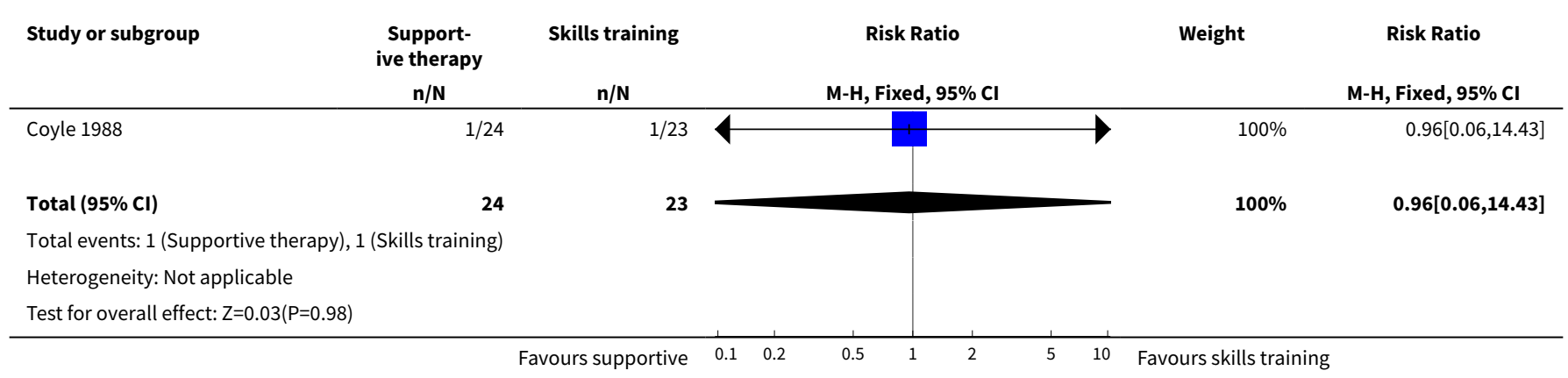

Analysis 7.2. Comparison 7 SUPPORTIVE THERAPY versus SKILLS TRAINING, Outcome 2 Leaving the study early.

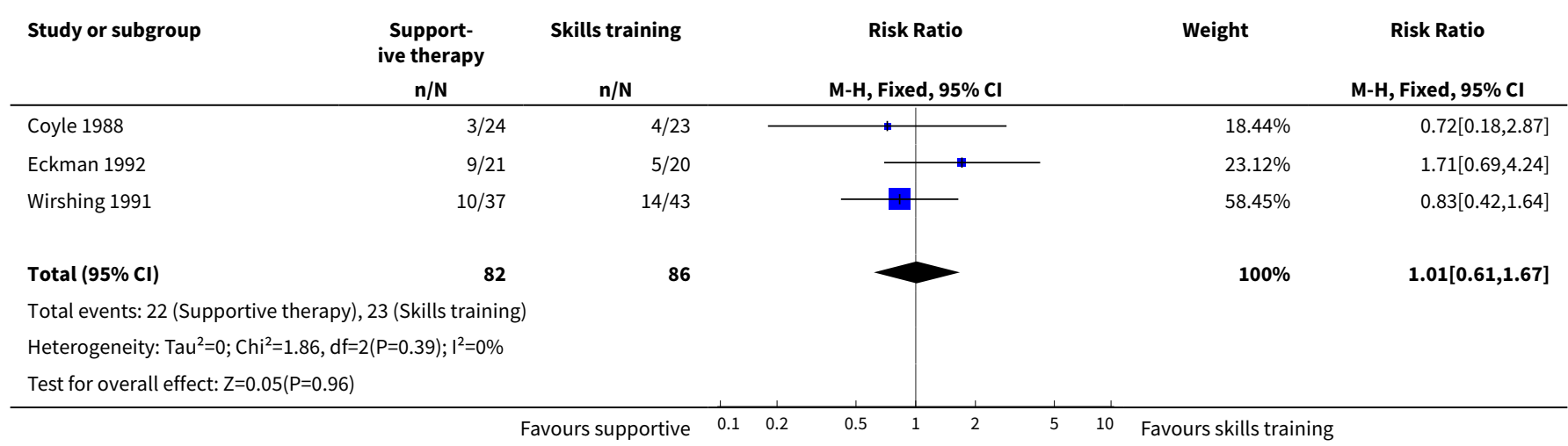


Analysis 7.3. Comparison 7 SUPPORTIVE THERAPY versus SKILLS TRAINING, Outcome 3 Death.

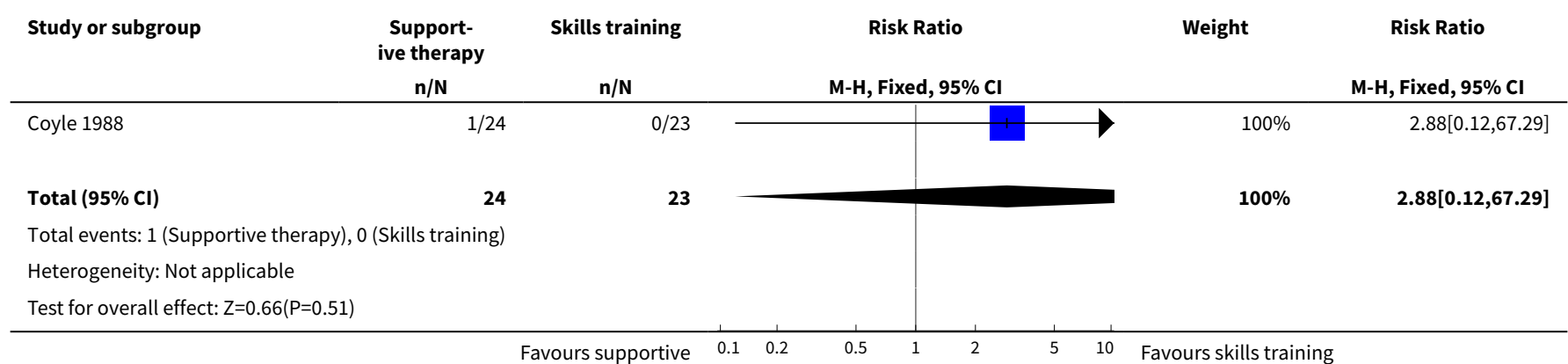

\section{Comparison 8. SUPPORTIVE THERAPY versus PSYCHODYNAMIC PSYCHOTHERAPY}

\begin{tabular}{lllll}
\hline $\begin{array}{l}\text { Outcome or subgroup ti- } \\
\text { tle }\end{array}$ & No. of studies & $\begin{array}{l}\text { No. of partici- } \\
\text { pants }\end{array}$ & Statistical method & Effect size \\
\hline 1 Leaving the study early & 1 & & Risk Ratio (M-H, Fixed, 95\% Cl) & Subtotals only \\
\hline 1.1 medium term & 1 & 164 & Risk Ratio (M-H, Fixed, 95\% Cl) & $0.62[0.42,0.91]$ \\
\hline 1.2 long term & 1 & 164 & Risk Ratio (M-H, Fixed, 95\% Cl) & $0.89[0.73,1.09]$ \\
\hline
\end{tabular}

Analysis 8.1. Comparison 8 SUPPORTIVE THERAPY versus PSYCHODYNAMIC PSYCHOTHERAPY, Outcome 1 Leaving the study early.

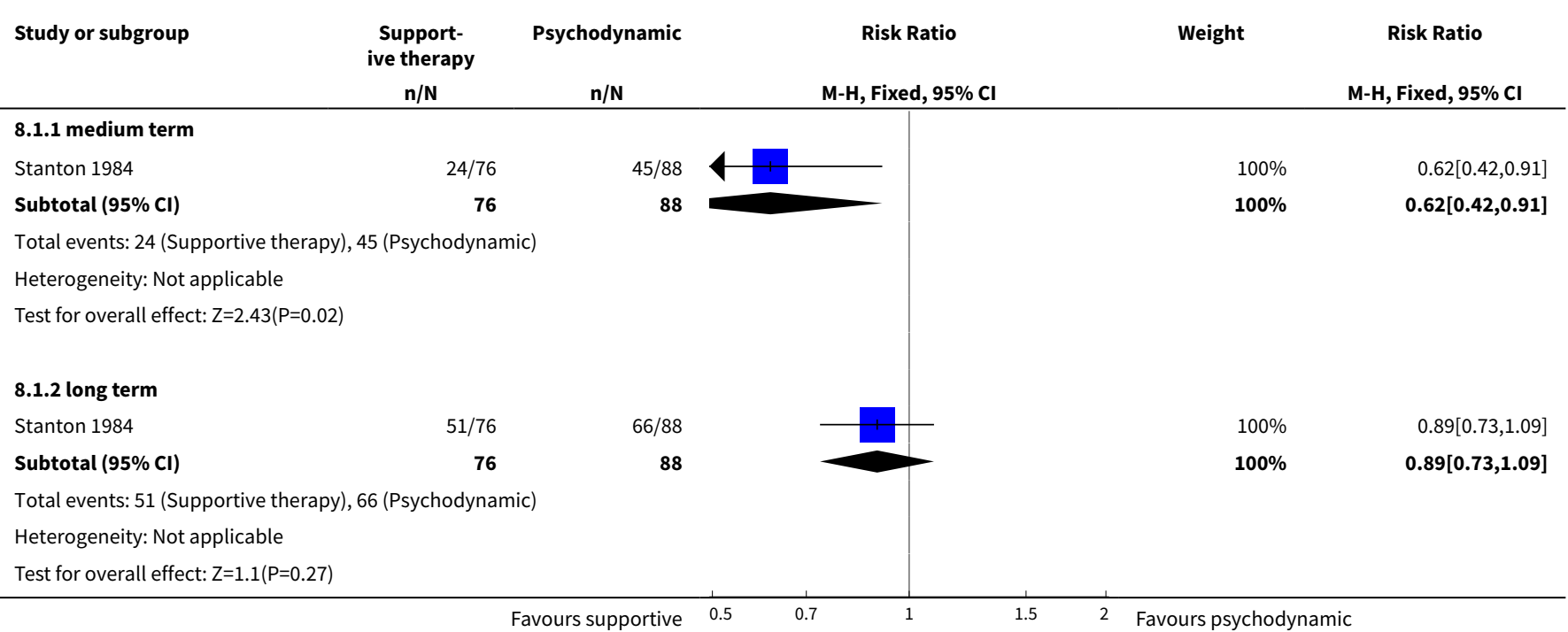


Comparison 9. SUPPORTIVE THERAPY verSUS COMBINATION OF OTHER PSYCHOSOCIAL INTERVENTIONS

\begin{tabular}{|c|c|c|c|c|}
\hline Outcome or subgroup title & No. of studies & $\begin{array}{l}\text { No. of partici- } \\
\text { pants }\end{array}$ & Statistical method & Effect size \\
\hline 1 Global state: Relapse & 1 & & Risk Ratio (M-H, Fixed, 95\% Cl) & Subtotals only \\
\hline 1.1 long term & 1 & 50 & Risk Ratio (M-H, Fixed, 95\% Cl) & $1.48[0.86,2.55]$ \\
\hline $\begin{array}{l}2 \text { Mental state: Episode of affec- } \\
\text { tive symptoms }\end{array}$ & 1 & & Risk Ratio (M-H, Fixed, 95\% Cl) & Subtotals only \\
\hline 2.1 long term & 1 & 50 & Risk Ratio (M-H, Fixed, 95\% Cl) & $1.63[0.81,3.28]$ \\
\hline $\begin{array}{l}3 \text { Leaving the study early: Treat- } \\
\text { ment-related reasons }\end{array}$ & 1 & 50 & Risk Ratio (M-H, Fixed, 95\% Cl) & $8.67[1.17,64.26]$ \\
\hline
\end{tabular}

Analysis 9.1. Comparison 9 SUPPORTIVE THERAPY versus COMBINATION OF OTHER PSYCHOSOCIAL INTERVENTIONS, Outcome 1 Global state: Relapse.

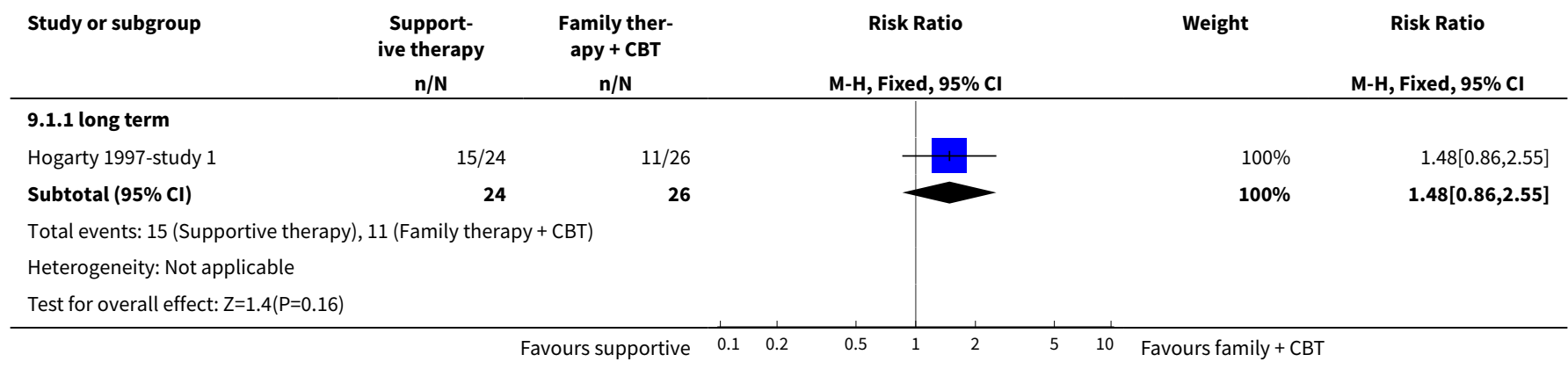

Analysis 9.2. Comparison 9 SUPPORTIVE THERAPY versus COMBINATION OF OTHER PSYCHOSOCIAL INTERVENTIONS, Outcome 2 Mental state: Episode of affective symptoms.

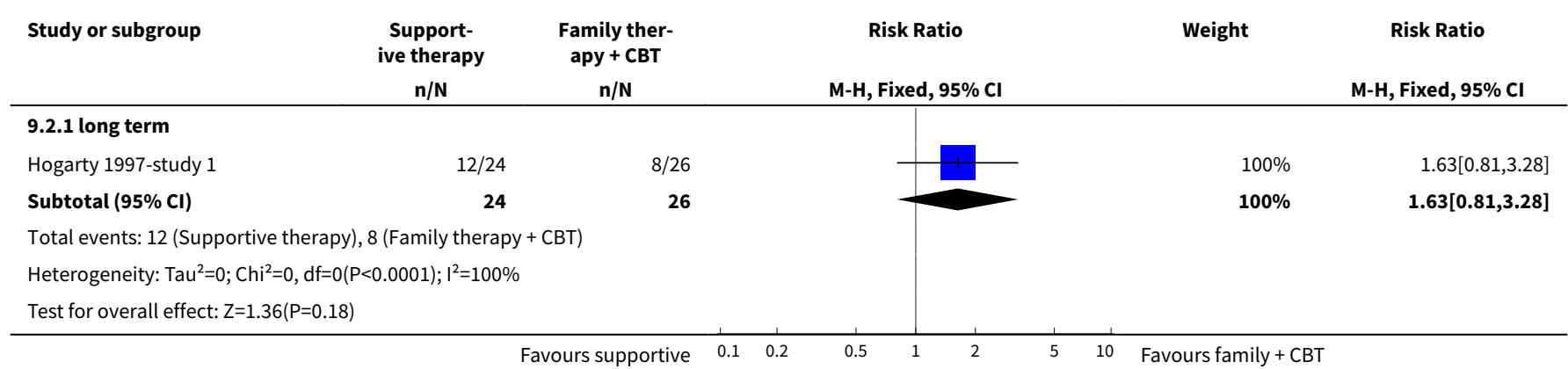


Analysis 9.3. Comparison 9 SUPPORTIVE THERAPY versus COMBINATION OF OTHER

PSYCHOSOCIAL INTERVENTIONS, Outcome 3 Leaving the study early: Treatment-related reasons.

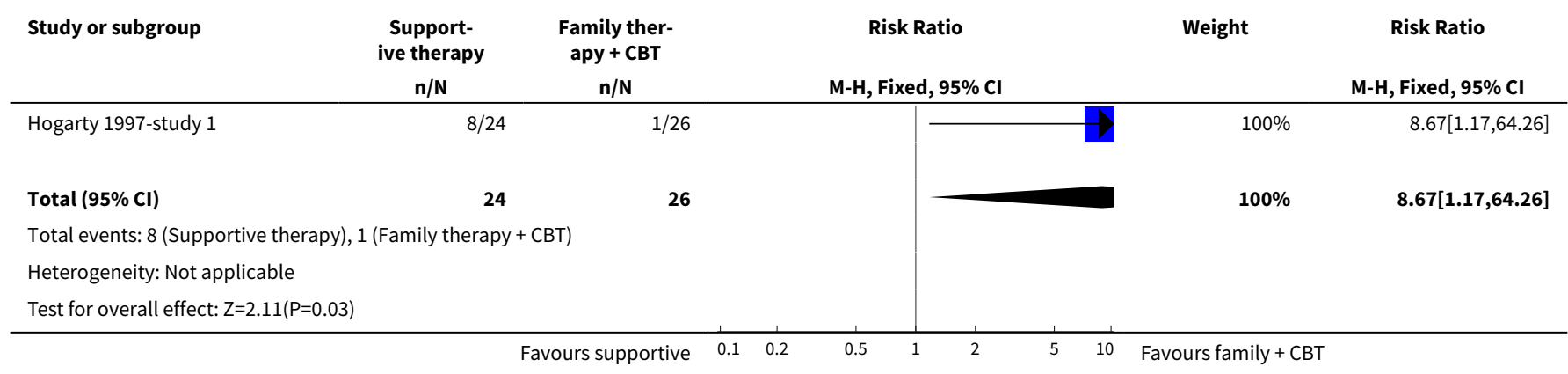

\section{Comparison 10. SUPPORTIVE THERAPY PLUS CLIENT-FOCUSED CASE MANAGEMENT versus CLIENT-FOCUSED CASE MANAGEMENT}

\begin{tabular}{lllll}
\hline $\begin{array}{l}\text { Outcome or subgroup } \\
\text { title }\end{array}$ & No. of studies & $\begin{array}{l}\text { No. of partici- } \\
\text { pants }\end{array}$ & Statistical method & Effect size \\
\hline 1 Global state: Relapse & 1 & & Risk Ratio (M-H, Fixed, 95\% Cl) & Subtotals only \\
\hline 1.1 long term & 1 & 61 & Risk Ratio (M-H, Fixed, 95\% Cl) & $0.32[0.05,2.14]$ \\
\hline 2 Leaving the study early & 2 & 145 & Risk Ratio (M-H, Fixed, $95 \% \mathrm{Cl})$ & $2.38[1.15,4.93]$ \\
\hline 3 Death & 1 & Risk Ratio (M-H, Fixed, 95\% Cl) & Subtotals only \\
\hline 3.1 long term & 1 & Risk Ratio (M-H, Fixed, 95\% Cl) & $2.61[0.11,62.26]$
\end{tabular}

Analysis 10.1. Comparison 10 SUPPORTIVE THERAPY PLUS CLIENT-FOCUSED CASE MANAGEMENT versus CLIENT-FOCUSED CASE MANAGEMENT, Outcome $1 \mathrm{Global}$ state: Relapse.

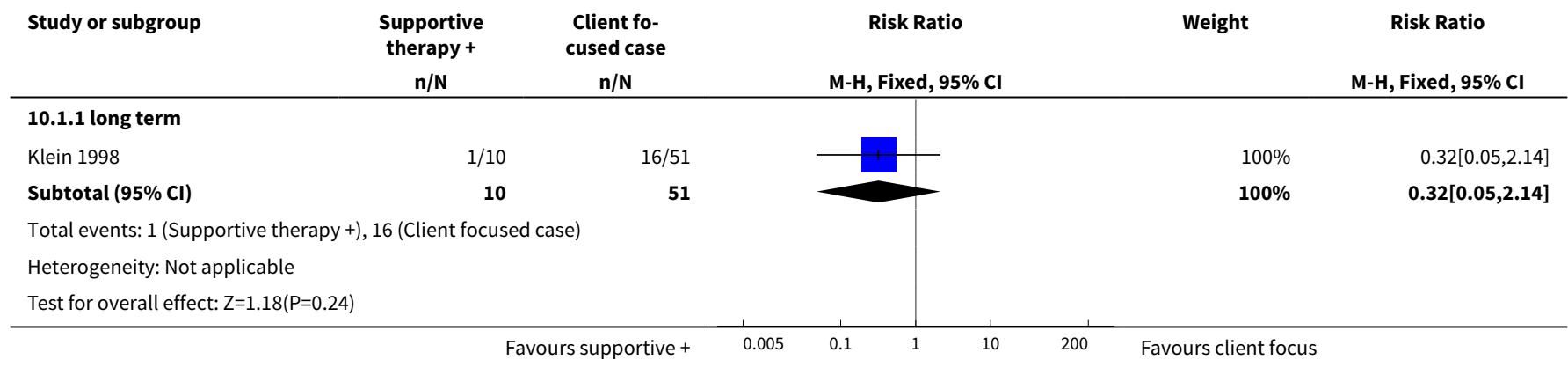


Analysis 10.2. Comparison 10 SUPPORTIVE THERAPY PLUS CLIENT-FOCUSED CASE MANAGEMENT versus CLIENT-FOCUSED CASE MANAGEMENT, Outcome 2 Leaving the study early.

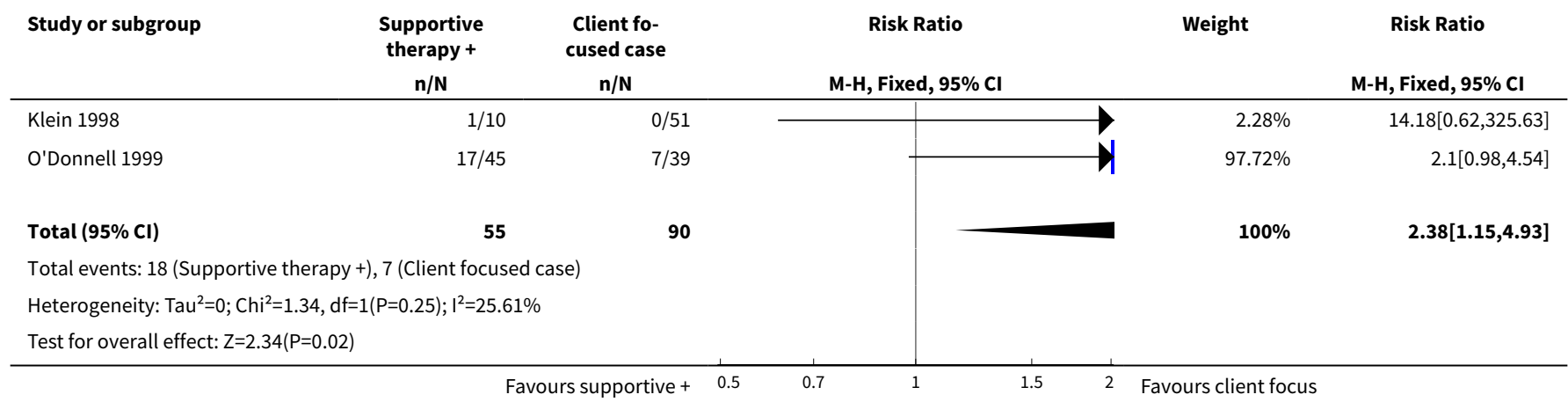

Analysis 10.3. Comparison 10 SUPPORTIVE THERAPY PLUS CLIENT-FOCUSED CASE MANAGEMENT versus CLIENT-FOCUSED CASE MANAGEMENT, Outcome 3 Death.

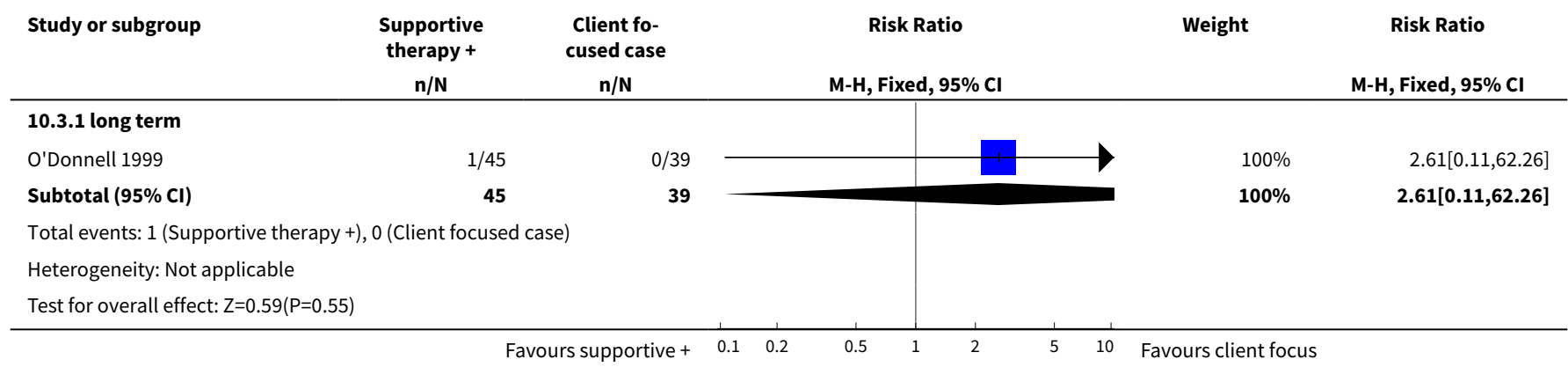

Comparison 11. SUPPORTIVE THERAPY PLUS CLIENT-FOCUSED CASE MANAGEMENT versUS STANDARD CASE MANGEMENT

\begin{tabular}{lllll}
\hline $\begin{array}{l}\text { Outcome or subgroup } \\
\text { title }\end{array}$ & No. of studies & $\begin{array}{l}\text { No. of partici- } \\
\text { pants }\end{array}$ & Statistical method & Effect size \\
\hline 1 Leaving the study early & 1 & 80 & Risk Ratio (M-H, Fixed, 95\% Cl) & $0.88[0.52,1.51]$ \\
\hline 2 Death & 1 & Risk Ratio (M-H, Fixed, 95\% Cl) & Subtotals only \\
\hline 2.1 long term & 1 & 80 & Risk Ratio (M-H, Fixed, 95\% Cl) & $2.35[0.10,55.94]$ \\
\hline
\end{tabular}

Analysis 11.1. Comparison 11 SUPPORTIVE THERAPY PLUS CLIENT-FOCUSED CASE MANAGEMENT versus STANDARD CASE MANGEMENT, Outcome 1 Leaving the study early.

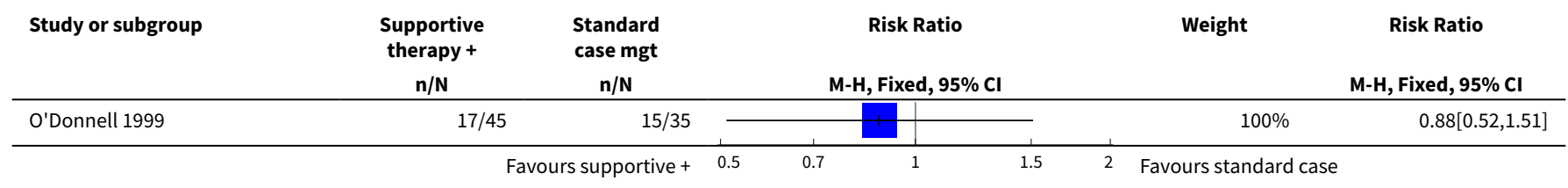




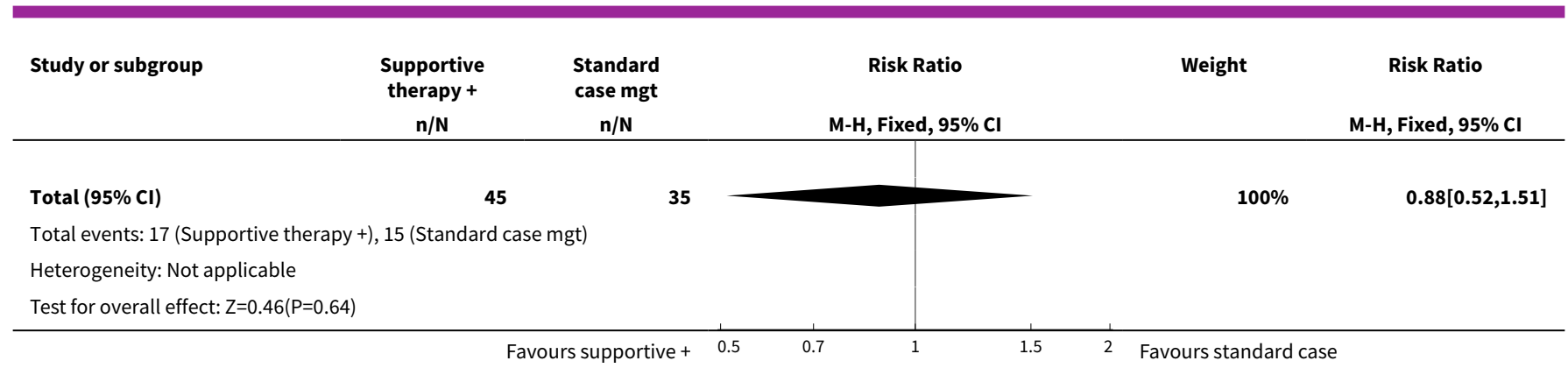

Analysis 11.2. Comparison 11 SUPPORTIVE THERAPY PLUS CLIENT-FOCUSED CASE MANAGEMENT versus STANDARD CASE MANGEMENT, Outcome 2 Death.

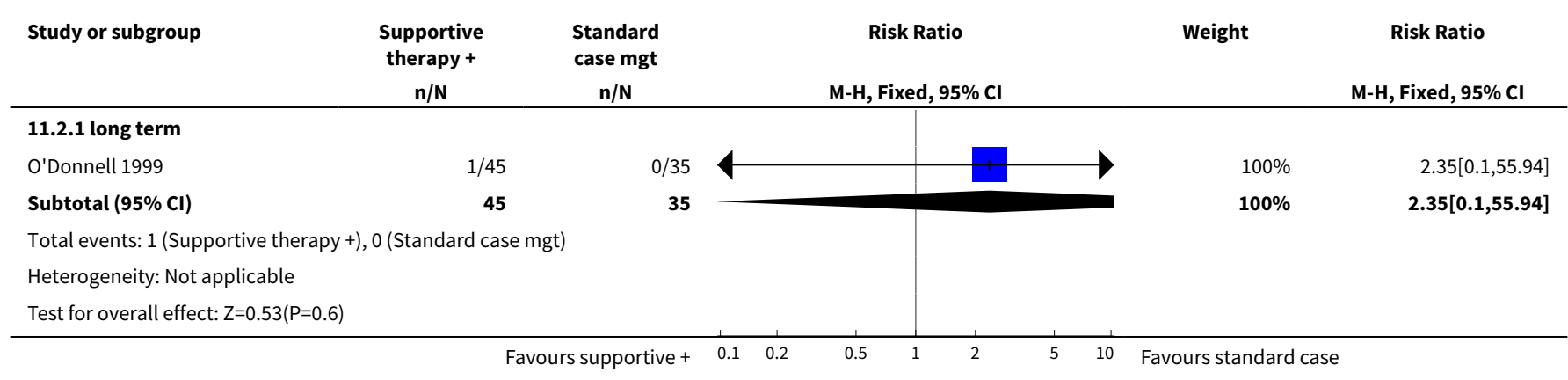

\section{Comparison 12. SUPPORTIVE THERAPY PLUS SKILLS TRAINING versus SKILLS TRAINING}

\begin{tabular}{|c|c|c|c|c|}
\hline Outcome or subgroup title & No. of studies & $\begin{array}{l}\text { No. of partici- } \\
\text { pants }\end{array}$ & Statistical method & Effect size \\
\hline 1 Global state: 1 . Relapse & 1 & 80 & $\begin{array}{l}\text { Risk Ratio (M-H, Fixed, } \\
95 \% \mathrm{Cl})\end{array}$ & $1.0[0.49,2.04]$ \\
\hline 2 Global state: 2 . No remission & 1 & & $\begin{array}{l}\text { Risk Ratio (M-H, Fixed, } \\
95 \% \mathrm{Cl})\end{array}$ & Subtotals only \\
\hline 2.1 long term & 1 & 80 & $\begin{array}{l}\text { Risk Ratio (M-H, Fixed, } \\
95 \% \mathrm{Cl})\end{array}$ & $0.78[0.54,1.12]$ \\
\hline $\begin{array}{l}3 \text { Service outcomes: Not discharged } \\
\text { from hospital }\end{array}$ & 1 & 80 & $\begin{array}{l}\text { Risk Ratio (M-H, Fixed, } \\
95 \% \mathrm{Cl})\end{array}$ & $1.14[0.46,2.85]$ \\
\hline $\begin{array}{l}4 \text { Mental state: } 1 \text {. Average endpoint } \\
\text { score for 'inability to feel' (CPRS, high = } \\
\text { poor) }\end{array}$ & 1 & & $\begin{array}{l}\text { Mean Difference (IV, Fixed, } \\
95 \% \mathrm{CI})\end{array}$ & Subtotals only \\
\hline 4.1 long term & 1 & 80 & $\begin{array}{l}\text { Mean Difference (IV, Fixed, } \\
95 \% \mathrm{CI})\end{array}$ & $0.10[-0.08,0.28]$ \\
\hline $\begin{array}{l}5 \text { Mental state: } 2 \text {. Average endpoint } \\
\text { score for 'derealisation' (CPRS, skewed } \\
\text { data, high = poor) }\end{array}$ & & & Other data & No numeric data \\
\hline
\end{tabular}




\begin{tabular}{|c|c|c|c|c|}
\hline Outcome or subgroup title & No. of studies & $\begin{array}{l}\text { No. of partici- } \\
\text { pants }\end{array}$ & Statistical method & Effect size \\
\hline 5.1 long term & & & Other data & No numeric data \\
\hline 6 Leaving the study early & 1 & 80 & $\begin{array}{l}\text { Risk Ratio (M-H, Fixed, } \\
95 \% \mathrm{Cl})\end{array}$ & $1.0[0.35,2.84]$ \\
\hline $\begin{array}{l}7 \text { General functioning: } 1 \text {. Average end- } \\
\text { point score for 'free time activities' (KAS, } \\
\text { high = good) }\end{array}$ & 1 & & $\begin{array}{l}\text { Mean Difference (IV, Fixed, } \\
95 \% \mathrm{CI})\end{array}$ & Subtotals only \\
\hline 7.1 long term & 1 & 80 & $\begin{array}{l}\text { Mean Difference (IV, Fixed, } \\
95 \% \mathrm{CI} \text { ) }\end{array}$ & $0.10[0.02,0.18]$ \\
\hline $\begin{array}{l}8 \text { General functioning: } 2 \text {. Average end- } \\
\text { point score for 'withdrawal' (KAS, } \\
\text { skewed data, high = good) }\end{array}$ & & & Other data & No numeric data \\
\hline 8.1 long term & & & Other data & No numeric data \\
\hline 9 Death & 1 & 80 & $\begin{array}{l}\text { Risk Ratio (M-H, Fixed, } \\
95 \% \mathrm{Cl})\end{array}$ & $2.0[0.19,21.18]$ \\
\hline
\end{tabular}

Analysis 12.1. Comparison 12 SUPPORTIVE THERAPY PLUS SKILLS TRAINING versus SKILLS TRAINING, Outcome 1 Global state: 1 . Relapse.

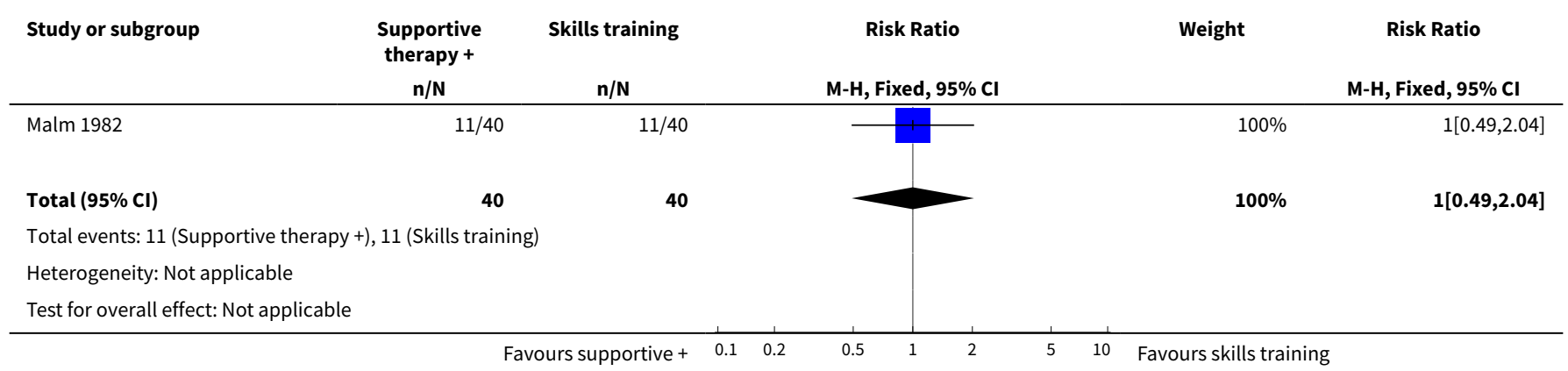

Analysis 12.2. Comparison 12 SUPPORTIVE THERAPY PLUS SKILLS TRAINING versus SKILLS TRAINING, Outcome 2 Global state: 2. No remission.

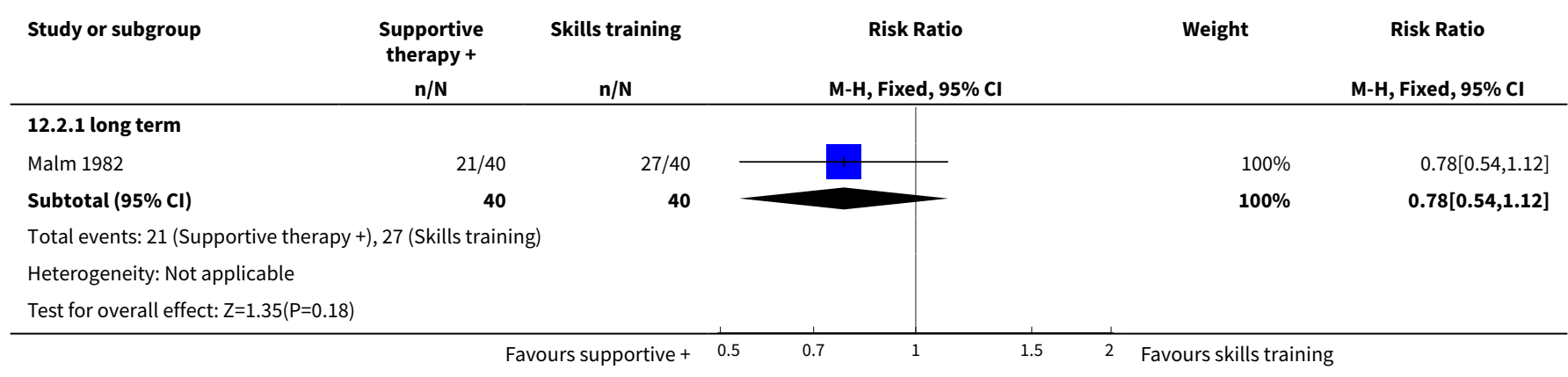


Analysis 12.3. Comparison 12 SUPPORTIVE THERAPY PLUS SKILLS TRAINING versus SKILLS TRAINING, Outcome 3 Service outcomes: Not discharged from hospital.

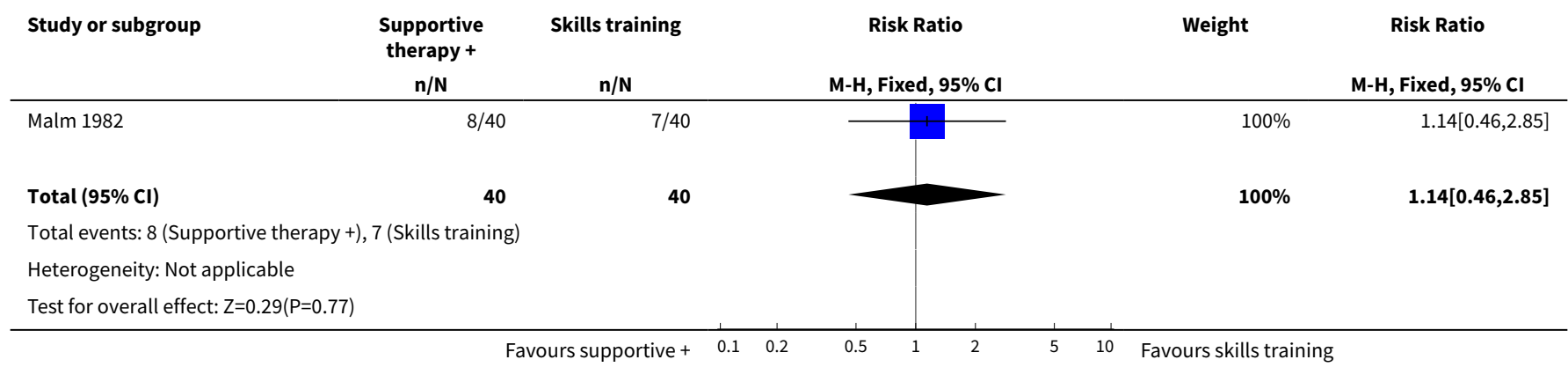

Analysis 12.4. Comparison 12 SUPPORTIVE THERAPY PLUS SKILLS TRAINING versus SKILLS TRAINING, Outcome 4 Mental state: 1. Average endpoint score for 'inability to feel' (CPRS, high = poor).

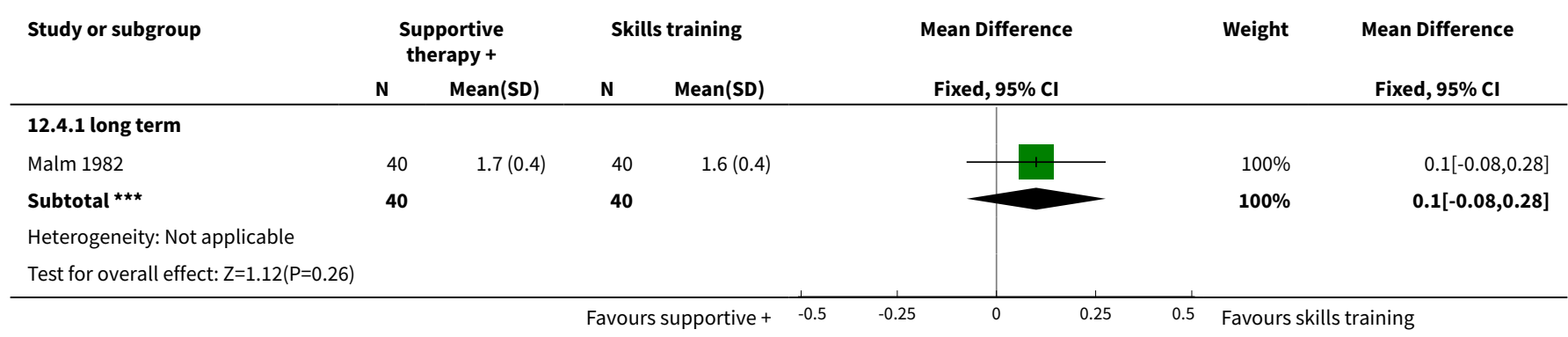

Analysis 12.5. Comparison 12 SUPPORTIVE THERAPY PLUS SKILLS TRAINING versus SKILLS TRAINING, Outcome 5 Mental state: 2. Average endpoint score for 'derealisation' (CPRS, skewed data, high = poor).

\begin{tabular}{|c|c|c|c|c|c|}
\hline \multicolumn{6}{|c|}{ Mental state: 2. Average endpoint score for 'derealisation' (CPRS, skewed data, high = poor) } \\
\hline Study & Intervention & & $\mathbf{N}$ & Mean & SD \\
\hline \multicolumn{6}{|c|}{ long term } \\
\hline Malm 1982 & $\begin{array}{l}\text { Supportive therapy + skills } \\
\text { training }\end{array}$ & 40 & & 0.5 & 0.27 \\
\hline Malm 1982 & Skills training & 40 & & 0.7 & 0.27 \\
\hline
\end{tabular}

Analysis 12.6. Comparison 12 SUPPORTIVE THERAPY PLUS SKILLS TRAINING versus SKILLS TRAINING, Outcome 6 Leaving the study early.

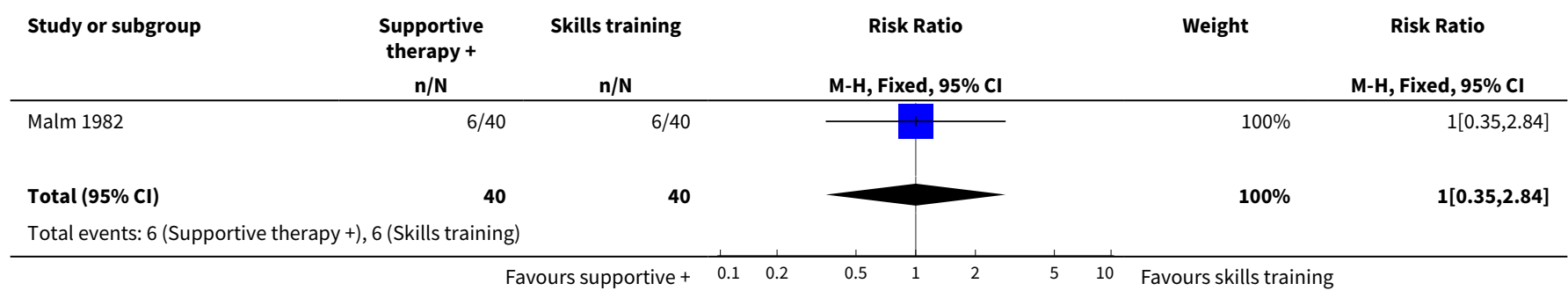




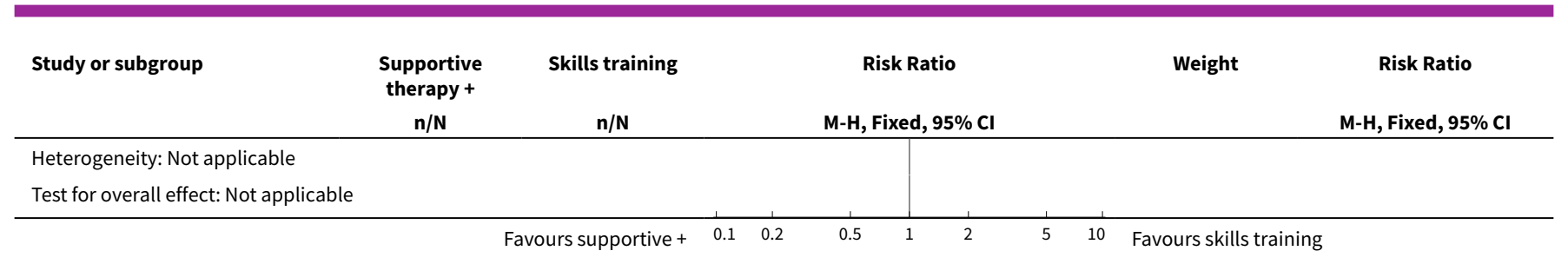

Analysis 12.7. Comparison 12 SUPPORTIVE THERAPY PLUS SKILLS TRAINING versus SKILLS TRAINING, Outcome 7 General functioning: 1. Average endpoint score for 'free time activities' (KAS, high = good).

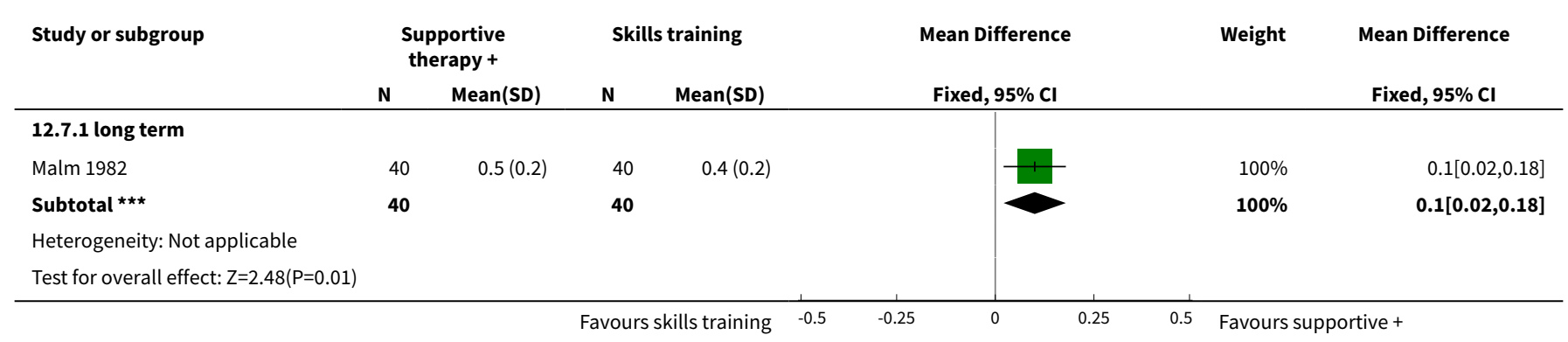

Analysis 12.8. Comparison 12 SUPPORTIVE THERAPY PLUS SKILLS TRAINING versus SKILLS TRAINING, Outcome 8 General functioning: 2. Average endpoint score for 'withdrawal' (KAS, skewed data, high = good).

\begin{tabular}{|c|c|c|c|c|c|}
\hline \multicolumn{6}{|c|}{ General functioning: 2 . Average endpoint score for 'withdrawal' (KAS, skewed data, high = good) } \\
\hline Study & Intervention & & $\mathbf{N}$ & Mean & SD \\
\hline \multicolumn{6}{|c|}{ long term } \\
\hline Malm 1982 & $\begin{array}{l}\text { Supportive therapy + skills } \\
\text { training }\end{array}$ & 40 & & 0.7 & 0.45 \\
\hline Malm 1982 & Skills training & 40 & & 0.5 & 0.45 \\
\hline
\end{tabular}

Analysis 12.9. Comparison 12 SUPPORTIVE THERAPY PLUS SKILLS TRAINING versus SKILLS TRAINING, Outcome 9 Death.

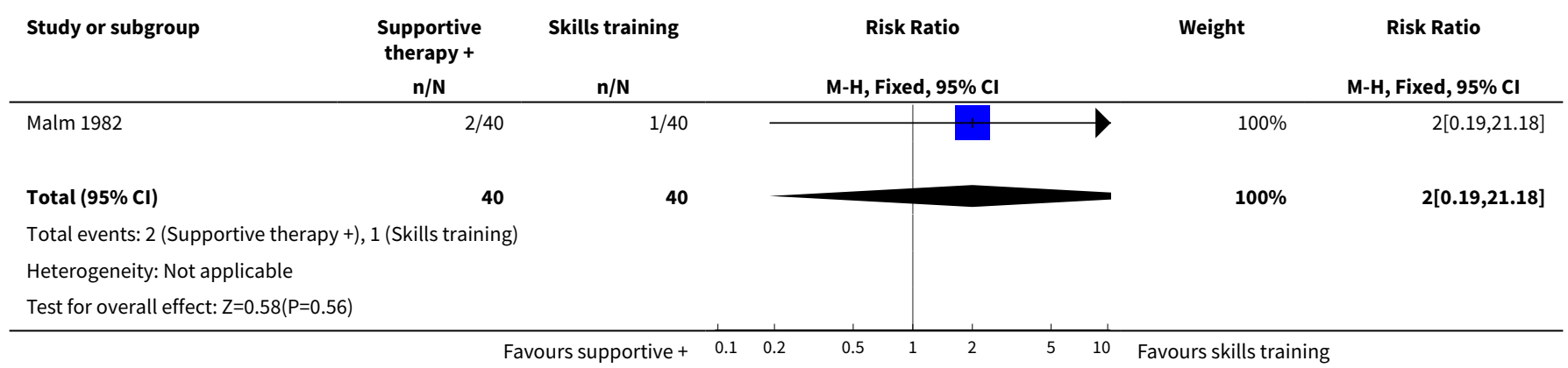

\section{ADDITIONAL TABLES}




\section{Table 1. Design of a future study}

\section{Methods}

Allocation: centralised sequence generation with table of random numbers or computer-generated code, stratified by severity of illness, sequence concealed till interventions assigned.

Blinding: those recruiting and assigning participants, those assessing outcomes, all blind to allocated group, blinding could be tested.

Duration: minimum of 24 weeks.

\section{Participants}

Diagnosis: schizophrenia, if operational criteria used these should be in the context of routine care. $\mathrm{N}=450^{*}$.

Age: adults.

Sex: men and women.

Setting: anywhere.

\section{Interventions}

1. Supportive therapy: the nature and frequency of this approach should be clearly described. $\mathrm{N}=$ 150.

2. Routine therapy. $N=150$. This also should be clearly described.

3. Cognitive behavioural therapy. $\mathrm{N}=150$.

\section{Outcomes}

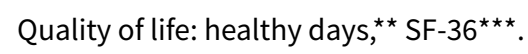

Service outcomes: days in hospital, time attending psychiatric outpatient clinic.

Satisfaction with care: patients/carers.

Global state: CGI.**

Mental state: $\mathrm{CGI}$.

Social functioning: to include occupational status.

Adverse effects: including mortality.

Economic data.

Notes

${ }^{*}$ size of study to detect a $10 \%$ difference in improvement with $80 \%$ certainty.

** Primary outcome.

*** If scales are used to measure outcome then there should be binary cut off points, defined before study starts, of clinically important improvement.

$\mathrm{N}=$ number of participants, $\mathrm{BPRS}=$ Brief Psychiatric Rating Scale, $\mathrm{CGI}$ = Clinical Global Impressions Scale

\section{AP PEN DICES}

\section{Appendix 1. Previous searches}

1. Electronic searches

We searched the Cochrane Schizophrenia Group's study-based register (January 2004) using the phrase:

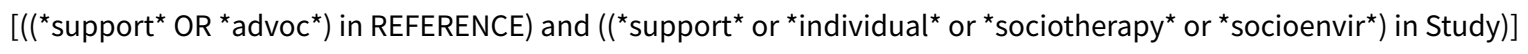

This register is compiled by systematic searches of major databases, hand searches and conference proceedings (see Group Module).

2. Reference searching

We inspected reference lists of all identified studies (included or excluded) for more studies.

\section{Personal contact}

We contacted authors of relevant reviews or studies for other sources of relevant information.

\section{Appendix 2. Previous data analysis methods}

\section{Selection of trials}

We (LB, TP) independently inspected the abstracts of all citations identified from the search. We identified potentially relevant abstracts and ordered full papers and reassessed these for inclusion and methodological quality. We resolved any disagreement by discussion and reported the outcome. 
2. Assessment of quality

We (LB, TP) independently allocated trials to three quality categories, as described in the Cochrane Collaboration Handbook (Higgins 2005), which is based on the evidence of a strong relationship between allocation concealment and direction of effect (Schulz 1995). Where disagreement arose as to which category a trial should be allocated, we attempted to resolve this by discussion. If doubt remained we added the study to the list of trials awaiting assessment until further information could be obtained. Only trials in Category A or B were included in the review.

A. Low risk of bias (adequate allocation concealment)

B. Moderate risk of bias (some doubt about the results)

C. High risk of bias (inadequate allocation concealment).

For the purpose of the analysis in this review, trials were included if they met the Cochrane Handbook criteria A or B.

3. Data management

3.1 Data extraction

We (LB, TP) independently extracted data and contacted authors' of trials to provide missing data.

\subsection{Intention-to-treat analysis}

We excluded data from studies where more than $50 \%$ of participants in any group were lost to follow-up (this did not include the outcome of 'leaving the study early'). In studies with less than 50\% dropout rate, people leaving early were considered to have had the negative outcome, except for the event of death. The impact of including studies with high attrition rates (25\% to $50 \%$ ) were analysed in a sensitivity analysis. If inclusion of data from this latter group did result in a substantive change in the estimate of effect, their data were not added to trials with less attrition, but were presented separately.

4. Data analysis

4.1 Binary data

For binary outcomes we calculated the relative risk (RR) and its $95 \%$ confidence interval (Cl). The number needed to treat statistic (NNT) and number needed to harm (NNH) were also calculated. If data were heterogeneous (see section 5) we used a random-effects model.

\subsection{Continuous data}

4.2.1 Skewed data: continuous data on clinical and social outcomes are often not normally distributed. To avoid the pitfall of applying parametric tests to non-parametric data the following standards were applied to all data before inclusion: (a) standard deviations and means were reported in the paper or obtainable from the authors, (b) when a scale started from a finite number (such as zero), the standard deviation, when multiplied by two, should be less than the mean (as otherwise the mean was unlikely to be an appropriate measure of the centre of the distribution (Altman 1996)). Endpoint scores on scales often have a finite start and end point and this rule can be applied to them.

4.2.2 Summary statistic: for continuous outcomes we estimated the weighted mean difference (WMD) between groups. Again, if data were heterogeneous (see section 5) we used a random effects model. In circumstances where trials assessed the same outcome but measured it in a variety of ways, then we analysed data using the standardised mean difference (SMD).

4.2.3 Valid scales: Unpublished scales are known to be subject to bias in trials of treatments for schizophrenia (Marshall 2000) therefore, we only included continuous data from rating scales were if the measuring instrument had been described in a peer-reviewed journal and the instrument was either a self report or completed by an independent rater or relative (not the therapist).

4.2.4 Endpoint versus change data: where possible we presented endpoint data and if both endpoint and change data were available for the same outcomes then we only reported the former in this review.

4.2.5 Cluster trials: studies increasingly employ 'cluster randomisation' (such as randomisation by clinician or practice) but analysis and pooling of clustered data poses problems. Authors often fail to account for intra class correlation in clustered studies, leading to a 'unit of analysis' error (Divine 1992) whereby $\mathrm{p}$ values are spuriously low, confidence intervals unduly narrow and statistical significance overestimated causing type I errors (Bland 1997, Gulliford 1999).

Where clustering was not accounted for in primary studies, we presented the data in a table, with a $\left({ }^{\star}\right)$ symbol to indicate the presence of a probable unit of analysis error. In subsequent versions of this review we will seek to contact first authors of studies to obtain intra class correlation co-efficients of their clustered data, and to adjust for this using accepted methods (Gulliford 1999). Where clustering has been incorporated into the analysis of primary studies, we will also present these data as if from a non-cluster randomised study, but adjusted for the clustering effect.

We have sought statistical advice and have been advised that the binary data as presented in a report should be divided by a 'design effect'. This is calculated using the mean number of participants per cluster $(\mathrm{m})$ and the intraclass correlation co-efficient $($ ICC) [Design effect $=$ $\left.1+(m-1)^{\star} I C C\right]$ (Donner 2002). If the ICC was not reported, it was assumed to be 0.1 (Ukoumunne 1999). 
Where cluster studies were appropriately analysed taking into account intra-class correlation coefficients and relevant data documented in the report, synthesis with other studies was possible using the generic inverse variance technique.

\section{Investigation for heterogeneity}

Firstly, we considered all included studies within any comparison to judge clinical heterogeneity. We also inspected the graphs to investigate the possibility of statistical heterogeneity. We supplemented this using the I-squared statistic. This provides an estimate of the percentage of variability due to heterogeneity rather than chance alone. Where the I-squared estimate is equal to or greater than $75 \%$, we interpreted this as indicating the presence of high levels of heterogeneity (Higgins 2003). We did not summate data if inconsistency were high, but presented the data separately and investigated reasons for heterogeneity.

\section{Addressing publication bias}

We had planned to enter data from all included studies into a funnel graph (trial effect against trial size) in an attempt to investigate the likelihood of overt publication bias (Davey 1997). However, we decided not to undertake funnel graphs, due to insufficient data being available to enable meaningful conclusions to be drawn.

\section{Sensitivity analyses}

We analysed the effect of including studies with high attrition rates in a sensitivity analysis.

\section{General}

Where possible, we entered data in such a way that the area to the left of the line of no effect indicated a favourable outcome for supportive therapy and supportive care.

\section{WHAT'S NEW}

\begin{tabular}{lll}
\hline Date & Event & Description \\
\hline 8 November 2017 & Amended & $\begin{array}{l}\text { New author team updating this review. It will be split into three } \\
\text { new titles, creating a 'family' of Supportive Therapy reviews. } \\
\text { New protocols to be published. }\end{array}$ \\
& & \\
\hline
\end{tabular}

\section{H IS T ORY}

Protocol first published: Issue 1, 2004

Review first published: Issue 1, 2007

\begin{tabular}{lll}
\hline Date & Event & Description \\
\hline 5 February 2015 & $\begin{array}{l}\text { New citation required but conclusions } \\
\text { have not changed }\end{array}$ & $\begin{array}{l}\text { Results from 2012 search added to review, four new studies } \\
\text { added. No new conclusions. }\end{array}$ \\
\hline 28 November 2012 & New search has been performed & 2012 update search run 58 new references found. \\
\hline 25 April 2008 & Amended & Converted to new review format. \\
\hline 27 April 2007 & $\begin{array}{l}\text { New citation required and conclusions } \\
\text { have changed }\end{array}$ & Substantive amendment \\
\hline
\end{tabular}

\section{CONTRIBUTIONS OF AUTHORS}

Lucy Buckley - primary author for original version of review. Protocol development, main author of text of review, quality rating and selection of studies, data extraction, data entry and analysis using RevMan.

Nicola Maayan - screening, data extraction and analysis of new studies from the 2012 search, updated the results, risk of bias of all studies and 'Summary of findings' tables. 
Karla Soares-Weiser - screening, data extraction and analysis of new studies from the 2012 search, contributed to 'Summary of findings' tables.

Clive Adams - protocol development, contributed to text of review, provided advice and motivation.

\section{DECLARATIONS OF INTEREST}

Lucy Buckley - none known.

Karla Soares-Weiser and Nicola Maayan - work for Enhance Review Ltd, a company that carries out systematic reviews mostly for the public sector. We currently do not provide services for the pharmaceutical industry.

Clive Adams - none known.

\section{SOURCES OF SUPPORT}

\section{Internal sources}

- Affinity Healthcare, Cheadle Royal Hospital, UK.

\section{External sources}

- Leeds Community and Mental Health Services, NHS Teaching Trust, UK.

- Northumberland Tyne and Wear NHS Trust, UK.

\section{DIFFERENCES BETWEEN PROTOCOLANDREVIEW}

The new methods template includes new methodology now required for Cochrane reviews, as such the methods section includes updates and differences from the original protocol.

The outcomes related to medication (insight, compliance, change in medication and attitude to medication) have been added to the included outcomes.

\section{INDEX TERMS}

\section{Medical Subject Headings (MeSH)}

Antipsychotic Agents [therapeutic use]; Family Therapy; Intention to Treat Analysis; Mental Health Services; Patient Satisfaction; Psychotherapy [methods]; Randomized Controlled Trials as Topic; Schizophrenia [therapy]; Schizophrenic Psychology; Social Support

\section{MeSH check words}

Humans 